\title{
Statistical Assessment of the Significance of Fracture Fits in Trace Evidence
}

\author{
Evie K. Brooks \\ West Virginia University, ekb0011@mix.wvu.edu
}

Follow this and additional works at: https://researchrepository.wvu.edu/etd

Part of the Forensic Science and Technology Commons

\section{Recommended Citation}

Brooks, Evie K., "Statistical Assessment of the Significance of Fracture Fits in Trace Evidence" (2020). Graduate Theses, Dissertations, and Problem Reports. 7704.

https://researchrepository.wvu.edu/etd/7704

This Thesis is protected by copyright and/or related rights. It has been brought to you by the The Research Repository @ WVU with permission from the rights-holder(s). You are free to use this Thesis in any way that is permitted by the copyright and related rights legislation that applies to your use. For other uses you must obtain permission from the rights-holder(s) directly, unless additional rights are indicated by a Creative Commons license in the record and/ or on the work itself. This Thesis has been accepted for inclusion in WVU Graduate Theses, Dissertations, and Problem Reports collection by an authorized administrator of The Research Repository @ WVU. For more information, please contact researchrepository@mail.wvu.edu. 
Graduate Theses, Dissertations, and Problem Reports

2020

Statistical Assessment of the Significance of Fracture Fits in Trace Evidence

Evie K. Brooks

Follow this and additional works at: https://researchrepository.wvu.edu/etd

Part of the Forensic Science and Technology Commons 
Statistical Assessment of the Significance of Fracture Fits in Trace Evidence

Evie K. Brooks

Thesis submitted

to the Eberly College of Arts and Sciences

at West Virginia University

in partial fulfillment of the requirements for the degree of

Master of Science in

Forensic and Investigative Science

\author{
Tatiana Trejos, Ph.D., Chair \\ Keith Morris, Ph.D. \\ Andria Mehltretter, M.S.
}

Department of Forensic and Investigative Science

Morgantown, West Virginia

2020

Keywords: trace evidence, physical fit, duct tape, inter-laboratory study, textiles, X-ray

fluorescence, electrical tape

Copyright 2020 Evie K. Brooks 


\title{
ABSTRACT \\ Statistical Assessment of the Significance of Fracture Fits in Trace Evidence
}

\author{
Evie K. Brooks
}

Fracture fits are often regarded as the highest degree of association of trace materials due to the common belief that inherently random fracturing events produce individualizing patterns. Often referred to as physical matches, fracture matches, or physical fits, these assessments consist of the realignment of two or more items with distinctive features and edge morphologies to demonstrate they were once part of the same object. Separated materials may provide a valuable link between items, individuals, or locations in forensic casework in a variety of criminal situations. Physical fit examinations require the use of the examiner's judgment, which rarely can be supported by a quantifiable uncertainty or vastly reported error rates.

Therefore, there is a need to develop, validate, and standardize fracture fit examination methodology and respective interpretation protocols. This research aimed to develop systematic methods of examination and quantitative measures to assess the significance of trace evidence physical fits. This was facilitated through four main objectives: 1) an in-depth review manuscript consisting of 112 case reports, fractography studies, and quantitative-based studies to provide an organized summary establishing the current physical fit research base, 2) a pilot inter-laboratory study of a systematic, score-based technique previously developed by our research group for evaluation of duct tape physical fit pairs and referred as the Edge Similarity Score (ESS), 3) the initial expansion of ESS methodology into textile materials, and 4) an expanded optimization and evaluation study of X-ray Fluorescence (XRF) Spectroscopy for electrical tape backing analysis, for implementation in an amorphous material of which physical fits may not be feasible due to lack of distinctive features.

Objective 1 was completed through a large-scale literature review and manuscript compilation of 112 fracture fit reports and research studies. Literature was evaluated in three overall categories: case reports, fractography or qualitative-based studies, and quantitative-based studies. In addition, 12 standard operating protocols (SOP) provided by various state and federal-level forensic laboratories were reviewed to provide an assessment of current physical fit practice. A review manuscript was submitted to Forensic Science International and has been accepted for publication. This manuscript provides for the first time, a literature review of physical fits of trace materials and served as the basis for this project.

The pilot inter-laboratory study (Objective 2) consisted of three study kits, each consisting of 7 duct tape comparison pairs with a ground truth of 4 matching pairs ( 3 of expected $\mathrm{M}+$ qualifier range, 1 of the more difficult M- range) and 3 non-matching pairs (NM). The kits were distributed as a Round Robin study resulting in 16 overall participants and 112 physical fit comparisons. Prior to kit distribution, a consensus on each sample's ESS was reached between 4 examiners with an agreement criterion of better than $\pm 10 \%$ ESS. Along with the physical comparison pairs, the study 
included a brief, post-study survey allowing the distributors to receive feedback on the participants' opinions on method ease of use and practicality. No misclassifications were observed across all study kits. The majority $(86.6 \%)$ of reported ESS scores were within \pm 20 ESS compared to consensus values determined before the administration of the test. Accuracy ranged from $88 \%$ to $100 \%$, depending on the criteria used for evaluation of the error rates. In addition, on average, $77 \%$ of ESS attributed no significant differences from the respective pre-distribution, consensus mean scores when subjected to ANOVA-Dunnett's analysis using the level of difficulty as blocking variables. These differences were more often observed on sets of higher difficulty (M-, 5 out of 16 participants, or $31 \%)$ than on lower difficulty sets $(\mathrm{M}+$ or $\mathrm{M}-, 3$ out of 16 participants, or 19\%). Three main observations were derived from the participant results: 1) overall good agreement between ESS reported by examiners was observed, 2) the ESS score represented a good indicator of the quality of the match and rendered low percent of error rates on conclusions 3) those examiners that did not participate in formal method training tended to have ESS falling outside of expected pre-distribution ranges. This interlaboratory study serves as an important precedent, as it represents the largest inter-laboratory study ever reported using a quantitative assessment of physical fits of duct tapes. In addition, the study provides valuable insights to move forward with the standardization of protocols of examination and interpretation.

Objective 3 consisted of a preliminary study on the assessment of 274 total comparisons of stabbed $(\mathrm{N}=100)$ and hand-torn $(\mathrm{N}=174)$ textile pairs as completed by two examiners. The first 74 comparisons resulted in a high incidence of false exclusions (63\%) on textiles prone to distortion, revealing the need to assess suitability prior to physical fit examination of fabrics. For the remaining dataset, five clothing items were subject to fracture of various textile composition and construction. The overall set consisted of 100 comparison pairs, 20 per textile item, 10 each per separation method of stabbed or hand-torn fractured edges, each examined by two analysts. Examiners determined ESS through the analysis of 10 bins of equal divisions of the total fracture edge length. A weighted ESS was also determined with the addition of three optional weighting factors per bin due to the continuation of a pattern, separation characteristics (i.e. damage or protrusions/gaps), or partial pattern fluorescence across the fractured edges. With the addition of a weighted ESS, a rarity ratio was determined as the ratio between the weighted ESS and nonweighted ESS. In addition, the frequency of occurrence of all noted distinctive characteristics leading to the addition of a weighting factor by the examiner was determined. Overall, 93\% accuracy was observed for the hand-torn set while 95\% accuracy was observed for the stabbed set. Higher misclassification in the hand-torn set was observed in textile items of either $100 \%$ polyester composition or jersey knit construction, as higher elasticity led to greater fracture edge distortion. In addition, higher misclassification was observed in the stabbed set for those textiles of no pattern as the stabbed edges led to straight, featureless bins often only associated due to pattern continuation. The results of this study are anticipated to provide valuable knowledge for the future development of protocols for evaluation of relevant features of textile fractures and assessments of the suitability for fracture fit comparisons.

Finally, the XRF methodology optimization and evaluation study (Objective 4) expanded upon our group's previous discrimination studies by broadening the total sample set of characterized 
tapes and evaluating the use of spectral overlay, spectral contrast angle, and Quadratic Discriminant Analysis (QDA) for the comparison of XRF spectra. The expanded sample set consisted of 114 samples, 94 from different sources, and 20 from the same roll. Twenty sections from the same roll were used to assess intra-roll variability, and for each sample, replicate measurements on different locations of the tape were analyzed $(n=3)$ to assess the intra-sample variability. Inter-source variability was evaluated through 94 rolls of tapes of a variety of labeled brands, manufacturers, and product names. Parameter optimization included a comparison of atmospheric conditions, collection times, and instrumental filters. A study of the effects of adhesive and backing thickness on spectrum collection revealed key implications to the method that required modification to the sample support material Figures of merit assessed included accuracy and discrimination over time, precision, sensitivity, and selectivity. One of the most important contributions of this study is the proposal of alternative objective methods of spectral comparisons. The performance of different methods for comparing and contrasting spectra was evaluated. The optimization of this method was part of an assessment to incorporate XRF to a forensic laboratory protocol for rapid, highly informative elemental analysis of electrical tape backings and to expand examiners' casework capabilities in the circumstance that a physical fit conclusion is limited due to the amorphous nature of electrical tape backings.

Overall, this work strengthens the fracture fit research base by further developing quantitative methodologies for duct tape and textile materials and initiating widespread distribution of the technique through an inter-laboratory study to begin steps towards laboratory implementation. Additional projects established the current state of forensic physical fit to provide the foundation from which future quantitative work such as the studies presented here must grow and provided highly sensitive techniques of analysis for materials that present limited fracture fit capabilities. 


\section{ACKNOWLEDGEMENTS}

I would first like to express my appreciation to my research advisor and committee chair, Dr. Tatiana Trejos. Over the past two years the guidance, time, and commitment she has put into assisting me in my research endeavors has shaped who I am as a student, as well as the project into what it is today. I am very grateful for the support and encouragement she provided me, as well as for the academic and professional lessons she has taught me through the years.

I would also like to thank my committee members, Dr. Keith Morris and Andria Mehltretter for the support and assistance they provided throughout the project. Your insight was always appreciated and greatly furthered the progression and growth of my ideas.

In addition, I would like to specifically thank Andria for the guidance and dedication she has shown during my graduate career as well as my time as her intern. The personal and professional growth you inspired as a supervisor has broadened my path and strengthened my commitment to the field.

I am thankful to my fellow research group members for their comradery and support throughout our time together. I would also like to thank my departmental peers for the friendships that have lifted me up and helped me to navigate my time at West Virginia University.

Finally, I would like to express my gratitude to my incredible support system: my parents Jeff and Lisa, my sister Katie, my brother Grayson, and my fiancé Brandon. Thank you for the endless encouragement and unconditional love you have always shown that was only greater magnified by this experience. Everything I am I owe to you. 


\section{TABLE OF CONTENTS}

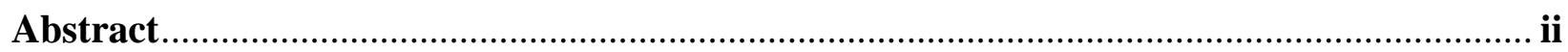

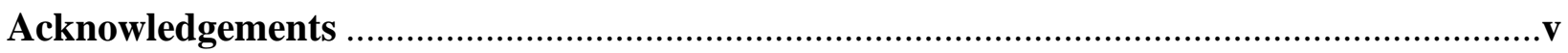

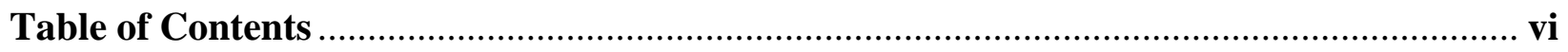

Table of Figures................................................................................................................. viii

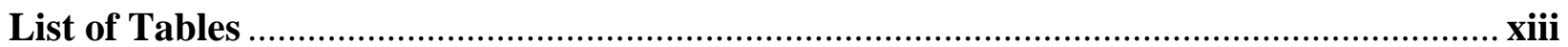

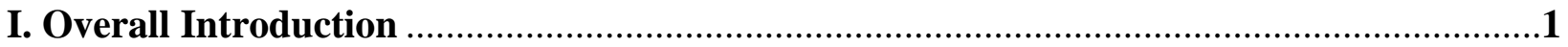

II. Chapter 1. Forensic Physical Fits in the Trace Evidence Discipline: A Review ...............5

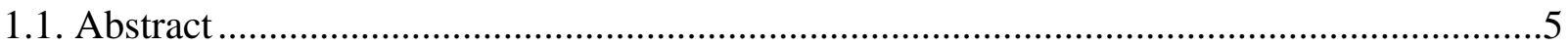

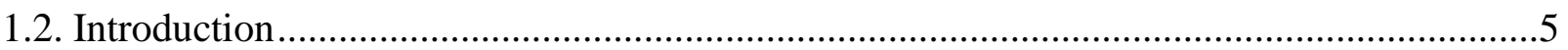

1.3. Physical Fits in Trace Evidence - Current Protocol Examples ........................................9

1.4. Established Physical Fit Research .............................................................................12

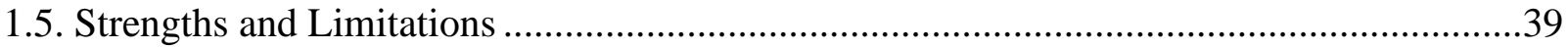

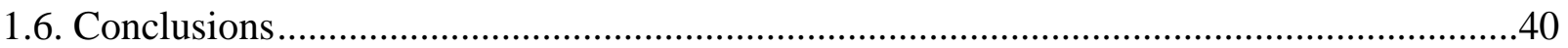

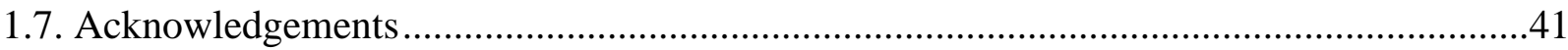

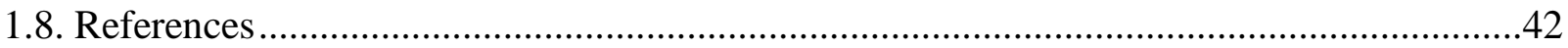

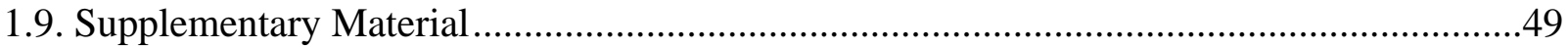

III. Chapter 2. Inter-Laboratory Assessment of the Utility of the Edge Similarity Score (ESS)

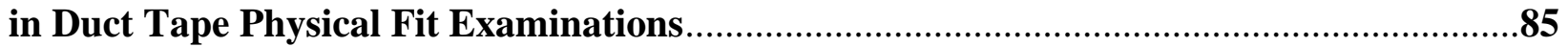

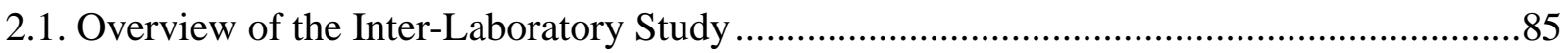

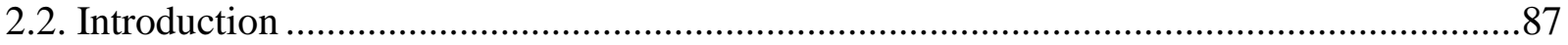

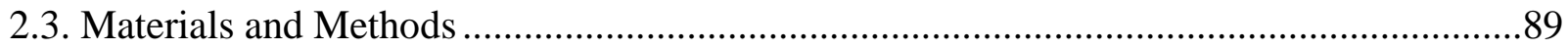

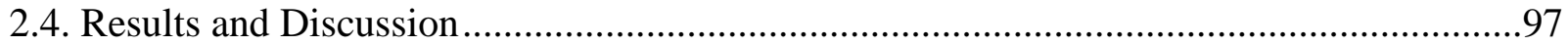

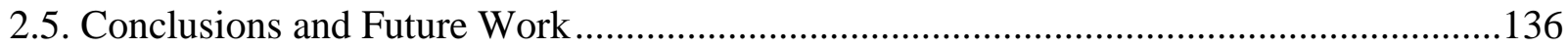

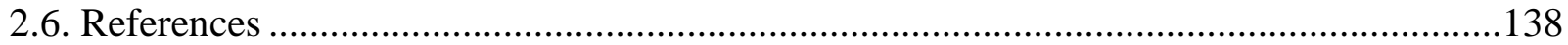

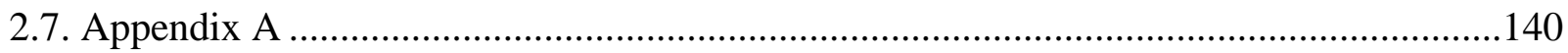

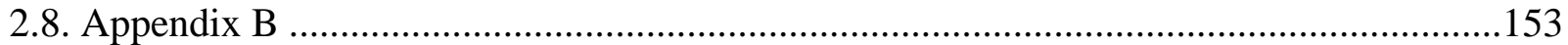

IV. Chapter 3. Steps Toward Quantitative Assessment of Textile Physical Fits - Expansion

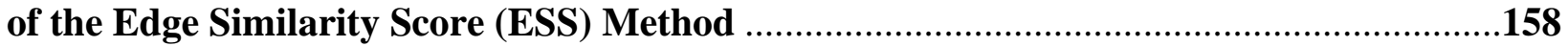

3.1. Overview of the Textile Fracture Study ..................................................................158 


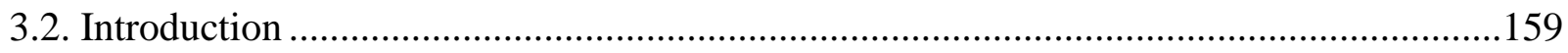

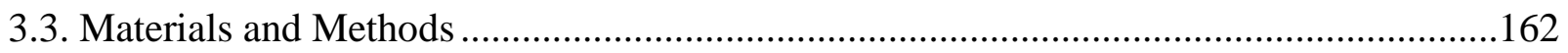

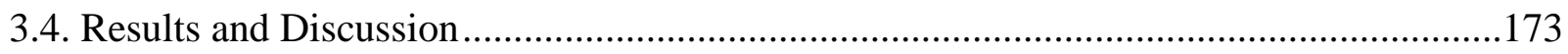

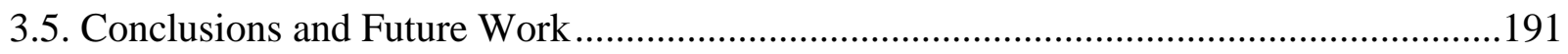

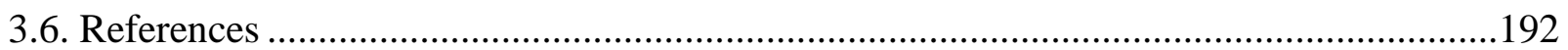

V. Chapter 4. Optimization and Evaluation of Spectral Comparisons of Electrical Tape

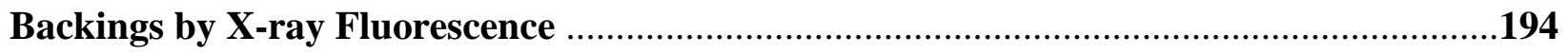

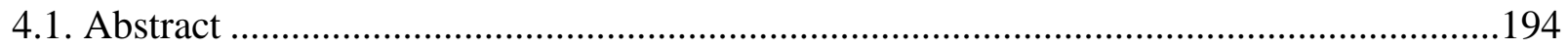

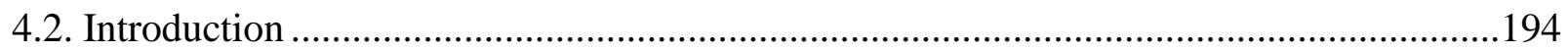

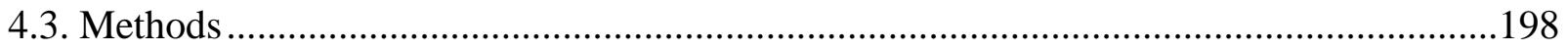

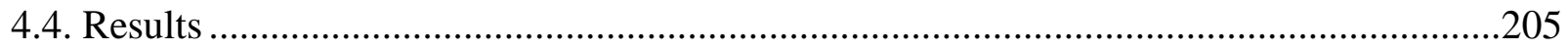

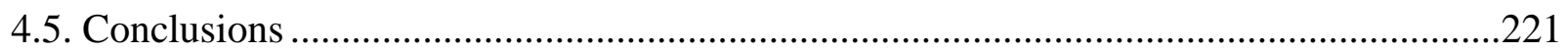

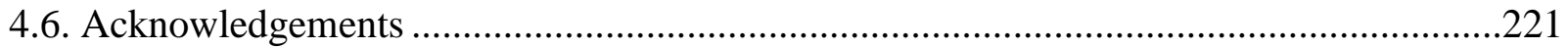

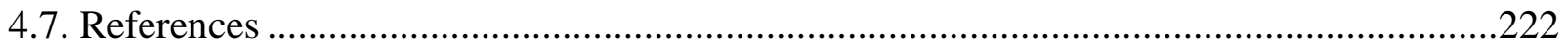

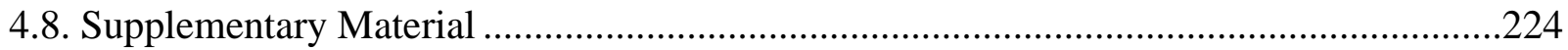

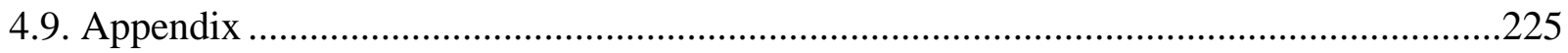

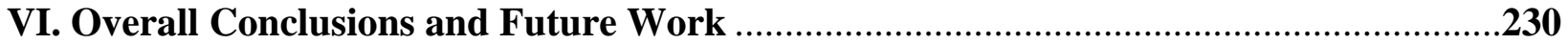

VII. Overall References (Introduction and Conclusions/Future Work Sections) ..............234 


\section{TABLE OF FIGURES}

\section{Chapter 1}

Figure 1. Reviewed physical fit literature by category and material type ( $n=79$ publications; articles discussing more than one material type are duplicated in the count of each relevant category)

\section{Chapter 2}

Figure 1. Comparison edge morphology classification for two examples of matching pairs (A and C) and one example of a non-matching pair (B)

Figure 2. Inter-laboratory modified petal test distribution

Figure 3. Backing physical feature examples: A) dimpling, B) calendering striae, C) backing distortion

Figure 4. Adhesive and scrim physical feature examples: A) warp scrim alignment/continuation of scrim pattern, B) protruding warp yarns, C) adhesive distortion, D) double weft edge scrim, E) missing scrim

Figure 5. Pre-distribution, consensus ESS values per sample per kit ( $\mathrm{N}=4$ examiners)

Figure 6. Kit 1 examiner ESS variation as compared to pre-distribution mean (consensus: $\mathrm{N}=4$ examiners)

Figure 7. Kit 2 examiner ESS variation as compared to pre-distribution mean (consensus: $N=4$ examiners)

Figure 8. Kit 3 examiner ESS variation as compared to pre-distribution mean (consensus: $N=4$ examiners)

Figure 9. Kit 1 examiner ESS variation as compared to consensus mean $\pm 20 \%$ threshold

Figure 10. Kit 2 examiner ESS variation as compared to consensus mean $\pm 20 \%$ threshold

Figure 11. Kit 3 examiner ESS variation as compared to consensus mean $\pm 20 \%$ threshold

Figure 12. Kit 1 examiner ESS variation as compared to expected comparison edge qualifier thresholds

Figure 13. Kit 2 examiner ESS variation as compared to expected comparison edge qualifier thresholds

Figure 14. Kit 3 examiner ESS variation as compared to expected comparison edge qualifier thresholds

Figure 15. Boxplot ESS distributions of inter-laboratory sample pairs grouped as $\mathrm{M}+, \mathrm{M}-$, and NM

Figure 16. Dunnett's test examiner control differences results, M+, M-, and NM samples 
Figure 17. Kit 1 ESS distribution by overall conclusion ( $\mathrm{N}=6$ examiners, $\mathrm{n}=42$ total comparisons). Numbering indicates discrepancy instances, points of discussion in which results varied from those expected.

Figure 18. Kit 1 samples, treatment of "featureless" scrim bins, red areas indicate bins marked "0" by participant

Figure 19. Kit 1 samples, treatment of distorted scrim bins, red areas indicate bins marked " 0 ” by participant

Figure 20. Kit 2 ESS distribution by overall conclusion ( $N=3$ examiners, $n=21$ total comparisons)

Figure 21. Kit 3 ESS distribution by overall conclusion ( $N=7$ examiners, $n=49$ total comparisons). Numbering indicates discrepancy instances, points of discussion in which results varied from those expected.

Figure 22. Kit 3 sample, treatment of "featureless" scrim bins, green areas indicate bins marked " 1 " by participant

Figure 23. Kit 3 sample, treatment of distorted scrim bins, green areas indicate bins marked "1" by participant

Figure 24. Kit 1 ESS distribution by qualifier ( $N=6$ examiners, $n=42$ total comparisons). Numbering indicates discrepancy instances, points of discussion in which results varied from those expected.

Figure 25. Kit 1 samples, qualifiers out of expected ranges, red areas indicate bins marked "0" by participant

Figure 26. Kit 2 ESS distribution by qualifier ( $N=3$ examiners, $n=21$ total comparisons). Numbering indicates discrepancy instances, points of discussion in which results varied from those expected.

Figure 27. Kit 2 samples, qualifiers out of expected ranges, red areas indicate bins marked " 0 " by participant while green areas indicate bins marked " 1 "

Figure 28. Comparison of Kit 2 samples assigned same ESS but different comparison edge qualifiers by same participant, red areas indicate bins marked " 0 " by participant

Figure 29. Kit 3 ESS distribution by qualifier ( $\mathrm{N}=7$ examiners, 49 total comparisons). Numbering indicates discrepancy instances, points of discussion in which results varied from those expected.

Figure 30. Comparison of Kit 3 samples assigned same ESS but different comparison edge qualifiers by same participant, green areas indicate bins marked "1" by participant

Figure 31. Comparison of Kit 3 samples assigned same ESS but different comparison edge qualifiers by same participant, red areas indicate bins marked " 0 " by participant

Figure 32. Kit 3 samples, qualifiers out of expected ranges, red areas indicate bins marked "0" by participant while green areas indicate bins marked " 1 " 
Figure 33. Overall inter-laboratory study ESS distribution

Figure 34. Prusinowski et al. ${ }^{1}$ medium quality, hand torn duct tape physical fit dataset $(\mathrm{N}=508$ comparison pairs per analyst)

\section{Chapter 2: Appendix B}

Figure i. Survey question 1 results

Figure ii. Survey question 2 results

Figure iii. Survey question 3 results

Figure iv. Survey question 4 results

Figure v. Survey question 5 results

Figure vi. Survey question 6 results

Figure vii. Survey question 7 results

Figure viii. Survey question 8 results

Figure ix. Survey question 9 results

\section{Chapter 3}

Figure 1. Foam human form fracturing substrate

Figure 2. Textile sample set experimental design schematic

Figure 3. General characteristic example - color

Figure 4. General characteristic example - fabric construction (twill weave)

Figure 5. General characteristic example - general fiber size/shape

Figure 6. General characteristic example - fiber twist ("Z" twist)

Figure 7. General characteristic example - alignment of long short threads. Note: Region highlighted indicates an area considered a distinctive characteristic (i.e. gap/protrusion)

Figure 8. General characteristic example - general fluorescence (Note: The dark square regions on the right and left image are sample labels, not a region within the fabric's pattern.)

Figure 9. Distinctive characteristic example - pattern continuation across fracture

Figure 10. Distinctive characteristic example - separation characteristics (e.g. fabric damage continuation across fracture - a "gather" or pulled thread within the fabric weave)

Figure 11. Distinctive characteristic example - separation characteristics (e.g. protrusions/gaps consistent across fracture)

Figure 12. Distinctive characteristic example - partial pattern fluorescence 
Figure 13. Edge curling in preliminary set fabric

Figure 14. Overall conclusion and comparison edge qualifier comparison between two examiners, preliminary Set A (100\% hand-torn, jersey knit polyester)

Figure 15. Preliminary textile set false negative examples

Figure 16. Item A edge morphology true match examples - a) hand-torn edges, b) stabbed edges

Figure 17. Examiner B false positive - Item D

Figure 18. Examiner B false negative - Item D

Figure 19. Examiner $B$ false negative - Item $E$

Figure 20. Examiner B inconclusive (true match sample) - Item E

Figure 21. Examiner B inconclusive (true match sample) - Item D

Figure 22. Examiner B false negative - Item D

Figure 23. Examiner B inconclusive (true non-match sample) - Item A

Figure 24. Examiner B inconclusive (true match sample) - Item B

Figure 25. Examiner A inconclusive (true match sample) - Item B

Figure 26. Hand-torn sample set ESS distribution boxplots

Figure 27. Stabbed sample set ESS distribution boxplots

Figure 28. Rarity ratio distribution - hand-torn sample set

Figure 29. Rarity ratio distribution - stabbed sample set

Figure 30. Graphical display of relative frequency of occurrence of weighting factor assignment (Note: fluorescence observations for Item B are being revisited in future work)

\section{Chapter 4}

Figure 1. Spectra overlay comparison of tape 45 run both in air (3 reps) and under vacuum (3 reps), low Zc filter

Figure 2. Spectra overlay of Be and lucite planchets, low Zc filter

Figure 3. Spectra overlay comparison of tape 33 run both with adhesive ( 3 reps) and without adhesive (3 reps), low Zc filter

Figure 4. Spectra overlay of stretched and pristine sample 12 run with the Be planchet, low Zc filter

Figure 5. $\mathrm{Ca} / \mathrm{Sb}$ low $\mathrm{Zc}$ interference and high $\mathrm{Zb}$ resolved $\mathrm{Sb}$, sample 91 
Figure 6. Comparison of ranges of contrast angle ratios variation for intra-samples (indistinguishable subgroup samples, same roll samples), and inter-samples (between groups and between subgroup samples). The inset shows a zoomed area of the plot.

Figure 7. QDA canonical plot by manufacturing origin for optimized filter overall tape data set $(\mathrm{N}=94)$

Figure 8. Spectral contrast angle intra-roll sample variation as compared to inter-group variation. 8a: Box plots of intra-roll (low Zc and high Zb and inter-group. 8b: Display of spectral contrast angle ratio for 190 comparison pairs of tape samples from the same roll.

\section{Chapter 4: Appendix}

Figure A.1. Inter-group SNR differences in present vs. absent elements: sample 65 (Pb present with $\mathrm{SNR}=301.28)$ and sample $75(\mathrm{~Pb}$ absent with $\mathrm{SNR}=0.74)$, mid Zc filter

Figure A.2. Inter-subgroup SNR difference in peak height/shape: sample 65 (higher Pb with $\mathrm{SNR}=301.28$ ) and sample 69 (lower $\mathrm{Pb}$ with $\mathrm{SNR}=167.67$ ), mid $\mathrm{Zc}$ filter

Figure A.3. Sample 14 - various SNR value examples: SNR < 3 (Zn SNR=1.36), SNR 3 (Pb $\mathrm{SNR}=2.98), \mathrm{SNR}>3(\mathrm{Si} \mathrm{SNR}=12.9), \mathrm{SNR}>3(\mathrm{Ca} \mathrm{SNR}=522)$

Figure A.4. QDA biplots displaying sample variation by element for optimized filter overall tape data set $(\mathrm{N}=94)$ 


\section{LIST OF TABLES}

\section{Chapter 1}

Table 1. Comparisons Between Physical Fit Standard Operating Procedures $(n=12)$

\section{Chapter 1: Supplementary Material}

Table A. Case Report Articles Summary

Table B. Fractography Articles Summary

Table C. Quantitative Articles Summary

\section{Chapter 2}

Table 1. Initial sample set classification ( $n=75$ fracture edge pairs)

Table 2. Optimized sample set classification

Table 3. Options for comparison pair overall conclusion and qualifiers, as well as expected ESS ranges per qualifier

Table 4. Performance rate equation summary

Table 5. Pre-distribution consensus ESS means per tape pair ( $\mathrm{N}=4$ examiners)

Table 6. Sample group pre-distribution characteristics across samples between the 3 kits

Table 7. Overall performance rates using the examiner reported conclusion and the ESS threshold conclusion

\section{Chapter 3}

Table 1. Textile item composition and construction summary

Table 2. Measurements of the foam human form fracturing substrate

Table 3. Observed alignment feature summary

Table 4. Options for comparison pair overall conclusions and comparison edge qualifiers

Table 5. Performance rate equation summary

Table 6. Preliminary textile set error rates, $\mathrm{N}=74$ total comparisons

Table 7. Performance rate summary by separation method

Table 8. Performance rate breakdown - hand-torn samples

Table 9. Performance rate breakdown - stabbed samples

Table 10. Performance rate summary by textile item - hand-torn samples

Table 11. Performance rate summary by textile item - stabbed samples 
Table 12. Proposed rarity ratio thresholds for verbal interpretation scale

Table 13. Relative frequency of occurrence of weighting factor assignment

\section{Chapter 4}

Table 1. XRF instrumental specifications

Table 2. Energy ranges (keV) for NIST SRM 1831 elements

Table 3. Energy ranges $(\mathrm{keV})$ for tape elements

Table 4. Filter comparison experiment results

Table 5. NIST SRM 1831 mean SNRs per element over all filters $(n=24)$

Table 6. Comparison of elements detected in different methods and instrumental configurations

Table 7. Estimated LODs for NIST SRM 1831 as a quality control standard for daily instrument performance $(\mathrm{n}=24)$

Table 8. $\mathrm{Cl} / \mathrm{Ca}$ repeatability and intermediate precision: sample 10

Table 9. Tape set (N=94) XRF characterization groups

\section{Chapter 4: Appendix}

Table A.1. Tape set product information for samples originating from different sources

Table A.2. Examples of spectral contrast angle ratio comparison. Refer to table 10 for subgroup additional information 


\section{OVERALL INTRODUCTION}

According to the American Society of Trace Evidence Examiners (ASTEE), a physical fit or fracture match is "the realignment of two or more objects to prove that they at one time formed a single object". ${ }^{1}$ For the purposes of this study, physical fits will be referred to as fracture fits. Fracture fits can appear in forensic casework through the separation of many materials including tapes, textiles, plastics, paints, and glass, to name a few. The analysis consists of examinations of compared items with fractured edges to determine if the items re-align with distinctive features. This is determined through macro- and micro-level analyses of the material's general characteristics such as color, morphology, and surface characteristics as well as more distinctive features such as surface striations, pattern alignment, or damage continuation that may allow higher confidence in an examiner's overall physical fit conclusion.

A fracture fit can serve as a powerful tool to link two items, individuals, or locations within an investigation. The determination of a positive fracture fit is the only conclusion within the trace evidence discipline that can associate two items to a specific single source beyond the limitation of other materials manufactured in a similar manner and time frame. The evidential value of physical match has been established in multiple case studies with application in a wide range of matrices from paints, metals and match sticks to even skin and fingernails. ${ }^{2-6}$ As fracture fits are regarded as the highest degree of association between a questioned and known sample, it is common that no further chemical comparative analyses are performed following a positive physical fit conclusion. In fact, in a 2012 survey by the tapes subgroup of the Scientific Working Group for Materials Analysis (SWGMAT), 78\% of respondents indicated no further analysis is performed on tape samples when a fracture fit is determined. Survey responses were received from 130 laboratories across 18 different countries. ${ }^{7}$ In a more recent study, conducted by the newly formed NIST-OSAC Physical Fit Task Group, out of 121 respondents, 76\% reported the examinations cease once a physical fit is found. The same survey revealed that although $92 \%$ of the participants have standard operating procedures for physical fit examinations, only $21 \%$ have procedures specific for different types of materials. ${ }^{8}$ Moreover, the lack of consensus-based standard methods makes the evaluation of the quality of a physical fit subjective and often reported without its respective uncertainty.

The 2009 National Academy of Sciences (NAS) report, ${ }^{9}$ the 2016 President's Council of Advisors on Science and Technology (PCAST) report, ${ }^{10}$ and more recently a statement from the American Statistical Association (ASA), ${ }^{11}$ have called attention to the need for reporting error rates and uncertainties associated with comparative forensic analyses that tend to be more subjective or based mostly upon practitioner experience and opinion. Error rates are a particularly critical aspect in determining scientific validity of a method and are recommended in Daubert guidelines that provide judges a means to evaluate the credibility of a scientific technique. ${ }^{12}$

As a response to recent criticism, the research basis of physical fits has greatly expanded in recent years through three main avenues: case reports, fractography studies, and quantitative-based studies. Case reports provide valuable insight to researchers on the actual materials and 
circumstances surrounding physical fit casework received in forensic laboratories. Fractography studies provide an understanding of the mechanism by which certain materials fracture and lay a foundation for determining the formation of distinctive fracture edge features that may become valuable in the alignment of two separated items. Most recently, physical fit research has shifted to more quantitative methods of fit assessment including establishment of error rates through performance-based studies; systematic, score-based assessment of fracture fit comparison pairs; statistical assessment of physical fits through score likelihood ratio assessment and populationalbased studies; and automatic assessment of fractured materials through the development of automated algorithms. Chapter 1 of this thesis serves as an in-depth literature review of the current fracture fit research base, dating back to the $1700 \mathrm{~s} .{ }^{13}$ In addition to organizing and summarizing 112 relevant items of literature, the chapter provides a description of strengths, limitations, and future directions of physical fit research. Chapter 1 has been accepted for publication in Forensic Science International.

Regardless of the basis of our understanding of fracture matches, there are still some significant knowledge gaps in the discipline. Specifically, the majority of published studies a) are focused on evaluating the factors that affect the fracture type but no the informative value of the features, $b$ ) have limited number of samples that prevent generalization of conclusions, b) have been conducted in a limited type of trace materials, c) have not followed a systematic method of analysis or established a defined comparison criteria, d) have used experimental designs that are statistically underpowered, d) do not develop a blind process, e) do not provide quantitative assessment of the quality of a match, or $\mathrm{f}$ ) do not report probabilistic evaluation of the significance of a fracture fit. Therefore, there is a need to develop systematic, quantitative, score-based methodology for assessing and interpreting physical matches in a variety of trace materials. Techniques that can provide transparent and repeatable means of assessing physical fits will lead to higher levels of examiner agreement, more efficient technical review processes, established error rates per material type, and overall a more solid foundation for the credibility of physical fit analyses in expert courtroom testimony.

To close this gap in the research basis, our research group has developed an edge similarity score (ESS) as a quantitative, score-based method by which to examine trace materials and to compute experimental error rates. The method was previously applied to duct tapes of various qualities (low, medium, or high), separation methods (hand-torn or scissor cut), and sample conditions (stretched or pristine samples). ${ }^{14} \mathrm{~A}$ set of 2280 duct tape comparison pairs were assessed with overall accuracy ranging from $84.9 \%$ to over $99 \%$. No false positives were reported for any of the sets examined. This study also introduced a quantitative means of interpretation for duct tape end matches through the score likelihood ratio. ${ }^{14}$

Chapter 2 serves as an expansion of this research into the development of ESS methodology for duct tape fracture fits. In order to begin the process of eventual implementation into forensic laboratories, the first step began as an inter-laboratory study of the novel duct tape ESS method. Three kits of seven duct tape comparison pairs each were distributed to 16 participants overall. Few misclassifications were observed in any of the kits and overall accuracy ranged from 88- 
$100 \%$, depending on the evaluation criteria. In addition to the comparison samples, the kit documentation included a brief survey allowing our group to receive feedback on the method's utility and practicality and as a means to implement improvements. The feedback provided insight into areas of the methodology that require further formal training prior to method implementation as well as areas of the protocol that need to be optimized to allow for full validation. Future work will include an expanded inter-laboratory study incorporating the modifications needed as indicated by this groundwork research. Chapter 2 provides a detailed look into the study results through the evaluation of ESS distributions compared to consensus values, statistical analysis, and observations of examiner feedback as related to individual ESS determinations and the method overall.

An additional goal of our group's physical fit ESS method research is to expand the methodology for use in other material types commonly received as evidence in trace evidence units. Chapter 3 outlines the first expansion of the method into use for textile physical fit examinations. Textiles present an additional challenge to physical fit interpretation as they introduce greater variability within the potential fracture features due to their wide variety in general characteristics such as composition, construction, color, fiber size/shape, fiber twist, alignment of long/short threads, and fluorescence; as well as more distinctive characteristics that arise due to the separation mechanism such as consistent gaps and protrusions or damage across the fractured edges. Due to this variability, the textile fracture study served as a baseline in which performance of the adapted ESS methodology was assessed for various fabric compositions, constructions, and separation methods. This preliminary study consisted of a total of 200 comparisons of stabbed and hand-torn textile pairs as completed by two examiners blind to the ground truth of the sample set. Overall, sample sets of both separation methods resulted in low error rates with accuracies ranging from 85-100\% depending on the textile item. This study also introduced a metric for interpretation of the added textile fracture features through use of weighting factors leading to a weighted ESS value to be represented as a rarity ratio. Values of the rarity ratios reported throughout the study resulted in a proposed verbal interpretation scale for textile physical fits. The study represents a successful first expansion of the ESS methodology into a new material type.

Physical fits have been shown to be problematic in more amorphous materials such as electrical tapes. Within an electrical tape end match sample set created by Bradley et al., of 106 known end matches one pair was reported as a false positive by one of three examiners blind to the samples' ground truth. Additionally, a secondary reviewer also reported a false positive on the same tape pair. The findings of this study led the FBI to change their protocols to continue in the analytical scheme of all tapes regardless of the discovery of a fracture fit. ${ }^{15}$ This change assures that in the case of a false positive physical fit conclusion, the sample pairs still have potential to be discriminated by other sensitive chemical analyses before a final conclusion is determined.

In the circumstance that a physical fit is not discovered between two evidence items, or that an examiner's laboratory protocol requires them to provide additional analyses along with a physical fit examination, it is crucial that practitioners have access to highly discriminatory and informative techniques of analysis to best assess the physical evidence. In terms of electrical tapes, X-ray 
fluorescence (XRF) spectroscopy presents high discrimination as a screening method to complement conventional analytical schemes for electrical tape backing analysis. ${ }^{16-18}$ XRF has the advantage of being easy to operate, non-destructive, and widely available in forensic laboratories. Previous work by our research group characterized a set of 40 electrical tape backing samples of known different sources utilizing three different XRF instrumental configurations. XRF was found to be comparable to LA-ICP-MS when considering the same $\mathrm{N}=40$ sample set, as the most sensitive XRF configuration achieved a discrimination power of $90.1 \%$ as opposed to LA-ICP-MS at $84.6 \% .^{18,19}$

Chapter 4 provides an expansion of the previous XRF electrical tape methodology. The aim of the study expansion was to evaluate the XRF method for use within a forensic laboratory following optimization of atmospheric condition, collection time, sample support material, filters used, adhesive effects, and backing thickness effects. Further experimentation and evaluation of the method's potential for laboratory implementation included assessments of accuracy and discrimination over time, precision, sensitivity, and selectivity. In addition, the initial sample set $(\mathrm{N}=40)$ was increased to a full characterization of 94 electrical tape backing samples originating from known different sources, both by roll and product. The study also included an intra-roll variability study of 20 same roll samples utilizing the newly optimized XRF parameters. This study was performed as an internship and collaboration with the Federal Bureau of Investigation, with the aim of assisting in the validation of the method and implementation in their laboratory.

Overall, the XRF technique achieved discrimination power comparable to that achieved after conducting a full analytical scheme (physical examination, SEM-EDS, FTIR, and Py-GC-MS). The discrimination was also comparable to LA-ICP-MS alone, with a value of $96.7 \%$ for XRF as compared to values of $94.3 \%$ (full protocol ${ }^{20}$ ) and $93.9 \%\left(\right.$ LA-ICP-MS $^{19}$ ), respectively. The method showed to be well suited for quick screening with suitable figures of merit for laboratory implementation, all while demonstrating the high inter-sample variability and low intra-sample variability of electrical tape backings. In addition, this study assessed the application of spectral contrast angle interpretation to spectral comparisons as a useful tool for supporting examiner opinion and providing an objective support to commonly used spectral overlay assessments. Chapter 4 has been submitted to Elsevier's Journal, Forensic Chemistry.

It should be noted that throughout this document, the term "consistent" is often used to describe features along the edges of two fractured items considered to be in alignment. It is also utilized when referencing two items determined to be associated to one another through a physical fit. The limitations of the term must be mentioned to avoid misconception. The use of "consistent" when describing physical fit features does not indicate "to the exclusion of all others." As a proper background study of all variations of physical fit features, orientations, materials, and scenarios initiating a fracture is not available, it is not known to what degree specific features may repeat themselves within a given population. Although the variable nature of physical fits provides their higher level of association in trace evidence analysis, it should not be assumed that features and pairs described within this research as "consistent" may never be replicated under similar conditions. 


\title{
II. CHAPTER ONE
}

\section{Forensic Physical Fits in the Trace Evidence Discipline: A Review}

The following chapter has been published in Forensic Science International (C2020: Brooks E, Prusinowski M, Gross S, Trejos T. Forensic physical fits in the trace evidence discipline: A review. Forensic Science International. 2020. doi:10.1016/j.biteb.2019.100321

We acknowledge the editor's permission to reproduce in part the publication for purposes of this thesis.

\begin{abstract}
Physical fit examinations have long played a critical role in forensic science, particularly in the trace evidence, toolmark, and questioned documents disciplines. Specifically, in trace evidence, physical fits arise in various instances such as separated pieces of duct tape, torn textile fragments, and fractured polymeric items to name a few. The case report and research basis for forensic physical fit dates to the late 1700s and varies by material type. Three main areas of physical fit appear within the literature: case reports, fractography studies, and quantitative assessment of a fracture fit. A strong foundation within the discipline lies in case reports, articles demonstrating occurrences of physical fit the authors have experienced in their laboratories. Fractography research offers information about the fracturing mechanism of a given material for purposes of identifying a potential breaking source. Also, fractography studies demonstrate variation in fracture morphology per material types, with a qualitative basis for comparison and reporting. The current shift in the research appears to be more quantitative or performance-based, assessing the error rates associated with physical fit examinations, the application of likelihood ratios as a means to determine evidential weight, probabilistic interpretations of large sample sets, and the implementation of automatic edge-detection algorithms to support the examiner's expert opinion. This review aims to establish the current state of physical fit research through what has been accomplished, the limitations faced due to the unpredictable nature of casework, and the future directions of the discipline. In addition, current practice in the field is evaluated through a review of standard operating procedures.
\end{abstract}

\section{Introduction}

The American Society of Trace Evidence Examiners (ASTEE) defines a physical match or end match as "the realignment of two or more objects to prove that they at one time formed a single object"1. This concept has been referred to as physical match, fracture match, or fracture fit. For the purposes of this article, the term physical fit is used. Physical fits appear in forensic casework through the separation of many materials including tapes, textiles, plastics, paints, and glass. The realignment between portions left at the scene and those recovered from an individual or object of interest can be important evidence during the investigation. For instance, the physical fit of a piece of duct tape recovered from a bound victim to a roll in the possession of a suspect can provide an association. In a hit-and-run case, the alignment of a broken automotive headlight discovered at 
the scene with a seized vehicle is another example of evidence that can demonstrate the items were once part of a single object.

The analysis of a potential physical fit involves an examination of edges to determine if they realign with distinctive features. The most common observations made between two objects in the course of a fit assessment include material thickness, color and pattern, fracture morphology, irregularities in the fracture, and any striations or imperfections present across the fracture ${ }^{2}$. The evidential value of physical fits has been established in multiple case studies with application in a wide range of matrices from paints, metals and polymers to even skin and fingernails ${ }^{3-7}$.

Many examiners recognize two types of physical fit: direct and indirect. One description of these fits comes from De Forest et al. ${ }^{8}$. A sufficient number of individual characteristics can demonstrate the two items were at one point a single object. The level of significance depends on the nature of the fracture morphology, and presence of additional features such as writing, printing, design, surface topography, grain structure, pigmentation pattern, or irregularities consistent across the fracture. A direct physical fit is defined as occurring when known and questioned materials fit together using the edges. Direct physical fits are referred to as "jigsaw fit matches" demonstrating common origin. Indirect physical fits arise when inadequate detail is present to allow a direct match, such as when a very smooth cut lacks the previously described "jigsaw-like" nature or when material loss causes an intervening piece between two items to be missing.

Indirect matching involves the comparison of continuity of features (both surface and internal), markings, or internal inhomogeneities. For example, a cut newspaper could be indirectly matched to a known piece of paper through surface fiber pattern, crease lines, printing, and inclusions and flaws across the cut line. In cut fabric, indirect matching could occur between thread size, flaws, dyes, and surface printing. Plastic bags can be indirectly matched through their surface striae and pigmentation. Common pattern continuity examples include fabric weave, wood grain, sheet glass striae or ream marks, surface scratches on paint flakes, die marks on wires, and extrusion marks on plastic or metal. Examples include the indirect physical fit of plastic garbage bags over their manufacturer-cut edges due to pigmentation patterns continuing across the cut edge, or two wood pieces cut evenly with a circular saw, realigned due to wood grain, surface markings, surface contours, and external dimensions rather than by the "jigsaw" alignment of the two fractured edges ${ }^{8}$.

Through the years, the value of physical fits has been continually established through case reports and further supported through research studies. This approach has shifted from fractography studies providing an understanding of the separation of materials to qualitative-based fit comparison recommendations, and most recently to more quantitative, score-based approaches through the support of automated algorithms. Literature published during the 1960s-1970s consisted of methodology-focused publications from practitioners illustrating techniques utilized. Examples include studies describing how glass fracture marks can be used to demonstrate a physical fit, a dyeing method for revealing matchstick correspondence, and the application of ultraviolet lighting to illustrate shoe heel and sole fit through fluorescing adhesive ${ }^{9-11}$. 
During the 1980s, while further case reports were published to provide reference to actual casework scenarios, a rise propagated in studies with sample sets of known ground truth (e.g., sets of known non-matches and known matches) to assess fit comparison methodology. For example, a major physical fit study of the decade involved a systematic method introduced by Von Bremen et $a l .{ }^{12}$ in which the order of manufacture of garbage bags can be assessed based on increasing slope of die lines. The authors obtained ten packages of bags from local stores along with 13 known consecutively-manufactured bags and three packages of known consecutively-manufactured bags from a plant in order to create the sample sets for this study ${ }^{12}$. This method was later a key technique utilized in a homicide case as published by Ryland et al. in $2001^{5}$. The first instance of computer-based modeling of fracture fits also appeared during the 1980s with a study on fractal surfaces by Thornton ${ }^{13}$. Another study by Gummer et al. ${ }^{14}$ described two known contact points between the hinge and the door of six vehicles that were compared to identify features adding strength to fit visualization.

The early 2000s brought increased growth in available physical fit literature including case reports, fractography and qualitative-based studies, as well as the emergence of more blind, performancebased studies for fit determination. Studies involved the blind presentation of comparison pairs of various materials including duct tapes, metals, and bones to examiners for the purposes of assessing their accuracy and any observed misclassification rates (false positives or false negatives $)^{15-18}$. The 2000 s were also a time that automated algorithm methods began to be reported in the literature. Some examples are within the questioned documents discipline to reconstruct shredded paper items ${ }^{19}$, as well as an algorithm attributing similar fragment shapes in broken ceramics ${ }^{20}$.

While the 2010s have given rise to one of the first major duct tape end matching studies with a sample size of 1600 comparison pairs ${ }^{21,22}$, this decade is characterized by a significant expansion in automated algorithm research. Studies of note utilize a type of morphological image processing known as content based image retrieval (CBIR) ${ }^{23}$ to initiate a set of coordinates describing a fractured edge to which similarity metrics can then be applied ${ }^{20,24,25}$. In addition, the 2010s are noted for a rise in application of the Bayesian approach in comparative forensic evidence ${ }^{26-30}$, moving towards the potential for a likelihood ratio approach to physical fit conclusions.

Pioneers of the field had initially recognized the strength of physical fits in forensic casework. Walls recognized, "the fitting together of the broken edges may provide the most incontrovertible evidence possible" 31 . In a similar statement by Kirk, he described physical fits as, "evidence being so strong as to constitute almost absolute proof" ${ }^{32}$. De Forest et al. described physical pattern comparison in general as "the most effective approach to many individualizations" 8 . In a letter to the editor to the Journal of Forensic Science in 1986, Thornton expressed his opinion on the evidential value and significance of physical fits by using the analogy of the frequency of occurrence of snowflake patterns in nature ${ }^{33}$. This seems to be an early hint of population-based thinking that has recently been furthered in studies by Lograsso ${ }^{34}$ and Stone ${ }^{35}$. A similar hint towards algorithm and database technology is given by De Forest ${ }^{36}$. While the author noted that macro-scale physical fits provide "unequivocal associations" to negate the need of databases, he 
claimed "micro-physical matching" may benefit from this type of technology. Database and rapidscanning technology may be extremely beneficial for microscopic fragments for which identifying physical fits is difficult and examining all possible edge matches is tedious ${ }^{36}$. Nonetheless, nowadays the criminal justice system is more aware of the risks of wrongful convictions when overstating the value of the evidence. More stringent methods to assess the reliability of forensic examinations are needed to support any individualizing assumption. As a result, assessing the scientific validity of physical fits has become critical and statements such as the ones described by pioneers in this field should be proven experimentally.

Many other forensic disciplines carry out pattern comparison-type examinations. These include latent prints, questioned documents, and footwear. Others involve more impression-based comparisons of indentations and subsequent protrusions, such as in toolmarks. While these types of contour comparisons may not necessarily involve two fractured items, the principles surrounding the interpretation and method of examination assist in laying a foundation for forensic physical fits. In addition, these disciplines have experienced a similar shift towards automation. For instance, studies have established methodology for determining similarity of written signatures ${ }^{30}$, performing spatial statistics to attribute a similarity metric to footwear impressions $\mathrm{s}^{37}$, and improving automatic comparison of fingerprints ${ }^{38}$. Similar techniques have been applied in forensic anthropology, specifically with situations involving mass skeletal remains. Automated pair-matching systems helped to pair compatible bone types by size and morphology for a more efficient method of sorting ${ }^{39-41}$. Anthropological bone comparisons typically focus more on similarities between size and structure rather than fractured edges; however, as with toolmarks, these disciplines provide similar foundations to human-based pattern recognition and comparison. Therefore, some studies from these disciplines will be introduced within this article as well.

The 2009 National Academy of Sciences report, the 2016 President's Council of Advisors on Science and Technology report, and more recently a statement from the American Statistical Association have called attention to the need for reporting error rates and uncertainties associated with some forensic analyses such as fingerprint, firearm, and other examinations involving featurebased comparisons such as physical fit ${ }^{42-44}$. However, standardizing evaluation of the quality of a physical fit is challenging. One way of assessing the performance of qualitative, comparative methods is by evaluating error rates in datasets of known ground truth. Error rates can be a crucial component to determining scientific validity. Further, error rates, while not necessarily a requirement for court admissibility, are recommended in the Daubert Standard as a guideline by which judges can evaluate the credibility of a scientific technique ${ }^{45}$.

In terms of physical fit examinations, the error rate could be considered as the rate of misclassification of true matches or true non-matches, known as false negatives and false positives, respectively. These types of studies can be a useful reference for an examiner to demonstrate the validity of their method. However, it should be noted that error rates are difficult to quantify in terms of physical fits due to the many factors associated with fracturing events. These include the material type, circumstances and force of the separation, and known population information. It is difficult to encompass each of these factors for many material types in a research study. 
This article establishes the current state of forensic physical fits through two avenues: current practice in the field and research studies. Practice in the field is illustrated through a summary of typical end match protocols implemented in various forensic laboratories. Research is presented in terms of three main approaches existing in current studies. These include a) case reviews, b) fractography studies or qualitative-based fit reporting, and c) quantitative assessments of physical fits. Through this, the foundation and future directions in the field are discussed.

\section{Physical Fits in Trace Evidence - Current Protocol Examples}

In a recent small survey distributed by our research group to U.S. trace evidence examiners, eight respondents were able to share twelve standard operating procedures (SOP) used for physical fit examinations at their laboratories. While most of the reviewed protocols appeared to outline general approaches to physical fit examinations regardless of material type, two documents were received in which the procedure was separated based on material. One document (consisting of five SOPs) included sections for fabric comparisons, cordage comparisons, polymeric materials, paint, and brittle materials. Another included specific instructions for fabric and polymeric materials. Additionally, while not necessarily categorized as material-specific due to separation of SOP sections, two protocols included brief examples of features for a few material types that could become useful in the physical fit examination.

Of the more general protocols, all shared the way in which the approach to a physical fit examination was described. Each provided a process of initially orienting the samples together as well as general physical features to examine during the physical fit analysis such as color, construction, texture, and surface appearance. Every procedure also indicated that physical fits should be documented through notes, sketches, or digital images. Most protocols mentioned that the examination ends and a conclusion is made when a fit is discovered, while further analysis should take place if no fit is discovered.

While the general procedures did not focus on specific material types, some provided additional information based on considerations for different item morphologies. For example, two protocols provided different examination recommendations depending upon if the material presented twodimensional or three-dimensional junctions. Two-dimensional fits were to be examined under stereomicroscopy for corresponding textures, scratches, or defects on the surface of the samples across the fractured edge. Three-dimensional fits were instructed to be examined under stereomicroscopy for each of multiple corresponding surfaces. In addition, the methodologies recommended that the examiner should look within the fracture edge itself for any corresponding defects or features, such as rib markings in glass.

The general procedures also differed in the level of detail they provided for the process of conducting the examination. For instance, a few protocols provided specific lighting configurations that could assist in the establishment of consistency of physical features. Specifically, one protocol explicitly mentioned using a light box with optional polarizing filters to examine thin polymer films. Another protocol required a stereomicroscope with up to $100 \mathrm{x}$ 
magnification as well as transmitted and incident lighting. A few others mentioned utilizing fluorescence to orient float glass samples. Other protocols more generally recommend utilizing various light sources.

The main difference that became apparent between procedures was the way in which an examiner was instructed to fit the samples to one another. While three protocols instructed the examiner to attempt to physically slide the samples past one another to observe if a fit exists, three others specifically mentioned to never let the samples touch one another or to match edges "without inflicting further damage" to preserve microscopic edge characteristics that could assist in assessing a fit. Another key difference was that as the majority of the protocols were mainly qualitative in their recommendations, one protocol did mention that measurements and pattern counts should be completed if necessary. While not as contrasting, six protocols mentioned only to perform physical fits if the materials were "suitable" for analysis. One protocol mentioned physical fits should not be performed on crystalline structures that fracture "in a predictable manner." Another mentions that an indirect physical fit should be attempted if a direct cannot be established. Table 1 below further summarizes key similarities and differences between the reviewed standard operating procedures.

Table 1. Comparisons Between Physical Fit Standard Operating Procedures $(n=12)$

\begin{tabular}{|c|c|}
\hline Similarities & Differences \\
\hline $\begin{array}{c}\text { All protocols discussed proper orientation of samples } \\
\text { for analysis - "siding" }\end{array}$ & $\begin{array}{c}\text { Two documents (6 SOPs total) were material-specific, } \\
\text { all others were generic }\end{array}$ \\
\hline $\begin{array}{c}\text { All provided a list of general physical features to } \\
\text { examine for consistency (i.e., color, construction, } \\
\text { texture) }\end{array}$ & $\begin{array}{c}\text { Two protocols mentioned differences in examinations } \\
\text { between 2D and 3D fits }\end{array}$ \\
\hline $\begin{array}{c}\text { All protocols mentioned necessary documentation of } \\
\text { an established fit (i.e., notes, sketches, photographs) }\end{array}$ & $\begin{array}{c}\text { Five protocols gave specific methods to use (i.e., } \\
\text { fluorescence) rather than more general guidelines (i.e., } \\
\text { "different lighting conditions") }\end{array}$ \\
\hline $\begin{array}{c}\text { All mentioned further physical and/or chemical } \\
\text { analyses should be completed when no fit is discovered }\end{array}$ & $\begin{array}{c}\text { Only one protocol mentioned a quantitative aspect (i.e., } \\
\text { sample measurements and pattern count) }\end{array}$ \\
\hline & $\begin{array}{c}\text { One protocol mentioned attempting an indirect } \\
\text { physical fit if a direct is not established }\end{array}$ \\
\hline & $\begin{array}{c}\text { Six protocols recommended fits on only materials } \\
\text { "suitable for analysis" (e.g., adequate sample size, } \\
\text { substrate composition, and/or condition) }\end{array}$ \\
\hline & $\begin{array}{c}\text { Three protocols explicitly stated not to allow the two } \\
\text { items to touch, while three protocols recommended } \\
\text { sliding the items past one another to "feel" alignment }\end{array}$ \\
\hline Ten protocols mentioned review by a second examiner \\
\hline
\end{tabular}

In one document (five SOPs within) in which the examination protocols were separated by specific material type, the fabric comparisons SOP described first how to "side" and orient the fabric samples by their lengthwise (warp) and crosswise (weft) fibers. Macroscopic characteristics that can quickly eliminate a non-match are then established. These included yarn thickness, printed design, or stains across the fractured edge, followed by color and construction of individual yarns 
and continuation of the weave/knit pattern. Cordage examinations were established similarly, as macroscopic characteristics such as width and ply thickness were to be examined first followed by characteristics of the plastic edges and core fractured ends. The cord should then be opened to lie flat for examination of the core and allow for examination of core characteristics for compatibility between pieces when applicable. Another SOP focused on physical fits of polymeric materials. This SOP recommended to begin with orientation of the samples based on manufacturer markings or surface anomalies that are consistent across the fractured edges. Along with the overall broken edges, these distinctive characteristics assist in the establishment of a fit. Along with polymeric materials in general, an additional SOP was provided for tapes in which instructions are provided for straightening distorted edges, observing both backing and fabric reinforcement features, as well as examining any distinguishing characteristics such as backing defects or protruding fabric reinforcement portions that extend across the fracture. A similar approach was described in the SOP for paint chip physical fit examinations, in which broken-edge characteristics as well as surface anomalies are used to establish a fit beyond consistent physical features. An SOP was provided for physical fits of brittle materials as well. Within this protocol, features due to low and high velocity impacts, thermal stresses, and bending are described that may become useful in a physical fit examination.

The second material-specific document consisted of one SOP. This document initially described differences in observable features in 2D and 3D junctions, providing examples for each. Specific instructions were then provided for physical fit examinations of fabric and flexible materials such as tape and other polymeric materials.

Although the majority of reviewed protocols appeared as more generic than material-specific, it is important to note that a laboratory's standard operating procedure is a document referenced by trained examiners during casework. Forensic laboratories have formal training programs examiners must complete before beginning casework. Specific physical fit techniques are more thoroughly explained during training, as is evident in a laboratory training guide provided by one participant. Although this participant had a general physical fit SOP, their physical fit training manual included detail on specific casting techniques, lighting conditions, and features associated with fractured items in each of crystalline, amorphous (brittle or plastic), fibrous, and composite materials. In summary, while this information may not be explicitly stated in an SOP, this does not necessarily indicate the examiner has never been given more direct instruction.

Although we recognize the sample size is small, the protocol review demonstrated a critical need to standardize the fracture fit examination methods across laboratories. Currently, there are no standard guides or standard methods available for the examination of fracture fits of trace materials. Also, there is lack of specific criteria to support the examiner's opinion on when the observed features are substantial enough to conclude a match. Some of the research discussed below can serve as a basis for the harmonization of procedures and demonstration of validity of the examinations. 


\section{Established Physical Fit Research}

Studies involving forensic physical fits are numerous and date as far back as the late 1700s. Gehl and Plecas summarized one of the earliest documented instances of physical fit in which a group of volunteer citizens organized by Henry Fielding known as the "Bow Street Runners" discovered a piece of wadding paper in the gunshot wound of a murder victim shot with a muzzle loading weapon. When the suspect was searched, he was in possession of wadding paper. Investigators physically fit the torn edges of the questioned wadding paper fragment to the known paper recovered from the suspect to link him to the crime ${ }^{46}$. These studies serve to lay the foundation of physical fits. Figure 1 below outlines the reviewed literature in terms of category and material type.

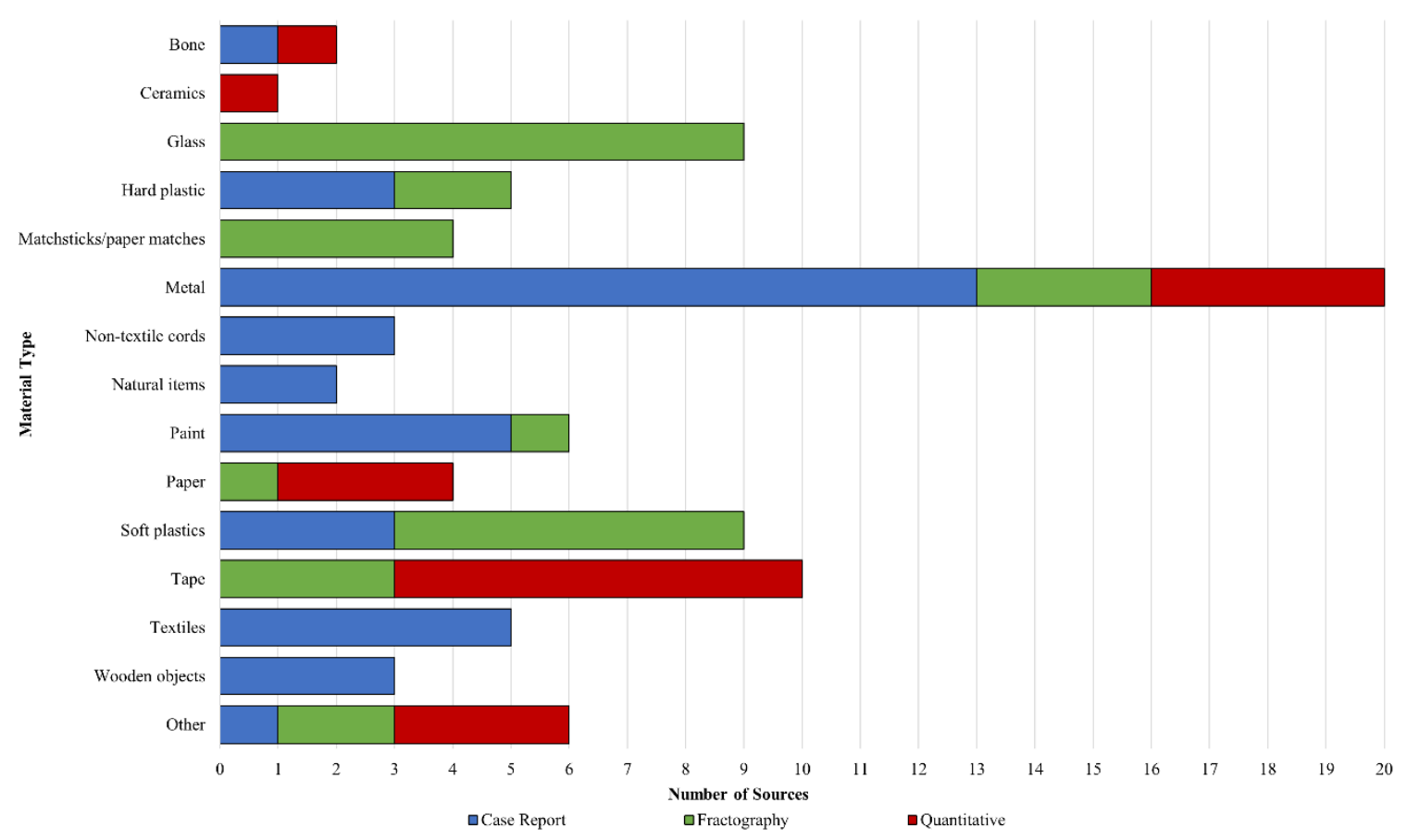

Figure 1. Reviewed physical fit literature by category and material type ( $\mathrm{n}=79$ publications; articles discussing more than one material type are duplicated in the count of each relevant category)

Extensive tables summarizing all reviewed literature in terms of article category (i.e., case report, fractography, or quantitative), material type, study population size, qualitative or quantitative components, experimental design, statistical performance measures, and main findings are provided in the supplementary information, which can be cited by forensic examiners or researchers as support to their opinions or protocols. However, it is recommended that the reader carefully evaluate the experimental designs and populations used in any cited studies in terms of applicability to a specific case. 


\subsection{Case Reports}

A majority of early physical fit literature exist as case reports demonstrating noteworthy instances of physical fit cases in forensic laboratories. These case-based studies have illustrated the relevance of physical fits in many forensic applications. Currently published case reports represent a vast array of materials. These include but are not limited to metal, textiles, hard and soft plastics, paint, wooden objects, non-textile cords, natural items, and other miscellaneous examples. Existing case reports are described by material below.

\subsubsection{Metal}

Many articles appear within the firearms and toolmarks discipline, especially in the case of metal physical fit case reports. For the purposes of this article, the review will focus on realignment of objects rather than impressions (e.g., toolmarks). To illustrate this, an article by Finkelstein et al. ${ }^{47}$ described a case in which a seemingly traditional toolmark examination became a physical fit examination. Toolmark examiners typically associate a tool to a surface by the characteristic markings imparted on the substrate. In the situation of a forced entry and robbery of a grocery store, individual markings were not present around the point of entry. However, a small metallic chip was discovered on the blade of bolt cutters recovered from the suspects' vehicle. This metallic chip was of similar chemical composition to the material of the fractured padlock, as determined via X-ray fluorescence (XRF) spectroscopy. Furthermore, the metallic chip appeared to be of similar morphology to the fractured edge of the padlock. According to manufacturer-provided hardness values, the bolt cutters theoretically should not have been able to cut a material with the hardness value of the padlock. Due to this implication, the discovered physical fit was used to associate two items that otherwise may have been discriminated based on manufacturing specifications alone ${ }^{47}$. This study drew attention to a physical fit opportunity that could be overlooked, and recommended toolmark examiners keep this in mind and work to preserve any metallic chips found on tools for this purpose.

In many cases, the combination of fractured edge alignment and any manufacturer striations lead to an association. Tenorio ${ }^{48}$ provided an example of this through a case report involving an empty beer can found next to a murder victim and a questioned "pop-top" tab. Comparison microscopy revealed that striations observed on the tops of both items were in alignment. Additionally, the tab was flattened and placed in the opening of the beer can, to which the separation patterns aligned as well ${ }^{48}$.

It also often occurs that physical fit examinations involve comparison of fracture morphology, manufacturer striations or features, and striations appearing as a result of use. This scenario occurred during a case report by Streine ${ }^{49}$ in which pieces of a knife blade recovered from a crime scene were compared to determine if they could have originated from the same blade. The pieces were examined under a microscope. The edges of the pieces were puzzle-like in nature and found to align with one another. In addition, striated marks both from the manufacturer and those imparted during use were found to align across the fracture. The discovered striae assisted the physical fit conclusion ${ }^{49}$. A similar situation involving striae from both manufacturing and use 
occurred in a case report by Moran ${ }^{50}$ in which a victim had broken the suspect's car antenna from the vehicle. When observing the two pieces under a comparison microscope, toolmark striations on the interior of the antenna fragments aligned across the fractured edge, as did external scratches and markings. While the fractured edges themselves were distorted leading to a limited physical fit comparison, the presence of the interior and exterior markings added additional value for an association of the two antenna pieces ${ }^{50}$.

Another casework scenario involving a knife blade is provided in a case report by McKinstry ${ }^{51}$. A questioned, broken knife blade was submitted to the laboratory that had been recovered from the chest of a stab victim. A month later, investigators submitted a knife with a melted handle and unknown length of blade apparently missing. The examiner was able to physically fit the broken blade edges to one another with distinctive fracture edge morphology. Additionally, consistency between striations present on each blade surface were discovered through a toolmark examination $^{51}$.

Karim $^{52}$ shared a case report involving a broken piece of vehicular tailpipe and alignment assisted by the manufacturer-sealed seam. In this report, a broken piece of tailpipe was recovered from the scene of a homicide. Over a year later, a vehicle was recovered with a seemingly broken tailpipe. The previous piece from the scene was compared to the intact piece on the vehicle for a physical fit to find that the edges were in alignment despite accumulated mud on the intact piece from continued use post-crime that was not present on the broken fragment. Additionally, the questioned piece aligned with a bracket on the tailpipe corresponding to a location with a hook designed to hold the intact tailpipe in place. The known tailpipe piece was removed from the vehicle for closer examination of fracture morphology. It was found the pieces aligned with a distinctive separation pattern and the manufacturer-sealed seam corresponded across both tailpipe pieces ${ }^{52}$.

Striations imparted to metals due to wear become useful points of comparison during physical fit examinations. An example of this examination scenario is given in a case report by $\operatorname{Reich}^{53}$ in which a screwdriver tip was recovered from a door frame in the case of a forced entry. The broken screwdriver was later discovered in the suspect's car. Under examination, both the fracture morphology and use-imparted striae appeared in alignment between the two items ${ }^{53}$. A similar examination involving striations was reported by Smith in which a broken antenna fragment from a hit-and-run was compared via comparison microscopy to the antenna removed from the suspect's car. The fractured ends were found to correspond, and linear marks on the outside of the antenna were found to align across the edges ${ }^{54}$.

Other physical fits of metals are able to demonstrate alignment through fracture edge morphology alone. This level of examination is exhibited in several instances throughout the current literature. Within a case review by Jayaprakash et $a l .{ }^{4}$, one of the reviewed cases described the reconstruction of a questioned improvised explosive device (IED) tin sheet container and known suspect tin sheet fragments which revealed a consistency leading to a break-through in the case. In a report by Streine ${ }^{55}$, broken pieces of a wheel well were recovered from a homicide scene. The pieces were later compared to the remaining wheel well of the suspect's vehicle. Visual alignment was 
determined between the questioned and known pieces ${ }^{55}$. Caine et al. ${ }^{56}$ described a scenario in which a roof located at a chop shop was physically fit to the roof beams of a known vehicle.

In a case review by Klein et al. ${ }^{57}$, two cases were presented involving physical fits of bullet fragments that played crucial roles in their respective investigations. The first case involved a shooting between gang members. All cartridge casings recovered from the scene appeared to be of the same type, but investigators wanted to determine if the projectile fragment lodged in the victim was consistent, meaning fragments found on scene were from the same bullet, fired from the same gun so as to help establish the number of shooters at the crime scene. Forensic examiners were asked to compare fragments found at the scene with the one removed from the victim's leg. A physical fit was crucial for the fragments in this circumstance as the fracture occurred between land impressions on the bullet, eliminating the possibility of an association due to corresponding land impressions on each side of the fracture. Through examination under a comparison microscope and experimentation with several lighting conditions, the examiner was able to determine a fit existed between two fragments. In the second case, a victim was shot five times by a suspect wielding two different firearms. Investigators wanted to determine that a third was not involved. Therefore, bullet fragments found at the scene were again compared to a fragment recovered from the body. As in the last case example, a land impression comparison was not possible. A physical fit was determined and agreed upon by an expert hired by the defense council $^{57}$.

Robinson ${ }^{58}$ presented a case report in which a robber assaulted a store owner with a rifle which then broke into three pieces. The assailant fled the scene with the barreled action and trigger guard. A suspect rifle was found with a broken trigger guard which was then compared with the recovered pieces at the scene. Visual alignment was established between the known and questioned pieces. In addition, surface material on the outside of the trigger guard indicated that the stock was refinished and the gun reassembled while wet, assisting with the fit assessment ${ }^{58}$.

An additional case report by Townshend ${ }^{59}$ involved a slammer tool and two vehicle ignition locks. The examiner was requested to assess whether or not one of the locks could be identified with an ignition wing cap found in possession of the suspect. To do so, casts were made of the ignition lock cores and dusted with gray fingerprint powder to reduce transparency and glare. The cast was then compared microscopically to the wing cap. Fracture marks on the cast were found to correspond to one of the ignition locks ${ }^{59}$.

\subsubsection{Textiles}

For the purposes of this article, textile materials will include clothing, artistic canvas, shoe insoles, and rope.

Fisher et al. ${ }^{60}$ introduced a few examples of textile physical fit cases. For example, a rape case is described in which a victim cut her hands while reaching for a knife. The suspect tore off a piece of his shirt to bandage her hands. These fragments from the victim's hands were later compared to the suspect's recovered torn shirt. Another situation was presented in which a hit-and-run victim's 
torn coat was compared to a piece of fabric collected from the front fender of the suspect's car. An additional scenario provided by the authors involved a torn fabric fragment discovered at the point of entry of a burglary scene that was later compared to the suspect's torn clothing ${ }^{60}$.

Shor et $a l .{ }^{61}$ presented a case in which a physical fit examination was responsible for the confirmation of stolen artwork. Initially, the only known samples provided to the examiners were photographs of the original art samples from the owners. Upon examination of the questioned, stolen paintings, examiners recognized under UV illumination that there had been an over-painting from the canvas edges to their wooden frames with a brown tint not original to the painting surface. Examiners removed the questioned paintings from their frames and utilized acetone and glue remover on the canvas edges to reveal original edges indicating they had been retouched. This discovery prompted investigators to request the original frames from the owners, from which the stolen paintings had been cut. Examiners were able to physically fit the cut canvas edges to the known original frames due to the complex morphology of the distorted canvas ${ }^{61}$.

Several manuscripts involved an association of separated shoe insole material. An article by Shor et al. ${ }^{62}$ presented a case in which an original shoe impression comparison transformed to a physical fit examination. In this case, castings of three family members' bare feet were made to determine which of three pairs of shoes belonged to each individual. It was suspected that the insoles of the three pairs of shoes had been switched in previous examinations within the laboratory. Examiners were able to discover and document a physical fit about $2 \mathrm{~cm}$ long between a questioned insole and inner shoe bottom. Due to wear pattern, parts of the insole had adhered to the inside of the shoe, leaving a characteristic contour pattern appearing as mirror images between the insole and shoe. The fit of the insole fragments remaining inside the shoe to the suspected mislabeled insole revealed that insoles had in fact been mixed up between shoes previously in the chain of custody. This case was critical to the authors' laboratory as it led to a protocol change for documentation of both sides of shoe insoles, to prevent any further misconstruing of evidence ${ }^{62}$.

In a case report by Laux ${ }^{63}$, questioned and known rope fragments were compared to one another. Examination began with a stereomicroscopical examination of the cut edges. The ropes were examined qualitatively for consistency in color, direction of twist, and comprising material (e.g., the rope samples contained two consistent orange fiberglass cords). Quantitative measures were also employed in the analysis including diameter measurements, number of twists per unit length, as well as the number of strands, thread, and fibers within the ropes ${ }^{63}$. While quantitative features were a part of the analysis, it was not utilized in the physical fit of the inner core.

\subsubsection{Hard and soft plastics}

In terms of physical fits, polymeric materials are typically classified as soft or brittle in nature. The nature of the polymer often determines the manner in which it separates and how its pieces are examined in a forensic context. For example, soft polymeric material typically undergoes an extrusion process during its manufacture, leaving behind striations that can add a significant point of comparison during a physical fit examination. This is useful as soft polymeric materials tend to distort to a greater degree, sometimes limiting comparison of the fractured edge. These 
characteristics add an additional feature to examine despite edge damage. Alternatively, brittle polymeric materials often fracture with more distinctive edges, offering more fortuitous comparison possibilities. Examples of the differences in examination between soft and brittle polymeric material are provided below.

In a case report by Dillon ${ }^{64}$, an individual had been suspected of fishing without a license. A fishing pole with no tackle was found in possession of the suspect. The officer discovered a section of fishing line on the ground outside the suspect's car that was connected to baited tackle in the water. The fishing pole, recovered line, and a knife found in the suspect's car were submitted to attempt to see if the fishing line was originally joined. The knife was not found to impart any distinct features/residues on the line. The lines were severed in one straight pass, and so there were not any distinct features or irregularities. To examine the thin line, the questioned and known line were inserted into hypodermic needles to hold the line in place. The examiner observed extrusion striae patterns in the line that corresponded across the edges. It was concluded that the two sections of fishing line were once part of the same line ${ }^{64}$.

Soft polymeric manufacturing features were well established in a case report by Kopec et al. ${ }^{65}$ involving a homicide case in which a young girl's body and belongings were recovered in multiple trash bags. The bags from the scene were submitted for comparison to bags discovered in the suspect's possession. Features imparted on trash bags during manufacturing include melt pattern characteristics such as lines and arrowheads originating due to a mixture of recycled and virgin polymer pellets in the extrusion process, resulting in varied pigmentation. Transmitted lighting was used to reveal these characteristic melt markings and striae were contiguous across trash bag edges, revealing consecutive manufacture ${ }^{65}$.

A physical fit is presented by Moran ${ }^{66}$ involved a breaking and entering at a jewelry store. Four small, black, rubber fragments were recovered from a broken glass doorway. It was noted the rubber fragments and the rubber part of the bottom of the suspect's shoes appeared to be of similar material. Examination under the microscope revealed striations on the surface of the fragments. Examination of the shoe soles revealed similar striations and missing portions. Direct attempts to physically match the fragments were inconclusive. The authors then cast the voids in the soles of the shoes with Mikrosil and compared the casts to the fragments. The casts reproduced the striations and allowed for comparison of fragment shape and striae. The fragments were ultimately concluded as having originated from the suspect's shoes. It was hypothesized that the suspect kicked the glass door to enter the store, and the broken glass gouged out pieces of the sole, imparting striations to both the soles and fragments ${ }^{66}$.

In a case report by White et al. ${ }^{11}$, examiners received a questioned heel piece and a known suspect shoe sole from an armed robbery and rape scene. The questioned heel and known sole were initially aligned by nail hole location and physical size. However, the comparison was enhanced by examining the heel and sole for fluorescent adhesives. The applied UV-light was able to establish "excellent points of comparison" between the samples. This report additionally mentioned that multiple examiners reviewed the match to come to a consensus ${ }^{11}$. 
Garcia $^{67}$ provided an example of a physical fit examination of a brittle polymeric material in a case report of an individual shot by police. The officer had claimed the individual had threatened him with two knives. Two knives were recovered from the scene, one of which had a broken handle. A small piece of material was found embedded in the deceased individual's hand. The piece was collected and compared to the broken knife handle to determine if there was support for the victim carrying the knives. Visual observation revealed that both pieces of known knife handle and the questioned piece were composed of a similar black, polymer material. In addition, a milling pattern was seen on the inside of all pieces. The questioned samples and a section of the broken knife handle were cast using Mikrosil to evaluate a potential physical fit. The cases were found to have similar features, and when the pieces were directly compared with reverse lighting they were found to correspond ${ }^{67}$.

\subsubsection{Paint}

Paint physical fits may arise in casework through the fracturing of automotive, architectural, or even safe door paint when tampered with. For example, Osterburg ${ }^{68}$ presented several examples of paint chip physical fit cases including corresponding architectural paint chips from a housebreaking case, paint chips from a burglarized safe, fragments from a torn price tag in comparison to flaking crow bar paint, as well as a paint chip on a screwdriver head corresponding to the mold of a door frame ${ }^{68}$.

Another example of a paint physical fit was presented by Walsh et al. ${ }^{3}$ regarding paint flakes from a safe door. In this case, questioned paint flakes were discovered in the suspect's workshop that appeared to be consistent with missing paint from six welding beads in the safe door at the crime scene. Casts were taken of the welding beads and pattern associations were made between the ridges in the casts and the paint flakes. In this situation, a physical match was made as the welding ridges were determined to be unique due to the suspected high variability of pattern formation in the welding process, mainly due to the manual action of a welder along with external factors such as ambient temperature, metals used, speed of the process, and type of weld ${ }^{3}$.

An article by Vanhoven et al. ${ }^{69}$ reviewed two cases where external striations on automotive paint chips were used to connect questioned paint chips to a vehicle. In both cases, a comparison microscope was utilized to view the questioned and known fragments of paint. In the first case, a paint chip collected from a body was found to correspond to a suspect's vehicle. The fragment generally fit damage in the fender, only a small section of topcoat remained for realignment. In the second case, a car struck by a bullet was found to have missing paint on the fender. Paint chips from the scene were found and compared to the vehicle. In both cases, the external striations were found to align across the edges of the fragments ${ }^{69}$.

An interesting paint physical fit case is given in the case review by Jayaprakash et al. ${ }^{4}$ involved a stolen van that was suspected of being altered so that its registration details matched that of a broken-down van. The broken-down van was missing its chassis registration plate, and on the painted metal surface beneath where the plate was adhered, a trickled, dried paint droplet was present. An impression of this droplet was discovered on the back of the questioned registration 
plate on the stolen vehicle. The droplet was found to fit into the impression, and the physical fit was determined ${ }^{4}$.

\subsubsection{Wooden objects}

Physical fit examinations of wood materials are similar to those of metals, as fracture edge morphology alignment can be complimented by naturally occurring features such as wood grain and growth rings. This is demonstrated in a case report by Townshend ${ }^{70}$ in which a large black walnut tree was stolen. A section of the stump and a wedge piece of wood from the scene was compared to the end of a tree in possession of the suspects. Examiners observed the grain, rings, and fracture pattern to determine if the pieces were once joined. It was concluded that the wedge piece found at the scene aligned to the end of the tree from the suspects. In addition, the examiners cast a section of the stump and compared the cast to the suspected tree end, finding it to be in alignment in microscopic features ${ }^{70}$. A case report by Hathaway ${ }^{71}$ outlined additional methods that can be used for wood examinations including xylem and phloem tissue comparisons, along with the previously established physical fit and growth ring comparisons. In this case, four fragments of a broken pool cue stick were physically fit together to reveal they were likely once a part of the same item. The examination was performed in response to a defense attorney's concern that the fragments indicated multiple cue sticks were involved in the homicide under investigation ${ }^{71}$.

It is common in case reports that along with presenting their evidential findings, authors share a useful technique that assisted in optimal demonstration of alignment, or the typical methodology they tend to follow in their examinations. In a case report by Christophe et al. ${ }^{72}$, the authors exhibited how they were able to utilize Photoshop techniques to best visualize a physical fit of a questioned wood chip to a damaged wooden pallet. The described scenario involved a hit-and-run in which the suspect was carrying a wooden pallet in the back of his truck. A wood chip was discovered at the scene. The questioned fragment was scanned with a high-quality photo-scanner, enhanced, and overlaid to a scan of the known pallet section. Markers were used to highlight points of significance along the corresponding fractured edges for illustration to the jury ${ }^{72}$.

\subsubsection{Non-textile cords}

Cable or wire physical fit examinations often involve a comparison of multiple material types on the fractured edge, as most cabling consists of a metal core and polymeric outer insulation material. An example of this is provided in a case report by Kenny ${ }^{73}$ of a stolen truck radio. The stolen radio was recovered from a group of suspects, and the victim was unable to positively identify the radio. The radio was then submitted to the laboratory for a physical fit comparison between the severed wires on the questioned radio to those remaining in the victim's vehicle. Visual observation of the wires revealed air pockets in the insulation layer of the wires, present in the severed edges of both the known and questioned samples. The air pockets were determined to correspond across the fractured edge ${ }^{73}$. A similar examination is presented by Striupaitis ${ }^{74}$ in which eight sections of cable were received from a theft from a public utility company. Law enforcement submitted these wire pieces in cut portions: two standard portions from the scene and six portions from the suspects. To look for a fit, the examiner cut the sections horizontally in order to lay the material flat and examine the entire fractured edge at once. The examiner was able to observe a fit between 
one of the standard sections and one of the evidence sections on the outer layer of the wire. In addition, the examiner was able to observe an inner layer of the wire with printed wording that also aligned ${ }^{74}$.

\subsubsection{Natural items}

Interesting case reports involving physical fits of biological materials are also provided in the literature. Examples include those of skin and fingernails, as described in publications by Perper et al. ${ }^{6}$ and Bisbing et al. ${ }^{7}$, respectively. In the case of the skin physical fit, a questioned skin sample discovered at the crime scene appeared consistent to a known injury on the suspect's thumb. The examination consisted of overlaying the questioned skin on the known injury for observation as well as fingerprinting the questioned and known sample for assessment of friction ridge consistency. Serological testing was also performed on both samples, and the authors claim this factor is an objective support to any subjectivity of their physical fit examination ${ }^{6}$. In another instance of a physical fit, examiners received a questioned fingernail fragment from the crime scene that appeared consistent with the damaged edge of one of the suspect's nails. A clipping was taken for a known sample and the grooves in the nail plate between the two samples were examined for alignment under the microscope. As the basis for the individuality of one's fingernail grooves was not established, examiners reported the match as probable rather than definitive ${ }^{7}$.

\subsubsection{Other}

Unconventional methods of physical fit involve overlays of digital images to best visualize alignment. Another case shared in the Jayaprakash et al. ${ }^{4}$ case review was an interesting application of physical fit in which an unidentified body was determined to be that of a missing child due to consistencies in suture pattern and contour of the Wormian bone in the skull through comparison of the questioned skull and known victim ante-mortem X-rays. The fit was crucial in this case, as DNA analysis was impossible due to decay of the body. Another case in Jayaprakash et al. involved another identity determination in which video superimposition of known victim facial footage and a questioned skull from an unidentified body were compared. The alignment of dentition led to a positive conclusion. This review article, while also pointing out unique applications of forensic physical fits, also discussed one of the key limitations of this type of research - that probabilistic statements regarding physical fit are challenging due to variable circumstances surrounding the match "population", as materials and events surrounding the fracture vary on a case-by-case basis ${ }^{4}$.

\subsubsection{Summary}

Case reports are well established in the literature, as evident in the large portion of case reports reviewed in this paper as shown in Figure 1. Despite their vast presence, it is critical that physical fit case reports continually be published to allow the documentation of the types of materials received in crime laboratories to stay current. These reports provide an important knowledge base regarding the presence of distinctive features along fractures of various substrates, as well as demonstrate to researchers the vast array of unusual circumstances in which physical fit cases arise in forensic laboratories. Through reviewing case reports, researchers gain a better understanding 
of prevalent materials and features from which to base their research on in order to best assist, support, and advance the discipline.

In addition, while case reports tended to thoroughly explain the circumstances of the case as well as the examination results, few detailed the methodology used to come to their conclusions. Examiners publishing future case reports might consider describing their basis and rationale for their decision-making and fracture edge feature interpretation processes to better inform the endusers. Further, the majority of case reports reviewed in this paper were based on metallic evidential materials. In order to provide a better understanding of frequent physical fit examinations performed in forensic laboratories, there is a need for increased publication of case reports for physical fit examinations for other material types often received in trace evidence units.

However, due to the limited nature of evidential samples, case reports unavoidably are based upon a limited sample size and rarely can report statistical performance rates of the physical fit analyses. This illustrates the importance of research studies establishing large population sample sets from which probabilistic interpretations can be made, to provide reference and support for forensic examiners when working with similar material types. Therefore, while it is crucial for forensic examiners to publish their experiences to establish the realistic state of evidence received in the field, it is equally important for researchers to educate themselves on the prevalence of material types in casework and take their findings into account with their experimental designs. The close collaboration of academia, researchers, law enforcement personnel, and practitioners is vital for the advancement of the discipline. Also, due to the large variety of materials processed for fracture fit analysis, a multi-disciplinary approach to evaluation of casework items would be beneficial.

\subsection{Fractography and Qualitative-Based Studies}

Existing forensic fractography studies aim to understand the mechanism of the fracture as well as to determine the source of damage (whether it be shearing, tearing, sawing, etc.) based on morphological characteristics. These studies establish features due to the fracture morphology for qualitative-based comparison techniques. A variety of fractography-based studies exist for materials including hard and soft plastics, glass, matchsticks and paper matches, metal, paper, paint, and other miscellaneous items, listed in decreasing quantity.

The nature of fracturing, features, and methods of evaluation, especially for brittle materials such as glass, are covered in fractography textbooks and practice guides ${ }^{75,76}$. Fréchette ${ }^{75}$ discussed the fundamental markings on cracked surfaces by initially explaining the concept of the origin flaw, the flaw or discontinuity in a brittle solid surface from which cracking begins. The origin flaw can be imparted on a material by chemical, thermal, or mechanical means. Cracks propagate by forming a new surface perpendicular to the axis of principal tension, beginning at the origin flaw. The more stress applied at the origin flaw, the quicker the crack will propagate. At any point during crack propagation, an external influence may cause a change in direction of the axis of principal tension, resulting in an alteration to the morphology of the running crack front. Events such as this influence the variability of a resulting fracture pattern ${ }^{75}$. Quinn further discussed the origin of 
different fractures, including whether or not pre-existing flaws that contribute to fractures are a result of external manufacturing (extrinsic), or are a result of the internal structure of the material (intrinsic) $^{76}$.

Fréchette $^{75}$ also described the types of markings that can result in brittle materials from fractures, starting with the rib and hackle markings imparted in glass. The author highlighted markings found within the rib mark family (markings concave in the direction from which the crack came from) including arrest lines, three types of Wallner lines, and scarps. For a more extensive description of these fracture details, the reader can refer to Fréchette ${ }^{75}$.

The literature also discusses how features in brittle materials can lead to fracture variability. Fréchette stated that inclusions in brittle materials are subject to spontaneous cracking during a fracture event as in wake hackle, for example. Inclusions also lead to crack variability as cracks tend to deviate from the axis of principal tension in order to avoid intersecting with an inclusion under tensile stress, in turn tending to intersect with inclusions under compression ${ }^{75}$.

Quinn's practice guide highlighted common tools and instruments that can be used to examine fractures. Jewelers' loupes and various microscopes allow for closer magnification of overall fracture structure, while instruments such as scanning electron microscopy, confocal microscopy, and X-ray topography can be utilized to observe obscure features or perform chemical analysis on the material $^{76}$.

\subsubsection{Hard and soft plastics}

In terms of polymeric material, fractography studies tend to examine the fracture mechanisms of brittle materials and report techniques for best handling and visualization of soft fractured materials for purposes of physical fit examination. For example, within a study on fracturing of various materials by Katterwe ${ }^{77}$, polymethyl methacrylate (PMMA) sheet fractures were studied. Fracturing occurred using an impact "hail-stone gun". Plastic balls of two different sizes (20- and 40-mm diameter) were discharged at the PMMA sheets. The velocity of the balls was measured to determine the kinetic energy of each fired projectile. The cracks from the impact revealed that fracture features varied even when struck with plastic balls at the same kinetic energy, revealing the characteristic nature of polymeric fracture surfaces ${ }^{77}$.

Studies suggesting methodology to best handle fractured soft polymeric materials often occur for tapes and plastic bags. For example, an article by Weimar ${ }^{78}$ demonstrated a method for reducing distortion or stretching on the edges of PVC-tapes (electrical tapes). Tapes from six different manufacturers were torn by hand and their ends were observed with a comparison microscope. The edges were then treated with $100^{\circ} \mathrm{C}$ hot air for a few seconds. This temperature was chosen to prevent melting of polyvinyl chloride often used in the tape backings. After treatment, the tapes were re-observed under comparison microscopy. The heat treatment was found to make it easier to find the corresponding edge, and to improve examiner confidence in the conclusion. The author did note however that applying heat treatment may destroy other evidence such as DNA or fingerprints ${ }^{78}$. 
Specific methodology is also established for the comparison of castings of electrical tape ends in a study by Weimar ${ }^{79}$. Tape samples were either sheared or torn for the creation of match pairs. In order to obtain castings, tape ends were heat-treated at $100^{\circ} \mathrm{C}$ with demineralized water to undo any plastic deformation occurring after the fracture. Ends were then able to be recreated with casting material. Corresponding end casting pairs were examined under a comparison microscope for the fracture matching process. The author concluded that each fracture cast generated a distinctive pattern for nearly mirror-image comparison microscopy results ${ }^{79}$.

While technically a case report, a fractography study was completed within a publication by Agron et al. ${ }^{80}$, in which the authors described their process of recreating electrical tape fracture pairs to demonstrate distinctiveness. The recreated fractures were used to support their determined physical fit in an investigation of an explosion involving a hand grenade. Various examples of torn and sheared electrical tape samples were photographed to provide a demonstration to the jury of distinguishing features along the fractures ${ }^{80}$.

Comparably, a study by von Bremen et al. ${ }^{12}$ proposed criteria for revealing sequential relationships in plastic garbage and sandwich bags. Bags were purchased from various local retailers as well as known consecutive samples obtained from manufacturing plants. Recommended comparison points were mainly qualitative regarding bag color, size, perforations, construction, and any colored individual striations including fisheyes, arrowheads, streaks, and tiger stripes. These individual pigmentation characteristics can be viewed utilizing polarized light microscopy. The authors did introduce a quantitative factor for consecutive manufacture determination. This involved calculating the slope of any prominent markings present across all known consecutive bags. Slope was ranked increasingly to determine sequence of manufacture. Questioned samples obtained from the same manufacturer could then be used to determine the number of missing bags in the sequence by taking the difference of the height of the striation on the questioned bag and the highest known sample, then comparing this value to the average height of the known sample striations ${ }^{12}$.

Vanderkolk ${ }^{81}$ published a similar article regarding the determination of consecutively manufactured garbage bags; however, the article was an illustrative review of methodology and general features to observe during an examination rather than a study involving physical samples. Alignment was recommended according to the heat-sealed edges of the bags. Striations imparted during the manufacturing process, as those described by von Bremen et al. ${ }^{12}$, can be visualized by transmitted light beneath the sample and used to make a physical $\mathrm{fit}^{81}$. The different types of markings that can be used to establish sequential relationships in plastic films were also demonstrated in an article by Pierce ${ }^{82}$. The pigmentation in these additives create patterning or striations that can be used to fit films together to reveal sequential relationships. The article also mentioned these additives can cause abrasion to production machinery, leading to differences in film perforations, cut edges, and roller imprints ${ }^{82}$. 
Denton ${ }^{83}$ shared in a similar article a method for photographing extrusion marks in polyethylene films. As discussed previously, extrusion marks are left behind as a result of debris on the extrusion die in the manufacturing process. The marks are discontinuous, and so therefore can be used to assist fracture matching across consecutive bags. To photograph them, a black card was cut to have $1 / 8$ inch $\times 6 \frac{1}{2}$ inch slots. Two sheets of glass were put together and placed above the grid. The grid was illuminated by a 500-watt lamp at a right angle. Extraneous light was reduced by a black shield. The camera was focused on the glass in the frame so that the whole area of glass would be in the negative. The piece of polyethylene was sandwiched between the glass sheets with the extrusion marks on the short side. The authors found this set up allowed them to optimally capture the extrusion marks ${ }^{83}$.

Ford ${ }^{84}$ provided an additional article establishing methods to best photograph features for comparison of plastic bags and film that have potential to be used to denote matching edges or connected pieces of evidence. Extrusion marks were recommended to be photographed using a secondary lens system so that the extrusion marks can be focused at any magnification. Heat marks originate from bags that are sealed together by an individual separately from the manufacturing heat seals. Secondary heat marks were often created using a soldering iron or laundry iron, or by commercially made sealing machines. For sealing machines, conclusions were made by examining the patterns left by the heat proof fabric on the machine, by observing inclusions and irregularities created in consecutive seals made by the same machine, and by hot spots (unique areas of deformation caused by heat). Cut edges of films offered some additional details if the instrument used to sever the edges left similar characteristics (snags, changes in direction of cut, etc.) ${ }^{84}$.

While multiple articles establish methodology for the comparison of plastic bags and films, an article by Castle et $\mathrm{al}^{85}$ provided a summary of a variety of methods that can be used to visualize and assess physical properties of plastic bags and cling film. In addition, it also summarized the manufacturing of plastic bags and film. In short, three methods were provided for feature visualization such as color and variation of die lines, polarization patterns, and striations from manufacturing. These methods included utilization of a polarization table, shadowgraphy/Schlieren imaging, and incident/transmitted light microscopy. The article also provided four case examples in which these methods proved useful in the analysis of polymeric materials. For further detail on the use of these methods, refer to Castle et al. ${ }^{85}$.

\subsubsection{Glass}

Numerous articles exist in forensic literature discussing the fracturing mechanics of glass as well as resulting patterns. A study by McJunkins et al. ${ }^{86}$ described multiple experiments in which glass is fractured, focusing more on the mechanism by which the glass fractures rather than the process of fitting samples back together. The article described the two major types of glass fracture patterns - radial and concentric patterns. The article also described the appearance of fracture patterns when a bullet has travelled through safety or tempered glass - the entrance plane of the glass bullet hole will exhibit perpendicular chips while the bullet exit plane will show angled chips on the glass ${ }^{86}$. 
Another glass fractography study was completed by Harshey et $a l .{ }^{87}$ through the analysis of fracture patterns made in glass from a projectile fired from an air rifle. The authors fired a $4.5 \mathrm{~mm}$ air rifle at windowpanes with three different thicknesses. Each type of windowpane was available with and without sun control film (SCF). They then recorded various measurements on the fracture patterns including radial fracture count, concentric fracture count, bullet hole diameter, mist zone thickness, and mist zone diameter. Generally, more radial fractures were observed than concentric in each of the glass types. It was determined through the chi-squared test that no significant differences were present in fracture pattern measurements between the thicknesses, regardless of SCF.

A study by Thornton et al. ${ }^{88}$ described glass fractures occurring due to being shot with projectiles in which there is no obvious distortion. Characteristic striations occur under quasi-static loading. In essence, the fracture occurs when the glass fails at a Griffith crack, minute flaws that are often a point of stress concentration. The author's goal was to demonstrate that glass can break under tension even if deformation is not visible. This is described in terms of dynamic loading through the projectile and mechanical waves that propagate through glass when shot. These waves have enough stress to produce a crater in the glass even if the projectile does not cause full penetration. For further information on this phenomenon, refer to Thornton et al. ${ }^{88}$.

An extensive glass fractography study is provided by Baca et al. ${ }^{89,90}$ in which the researchers fractured 60 replicates each of double strength glass windowpanes, wine bottles, and taillight lenses. Both dynamic and static impact fracturing devices in controlled conditions were utilized. Of the glass samples, the $608 \times 8$ inch windowpane fragments were all cut from the same sheet of glass, and all wine bottles were donated from the manufacturer, all taken from the production line on the same day. This was done to assure all samples originated from the same batch. For dynamic impact, a device was constructed utilizing a drop weight at adjustable heights to initiate fracture through an attached indenter tip without penetrating the sample. Static impact was applied through compression with a tensile tester also fitted with indenter tips. Each experiment used three indenter tips interchangeably - a sharp tip, a round tip, and a blunt tip. Of the plastic samples, polymeric taillight lens covers of the same brand and part number were utilized. Indenter tips differed for the polymeric samples as sufficient velocity to break the samples with the previously used tips could not be obtained. Indenter tips consisted of a 2 -inch diameter flat disc for the static impact tests. For polymeric dynamic impact tests, a dropping pipe device was used that is typically used to induce filament deformation in automotive lamps. Fracture velocities were measured using both a video of the event analyzed in MATLAB software as well as wavelength sensors and a timing mechanism. Maximum extension and maximum load value determinations were also recorded. After fracturing, samples were reassembled and covered with clear tape for ease of fracture morphology documentation via hand-sketching, scanning, and digital CAD representation by tablet drawing. Fracture patterns were compared by overlay to all other fracture patterns within their respective sample type. This led to a total of 5,310 pairwise comparisons over all sample sets. Visual examinations were reported to reveal differentiable fracture patterns between similar samples under reproducible conditions. It was also observed the blunt fracture tips typically required the most velocity and load to initiate a fracture, while the round tips required the least. 
This reflected in the number of fracture lines, as the tips requiring the highest velocity imparted the most fracture lines on the sample ${ }^{89,90}$.

A similar fractography study is provided by Katterwe $\mathrm{e}^{77}$ in which reproducible fracturing of glass was examined for variation in fracture morphology. In a static fracture experiment, small slides of plate glass were used in conjunction with three different loads, represented in units of Newtons $(\mathrm{N}): 0.98 \mathrm{~N}, 2.0 \mathrm{~N}$, and $2.9 \mathrm{~N}$. A hard indenter was used to apply each load, creating fractures in a reproducible fashion. The fractures were found to have random distributions of cracks. The cracks themselves were found to be in random quantities, lengths, propagations, directions, shapes, and orientations. The second part of the study was bending of glass, in which a universal testing machine was used to create reproducible load distributions. The resulting curves and fractures were also randomly distributed, illustrating the distinctive nature of glass fracture ${ }^{77}$.

Nelson ${ }^{9}$ described qualitative features that can be used to exhibit glass fragment alignment, referencing a recent hit-and-run case. The author first described the two types of glass fracture markings that can be utilized for this purpose. These included rib markings, those appearing as oyster shell-like fractures, and hackle markings, appearing as small striae normal to rib markings. Hackle markings were found to be most useful for alignment. The method the authors demonstrated for glass physical fits was facilitated by placing a convex glass chip into its original, concave medium and viewing alignment under the microscope through the chip surface, normal to the fracture. It was recommended to photograph the fit with surfaces aligned as well as slightly displaced, so hackle marks were revealed. The author referenced a hit-and-run case in which this method was applied, placing two 3/8 inch glass fragments within larger broken headlamp fragments to identify corresponding features ${ }^{9}$.

Glass fractography features useful for examination purposes are further explored in Thornton's chapter of "Forensic Examination of Glass and Paint: Analysis and Interpretation"91. In his chapter, noted methods beyond traditional aligning of irregular surfaces included microscopic alignment of rib or hackle marks, identification of continuous ream or cord via shadowgraph, and visualization of surface irregularities through laser interferometry. Ream is the typical term for these markings in sheet glass while cord is used for container glass. Ream (or cord) are markings imparted due to physical and chemical property variations within the glass, potentially forming due to poor melting and batch separation within the furnace at the manufacturing plant ${ }^{92}$. These additional techniques arise due to the three-dimensional nature of glass physical fit. Thornton also established the random formation of glass fractures by explaining how fractures propagate through the randomly oriented crystal lattice composing glassy materials. He claimed this understanding provides a "universal acceptance of the uniqueness of a match" $"$.

Indirect glass physical fit is explored in a study by von Bremen ${ }^{92}$. Within the article, the author described a method utilizing ream or cord markings to establish associations between noncontiguous glass fragments. These markings appear as striations within the glass and were visualized in the article by shadowgraph photography. This method involved placing photographic film beneath a glass sample and placing a light source above it to cast a shadow onto the film. The 
shadow pattern was developed as a photograph that allowed visualization of any ream of cord markings. Along with sheet glass, von Bremen also examined 14 glass bottles for cord, which was identified in all samples with varying patterns between bottles. Shadowgraphs were also used to image patterns of six transparent plastic samples and five automotive bulbs. After demonstrating successful images produced via shadowgraph, von Bremen outlined a study utilizing window glass obtained from a known manufacturer to examine the frequency and persistence of ream markings. Four sheets of glass were used to create $1.8-\mathrm{cm}$ wide strips examined in various combinations of non-contiguous distances between one another. Twenty-one strips were examined that originated $1.8-\mathrm{cm}$ apart in the original sheet, 12 were examined at the $13-\mathrm{cm}$ distance, and the two extreme edges of each glass sheet were used to compare strips $70-\mathrm{cm}$ apart. $90 \%$ of ream marks persisted at $1.8-\mathrm{cm}, 33 \%$ persisted at $13-\mathrm{cm}, 10 \%$ persisted over $70 \mathrm{~cm}$, and at $140 \mathrm{~cm}$ none were identified as matching. From these results, von Bremen demonstrated that ream can be used to associate two sheet glass fragments even when a direct physical match is not present ${ }^{92}$.

\subsubsection{Matchsticks and paper matches}

Many fractography articles involving matchsticks share specific techniques that may assist in visualizing qualitative features during examination, such as the method reported by Gerhart et al. ${ }^{93}$ involving matchstick to match book comparisons. Suspected match to matchbook samples were first compared for size, color, wax dip line, and cut or torn edges. The samples were then submerged in a high refractive index liquid in order to make the cellulosic surface fibers of the matchsticks transparent, to allow for ease of viewing further fracture edge detail. The authors claimed this approach has proven highly effective in roughly 40 casework comparisons through the years ${ }^{93}$. In another article involving the comparison of match sticks and booklets, Funk ${ }^{10}$ described a method used to establish consistencies between matchsticks as tested on eight total booklets: four Canadian, two American, one Brazilian, and one Japanese in manufacture. The method was similar in that the surface fiber continuations across consecutive matches are being examined, however the technique used involved dyeing the matchsticks via stain on a wooden roller, mounting the dyed matches on wooden blocks, and examining them under both stereo and comparison microscopes. The authors concluded this method is reliable, cheap, easy, and effective as they claimed the technique has yet to be reported to cause false positives ${ }^{10}$.

An additional method for examination of paper match sticks was presented by von Bremen ${ }^{94}$ utilizing laser excited luminescence. In this study, match boards were removed from books and both surfaces of book were searched for luminescing inclusions and fibers. The manufacturer-cut sides of 120 matches from 6 books were searched for inclusions with stereomicroscope. During both search types, both an argon and dye laser were used for illumination. Images were taken of all observed inclusions. Results showed that the argon laser produced more luminescing inclusions than the dye laser, even though the dye laser seemed to excite more fibers. Although the dye laser was able to reveal some inclusions that were not shown by the argon laser, the argon still performed optimally. The dye laser also had the capability to show cross-sections of a single fiber ${ }^{94}$.

In a study by Dixon, the author provided a recommendation for the minimum number of features to be determined consistent for a positive fit conclusion ${ }^{95}$. Dixon first highlighted ten major points of comparison in analysis of torn or burned matchstick fragments. These included the length, 
width, thickness, waxing, color and thickness of coloring material, the fluorescence of filler materials or sizing, cut edges, torn edges, inclusions, and cross-cut and torn fiber relationships, both horizontal and vertical. The author provided the recommendation that a minimum of four cross-cut or torn fibers must be associated using these comparative points between the questioned and known samples for a positive identification, but only if the match head is still intact ${ }^{95}$. This provided a basis for consideration of comparison requirements.

\subsubsection{Metal}

Fractography studies for metals consist of breaking source determination studies as well as studies looking into the fracture edge variation of metallic materials. These studies examine the morphology changes in their respective matrices in a fracturing event, which provides an important foundation to the understanding of physical fits. In a study by Matricardi et al. ${ }^{96}$, various metal wires were fractured through five methods including tension, shearing, torsion, diagonal cutting and sawing. Their respective ends were then compared via Scanning Electron Microscopy (SEM) to determine if fracture source could be attributed from the cross-sectional shapes. The authors reported that "sufficient detail" for breaking source determination was shown in the tension, torsion, and diagonally cut wires, but not in the sheared samples ${ }^{96}$.

Another fractography study considering wires is that of Katterwe ${ }^{77}$, which was completed to study the variation of fractured wire edges. Tensile tests were performed on steel wires until failure was achieved. The steel wires were found to allow for a fracture match between the edges. The curves and fracture surfaces were random and varied between the different wires, despite being made of the same material ${ }^{77}$.

In addition to studying the way in which materials fracture, many studies then include qualitativebased reporting to highlight features resulting from the fracture that can be used by the examiner to illustrate that two items were once part of the same object. A study of this type was completed for metal keys by Miller et al. ${ }^{97}$ in which six sample sets of five keys each were broken either by bending or sharp impact. Known matches were first microscopically examined and photographed to demonstrate distinctive features, followed by a verification that known non-match pairs did not appear consistent due to similar features. Examinations were completed in the following sequence. The overall fit pattern was first observed for alignment, followed by the correspondence of the toolmarks across the fracture as subclass characteristics. Scientists then examined the internal fracture pattern, making note of any abstract features, ridges, or furrows consistent across both samples through observation under a comparison microscope. By propagating their analyses in this manner, the authors concluded that known match pairs appeared to share a high level of agreement based on qualitative features ${ }^{97}$.

\subsubsection{Paper}

An article by Barton ${ }^{98}$ described a method for more efficient visualization of paper delamination, the unequal tearing of paper layers. This method was discovered during a typical electrostatic detection apparatus (ESDA) analysis for writing impressions on a torn piece of document paper and was later studied through examiner-torn paper. When the torn papers were placed into the 
ESDA with their delaminated edges facing up, the delaminated regions appeared dark in contrast to the remainder of the page in the resulting ESDA image. This technique was useful for rapid visualization of corresponding paper tears and was not affected by the routine humidification imparted on paper being examined for writing indentations ${ }^{98}$.

\subsubsection{Paint}

A study to determine a method for association of separated vehicle parts was shared by Gummer et al. ${ }^{14}$ Through their research, door hinges were examined qualitatively to determine if matches could be established between a vehicle's driver-side door and hinges by the patterns associated to each. Patterns formed between door and hinge as any gaps between the panels allowed capillaries to form in the surface coating of the paint. This caused striations to form that could assist in alignment. Six vehicles of two models were examined, both Ford Telstars and Ford Lasers. Two points of contact of the hinge in the driver's door were analyzed. The authors found that surface coating striations were distinguishable between vehicles. However, if electro-coating between panels was poor, these patterns would not appear at all. ${ }^{14}$. This study revealed a unique method of establishing alignment between vehicular door panels and door hinges.

\subsubsection{Other}

A method meant to be applied to many fractured material types was provided in a review article by Zieglar ${ }^{99}$. The article highlighted two optical techniques to aid in comparing fractures when one is a mirror/negative of the other. Under most cases, overlays would be done using photographic overlays or surface molds, but often detail is lost. The two optical techniques highlighted by the author are a beam splitter technique and reverse lighting. Beam splitters are optical devices designed to split light in half, one portion being reflected, and the other being transmitted. The divided light allowed the observer to examine the object directly and/or a reflected image of the object. Beam splitting helped with recessed fractures and allowed for an overlay. Reverse lighting inverted the surface of one object being examined and could be used correspondingly with beam splitting. These methods allowed for an easier examination of difficult fractures, either by the nature of the fracture or by highlighting features that would be lost under standard comparison microscopy techniques ${ }^{99}$.

\subsubsection{Summary}

As shown above, fractography studies provide a deeper look into the specific features that may assist in assessing a potential physical fit between two fractured items. Studies involving controlled fracture of various materials for assessment of any resulting features, as well as studies outlining a methodology for best contrast and visualization of alignment features are critical to the forensic science community. These studies assist forensic practitioners in sharing alternate viewpoints for assessing certain material types and assist researchers in understanding the features considered by examiners to evaluate a physical fit. Further, studies initiating controlled fractures provide an essential foundation for the knowledge of the separation tendencies of specific material types. By observing the fracturing process, researchers understand the development of features that may be useful in the alignment of separated items. For the physical fit discipline to progress, more fractography studies must be initiated, attempting to understand fracture mechanisms and the 
features imparted to the items during the separation or fracture of the materials. Practitioners must also continue to share their comparison processes to facilitate further conversation and consensus into the decision-making involved in physical fit examinations. Determining which fracture features are class characteristics and which are distinct has not been specifically addressed in a consensus-based protocol. One reason may be that it depends on each material's physical and chemical properties. This remains by far one of the main challenges towards the harmonization of decision-making in current practice. Studies based on fractography, provide a body of knowledge to set the basis of such comparison criteria.

\subsection{Quantitative Assessments of Physical Fits}

\subsubsection{Performance rates}

Studies observing performance of methods to compare fractured items utilize validation sets in which the true origin of the samples (the original matching piece) is known. To mitigate bias, examiners usually remain blind to the origin of the samples during the comparisons. When utilizing validation sets, four outcomes can be identified. A true positive is an outcome where the examiner correctly identifies as a match a pair of items that originated from the same piece. A true negative result is when the examiner correctly reports the pair as a non-match when the items originate from different pieces or objects. False negatives result when the examiner incorrectly reports a pair that was once the same piece as a non-match. A false positive is the outcome when an examiner incorrectly reports a match between objects originating from different items or pieces. In addition to those outcomes, some studies also separate misidentifications - false positives and negatives from inconclusive results, in which there were not enough distinct features for the examiner to reach a conclusion of match or non-match. Performance rates such as sensitivity, specificity and accuracy can be calculated based on the results of the validation sets. Sensitivity, or the true positive rate, is the number of true positive pairs out of the total number known matching pairs in the set. Specificity, or the true negative rate, is the number of true negative pairs out of the all the known non-matching pairs. Accuracy would be calculated by the total number of true positive and true negative pairs out of all the pairs in the set.

Physical fit literature involving performance-based assessment includes materials such as bones, metal-coated papers and silicon cast sheeting, metals, and polymeric material including tapes. In a study by Christensen et al. ${ }^{15}$, volunteer examiners performed physical fit comparisons of various bone, shell, and tooth fragments. Overall, the positive association rate was found to be $92.5 \%$ with only four negative associations reported at a rate of $0.1 \%{ }^{15}$. Performance rates were also evaluated for metal-coated papers and silicon cast sheeting in a study by Tsach et al. ${ }^{16}$ in which samples were torn on a tensile machine and a double-blind physical fit analysis was performed. Of the 24 fracture pairs examined, all were correctly matched for the entire length of the fracture. Twelve of the pairs were attempted to be matched according to transparencies of only $1 \mathrm{~cm}$ of the fracture edge. Of these, $66 \%$ were correctly identified. When examiners were provided with the actual

materials for analysis rather than transparencies, all were correctly identified at $1 \mathrm{~cm}^{16}$. 
Performance rates were examined for the comparison of hacksaw blade physical fits in an article provided by Claytor et al. ${ }^{100}$ This study was conducted to look at the fracturing of metal using a repeatable technique. The authors used a measuring software to document fracture characteristics and also conducted a proficiency test of the comparison process. Twelve consecutively manufactured hacksaw blades were used. Two blades (A and B) were labeled at 1-inch segments (e.g. A1-A22) and broken into 12-inch segments. A cast was made of each evenly numbered edge. Images were taken of each edge, and then the odd edges were compared to every even edge and documented. To conduct the proficiency tests, four consecutively manufactured blades were broken in the same manner, casts of the edges were taken, and all the items were labeled with a test number and item number. 253 comparisons were made using A and B (33 within each blade, and 187 between). The authors found more points of alignment using topographical evaluation of the edges compared to the physical fit of the edges. Of the proficiency testing, 330 test results were returned. 157 of 173 true matches were reported (90.8\%). 109 out of 157 true negatives were reported $(69.4 \%)$. If inconclusive results were included, the true negative rate increases to $98 \%$ $(154 / 157)^{100}$.

A study by Orench ${ }^{18}$ attempted to demonstrate the high degree of variability possible in the fracture patterns of metals. The authors first established the potential for variation by describing the way in which metal specimens fail. When a load applied in either tension, compression, shear, torsion, or bending was applied to a metal, it in turn experiences a strain due to planes of atoms moving relative to each other, known as dislocation movement. Crystal morphology of the metal alters the way in which dislocation occurs. Fracture morphology will change at areas of crystal imperfections known generally as point defects, line defects, planar defects, and bulk defects. Within these categories are 15 types of defects, meaning any given grain of a metal can have any number or combination of these defects. This allows for great variability in the overall fractured edge, increasing with fracture length. Possibilities increase even further when considering the five load types that may be applied in any given combination. The aim of this study was to provide error rate data specifically dealing with metal fracture to conform to Daubert criteria. Twenty sample sets of ten 0.25 -inch diameter steel fracture fragments each were created. A random number generator was used to select a three-digit number to engrave on the end of each piece to mark a true match pair. Fracture fragments were established by notching each original sample 50\% of its diameter halfway down their length with a diamond cutter and pulling them apart with a tensile tester. Of each sample set, two of the ten fragments were true non-matches to all other possible ends in the set. Ten examiners participated in the blind comparison process. Each was randomly assigned two sample sets to complete. Examination followed typical comparison procedure via a comparison microscope with a digital camera and fluorescent light source. All examiners had a $100 \%$ success rate with no false positives reported. This study indicates the high variability of metal fracture morphology leading to high success in metal fracture fit examinations ${ }^{18}$.

The correct association rates of duct tape fracture fits were assessed in a study by Bradley et al. ${ }^{17}$ in which four examiners performed fracture fit analyses on five comparison sets, three of which were hand torn and two were scissor cut. The authors reported that $92 \%$ of hand torn samples and $81 \%$ of scissor cut were correctly identified. No false positives or false negatives occurred; the 
remaining fraction of pairs were reported as inconclusive. When examiners were asked to reexamine the scissor cut set due to the lower matching percentage, two misidentifications did occur. The authors also stressed the importance of the peer review process in these types of comparisons $^{17}$.

In an additional study by Bradley et al. ${ }^{101}$, the association rates of electrical tape end matches were examined. Three examiners performed end matches on 10 sets each of electrical tape fracture pairs created from 7 rolls of constant color and width. Each set design consisted of factor variation between tape brand, test set preparer, and mode of separation (tear, nick then tear, and dispensertorn). Between the 30 total test sets distributed, a total of 2142 end comparisons were possible due to various combinations of tape ends. Of these, 106 known end matches existed of which 98 were correctly identified. Of the remaining pairs, 7 were inconclusive and one was a false positive. A secondary reviewer also reported a false positive on the same tape pair. Given the overall number of possible comparison pairs in the dataset, the determined error rate was $0.049 \%{ }^{101}$.

One of the first reports providing a quantitative assessment of the quality of a physical fit was Tulleners and Braun's ${ }^{21}$ study in which duct tape fracture edges were attributed a match percentage by using a ruler to measure the proposed match area lengths along the fracture edge and then dividing the total match area lengths by the width of the tape. In addition, fractures were categorized according to the following conclusions: match, non-match, or inconclusive. Tape fractures were generated through various methods including hand torn, Elmendorf torn, scissor cut, and box cutter knife cut. This study has been the first to evaluate error rates in large duct tape data sets ( $\geq 1600$ samples). While this process revealed relatively low error rates, the process of hand-measuring a stretched uneven fracture edge remains subjective and difficult to standardize ${ }^{21}$.

More recently, Prusinowski et al. ${ }^{102}$ contributed to the effort of determining a systematic and quantifiable method of duct tape physical fit assessment through the determination of a similarity score based on the relative percentage of consistent scrim areas along the width of the tape. Because the number and position of yarns has been found to be consistent within a roll, establishing the scrim areas as the smallest unit of comparison provided a practical alternative for a systematic comparison approach ${ }^{103}$. The proposed method not only allowed for the reporting of relative edge similarity scores (ESS) but also provided a transparent method for documenting comparison criteria decisions and the peer-review process. A set of 2280 duct tape end comparison scores were obtained from student examiners for low, medium, and high-grade tapes. Separation method was also assessed with the creation of hand torn and scissor cut sets to observe any shifts in the distributions of the scores. Varying degrees of stretching were applied to mid-grade hand-torn set to additionally evaluate how stretching changed the score distributions. Resulting ESS were assessed according to performance rates. The accuracy ranged from $84.9 \%$ to over $99 \%$. No false positives were reported for any of the sets examined. This study also introduced a quantitative interpretation for duct tape end matches through the score likelihood ratio ${ }^{102}$, previously used in questioned documents, latent prints, and trace disciplines ${ }^{28-30,104-106}$ among others, as outlined below. 


\subsubsection{Score likelihood ratios}

The articles outlined below, while not necessarily physical fit specific, provide examples of how score likelihood ratios have been incorporated into other disciplines for quantitative interpretation of qualitative comparisons. Disciplines covered include questioned documents, latent prints, and trace $28-30,104-106$, among others. For a general introduction to likelihood ratios and Bayes' Theorem as a whole, please refer to "Interpreting Evidence: Evaluating Forensic Science in the Courtroom" by Robertson et al. ${ }^{107}$

Within questioned documents, research efforts have attributed and evaluated score likelihood ratios to automated document comparison methodology. An article by Chen et al. ${ }^{30}$ introduced a new automated system for signature comparison in which features such as width, grayscale, radian, and writing sequence were extracted by an algorithm and used to assign a correlation coefficient between signature pairs. Density distributions of these coefficients in relation to the ground truth were derived in order to determine a likelihood ratio ${ }^{30}$.

Further questioned documents studies delve deeper into possible alternate interpretations of the score likelihood ratio format as applied within the discipline. A study completed by Hepler et al. ${ }^{29}$ discussed and applied three different denominator interpretations for the score likelihood ratio (SLR) to automated comparisons between hand-written documents. Score likelihood ratios were calculated for a dataset of writing samples and general trends showed that none of the SLR interpretations resulted in a false positive or false negative rate. However, disagreement rates in overall proposition between SLR types tended to increase as character size of the document increased $^{29}$. An additional study by Davis et al. ${ }^{28}$ highlighted the considerations involved within SLR numerator interpretation for questioned documents. The authors addressed the key requirement for within-source variability information of document scores from samples known to have originated from the suspect. As handwriting samples known to have been generated under the same conditions as the questioned samples are nearly impossible to obtain through the course of an investigation, a sub-sampling method was introduced in which individual, randomly-selected characters from the available known documents or "template" were compared to those randomly selected from a total population of both the suspect and a secondary writer for the propagation of a score likelihood ratio ${ }^{28}$.

Score likelihood ratio application within latent prints is demonstrated in a study by Leegwater et $a l .{ }^{104}$ in which an SLR approach is provided for evaluating the significance of similarity scores assigned to latent print pairs by AFIS. An anonymous copy of the HAVANK2 Dutch National fingerprint database was utilized to obtain AFIS scores. Given the ground truth, these scores were input into score likelihood ratios. Performance assessment resulted in a $6.9 \%$ false negative rate and a $0.1 \%$ false positive rate. Due to the variation and misleading evidence rates shown in the SLR, the authors indicated further research is planned to compare the SLR approach to the performance rates of latent examiners, who possibly consider more or different features of the print than an automated system ${ }^{104}$. 
Martyna et al. ${ }^{106}$ described a method of applying score-based likelihood ratios to pyrograms, especially those used within the trace discipline to analyze paints, plastics, and fibers, but also applicable for pyrograms of drugs, fire debris, and explosives. As all samples are of similar polymeric materials, their pyrograms were expected to be highly similar with variance both within and between samples to be small. Therefore, before deriving score likelihood ratios, the pyrograms had to be transformed via statistical methodology that both maximized inter-sample variability and minimized intra-sample variability. The three methods utilized included ANOVA simultaneous component analysis (ASCA), regularized MANOVA (rMANOVA), and ANOVA target projection partial least squares (ANOVA-TP). Score likelihood ratios were formed as both the traditional score-based model as described in the questioned document and latent examples above, as well as the logistic regression SLR, which attempts to link prior and posterior probabilities through the application of Bayes equation. Overall, the technique of applying an rMANOVA transformation to the chromatographic data implementing the logistic regression SLR showed optimal performance with lowest false positive and false negative rates. Therefore, this technique was recommended by the authors although they mention further research and calibration is needed ${ }^{106}$.

Along with the examples provided above, an article by Morrison et al. ${ }^{108}$ provided an overview of the key considerations for applying score-based likelihood ratios to forensic examinations and provided additional examples of SLR use with voice recordings, face images, digital camera images, ink, identity documents, smokeless powders, and pharmaceutical tablets ${ }^{108}$.

While the score likelihood ratio is prevalent in multi-disciplinary research, it shows promise for increased application within physical fit research. For instance, the previously mentioned study by Prusinowski et al. ${ }^{102}$ applied the score likelihood ratio for interpretation of the edge similarity score (ESS) for comparison pairs. It was found that high similarity scores generally resulted in SLRs supporting the conclusion of a match, while low ESS resulted in SLRs supporting the conclusion of a non-match. This study highlighted one application of the SLR within physical fit materials, introducing the possibility of applying the method to extended material types ${ }^{102}$.

\subsubsection{Probabilistic interpretations}

In addition to the score likelihood ratio, research is beginning to emerge involving physical fit probabilistic interpretations of feature occurrence. This was introduced through probabilistic interpretation of metal fractures within a study by Lograsso ${ }^{34}$ in which Electron Backscattered Diffraction/Orientation Imaging Microscopy (EBSD/OIM) was used to characterize crystal orientation along the fractured edge. Fractures in metallic materials can orient in two directions relative to the grain of the substrate. If the stress applied to the material exceeds its atomic bond strength, the atomic planes of the substrate separate from one another. If a fracture travels through a crystal, it is a transgranular or intracrystalline fracture. However, if grain boundaries are weaker than atomic bond strength, the fracture will travel through grain boundaries as an intergranular fracture. The proposed method was effective for transgranular or intracrystalline fracture. 
The fractured edge was scanned via EBSD/OIM and a sequence of grain orientation was developed along the edge length. From the orientation sequence, a series of misorientation vectors was derived for the fractured edge dependent upon representation of crystal orientation by Euler angles. These angles provided a coordinate system for crystal rotation and angle, relative to an origin crystal. These misorientation vectors were then compared to determine similar or dissimilar edges, helping to attribute to a potential physical fit. This analysis method added value to a physical fit examination as the number of possible crystal orientations along a fractured edge could be calculated, and when combined with the potential population for the evidential material (e.g., the potential population of kitchen knives in the United States), the likelihood of obtaining the same misorientation sequence in another sample pair could be established. Further, due to the large number of potential orientations, the probability of reoccurrence of a given grain pattern was shown to be relatively low depending on the circumstances in question. The author provided examples of how to determine these probabilities depending upon the ordering of the sequence, number of grains in the sequence, and whether the assumption was being made that grain orientations are repeated $^{34}$. However, the estimated probabilities (e.g., 1 to nonillion) need to be calibrated for more realistic interpretation of casework samples to avoid overstatement of evidential value, a key consideration for examiners referencing these studies.

A similar probabilistic interpretation of metal fractures was provided by Stone ${ }^{35}$. This article introduced a theoretical model for developing a probabilistic interpretation of metal fracture fits at both the two- and three-dimensional levels. A fracture "unit" was first defined as the "smallest discernible variations in either directional change or height." For two-dimensional edge fractures, the model assumed a 50\% chance of propagation in each of the vertical and horizontal directions. Depending upon the number of units across the fractured edge, directional combinations increased exponentially. This occurred even more so in three-dimensional edge considerations, where height was incorporated as a third level. For simplicity, the author included only two height possibilities at this time. To provide an example of the degree of probability of occurrence calculated in this manner, an individual metal fracture with unit length of 100 was stated to occur in only 1 out of 1.27 nonillion fractures of the same length. Stone provided the caveat that this model was to be considered tentative, but revealed the potential for probabilistic interpretation of physical fit in metallic materials ${ }^{35}$.

\subsubsection{Automated algorithms}

A more recent approach in physical fit examination research has been the development of quantitative algorithms for an objective method of analysis to support examiner conclusions ${ }^{20,24,25}$. The groundwork for the modeling of fractured edges was studied by Thornton in which computer software was used to model fractured edges as fractal surfaces. The theory used Walls' model, which indicates that each fracture contains inflection points. These points form the course a fracture follows within one plane. The author explained that fractures should be described by fractal surfaces of n-dimensions, as fractals are dimensionally discordant figures. This means fractals do not have dimensions that are integers. The idea of representing fractures as fractals would be that the complexity or individuality of the fractal surface can be calculated as a value to later attribute to association between two sample models. Although the author ultimately 
discovered that the processing time required to generate an accurate fractal surface exceeded the capabilities of computers at the time of publication, this article laid the foundation for developing automation of fractured edge comparisons ${ }^{13}$.

In a study by Yekutieli et al. ${ }^{25}$, automatic physical fit was attempted through the development of two computerized systems. One system extracted contour representation from an input digital fracture image in the form of local angle representation vectors along the fracture edge. This was done by utilizing a "chain code" contour representation, a discrete representation of angle changes along a contour. The interface first presented each sample as black and white, edge-detected images. The user then selected if the white or black region of the image was the sample, rather than the background. The contour of the sample was then extracted as an outline in a separate window. The user then selected a target area on the contour of one sample and the area for the computer to search for matching contours on the other sample. The algorithm compared all segment possibilities along the contour by first translating and aligning the curves according to the angle that minimizes the distance between the two curves. The sum of minimal distances between the curves was calculated and the user was presented with the region with the lowest $2 \mathrm{D}$ match error as the best fit. The other system introduced in the article compared a given fracture contour to a database of fracture contours of the same substrates to generate statistical probability of the match through a similarity value. The digital fracture images were created from 24 silicon casting material fracture pairs, 24 metal-coated paper pairs, and 22 Perspex plate pairs that had been fractured using a tensile machine. To create a large number of fractures for the respective substrate databases, combinations of various matching and non-matching points along the established known match and non-match pair fracture contours were created by shuffling match points marked manually on each digital contour, as well as varying the lengths of each contour segment used. Pixel lengths between known matches and non-matches were used to generate criteria for classification of a questioned fracture. Probabilities of occurrence within generated databases were used to determine optimal separation criterion for this purpose. Overall, the system's correct match classification probability was found to be 0.968 while the false positive classification probability was found to be $0.0519^{25}$. This study demonstrated potential for a useful forensic tool. While performed on very specific types of polymer sheeting and metal-coated paper, it shows potential for future application in other trace materials present in evidential samples.

Another study dealing with edge-detection algorithms was presented by Leitão et al. ${ }^{20}$ in which the performance of current algorithms with scaled-up sample quantity was assessed. This is especially important as forensic materials such as glass or ceramics may fracture with fragment numbers in the magnitude of $10^{3}-10^{5}$. For example, when a rigid object such as a ceramic container breaks, it could shatter into a thousand fragments resulting in about half a million potential comparison pairs, considering the multiple sides of each fragment that could potentially have been adjacent to each other in the original object. This indicates a larger number of nonmatching pairs will exist in the dataset as well. This issue differs from other previously described algorithms in which samples possessing one fractured side for comparison each were assessed, resulting in algorithm success on a dataset of less dimensions than those that glass or ceramic fragments would present. 
In this study ${ }^{20}$, five ceramic tiles were shattered into roughly 100 fragments each. Fragments were scanned and images were then applied to an edge-detection algorithm. Fifty true match fragments were used to train the algorithm, with 50 true non-match fragments used as a control experiment. The specific algorithm quantified fragment shape by transforming each edge curve as a signal. This was done by applying a shape function to the fracture curvature that reads the contour as vectors between individual points along the edge. Matching contours were determined by the amount of variation between the shape values. This was first established by using variation between known matching contours to set a maximum threshold for matching pairs.

Each segment along the shape contour was considered a "bit" of useful edge information. The authors presented a calculation for determining the minimum number of bits expected in a fracture depending on its length. From this minimal bit number, the number of expected false positives reported by the algorithm could be determined as the probability that a randomly selected segment along a contour randomly selected from the database would resemble a given contour as well as the original 50 true match pairs used to train the algorithm. It was found that the higher the number of bits, or amount of significant detail contained on a fragment led to a lower chance of a false positive. The authors mentioned applying this probabilistic interpretation of the rarity of the match of two fragments is a subject of future work ${ }^{20}$.

A similar algorithm-based approach was taken for duct tape physical fits by Ristenpart et $a .^{24}$ using the duct tape fracture pairs generated in McCabe et al.'s 2013 study $^{22}$. In this study, an algorithm was developed utilizing morphological image processing to extract the coordinates of fractured duct tape ends from digital images of the samples to produce a binary image of the fracture, adjusted for noise, image illumination, tape color, and protruding scrim fiber removal. The coordinate system used was two-dimensional, with the x-direction being the fracture direction and the y-direction being the warp direction of the tape sample. The distance between the assigned coordinates along the fracture edge of two tape samples was calculated in the form of a sum of squares residuals (SSR) value. A lower SSR value indicated more similar fracture edges between samples $^{24}$. While generally it was found that the SSR values for known non-matching pairs were orders of magnitude larger than the SSR values determined for known matching pairs, there were a few circumstances in which a non-matching SSR was even smaller than a matching SSR, especially if the fracture edges appeared visually similar. In addition, scissor cut tape samples had higher error rates than hand torn. False positive rates ranged from $0.5 \%$ for hand-torn to $61.5 \%$ for scissor-cut ${ }^{24}$. This study took an important step forward by attempting to apply an automatic algorithm to a more forensically relevant material. However, error rates were much higher than those typically observed in human examinations of the same samples. As reported by McCabe $e t$ $a l$, human analysts obtained false positive rates ranging from $0-8 \%{ }^{22}$. Therefore, the algorithm was not truly superior to the comparison process used by forensic practitioners.

Algorithm-based research has also emerged in the Questioned Documents discipline. In terms of physical fit, comparative algorithms have been applied to torn documents for reconstruction purposes. In an article by Lotus et al. ${ }^{109}$, an algorithm comparing the hand torn edges of fragments from a single document was established as follows. Hand-torn paper fragments were scanned for 
digital images and stored in an array. The contours of the torn edges were extracted utilizing the Douglas and Peucker polyline simplification algorithm, giving a smoothed polygon representation. The extracted polygon sides were then classified by either frame part (exterior, machine-cut paper edges) or inner part (hand torn edge). This was done by comparing the angle values of the pixels within the contour polygons and classifying them into two different arrays depending on predefined thresholds for frame and inner sides. The polygons were then subjected to a feature extraction process in which the number of sudden changes in the contour orientation with respect to the extracted polygon were counted and the Euclidean distance between the inner side polygon vertices was calculated. A decision matrix was then created to identify which fragment pairs were to be compared. During the matching phase, a high score was received if the Euclidean distance between the inner line segments was small and the number of sudden changes in contour orientation between the two sides was equal. The purpose of factoring both the Euclidean distance and the changes in contour orientations into the score was to account for any fragments with similar Euclidean distances that are true non-matches. The authors stated the proposed algorithm has the potential to be applied to all types of shred patterns associated with fragmented documents. However, the algorithm performed better with hand-torn fragments as opposed to those with sheared edges ${ }^{109}$.

An additional automated algorithm for torn paper fragments was presented by Kleber et al. ${ }^{110}$ The algorithm assessed the rotational and gradient orientation of the paper as the previously discussed algorithm, but with the addition of the color of the ink/paper to cluster torn pieces of paper together. The algorithm was tested with 690 images of torn documents. The rotational analysis assessed 678 images (32 could not be assigned an orientation). The color segmentation was tested using 13 samples, and the algorithm was able to distinguish color from black/grey text. In the end, the algorithm could be used to assess general information like the orientation and distinguish between colors and black writing on paper. At this time the algorithm could not be used to match samples together, but future work on the algorithm could include that aspect, as well as additional informing characteristics such as writing type, line spacing, and paper type to name a few ${ }^{110}$.

The development of objective algorithms capable of producing similarity values for fracture pairs in combination with the establishment of comparison criteria for the systematic evaluation of physical fits can provide examiners with quantitative, statistical-based support. However, it should be noted that many of these automated algorithms are still in the research phase. While these techniques show potential for eventual forensic utilization, it should be noted that current studies have shown that human examiners still achieve lower error rates than automated algorithms ${ }^{22,24}$. The future implementation of these techniques could prove beneficial, as the judicial system is becoming interested in a statistical, quantitative approach versus qualitative, opinion-based results.

\subsubsection{Summary}

As demonstrated by the various quantitative methods represented above, multiple approaches have been taken moving towards objective techniques of physical fit assessment. The publication of performance rates is an important aspect of assessing examiner consensus and error rates per material type. These studies also provide valuable insight into what factors may influence the 
quality of a fracture fit. They also raise the awareness that the determination of a fracture fit has an uncertainty associated with the examination process, including the much-needed judgment of the expert.

Likelihood ratios provide an alternative approach for the interpretation and of the weight of evidence. While probabilistic interpretation can be a challenging undertaking due to the various factors affecting fracture feature formation, their expansion may eventually provide useful references to examiners in conveying the rarity of a physical fit association in a particular material type. However, these studies will require large sample populations and incorporate various experimental factors such as separation method, separation force, and sample condition before fracture (i.e., degradation, distortion, external contaminants). Therefore, more research is needed before these studies can be considered admissible in a court setting.

On the other hand, automatic algorithms are quickly developing that have the capability for rapid assessment of similarity of fractured edges, providing an objective support to inform or substantiate the examiner's opinion. Overall, the research basis of quantitative physical fit assessment techniques is demonstrating promising development. These techniques may soon prove valuable in supporting examiner opinion during comparative examinations facing scrutiny within the forensics field, particularly with advances in computational capacity and the speed of self-learning algorithms such as machine learning neural networks. We hope to see a growth in the implementation of 2D and 3D imaging algorithms to aid examiners with the comparative analysis of fracture edges.

\section{Strengths and Limitations}

A few unavoidable limitations are encountered during physical fit examinations, as is true in most techniques. For example, material loss can occur during the fracturing event that can result in a limited physical examination. This is more common in materials that tend to fracture to a greater degree such as glass or ceramics, and with materials that have the potential to fray at their damaged edge, such as textiles. This leads to the loss of microscopic edge detail that can be used to establish alignment and fit. The limitation of potential material loss is corroborated by Shor et al. ${ }^{111}$ Often, when a physical fit is not determined, the items may still share class characteristics and a laboratory will continue with a full analytical scheme of the material. If the two items had originated from the same original object, these items would still be associated due to physical and/or chemical characteristics, just to a lower significance than would be possible with the physical fit.

Another limitation arises through any distortion of the fractured edges that may occur before the items are submitted to the laboratory. For example, more amorphous polymeric material such as duct tapes and electrical tape can undergo extensive alteration during the events of a crime. Alteration could occur through the prolonged tearing of the tape, wadding up of the tape, or stretching of the tape by a potential bound victim. Although there are documented methods to assist in the disentanglement of tapes, areas of the fractured edges that have been distorted to a reasonable degree are likely to be deemed unsuitable for comparison by the examiner. Another 
example of fracture edge alteration would be medical cuts through a victim's clothing. Emergency personnel attempting to assist a victim are rightfully not concerned with preserving the fractured edges of an individual's clothing, leading to unsuitable comparison edges if a fabric fragment were to be recovered from the suspect. The limitation of distortion to the fractured edge beyond the examiner's control is corroborated by De Forest et al. ${ }^{8}$

Despite limitations, physical fits are still considered the highest level of association of two items due to the probative value they provide and present multiple strengths due to their unique nature. The fracturing of various materials tends to produce an array of features, giving examiners multiple comparison points of which to base their physical fit conclusions on. This is especially revealed in performance rate-based studies, as low to non-existent false positive rates have been demonstrated for materials such as bones, metals, and polymeric material ${ }^{15,18,21,100,102}$. Further, fractography studies demonstrating the random, characteristic nature of the separation of materials have been established, most significantly in glass and brittle polymeric material ${ }^{77,89,90,112}$.

Numerous case reports previously established in this article demonstrate the value that physical fit examinations can add to an investigation. Determining a fit between items can establish support for a single source. Specifically, physical fits have been shown to be the sole examination linking the suspects to the crime scene or victim ${ }^{47,57,61}$ Additionally, physical fits are easily demonstrable to a jury either through digital documentation or by the examiner physically demonstrating the fit between items during the testimony. Due to the nature of mass-manufactured materials, establishing a single common source can be difficult - many items manufactured in the same lot will share consistent class characteristics and composition, lending to associations that are valuable but restricted in their overall interpretation within a case context. Physical fits establish stronger support for a single source by utilizing the distinct and random features left by the fracture to establish a connection between the separated fragments. However, to hold such a probative value, the quality of a physical fit must be demonstrated. In addition, new research is emerging to study probabilistic interpretation of physical fit pairs through large databases and automated algorithms.

\section{Conclusions}

Overall, forensic physical fit has a diverse and well-established research base that continues to evolve to meet the modern demands faced by the forensic field. While many different approaches have been taken to study physical fits, all provide foundational information that assist examiners and researchers alike in understanding both the nature of the materials and their prevalence in forensic laboratories. A strong foundation in case examples and qualitative reporting exists, with strides in quantitative assessment through automatic algorithms and probabilistic interpretation strategies. While case reports and fractography studies lay a crucial foundation in the understanding of feature formation and assessment, they also initiate important conversations between examiners and researchers into the decision-making and interpretation process associated to physical fit examinations. Further, studies have emerged creating databases of fractured materials that may allow for probabilistic assessment of physical fits in the future. Automated methodology is being developed to provide examiners the objective support needed to uphold the 
significance of their findings when challenged by increased statistical expectations in court. These quantitative aspects are placing the discipline more in line with NAS, PCAST, and ASA recommendations ${ }^{42-44}$.

In response to this recent scrutiny, organizations have come together to provide resources to forensic laboratories to initiate the standardization process of comparative examinations. In the United States, at the forefront of this effort is the Organization of Scientific Area Committees for Forensic Science (OSAC), as administered by the National Institute of Standards and Technology (NIST). Within OSAC, the Materials (Trace) Subcommittee has recently initiated a Physical Fit Task Group to develop consensus based standard protocols for physical fit examinations as well as identify research needs within the subdiscipline.

Physical fits are a complex research topic as the separation of materials has been demonstrated to be inherently random and dependent on multiple factors involved in the breaking event and the material. The force of the fracture, directionality, object used to impart the break, manipulation following the breaking event, and even temperature may influence the resulting fracture edge features. While large databases of fractures can be created for commonly encountered forensic materials, the nature of materials received for physical fit examination in forensic laboratories is incredibly vast. However, this inherent randomization of physical fit events is precisely what adds significance to their occurrence. Furthermore, physical fit examinations can never be truly objective, as the examiner's expert opinion is an essential input in the overall assessment. Although, with added statistical capabilities and automated algorithm support, the high associative power of physical fit examinations can be more transparently and credibly validated instances of forensic evidence.

\section{Acknowledgements}

The authors would like to thank the forensic laboratories that allowed us to review any standard operating procedures they were able to share, enabling us to learn from your experiences and expertise. The authors would also like to thank West Virginia University undergraduate students Megan Bradley and Paige Schmitt, who assisted in compiling and editing the supplementary literature tables. The West Virginia University Research Program is acknowledged for the internal PSCoR funding to our project. 


\section{References}

1. American Society of Trace Evidence Examiners (ASTEE). ASTEE Trace 101. 2018 [accessed 2018 Dec 12]. http://www.asteetrace.org/

2. Gupta SR. Matching of fragments. International Criminal Police Review. 1970;(June-July):198200.

3. Walsh K, Gordon A. Pattern Matching of a Paint Flake to its Source. AFTE Journal. 2001;33(2):143-145.

4. Jayaprakash PT. Practical relevance of pattern uniqueness in forensic science. Forensic Science International. 2013;231:403.e1-403.e16. doi:10.1016/j.forsciint.2013.05.028

5. Ryland S, Houck MM. Only Circumstantial Evidence. In: Houck MM, editor. Mute Witnesses: Trace Evidence Analysis. San Diego, CA: Academic Press; 2001. p. 117-137.

6. Perper JA, Prichard W, McCommons P. Matching the Lost Skin of a Homicide Suspect. Forensic Science International. 1985;29:77-82.

7. Bisbing RE, Willmer JH, LaVoy TA, Berglund JS. A Fingernail Identification. AFTE Journal. 1980;12(1):27-28.

8. De Forest PR, Gaensslen RE, Lee HC. Forensic Science: An Introduction to Criminalistics. Munson EM, Mediate C, Satloff J, editors. New York, NY: McGraw-Hill, Inc.; 1983.

9. Nelson DF. Illustrating the Fit of Glass Fragments. The Journal of Criminal Law, Criminology, and Police Science. 1959;50(3):312-314.

10. Funk HJ. Comparison of Paper Matches. Journal of Forensic Sciences. 1968;13(1):37-43. doi:10.2174/0929866525666171214111007

11. White R, Arrowood M. Ultraviolet Fluorescence and a Physical Match. AFTE Journal. 1975;7(2):105-106.

12. Von Bremen UG, Blunt LKR. Physical Comparison of Plastic Garbage Bags and Sandwich Bags. Journal of Forensic Sciences. 1983;28(3):644-654. doi:10.1111/j.1365-313X.2011.04857.x

13. Thornton JI. Fractal Surfaces as Models of Physical Matches. Journal of Forensic Sciences. 1986;31(4):1435-1438.

14. Gummer T, Walsh K. Matching vehicle parts back to the vehicle: a study of the process. Forensic Science International. 1996;82:89-97. doi:10.1016/0379-0738(96)01970-6

15. Christensen AM, Sylvester AD. Physical Matches of Bone, Shell and Tooth Fragments: A Validation Study. Journal of Forensic Sciences. 2008;53(3):694-698. doi:10.1111/j.15564029.2008.00705.x

16. Tsach T, Wiesner S, Shor Y. Empirical proof of physical match: Systematic research with tensile machine. Forensic Science International. 2007;166:77-83. doi:10.1016/j.forsciint.2006.04.002 
17. Bradley MJ, Keagy RL, Lowe PC, Rickenbach MP, Wright DM, LeBeau MA. A validation study for duct tape end matches. Journal of Forensic Sciences. 2006;51(3):504-508. doi:10.1111/j.1556-4029.2006.00106.x

18. Orench JA. A Validation Study of Fracture Matching Metal Specimens Failed in Tension. AFTE Journal. 2005;37(2):142-149.

19. Ukovich A, Ramponi G. Features for the Reconstruction of Shredded Notebook Paper. IEEE. 2005:93-96.

20. Leitão HCG, Stolfi J. Measuring the information content of fracture lines. International Journal of Computer Vision. 2005;65(3):163-174. doi:10.1007/s11263-005-3226-8

21. Tulleners FA, Braun J. The Statistical Evaluation of Torn and Cut Duct Tape Physical End Matching. National Institute of Justice 2011; Jul. Report No. 235287.

22. McCabe KR, Tulleners FA, Braun J V, Currie G, Gorecho EN. A Quantitative Analysis of Torn and Cut Duct Tape Physical End Matching. Journal of Forensic Sciences. 2013;58(S1):S34S42.

23. Baji F, Mocanu M. Chain Code Approach For Shape Based Image Retrieval. Indian Journal of Science and Technology. 2018;11(3):1-17. doi:10.17485/ijst/2018/v11i3/119998

24. Ristenpart W, Tulleners FA, Alfter A. Quantitative Algorithm for the Digital Comparison of Torn Duct Tape. Final Report to the National Institute of Justice Grant 2013-R2-CX-K009; University of California at Davis: Davis, CA. 2017.

25. Yekutieli Y, Shor Y, Wiesner S, Tsach T. Physical Matching Verification. Final Report to United States Department of Justice on Grant 2005-IJ-R-051; National Criminal Justice Reference Service: Rockville, MD. 2012.

26. Andersson MG, Ceciliason AS, Sandler H, Mostad P. Application of the Bayesian framework for forensic interpretation to casework involving postmortem interval estimates of decomposed human remains. Forensic Science International. 2019;301:402-414. doi:10.1016/j.forsciint.2019.05.050

27. Bunch S, Wevers G. Application of likelihood ratios for firearm and toolmark analysis. Science and Justice. 2013;53(2):223-229. doi:10.1016/j.scijus.2012.12.005

28. Davis LJ, Saunders CP, Hepler A, Buscaglia JA. Using subsampling to estimate the strength of handwriting evidence via score-based likelihood ratios. Forensic Science International. 2012;216(1-3):146-157. doi:10.1016/j.forsciint.2011.09.013

29. Hepler AB, Saunders CP, Davis LJ, Buscaglia J. Score-based likelihood ratios for handwriting evidence. Forensic Science International. 2012;219(1-3):129-140. doi:10.1016/j.forsciint.2011.12.009

30. Chen XH, Champod C, Yang X, Shi SP, Luo YW, Wang N, Wang YC, Lu QM. Assessment of signature handwriting evidence via score-based likelihood ratio based on comparative measurement of relevant dynamic features. Forensic Science International. 2018;282(2018):101110. doi:10.1016/j.forsciint.2017.11.022 
31. Walls HJ. Forensic science. London: Sweet and Maxwell Limited; 1968.

32. Kirk PL. Crime investigation. 2nd ed. New York, NY: John Wiley and Sons; 1974.

33. Thornton JI. The Snowflake Paradigm. Journal of Forensic Sciences. 1986;31(2):399-401.

34. Lograsso BK. Physical Matching of Metals: Grain Orientation Association at Fracture Edge. Journal of Forensic Sciences. 2015;60(S1):S66-S75. doi:10.1111/1556-4029.12607

35. Stone RS. A Probabilistic Model of Fractures in Brittle Metals. AFTE Journal. 2004;36(4):297-301.

36. De Forest PR. What is Trace Evidence. In: Caddy B, editor. Forensic Examination of Glass and Paint. New York, NY: Taylor \& Francis; 2001. p. 8-9.

37. Luostarinen T, Lehmussola A. Measuring the accuracy of automatic shoeprint recognition methods. Journal of Forensic Sciences. 2014;59(6):1627-1634. doi:10.1111/1556-4029.12474

38. Cao K, Jain AK. Automated Latent Fingerprint Recognition. IEEE Transactions on Pattern Analysis and Machine Intelligence. 2019;41(4):788-800. doi:10.1109/TPAMI.2018.2818162

39. Warnke-Sommer JD, Lynch JJ, Pawaskar SS, Damann FE. Z-Transform Method for Pairwise Osteometric Pair-matching. Journal of Forensic Sciences. 2019;64(1):23-33. doi:10.1111/15564029.13813

40. Karell MA, Langstaff HK, Halazonetis DJ, Minghetti C, Frelat M, Kranioti EF. A novel method for pair-matching using three-dimensional digital models of bone: mesh-to-mesh value comparison. International Journal of Legal Medicine. 2016;130(5):1315-1322. doi:10.1007/s00414-016-1334-3

41. LaPorte K, Weimer R. Evaluation of Duct Tape Physical Characteristics: Part I - Within-Roll Variability. Journal of the American Society of Trace Evidence Examiners. 2017;7(1):15-34. doi:10.1111/1556-4029.13787

42. National Academy of Sciences (NAS). Strengthening Forensic Science in the United States: A Path Forward. 2009. doi:0.17226/12589

43. President's Council of Advisors on Science and Technology. Forensic Science in Criminal Courts: Ensuring Scientific Validity of Feature-Comparison Methods. 2016.

44. American Statistical Association. American Statistical Association Position on Statistical Statements for Forensic Evidence. [accessed 2019 Jan 30]. https://www.amstat.org/asa/files/pdfs/POL-ForensicScience.pdf

45. \{US Supreme Court\}. Daubert vs Merrell Dow Pharmaceuticals, Inc. 509 U.S. 579 (1993). JUSTIA US Supreme Couts. 1993.

46. Gehl R, Plecas D. Chapter 1: Introduction. In: Introduction to Criminal Investigation: Processes, Practices and Thinking. New Westminster, BC: Justice Institute of British Columbia; 2016. p. $1-10$. 
47. Finkelstein N, Volkov N, Novoselsky Y, Tsach T. A Physical Match of a Metallic Chip Found on a Bolt Cutters' Blade. Journal of Forensic Sciences. 2015;60(3):787-789. doi:10.1111/15564029.12735

48. Tenorio FS. Identification of a "Pop-Top" Tab and Beer Can. AFTE Journal. 1983;15(2):5657.

49. Streine KM. Striated Marks Encountered While Attempting a Physical Fracture Match. AFTE Journal. 2010;42(3):293-294.

50. Moran B. An Interesting Physical Match. AFTE Journal. 1996;28(1):19-20.

51. McKinstry EA. Fracture Match - A Case Study. AFTE Journal. 1998;30(2):343-344.

52. Karim G. A Pattern-fit Identification of Severed Exhaust Tailpipe Sections in a Homicide Case. AFTE Journal. 2004;36(1):65-66.

53. Reich JE. A Comparative Photography Case. AFTE Journal. 1978;10(3):23.

54. Smith RM. Another Hit and Run Tool Mark Case. AFTE Journal. 1972;4(5):31.

55. Streine KM. An Interesting Physical Fracture Match. AFTE Journal. 2007;39(1):68-69.

56. Caine C, Thompson E. Physical Match of an Automobile Roof to the Body Section. AFTE Journal. 1989;21(4):632-634.

57. Klein A, Nedivi L, Silverwater H. Physical Match of Fragmented Bullets. Journal of Forensic Sciences. 2000;45(3):722-727. doi:10.1520/jfs14757j

58. Robinson M. Comparison of Gunstock Parts to Barreled Action. Herpetological Review. 1976;8(1):65-69.

59. Townshend DG. Identification of Fracture Marks. Herpetological Review. 1976;8(2):74-75.

60. Fisher BAJ, Svensson A, Wendel O. Techniques of Crime Scene Investigation. 4th ed. Fisher BAJ, editor. New York, NY: Elsevier Science Publishing Co., Inc.; 1987.

61. Shor Y, Novoselsky Y, Klein A, Lurie DJ, Levi JA, Vinokurov A, Levin N. The Identification of Stolen Paintings Using Comparison of Various Marks. Journal of Forensic Sciences. 2002:633637.

62. Shor Y, Kennedy RB, Tsach T, Volkov N, Novoselsky Y, Vinokurov A. Physical match: insole and shoe. Journal of forensic sciences. 2003;48(4):1-3.

63. Laux DL. Identification of a Rope by Means of a Physical Match Between the Cut Ends. Journal of Forensic Sciences. 1984;29(4):1246-1248.

64. Dillon DJ. Comparisons of Extrusion Striae to Individualize Evidence. AFTE Journal. 1976;8(2):69-70.

65. Kopec RJ, Meyers CR. Comparative Analysis of Trash Bags - A Case History. AFTE Journal. 1980;12(1):23-26. 
66. Moran B. Physical Match/Tool Mark Identification Involving Rubber Shoe Sole Fragments. AFTE Journal. 1984;16(3):126-128.

67. Garcia Y. A Fracture Match in a Police-Involved Shooting Investigation. AFTE Journal. 2012;44(2):182-183.

68. Osterburg JW. The Crime Laboratory, Case Studies of Scientific Criminal Investigation. 2nd ed. Bloomington, IN: Indiana University Press; 1968. p. 96-115.

69. VanHoven HA, Fraysier HD. The Matching of Automotive Paint Chips by Surface Striation Alignment. Journal of Forensic Sciences. 1983;28(2):11530J. doi:10.1520/jfs11530j

70. Townshend DG. Examination of Tree Stumps. AFTE Journal. 1981;13(4):32-36.

71. Hathaway RA. Physical Wood Match of Broken Pool Cue Stick. AFTE Journal. 1994;26(3):185-186.

72. Christophe DP, Daniels C. An Unusual Technique for Physical Match Comparison. AFTE Journal. 2008;40(4):396-398.

73. Kenny RL. Identification of Insulating Material Surrounding Wires. AFTE Journal. 1978;10(2):64.

74. Striupaitis P. Physical Fit - Public Utility Cable. AFTE Journal. 1981;13(4):48-49.

75. Fréchette VD. Failure Analysis of Brittle Materials. 28th ed. Westerville, OH: The American Ceramic Society, Inc.; 1990.

76. Quinn GD. Fractography of Ceramics and Glasses. Gaithersburg, MD; 2016.

77. Katterwe HW. Fracture Matching and Repetitive Experiments: A Contribution of Validation. AFTE Journal. 2005;37(3):229-241.

78. Weimar B. Physical Match Examinations of Adhesive PVC-Tapes: Improvement of the Conclusiveness by Heat Treatment. AFTE Journal. 2008;40(3):300-302.

79. Weimar B. Physical Match Examination of the Joint Faces of Adhesive PVC-Tapes. AFTE Journal. 2008;40(3):300-302.

80. Agron N, Schecter B. Physical Comparisons and Some Characteristics of Electrical Tape. AFTE Journal. 1986;18(3):53-59. doi:10.2174/0929866525666171214111007

81. Vanderkolk JR. Identifying Consecutively Made Garbage Bags Through Manufactured Characteristics. Journal of Forensic Identification. 1995;45(1):38-50. doi:10.2174/0929866525666171214111007

82. Pierce DS. Identifiable Markings on Plastics. Journal of Forensic Identification. 1990;40(2):51-59.

83. Denton S. Extrusion Marks in Polythene Film. Journal of Forensic Science Society. 1981;21:259-262.

84. Ford KN. The Physical Comparison of Polythene Film. Journal of Forensic Science Society. 1975;15:107-113. 
85. Castle DA, Gibbins B, Hamer PS. Physical methods for examining and comparing transparent plastic bags and cling films. Journal of Forensic Science Society. 1994;34:61-68.

86. McJunkins SP, Thornton JI. Glass Fracture Analysis: A Review. Forensic Science. 1973;2:127. doi:10.2174/0929866525666171214111007

87. Harshey A, Srivastava A, Yadav VK, Nigam K, Kumar A, Das T. Analysis of glass fracture pattern made by.177" (4.5 mm) Caliber air rifle. Egyptian Journal of Forensic Sciences. 2017;7(20):1-8. doi:10.1186/s41935-017-0019-5

88. Thornton JI, Cashman PJ. Glass Fracture Mechanism--A Rethinking. Journal of forensic Sciences. 1986;31(3):818-824.

89. Baca AC, Thornton JI, Tulleners FA. Determination of Fracture Patterns in Glass and Glassy Polymers. Journal of Forensic Sciences. 2016;61:92-101. doi:10.1111/1556-4029.12968

90. Tulleners FA, Thornton J, Baca AC. Determination of Unique Fracture Patterns in Glass and Glassy Polymers. Final Report to the National Institute of Justice Grant 2010-DN-BX-K219; University of California at Davis: Davis, CA. 2013.

91. Thornton JI. Interpretation of physical aspects of glass evidence. In: Caddy B, Robertson J, editors. Forensic Examination of Glass and Paint. New York, NY: Taylor \& Francis; 2001. p. 94118.

92. von Bremen U. Shadowgraphs of Bulbs, Bottles, and Panes. Journal of Forensic Sciences. 1975;20(1):109-118. doi:10.1520/jfs10246j

93. Gerhart FJ, Ward DC. Paper Match Comparisons by Submersion. Journal of Forensic Sciences. 1986;31(4):1450-1454.

94. Von Bremen UG. Laser Excited Luminescence of Inclusions and Fibers in Paper Matches. Journal of Forensic Sciences. 1986;31(2):455-463. doi:10.1142/9789814307505_0001

95. Dixon KC. Positive Identification of Torn Burned Matches with Emphasis on Crosscut and Torn Fiber Comparisons. Journal of Forensic Sciences. 1983;28(2):351-359.

96. Matricardi VR, Clark MS, DeRonja FS. The comparison of broken surfaces: a scanning electron microscopic study. Journal of Forensic Sciences. 1975;20(3):507-523.

97. Miller J, Kong H. Metal Fractures: Matching and Non-Matching Patterns. AFTE Journal. 2006;38(2):133-165.

98. Barton BC. The use of an electrostatic detection apparatus to demonstrate the matching of torn paper edges. Journal of Forensic Science Society. 1989;29(1):35-38.

99. Zieglar PA. Examination Techniques: The Beam Splitter and Reverse Lighting. AFTE Journal. 1983;15(2):37-41.

100. Claytor LK, Davis AL. A Validation of Fracture Matching Through the Microscopic Examination of the Fractured Surfaces of Hacksaw Blades. AFTE Journal. 2010;42(4):323-334. 
101. Bradley MJ, Gauntt JM, Mehltretter AH, Lowe PC, Wright DM. A Validation Study for Vinyl Electrical Tape End Matches. Journal of Forensic Sciences. 2011;56(3):606-611. doi:10.1111/j.1556-4029.2011.01736.x

102. Prusinowski M, Brooks E, Trejos T. Development and validation of a systematic approach for the quantitative assessment of the quality of duct tape physical fits. Forensic Science International. 2020;307.

103. LaPorte K, Weimer R. Evaluation of Duct Tape Physical Characteristics: Part I - Within-Roll Variability. JASTEE. 2017;7(1):15-34.

104. Leegwater AJ, Meuwly D, Sjerps M, Vergeer P, Alberink I. Performance Study of a Scorebased Likelihood Ratio System for Forensic Fingermark Comparison. Journal of Forensic Sciences. 2017;62(3):626-640. doi:10.1111/1556-4029.13339

105. Rodriguez CM, De Jongh A, Meuwly D. Introducing a Semi-Automatic Method to Simulate Large Numbers of Forensic Fingermarks for Research on Fingerprint Identification. Journal of Forensic Sciences. 2012;57(2):334-342. doi:10.1111/j.1556-4029.2011.01950.x

106. Martyna A, Zadora G, Ramos D. Forensic comparison of pyrograms using score-based likelihood ratios. Journal of Analytical and Applied Pyrolysis. 2018;133:198-215.

107. Robertson B, Vignaux GA, Berger CEH. Interpreting evidence : evaluating forensic science in the courtroom. Chichester, West Sussex, UK ; Hoboken : Wiley, 2016.; 2016.

108. Morrison GS, Enzinger E. Score based procedures for the calculation of forensic likelihood ratios - Scores should take account of both similarity and typicality. Science and Justice. 2018;58(1):47-58. doi:10.1016/j.scijus.2017.06.005

109. Lotus R, Varghese J, Saudia S. An approach to automatic reconstruction of apictorial hand torn paper document. International Arab Journal of Information Technology. 2016;13(4):457-461.

110. Kleber F, Diem M, Sablatnig R. Torn Document Analysis as a Prerequisite for Reconstruction. VSMM 2009 - Proceedings of the 15th International Conference on Virtual Systems and Multimedia. 2009:143-148. doi:10.1109/VSMM.2009.27

111. Shor Y, Yekutieli Y, Wiesner S, Tsach T. Physical Match. 2nd ed. Published by Elsevier Inc.; 2013. doi:10.1016/B978-0-12-382165-2.00281-6

112. Rhodes EF, Thornton JI. The Interpretation of Impact Fractures in Glassy Polymers. Journal of Forensic Sciences. 1975;20(2):274-282. doi:10.1520/jfs 10274j 
CHAPTER 1: SUPPLEMENTARY MATERIAL

Table A. Case Report Articles Summary

\begin{tabular}{|c|c|c|c|c|c|c|c|}
\hline Category & $\begin{array}{c}\text { Material } \\
\text { Type }\end{array}$ & $\begin{array}{c}\text { Population } \\
\text { Size }\end{array}$ & $\begin{array}{c}\text { Qualitative or } \\
\text { Quantitative } \\
\text { Assessment? }\end{array}$ & Experimental Design & $\begin{array}{c}\text { Statistical } \\
\text { Performance } \\
\text { Measures }\end{array}$ & Main Findings & $\begin{array}{c}\text { Reference } \\
\text { Number }\end{array}$ \\
\hline $\begin{array}{l}\text { Case } \\
\text { Report }\end{array}$ & Paint & $\begin{array}{c}\text { Multiple } \\
\text { questioned, } \\
1 \text { known }\end{array}$ & Qualitative & $\begin{array}{l}\text {-Paint flakes examined for most } \\
\text { likely physical match } \\
\text { candidates, three with curved } \\
\text { surfaces selected } \\
\text {-6 weld beads on the safe door } \\
\text { were missing paint, these were } \\
\text { cast and images were taken of } \\
\text { casts as well as paint flake } \\
\text { backs for comparison of ridges }\end{array}$ & None & $\begin{array}{l}\text {-Pattern associations between the paint flake backs } \\
\text { and the weld beads from the safe door were } \\
\text { discovered upon zoomed photography and casting. } \\
\text {-Welding ridges were concluded to be "unique" } \\
\text { due to the high variability of pattern formation in } \\
\text { the welding process due to manual action of } \\
\text { welder along with external factors such as ambient } \\
\text { temp, metals used, speed of process, and type of } \\
\text { weld. }\end{array}$ & 3 \\
\hline $\begin{array}{l}\text { Case } \\
\text { Report }\end{array}$ & $\begin{array}{l}\text { Metal, } \\
\text { Paint, } \\
\text { Bone, } \\
\text { Other }\end{array}$ & $\begin{array}{l}\text { Multiple } \\
\text { questioned } \\
\text { and } \\
\text { knowns for } \\
\text { each case } \\
\text { presented }\end{array}$ & Qualitative & $\begin{array}{l}\text {-Comparison of known and } \\
\text { questioned items in } 4 \text { cases } \\
\text {-No clear methodology shared } \\
\text { except for a video } \\
\text { superimposition method }\end{array}$ & None & $\begin{array}{l}\text {-Case 1: Reconstruction of questioned IED tin sheet container } \\
\text { and known suspect tin sheet fragments reveal a physical fit } \\
\text {-Case 2: A trickled, dried paint droplet beneath where the } \\
\text { chassis registration plate would lie on a broken-down van } \\
\text { discovered to physically fit to an impression discovered on the } \\
\text { back of the questioned chassis registration plate fitted into the } \\
\text { stolen van } \\
\text {-Case 3: Unidentified body was determined to be that of a } \\
\text { missing child due to consistencies in suture patterns and } \\
\text { contour of the Wormian bone in the skull through comparison } \\
\text { of questioned skull image and known victim ante-mortem X- } \\
\text { rays } \\
\text {-Case 4: A video-superimposition of known victim facial } \\
\text { footage and questioned skull led to a positive identification due } \\
\text { to dental alignment } \\
\text {-There is a need to determine a minimum area requirement for a } \\
\text { physical match, or a minimum probability for negative } \\
\text { association, as determining the strength of a positive } \\
\text { association is difficult. }\end{array}$ & 4 \\
\hline
\end{tabular}




\begin{tabular}{|c|c|c|c|c|c|c|c|}
\hline $\begin{array}{c}\text { Case } \\
\text { Report }\end{array}$ & $\begin{array}{c}\text { Soft } \\
\text { plastic }\end{array}$ & $\begin{array}{l}1 \\
\text { questioned, } \\
\text { multiple } \\
\text { known } \\
\text { exhibits }\end{array}$ & $\begin{array}{l}\text { Qualitative } \\
\text { comparison } \\
\text { with } \\
\text { quantitative } \\
\text { measurements }\end{array}$ & $\begin{array}{l}\text {-Observations of physical } \\
\text { features of the questioned and } \\
\text { known bags } \\
\text {-Elemental analysis via XRF } \\
\text {-Visit to the manufacturer to } \\
\text { gain information on the } \\
\text { production process } \\
\text {-Determined frequency of } \\
\text { individual bag type } \\
\text {-Collected reference samples } \\
\text { for determination of period of } \\
\text { manufacture time before feature } \\
\text { change } \\
\text {-Die line slope method } \\
\text { described by Von Bremen and } \\
\text { Blunt used to determine order } \\
\text { of manufacture }\end{array}$ & $\begin{array}{c}\text { Population } \\
\text { frequency } \\
\text { provided }\end{array}$ & $\begin{array}{l}\text {-Both questioned and known bags were the results } \\
\text { of "J sheets" during the manufacturing process, a } \\
\text { characteristic appearing on only } 2 \text { of } 4 \text { stock sheet } \\
\text { rolls produced at once } \\
\text {-A bag with the same slope as the questioned bag } \\
\text { was produced only once every } 412 \text { bags produced } \\
\text {-Changes in die striae and chemistry are observed } \\
\text { in two hour intervals, in which } 254 \text { bags of similar } \\
\text { characteristics are produced which are spread over } \\
16 \text { rolls of stock film, and randomly loaded into } \\
\text { different bag machines. } \\
\text {-Consistency demonstrated in persistent die striae, } \\
\text { elemental composition, tie flap offset, bag width, } \\
\text { degree of tie-flap centering and the presence of die } \\
\text { flap over-tucks (due to origination from "J- } \\
\text { sheets") between the questioned and known bags }\end{array}$ & 5 \\
\hline $\begin{array}{l}\text { Case } \\
\text { Report }\end{array}$ & $\begin{array}{l}\text { Natural } \\
\text { items }\end{array}$ & $\begin{array}{c}1 \\
\text { questioned, } \\
1 \text { known }\end{array}$ & Qualitative & $\begin{array}{l}\text {-Questioned skin sample } \\
\text { overlaid to known suspect } \\
\text { injury and photographed } \\
\text {-Fingerprints taken of } \\
\text { questioned and known for } \\
\text { comparison } \\
\text {-Blood grouping and enzyme } \\
\text { profiling of blood samples from } \\
\text { questioned skin and known } \\
\text { suspect sample }\end{array}$ & $\begin{array}{l}\text {-None in } \\
\text { terms of } \\
\text { physical } \\
\text { match } \\
\text {-Frequency of } \\
\text { occurrence } \\
\text { for } \\
\text { serological } \\
\text { results } \\
\text { reported }\end{array}$ & $\begin{array}{l}\text {-Questioned and known samples appeared } \\
\text { consistent through visual overlays and fingerprint } \\
\text { void/fill of injured thumb to questioned sample } \\
\text {-Serological testing attributed match between } \\
\text { questioned and known as well }\end{array}$ & 6 \\
\hline $\begin{array}{c}\text { Case } \\
\text { Report }\end{array}$ & $\begin{array}{l}\text { Natural } \\
\text { items }\end{array}$ & $\begin{array}{c}1 \\
\text { questioned, } \\
1 \text { known }\end{array}$ & Qualitative & $\begin{array}{l}\text {-Comparison attempted } \\
\text { between grooves on underside } \\
\text { of questioned and known nail } \\
\text { plates with a comparison } \\
\text { microscope }\end{array}$ & None & $\begin{array}{l}\text {-Examiners offered a probable match due to visual } \\
\text { similarity }\end{array}$ & 7 \\
\hline
\end{tabular}




\begin{tabular}{|c|c|c|c|c|c|c|c|}
\hline $\begin{array}{c}\text { Case } \\
\text { Report }\end{array}$ & Textiles & $\begin{array}{l}1 \\
\text { questioned, } \\
1 \text { known }\end{array}$ & Qualitative & $\begin{array}{l}\text {-Heel aligned to sole by nail } \\
\text { hole location and physical size } \\
\text {-Examined heel and sole for } \\
\text { fluorescent adhesive in } \\
\text { consistent patterns }\end{array}$ & None & $\begin{array}{l}\text {-By applying UV-light, points of comparison were } \\
\text { able to be shown between the questioned heel and } \\
\text { known sole, leading to a physical fit conclusion }\end{array}$ & 11 \\
\hline $\begin{array}{c}\text { Case } \\
\text { Report }\end{array}$ & Metal & $\begin{array}{l}1 \\
\text { questioned, } \\
1 \text { known }\end{array}$ & Qualitative & $\begin{array}{l}\text {-Physical examination of edges } \\
\text { and morphology } \\
\text {-X-ray fluorescence to confirm } \\
\text { elemental composition }\end{array}$ & None & $\begin{array}{l}\text {-Metallic chip was of similar elemental } \\
\text { composition to the material of the fractured } \\
\text { padlock } \\
\text {-Metallic chip appeared to be of similar } \\
\text { morphology to the fractured edge of the padlock }\end{array}$ & 47 \\
\hline $\begin{array}{l}\text { Case } \\
\text { Report }\end{array}$ & Metal & $\begin{array}{l}1 \\
\text { questioned, } \\
1 \text { known }\end{array}$ & Qualitative & $\begin{array}{l}\text {-Pop-top tab compared to } \\
\text { empty beer can using } \\
\text { comparison microscopy } \\
\text {-Striations observed as well as } \\
\text { separation/tear patterns on rim } \\
\text { of can's opening and rim of tab }\end{array}$ & None & $\begin{array}{l}\text {-Striations found to be in alignment } \\
\text {-Separation/tear pattern of pop-tab was also found } \\
\text { to be in alignment with rim of the can's opening }\end{array}$ & 48 \\
\hline $\begin{array}{c}\text { Case } \\
\text { Report }\end{array}$ & Metal & Not given & Qualitative & $\begin{array}{l}\text {-Blade pieces examined under } \\
\text { the microscope }\end{array}$ & None & $\begin{array}{l}\text {-Edges of pieces were found to align (puzzle-like } \\
\text { edges) } \\
\text {-Striated marks both from manufacturer and use } \\
\text { were observed and found to align across fracture }\end{array}$ & 49 \\
\hline $\begin{array}{l}\text { Case } \\
\text { Report }\end{array}$ & Metal & $\begin{array}{l}1 \\
\text { questioned, } \\
1 \text { known }\end{array}$ & Qualitative & $\begin{array}{l}\text {-Fractured antenna edges } \\
\text { compared using a comparison } \\
\text { microscope } \\
\text {-Tool mark striations on } \\
\text { interior of the antenna pieces } \\
\text { observed }\end{array}$ & None & $\begin{array}{c}\text {-Fractured edges distorted so physical fit } \\
\text { examination was inconclusive } \\
\text {-Striations were found to align across fracture } \\
\text {-External surface scratches/marks also in } \\
\text { alignment } \\
\text {-Questioned antenna piece was concluded to have } \\
\text { come from suspect's car }\end{array}$ & 50 \\
\hline $\begin{array}{l}\text { Case } \\
\text { Report }\end{array}$ & Metal & $\begin{array}{l}1 \\
\text { questioned, } \\
1 \text { known }\end{array}$ & Qualitative & $\begin{array}{l}\text {-Questioned blade piece compared to known } \\
\text { knife } \\
\text {-Blood present on both items collected for } \\
\text { testing } \\
\text {-Both a physical fit and tool mark } \\
\text { examination were completed }\end{array}$ & None & $\begin{array}{l}\text {-Physical fit discovered between questioned blade } \\
\text { fragment and known knife through fracture edge } \\
\text { morphology and consistency in blade striations }\end{array}$ & 51 \\
\hline
\end{tabular}




\begin{tabular}{|c|c|c|c|c|c|c|c|}
\hline $\begin{array}{c}\text { Case } \\
\text { Report }\end{array}$ & Metal & $\begin{array}{l}1 \\
\text { questioned, } \\
1 \text { known }\end{array}$ & Qualitative & $\begin{array}{l}\text {-Broken piece of tailpipe } \\
\text { compared to the intact } \\
\text { remainder on vehicle } \\
\text {-Edges were compared visually }\end{array}$ & None & $\begin{array}{c}\text {-Edges of tailpipe pieces corresponded while } \\
\text { muffler was still attached to car } \\
\text {-Questioned piece aligned with a bracket on } \\
\text { tailpipe corresponding in location to a hook } \\
\text { attached to the underside of the car designed to } \\
\text { hold tailpipe in place } \\
\text {-When removed from car for closer inspection, } \\
\text { edges fit together and metal seam corresponded } \\
\text { across known and questioned pieces } \\
\text {-The tailpipe was concluded to have come from } \\
\text { the vehicle }\end{array}$ & 52 \\
\hline $\begin{array}{c}\text { Case } \\
\text { Report }\end{array}$ & Metal & $\begin{array}{c}1 \\
\text { questioned, } \\
1 \text { known }\end{array}$ & Qualitative & $\begin{array}{l}\text {-Pieces of screwdriver aligned } \\
\text { side by side }\end{array}$ & None & $\begin{array}{c}\text {-Fracture pattern and striae found to correspond } \\
\text { visually }\end{array}$ & 53 \\
\hline $\begin{array}{c}\text { Case } \\
\text { Report }\end{array}$ & Metal & $\begin{array}{l}1 \\
\text { questioned, } \\
1 \text { known }\end{array}$ & Qualitative & $\begin{array}{l}\text {-Questioned antenna piece } \\
\text { compared by comparison } \\
\text { microscope to the antenna from } \\
\text { car }\end{array}$ & None & $\begin{array}{l}\text {-Ends were found to correspond } \\
\text {-Linear marks on outside of antenna were found to } \\
\text { align across the edges }\end{array}$ & 54 \\
\hline $\begin{array}{c}\text { Case } \\
\text { Report }\end{array}$ & $\begin{array}{c}\text { Hard } \\
\text { plastic }\end{array}$ & $\begin{array}{l}2 \\
\text { questioned, } \\
2 \text { known }\end{array}$ & Qualitative & $\begin{array}{l}\text {-Broken pieces of a wheel well } \\
\text { from scene were visually } \\
\text { compared to wheel well of a } \\
\text { suspect's car }\end{array}$ & None & $\begin{array}{l}\text {-Questioned pieces were found to visually align } \\
\text { with known wheel well }\end{array}$ & 55 \\
\hline $\begin{array}{c}\text { Case } \\
\text { Report }\end{array}$ & $\begin{array}{l}\text { Metal, } \\
\text { hard } \\
\text { plastic }\end{array}$ & $\begin{array}{l}1 \\
\text { questioned, } \\
1 \text { known }\end{array}$ & Qualitative & $\begin{array}{l}\text {-A roof located at a chop shop } \\
\text { was compared to the roof } \\
\text { beams of a known vehicle }\end{array}$ & None & $\begin{array}{c}\text {-A physical fit was discovered due to physical } \\
\text { examination and measurements }\end{array}$ & 56 \\
\hline $\begin{array}{l}\text { Case } \\
\text { Report }\end{array}$ & Metal & $\begin{array}{l}\text { Multiple } \\
\text { questioned, } \\
1 \text { known } \\
\text { for each } \\
\text { case } \\
\text { presented }\end{array}$ & Qualitative & $\begin{array}{l}\text {-Questioned bullet fragments from } \\
\text { scene were compared to known } \\
\text { fragments removed from victim's } \\
\text { body via comparison microscopy } \\
\text { and experimentation with various } \\
\text { lighting conditions in each of two } \\
\text { cases }\end{array}$ & None & $\begin{array}{c}\text { Two cases covered: } \\
\text {-A physical fit was determined between scene fragments } \\
\text { and fragment recovered from victim's leg } \\
\text {-A physical fit was determined by two independent } \\
\text { examiners between scene fragments and fragment } \\
\text { recovered from victim's body }\end{array}$ & 57 \\
\hline
\end{tabular}




\begin{tabular}{|c|c|c|c|c|c|c|c|}
\hline $\begin{array}{c}\text { Case } \\
\text { Report }\end{array}$ & Metal & $\begin{array}{l}3 \\
\text { questioned, } \\
1 \text { known }\end{array}$ & Qualitative & $\begin{array}{l}\text {-Three broken rifle pieces } \\
\text { recovered from robbery scene } \\
\text { were examined visually in } \\
\text { comparison to suspect's broken } \\
\text { trigger guard }\end{array}$ & None & $\begin{array}{l}\text {-Pieces fit together visually along the fracture } \\
\text { edges } \\
\text {-Surface material on outside of trigger guard } \\
\text { indicated that the stock was refinished and the gun } \\
\text { reassembled while wet, adding additional } \\
\text { probative value to fit }\end{array}$ & 58 \\
\hline $\begin{array}{l}\text { Case } \\
\text { Report }\end{array}$ & Metal & $\begin{array}{l}2 \\
\text { questioned, } \\
1 \text { known }\end{array}$ & Qualitative & $\begin{array}{l}\text {-Casts were made of questioned } \\
\text { lock core and dusted with grey } \\
\text { fingerprint powder to reduce } \\
\text { translucency and glare } \\
\text {-Cast was then compared } \\
\text { microscopically to known } \\
\text { ignition wing cap }\end{array}$ & None & $\begin{array}{l}\text {-Fracture marks on wing cap were found to } \\
\text { correspond to one out of two questioned locks }\end{array}$ & 59 \\
\hline $\begin{array}{l}\text { Case } \\
\text { Report }\end{array}$ & Textiles & $\begin{array}{l}\text { Questioned } \\
\text { fragment(s) } \\
, 1 \text { known } \\
\text { item for } \\
\text { each case } \\
\text { presented }\end{array}$ & Qualitative & $\begin{array}{l}\text {-Comparison of questioned } \\
\text { textile fragment(s) to known } \\
\text { item }\end{array}$ & None & $\begin{array}{c}\text { Two cases are presented: } \\
\text {-Torn textile fragments used to bandage victim's } \\
\text { hand during crime were discovered to physically } \\
\text { fit to suspect's shirt } \\
\text {-A textile fragment found on bumper of suspect's } \\
\text { vehicle was found to physically fit to victim's torn } \\
\text { coat }\end{array}$ & 60 \\
\hline $\begin{array}{l}\text { Case } \\
\text { Report }\end{array}$ & $\begin{array}{l}\text { Paint, } \\
\text { Textiles }\end{array}$ & $\begin{array}{l}4 \\
\text { questioned, } \\
4 \text { known }\end{array}$ & Qualitative & $\begin{array}{c}\text {-Physical match examination, } \\
\text { comparison of depression } \\
\text { marks, and comparison of } \\
\text { micro-topography } \\
\text {-Paintings examined under UV } \\
\text { illumination to recognize edges } \\
\text { had been painted over } \\
\text {-Acetone used to remove added } \\
\text { paint and original, known } \\
\text { canvas edges were compared to } \\
\text { questioned cut stretchers }\end{array}$ & None & $\begin{array}{l}\text {-Examiners discovered distinct physical fits due to } \\
\text { the complex morphology of the distorted canvas as } \\
\text { compared to the cut stretchers }\end{array}$ & 61 \\
\hline
\end{tabular}




\begin{tabular}{|c|c|c|c|c|c|c|c|}
\hline $\begin{array}{c}\text { Case } \\
\text { Report }\end{array}$ & Textiles & $\begin{array}{c}\text { Multiple } \\
\text { questioned } \\
\text { and known }\end{array}$ & Qualitative & $\begin{array}{l}\text {-Castings of three family } \\
\text { members' bare feet were made } \\
\text { to determine which of three } \\
\text { pairs of shoes belonged to each } \\
\text { individual } \\
\text {-It was noticed insoles of } \\
\text { questioned pair of shoes } \\
\text { appeared slightly different in } \\
\text { coloration and wear. Therefore, } \\
\text { it was suspected that the insoles } \\
\text { of the three pairs of shoes had } \\
\text { been switched in previous } \\
\text { examinations } \\
\text {-Insoles and shoes then } \\
\text { examined in all combinations }\end{array}$ & None & $\begin{array}{c}\text {-Examiners were able to discover a physical fit } \\
\text { about } 2 \mathrm{~cm} \text { long between a questioned insole and } \\
\text { inner shoe bottom } \\
\text {-Due to wear pattern, parts of insole had adhered } \\
\text { to inside of shoe, leaving a characteristic contour } \\
\text { pattern appearing as mirror images between the } \\
\text { insole and shoe }\end{array}$ & 62 \\
\hline $\begin{array}{c}\text { Case } \\
\text { Report }\end{array}$ & Textiles & $\begin{array}{c}1 \\
\text { questioned, } \\
1 \text { known }\end{array}$ & $\begin{array}{l}\text { Qualitative } \\
\text { comparison } \\
\text { with } \\
\text { quantitative } \\
\text { measurement }\end{array}$ & $\begin{array}{l}\text {-Ropes examined by diameter, } \\
\text { direction of twist, number of } \\
\text { twists per unit length, material } \\
\text { used to construct the rope, } \\
\text { number of strands, threads, and } \\
\text { fibers }\end{array}$ & None & $\begin{array}{l}\text {-Examination of ropes and cords should always } \\
\text { begin with a stereoscopic examination of cut edges } \\
\text {-Rope contained two orange fiberglass cords, one } \\
\text { of which matched the spool }\end{array}$ & 63 \\
\hline $\begin{array}{c}\text { Case } \\
\text { Report }\end{array}$ & $\begin{array}{l}\text { Non- } \\
\text { textile } \\
\text { cords }\end{array}$ & $\begin{array}{c}1 \\
\text { questioned, } \\
1 \text { known }\end{array}$ & Qualitative & $\begin{array}{l}\text {-Comparison requested } \\
\text { between questioned fishing line } \\
\text { fragment, known knife blade, } \\
\text { and known broken fishing line } \\
\text {-Questioned and known line } \\
\text { pieces were inserted into } \\
\text { hypodermic needles to hold line } \\
\text { in place }\end{array}$ & None & $\begin{array}{l}\text {-Knife was not found to impart any distinct } \\
\text { features/residues on the line } \\
\text {-Lines were severed in one straight pass, so there } \\
\text { were not any distinct features or irregularities } \\
\text {-Examiner observed extrusion/striae patterns } \\
\text { corresponded across the edges of the fishing line } \\
\text { pieces } \\
\text {-A physical fit was determined between the lines }\end{array}$ & 64 \\
\hline
\end{tabular}




\begin{tabular}{|c|c|c|c|c|c|c|c|}
\hline $\begin{array}{c}\text { Case } \\
\text { Report }\end{array}$ & $\begin{array}{c}\text { Soft } \\
\text { plastics }\end{array}$ & $\begin{array}{l}1 \\
\text { questioned, } \\
1 \text { known }\end{array}$ & Qualitative & $\begin{array}{l}\text {-Trash bag examination for } \\
\text { consecutive manufacture } \\
\text { determination between } \\
\text { questioned bags and known roll } \\
\text {-Manufacturing plant to learn } \\
\text { of melt pattern characteristics } \\
\text { that can be used to associate } \\
\text { consecutive trash bags }\end{array}$ & None & $\begin{array}{c}\text {-Manufacturer-imparted, melt pattern } \\
\text { characteristics of trash bags such as lines and } \\
\text { arrowheads can be used to associate consecutive } \\
\text { trash bags } \\
\text {-These features can be revealed with transmitted } \\
\text { lighting }\end{array}$ & 65 \\
\hline $\begin{array}{c}\text { Case } \\
\text { Report }\end{array}$ & $\begin{array}{c}\text { Soft } \\
\text { plastics }\end{array}$ & $\begin{array}{l}4 \\
\text { questioned, } \\
1 \text { known }\end{array}$ & Qualitative & $\begin{array}{l}\text {-Examination under the } \\
\text { microscope revealed striations } \\
\text { on surface of questioned sole } \\
\text { fragments } \\
\text {-Examination of soles of } \\
\text { suspect's boots revealed similar } \\
\text { striations and missing portions } \\
\text {-Voids in soles cast in Mikrosil } \\
\text { and then compared to the } \\
\text { fragments }\end{array}$ & None & $\begin{array}{l}\text {-Direct physical fit inconclusive before casting } \\
\text {-Fragments were concluded as having come from } \\
\text { the suspect's soles due to alignment in striations } \\
\text { between cast voids and sole fragments }\end{array}$ & 66 \\
\hline $\begin{array}{c}\text { Case } \\
\text { Report }\end{array}$ & $\begin{array}{c}\text { Hard } \\
\text { plastic }\end{array}$ & $\begin{array}{l}1 \\
\text { questioned, } \\
1 \text { known }\end{array}$ & Qualitative & $\begin{array}{l}\text {-Questioned blade fragments } \\
\text { were compared visually to two } \\
\text { known knives } \\
\text {-Questioned sample and a } \\
\text { section of one of the broken } \\
\text { blade fragments were cast using } \\
\text { Mikrosil }\end{array}$ & None & $\begin{array}{c}\text {-Casts were found to have similar features } \\
\text {-Direct comparison with reverse lighting revealed } \\
\text { a physical fit }\end{array}$ & 67 \\
\hline $\begin{array}{c}\text { Case } \\
\text { Report }\end{array}$ & Paint & $\begin{array}{l}\text { Multiple } \\
\text { questioned } \\
\text { and known } \\
\text { evidence } \\
\text { items for } \\
\text { each case } \\
\text { presented }\end{array}$ & Qualitative & $\begin{array}{l}\text {-Multiple case examples of } \\
\text { paint physical fits are covered, } \\
\text { demonstrating photographic } \\
\text { techniques }\end{array}$ & None & $\begin{array}{c}\text { Multiple paint physical fits are demonstrated: } \\
\text {-Physical fit discovered between architectural paint chips in a } \\
\text { housebreaking case } \\
\text {-Physical fit discovered between paint chips from a burglarized } \\
\text { safe } \\
\text {-Physical fit discovered between a torn price tag and flaking } \\
\text { crow bar paint } \\
\text {-Physical fit discovered between a paint chip recovered from a } \\
\text { screwdriver head and a damaged door frame }\end{array}$ & 68 \\
\hline
\end{tabular}




\begin{tabular}{|c|c|c|c|c|c|c|c|}
\hline $\begin{array}{c}\text { Case } \\
\text { Report }\end{array}$ & Paint & $\begin{array}{c}1 \\
\text { questioned, } \\
1 \text { known } \\
\text { for each } \\
\text { case } \\
\text { presented }\end{array}$ & Qualitative & $\begin{array}{l}\text {-Two cases reviewed where } \\
\text { external striations on } \\
\text { automotive paint chips were } \\
\text { used to connect questioned } \\
\text { paint chips to a vehicle } \\
\text {-Comparison microscopy } \\
\text { utilized in both cases }\end{array}$ & None & $\begin{array}{l}\text {-In the first case, a paint chip collected from a } \\
\text { body was found to correspond to the damaged } \\
\text { fender of a suspect's vehicle by alignment in } \\
\text { topcoat between fragments } \\
\text {-In the second case, external striations were found } \\
\text { to align across the edges of both questioned paint } \\
\text { chips and known vehicular damage }\end{array}$ & 69 \\
\hline $\begin{array}{c}\text { Case } \\
\text { Report }\end{array}$ & $\begin{array}{l}\text { Wooden } \\
\text { Objects }\end{array}$ & $\begin{array}{l}2 \\
\text { questioned, } \\
1 \text { known }\end{array}$ & Qualitative & $\begin{array}{l}\text {-Questioned section of stump } \\
\text { was compared to the end of a } \\
\text { tree in the possession of the } \\
\text { suspects as well as a piece of } \\
\text { wood found at the scene } \\
\text {-Examiners observed grain, } \\
\text { rings, and pattern of fracture } \\
\text {-Examiners cast a section of the } \\
\text { stump in molding material, and } \\
\text { then compared to suspect log }\end{array}$ & None & $\begin{array}{l}\text {-Examiners concluded wedge piece found at scene } \\
\text { physically fit to log from the suspects } \\
\text {-Cast and known log found it to be in alignment in } \\
\text { microscopic characteristics }\end{array}$ & 70 \\
\hline $\begin{array}{c}\text { Case } \\
\text { Report }\end{array}$ & $\begin{array}{l}\text { Wooden } \\
\text { Objects }\end{array}$ & $\begin{array}{c}4 \text { items, } \\
\text { unclear } \\
\text { which are } \\
\text { questioned } \\
\text { vs. known }\end{array}$ & Qualitative & $\begin{array}{l}\text {-Four fragments of a broken } \\
\text { pool cue stick were compared } \\
\text { to determine if they originated } \\
\text { from the same or multiple items }\end{array}$ & None & $\begin{array}{l}\text {-A physical fit was discovered between each of the } \\
\text { four pieces, revealing they likely originated from } \\
\text { the same cue stick }\end{array}$ & 71 \\
\hline $\begin{array}{c}\text { Case } \\
\text { Report }\end{array}$ & $\begin{array}{l}\text { Wooden } \\
\text { Objects }\end{array}$ & $\begin{array}{l}1 \\
\text { questioned, } \\
1 \text { known }\end{array}$ & Qualitative & $\begin{array}{l}\text {-Questioned wood chip from scene and } \\
\text { damaged pallet piece from suspect's vehicle } \\
\text { were scanned at various resolutions using } \\
\text { photography and blending techniques } \\
\text {-Scanned images were opened in Adobe } \\
\text { Photoshop CS2 and red dots placed on } \\
\text { known pallet image used to overlay and } \\
\text { orient image of questioned wood chip } \\
\text {-Varying levels of opacity used to achieve } \\
\text { optimal viewing of the corresponding } \\
\text { striations and contours of the wood }\end{array}$ & None & $\begin{array}{c}\text {-Examiners determined a physical fit between the } \\
\text { questioned wood fragment and known pallet }\end{array}$ & 72 \\
\hline
\end{tabular}




\begin{tabular}{|c|c|c|c|c|c|c|}
\hline $\begin{array}{c}\text { Case } \\
\text { Report }\end{array}$ & $\begin{array}{c}\text { Non- } \\
\text { textile } \\
\text { cords }\end{array}$ & Not given & Qualitative & $\begin{array}{c}\text {-Known wire ends from the } \\
\text { scene of a stolen truck radio } \\
\text { were compared visually to } \\
\text { questioned wires from a } \\
\text { recovered radio }\end{array}$ & None & $\begin{array}{c}\text {-Air pockets were observed on both sides of the } \\
\text { severed edges in the insulation that were found to } \\
\text { correspond across severed edges }\end{array}$ \\
\hline Case & $\begin{array}{c}\text { Non- } \\
\text { textile } \\
\text { cords }\end{array}$ & $\begin{array}{c}\text { questioned, } \\
\text { known }\end{array}$ & Qualitative & $\begin{array}{c}-6 \text { stolen cable fragments } \\
\text { compared visually to } 2 \text { sections } \\
\text { cut from the scene } \\
\text {-Examiners cut cable sections } \\
\text { horizontally to lay material flat } \\
\text { for examination of whole } \\
\text { fracture }\end{array}$ & None & $\begin{array}{c}\text {-The examiner discovered a fit between one of the } \\
\text { standard sections and one of the evidence sections } \\
\text { on the outer layer of the wire } \\
\text {-The examiner was able to observe an inner layer } \\
\text { of the wire with wording that also aligned }\end{array}$ \\
\hline
\end{tabular}


Table B. Fractography Articles Summary

\begin{tabular}{|c|c|c|c|c|c|c|c|}
\hline Category & $\begin{array}{c}\text { Material } \\
\text { Type }\end{array}$ & $\begin{array}{c}\text { Population } \\
\text { Size }\end{array}$ & $\begin{array}{l}\text { Qualitative } \\
\text { or } \\
\text { Quantitative } \\
\text { Assessment? }\end{array}$ & Experimental Design & $\begin{array}{c}\text { Statistical } \\
\text { Performance } \\
\text { Measures }\end{array}$ & Main Findings & $\begin{array}{c}\text { Reference } \\
\text { Number }\end{array}$ \\
\hline $\begin{array}{c}\text { Fractography/ } \\
\text { Qualitative }\end{array}$ & Glass & NA & Qualitative & $\begin{array}{l}\text {-A convex glass chip is placed } \\
\text { in its concave original medium } \\
\text { and the alignment is viewed } \\
\text { under the microscope through } \\
\text { the chip surface (normal to the } \\
\text { fracture) } \\
\text {-Photos are taken both with the } \\
\text { surfaces aligned and slightly } \\
\text { displaced to reveal both sets of } \\
\text { hackle marks }\end{array}$ & None & $\begin{array}{c}\text {-Aligned glass fractures should be } \\
\text { photographed both in alignment and } \\
\text { slightly displaced } \\
\text {-There are two types of glass fracture } \\
\text { markings: rib (the main, oyster shell-like } \\
\text { fractures) and hackle (small striae normal } \\
\text { to rib markings) } \\
\text {-Hackle markings are most useful in } \\
\text { establishing alignment }\end{array}$ & 9 \\
\hline $\begin{array}{c}\text { Fractography/ } \\
\text { Qualitative }\end{array}$ & $\begin{array}{c}\text { Matchsticks/ } \\
\text { paper } \\
\text { matches }\end{array}$ & $\begin{array}{c}8 \text { match } \\
\text { booklets; } 4 \\
\text { Canadian, } 2 \\
\text { American, } 1 \\
\text { Brazilian, } 1 \\
\text { Japanese }\end{array}$ & Qualitative & $\begin{array}{l}\text {-Methods of comparison for } \\
\text { consecutive match fractures are } \\
\text { explored, as well as effect of } \\
\text { dye on match surface fibers } \\
\text {-Matches are dyed with stain } \\
\text { and wooden roller, mounted on } \\
\text { wooden blocks, and compared } \\
\text { under both stereo and } \\
\text { comparison microscopes }\end{array}$ & None & $\begin{array}{c}\text {-Consecutive match comparisons in this set } \\
\text { were not reported to cause false positives } \\
\text {-Concluded a reliable, cheap, and easy } \\
\text { technique }\end{array}$ & 10 \\
\hline
\end{tabular}




\begin{tabular}{|c|c|c|c|c|c|c|c|}
\hline $\begin{array}{c}\text { Fractography/ } \\
\text { Qualitative }\end{array}$ & Soft plastics & $\begin{array}{c}-13 \text { packages of } \\
\text { garbage bags: } \\
10 \text { packages of } \\
\text { various brands } \\
\text { purchased from } \\
\text { local stores; } 3 \\
\text { retail packages } \\
\text { obtained from } 2 \\
\text { manufacturing } \\
\text { plants } \\
-13 \\
\text { consecutively } \\
\text { made garbage } \\
\text { bags obtained } \\
\text { from a } \\
\text { manufacturing } \\
\text { plant } \\
-7 \text { packages of } \\
\text { sandwich bags: } \\
5 \text { of various } \\
\text { brands } \\
\text { purchased from } \\
\text { local stores; } 2 \\
\text { obtained from a } \\
\text { manufacturing } \\
\text { plant }\end{array}$ & $\begin{array}{c}\text { Qualitative } \\
\text { comparison } \\
\text { with } \\
\text { quantitative } \\
\text { measurement }\end{array}$ & $\begin{array}{c}\text {-Bags first examined for color, } \\
\text { size, perforations, construction, } \\
\text { code, pigment bands, and } \\
\text { hairline marks presence or } \\
\text { absence } \\
\text {-For garbage bags, production } \\
\text { sequence determined by finding } \\
\text { slope of a prominent marking } \\
\text { across all bags } \\
\text {-Bags then examined for } \\
\text { colored striations under crossed } \\
\text { polars, as well as individual } \\
\text { characteristics including } \\
\text { fisheyes, arrowheads, streaks, } \\
\text { and tiger stripes } \\
\text {-Individual characteristics } \\
\text { examined on sandwich bags } \\
\text { include surface scratches and } \\
\text { colored bands }\end{array}$ & None & $\begin{array}{l}\text {-Knowledge from the manufacturing } \\
\text { process can be utilized to discern the order } \\
\text { or markings across multiple plastic bags } \\
\text {-Bags can be thought of as consecutive } \\
\text { when both class and individual } \\
\text { characteristics align }\end{array}$ & 12 \\
\hline
\end{tabular}




\begin{tabular}{|c|c|c|c|c|c|c|c|}
\hline $\begin{array}{c}\text { Fractography/ } \\
\text { Qualitative }\end{array}$ & Paints & $\begin{array}{l}6 \text { vehicles, } 2 \\
\text { models (Ford } \\
\text { Telstar and } \\
\text { Ford Laser), } \\
\text { two points of } \\
\text { contact in hinge } \\
\text { of driver's door } \\
\text { per vehicle }\end{array}$ & Qualitative & $\begin{array}{c}\text {-Two points of contact were } \\
\text { photographed in driver door } \\
\text { hinge area of } 6 \text { vehicles at a } \\
\text { production plant } \\
\text {-Photographs, as well as their } \\
\text { negatives, were compared over } \\
\text { a light box for pattern } \\
\text { consistency between known } \\
\text { door and hinge, and also } \\
\text { between vehicles }\end{array}$ & None & $\begin{array}{l}\text {-Gaps between panels allowed capillaries } \\
\text { of the surface coating to form, revealing } \\
\text { striations that could be aligned between } \\
\text { door and hinge } \\
\text {-Corresponding pattern would appear on a } \\
\text { panel beside door if capillaries broke } \\
\text { unevenly } \\
\text {-If there was poor electro coating between } \\
\text { panels, these patterns would not be } \\
\text { displayed at all } \\
\text {-Patterns were distinguishable between } \\
\text { vehicles } \\
\text {-Methods of court presentation: mounting } \\
\text { photographs to reveal the mirror image, } \\
\text { reversing one of the images to directly } \\
\text { show points of comparison, or producing a } \\
\text { high contrast transparency of one of the } \\
\text { photographs to be overlaid on the other }\end{array}$ & 14 \\
\hline $\begin{array}{c}\text { Fractography/ } \\
\text { Qualitative }\end{array}$ & $\begin{array}{c}\text { Glass, } \\
\text { Metal, Hard } \\
\text { plastics }\end{array}$ & Not given & Qualitative & $\begin{array}{l}\text {-Three different loads were used } \\
(0.98 \mathrm{~N}, 2.0 \mathrm{~N} \text {, and } 2.9 \mathrm{~N}) \text { for a hard } \\
\text { indenter to reproducibly create } \\
\text { fractures } \\
\text {-The second part of the study was } \\
\text { bending of glass, in which a } \\
\text { universal testing machine was used } \\
\text { to create reproducible load } \\
\text { distributions } \\
\text {-The third test was with polymers } \\
\text { using an impact "hail-stone gun". } \\
\text { Plastic balls were discharged at } \\
\text { polymethyl methacrylate (PMMA) } \\
\text { sheets } \\
\text {-Tensile tests completed on steel } \\
\text { wires }\end{array}$ & None & $\begin{array}{l}\text {-Fractures were found to have random } \\
\text { distributions of cracks } \\
\text {-Cracks themselves were found to have } \\
\text { random number, lengths, propagations, } \\
\text { directions, shapes, and orientations } \\
\text {-Curves and fractures made in the second } \\
\text { study were also randomly distributed } \\
\text {-Cracks from the impact (third study) was } \\
\text { found to also be random } \\
\text {-Curves and fracture surfaces of the wires } \\
\text { were random and varied between the } \\
\text { different wires, despite being made of the } \\
\text { same material } \\
\text {-The steel wires were found to allow for a } \\
\text { fracture match between the edges }\end{array}$ & 77 \\
\hline
\end{tabular}




\begin{tabular}{|c|c|c|c|c|c|c|c|}
\hline $\begin{array}{c}\text { Fractography/ } \\
\text { Qualitative }\end{array}$ & Tape & Not given & Qualitative & $\begin{array}{l}\text {-Tapes from six different } \\
\text { manufacturers were torn by } \\
\text { hand and observed with a } \\
\text { comparison microscope } \\
\text {-The edges treated with } 100 \\
\text { Celsius hot air for a few } \\
\text { seconds } \\
\text {-After treatment the tapes were } \\
\text { re-observed under comparison } \\
\text { microscopy }\end{array}$ & None & $\begin{array}{l}\text {-Heat treatment was found to make it } \\
\text { easier to find the corresponding edge, and } \\
\text { improved confidence in the conclusion } \\
\text {-The author did note however that } \\
\text { applying heat treatment may destroy other } \\
\text { evidence (DNA, fingerprints) }\end{array}$ & 78 \\
\hline $\begin{array}{c}\text { Fractography/ } \\
\text { Qualitative }\end{array}$ & Tape & NA & Qualitative & $\begin{array}{l}\text {-Tapes were either sheared or } \\
\text { torn, heat-treated at } 100^{\circ} \mathrm{C} \text { with } \\
\text { demineralized water to undo } \\
\text { any plastic deformation } \\
\text { occurring after fracture, cast } \\
\text { with casting material, and each } \\
\text { edge of the fracture cast was } \\
\text { examined using comparison } \\
\text { microscopy for fracture } \\
\text { matching }\end{array}$ & None & $\begin{array}{l}\text {-Each tested fracture generated an } \\
\text { individual fracture pattern of which a cast } \\
\text { could be taken for nearly mirror-image } \\
\text { comparison microscopy results }\end{array}$ & 79 \\
\hline $\begin{array}{c}\text { Fractography/ } \\
\text { Qualitative }\end{array}$ & Tape & Not given & Qualitative & $\begin{array}{l}\text {-Tapes torn by hand and cut } \\
\text { with scissors to demonstrate } \\
\text { non-reproducibility }\end{array}$ & None & $\begin{array}{c}\text {-Tearing and shearing black electrical tape } \\
\text { samples left distinct tears that were non- } \\
\text { reproducible }\end{array}$ & 80 \\
\hline $\begin{array}{c}\text { Fractography/ } \\
\text { Qualitative }\end{array}$ & Soft plastics & NA & Qualitative & $\begin{array}{l}\text {-A review/recommendation for analysis } \\
\text { of garbage bags for consecutive } \\
\text { manufacturing identification rather than } \\
\text { a study with actual samples } \\
\text {-Garbage bags can be aligned according } \\
\text { to their heat-sealed edges/ending. } \\
\text { Transmitted light from underneath can } \\
\text { reveal striations from the } \\
\text { manufacturing process that can attribute } \\
\text { to a common source }\end{array}$ & None & $\begin{array}{c}\text {-Horizontal streaks in plastic bag material } \\
\text { formed during the manufacturing process are in } \\
\text { the following categories: } \\
\text { 1-fisheyes (randomly-distributed dark } \\
\text { pigments) } \\
\text { 2-arrowheads (triangular striae of dark pigment) } \\
\text { 3-tiger stripes (horizontal striae of dark } \\
\text { pigment) } \\
\text { 4-die lines (become visible in the blowing and } \\
\text { stretching process, straight horizontal lines) }\end{array}$ & 81 \\
\hline
\end{tabular}




\begin{tabular}{|c|c|c|c|c|c|c|c|}
\hline $\begin{array}{l}\text { Fractography/ } \\
\text { Qualitative }\end{array}$ & Soft plastics & NA & Qualitative & $\begin{array}{l}\text {-Summary of characteristics of } \\
\text { polyethylene films that can be } \\
\text { used for comparisons and } \\
\text { manufacturing processes }\end{array}$ & NA & $\begin{array}{l}\text {-Additives to films from manufacturing } \\
\text { appear as striations/patterning } \\
\text {-Extrusion marks originate from the roller } \\
\text {-Additional scratches and surface striations } \\
\text { come from machine wear } \\
\text {-Dye variations come from uneven } \\
\text { applications of dye }\end{array}$ & 82 \\
\hline $\begin{array}{l}\text { Fractography/ } \\
\text { Qualitative }\end{array}$ & Soft plastics & NA & Qualitative & $\begin{array}{l}\text {-Black card was cut to have } 1 / 8 \\
\text { in X } 6 \frac{1}{2} \text { slots. Two sheets of } \\
\text { glass were put together and } \\
\text { placed above the grid. The grid } \\
\text { was illuminated by a } 500 \text {-watt } \\
\text { lamp at a right angle } \\
\text {-Camera was focused on the } \\
\text { glass in the frame so that the } \\
\text { whole area of glass would be in } \\
\text { the negative } \\
\text {-Polyethylene piece was } \\
\text { sandwiched between the glass } \\
\text { sheets with the extrusion marks } \\
\text { on the short side }\end{array}$ & NA & $\begin{array}{l}\text {-The photography method was found to be } \\
\text { useful for visualizing and documenting } \\
\text { extrusion marks in polyethylene film }\end{array}$ & 83 \\
\hline
\end{tabular}




\begin{tabular}{|c|c|c|c|c|c|c|c|}
\hline $\begin{array}{c}\text { Fractography/ } \\
\text { Qualitative }\end{array}$ & Soft plastics & NA & Qualitative & $\begin{array}{c}\text {-This paper focuses on } \\
\text { photographing physical } \\
\text { characteristics of plastic bags } \\
\text { and film that have potential to } \\
\text { be used to denote matching } \\
\text { edges or connected pieces of } \\
\text { evidence }\end{array}$ & None & $\begin{array}{l}\text {-Extrusion marks are recommended to be } \\
\text { photographed using a secondary lens } \\
\text { system so that the extrusion marks can be } \\
\text { focused at any magnification } \\
\text {-Heat marks originate from bags that are } \\
\text { sealed together by an individual separately } \\
\text { from the manufacturing heat seals } \\
\text {-Secondary heat marks were often created } \\
\text { using a soldering iron or laundry iron, or } \\
\text { by commercially made sealing machines } \\
\text {-For sealing machines, conclusions could } \\
\text { be made by examining the patterns left by } \\
\text { the heat proof fabric on the machine, by } \\
\text { observing inclusions and irregularities } \\
\text { created in consecutive seals made by the } \\
\text { same machine, and by hot spots (unique } \\
\text { areas of deformation caused by heat) } \\
\text {-Cut edges of films could offer some } \\
\text { additional details if the instrument used to } \\
\text { sever the edges left similar characteristics } \\
\text { (snags, changes in direction of cut, etc.) }\end{array}$ & 84 \\
\hline
\end{tabular}




\begin{tabular}{|c|c|c|c|c|c|c|c|}
\hline $\begin{array}{c}\text { Fractography/ } \\
\text { Qualitative }\end{array}$ & Soft plastics & NA & Qualitative & $\begin{array}{l}\text {-Summary of a variety of } \\
\text { methods that can be used to } \\
\text { visualize and assess physical } \\
\text { properties of plastic bags and } \\
\text { cling film } \\
\text {-Kinds of properties that can be } \\
\text { utilized include color and } \\
\text { variation of die lines, } \\
\text { polarization patterns, striations } \\
\text { from manufacturing } \\
\text {-Summary as well of the } \\
\text { manufacturing of plastic bags } \\
\text { and film: } \\
\text {-Manufacturing: plastic bags } \\
\text { are made by blowing polymer } \\
\text { through a circular tube and then } \\
\text { flattened. Cling film is also } \\
\text { made by a blown film } \\
\text { extrusion, but forms a single } \\
\text { sheet that is wound up } \\
\text {-Finally, four cases mentioned } \\
\text { in which characteristics of } \\
\text { plastic bags were viewed to } \\
\text { allow for matching }\end{array}$ & None & $\begin{array}{l}\text {-Polarization (polarization table): used } \\
\text { because many polymeric films are } \\
\text { birefringent. Consecutively produced bags } \\
\text { often have similar or consecutive colors } \\
\text { under cross-polars, and the patterns can be } \\
\text { compared to fit matching bags together } \\
\text {-Shadowgraph and Schlieren imaging: } \\
\text { shadowgraphs involve a point light source } \\
\text { at an angle to the film, highlighting } \\
\text { discontinuities and defects within the film. } \\
\text { The film is photographed in front of the } \\
\text { light. For Schlieren, point source is } \\
\text { directed through a convex lens or spherical } \\
\text { mirror so that a parallel beam of light } \\
\text { passes through the film. A matching lens } \\
\text { or mirror catches the light and allows for } \\
\text { photography } \\
\text {-Incident and transmitted light microscopy: } \\
\text { microscopes that can be adjusted to allow } \\
\text { for visualization of inhomogeneities of the } \\
\text { films } \\
\text {-Four cases include an instance of printing } \\
\text { defects showing bags produced on the } \\
\text { same production line, a case where the } \\
\text { polarizations colors demonstrated the bags } \\
\text { were produced consecutively, a case where } \\
\text { the polarization, die lines, and striations } \\
\text { demonstrated consecutive manufacturing, } \\
\text { and finally a case where cling film die } \\
\text { lines demonstrated consecutive } \\
\text { manufacturing }\end{array}$ & 85 \\
\hline
\end{tabular}




\begin{tabular}{|c|c|c|c|c|c|c|c|}
\hline $\begin{array}{c}\text { Fractography/ } \\
\text { Qualitative }\end{array}$ & Glass & NA & Qualitative & $\begin{array}{l}\text {-Multiple experiments } \\
\text { described without much } \\
\text { information on methodology } \\
\text {-Looking at how glass fractures } \\
\text { rather than how to piece broken } \\
\text { glass back together }\end{array}$ & None & $\begin{array}{l}\text {-Two major types of fractures: radial and } \\
\text { concentric } \\
\text {-Arcs on radial fractures present concave } \\
\text { opposite the origin of the breaking force, } \\
\text { while the opposite is true of concentric } \\
\text {-Only occurrences of first-order fracture } \\
\text { surfaces (fracture center and first } \\
\text { concentric fracture) should be considered } \\
\text { reliable } \\
\text {-Bullet holes in safety glass have different } \\
\text { chipping - the entrance pane will have } \\
\text { perpendicular chips, the exit will have } \\
\text { chips at an angle with the surface }\end{array}$ & 86 \\
\hline $\begin{array}{c}\text { Fractography/ } \\
\text { Qualitative }\end{array}$ & Glass & $\begin{array}{c}16 \text { glass } \\
\text { samples (4 } \\
\text { types) }\end{array}$ & Quantitative & $\begin{array}{l}\text {-Window panes at three } \\
\text { different thicknesses were shot } \\
\text { with a } 4.5 \mathrm{~mm} \text { air rifle } \\
\text {-Various measurements } \\
\text { recorded on the fracture } \\
\text { patterns including radial } \\
\text { fracture count, concentric } \\
\text { fracture count, bullet hole } \\
\text { diameter, mist zone thickness, } \\
\text { and mist zone diameter }\end{array}$ & $\begin{array}{l}\text {-Chi-Square } \\
\text { Test used to } \\
\text { assess } \\
\text { goodness of } \\
\text { fit or minimal } \\
\text { variation for } \\
\text { measurement } \\
\text { trend lines }\end{array}$ & $\begin{array}{l}\text {-No significant differences were present in } \\
\text { fracture pattern measurements between } \\
\text { both all glass thicknesses, regardless of sun } \\
\text { control film } \\
\text {-Bullet hole diameters in regular rifles tend } \\
\text { to be double the caliber of the firearm } \\
\text { while those of air rifles tend to be similar } \\
\text { to the weapon's caliber. This may be useful } \\
\text { in distinguishing between weapon type }\end{array}$ & 87 \\
\hline
\end{tabular}




\begin{tabular}{|c|c|c|c|c|c|c|c|}
\hline $\begin{array}{c}\text { Fractography/ } \\
\text { Qualitative }\end{array}$ & Glass & NA & Qualitative & $\begin{array}{l}\text {-Quasi-static loading can result } \\
\text { in glass fractures with no } \\
\text { obvious distortions in the glass } \\
\text {-Fracture occurs when the glass } \\
\text { fails at a Griffith crack (minute } \\
\text { flaws that are often a point of } \\
\text { stress concentration) }\end{array}$ & None & $\begin{array}{l}\text {-Dynamic loading is discussed, including } \\
\text { how kinetic energy is transferred to glass - } \\
\text { mainly through direct force by the } \\
\text { projectile and mechanical waves } \\
\text {-The waves produce stress on the glass } \\
\text { structure as the waves reflect off the back } \\
\text { and front of the glass } \\
\text {-The high stress impact of the mechanical } \\
\text { waves creates a crater in the glass, } \\
\text { although penetration of the glass is not } \\
\text { necessary for crater formation as long as } \\
\text { there is enough stress applied to a weak } \\
\text { point/flaw } \\
\text {-Though high amounts of energy may be } \\
\text { transferred, if the velocity of the crack } \\
\text { propagation is not propagated for long, the } \\
\text { extent of the fracturing may be minimal } \\
\text { around the crater } \\
\text {-While cratering can be useful in } \\
\text { reconstruction if the calibers are known, } \\
\text { the size and distribution of the crater and } \\
\text { resulting fractures cannot be used to } \\
\text { provide definitive information about the } \\
\text { calibers if unknown }\end{array}$ & 88 \\
\hline
\end{tabular}




\begin{tabular}{|c|c|c|c|c|c|c|c|}
\hline $\begin{array}{c}\text { Fractography/ } \\
\text { Qualitative }\end{array}$ & $\begin{array}{c}\text { Glass, hard } \\
\text { plastic }\end{array}$ & $\begin{array}{c}60 \text { panes } \\
\text { double-strength } \\
\text { glass, } 60 \text { clear } \\
\text { glass wine } \\
\text { bottles, } 60 \\
\text { polymer } \\
\text { taillight lenses }\end{array}$ & Qualitative & $\begin{array}{l}\text {-60 each of three sample types, } \\
\text { two fracture methods: dynamic } \\
\text { impact and static pressure, } 30 \\
\text { samples each, three fracture tips } \\
\text { (blunt, round, sharp) } \\
\text {-Dynamic: } 8 \text { x } 8 \text { " glass panes, } \\
\text { wine bottles coated with RTV } \\
\text { urethane, } 5.5 / 8 \times 4.1 / 4 \text { " plastic } \\
\text { lens, } 10 \text { glass samples per } \\
\text { dropping weight impact tip, } 10 \\
\text { plastic lenses per dropping } \\
\text { height, reassembled, imaged, } \\
\text { and videoed for velocity } \\
\text { measurements } \\
\text {-Static: } 8 x 8 \text { " sample, wine } \\
\text { bottles coated with RTV } \\
\text { urethane, indenter crosshead } \\
\text { speed } 10 \text { mm/min, } 10 \text { samples } \\
\text { per indenter tip (only wide tip } \\
\text { used on plastic so all } 30 \text { were } \\
\text { the same), load vs extension } \\
\text { measured by Instron software, } \\
\text { reassembled and imaged } \\
\text {-Visual comparisons: fractures } \\
\text { traced onto acetate and overlay } \\
\text { one-to-one per sample at four } \\
\text { orientations (two for bottles) }\end{array}$ & None & $\begin{array}{c}\text {-Blunt fracture tip required the highest } \\
\text { velocity (dynamic) and force (static) while } \\
\text { sharp tips required the least } \\
\text {-Sharp tip fracture patterns contained } \\
\text { fewest lines, blunt tip pattern contained } \\
\text { most lines } \\
\text { Glass panes: Blunt tip created more radial } \\
\text { and concentric fractures, and dynamic } \\
\text { fracture patterns more simple than static } \\
\text { Wine bottles: Number of fractures } \\
\text { between impact tips more evenly } \\
\text { distributed, and fracture patterns between } \\
\text { dynamic and static samples did not vary as } \\
\text { much } \\
\text {-Linear relationship expected between } \\
\text { load and extension, curvature obtained } \\
\text { from load profiles } \\
\text {-In plastic lenses, velocity increased as } \\
\text { drop height increased, causing a center } \\
\text { crushing and edge fracturing } \\
\text {-Plastic extension value exceeds glass } \\
\text { values, however load is smaller }\end{array}$ & 89,90 \\
\hline
\end{tabular}




\begin{tabular}{|c|c|c|c|c|c|c|}
\hline & & & & & $\begin{array}{c}\text {-Noted methods beyond traditional } \\
\text { aligning of irregular surfaces include } \\
\text { microscopic alignment of rib or hackle } \\
\text { marks, identification of continuous ream or } \\
\text { cord via shadowgraph, and visualization of } \\
\text { surface irregularities through laser } \\
\text { interferometry } \\
\text { Qualitative }\end{array}$ \\
$\begin{array}{c}\text { Fractography/ } \\
\text { Glass }\end{array}$ & NA & Qualitative & $\begin{array}{c}\text {-Specific techniques for glass } \\
\text { physical fit examinations } \\
\text { discussed }\end{array}$ & NA & $\begin{array}{c}\text {-These additional techniques arise due to } \\
\text { the three-dimensional nature of glass } \\
\text { physical fit } \\
\text {-Established random formation of glass } \\
\text { fractures by explaining how fractures } \\
\text { propagate through the randomly-oriented } \\
\text { crystal lattice composing glassy materials }\end{array}$ \\
\hline
\end{tabular}




\begin{tabular}{|c|c|c|c|c|c|c|c|}
\hline $\begin{array}{c}\text { Fractography/ } \\
\text { Qualitative }\end{array}$ & Glass & NA & Qualitative & $\begin{array}{l}\text {-Ream (or cord) are markings } \\
\text { imparted due to physical and } \\
\text { chemical property variations } \\
\text { within the glass, and appear as } \\
\text { striations within the glass that } \\
\text { can be visualized by shadow } \\
\text { graphing } \\
\text {-Shadow pattern is developed } \\
\text { as a photograph that allows } \\
\text { visualization of any ream of } \\
\text { cord markings } \\
\text {-14 glass bottles examined for } \\
\text { cord, which was identified in } \\
\text { all samples with varying } \\
\text { patterns between bottles } \\
\text {-Shadowgraphs were also used } \\
\text { to image patterns of six } \\
\text { transparent plastic samples and } \\
\text { five automotive bulbs. } \\
\text {-A study utilizing window glass } \\
\text { obtained from a known } \\
\text { manufacturer was preformed to } \\
\text { examine the frequency and } \\
\text { persistence of ream markings: } \\
\text {-Four sheets of glass were used } \\
\text { to create 1.8-cm wide strips } \\
\text { examined in various } \\
\text { combinations of non- } \\
\text { contiguous distances between } \\
\text { one another }\end{array}$ & None & $\begin{array}{l}-90 \% \text { of ream marks persisted at } 1.8-\mathrm{cm} \\
33 \% \text { persisted at } 13-\mathrm{cm}, 10 \% \text { persisted } \\
\text { over } 70 \mathrm{~cm} \text {, and at a distance of } 140 \mathrm{~cm} \\
\text { none were identified as matching }\end{array}$ & 92 \\
\hline
\end{tabular}




\begin{tabular}{|c|c|c|c|c|c|c|c|}
\hline $\begin{array}{c}\text { Fractography/ } \\
\text { Qualitative }\end{array}$ & $\begin{array}{c}\text { Matchsticks/ } \\
\text { paper } \\
\text { matches }\end{array}$ & NA & Qualitative & $\begin{array}{l}\text {-Match-matchbook pairs } \\
\text { compared according to size, } \\
\text { color, wax dip line of head, and } \\
\text { cut or torn edges before } \\
\text { submersion } \\
\text {-Samples are then submerged } \\
\text { and photographed for further } \\
\text { fracture comparison }\end{array}$ & None & $\begin{array}{l}\text {-Cellulosic surface fibers on matches make } \\
\text { visual fracture comparisons difficult to see, } \\
\text { submersion in high refractive index-liquid } \\
\text { makes these fibers transparent and reveals } \\
\text { more fracture detail to provide inclusions } \\
\text { for matches in casework }\end{array}$ & 93 \\
\hline $\begin{array}{c}\text { Fractography/ } \\
\text { Qualitative }\end{array}$ & $\begin{array}{c}\text { Matchsticks/ } \\
\text { paper } \\
\text { matches }\end{array}$ & 41 matchbooks & Qualitative & $\begin{array}{c}\text {-Match boards (cut into } 10 \text { or } \\
\text { more sections by manufacturer) } \\
\text { removed from books and both } \\
\text { surfaces of book searched for } \\
\text { luminescing inclusions and } \\
\text { fibers } \\
\text {-Cut sides of } 120 \text { matches from } \\
6 \text { books searched for inclusions } \\
\text { with stereomicroscope } \\
\text {-During both search types, both } \\
\text { dye and argon lasers were used } \\
\text { for illumination. Images were } \\
\text { taken of all observed inclusions }\end{array}$ & None & $\begin{array}{c}\text {-Argon laser produced more luminescing } \\
\text { inclusions than the dye laser } \\
\text {-Dye laser excited more fibers } \\
\text {-Dye laser can reveal some inclusions not } \\
\text { shown by argon, but argon should be first } \\
\text { choice } \\
\text {-Dye laser can show cross-sections of a } \\
\text { single fiber }\end{array}$ & 94 \\
\hline $\begin{array}{c}\text { Fractography/ } \\
\text { Qualitative }\end{array}$ & $\begin{array}{c}\text { Matchsticks/ } \\
\text { paper } \\
\text { matches }\end{array}$ & NA & Qualitative & $\begin{array}{l}\text {-10 major points of } \\
\text { comparison: length, width, } \\
\text { thickness, waxing, color (front } \\
\text { and back, thickness of coloring } \\
\text { material), sizing (fluorescence } \\
\text { of filler materials), cut edges, } \\
\text { torn edges, inclusions, cross-cut } \\
\text { and torn fiber relationships } \\
\text { (horizontal and vertical) }\end{array}$ & NA & $\begin{array}{c}\text {-The US has } 7 \text { major match manufacturers, } \\
\text { all with an extremely similar } \\
\text { manufacturing process } \\
\text {-A minimum of } 4 \text { crosscut or torn fibers } \\
\text { must be associated for a positive } \\
\text { identification (as believed by the author), } \\
\text { only if the head is still in-tact. If not, more } \\
\text { are required } \\
\text {-The author suggests a staining agent for } \\
\text { match fibers is needed for ease-of } \\
\text { comparison }\end{array}$ & 95 \\
\hline
\end{tabular}




\begin{tabular}{|c|c|c|c|c|c|c|c|}
\hline $\begin{array}{l}\text { Fractography/ } \\
\text { Qualitative }\end{array}$ & Metal & 5 wire samples & $\begin{array}{l}\text { Qualitative } \\
\text { assessment } \\
\text { and } \\
\text { quantitative } \\
\text { measurement }\end{array}$ & $\begin{array}{l}\text {-5 sets of wire fractured } \\
\text { through different methods } \\
\text { (tension, shearing, torsion, } \\
\text { diagonal cutter, and sawing) } \\
\text {-Respective fracture ends } \\
\text { mounted on separate stubs and } \\
\text { viewed under the SEM } \\
\text { simultaneously } \\
\text {-Images taken perpendicular to } \\
\text { fracture surface for comparison. } \\
\text { Regular images, photographic } \\
\text { negatives, and mirror images } \\
\text { (reversed scan direction) } \\
\text { compared } \\
\text {-Elemental analysis (x-ray } \\
\text { spectra) on samples also } \\
\text { recorded }\end{array}$ & None & $\begin{array}{l}\text {-SEM is useful when fractured surfaces are } \\
\text { too small to be examined, or a conclusion } \\
\text { is unable to be drawn } \\
\text {-Most useful in examinations of fracture } \\
\text { surfaces less than } 50 \text { micrometers } \\
\text {-If samples are not differentiated by } \\
\text { elemental analysis, move on to SEM image } \\
\text { comparison } \\
\text {-Wire broken by tension has enough } \\
\text { fracture characteristics in SEM image to } \\
\text { show a match, shear wire doesn't have as } \\
\text { much detail } \\
\text {-Very characteristic patterns in torsion } \\
\text { wires } \\
\text {-Sufficient detail shown for diagonally cut } \\
\text { wires when viewed along the wire axis }\end{array}$ & 96 \\
\hline $\begin{array}{l}\text { Fractography/ } \\
\text { Qualitative }\end{array}$ & Metal & $\begin{array}{c}30 \text { keys ( } 6 \text { sets } \\
\text { of } 5)\end{array}$ & Qualitative & $\begin{array}{c}\text {-Metal keys were placed into a } \\
\text { vise and either broken by sharp } \\
\text { impact or bent twice in opposite } \\
\text { directions for breakage } \\
\text {-Each half was examined under } \\
\text { a stereomicroscope and } \\
\text { photographed } \\
\text {-Known matches first observed, } \\
\text { followed by verification of } \\
\text { known non-matches by } \\
\text { switching fragments among } \\
\text { pairs }\end{array}$ & None & $\begin{array}{l}\text {-Level of agreement (qualitative) of overall } \\
\text { break pattern appeared high between } \\
\text { known matches, with an apparent decrease } \\
\text { in agreement when observing known non- } \\
\text { matches } \\
\text {-Not all internal fracture patterns (key } \\
\text { cross-sections) provided enough detail for } \\
\text { inclusion at 10x. 15x magnification } \\
\text { minimum required }\end{array}$ & 97 \\
\hline
\end{tabular}




\begin{tabular}{|c|c|c|c|c|c|c|c|}
\hline $\begin{array}{l}\text { Fractography/ } \\
\text { Qualitative }\end{array}$ & Paper & $\begin{array}{l}4 \text { pieces of } \\
\text { paper (2 per } \\
\text { paper) }\end{array}$ & Qualitative & $\begin{array}{l}\text {-Method for more efficient } \\
\text { visualization of paper } \\
\text { delamination (unequal tearing } \\
\text { of paper layers) discovered } \\
\text { during a typical electrostatic } \\
\text { detection apparatus (ESDA) } \\
\text { analysis }\end{array}$ & None & $\begin{array}{l}\text {-When the torn papers are placed into the } \\
\text { ESDA with their delaminated edges facing } \\
\text { up, the delaminated regions appeared dark } \\
\text { in contrast to the remainder of the page in } \\
\text { the resulting ESDA image } \\
\text {-This technique is useful for rapid } \\
\text { visualization of corresponding paper tears } \\
\text { and is not affected by the routine } \\
\text { humidification imparted on paper being } \\
\text { examined for writing indentations }\end{array}$ & 98 \\
\hline $\begin{array}{l}\text { Fractography/ } \\
\text { Qualitative }\end{array}$ & NA & NA & Qualitative & $\begin{array}{l}\text {-Two optical techniques aid } \\
\text { comparing fractures when one } \\
\text { is a mirror/negative of the other } \\
\text {-Beam splitters are an optical } \\
\text { device designed to split light so } \\
\text { half is reflected and half is } \\
\text { transmitted. The divided light } \\
\text { allows the observer to examine } \\
\text { the object directly and/or a } \\
\text { reflected image of the object } \\
\text {-Reverse lighting inverts the } \\
\text { surface of one object being } \\
\text { examined, and can be used } \\
\text { correspondingly with beam } \\
\text { splitting }\end{array}$ & NA & $\begin{array}{l}\text {-Allowed for an easier examination of } \\
\text { difficult fractures, either by the nature of } \\
\text { the fracture or by highlighting features that } \\
\text { would be lost under standard comparison } \\
\text { microscopy techniques }\end{array}$ & 99 \\
\hline
\end{tabular}


Table C. Quantitative Articles Summary

\begin{tabular}{|c|c|c|c|c|c|c|c|}
\hline Category & $\begin{array}{l}\text { Material } \\
\text { Type }\end{array}$ & $\begin{array}{l}\text { Population } \\
\text { Size }\end{array}$ & $\begin{array}{c}\text { Qualitative } \\
\text { or } \\
\text { Quantitative } \\
\text { Assessment? }\end{array}$ & Experimental Design & $\begin{array}{c}\text { Statistical } \\
\text { Performance } \\
\text { Measures }\end{array}$ & Main Findings & $\begin{array}{l}\text { Reference } \\
\text { Number }\end{array}$ \\
\hline Quantitative & NA & NA & $\begin{array}{l}\text { Qualitative } \\
\text { assessment of } \\
\text { computer } \\
\text { software's } \\
\text { ability to } \\
\text { model } \\
\text { fractures as } \\
\text { fractal } \\
\text { surfaces. }\end{array}$ & $\begin{array}{l}\text {-Computer software } \\
\text { generation of fractal surfaces }\end{array}$ & NA & $\begin{array}{c}\text {-Walls' model: fracture } \\
\text { contains inflection points, a } \\
\text { particular path or course a } \\
\text { fracture follows in one plane } \\
\text {-Fractures should be } \\
\text { described by fractal surfaces } \\
\text { of n-dimensions } \\
\text {-Complexity/individuality of } \\
\text { fractal surface can be } \\
\text { calculated as a value } \\
\text {-Processing time required to } \\
\text { generate an accurate fractal } \\
\text { surface exceeded limits of } \\
\text { computers at the time }\end{array}$ & 13 \\
\hline Quantitative & $\begin{array}{l}\text { Bone, } \\
\text { Other }\end{array}$ & $\begin{array}{l}57 \text { bone } \\
\text { fragments }\end{array}$ & $\begin{array}{c}\text { Qualitative } \\
\text { comparison } \\
\text { with } \\
\text { quantitative } \\
\text { assessment }\end{array}$ & $\begin{array}{l}\text {-Bone types were fractured using } \\
\text { static and dynamic forces } \\
\text {-95 study participants were instructed } \\
\text { to tape believed physical matches } \\
\text { together } \\
\text {-Participants filled out a survey of } \\
\text { their background knowledge and } \\
\text { experience with physical match } \\
\text {-Test scored according to number of } \\
\text { positive associations, negative } \\
\text { associations, and non-associations } \\
-40 \text { known positive associations } \\
\text { possible (denominator of error and } \\
\text { accuracy rate determinations) }\end{array}$ & $\begin{array}{c}\text {-ANOVA } \\
\text {-Kruskal-Wallace } \\
\text {-Positive association rate } \\
\text { and standard deviations } \\
\text { determined per participant } \\
\text { group. Error rates also } \\
\text { determined. } \\
\text {-Mean, range, and standard } \\
\text { deviation for exercise } \\
\text { completion time per } \\
\text { participant group also } \\
\text { determined. }\end{array}$ & $\begin{array}{l}\text {-Positive association rate (pooled) = } \\
0.925 \\
\text {-Performance rates decreased with } \\
\text { decrease in experience. No significant } \\
\text { statistical difference between the } \\
\text { group rate differences } \\
-4 \text { total negative associations in the } \\
\text { study, rate of } 0.001 \\
\text {-Significant statistical difference in } \\
\text { completion time by those in expert } \\
\text { category as compared to those in no- } \\
\text { experience category }\end{array}$ & 15 \\
\hline
\end{tabular}




\begin{tabular}{|c|c|c|c|c|c|c|c|}
\hline Quantitative & Other & $\begin{array}{l}24 \text { metal- } \\
\text { coated, } \\
\text { twelve each } \\
\text { of silicon } \\
\text { sheets }\end{array}$ & $\begin{array}{c}\text { Qualitative } \\
\text { assessment } \\
\text { and } \\
\text { quantitative } \\
\text { measurement }\end{array}$ & $\begin{array}{l}\text {-Sample thickness measured } \\
\text { according to ASTM D645, } \\
\text { hardness measured according } \\
\text { to ASTM D2240A } \\
\text {-Samples torn on tensile } \\
\text { machine according to ASTM } \\
\text { D5735-95 at set rate of } 100 \\
\text { mm/min, shearing force } \\
\text { applied perpendicular to } \\
\text { sample } \\
\text {-Tearing stress from tensile } \\
\text { machine collected according } \\
\text { to ASTM D2240A } \\
\text {-Torn samples photographed, } \\
\text { transparencies prepared } \\
\text {-Double blind matching of } \\
\text { sample fracture edges } \\
\text { conducted on both whole } \\
\text { length of rim (8 cm) and a } 1 \\
\text { cm section of the rim }\end{array}$ & None & $\begin{array}{l}\text {-All } 24 \text { samples were matched } \\
\text { correctly for the whole length } \\
\text { of the fracture } \\
\text {-Only } 121 \mathrm{~cm} \text { comparisons } \\
\text { were performed due to } \\
\text { number involved in the full } \\
\text { set } \\
\text {-8 out of } 12 \text { matched correctly } \\
\text { for } 1 \mathrm{~cm} \text { comparisons (using } \\
\text { transparencies alone). } \\
\text { Remaining } 4 \text { correctly } \\
\text { matched when provided } \\
\text { actual materials for reference } \\
\text {-The authors conclude that } \\
\text { under reproducible } \\
\text { conditions, "unique" shears } \\
\text { are still generated leading to } \\
\text { high match accuracy }\end{array}$ & 16 \\
\hline Quantitative & Tape & $\begin{array}{c}5 \text { tests with } \\
10 \text { tape strips } \\
\text { per sets }\end{array}$ & Qualitative & $\begin{array}{l}\text {-5 test sets: hand-torn from } \\
\text { each of three rolls and scissor } \\
\text { cut from each of the two rolls } \\
\text {-Four examiners, individual } \\
\text { assessments of each set. } \\
\text { Separate sets per examiner, } \\
20 \text { prepared total }\end{array}$ & Performance rates & $\begin{array}{c}-46 / 50 \text { or } 92 \% \text { hand-torn end } \\
\text { matches identified correctly } \\
-25 / 31 \text { or } 81 \% \text { scissor-cut end } \\
\text { matches identified correctly } \\
\text {-No false positives or negatives, } \\
\text { remaining were inconclusive } \\
-2 \text { misidentifications occurred } \\
\text { when examiners re-evaluated the } \\
\text { scissor cut sets (due to lower } \\
\text { matching percentage) }\end{array}$ & 17 \\
\hline
\end{tabular}




\begin{tabular}{|c|c|c|c|c|c|c|c|}
\hline Quantitative & Metal & $\begin{array}{l}20 \text { sample } \\
\text { sets of } 10 \\
\text { fracture } \\
\text { fragments } \\
\text { each }(200 \\
\text { samples } \\
\text { total) }\end{array}$ & Qualitative & $\begin{array}{l}\text {-20 sample sets of } 10 \\
\text { fractured steel fragments } \\
\text { were created and pulled apart } \\
\text { using an MTS Tensile Tester } \\
-2 \text { out of the } 10 \text { pairs in each } \\
\text { sample set were known non- } \\
\text { matches. } 10 \text { examiners } \\
\text { completed the study, each } \\
\text { completing } 2 \text { randomly } \\
\text { assigned kits } \\
\text {-Examiners were given the } \\
\text { choice of } 3 \text { conclusions: } \\
\text { identification, elimination, or } \\
\text { no conclusion. Examiners } \\
\text { also asked to photograph the } \\
\text { fractured surfaces } \\
\text {-Participating examiners had } \\
\text { experience ranging from } 2.5- \\
13 \text { years } \\
\text {-Typical examination } \\
\text { protocol was followed, } \\
\text { involving digital photography } \\
\text { and a fluorescent light source } \\
\text {-Reverse lighting was used to } \\
\text { optimally illuminate surface } \\
\text { contours during examination }\end{array}$ & None & $\begin{array}{c}\text {-All examiners achieved } \\
100 \% \text { accuracy with no false } \\
\text { positives recorded } \\
\text {-Photographs of metal } \\
\text { fractures are provided to } \\
\text { demonstrate the variety of } \\
\text { patterns formed }\end{array}$ & 18 \\
\hline
\end{tabular}




\begin{tabular}{|c|c|c|c|c|c|c|c|}
\hline Quantitative & Paper & $\begin{array}{c}38 \text { remnants } \\
\text { of shredded } \\
\text { notebook } \\
\text { paper }\end{array}$ & Quantitative & $\begin{array}{l}\text {-Features are described as } 3 \\
\text { categories: color features, } \\
\text { features for detection of } \\
\text { squared/lined paper, and features } \\
\text { for handwriting style description } \\
\text {-Color histogram feature scaled } \\
\text { back to few coefficients applied } \\
\text { (such as the MPEG-7 Scalable } \\
\text { Color or dominant color } \\
\text { descriptors) } \\
\text {-For handwriting style } \\
\text { description features, descriptors } \\
\text { needed to detect general } \\
\text { preference in direction of } \\
\text { handwritten characters } \\
\text {-Modifications were made to } \\
\text { Hough transform, a squared } \\
\text { pattern detection feature, to } \\
\text { transform shredded strips into } \\
\text { Hough accumulation matrix } \\
\text {-Involves dividing strips into } \\
\text { multiple squares, as transform } \\
\text { performed best on square units } \\
\text {-To test the Hough transform on } \\
\text { shredded notebook paper strips, a } \\
\text { set of } 38 \text { remnants was prepared, } \\
\text { consisting of } 16 \text { squared } \\
\text { remnants and } 22 \text { non-squared } \\
\text { remnants from } 18 \text { different } \\
\text { documents and } 6 \text { different types } \\
\text { of squared paper } \\
\text {-The squared paper detection } \\
\text { feature assigns values to } \\
\text { remnants as an SP value. A value } \\
\text { above } 50 \text { indicates a squared } \\
\text { pattern while a value below } 50 \\
\text { indicates a non-squared pattern }\end{array}$ & None & $\begin{array}{l}\text {-All remnants were correctly } \\
\text { classified by the squared } \\
\text { paper detection feature } \\
\text {-However, the values were } \\
\text { high and disperse due to the } \\
\text { different types of squared } \\
\text { paper introduced } \\
\text {-Further classification can } \\
\text { occur due to the disperse } \\
\text { values as those with highest } \\
\text { values likely originated from } \\
\text { the same document } \\
\text {-Future work will involve } \\
\text { combining RGB data from the } \\
\text { color properties of the paper } \\
\text { and handwriting style } \\
\text { descriptors in with the } \\
\text { squared paper detection } \\
\text { feature }\end{array}$ & 19 \\
\hline
\end{tabular}




\begin{tabular}{|c|c|c|c|c|c|c|c|}
\hline Quantitative & Ceramics & $\begin{array}{l}500 \\
\text { fragments of } \\
\text { ceramic from } \\
5 \text { tiles }\end{array}$ & Quantitative & $\begin{array}{l}\text {-Five ceramic tiles were } \\
\text { scattered into roughly } 100 \\
\text { fragments each. Fragments } \\
\text { were scanned and images } \\
\text { were then applied to an edge- } \\
\text { detection algorithm } \\
-50 \text { true match fragments } \\
\text { were used to train the } \\
\text { algorithm, with } 50 \text { true non- } \\
\text { match fragments used as a } \\
\text { control experiment }\end{array}$ & $\begin{array}{c}\text { Frequency of } \\
\text { occurrence of } \\
\text { individual bits was } \\
\text { able to be expressed } \\
\text { probabilistically, but } \\
\text { conclusions on pairs } \\
\text { are a current } \\
\text { limitation }\end{array}$ & $\begin{array}{c}\text {-The specific algorithm used } \\
\text { quantified fragment shape by } \\
\text { "bits" of useful edge } \\
\text { information } \\
\text {-Higher number of bits } \\
\text { contained on a fragment led to } \\
\text { a lower chance of a false } \\
\text { positive }\end{array}$ & 20 \\
\hline Quantitative & Tape & $\begin{array}{l}1600 \text { torn } \\
\text { pairs for } \\
\text { hand-torn } \\
200 \\
\text { Elmendorf- } \\
\text { torn } \\
200 \text { scissor- } \\
\text { cut } \\
200 \text { box } \\
\text { cutter-cut }\end{array}$ & Qualitative & $\begin{array}{l}-4 \text { separation methods (hand } \\
\text { torn, Elmendorf torn, scissor } \\
\text { cut, box cutter cut) } \\
-3 \text { analysts, all peer- } \\
\text { reviewing each other }\end{array}$ & $\begin{array}{l}\text {-Contingency tables: } \\
\text { inconclusive rate, } \\
\text { accuracy rate, false- } \\
\text { positive rate, false- } \\
\text { negative rate } \\
\text {-Mean and standard } \\
\text { deviations calculated } \\
\text { for each analyst }\end{array}$ & $\begin{array}{c}\text { Peer review results: } \\
\text {-Hand-torn: } 9 \text { false negatives, } 2 \text { false } \\
\text { positives, } 37 \text { inconclusive } \\
\text {-Elmendorf-torn: } 3 \text { false negatives, } 0 \\
\text { false positives, } 11 \text { inconclusive } \\
\text {-Scissor-cut: } 4 \text { false positives, } 0 \text { false } \\
\text { negatives, } 1 \text { inconclusive } \\
\text {-Box cutter-cut: only one } \\
\text { misidentification } \\
\text {-Totals: Elmendorf = highest IN rates } \\
\text { across examiners; Hand torn NGB } \\
\text { NPB 3MGB 3MGG somewhat high; } \\
\text { scissor-cut relatively low; box cutter- } \\
\text { cut all } 0 \\
\text {-Mean accuracy torn tape: } 98.58 \text { - } \\
100.00 \% \\
\text {-Mean accuracy cut tape: } 98.15 \text { - } \\
99.83 \% \\
\text {-Mean false positive rate torn tape: } \\
0.00 \text { - } 0.67 \% \\
\text {-Mean false positive rate cut tape: } \\
0.00 \text { - } 3.33 \% \\
\text {-Mean false negative rate torn tape: } \\
0.00-2.67 \% \\
\text {-Mean false negative rate cut tape: } \\
0.33 \%\end{array}$ & 21,22 \\
\hline
\end{tabular}




\begin{tabular}{|c|c|c|c|c|c|c|c|}
\hline Quantitative & Tape & $\begin{array}{c}11 \text { tape sets, } \\
200 \text { tapes per } \\
\text { set, } 40,000 \\
\text { inter- } \\
\text { comparisons, } \\
\text { total of } \\
440,000 \\
\text { comparisons }\end{array}$ & Quantitative & $\begin{array}{l}\text {-Sets were } 200 \text { samples each } \\
\text { of the following fracture } \\
\text { methods: hand torn ( } 8 \text { sets), } \\
\text { Elmendorf torn ( } 1 \text { set), } \\
\text { scissor cut ( } 1 \text { set), and box } \\
\text { cutter ( } 1 \text { set) } \\
\text {-Digital images taken of all } \\
\text { individual ends and fracture } \\
\text { pair exemplars } \\
\text {-An algorithm was developed } \\
\text { to extract coordinates of } \\
\text { fracture ends, thresholds set } \\
\text { depending on image } \\
\text { illumination and tape color, } \\
\text { binary image generated, noise } \\
\text { from contamination filtered } \\
\text { out } \\
\text {-Similarity/distance between } \\
\text { coordinates of a fracture pair } \\
\text { calculated as the sum of } \\
\text { squared residuals (SSR) value } \\
\text { to quantify differences. } \\
\text { Lower values indicate more } \\
\text { similar }\end{array}$ & $\begin{array}{c}\text {-Frequency } \\
\text { histograms of true } \\
\text { match and non-match } \\
\text { SSR values } \\
\text {-Box plots for SSR } \\
\text { values among } \\
\text { comparisons } \\
\text {-Colored matrix plot } \\
\text { of SSR values (shows } \\
\text { that high and low } \\
\text { SSRs are not random } \\
\text { and common in } \\
\text { certain samples) } \\
\text {-SSR means and } \\
\text { standard deviations } \\
\text { between matches and } \\
\text { non-matches }\end{array}$ & $\begin{array}{c}\text {-True matching SSR values } \\
\text { were always below a critical } \\
\text { value } \\
\text {-Majority of non-matching } \\
\text { SSRs were orders of } \\
\text { magnitude larger than } \\
\text { matching } \\
\text {-In some samples, a non- } \\
\text { matching SSR could be even } \\
\text { smaller than a matching SSR } \\
\text { if fractures were somewhat } \\
\text { similar } \\
\text {-General grade tapes error } \\
\text { rates with } 40,000 \\
\text { intercomparisons: } 0.0025- \\
0.29 \% \\
\text {-General grade tapes error rate } \\
\text { with } 200 \text { intracomparisons: } \\
0.5-18.50 \% \\
\text {-Professional grade tapes } \\
\text { error rate with } 40,000 \\
\text { intercomparisons: } 0.085- \\
0.20 \% \\
\text {-Professional grade tapes } \\
\text { error rate with } 200 \\
\text { intracomparisons: } 7.0-7.5 \%\end{array}$ & 24 \\
\hline
\end{tabular}




\begin{tabular}{|c|c|c|c|c|c|c|c|}
\hline Quantitative & Other & $\begin{array}{c}12 \text { fracture } \\
\text { pairs from } \\
\text { silicon, } 24 \\
\text { metal-coated } \\
\text { paper } \\
\text { samples, and } \\
22 \text { Perspex } \\
\text { plates }\end{array}$ & Quantitative & $\begin{array}{l}\text {-Fractures illuminated with } \\
\text { oblique lighting and scanned } \\
\text {-Two computerized systems } \\
\text { developed: one extracts } \\
\text { contour representation from } \\
\text { fracture image/scan, other } \\
\text { compares to database to } \\
\text { generate statistical probability } \\
\text { of the match } \\
\text {-Individual similarity scores } \\
\text { against the databases } \\
\text { determined by algorithm } \\
\text {-Correct matches were } \\
\text { classified by human users } \\
\text { who marked match points on } \\
\text { the software. Pixel distances } \\
\text { between the proposed points } \\
\text { then calculated } \\
\text {-Classification process told } \\
\text { system correct matches and } \\
\text { non matches for different } \\
\text { material types and fracture } \\
\text { line lengths. Pixel lengths } \\
\text { between known matches and } \\
\text { non-matches used to generate } \\
\text { criteria for classification of a } \\
\text { questioned fracture } \\
\text {-Probabilities of occurrence } \\
\text { within generated databases } \\
\text { used to determine optimal } \\
\text { separation criterion for this } \\
\text { purpose }\end{array}$ & $\begin{array}{c}\text { Similarity measures } \\
\text { between sections of } \\
\text { fracture contour: } \\
\text {-Difference sum of } \\
\text { squares } \\
\text {-Difference standard } \\
\text { deviation } \\
\text {-Normalized cross- } \\
\text { correlation } \\
\text {-Histograms and } \\
\text { probability density } \\
\text { functions for correct } \\
\text { match and } \\
\text { populations } \\
\text {-Likelihood ratios of } \\
\text { match within material } \\
\text { population in database }\end{array}$ & $\begin{array}{c}\text {-Correct match classification } \\
\text { probability: } 0.968 \\
\text {-False positive classification } \\
\text { probability: } 0.0519 \\
\text {-Likelihood ratio of true } \\
\text { positive: } 18.66 \\
\text {-Positive predictive value: } \\
0.9491 \\
\text {-Bayes risk (false } \\
\text { classifications): } 0.084 \\
\text {-50\% correct criterion } \\
\text { positive likelihood ratio: } 529 \\
\text { (pairs with matching error } \\
\text { below } 0.775 \text { will be classified } \\
\text { as correct matches) } \\
\text {-Probability of correct } \\
\text { classification of a matching } \\
\text { pair with error values between } \\
1.05-1.15=0.0561 \\
\text {-Probability of a non-match } \\
\text { with these error values }= \\
0.0039 \\
\text {-0.93 probability of being a } \\
\text { correct pair within these error } \\
\text { ranges }\end{array}$ & 25 \\
\hline
\end{tabular}




\begin{tabular}{|c|c|c|c|c|c|c|c|}
\hline Quantitative & Metal & Not given & Quantitative & $\begin{array}{l}\text {-Electron Backscattered } \\
\text { Diffraction/Orientation } \\
\text { Imaging Microscopy } \\
\text { (EBSD/OIM) used to } \\
\text { characterize crystal } \\
\text { orientation along fractured } \\
\text { edge } \\
\text {-Fracture edge scanned and a } \\
\text { sequence of grain orientation } \\
\text { along the edge length } \\
\text { developed. A series of } \\
\text { misorientation vectors is } \\
\text { derived for the fractured edge } \\
\text { dependent upon } \\
\text { representation of crystal } \\
\text { orientation by Euler angles } \\
\text {-These misorientation vectors } \\
\text { are then compared to } \\
\text { determine similar or } \\
\text { dissimilar edges, helping to } \\
\text { attribute to a potential } \\
\text { fracture fit }\end{array}$ & $\begin{array}{c}\text { Probabilistic } \\
\text { statements based on } \\
\text { all possible grain } \\
\text { orientations } \\
\text { considered }\end{array}$ & $\begin{array}{l}\text {-Fractures in metallic } \\
\text { materials can orient in two } \\
\text { directions relative to the grain } \\
\text { of the substrate } \\
\text {-If the stress applied to the } \\
\text { material exceeds its atomic } \\
\text { bond strength, the atomic } \\
\text { planes of the substrate } \\
\text { separate from one another. If } \\
\text { a fracture travels through a } \\
\text { crystal it is a transgranular or } \\
\text { intracrystalline fracture } \\
\text {-However, if grain boundaries } \\
\text { are weaker than atomic bond } \\
\text { strength, the fracture will } \\
\text { travel through grain } \\
\text { boundaries as an intergranular } \\
\text { fracture } \\
\text { probabilistic interpretation of } \\
\text { the likelihood of obtaining the } \\
\text { same misorientation sequence } \\
\text { in another sample pair } \\
\text { potential population for the } \\
\text { match examination as the } \\
\text { number of possible crystal } \\
\text { orientations along a fractured } \\
\text { edge can be calculated, and } \\
\text { when combined with the }\end{array}$ & 34 \\
\hline
\end{tabular}




\begin{tabular}{|c|c|c|c|c|c|c|c|}
\hline Quantitative & Metal & NA & Quantitative & $\begin{array}{l}\text {-A fracture unit defined as the } \\
\text { "smallest discernible } \\
\text { variations in either directional } \\
\text { change or height" } \\
\text {-For 2D edge fractures, the } \\
\text { model assumed a } 50 \% \text { chance } \\
\text { of propagation in each of the } \\
\text { vertical and horizontal } \\
\text { directions } \\
\text {-Depending upon the number } \\
\text { of units across the fractured } \\
\text { edge, directional } \\
\text { combinations increase } \\
\quad \text { exponentially } \\
\text {-This occurs even more so in } \\
\text { three-dimensional edge } \\
\text { considerations, where height } \\
\text { is incorporated as a third level } \\
\text {-For simplicity, the author } \\
\text { included only two height } \\
\text { possibilities at this time }\end{array}$ & $\begin{array}{c}\text { Likelihood/probability } \\
\text { ratios }\end{array}$ & $\begin{array}{c}\text {-Probability of occurrence } \\
\text { calculated - e.g., length of } 100 \\
\text { was stated to occur in only } 1 \\
\text { out of } 1.27 \text { nonillion fractures } \\
\text { of the same length } \\
\text {-Provides potential for } \\
\text { probabilistic interpretation of } \\
\text { physical fit in metallic } \\
\text { materials }\end{array}$ & 35 \\
\hline Quantitative & Metal & $\begin{array}{c}2 \\
\text { consecutively } \\
\text { manufactured } \\
\text { hacksaw } \\
\text { blades, each } \\
\text { blade } \\
\text { fractured into } \\
12 \text { pieces }\end{array}$ & Quantitative & $\begin{array}{l}\text {-2 blades broken into twelve } \\
\text { 1-inch segments using a vice } \\
\text { and vice jaws } \\
\text {-Casts were made of each } \\
\text { even numbered edge } \\
\text {-Proficiency test: four } \\
\text { hacksaw blades were broken } \\
\text { as previously described, and } \\
\text { each edge cast using Mikrosil }\end{array}$ & Performance rates & $\begin{array}{l}\text {-The fractures produced in the } \\
\text { research created two surfaces } \\
\text { with characteristics that were } \\
\text { found to be distinctive } \\
\text {-Proficiency test: } 157 \\
\text { expected identifications out of } \\
173 \text { received. } 9 \text { eliminations } \\
\text { and } 1 \text { misidentification } \\
\text {-Total of } 109 \text { eliminations and } \\
45 \text { inconclusive responses } \\
\text {-Sensitivity }=0.908, \\
\text { specificity }=0.694\end{array}$ & 100 \\
\hline
\end{tabular}




\begin{tabular}{|c|c|c|c|c|c|c|c|}
\hline Quantitative & Tape & 30 test sets & Qualitative & $\begin{array}{c}-3 \text { examiners performed end } \\
\text { matches on } 10 \text { sets each of } \\
\text { electrical tape fracture pairs } \\
\text {-Each set design consisted of } \\
\text { factor variation between tape } \\
\text { brand, test set preparer, and } \\
\text { mode of separation }\end{array}$ & Performance rates & $\begin{array}{c}-2142 \text { end comparisons } \\
\text { possible due to various } \\
\text { combinations of tape ends } \\
-98 / 106 \text { true matches } \\
\text { identified } \\
-7 \text { pairs misidentified as } \\
\text { inconclusive and } 1 \text { was a false } \\
\text { positive } \\
\text {-A secondary reviewer also } \\
\text { reported a false positive on } \\
\text { the same tape pair } \\
\text {-False positive rate was } \\
0.049 \%\end{array}$ & 101 \\
\hline Quantitative & Tape & 2280 pairs & $\begin{array}{c}\text { Qualitative } \\
\text { comparison } \\
\text { with } \\
\text { quantitative } \\
\text { assessment }\end{array}$ & $\begin{array}{c}\text {-Tape pairs of various } \\
\text { qualities either hand-torn or } \\
\text { scissor-cut } \\
\text {-Number of areas between } \\
\text { scrim that matched across } \\
\text { tape edges counted (edge } \\
\text { similarity score) and } \\
\text { conclusion of non-match or } \\
\text { match determined } \\
\text {-Total population of known } \\
\text { non-matches and matches } \\
\text { used to evaluate score } \\
\text { distribution and performance } \\
\text { rates }\end{array}$ & $\begin{array}{c}\text {-Performance rates } \\
\text {-Score-based } \\
\text { likelihood ratios }\end{array}$ & $\begin{array}{c}\text {-No false positives reported } \\
\text {-Accuracy reported between } \\
84-99 \% \\
\text {-ESS higher than } 80 \% \\
\text { supported match, and ESS } \\
\text { lower than } 25 \% \text { supported } \\
\text { non-match }\end{array}$ & 102 \\
\hline
\end{tabular}




\begin{tabular}{|c|c|c|c|c|c|c|c|}
\hline Quantitative & Paper & NA & Quantitative & $\begin{array}{l}\text {-Hand-torn paper fragments } \\
\text { were scanned and he contours } \\
\text { of the torn edges were } \\
\text { extracted utilizing the } \\
\text { Douglas and Peucker polyline } \\
\text { simplification algorithm } \\
\text {-Polygon sides were then } \\
\text { classified by either frame part } \\
\text { or inner part } \\
\text {-The polygons subjected to } \\
\text { feature extraction process in } \\
\text { which the number of sudden } \\
\text { changes in the contour } \\
\text { orientation with respect to the } \\
\text { extracted polygon counted } \\
\text { and the Euclidean distance } \\
\text { between the inner side } \\
\text { vertices calculated } \\
\text {-A decision matrix was then } \\
\text { created to identify which } \\
\text { fragment pairs are to be } \\
\text { compared } \\
\text {-High score was received if } \\
\text { the Euclidean distance } \\
\text { between the inner line } \\
\text { segments is small and the } \\
\text { number of sudden changes in } \\
\text { contour orientation between } \\
\text { sides is equal }\end{array}$ & $\begin{array}{l}\text {-Efficacy factor } \\
\text {-Euclidean distance }\end{array}$ & $\begin{array}{l}\text {-Only accounted for single } \\
\text { page reconstruction rather } \\
\text { than multiple documents } \\
\text {-Factoring both the Euclidean } \\
\text { distance and the changes in } \\
\text { contour orientations into the } \\
\text { score accounts for any } \\
\text { fragments with similar } \\
\text { Euclidean distances that are } \\
\text { true non-matches } \\
\text {-Algorithm performed better } \\
\text { with hand-torn fragments } \\
\text { compared to sheared edges }\end{array}$ & 109 \\
\hline
\end{tabular}




\begin{tabular}{|c|c|c|c|c|c|c|c|}
\hline Quantitative & Paper & $\begin{array}{c}690 \text { snippets } \\
\text { of paper }\end{array}$ & Quantitative & $\begin{array}{l}\text {-The developed algorithm } \\
\text { assesses the rotational and } \\
\text { gradient orientation of the } \\
\text { paper, and the color of the } \\
\text { ink/paper to cluster torn } \\
\text { pieces of paper together }\end{array}$ & $\begin{array}{c}\text { Evaluation of } \\
\text { algorithms used: } \\
\text {-Mean error, median } \\
\text { error } \\
\text {-Thresholds/fitted } \\
\text { Gaussians } \\
\text {-Error rates }\end{array}$ & $\begin{array}{l}\text {-678 images assessed for } \\
\text { orientation ( } 32 \text { could not be } \\
\text { assigned an orientation) } \\
\text {-Mean error was } 1.95 \text { degrees, } \\
\text { Median error was } 0.37 \\
\text { degrees } \\
\text {-The color segmentation was } \\
\text { tested using } 13 \text { samples, and } \\
\text { distinguished color from } \\
\text { black/grey text } \\
\text {-Algorithm could be used to } \\
\text { assess general information } \\
\text { like the orientation and } \\
\text { distinguish between colors } \\
\text { and black writing on paper }\end{array}$ & 110 \\
\hline
\end{tabular}




\section{CHAPTER TWO}

\section{Inter-Laboratory Assessment of the Utility of the Edge Similarity Score (ESS) in Duct Tape Physical Fit Examinations}

\section{Overview of the Inter-laboratory Study}

As recent criticism of the forensic field has called for more quantitative methodology to reduce subjectivity in comparative analyses ${ }^{1-3}$, it is becoming crucial to implement new comparison methods to even the seemingly most straightforward of examinations, such as physical fit. To do so, a critical component of the process towards validation and standardization of a new method is to test it via inter-laboratory studies. This is done for purposes of establishing reproducibility and reliability of a method for implementation into practice. These collaborative studies are also effective to fine-tune the methods and arrive to consensus protocols.

In this project, an inter-laboratory study between trace evidence scientists was designed to assess a quantitative, score-based physical fit technique, known as the edge similarity score (ESS) ${ }^{4}$. This interlaboratory collaboration was focused on the evaluation of the quality of duct tapes fractured edges. A secondary purpose of this study was to evaluate the practitioners' feedback on the method for further improvements, which will be implemented in future collaborative exercises. Incorporating the examiners' comments on the applicability of the method is one of the essential processes to generate approaches that are practical and likely to be implemented by the scientific community.

As exact duct tape fractured edges cannot be experimentally reproduced, it was impractical to provide the same fractured edges to every participant in a sequential circulation. Instead, physical samples were created for each of three study kits in order to simulate items encountered in casework. Each kit consisted of seven duct tape comparison pairs each, distributed in a RoundRobin style to volunteer examiners at various federal, state, and local forensic laboratories. Each kit contained four matching pairs (3 of them with a good quality match $\mathrm{M}+$, one of them with a weaker quality match $\mathrm{M}-$ ) and 3 non-matching pairs (NM).

For each kit, the respective sample (e.g. sample 1 from Kits 1, 2 and 3) were prepared using the same duct tape roll and the same separation method. Also, they were chosen to exhibit the same macro edge pattern (e.g., puzzle, wavy or straight) and a similar ESS score. To establish maximum similarity between kit samples, the comparison tapes were selected according to pre-distribution, consensus ESS values established by four examiners. An agreement in the ESS better than $\pm 10 \%$ ESS was used as the criteria for pre-distribution consensus. The average consensus ESS for true good quality matches ranged from $86 \%$ to $99 \%(\mathrm{M}+)$, true matches of lower alignment ranged from $70 \%$ to $77 \%(\mathrm{M}-)$, and non-matches ranged from $0 \%$ to $11 \%(\mathrm{NM})$, depending on the tape sample. 
As a means to reduce inter-examiner variability, participants were provided instructions in the form of a detailed protocol document, and the majority also received an instructional presentation on the ESS method to be used in their physical fit examinations. The study distribution resulted in 16 completed kits overall, totaling 112 documented comparisons. Four approaches were used to assess the ILS results. The first two approaches evaluated error rates based on pre-determined thresholds or the overall examiner's conclusion. The other two methods assessed the level of interexaminer agreement in reporting the edge similarity scores.

The overall performance and error rates were estimated based on two varying interpretations of the reported ESS score and the respective correlation with the ground truth: 1) as per thresholds established based on larger population datasets ${ }^{4}$ in which an ESS score below 50 was considered a non-match, NM, and above 50, a match, M, and 2) as per the overall conclusion reported by the examiners (Match, Inconclusive, or Non-match). Overall, the observed error rates in the ILS study by threshold ESS values were $92 \%$ true positives (59/64), $8 \%$ false negatives (5/48), 100\% true negatives (48/48), and $0 \%$ false positives (0/64). Observed error rates by examiner-reported conclusion were as follows: $95 \%$ true positives (61/64), $0 \%$ false negatives $(0 / 48), 100 \%$ true negatives (48/48), and $0 \%$ false positives (0/64). The reduction in the true positive rate is the result of a $5 \%$ inconclusive rate ( 3 true positive samples were concluded as inconclusive across the sample set).

Next, we evaluated how close the study participants reported the ESS and comparison edge qualifiers in comparison to the consensus ranges. The majority $(86.6 \%)$ of reported ESS scores were within \pm 20 ESS compared to consensus values determined before the administration of the test, except for 15 out of 112 instances. We also observed that the majority (86 out of 112) of reported ESS scores fell within expected comparison edge qualifier ranges as established in a previous study by our research group ${ }^{4}$.

The proximity of reported ESS was also evaluated according to statistical significance testing via Analysis of Variance with the Dunnett's test at a 95\% confidence interval. $77 \%$ of the reported ESS showed no significant differences from the respective pre-distribution, consensus mean scores. Interestingly, it was found that 8 of 11 individuals who reported significantly different ESS scores from the consensus range received less instructional training.

ESS were also evaluated in terms of expected sample difficulty in relation to ground truth: true positive samples of less expected difficulty in the upper qualifier range $(\mathrm{M}+$, ESS between 80 and 100), true positive samples of more expected difficulty in the M- qualifier range (M-, ESS between $>50$ and <80), and non-matching samples (NM, ESS <50). It was observed that within the M+ and the NM groups, $81 \%$ of examiner ESS values were in agreement with consensus means according to the Dunnett's test. The M- group exhibited lower agreement of ESS scores according to Dunnett's (69\% of values) which was expected due to increased examination difficulty. The average ESS reported by participants for true good quality matches was $83 \pm 17 \%(\mathrm{M}+), 71 \pm 19 \%$ for $\mathrm{M}$-, and $7 \pm 11 \%$ for non-matches. 
Three main observations were derived from the participant results: 1) overall good agreement between ESS reported by examiners was observed, 2) the ESS score represented a good indicator of the quality of the match and rendered low percent of error rates on conclusions 3) those examiners that did not participate in formal method training tended to have ESS falling outside of expected pre-distribution ranges. Also, the survey responses revealed that: 1) further training is needed to standardize the reporting and interpretation of areas between scrim that contain less features to evaluate, and 2) further training is also needed to establish consistency in terms of the proper use of the comparison edge qualifier, as well as improving the understanding that the ESS is only one step in the overall assessment of a fractured edge comparison pair.

These results indicate the ESS methodology allows for a high rate of inter-examiner agreement in score value while still maintaining a correct pair classification (e.g., true match, true non-match) overall. The prevalent observed trends, as well as feedback received through the post-study survey, will be used to optimize the ESS methodology for the future development of a larger interlaboratory study which will be used to further validate the technique.

Most importantly, this pilot ILS represents the first time that a specific quantitative criterion is used for end-tape physical fit examinations to support and inform the examiner's opinion, to evaluate examiner error rates, and to provide a systematic peer review process. Indeed, most respondents reported the ESS approach was useful for documenting the basis for their findings, training new examiners, and allowing a transparent peer-review process. The implementation of the method is therefore anticipated to increase objectivity and help to move towards consensusbased guidelines.

\section{Introduction}

As covered in Chapter One, physical fits are considered the highest level of association between two materials in trace evidence. However, recent reports from the National Academy of Sciences $(\mathrm{NAS})^{1}$ and President's Council of Advisors on Science and Technology (PCAST), ${ }^{2}$ as well as a statement from the American Statistical Association ${ }^{3}$ have called for further research into the reporting of error rates and uncertainties associated with forensic analyses relying primarily upon visual, feature-based comparisons. In terms of physical fits, this is a challenging task due to the highly variable nature of circumstances faced in these examinations. To name a few, these varying factors include material type, size, quantity, and fracture source.

An approach to assessing the performance of comparative methods is by evaluating error rates observed in large datasets of known ground truth that are kept blind to the test takers. For duct tape physical fits, performance rate studies have been demonstrated by Bradley et al. in which no false positive or negatives were reported by any of the four participating examiners when assessing both hand torn and scissor cut sample sets ${ }^{5}$. These studies have also been shared by Tulleners and Braun in which low examiner error rates were demonstrated in an expanded sample set ( $\geq 1600$ samples) of various separation methods including hand torn, Elmendorf torn, scissor cut, and box cutter knife cut. Overall, the accuracy rate ranged from 98.15-100\% depending on separation method, 
while the false positive rate ranged from $0.00-3.33 \%$, and the false negative rate ranged from $0.00-$ $2.67 \%$.

Most recently, a study by Prusinowski et $a l .{ }^{4}$ introduced an alternative method to obtain a similarity score for a duct tape physical fit pair. The proposed method involves a relative percentage of consistent scrim areas along the total width of a tape pair, referred to as an edge similarity score (ESS) as demonstrated in Equation 1 below.

$$
\text { Edge similarity score }(\text { ESS })=\frac{\text { Number of consistent scrim areas }}{\text { Total number of scrim areas }} * 100
$$

Within the Prusinowski study ${ }^{4}$, a set of 2280 duct tape ESS were obtained from student examiners kept blind to sample ground truth for low, medium, and high-grade tapes of both hand torn and scissor cut separation methods. The resulting scores were evaluated in terms of performance rates. No false positives were observed in any of the sets and examiner accuracy ranged from $84.9 \%$ to over $99.0 \%$. The study also utilized the score likelihood ratio as a quantitative interpretation of the ESS within the sample set. ${ }^{4}$ This study demonstrated for the first time a systematic, quantitative method of score-based assessment of duct tape physical fits. This method provides several advantages including: 1) a method by which to inform the practitioner's opinion in difficult item alignment situations, 2) a method of providing further support to the practitioner's opinion of the physical fit, 3) the development of systematic criteria for a more transparent peer review process, 4) a method to assess experimental error rates, and 5) a means to assess factors that influence the quality of a fit.

Following the development of the ESS method for duct tape physical fit examinations by our research group, the expanding goals of the study included steps towards implementation of the method into forensic laboratories. Before implementation can occur, extensive verification of the method's utility, validity, reliability, and reproducibility between different examiners as well as different laboratories must be assessed. An effective approach for such assessment is via an interlaboratory study. According to ISO/IEC $17043,{ }^{7}$ these studies serve to evaluate methods or tests on the same or similar items by two or more laboratories in accordance with predetermined conditions. Inter-laboratory comparisons are utilized in several scientific disciplines such as biotechnology, environmental science, food science, forensics, and medicine. ${ }^{8-12}$ Purposes for inter-laboratory studies can take several forms. One of which is to establish reproducibility of a single analytical method as part of a validation process. These studies are referred to as collaborative trials or method performance studies. ${ }^{13}$ Inter-laboratory comparisons can also be utilized to reach a consensus on the characterization of a standard reference material or a protocol of analysis or interpretation, as is often reported in ASTM standard test methods. For example, ASTM E $177^{14}$ and E691 ${ }^{15}$ describe practices for the use of precision and bias in test methods and how to conduct an interlaboratory study to determine intra and inter-lab precision, respectively. Further, inter-laboratory studies can also be initiated for methods already standardized and routinely used in laboratories. This is done for purposes of laboratory performance assessment and identification of bias originating from either the method or between laboratories. This type of comparison is known as proficiency testing or laboratory performance studies. ${ }^{13}$ 
Inter-laboratory comparisons commonly occur in forensic laboratories during the assessment of new methods or through the route of proficiency testing. Due to the nature of forensic casework, demonstrated confidence in forensic laboratory performance is an essential aspect of a quality assurance. Interlaboratory testing is also critical for laboratory accreditation, which is recommended for all forensic laboratories in the United States by the National Commission on Forensic Science (NCFS). ${ }^{16}$ Furthermore, ISO/IEC 17025 requires calibrating and testing laboratories to participate in proficiency testing, and ISO/IEC 17011 requires that accrediting bodies further enforce this by mandating a laboratory's participation in proficiency testing, as well as monitor the laboratory's associated performance. ${ }^{17,18}$

These tests are supplied to forensic laboratories through external testing service providers, an example of US providers being Collaborative Testing Services, Inc. (CTS ${ }^{\odot}$ ) and Forensic Testing Services (FTS), who provide proficiency tests in a variety of disciplines, including physical fits. Summary reports help participants to compare their performance to the expected results, and to the results reported by other examiners in the field. This process is useful not only to demonstrate proficiency but also to identify areas of improvement.

Unlike proficiency testing, interlaboratory studies are less stringent in that the results are used as a refinement process of the early stages of a method rather than as quality control that needs to pass minimum standards to maintain the proficiency status. Volunteers often participate in an anonymous and blind process. However, the requirements for the design, distribution, and analysis of ILS often follow those specified for a proficiency test. These include, but are not limited to, test's design by a qualified expert panel, pre-distribution testing to demonstrate consensus of results, coordination and management by an independent entity that maintains traceability of the test, distributes the samples, and provides summary reports to the participants.

The aim of this study was to design and implement an inter-laboratory study of duct tape physical fits utilizing the ESS method previously developed by our research group. This was done to evaluate the practicality, reproducibility, and accuracy of the method through resulting ESS distributions and feedback provided by practitioners. By assessing the variability of responses received by examiners, our group can demonstrate the enhanced support of examiner opinion the method provides while establishing reproducibility estimates needed for laboratory implementation. The feedback received from the study can be used to clarify and improve the method to be of optimal utility to the field.

\section{Materials and Methods}

3.1. Interlaboratory study kits design: pool of duct tape fracture edge comparisons and sample preparation

To create the fractured duct tape samples, 150 tape fragments were hand-torn from a single roll of Duck Brand Electrician's Grade Gray Duct Tape (Duck Brand, ShurTech Brands, Avon, OH). The selected tape roll exhibited a 4.0 mils backing thickness, 2.5 mils adhesive thickness, and 20/8 
warp/weft scrim count. All torn samples were roughly $6-8 \mathrm{~cm}$ in length and were placed on individual acetate, overhead transparency film sheets following fracture. All samples were labelled as to denote their true matching pair. All sample pairs were then divided into 5 groups by both ground truth and macroscopic edge morphology. Initial group designations are as shown in Table 1, while Figure 1 demonstrates examples of edge morphology classification.

Table 1. Initial sample set classification ( $n=75$ fracture edge pairs)

\begin{tabular}{|c|c|c|}
\hline Group Number & Ground Truth & Edge Morphology \\
\hline 1 & Match & Mostly straight/wavy \\
\hline 2 & Match & $\begin{array}{c}\text { Curved/puzzle-like } \\
\text { (intermediate) }\end{array}$ \\
\hline 3 & Match & Puzzle-like \\
\hline 4 & Non-match & Mostly straight/wavy \\
\hline 5 & Non-match & $\begin{array}{c}\text { Curved/puzzle-like } \\
\text { (intermediate) }\end{array}$ \\
\hline
\end{tabular}

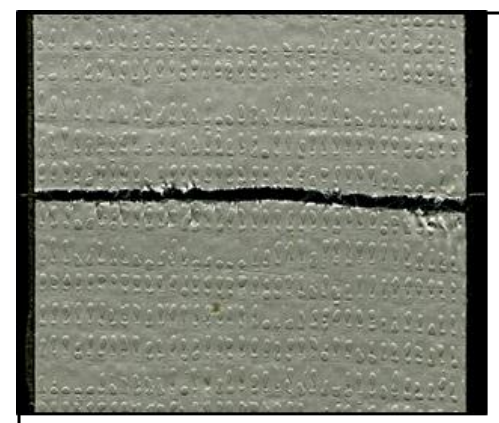

A. Straight/wavy

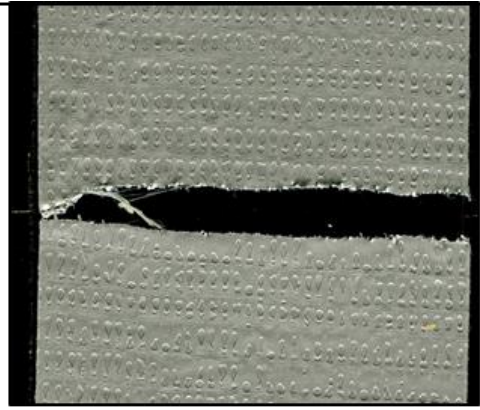

B. Curved/puzzle-like (Intermediate)

Figure 1. Comparison edge morphology classification for two examples of matching pairs (A and C) and one example of a non-matching pair (B)

While matching pairs were determined at the time of fracturing, non-matching pairs were assigned to one another through a random number generator function in Microsoft Excel® 2016. Nonmatching pairs were then separated into groups 4 and 5 based on edge morphology.

Initial tape pair groups were analyzed via the ESS method ${ }^{4}$ by four independent examiners using a blind process, where the ground truth was unknown by the analysts. The pre-distribution examination consisted of thorough assessment of each sample pair for alignment features on both the backing and adhesive sides under a stereomicroscope. Lighting conditions involved alternating between both transmitted and reflected light in order to observe varying features with optimal contrast. It was observed that adhesive detail was typically best viewed under transmitted lighting while backing detail was best viewed under oblique, reflected lighting. Magnification varied from 8-35x depending on the size of the edge feature under observation. Throughout the comparison process, examiners made annotations on a physical scrim bin template to indicate which bins were 
considered consistent (" $1 "=$ match) and inconsistent (" $0 "=$ non-match). The templates allowed for a more transparent discussion and review process when comparing examiner results to assess which samples resulted in the highest consensus in their ESS results. For a more detailed description of the edge features commonly assessed as well as the ESS method, please refer to Section 3.3 below.

Comparison pairs resulting in inter-examiner ESS relative standard deviations greater than $10 \%$ ESS were eliminated from the sample set as potential inter-laboratory kit sample. The remaining sample pairs meeting examiner agreement criteria were further rearranged into seven groups of three similar pairs each, to prepare 3 kits of seven comparison pairs. Classification of the seven optimized groups is provided in Table 2.

Table 2. Optimized sample set classification

\begin{tabular}{|c|c|c|c|}
\hline $\begin{array}{c}\text { Group Number } \\
(\mathrm{n}=3 \text { tape pairs per } \\
\text { group) }\end{array}$ & Ground Truth & $\begin{array}{c}\text { Expected } \\
\text { Comparison Edge } \\
\text { Qualifier }\end{array}$ & Edge Morphology \\
\hline 1 & Match & M+ & Straight/wavy \\
\hline 2 & Match & M- & Puzzle-like \\
\hline 3 & Match & M+ & Puzzle-like \\
\hline 4 & Non-match & NM+ & Straight/wavy \\
\hline 5 & Non-match & NM+ & $\begin{array}{c}\text { Curved/puzzle-like } \\
\text { (intermediate })\end{array}$ \\
\hline 6 & Match & M+ & Puzzle-like \\
\hline 7 & Non-match & NM+ & Straight/wavy \\
\hline
\end{tabular}

Kits were composed of one pair per optimized group. The pre-distribution score means provided a baseline for expected participant ESS values. The matching pairs consisted of 3 pairs with consensus ESS ranging from $86 \%$ to $99 \%(\mathrm{M}+)$ and one more difficult match pair with consensus ESS scores ranging from $70 \%$ to $77 \%(\mathrm{M}-)$; while the non-matching (NM) pairs had consensus scores from $0 \%$ to $11 \%$. The desired participant agreement threshold was set for $\pm 20 \%$ from the consensus mean.

\subsection{Design of test distribution}

The study kits consisted of the seven duct tape comparison pairs, a printed document outlining method protocol, and hard-copy templates for score documentation. Along with the physical kits sent by mail, participants received via email an instructional presentation, a digital copy of the protocol, and a digital template containing tabs for score documentation of each comparison pair. The final tab of the digital template file contained a post-study survey for each participant. Copies of these documents are provided in Appendix A. In addition, many study participants were present at a formal presentation of the proposed comparison method at which physical samples (none being used in the study kits) were available for hands-on instruction. Further, at the time of distribution, each participant was offered additional explanation of the protocol via phone or video conference. 
Study kits were distributed in a modified petal test design in which each kit would return to the coordination body before being re-distributed to the next participant as a Round Robin. A schematic of the study design is provided in Figure 2.

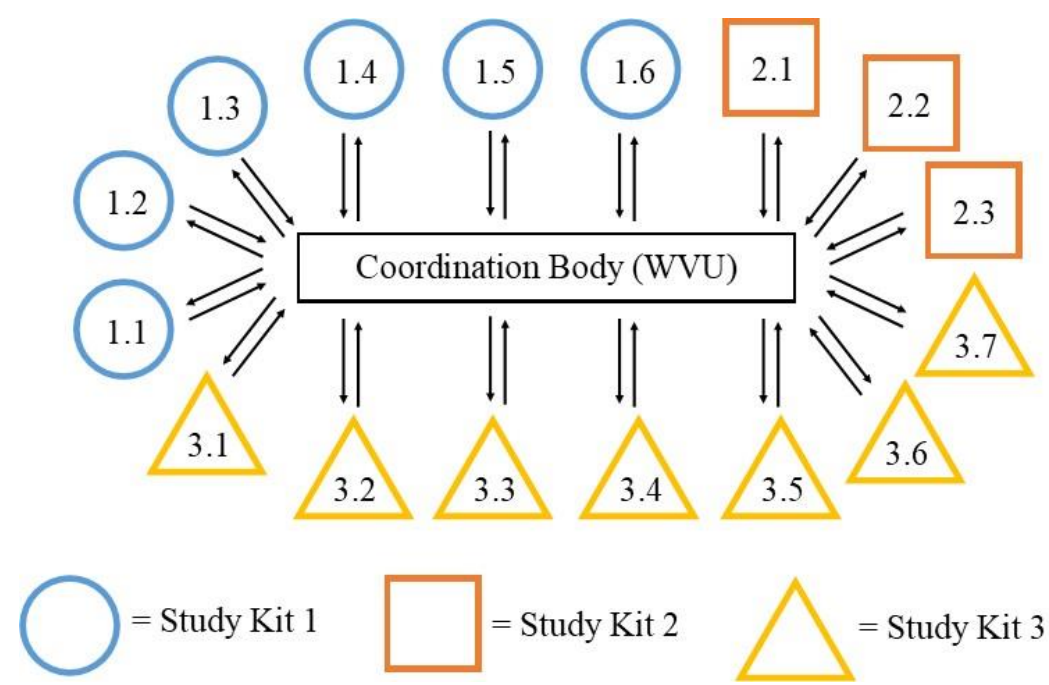

Figure 2. Inter-laboratory modified petal test distribution

We aimed for 7 participants per kit. However, due to uncontrolled circumstances, Kit 1 had six total participants, Kit 2 had three total participants, and Kit 3 had seven total participants. As kits were returned, sample pairs were examined under a stereomicroscope to assure tapes had not been manipulated or written upon before re-packaging the kit for continued distribution. The study distribution design allowed for simultaneous distribution of each of the three kits. Distribution took place over a period of about nine months. All participants were asked for a turnaround time of 3-4 weeks, although several took longer.

\subsection{Reporting instructions}

Participants were asked to follow the ESS method as outlined in Prusinowski et al. ${ }^{4}$ Within this method, participants begin their assessment by a general stereoscopic examination of both the backing and adhesive sides of a duct tape pair. For purposes of the inter-laboratory study, participants were given the specific physical feature examples of dimpling, calendering striae, backing distortion, warp scrim alignment, protruding warp yarns, adhesive distortion, continuation of scrim pattern, double weft edge scrim, and missing scrim to assess during their initial physical examinations. Images of the provided feature examples are shown in Figures $\mathbf{3}$ and $\mathbf{4}$ below. 


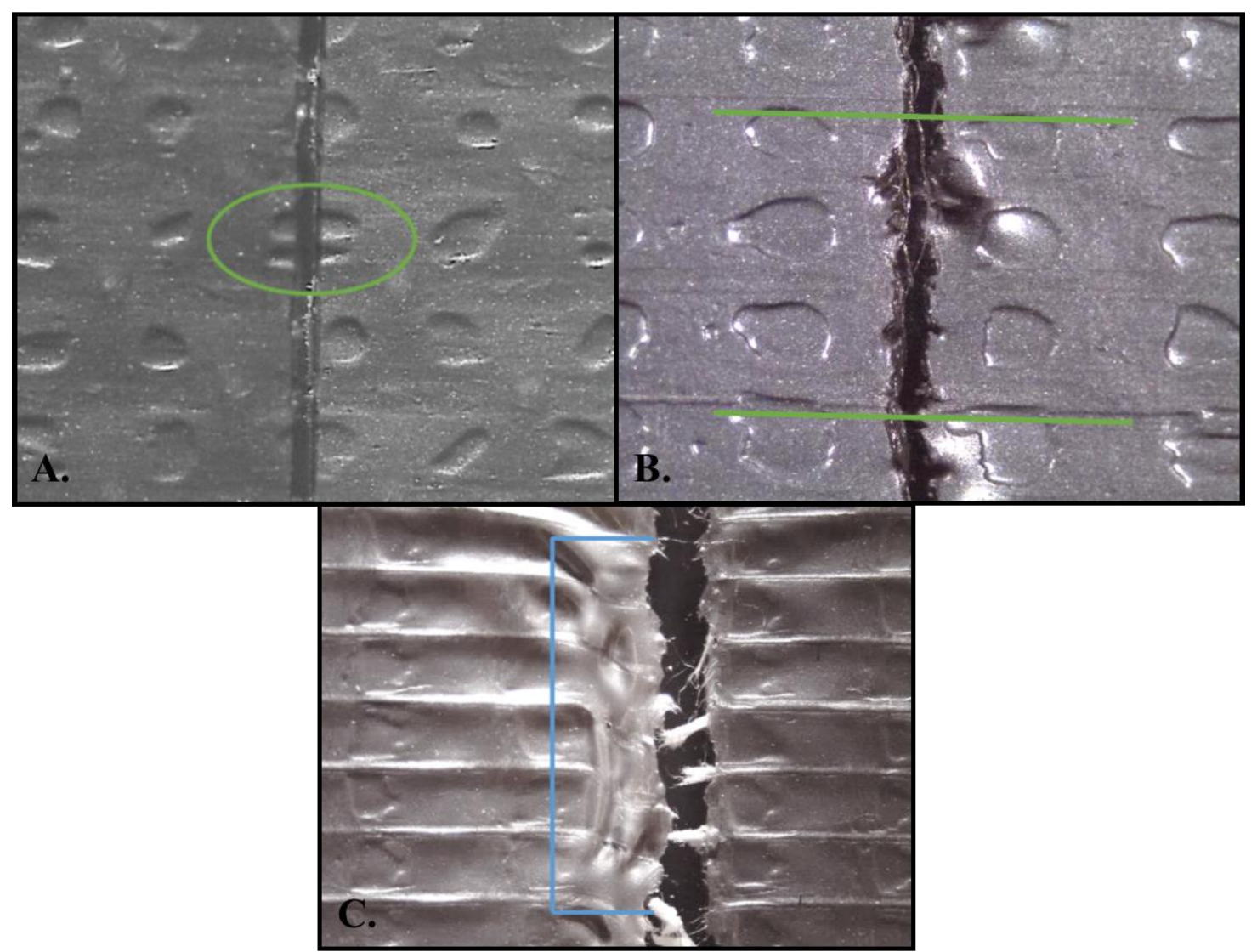

Figure 3. Backing physical feature examples: A) dimpling, B) calendering striae, C) backing distortion 


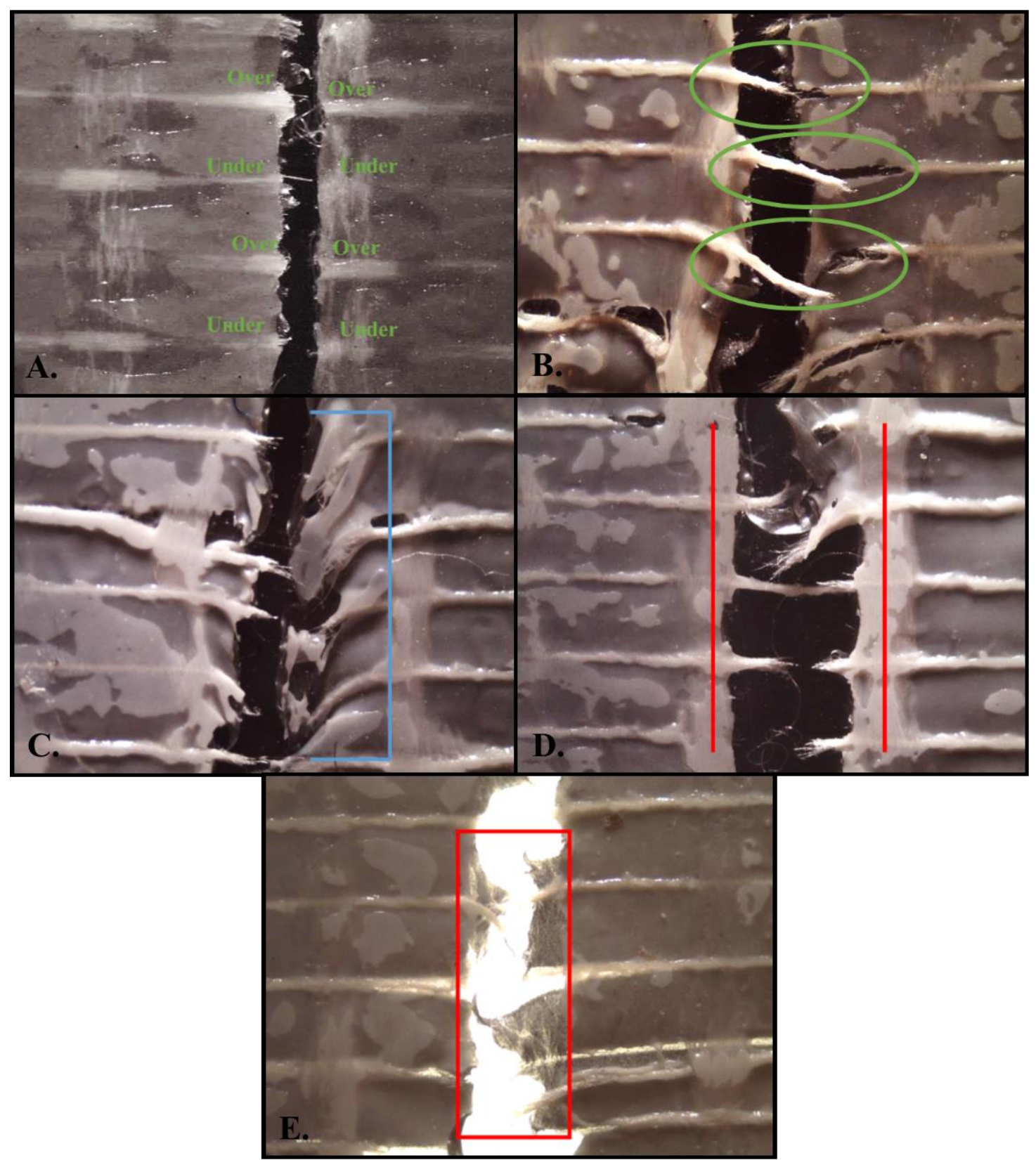

Figure 4. Adhesive and scrim physical feature examples: A) warp scrim alignment/continuation of scrim pattern, B) protruding warp yarns, C) adhesive distortion, D) double weft edge scrim, E) missing scrim

After initial assessment, participants will then assess the fracture edge using the scrim area or bin, the smallest unit of assessment bound by warp and weft scrim yarns which assures all participants are making decisions at the same areas along the edge of a tape pair. Examiners use the scrim bin to determine an edge similarity score (ESS) according to Equation $\mathbf{1}$ as shown above in the Introduction. 
Participants then determined comparison edge qualifiers and comparison pair overall conclusions with options as shown in Table 3 below:

Table 3. Options for comparison pair overall conclusion and qualifiers, as well as expected ESS ranges per qualifier

\begin{tabular}{|c|c|c|}
\hline $\begin{array}{c}\text { Comparison Pair Overall } \\
\text { Conclusion }\end{array}$ & Comparison Edge Qualifier & $\begin{array}{c}\text { Expected ESS Range per } \\
\text { Qualifier }\end{array}$ \\
\hline $1=$ Match & $\begin{array}{c}\text { M+ = Match with high } \\
\text { certainty }\end{array}$ & $80 \%-100 \%$ \\
\hline INC = Inconclusive & M- = Match with low certainty & $50 \%-<80 \%$ \\
\hline $0=$ Non-match & INC = Inconclusive & $25 \%-<50 \%$ \\
\hline & $\begin{array}{c}\text { NM- }=\text { Non-match with low } \\
\text { certainty }\end{array}$ & $0 \%-\leq 25 \%$ \\
\hline & $\begin{array}{c}\text { NM+ = Non-match with high } \\
\text { certainty }\end{array}$ \\
\hline
\end{tabular}

Table 3 above outlines expected ranges of ESS per qualifier according to previous SLR ranges in a publication by Prusinowski et $a .^{4}$. In the study, assessment of duct tape ESS via the score likelihood ratio (SLR) revealed that most ESS greater than $80 \%$ resulted in SLRs supporting a match conclusion, while ESS lower than $25 \%$ resulted in SLRs supporting a non-match conclusion. Samples were purposefully selected for the study kits that had been assigned a variation of ESS ranges in order to provide a range of scenarios for participants.

\subsection{Assessment of the inter-laboratory results}

Results were assessed through four main avenues: 1-2) error rate assessment based on predetermined thresholds or the overall examiner's conclusion, 3) ESS and qualifier consensus range analysis, and 4) distribution and statistical analysis of ESS as grouped by expected comparison difficulty in relation to ground truth. Each approach is outlined in further detail below. All calculations and range assessments were performed in Microsoft Excel (Version 19.08), while statistical analysis through Dunnett's testing was performed in JMP Pro 13 (v.2016, SAS Institute Inc., NC).

\subsubsection{Performance rate assessment}

The first assessment of study results was via performance rates including true positive rate (TPR), true negative rate (TNR), false positive rate (FPR), false negative rate (FNR), inconclusive rate, sensitivity, specificity, and accuracy. All rates were calculated according to the respective equations in Table 4. 
Table 4. Performance rate equation summary

\begin{tabular}{|c|c|}
\hline Performance rate & Equation \\
\hline True Positive Rate (TPR) & $T P R=\frac{T P}{T P+F N+I N C} * 100$ \\
\hline True Negative Rate (TNR) & $T N R=\frac{T N}{T N+F P+I N C} * 100$ \\
\hline False Positive Rate (FPR) & $F P R=\frac{F P}{F P+T N+I N C} * 100$ \\
\hline False Negative Rate (FNR) & $F N R=\frac{F N}{T P+F N+I N C} * 100$ \\
\hline Inconclusive Rate (TP) & INC $=\frac{I N C}{T P+F N+I N C} * 100$ \\
\hline Sensitivity & Sensitivity $=\frac{T P}{T P+F N} * 100$ \\
\hline Specificity & Specificity $=\frac{T N}{T N+F P} * 100$ \\
\hline Accuracy & Accuracy $=\frac{T P+T N}{T P+T N+F P+F N+I N C} * 100$ \\
\hline
\end{tabular}

Performance rates were assessed in two different interpretations: 1) according to a pre-established ${ }^{4}$ match/non-match ESS threshold in which ESS < 50\% indicate a non-match result and ESS > 50\% indicate a match result or 2) according to assigned overall examiner conclusion of match, nonmatch, or inconclusive - regardless of determined ESS value.

\subsubsection{ESS and qualifier consensus range analysis}

Resulting ESS distributions per kit were also examined to assess if scores fit within the predetermined \pm 20 threshold versus the consensus mean, and that participants were in agreement with the ground truth (e.g., match versus non-match). Distributions of comparison edge qualifiers between kits were also examined to observe if participant qualifiers fell within expected ranges as outlined in Table 3 above.

\subsubsection{ESS as grouped by expected comparison difficulty and ground truth}

ESS results were also assessed by grouping the resulting values in terms of the expected comparison difficulty in relation to ground truth: true positive samples of less expected difficulty $(\mathrm{M}+$ qualifier range, $\mathrm{M}+$ ), true positive samples of more expected difficulty (M- qualifier range, M-) and non-matching samples (NM). ESS distributions per group are examined through boxplots. Following exploratory ESS variation analysis, descriptive statistics were reported and Analysis of Variance (ANOVA) for a Randomized Complete Block Design (RBCD) was performed on the data to determine if significant differences existed between examiner results and the predistribution, consensus mean per difficulty grouping. This was done specifically through the utility of the Dunnett's test, which compares individual sample means to an established control mean to determine if any statistically significant differences arise. 
In addition to tape pair results, survey results were compiled to assess examiner feedback and comments that will be utilized to modify and improve the method to improve its practicality for future implementation into forensic laboratories. These results are provided at the end of the ESS result discussion.

\section{Results and Discussion}

\subsection{Pre-Distribution Results}

As is required for interlaboratory testing, pre-distribution analysis was conducted and documented. Prior to distribution of the study kits, four examiners analyzed tape pairs and assigned ESS values without knowing the origin of the samples (blind test). Table 5 below outlines the inter-examiner consensus mean estimated per sample pair, while Figure 5 displays boxplots of consensus ESS values per sample kit.

Table 5. Pre-distribution consensus ESS means per tape pair ( $\mathrm{N}=4$ examiners)

\begin{tabular}{|c|c|c|c|}
\hline Kit Number & Pair Number & Consensus ESS Mean & Standard Deviation \\
\hline \multirow{7}{*}{1} & 1 & 97 & 4 \\
\hline & 2 & 77 & 6 \\
\hline & 3 & 88 & 3 \\
\hline & 4 & 11 & 3 \\
\hline & 5 & 2 & 3 \\
\hline & 6 & 95 & 2 \\
\hline & 7 & 5 & 4 \\
\hline \multirow{7}{*}{2} & 1 & 99 & 3 \\
\hline & 2 & 70 & 3 \\
\hline & 3 & 86 & 2 \\
\hline & 4 & 10 & 4 \\
\hline & 5 & 0 & 0 \\
\hline & 6 & 96 & 3 \\
\hline & 7 & 3 & 3 \\
\hline \multirow{7}{*}{3} & 1 & 97 & 4 \\
\hline & 2 & 75 & 5 \\
\hline & 3 & 89 & 2 \\
\hline & 4 & 10 & 3 \\
\hline & 5 & 0 & 0 \\
\hline & 6 & 92 & 4 \\
\hline & 7 & 5 & 4 \\
\hline
\end{tabular}




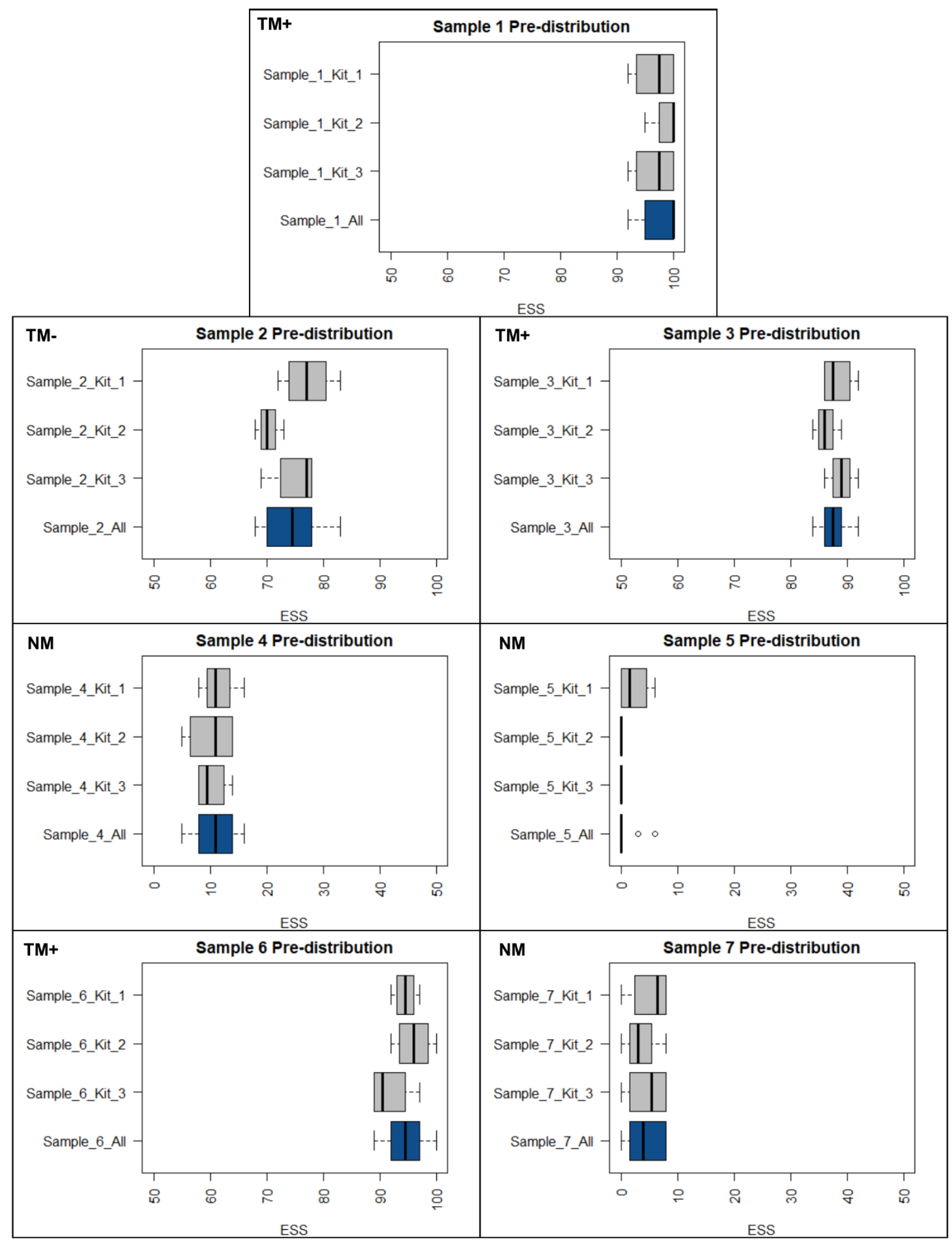

Figure 5. Pre-distribution, consensus ESS values per sample per kit ( $\mathrm{N}=4$ examiners) 
As observed in Table 5 and Figure 5, sample pairs were selected for use in the study kits in which the consensus mean had a standard deviation value lower than 10. In addition, samples were selected such that each respective pair would be of similar edge morphology and expected ESS range to its equivalent pair in all study kits. Sample groups were also assigned expected comparison edge qualifier ranges due to previously reported threshold values ${ }^{4}$. Table 6 below displays selected edge morphology, ground truth, expected qualifier range, and mean ESS across equivalent samples per kit.

Table 6. Sample group pre-distribution characteristics across samples between the 3 kits

\begin{tabular}{|c|c|c|c|c|c|c|c|}
\hline $\begin{array}{c}\text { Sample } \\
\text { group }\end{array}$ & 1 & 2 & 3 & 4 & 5 & 6 & 7 \\
\hline $\begin{array}{c}\text { Edge } \\
\text { morphology }\end{array}$ & $\begin{array}{c}\text { Mostly } \\
\text { straight/wavy }\end{array}$ & $\begin{array}{c}\text { Puzzle- } \\
\text { like }\end{array}$ & $\begin{array}{c}\text { Puzzle- } \\
\text { like }\end{array}$ & $\begin{array}{c}\text { Mostly } \\
\text { straight/wavy }\end{array}$ & $\begin{array}{c}\text { Curved/puzzle- } \\
\text { like } \\
\text { (intermediate) }\end{array}$ & $\begin{array}{c}\text { Puzzle- } \\
\text { like }\end{array}$ & $\begin{array}{c}\text { Mostly } \\
\text { straight/wavy }\end{array}$ \\
\hline $\begin{array}{c}\text { Ground } \\
\text { truth }\end{array}$ & Match & Match & Match & Non-match & Non-match & Match & Non-match \\
\hline $\begin{array}{c}\text { Expected } \\
\text { qualifier } \\
\text { range }\end{array}$ & M+ & M- & M+ & NM+ & NM+ & M+ & NM+ \\
\hline $\begin{array}{c}\text { Mean ESS } \\
\text { across kits }\end{array}$ & 97 & 74 & 88 & 11 & 1 & 94 & 4 \\
\hline $\begin{array}{c}\text { ESS } \\
\text { standard } \\
\text { deviation }\end{array}$ & 1 & 4 & 1 & 1 & 1 & 2 & 1 \\
\hline
\end{tabular}

\subsection{Performance Rate Assessment}

Performance rates were considered through two main interpretations: 1) according to thresholds established based on larger population datasets ${ }^{4}$ in which an ESS score below 50 was considered $\mathrm{NM}$, and above $50 \mathrm{M}$, and 2) according to the conclusion reported by the examiners ("1" = Match, "INC" = Inconclusive, " $0 "$ = Non-match). For each avenue, true positive rate (TPR), true negative rate (TNR), false positive rate (FPR), false negative rate (FNR), inconclusive rate (INC), sensitivity, specificity, and accuracy per kit were calculated according to the equations in Table 4. It should be noted that in this study there were three inconclusive conclusions, all of which were true match samples. Table 7 below provides TPR, TNR, FPR, FNR, INC, sensitivity, specificity, and accuracy rates overall and per kit for both overall examiner conclusion as well as conclusions by ESS based on the expected 50/50 non-match/match threshold. As observed, accuracy rates by examiner conclusion ranged between 90 and $100 \%$ across all kits with low error rates. Accuracy rates by ESS threshold ranged between 88 and $10 \%$ with error rates ranging from $0-21 \%$. Higher error rates arose with Kits 1 and 2, thereby also affecting the overall error rates. When considering Kit 1 classifications by ESS threshold, there were five samples with ESS scores reported below $50 \%$ that were still concluded as matches. However, this decreased the TPR and increased the FNR. Kits 1 and 2 exhibited the presence of inconclusive conclusions by the examiner for true match samples ( 1 within kit 1 and 2 within kit 2 ). While not necessarily a misclassification, this caused a slight decrease in the accuracy and TPR for each kit. 
Table 7. Overall performance rates using the examiner reported conclusion and the ESS threshold conclusion

\begin{tabular}{|c|c|c|c|c|c|c|c|c|}
\hline & $\begin{array}{c}\text { Kit 1 } \\
\text { examiner } \\
\text { conclusion }\end{array}$ & $\begin{array}{c}\text { Kit 1 } \\
\text { ESS } \\
\text { threshold }\end{array}$ & $\begin{array}{c}\text { Kit 2 } \\
\text { examiner } \\
\text { conclusion }\end{array}$ & $\begin{array}{c}\text { Kit 2 } \\
\text { ESS } \\
\text { threshold }\end{array}$ & $\begin{array}{c}\text { Kit 3 } \\
\text { examiner } \\
\text { conclusion }\end{array}$ & $\begin{array}{c}\text { Kit 3 } \\
\text { ESS } \\
\text { threshold }\end{array}$ & $\begin{array}{c}\text { Overall } \\
\text { examiner } \\
\text { conclusion }\end{array}$ & $\begin{array}{c}\text { Overall } \\
\text { ESS } \\
\text { threshold }\end{array}$ \\
\hline TPR & 96 & 79 & 83 & 100 & 100 & 100 & 95 & 92 \\
\hline TNR & 100 & 100 & 100 & 100 & 100 & 100 & 100 & 100 \\
\hline FPR & 0 & 0 & 0 & 0 & 0 & 0 & 0 & 0 \\
\hline FNR & 0 & 21 & 0 & 0 & 0 & 0 & 0 & 9 \\
\hline INC & 4 & NA & 17 & 0 & 0 & 0 & 5 & NA \\
\hline Sensitivity* & 100 & 82 & 100 & 100 & 100 & 100 & 100 & 92 \\
\hline Specificity* & 100 & 100 & 100 & 100 & 100 & 100 & 100 & 100 \\
\hline Accuracy & 98 & 88 & 90 & 100 & 100 & 100 & 97 & 96 \\
\hline
\end{tabular}

*It should be noted that inconclusive conclusions were not included in sensitivity and specificity rates as they were not considered as false negatives or false positives, respectively.

\subsection{ESS and Qualifier Consensus Range Analysis}

Figures 6-8 below display examiner ESS variation as compared to the pre-distribution, consensus mean for each of the three study kits. As shown Figure 6, much more score variation was observed in the true positive pairs (Samples 1-3 and 6) as compared to the true negative pairs (Sample 4-5 and 7) in Study Kit 1. In Study Kit 2 (Figure 7), while variation was observed in both the true positive and true negative pairs, the variability between examiners was lower than that of Study Kit 1. Study Kit 3 (Figure 8) exhibits good consistency in true positive pair ESS values. While more variation is observed in the true negative samples (Samples 4-5 and 7) than the true positive samples in Study Kit 3, all true negative ESS were below the expected 50\% threshold for a NM conclusion. 


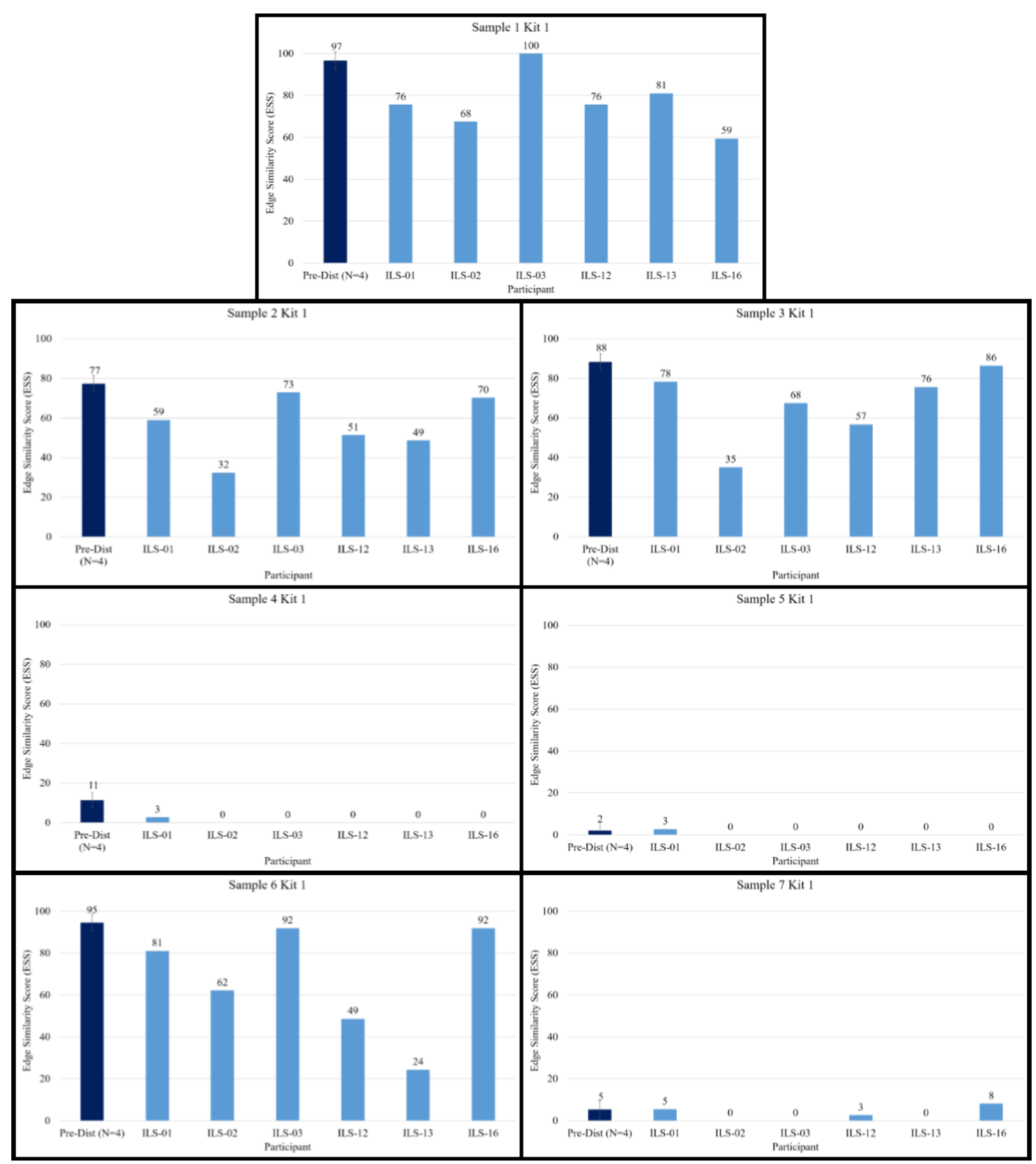

Figure 6. Kit 1 examiner ESS variation as compared to pre-distribution mean (consensus: $\mathrm{N}=4$ examiners) 


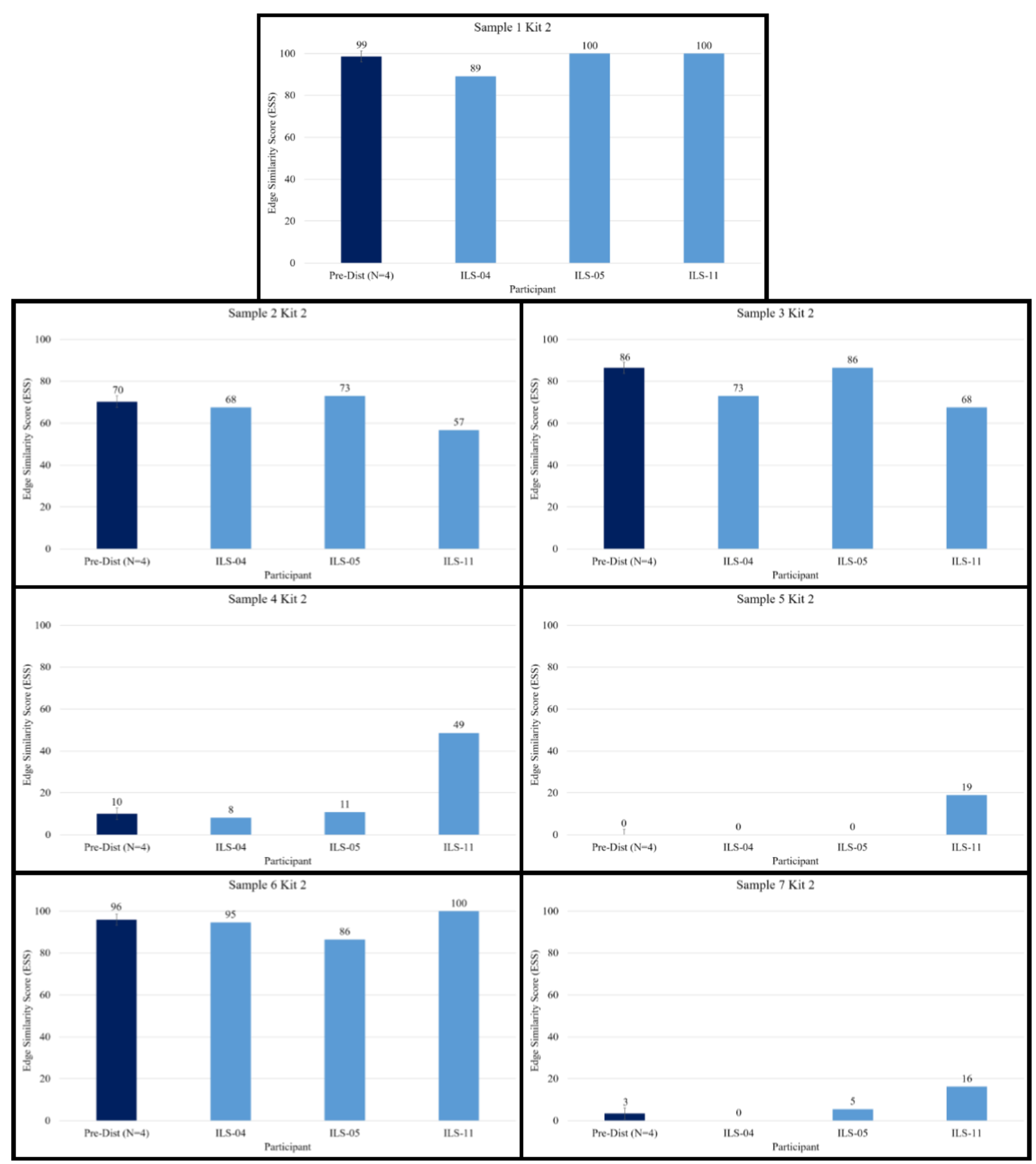

Figure 7. Kit 2 examiner ESS variation as compared to pre-distribution mean (consensus: $\mathrm{N}=4$ examiners) 


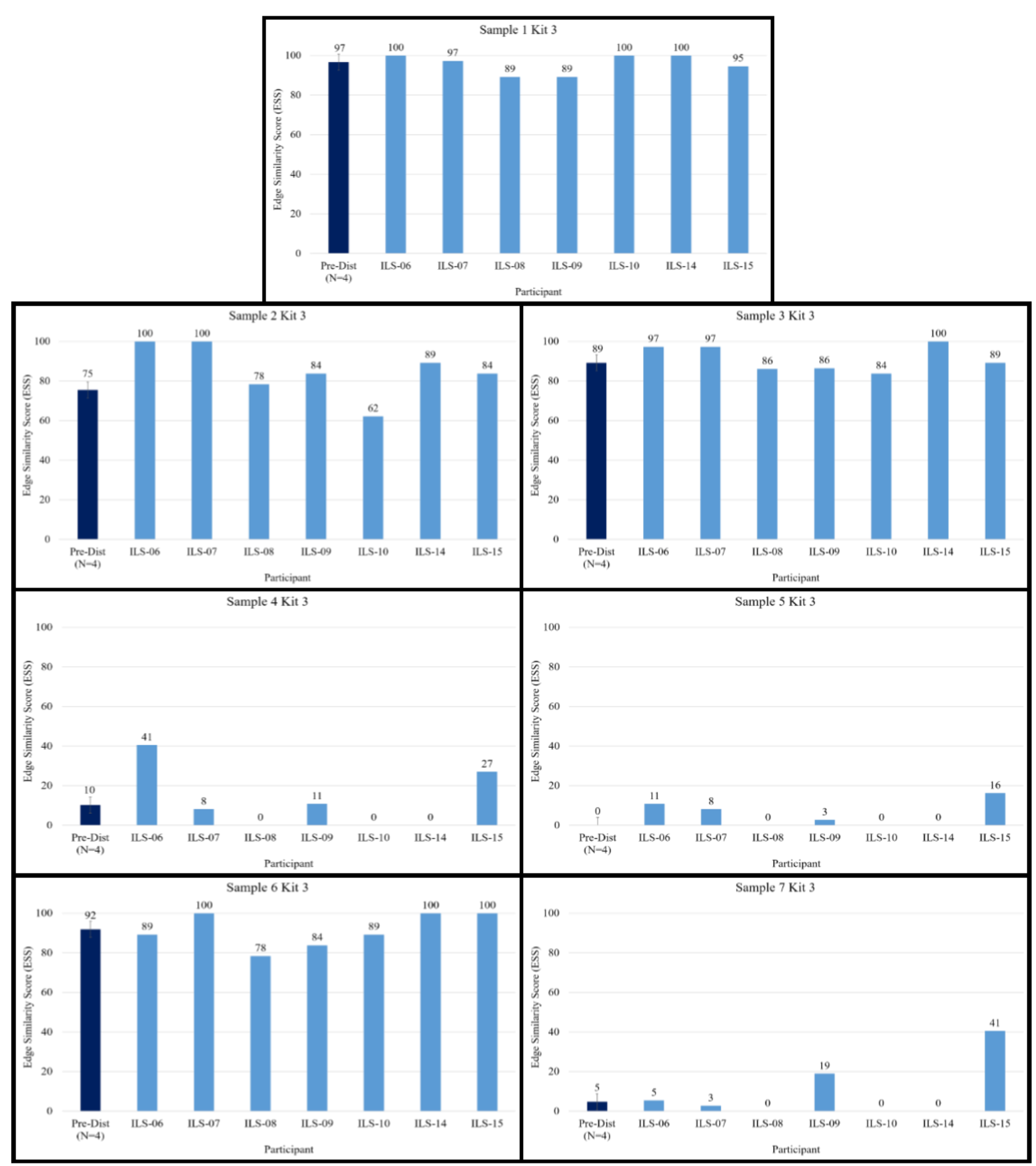

Figure 8. Kit 3 examiner ESS variation as compared to pre-distribution mean (consensus: $N=4$ examiners)

During the pre-distribution process, it was estimated participant ESS would tend to fall within a \pm 20 threshold from the consensus mean. Figures 9-11 below display examiner ESS variation as compared to consensus mean upper and lower limits based on the $20 \%$ threshold. It should be noted that the upper limit could not surpass 100 while the lower limit could not extend below 0 . Between all kits, the majority of participants fell within the expected ranges. Specifically, in Kit 1 
(Figure 9), while all examiner scores for the true negative samples fell within the expected range, four examiners fell outside the range in the true positive samples in 12 instances across all samples. Interestingly, three of these four examiners did not receive formal method training through either the in-person or teleconference options, indicating a lack of comprehension on the application of the ESS method. Indeed, 10 of the 12 instances of variation outside the consensus means could be identified as outliers via the Grubbs' test with a 95\% confidence interval.

For Study Kit 2 (Figure 10), all examiner scores fell within the expected range with the exception of one examiner (ILS-11) with Sample 4. While the examiner's overall conclusion (non-match) was still correct, the assigned ESS fell above the upper 20\% threshold limit (the examiner reported a $49 \%$ while the upper consensus range limit was $30 \%$. This participant was present for formal training, this was the only instance of a score not falling within the expected threshold in the overall kit results.

Figure 11 shows all examiner scores for Study Kit 3 fell within the expected range with the exception of two instances - one examiner with Sample 4 and another with Sample 7. However, both examiners' overall conclusions (non-match) were still correct. Neither of the participants reporting outside of the thresholds were present for formal training. Further, the deviation on the ESS scores for these participants/samples were less drastic than those observed on some of the examiners of Kit 1. 


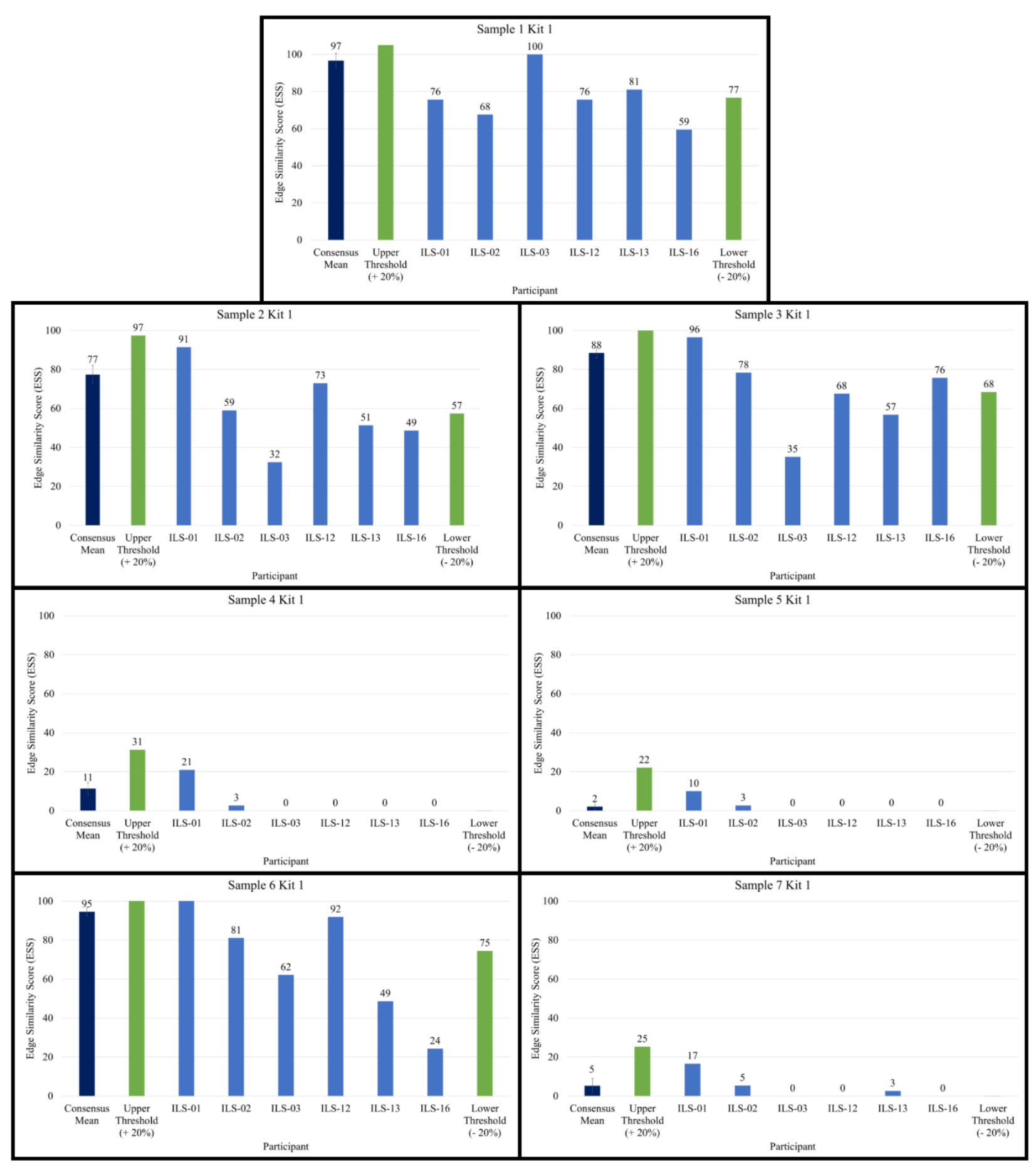

Figure 9. Kit 1 examiner ESS variation as compared to consensus mean $\pm 20 \%$ threshold 


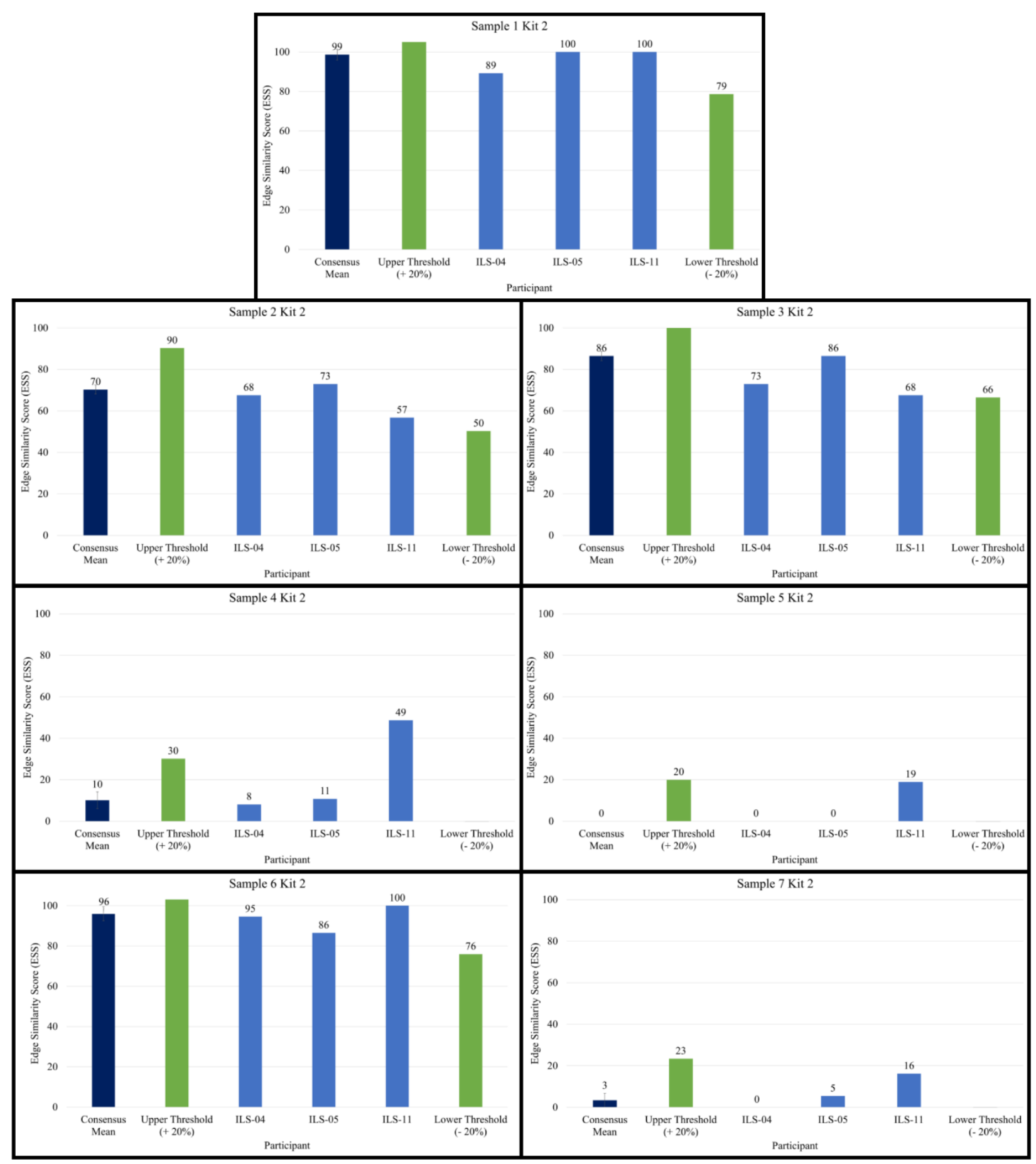

Figure 10. Kit 2 examiner ESS variation as compared to consensus mean $\pm 20 \%$ threshold 


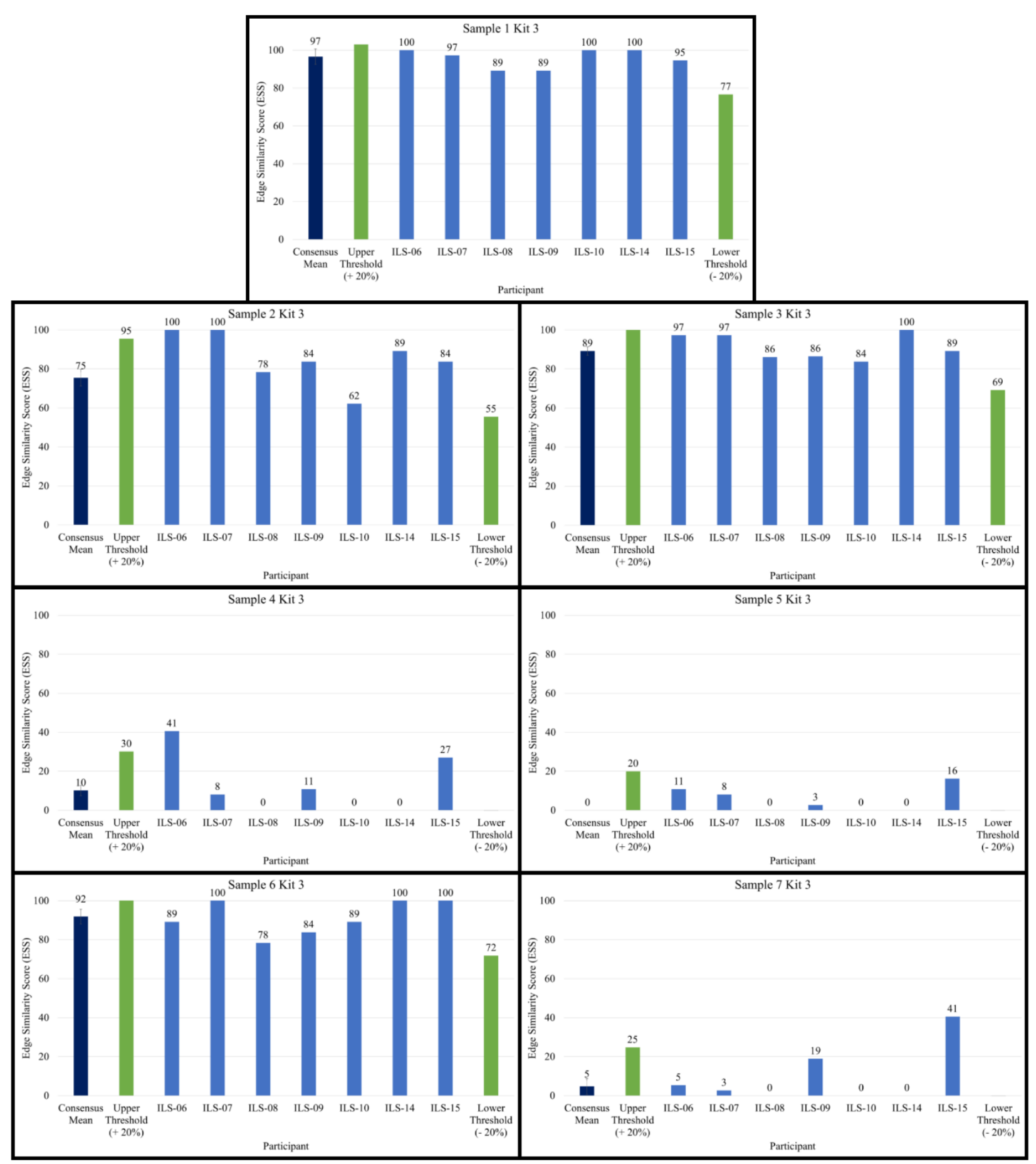

Figure 11. Kit 3 examiner ESS variation as compared to consensus mean $\pm 20 \%$ threshold

Examiner ESS scores were also evaluated based upon expected qualifier thresholds, as summarized in Table 3. Observations within these ranges per kit are provided in Figures 12-14 below. As observed in Study Kit 1 (Figure 12), all true negative samples fell within the expected $\mathrm{NM}+$ qualifier range. Again, more variation was observed in this kit for the true positive pairs. Of the participants with scores falling outside of the expected range, participants ILS-02, ILS-12, and 
ILS-13 provided ESS that were consistently lower than the expected range. As mentioned earlier, this seems to be a result of lack of formal training.

Within Study Kit 2 (Figure 13), all examiner scores fell within the expected qualifier range with the exception of two examiners for Sample 3 and one examiner for Sample 4. In Sample 3, both participant (ILS-04 and ILS-11) scores fell below the M+ threshold range by 7 and 12 ESS units, respectively. In addition, while Sample 3 was concluded a M+ by participant ILS-04, participant ILS-11 labeled Sample 3 as an INC, indicating they had experienced less confidence in the overall sample assessment. For Sample 4, the ESS assigned by ILS-11 was $49 \%$ while the upper expected qualifier range limit was 25\%. While these participants did attend formal training, no misclassifications were observed despite ESS out of expected comparison edge qualifier ranges.

Figure 14 below provides examiner ESS variation in Study Kit 3 as compared to the expected comparison edge qualifier threshold. As observed in the figure, six examiners had instances of scores falling outside of the expected qualifier range. Most of these occurrences were within Sample 2, the expected M- range sample. As this sample was anticipated to have a more difficult physical fit assessment, variation is expected. In addition, four out of these six examiners did not receive any formal training. 

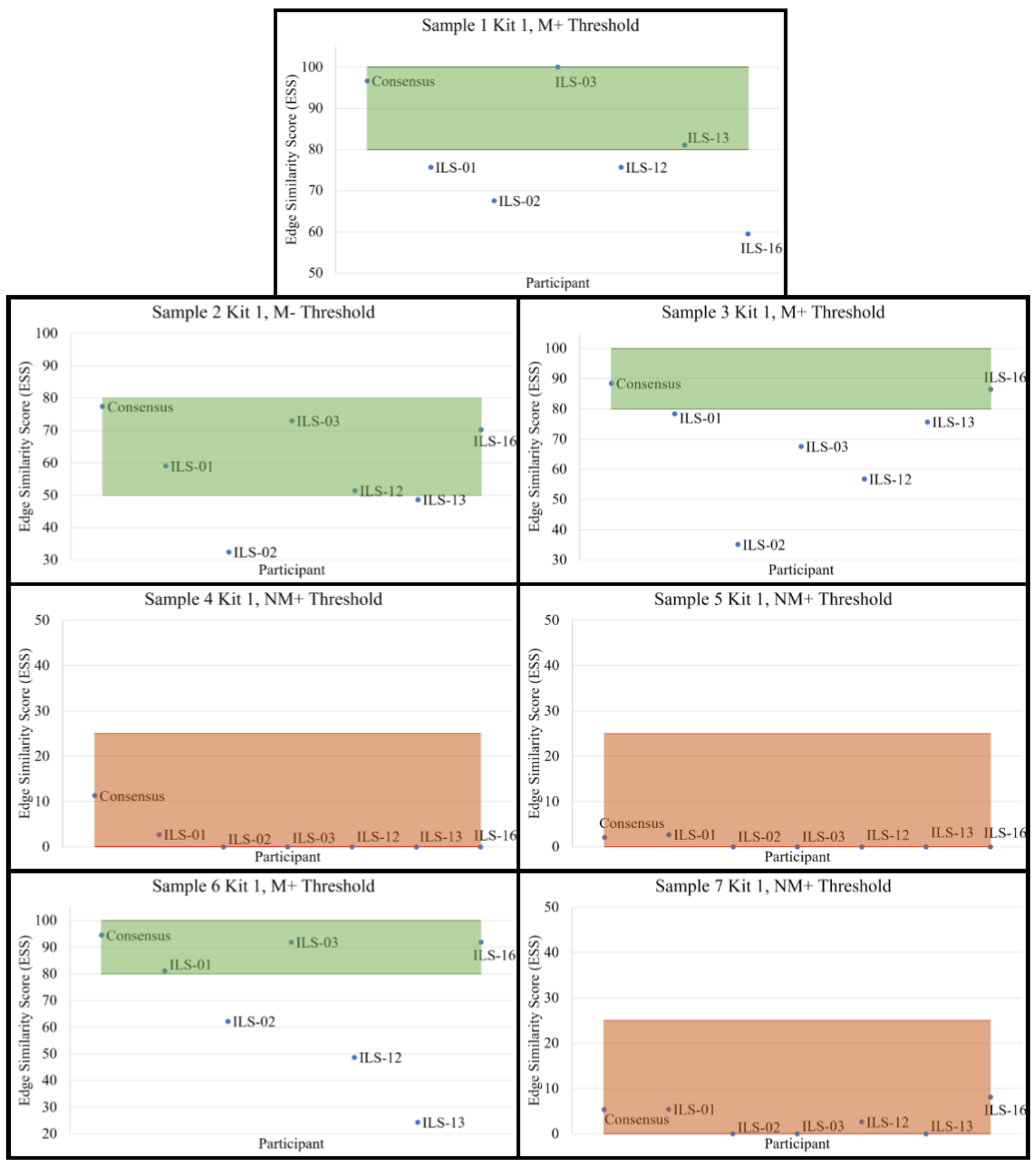

Figure 12. Kit 1 examiner ESS variation as compared to expected comparison edge qualifier thresholds 


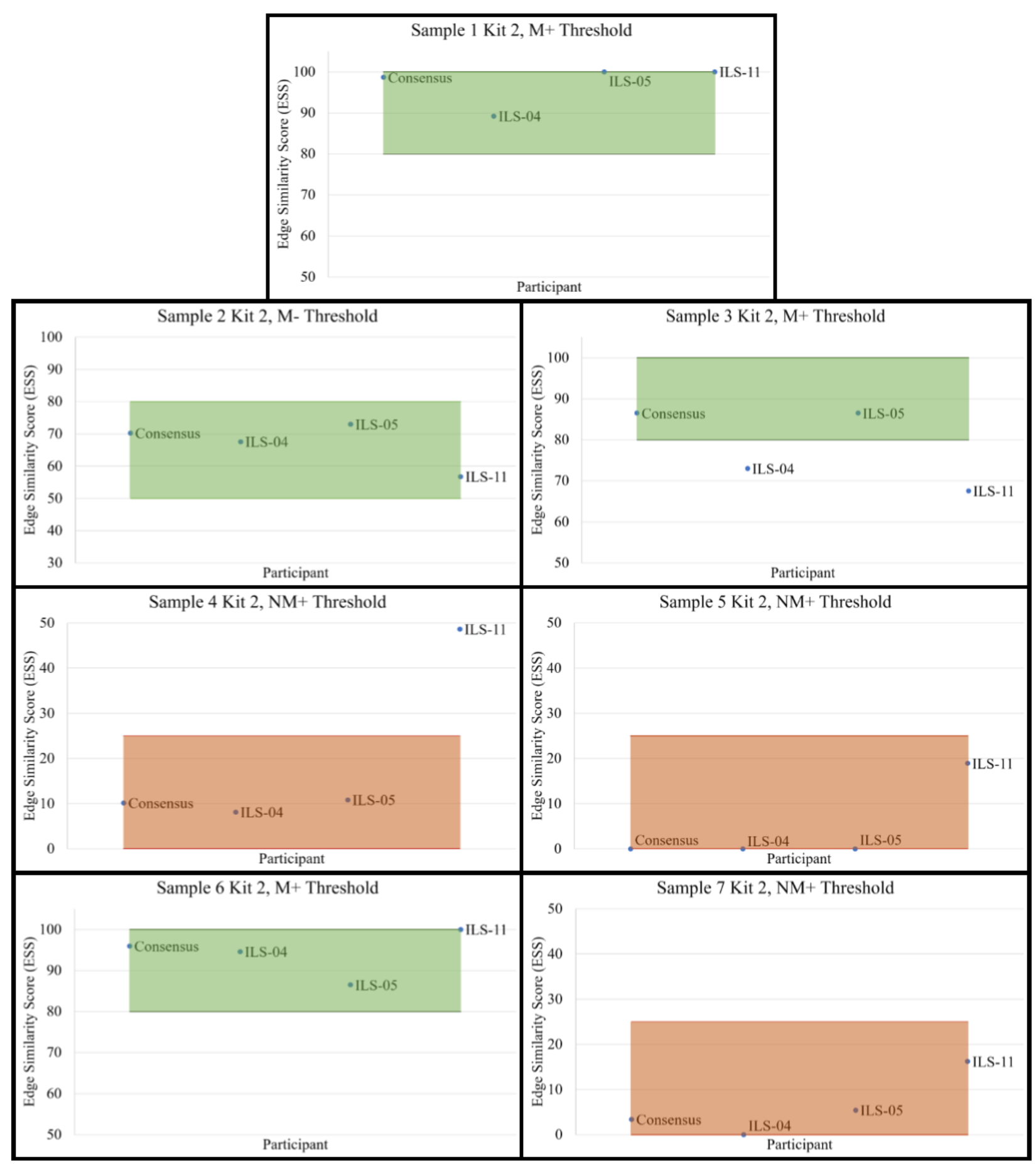

Figure 13. Kit 2 examiner ESS variation as compared to expected comparison edge qualifier thresholds 


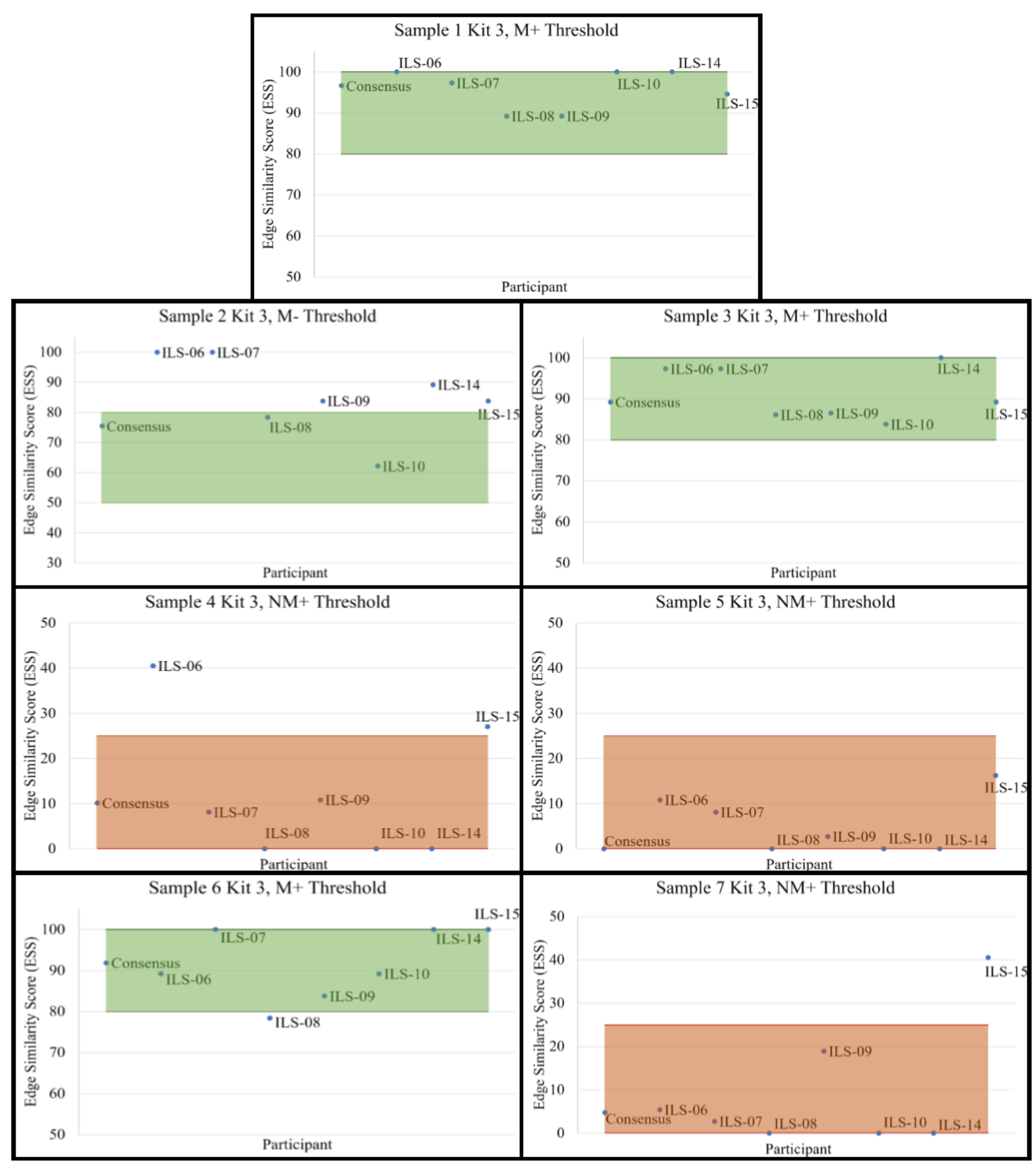

Figure 14. Kit 3 examiner ESS variation as compared to expected comparison edge qualifier thresholds

\subsection{ESS as Grouped by Expected Comparison Difficulty and Ground Truth}

The data of examiner ESS values were also grouped and analyzed by their ground truth and respective edge qualifiers, instead of per-kit assessment. Since the all true positive samples 
between kits were chosen to be between 80-100\% ESS, with the exception of Sample 2 (60-80\%) to provide a comparison of more difficulty, the data was further split into two separate match groups: M+ (16 participants, 38 samples) and M- (16 participants and samples). The third group consisted of all remaining 48 samples belonging to the non-match category.

The distribution of ESS values per group are provided below in terms of boxplots. Figure 15 below provides a boxplot for ESS distribution within the M+, M-, and NM groups. As shown, the majority of scores assigned the $\mathrm{M}+$ conclusion fell within the range of $75-100 \%$. This is only a 5\% difference from the expected range of $80-100 \%$ as predicted by previously-reported SLR ranges ${ }^{4}$. While a few outliers are exhibited with low ESS values below 50\%, these pairs were still correctly identified as matching pairs by the participant.

For the M- group, the majority of scores assigned this conclusion fell within the range of 55-90\%. This is about a $10 \%$ difference from the expected M- range of 50-80\% ${ }^{4}$. Overall, a shift in ESS ranges towards $50 \%$ was expected as this group consisted of true matching pairs considered of higher difficulty to assess than those of the M+ group. This shift was observed in the dataset. Additionally, as in the M+ group, a couple outliers are exhibited with low ESS values below 50\%. But again, these pairs were still correctly identified as matching pairs by the participant.

As shown, the majority of scores assigned the NM conclusion fell within the range of $0-20 \%$. This is a range 5\% more narrow than the expected $\mathrm{NM}+$ range of $0-25 \%$ as predicted by previously reported SLR ranges ${ }^{4}$.

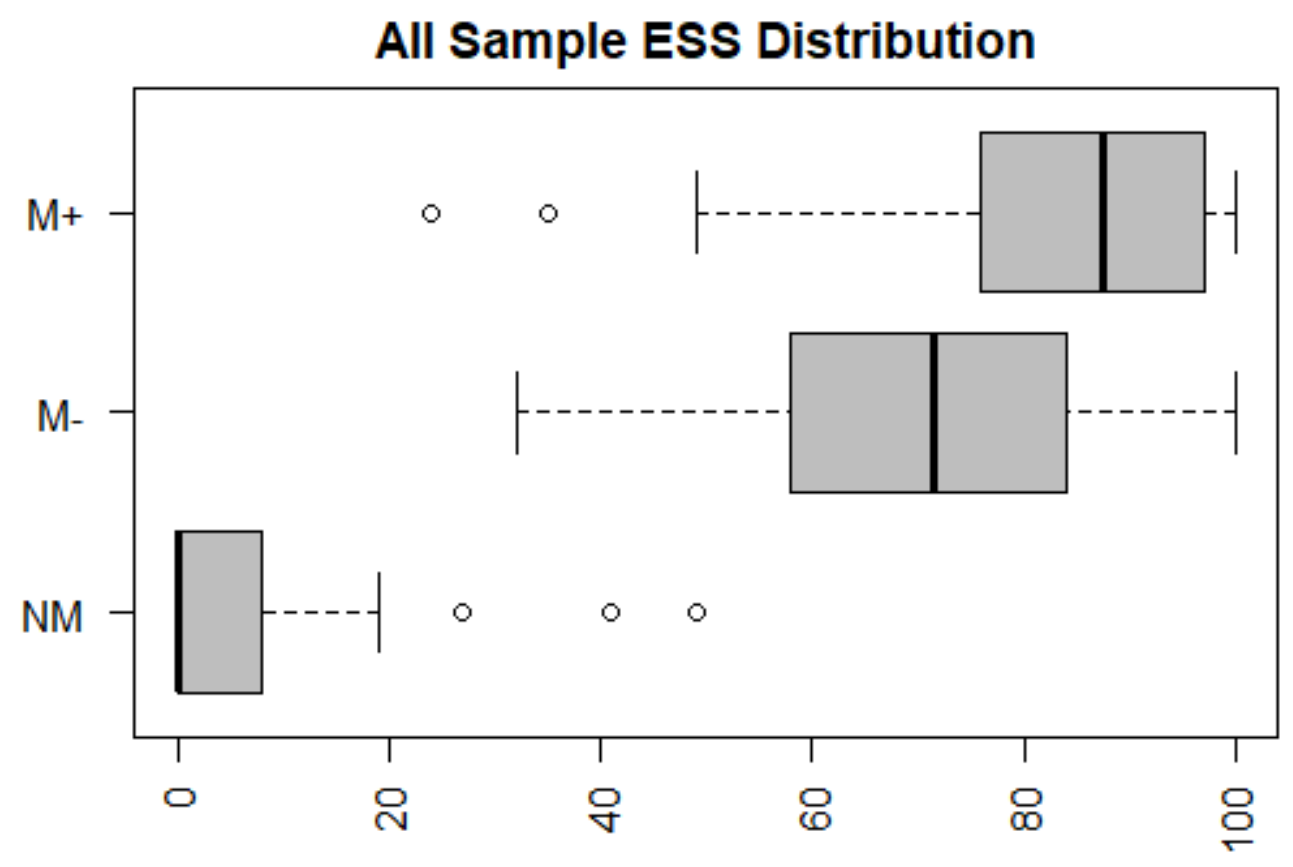

ESS

Figure 15. Boxplot ESS distributions of inter-laboratory sample pairs grouped as $\mathrm{M}+, \mathrm{M}-$, and $\mathrm{NM}$ 
In order to assess any significant ESS differences from the consensus mean by examiner, ANOVA was used from the randomized complete block design (RBCD) of the data set in which examiner was used as the treatment variable and tape sample per difficulty was used as the blocking variable. Dunnett's testing analysis was performed on each difficulty grouping (M+, M-, and NM). As tape pairs were selected in pre-distribution to encompass a wide variety of reported ESS, significant differences were expected when observing ESS differences by tape sample (for instance ESS score for a NM versus a $\mathrm{M}+, \mathrm{M}-$-).Therefore, for the purposes of this chapter analysis of the effects of examiner alone are reported.

Figure 16 below provides the results of Dunnett's testing on the M+, M-, and NM groups. As shown, out of 16 total study participants, only three examiners attributed significant differences in assigned ESS values as compared to the overall consensus mean for $M+$ sample pairs $(n=48)$. As discussed earlier, the same trend was observed in all three of these participants, as these variants also correlate with gaps on formal training.

Within the M- group, five examiners attributed significant differences in assigned ESS values as compared to the overall consensus mean for $M$ - sample pairs $(n=16)$. Of these five participants, four (ILS-02, ILS-06, ILS-12, and ILS-13) did not participate in formal method training.

As shown for the NM group, three examiners attributed significant differences. Of these three participants, one (ILS-06) did not participate in formal method training. Overall, it was shown that of 11 variants from control mean, 8 or $73 \%$ were associated to lack of formal training, further emphasizing its importance in future study expansion. 


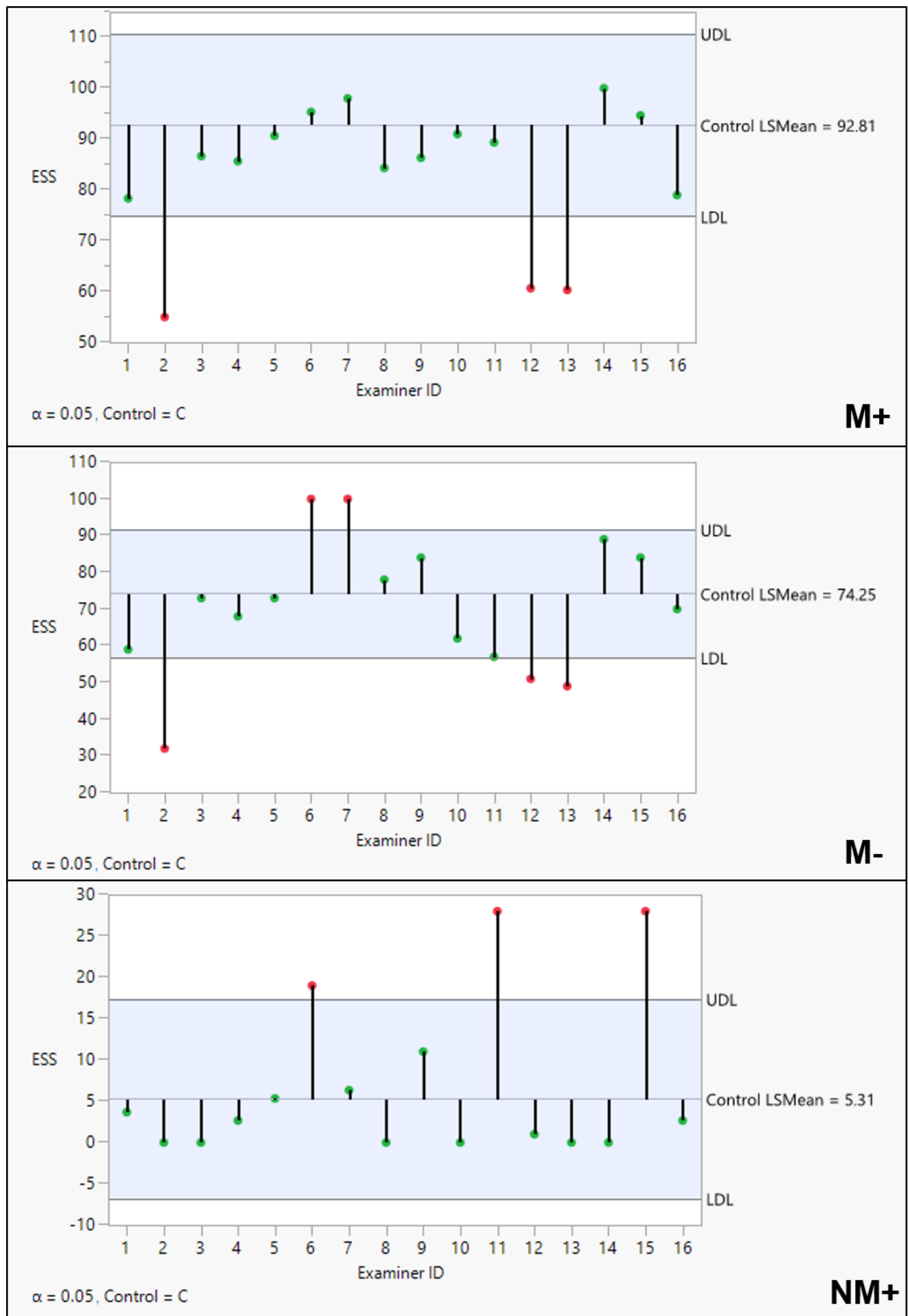

Figure 16. Dunnett's test examiner control differences results, $\mathrm{M}+, \mathrm{M}-$, and NM samples 


\subsection{Overall Observations}

In summary, three general trends were observed. First, those participants that did not participate in formal method training through either the in-person method presentation or teleconference tended to exhibit statistically significant score differences from the consensus $(\mathrm{N}=4)$, predistribution mean ESS. Some of those ESS differences, however, were not exclusionary when using a broader threshold criterion (e.g. 20\% ESS) or were not large enough to generate an erroneous conclusion. As shown in Figure 16, out of 48 consensus mean comparisons $(\mathrm{n}=16$ examiners per overall sample group - M+, M-, NM), only 11 instances (23\%) showed significant differences between mean reported ESS and consensus mean values, indicating a $77 \%$ agreement with the pre-distribution, consensus mean. From those, only 8 out of 48 (17\%) would provide a misclassification of the qualifier (i.e. all significantly different NM ESS were still within the expected range of a non-match, $0-50 \%$ ). Also, from those remaining 8 differing results, 3 of them were produced by analysts that did not elect to participate in formal method training beyond the protocol and instructional presentation provided at the time of kit receipt. This indicates the differences in reported values may be a result of lack of understanding of the proposed method. Moreover, the differences on the remaining instances in which the participants did receive training were not as drastic as to produce a false positive or false negative conclusion. For example, in two of the three instances within the NM group that significant differences from the control mean arose, both participants were present for formal training. In both situations, the examiners provided overall non-match conclusions but ESS values of $40 \%$. While the high ESS values as compared to consensus means of $\sim 5-11 \%$ resulted in significant statistical differences, neither instance resulted in a misclassification. Higher scores were likely due to inconsistency in interpretation of scrim bin features, as one examiner indicated even "featureless" bins were considered matching, leading to an overall higher ESS despite the true negative conclusion.

Other main observations across the study included the variation in how a featureless scrim bin was characterized for ESS purposes. This was made apparent through comments left by participants per sample. While some chose to consider bins observed as featureless as matches ("1), others chose to label them non-matches (" 0 ") due to the lack of edge features. Another key observation included the various interpretations in the use of the comparison edge qualifier between participants. These variances are best observed through ESS distributions by overall conclusion and by assigned qualifier, respectively. These distributions are discussed below. It should be noted that no matter the ESS variation, no misclassifications were made by the examiner of any samples in any kits. A thorough evaluation of the potential sources of differences among reported ESS is provided below.

\subsubsection{ESS distributions by overall conclusion - variance in featureless/distorted bins}

Figure 17 below provides the ESS distribution resulting from six participants completing Study Kit 1. Scores of interest, referred to as "discrepancy instances" or "differences", are numbered for reference. It should be noted that other relatively low ESS values, such as the inconclusive of ESS $\sim 25 \%$ and one of the true positives of ESS 60\% are not included in discussion as further investigation into comments left by respective participants revealed that each felt multiple bins of 
these samples did not correspond due to specific features (i.e. backing striae). Therefore, these low values are not due to examiner treatment of "featureless" or distorted edges.

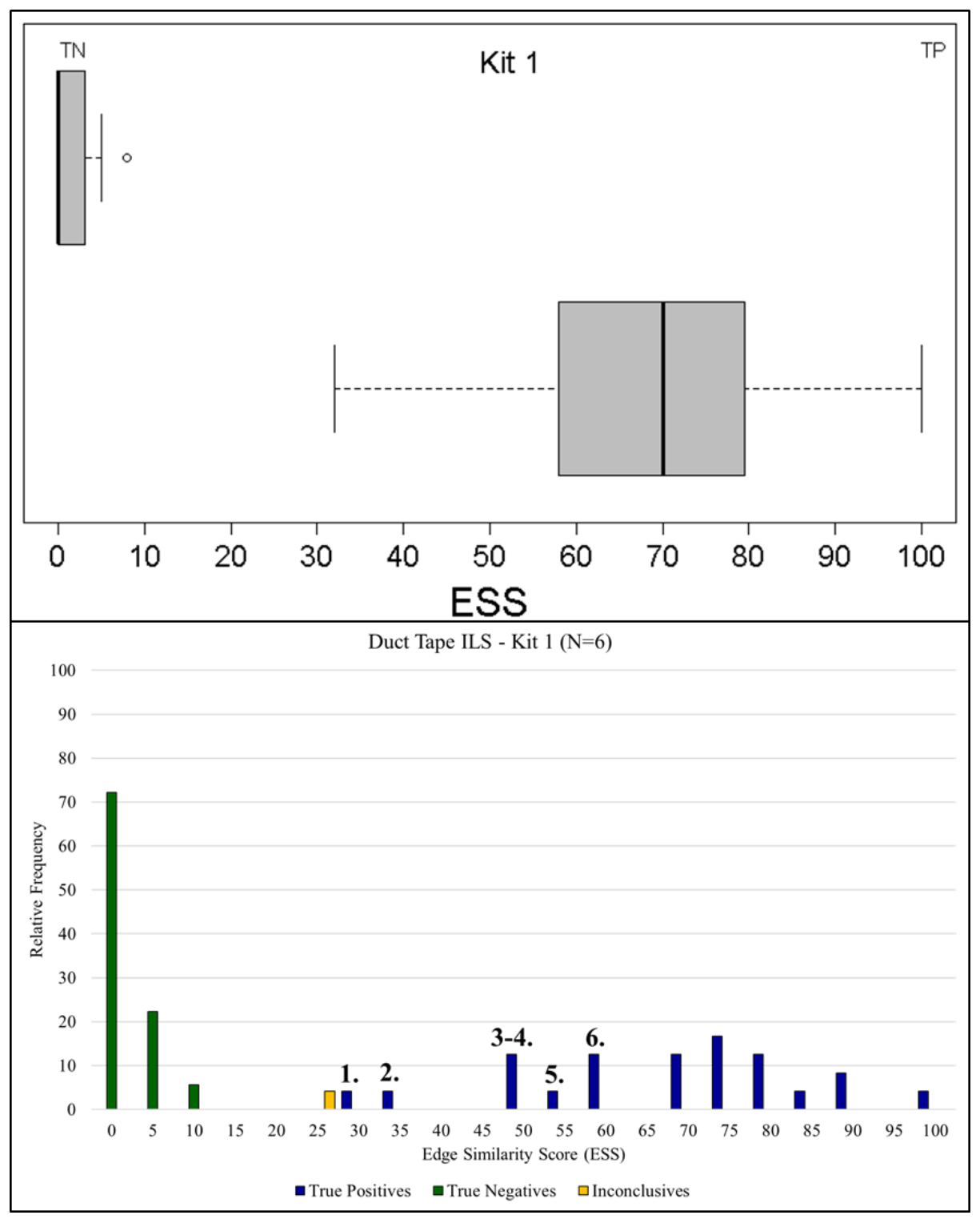

Figure 17. Kit 1 ESS distribution by overall conclusion $(\mathrm{N}=6$ examiners, $\mathrm{n}=42$ total comparisons). Numbering indicates discrepancy instances, points of discussion in which results varied from those expected.

Discrepancy instances 1 and 2 displayed in the above figure are examples of score determinations in which the participant assigned a zero to scrim bins that were determined aligned but "featureless." In other words, no specific adhesive, scrim, or backing features were considered present beyond a relatively straight edge morphology within the specific bin. Only those scrim bins with distinct consistent features were assigned ones. The specific features considered by the examiner can be observed according to their comments. Figure 18 below provides an image of the 
sample pair associated with each discussed discrepancy with the scrim bins considered featureless indicated, as well as any associated examiner comments.

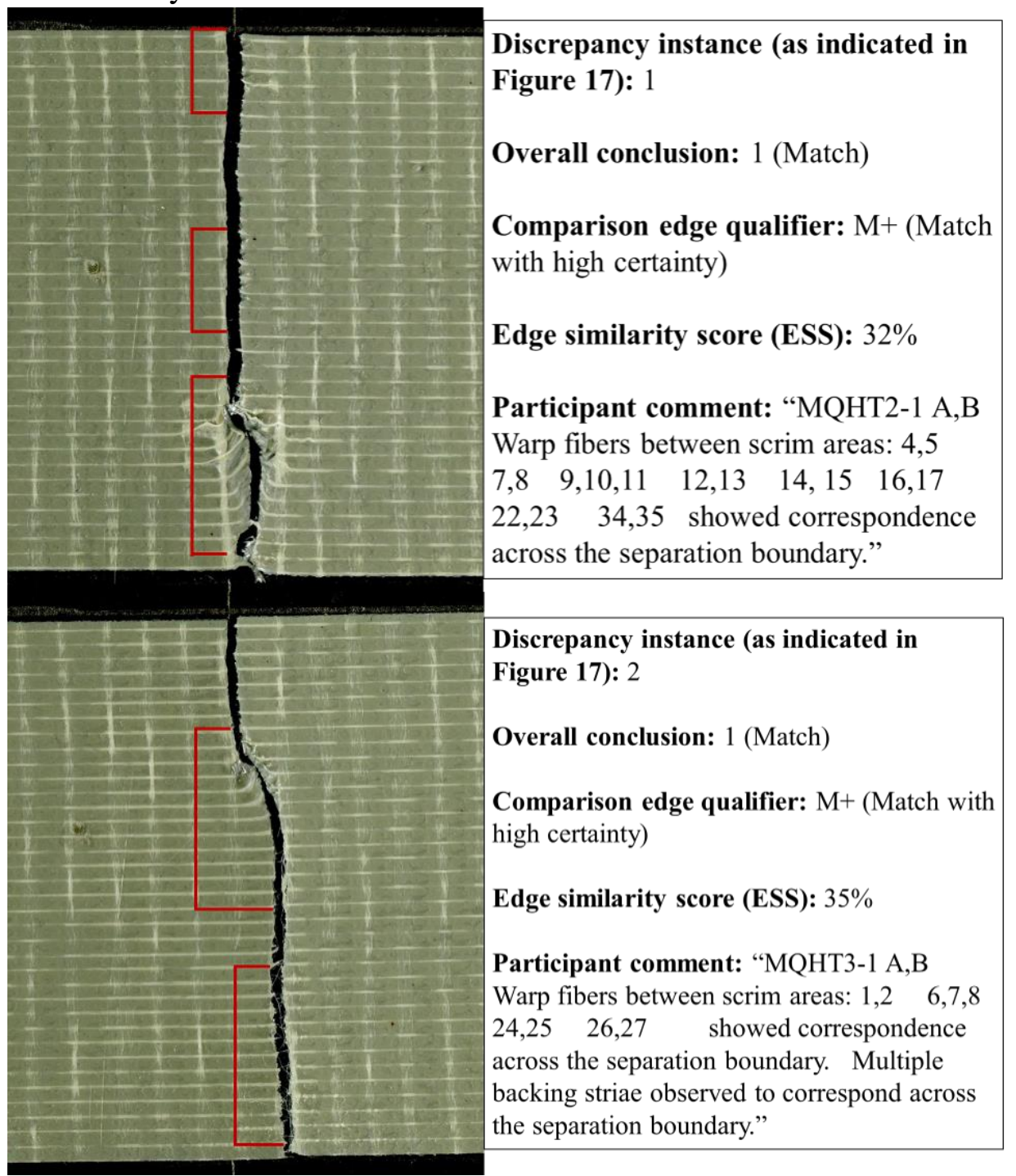

Figure 18. Kit 1 samples, treatment of "featureless" scrim bins, red areas indicate bins marked " 0 " by participant

Differences 3 to 6 in Figure 17 are examples of score determinations in which a zero was assigned to scrim bins in which the participant considered either the backing or adhesive to be distorted. Due to the obstruction of edge morphology presented by the distortion, these examiners remained more conservative in their score designations, leading to lower overall ESS. Figure 19 below provides an image of the sample pair associated with each discussed discrepancy with the scrim bins considered distorted indicated, as well as any associated examiner comments. 


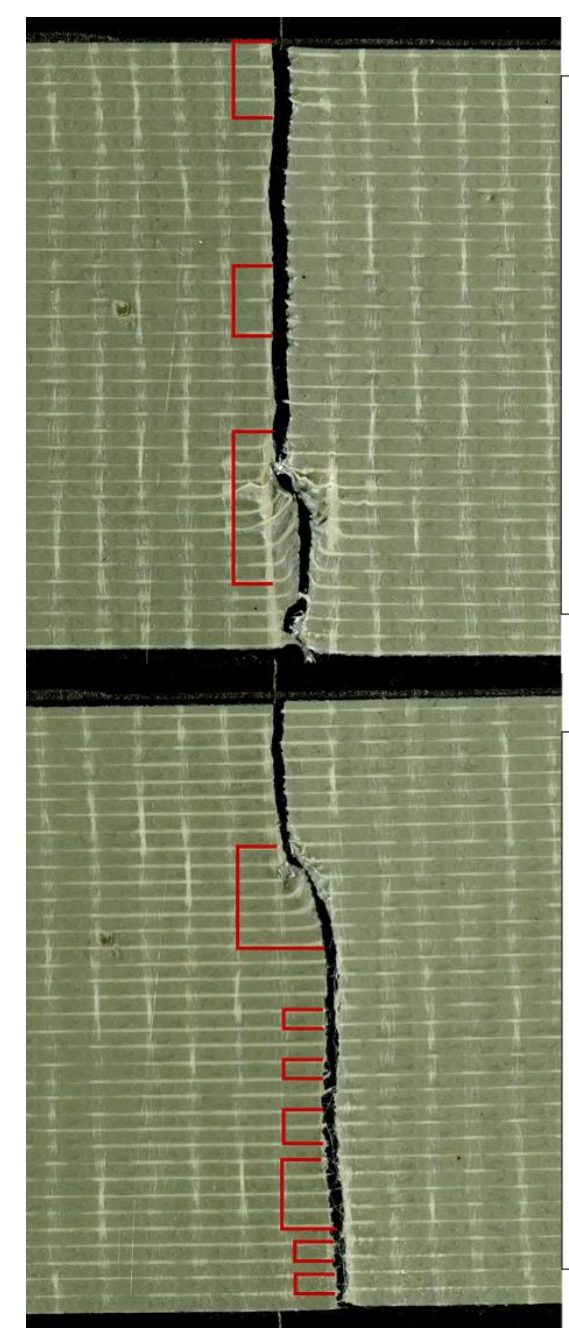

Discrepancy instance (as indicated

in Figure 17): 3

Overall conclusion: 1 (Match)

Comparison edge qualifier: $M-$

(Match with low certainty)

Edge similarity score (ESS): 51\%

Participant comment: "General adhesive distortion in multiple areas."

Discrepancy instance (as indicated in Figure 17): 5

Overall conclusion: 1 (Match)

Comparison edge qualifier: $\mathrm{M}+$

(Match with high certainty)

Edge similarity score (ESS): $57 \%$

Participant comment: "General adhesive distortion.'

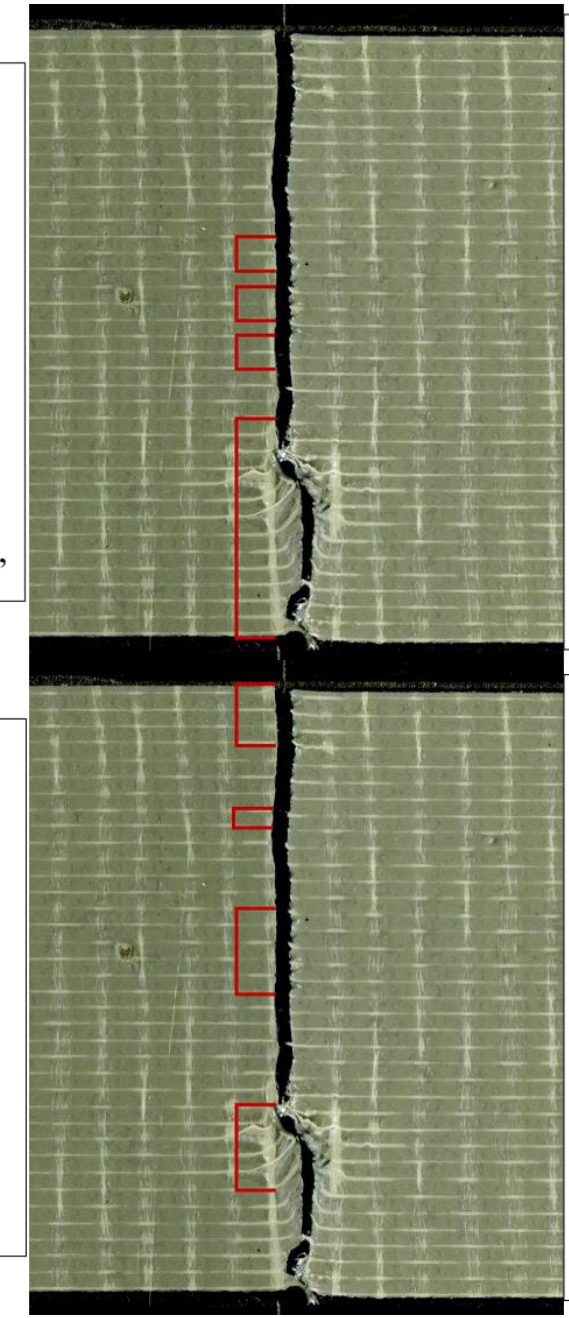

Discrepancy instance (as indicated in

Figure 17): 4

Overall conclusion: 1 (Match)

Comparison edge qualifier: M- (Match with low certainty)

Edge similarity score (ESS): $49 \%$

Participant comment: "Overall shape of fractured edges appear to correspond (scrim areas 25-37) but because those areas were wrinkled and/or folded over on the acetate, it was difficult to accurately assess the features and make a conclusion. Those areas were graded as "0 non-match."

Discrepancy instance (as indicated in Figure 17): 6

Overall conclusion: 1 (Match)

Comparison edge qualifier: M- (Match with low certainty)

Edge similarity score (ESS): 59\%

Participant comment: "Warp scrim alignment, continuation of scrim pattern, backing distortion, adhesive distortion, scrim distortion, protruding scrim, and calendaring alignment observed."

Figure 19. Kit 1 samples, treatment of distorted scrim bins, red areas indicate bins marked "0" by participant 
In the case of results of Study Kit 2, Figure 20 shows the ESS distribution with less incidences of discrepancies. While two inconclusive and a true negative with ESS 50\% are shown, the associated participants did not leave comments beyond their binary documentation of their scrim bin decisions. Therefore, conclusions cannot be drawn as to factors influencing their decision to mark certain bins as zero.

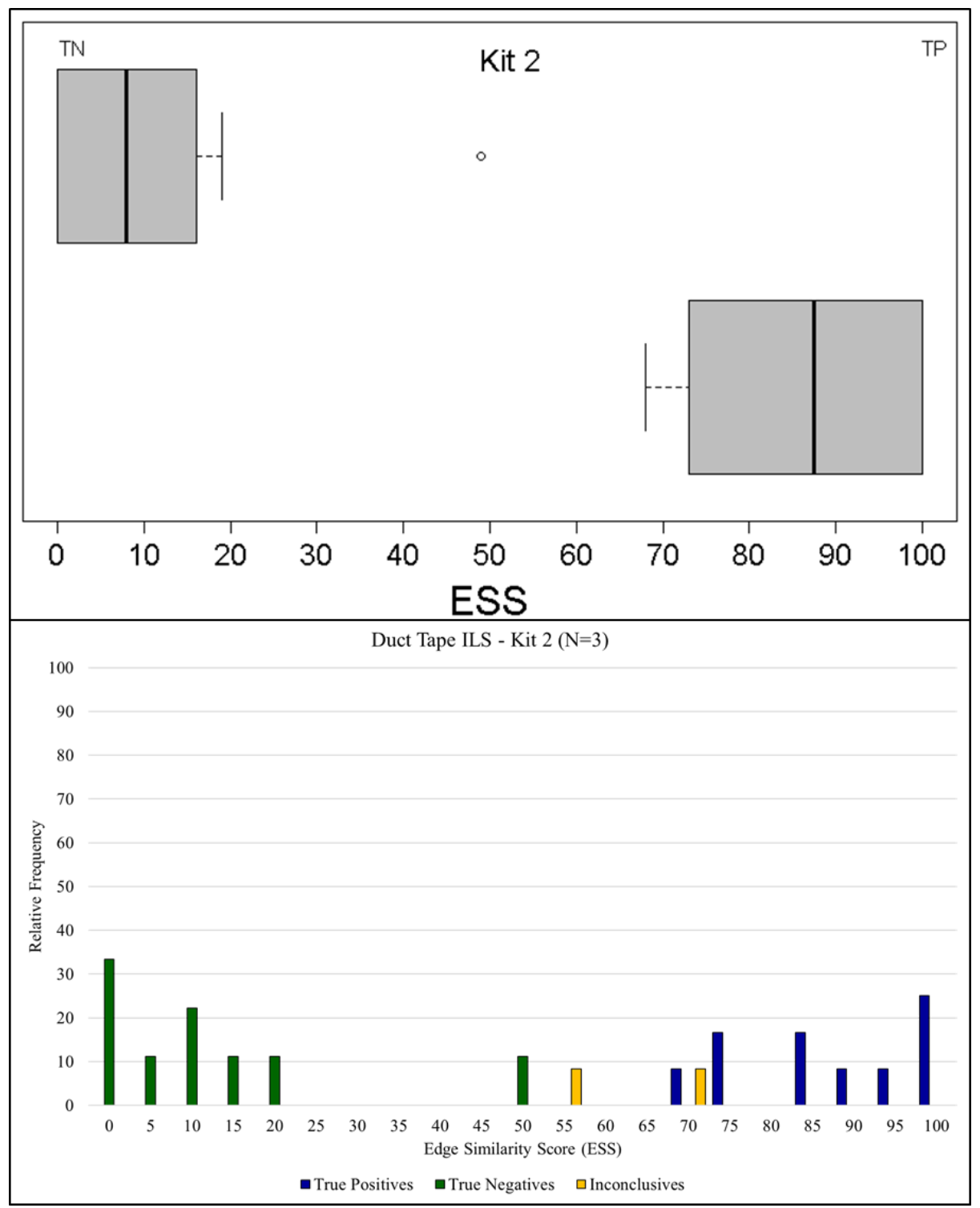

Figure 20. Kit 2 ESS distribution by overall conclusion ( $\mathrm{N}=3$ examiners, $\mathrm{n}=21$ total comparisons)

Finally, in the case of results of Study Kit 3, relatively good consistency is observed with some examples of different judgment in the ESS estimation (Figure 21). Discrepancy instances are numbered for reference. It should be noted that while a relatively high ESS value, one of the true negative assigned an ESS 40\% is not included in discussion as further investigation into comments left by the respective participant revealed that they felt multiple bins did not correspond 
due to specific features (i.e. dimpling, warp yarn misalignment). Therefore, this high value is not due to examiner treatment of "featureless" or distorted edges.

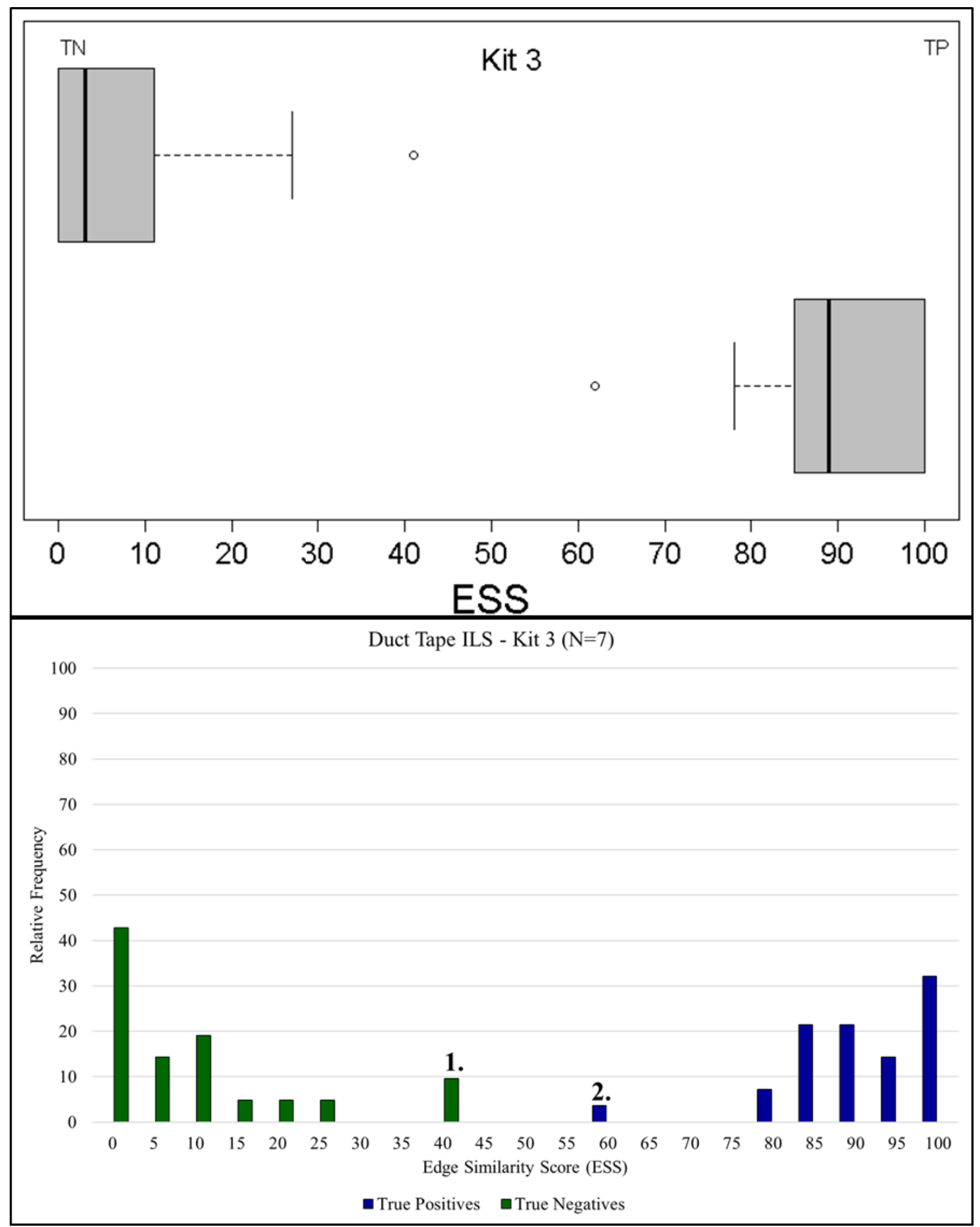

Figure 21. Kit 3 ESS distribution by overall conclusion ( $\mathrm{N}=7$ examiners, $\mathrm{n}=49$ total comparisons). Numbering indicates discrepancy instances, points of discussion in which results varied from those expected. 
Difference 1 displayed in the above figure is an example of a score determination in which the participant assigned a one, rather than a zero as discussed previously, to scrim bins that were determined "featureless." However, as the participant considered the insignificant edge morphology to still appear consistent, these bins were determined to correspond. These, along with scrim bins with distinct consistent features were assigned bin scores of one. The specific features considered by the examiner can be observed according to their comments. Figure 22 below provides an image of the sample pair associated with discrepancy instance 1 scrim bins considered featureless or consistent due to distinct features indicated, as well as any associated examiner comments.

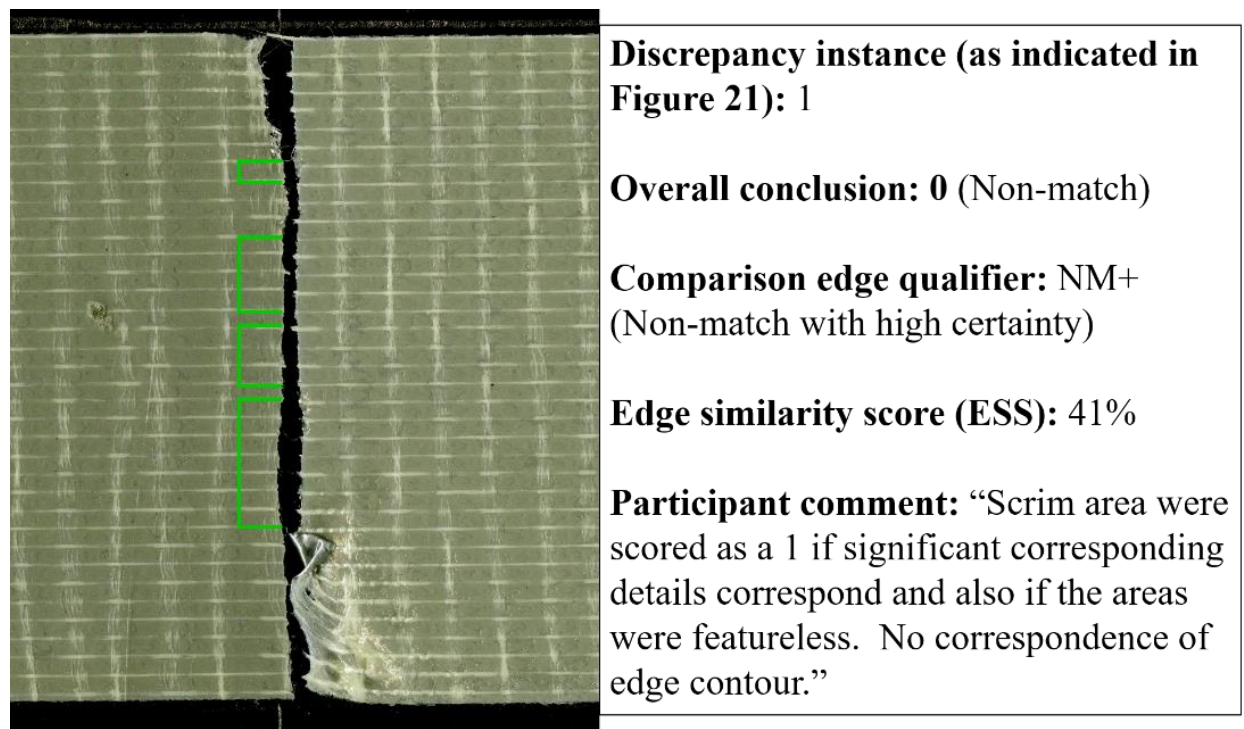

Figure 22. Kit 3 sample, treatment of "featureless" scrim bins, green areas indicate bins marked "1" by participant

Difference 2 in Figure 21 is an example of a score determination in which a zero was assigned to scrim bins in which the participant considered either the backing or adhesive to be distorted. Similar to examiners discussed within Kit 1 results, this examiner remained more conservative in their score determination by avoiding designating areas with obstructed edge morphologies as consistent, leading to a lower overall ESS. However, this examiner in particular indicated that they intended for areas of distortion to serve more as "inconclusive" areas. While there is not an "inconclusive" scrim bin option in the ESS method at this time, this feedback may lead to future modification of the method. Figure 23 below provides an image of the sample pair associated with each discussed discrepancy instance with the scrim bins considered distorted indicated, as well as any associated examiner comments. 


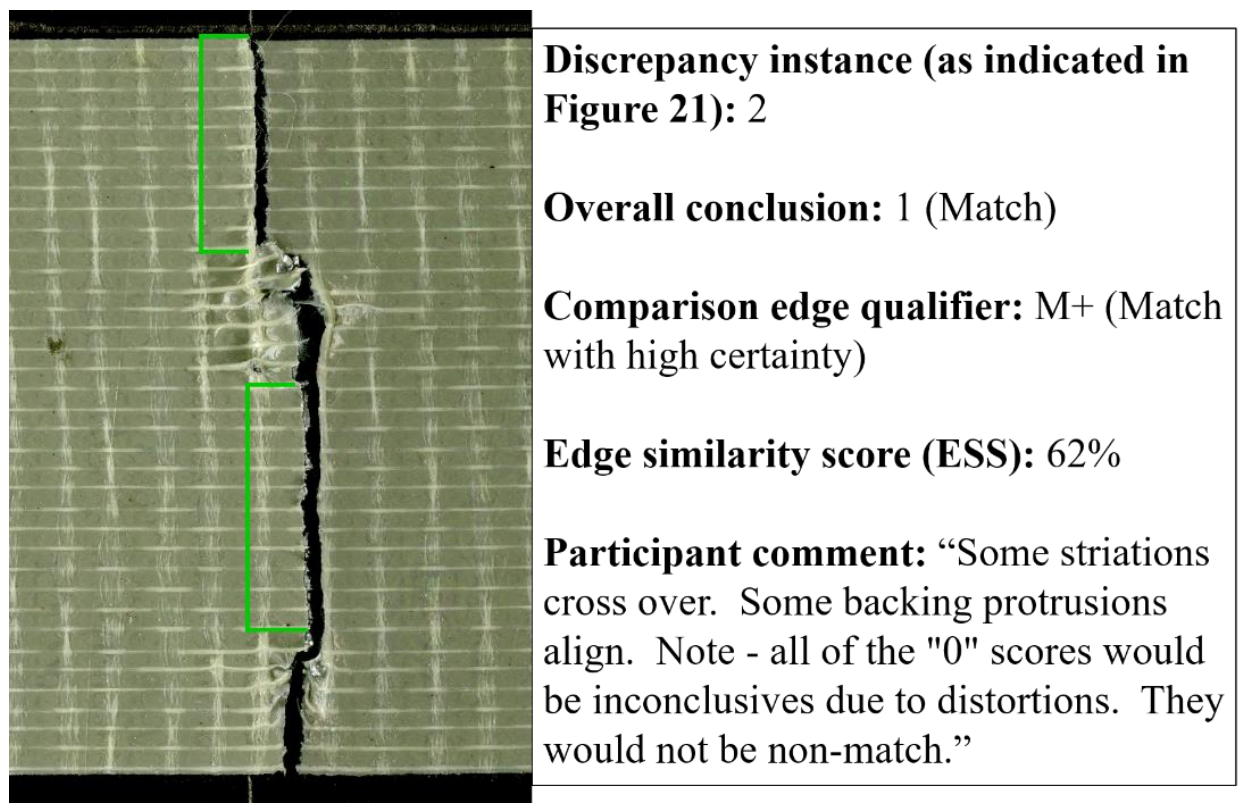

Figure 23. Kit 3 sample, treatment of distorted scrim bins, green areas indicate bins marked "1" by participant

\subsubsection{ESS distributions by comparison edge qualifier - variance in qualifier use}

While there were no misclassifications on overall conclusions, there were several instances throughout the study in which the participant assigned ESS did not fall within the expected ranges for the comparison edge qualifier selected. This is best observed in each individual sample pair per kit, as shown in Figures 12-14. To further explore these instances, ESS distributions by participant assigned comparison edge qualifier will be provided below, along with sample images and associated examiner comments.

Figure 24 below provides the ESS distribution by qualifier resulting from six participants completing Study Kit 1. Differences are numbered for reference, while discrepancy instances previously discussed in Section 3.6.1 are denoted with an asterisk. 


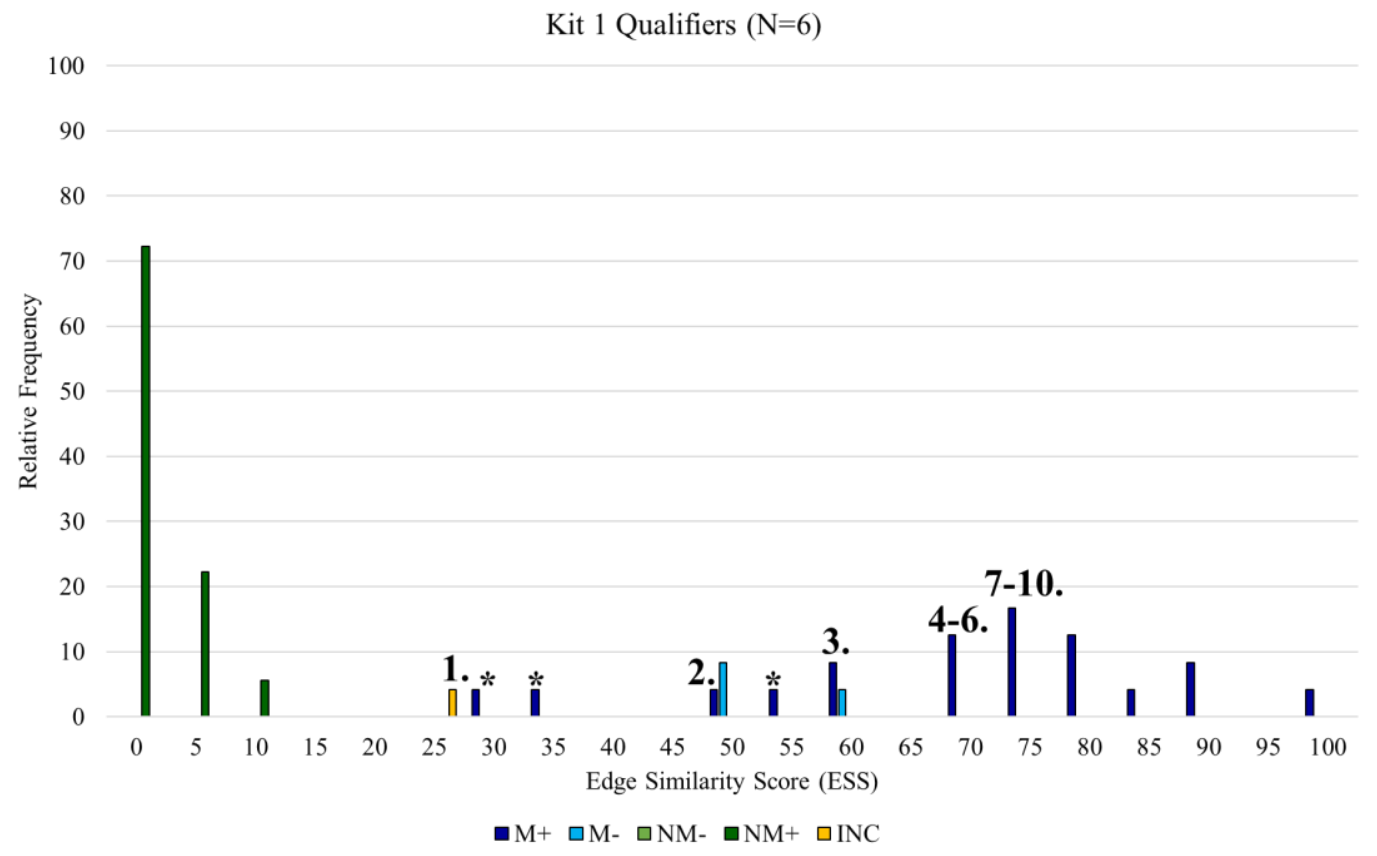

Figure 24. Kit 1 ESS distribution by qualifier ( $\mathrm{N}=6$ examiners, $\mathrm{n}=42$ total comparisons). Numbering indicates discrepancy instances, points of discussion in which results varied from those expected.

In Figure 24, discrepancy instances 1-3 are of the same sample pair, MQHT6-1. Instances 1 and 2 were both below the general 50\% threshold of a typical matching ESS value. However, difference 1 was denoted an inconclusive in the overall conclusion. The participant associated with discrepancy instance 1 noted that while overall morphology appeared consistent, they determined few scrim bins to align. However, participants responsible for differences 2 and 3 both noted scrim bin association was based upon alignment of backing striae. These two participants correctly classified the sample pairs as matches, despite the relatively lower ESS values, which reflects a lack of understanding of the ESS method.

Discrepancy instances 4, 7, and 9 were also of the same sample pair, MQHT1-1. While the participant associated to difference 9 did not leave any comments, participants from differences 4 and 7 both noted that consistent characteristics were observed between the samples, not mentioning which features may have led to the lower ESS assignment, yet still strong M+ comparison edge qualifier.

Discrepancy instances 5 and 10 were of the same sample pair. While the participant associated to difference 5 did not leave a comment, the individual responsible for difference 10 indicated that areas of distortion led to the lower ESS value, yet the overall match conclusion was still determined with high certainty.

Finally, discrepancy instances 6 and 8 were of the same sample pair. While neither participant left comments, these scores were in the 70s, whereas the lower bound for the expected M+ qualifier 

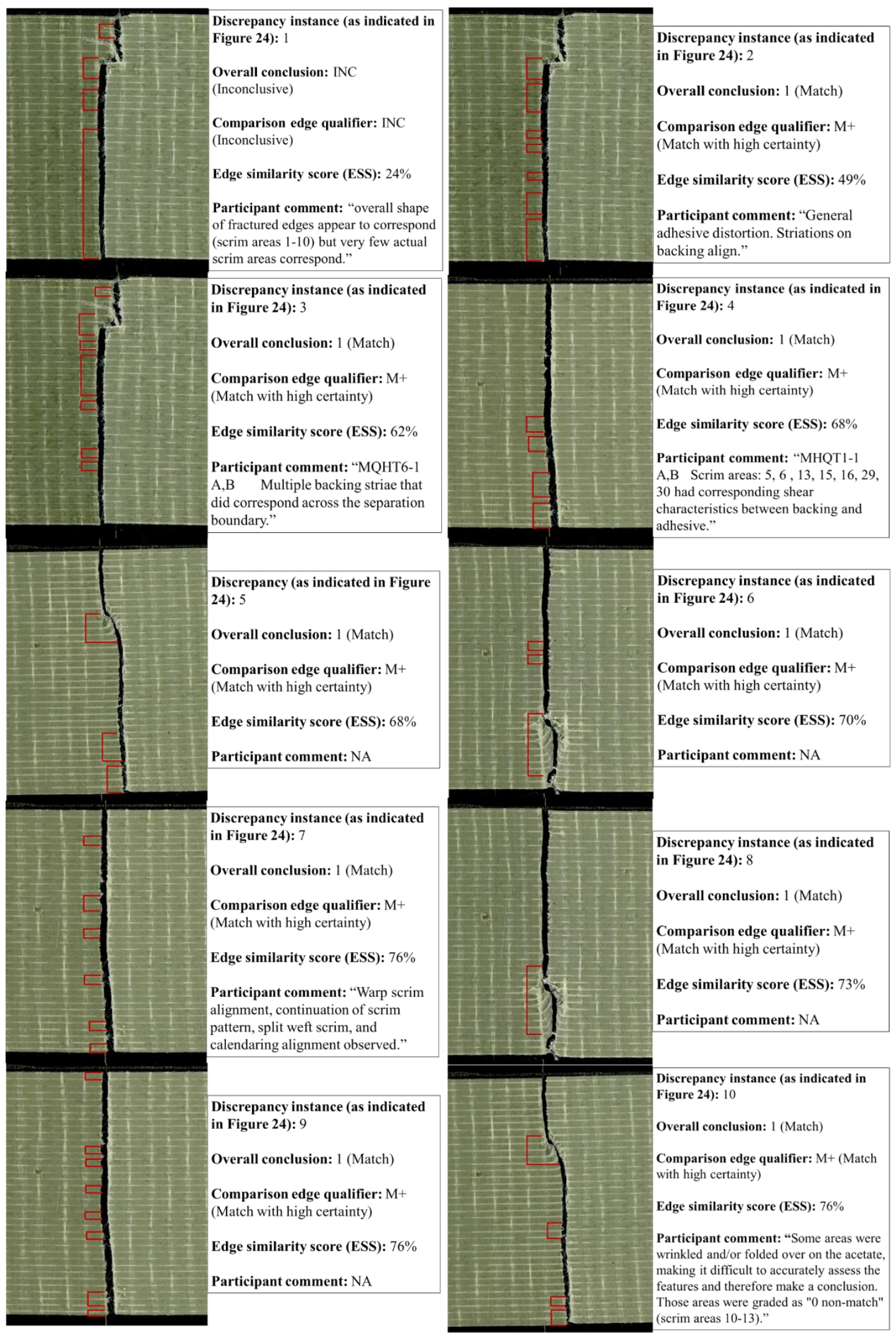

Figure 25. Kit 1 samples, qualifiers out of expected ranges, red areas indicate bins marked " 0 " by participant 
ESS range is $80 \%$. Figure 25 above provides an image of the sample pair associated with each discussed difference with the scrim bins considered inconsistent indicated, as well as any associated examiner comments.

Figure 26 below provides the ESS distribution by qualifier resulting from three participants completing Study Kit 2.

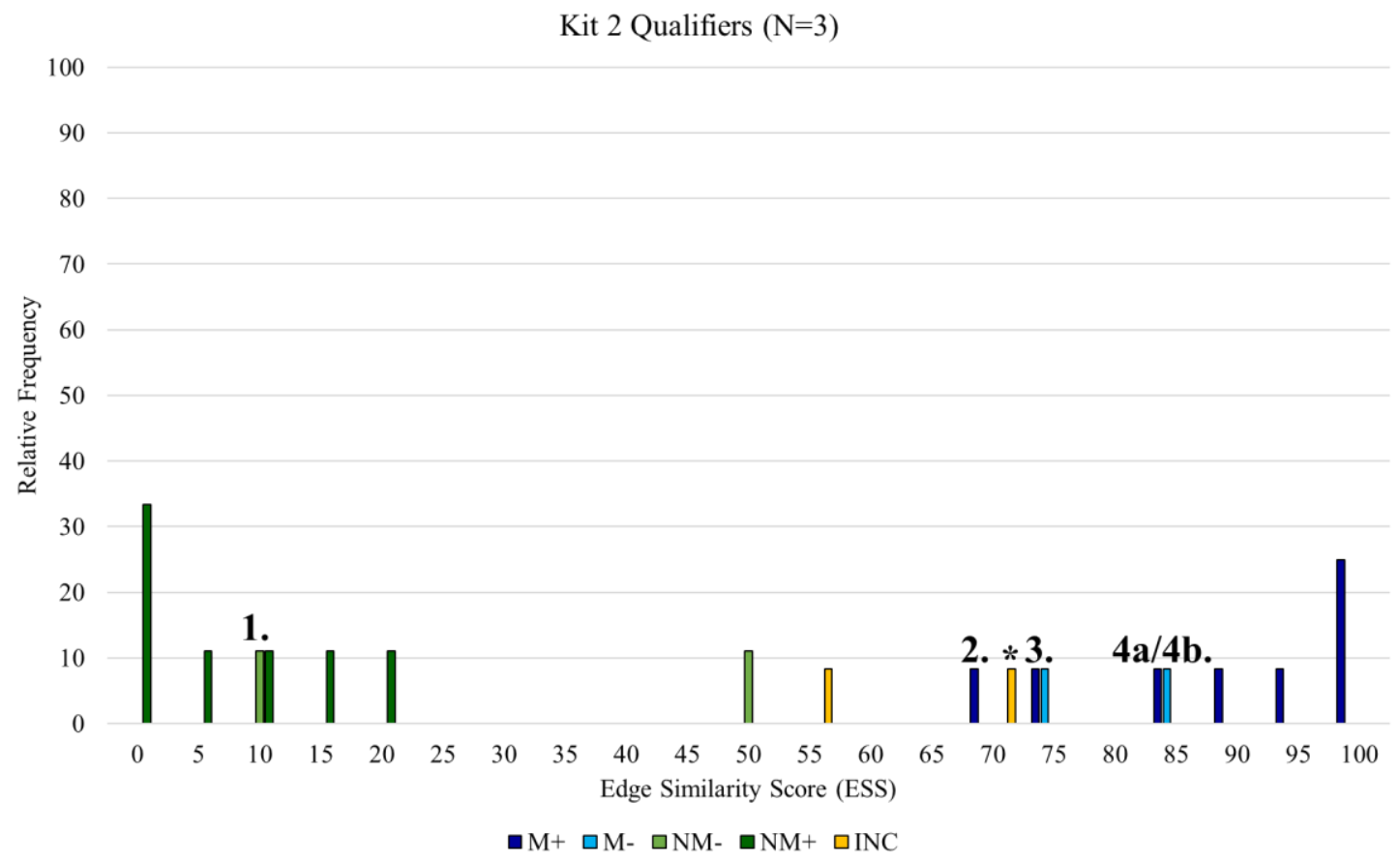

Figure 26. Kit 2 ESS distribution by qualifier ( $\mathrm{N}=3$ examiners, $\mathrm{n}=21$ total comparisons). Numbering indicates discrepancy instances, points of discussion in which results varied from those expected.

In Figure 26, difference 1 was assigned an ESS of $11 \%$ with a NM- comparison edge qualifier. While the associated examiner did not leave any comments, they did indicate a few areas in which scrim bins appeared to be consistent. Although the lower bound of the expected NM- ESS range is $25 \%$, this was an estimation not verified by SLR information ${ }^{4}$ and the examiner still arrived at the correct classification. The tape pair in question can be viewed in Figure 27.

While the participants associated to discrepancy instances 2 and 3 did not leave any comments, both pairs were assigned lower ESS values and high certainty M+ qualifiers. This indicated that a few scrim bins exhibited features causing the participants to exclude those areas, while their overall conclusion certainty was not affected. These tape pairs can also be viewed in Figure 27. 


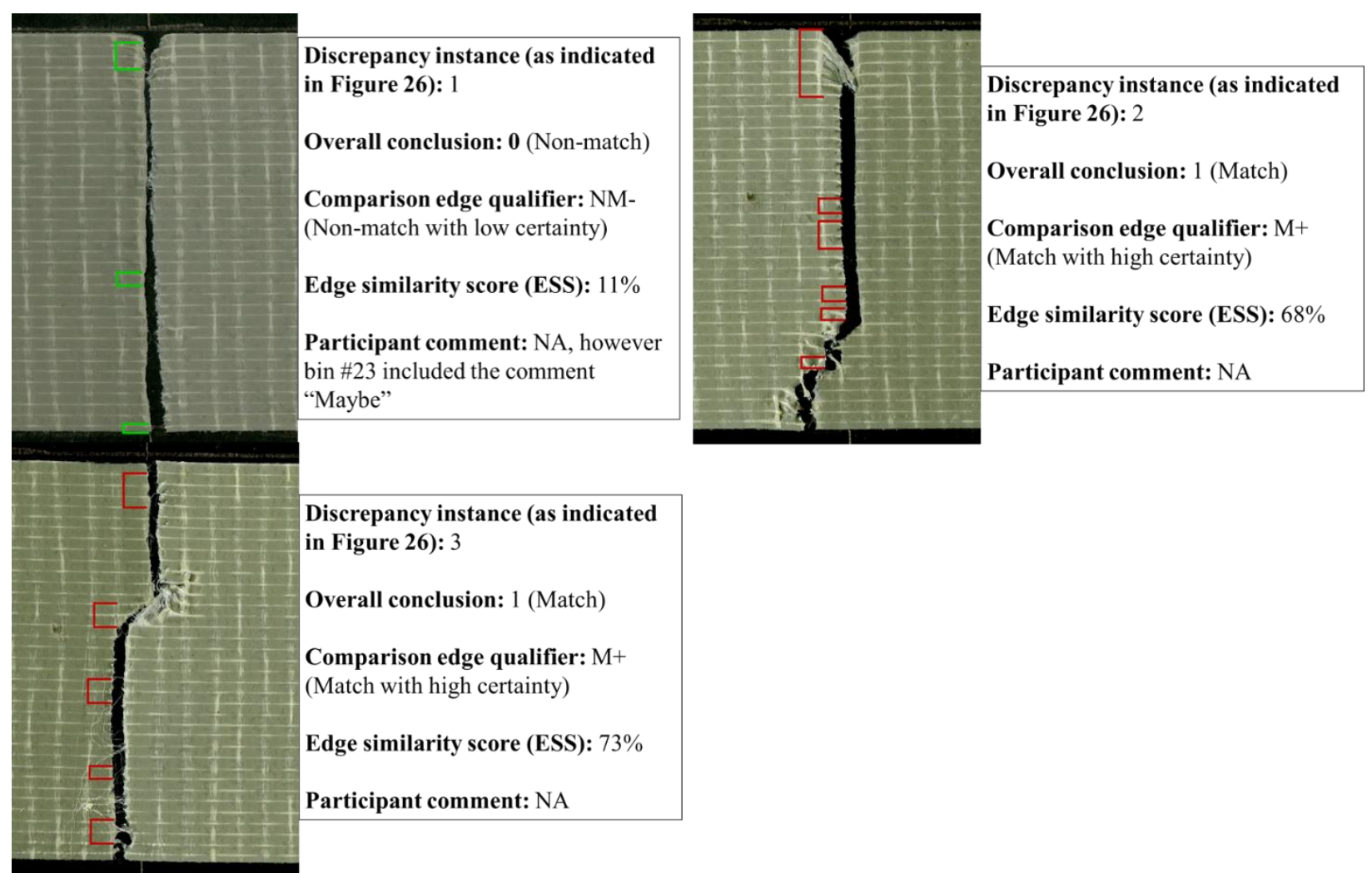

Figure 27. Kit 2 samples, qualifiers out of expected ranges, red areas indicate bins marked " 0 ” by participant while green areas indicate bins marked " 1 "

An interesting assignment of ESS vs comparison edge qualifier was observed in differences $4 \mathrm{a}$ and $4 \mathrm{~b}$ (as labeled in Figure 26), which were two different sample pairs analyzed by the same participant. While these differences were assigned the same ESS (86\%), 4a was assigned a M+ comparison edge qualifier while $4 \mathrm{~b}$ was assigned a M-. This appears to be due to varying degrees of distortion or deformation between the samples. According to the participant's notes, discrepancy instance 4a was considered to present distortion that lowered the examiner's certainty in the match conclusion, while difference $4 \mathrm{~b}$ also exhibited distortion, but with numerous other consistent features that upheld the examiner's certainty in the match. The comparison between these instances can be viewed in Figure 28 below. 


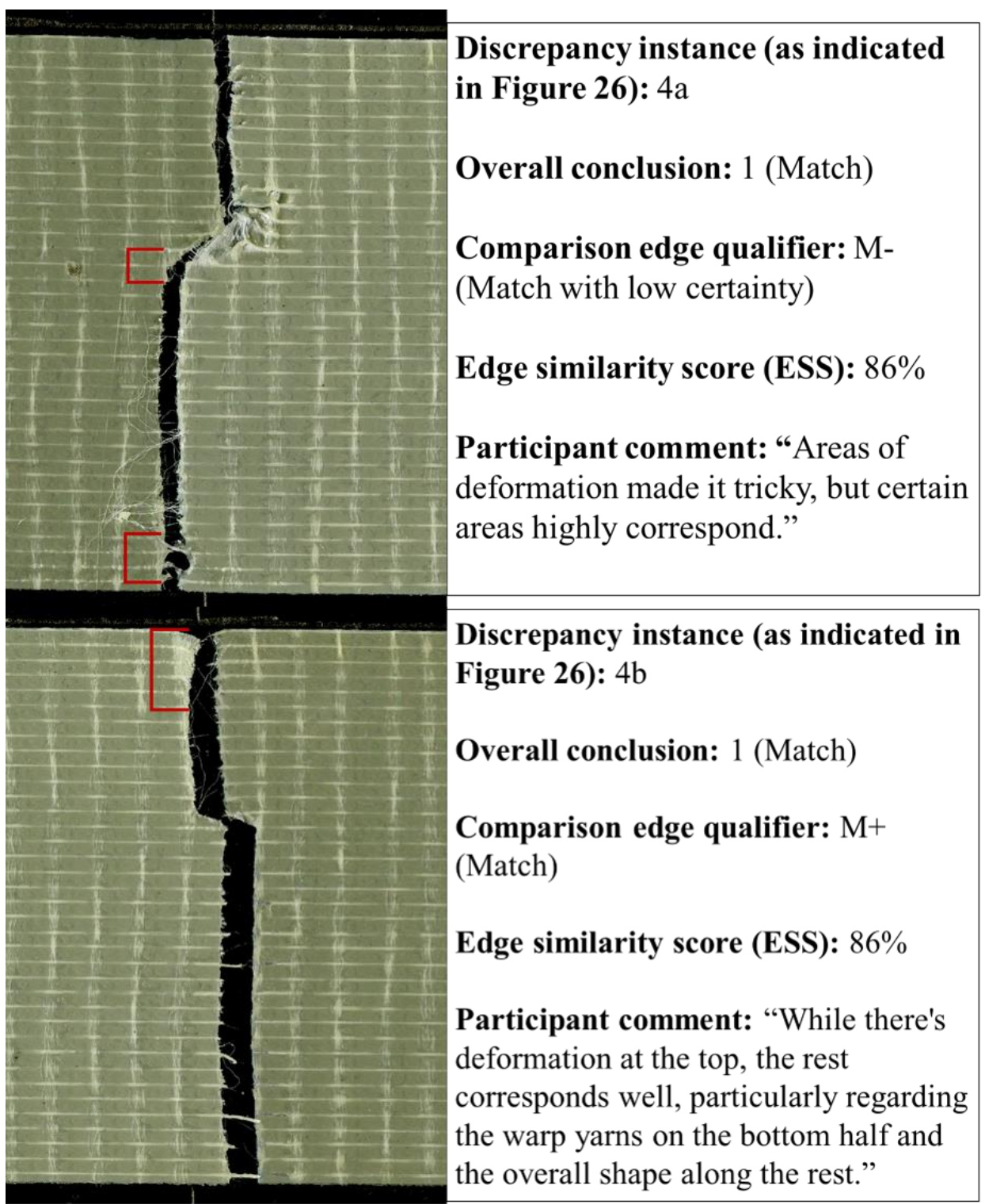

Figure 28. Comparison of Kit 2 samples assigned same ESS but different comparison edge qualifiers by same participant, red areas indicate bins marked "0" by participant

Figure 29 below provides the ESS distribution by qualifier resulting from seven participants completing Study Kit 3. 


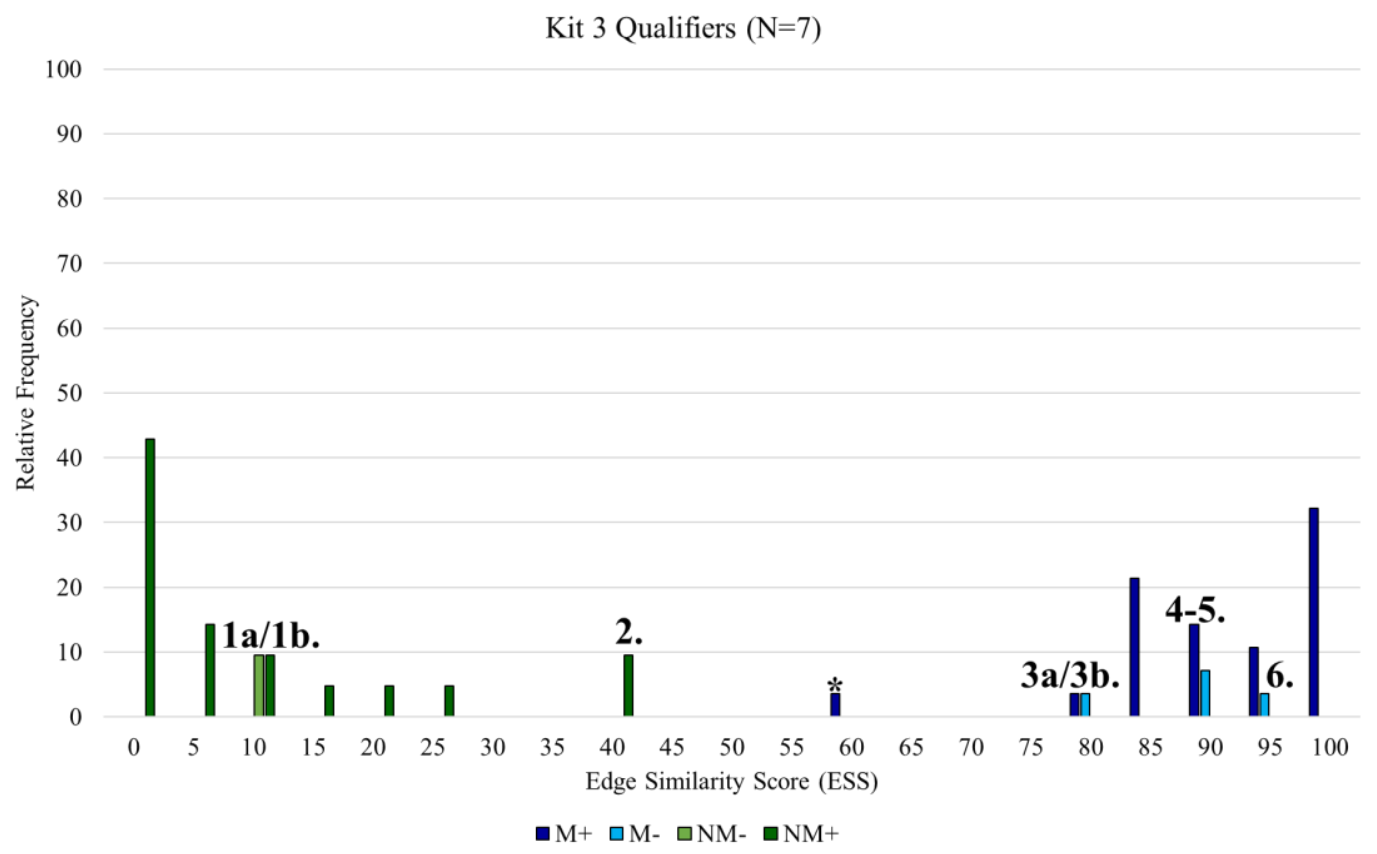

Figure 29. Kit 3 ESS distribution by qualifier ( $\mathrm{N}=7$ examiners, 49 total comparisons).

Numbering indicates discrepancy instances, points of discussion in which results varied from those expected.

As shown in Figure 29, difference 1a was assigned an ESS of $8 \%$ with a NM- comparison edge qualifier, while difference $1 \mathrm{~b}$ was also assigned an ESS of $8 \%$ but with a $\mathrm{NM}+$ qualifier. Interestingly, both of these score and qualifier determinations originated from the same participant. When examining the associated comments, it appears that the sample from discrepancy instance la presented more gross fracture edge morphology differences than that of difference $1 \mathrm{~b}$. Additionally, the sample pair associated to discrepancy instance $1 \mathrm{~b}$ presented edge distortion according to the participant, another factor that may have affected their certainty of the non-match conclusion. The tape pairs in question can be viewed in Figure 30. 


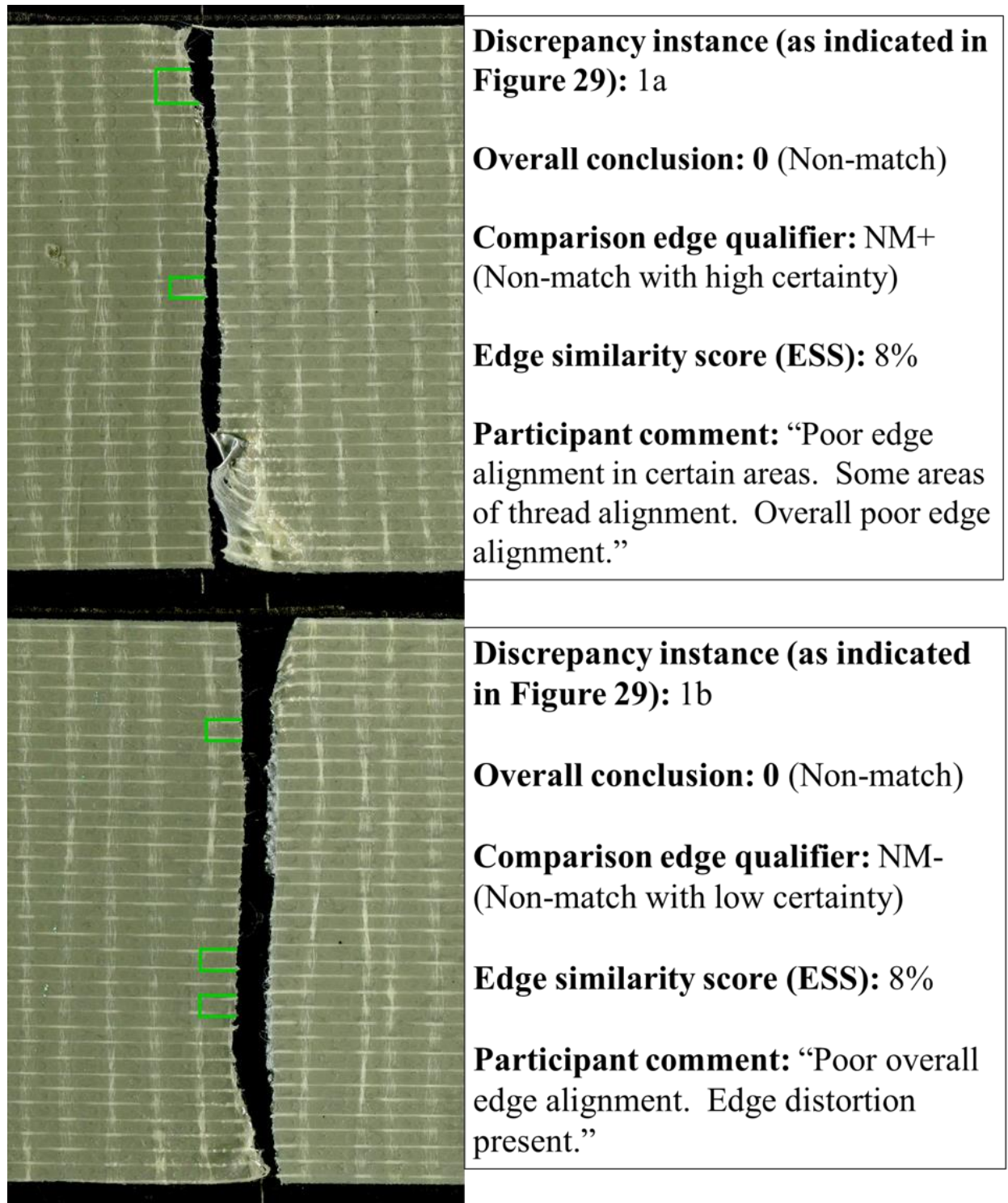

Figure 30. Comparison of Kit 3 samples assigned same ESS but different comparison edge qualifiers by same participant, green areas indicate bins marked "1" by participant

Similarly, Figure 29 also depicts differences 3a and 3b, which were both assigned ESS of 78\% by the same participant. However, discrepancy instance 3a was assigned a M-comparison edge qualifier while difference $3 \mathrm{~b}$ was assigned a $\mathrm{M}+$. In the comments for both sample pairs, the examiner notes that while some areas exhibited distortion that appeared consistent, others were distorted to the degree that edge detail was obstructed from view. In this circumstance, it is unclear the distinction in the varying qualifier assignment, other than the assumption that more edgeobstructing distortion was considered in difference $3 \mathrm{a}$ than difference $3 \mathrm{~b}$. These tape pairs can be viewed in Figure 31. 


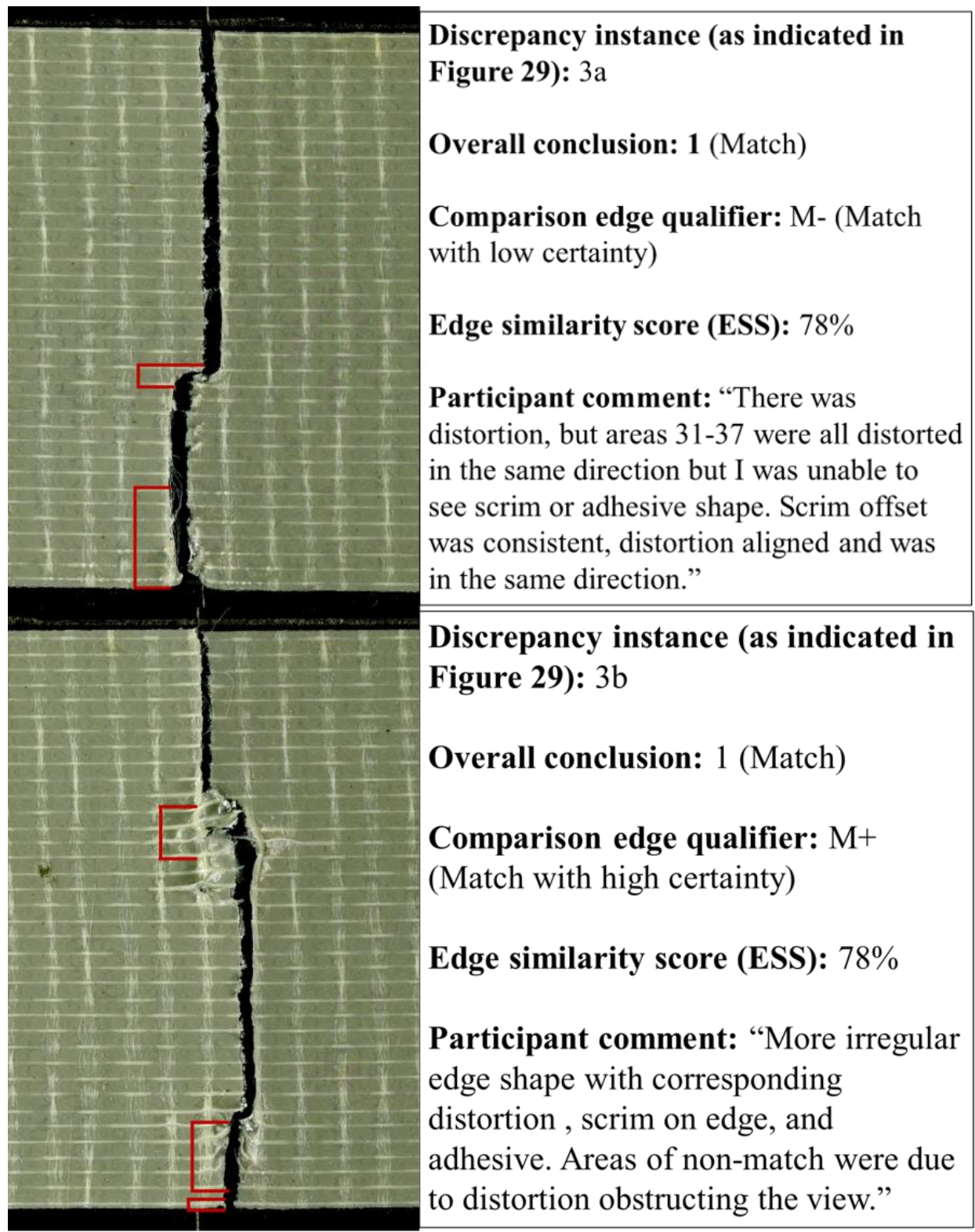

Figure 31. Comparison of Kit 3 samples assigned same ESS but different comparison edge qualifiers by same participant, red areas indicate bins marked " 0 ” by participant

Also shown in Figure 29 is discrepancy instance 2, a relatively high non-match ESS of $41 \%$ given the NM+ comparison edge qualifier. However, participant comments note all features along the tape that led to inconsistencies rather than those that led them to mark consistent scrim bins. Differences 4 and 5 are examples of relatively high ESS of $89 \%$ given M- comparison edge qualifiers. In the case of discrepancy instance 4, the examiner indicated that any inconsistent scrim bins were determined due to discrepancies in the adhesive-side detail in those regions. For difference 5, the participant indicated that while distortion was present, it was consistent across both sides of the fractured edge causing them to consider it "explainable." Discrepancy instance 6 was an interesting example as it was assigned an ESS value only 1 bin from $100 \%$. 


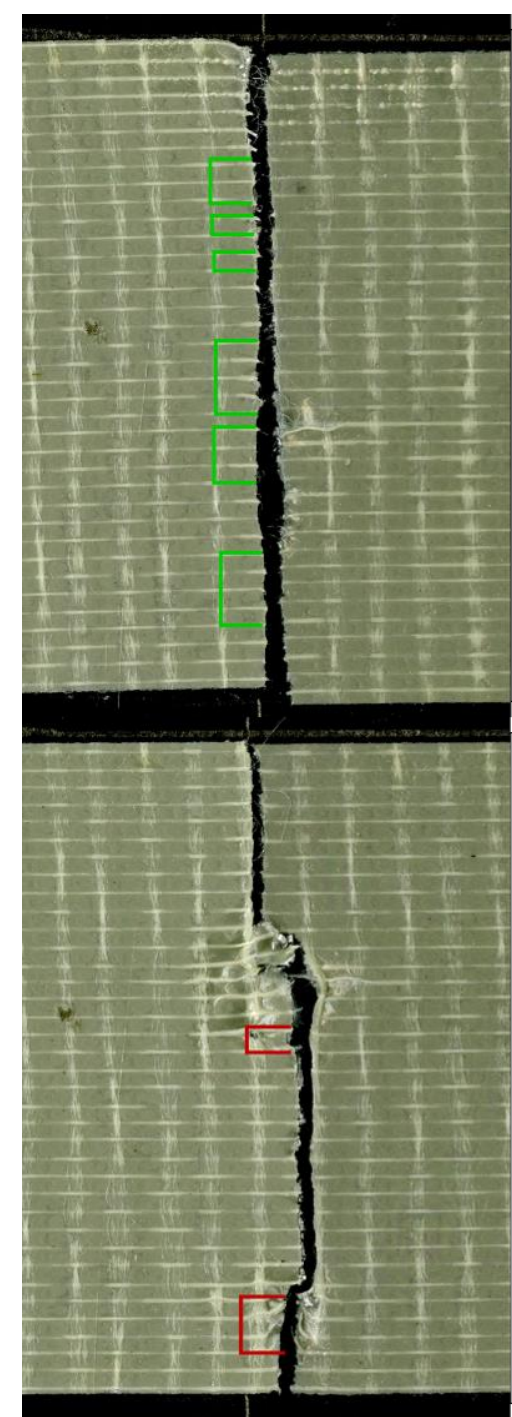

Discrepancy instance (as indicated in Figure 29): 2

Overall conclusion: 0 (Non-match)

Comparison edge qualifier: $\mathrm{NM}+$ (Non-match with high certainty)

Edge similarity score (ESS): $41 \%$

Participant comment: "Spacing dimples backing too close compared to rest of tape, spacing between warp \& edge different on one end, most of scrim yarn appears missing along fracture."

Discrepancy instance (as indicated in Figure 29): 5

Overall conclusion: 1 (Match)

Comparison edge qualifier: $\mathrm{M}$ (Match with low certainty)

\section{Edge similarity score (ESS): 89\%}

Participant comment: "Areas of distortion called inconsistent but since it's on both edges would say it's explainable."

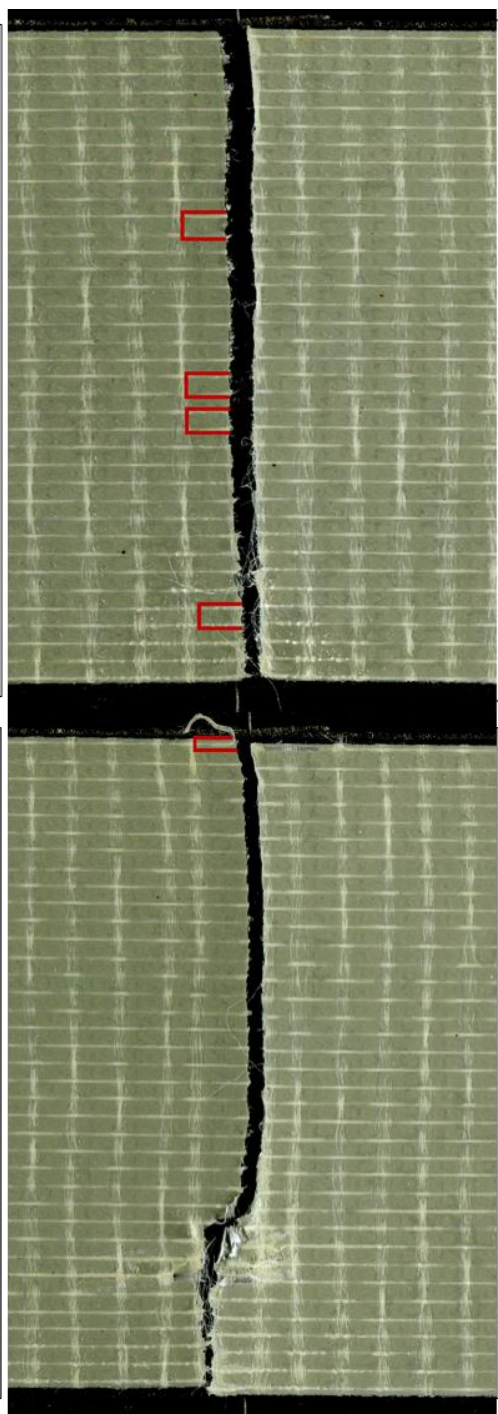

Discrepancy instance (as indicated in

Figure 29): 4

Overall conclusion: 1 (Match)

Comparison edge qualifier: M- (Match with low certainty)

Edge similarity score (ESS): 89\%

Participant comment: "Aligning calendaring striations and dimple shape across edge as expected as well as scrim. Though fairly straight tear. Areas where backing looked consistent but adhesive less so. And areas of non-match were based on adhesive-side slight differences."

Discrepancy instance (as indicated in Figure 29): 6

Overall conclusion: 1 (Match)

Comparison edge qualifier: $\mathrm{M}$ (Match with low certainty)

\section{Edge similarity score (ESS): 97\%}

Participant comment: "Good overall edge alignment and thread alignment. Minor edge distortion. Thread protruding between tape edges."

Figure 32. Kit 3 samples, qualifiers out of expected ranges, red areas indicate bins marked "0" by participant while green areas indicate bins marked " 1 " 
One bin was marked " 0 " due to a protruding yarn that was determined to be inconsistent with the corresponding edge. The examiner does denote that minor edge distortion was observed in addition to the protruding yarn, perhaps causing them to assign a qualifier of lower certainty. Images of these samples are provided in Figure 32 above.

In summary, a more in-depth assessment of the potential sources of dissimilarities between examiners' results and deviations from the consensus ESS scores was conducted by evaluating the comments each examiner documented on the ESS bin comparison sheets. Also, the respective tape images were carefully studied to identify which areas need further training to improve interexaminer agreement and to use the ESS method to its full potential. These types of assessments would not have been possible without the systematic analysis and documentation approach developed in this ILS. The bin-to-bin scores and corresponding notes, allowed us to do a thorough comparison of observed features and opinions between examiners, illustrating the utility of the ESS method for peer review process.

Specifically, the bin-to-bin evaluation revealed that the interpretation of the distinctiveness of features varied between some examiners. Less distinctive characteristics within a bin area, such as "featureless" straight edges or distorted edges were the most problematic. This feedback may indicate the need for a weighting factor to be applied to the method, in addition to the ESS, in order for examiners to best demonstrate a scrim bin that is consistent due to prominent physical features (e.g. corresponding protruding scrim or backing striae) versus a less distinctive scrim bin.

\subsubsection{Agreement of inter-laboratory ESS values and observed distributions in matched and non-matched pairs of larger datasets}

Despite any interpretation variances at the micro-level, the majority of overall ESS reported by participants were within approximate $\pm 20 \%$ ranges as compared to pre-distribution, consensus values with the exception of 15 out of 112 comparisons $(\mathrm{N}=16$ examiners overall, $\mathrm{n}=112$ total comparisons). When considering examiner overall conclusion despite assigned ESS value, no

misclassifications were observed throughout the study. When considering classification by the expected 50/50 ESS threshold, overall error rates were as follows: 92\% true positives (59/64), 8\% false negatives (5/64), 100\% true negatives (48/48), and $0 \%$ false positives (0/48). Moreover, overall agreement between examiners is shown in the boxplot distributions by ESS, provided in Figure 33 below. Additionally, as shown in Figure 34, overall study ESS distribution was similar to that of the true positives and true negatives of the larger population study, ${ }^{4}$ in which scores $>80 \%$ supported $\mathrm{M}+$ and scores $<25 \%$ supported NM. 


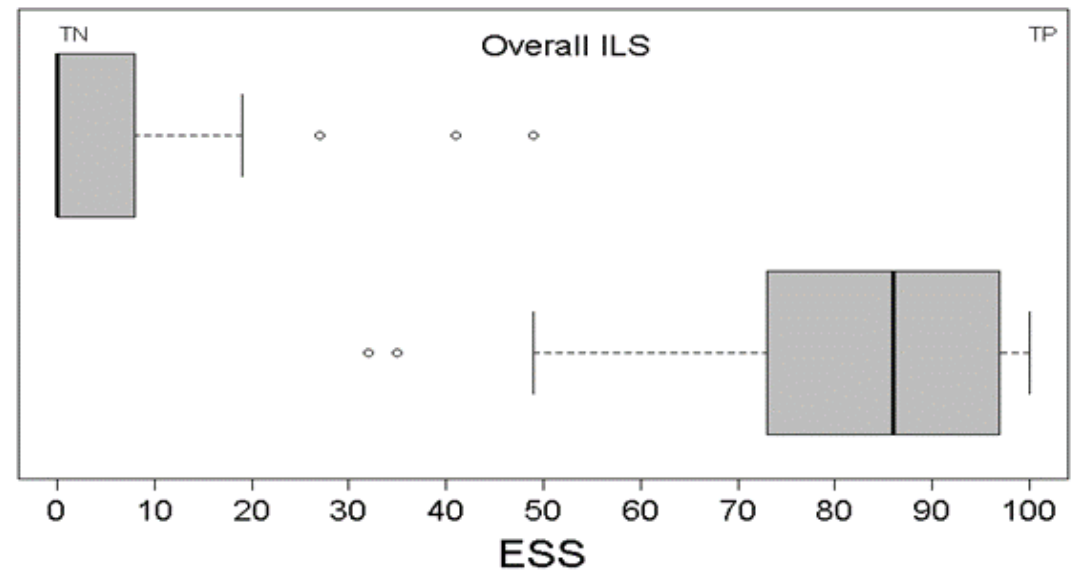

Figure 33. Overall inter-laboratory study ESS distribution

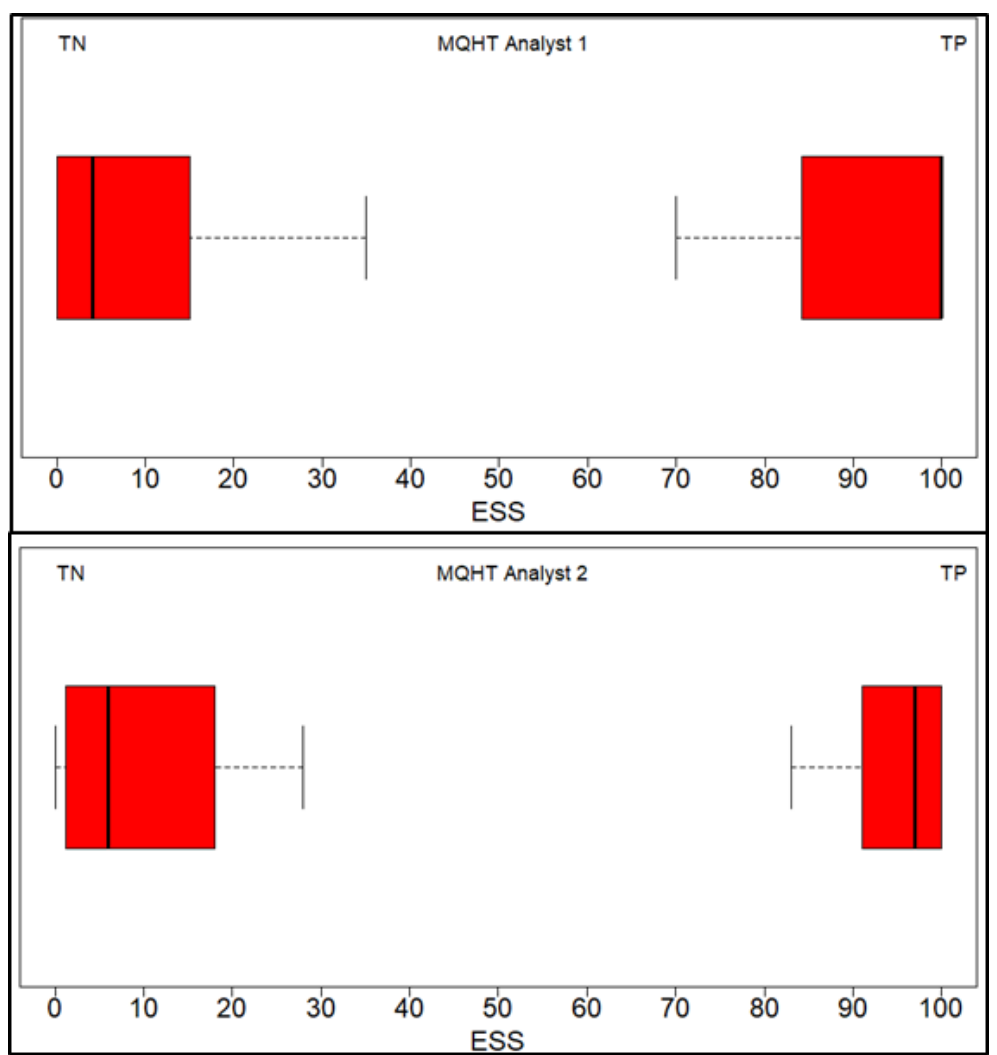

Figure 34. Prusinowski et al. ${ }^{4}$ medium quality, hand torn duct tape physical fit dataset $(\mathrm{N}=508$ comparison pairs per analyst)

Furthermore, comparison to 2019 Collaborative Testing Services (CTS), Inc. ${ }^{\odot}$ tape proficiency test results indicated that participants in the inter-laboratory study achieved higher accuracy rates. The CTS report revealed the following performance rates for comparisons of three K/Q tape physical fit pairs: a) K1/Q1 (true non-match): true negative rate of $84 \%, 16 \%$ false positive rate; b) K2/Q2 (true match): true positive rate of 95\%, 5\% false negative rate; and c) K3/Q3 (true nonmatch): true negative rate of $95 \%, 5 \%$ inconclusive. This indicates greater examiner accuracy utilizing the systematic, quantitative comparison method as compared to non-standardized, 
traditional methods used during proficiency testing. Furthermore, as discussed in Chapter One, as it is common for forensic laboratories to draw conclusions on evidence items once a physical match is determined, false positive conclusions are most detrimental to forensic casework. As this testing utilized non-standardized, traditional adhesive tape end match comparison methodology, these results indicate the need for exploration of examiner performance when adopting a systematic, quantitative method for duct tape physical fit examinations. Most importantly, it is critical to again demonstrate that the $16 \%$ false positive rate shown in CTS results is compared to a $0 \%$ false positive rate utilizing the proposed ESS method.

\subsection{Post-Study Survey Results}

Following the completion of the seven comparison pairs within a study kit, participants were asked to complete a brief survey to gauge their experience level and overall opinion on both the study kit as well as the duct tape physical fit ESS methodology. Survey questions were as follows:

1. Is your lab accredited?

2. Have you ever taken any of the following proficiency tests?

3. In terms of casework, about how much experience do you have with duct tape physical fits?

4. How is a physical fit usually represented in court?

5. About how much time do you typically spend on a physical fit examination?

6. About how long did it take you to work through the sample set?

7. Did you find the edge similarity score (ESS) approach easy to follow for duct tape end comparisons?

8. Did you find the edge similarity score metric useful to inform/support your opinion?

9. If you were to implement the ESS approach in your examinations, would you find the report templates for the score metric useful for a peer-review process?

While all survey questions were multiple choice, questions 3, 4, and 7-9 provided opportunities to leave supplementary comments for further elaboration. Survey results are presented graphically in Appendix B. Overall, survey responses indicated that participants all worked within accredited forensic laboratories, and only 6\% of examiners had not taken Tape Examination or Physical (Fracture) Match proficiency tests at the time of study completion. All participants had casework experience in physical fit, with only $13 \%$ of examiners claiming this experience was not related to tapes.

Of general physical fit casework information, $69 \%$ of participants indicated that photographs of physically fit evidence items are typically shown in court during their expert testimonies. The majority of participants (91\%) also indicated that they typically spend about 1-3 days working a physical fit examination.

Of study-related information, $94 \%$ of participants shared it took them more than 90 minutes to complete the examination of all seven sample pairs within a study kit, which seems fairly 
reasonable. The majority of participants also found the ESS approach average to easy in difficulty, indicating promise for smooth incorporation to current practice.

As far as examiner opinion, participants were split in their feelings of the assessment of usefulness of the ESS approach. Half of participants indicated the approach was not useful, with most of the comments revealed lack of understanding of the purpose of the ESS method or resistance to change, which is expected in the assessment of new approaches that differ from conventional protocols. As a result, we believe these negative perceptions are easy to correct in the future with further training and more detailed explanation of the scope and capabilities of the proposed approach. For instance, some of the expressed concerns were: 1) that the ESS would diminish the significance of a physical fit in the eyes of a jury if it is not $100 \%, 2$ ) that the examiner felt he/she had a bias in determining ESS due to their prior opinion of whether or not there was a match before estimating the ESS, and 3) that they did not feel their overall opinion should be based on a score.

As seen, these concerns, are easy to overcome with further training and communication with the end-users. For example, during a follow-up meeting with participants to discuss the ILS results, we stressed the ESS method is not intended to be the sole step on a physical match examination but rather a means to support and inform the examiner opinion. We also discussed the relevance of recognizing that not every match holds the same weight, and that a $100 \%$ perfect match is not always plausible, as demonstrated by our data. The ILS also demonstrated that as in any other discipline, it is impossible to be error-free. However, what is critical is we can identify and report sources of error and uncertainty. In addition, we noted that $63 \%$ of examiners that indicated "not useful" within the post-study survey did not receive the formal training and method interpretation discussion that allowed the researchers to be more familiar and open-minded with the proposed methodology.

On the other hand, the majority $(81 \%)$ of participants did feel that the ESS method and the scrim bin reporting templates would be useful tools for technical review of case reports and training of examiners. Indeed, the ESS method provides for the first time an opportunity for a blind, systematic, and transparent peer review process.

These comments are valuable as they draw to the researcher's attention the aspects of hesitation that some practitioners would demonstrate upon a decision to implement this methodology in their respective laboratories. As is common in this type of interlaboratory studies, the practitioners' feedback provided an opportunity to fine-tune the ESS method and most importantly, modify the training strategies to increase reproducibility in ESS between examiners and discuss crucial points of ESS interpretation. Therefore, this study provided the baseline from which future work may grow. 


\section{Conclusions and Future Work}

The purpose of this project was to develop and implement an inter-laboratory study in order to evaluate the performance of the proposed score-based method in assessing a potential duct tape physical fit. Of particular interest in this pilot study was the assessment of inter-examiner agreement, examiner error rates, and feedback from participants to facilitate the future adoption of the method to their laboratories. This study utilized the ESS methodology previously developed by Prusinowski et al. ${ }^{4}$ Three study kits were developed with sixteen forensic practitioner participants overall and ESS and conclusions reported for 112 duct tape fractured paired samples.

Overall, inter-examiner agreement with reporting ESS scores within $20 \%$ of the mean consensus values was observed. The participants' accuracy ranged from 88 to $100 \%$, depending on the quality of the match and test kit. Moreover, the inter-laboratory study highlighted the utility of the ESS score method to enhance future physical fit practice in several aspects:

a) Increased objectivity: Although human judgment will always be needed for physical fit examinations, the use of subjective decisions is risky when used without standardized criteria. The ESS score method allows, for the first time, established thresholds and standards that can be used for informing and supporting the examiners' opinion regarding the quality of a match.

b) Consensus: one of the challenges faced by forensic practitioners is to identify when a physical fit presents enough distinctive characteristics to decide between a match, a good match, an inconclusive, or a non-match conclusion. The ESS score has shown promise towards standardization of criteria and systematic documentation and peer review process. Most importantly, the reproducible bin-to-bin comparison of features leaves room for future improvement on the estimation of occurrence of rare or distinctive micro-features. Inter-laboratory studies using the ESS would help us in the near future identify which areas and features hold more weight during an examination and how and why we can arrive at consensus protocols.

c) Scientific reliability: the ESS scores and the ILS studies allow for estimation of performance rates, false positives, false negatives, overall accuracy, and inter-examiner agreement. Also, it provides a means to estimate which factors can affect the uncertainty of a physical fit. All of those measures provide a valuable empirically demonstrable basis to assess the significance of a fit.

A careful evaluation of the data, the bin-to-bin examiners' documentation, and the survey's feedback revealed three main observations across result sets. First, those participants that did not participate in formal method training through either the in-person method presentation or teleconference tended to exhibit statistically significant score differences from the consensus, predistribution mean ESS. This was shown through results of the Dunnett's test as well as distribution of scores. Of the $33 \%$ of participants presenting larger deviations with the consensus mean, $73 \%$ did not elect to participate in formal method training beyond the protocol and instructional presentation provided at the time of kit receipt. On the other hand, the majority of examiners who 
were exposed to formal instruction demonstrated agreement with consensus values and with distribution of score thresholds as compared with larger population datasets. As a result, future ILS would include more in-depth mandatory training as a pre-requisite to participation.

Other main observations across the study included variance in which examiners treated and interpreted a featureless or distorted region of scrim bins for ESS purposes. While some examiners assigned a binary classifier of 0 to these areas (non-matching, inconsistent bin determinations), others felt these areas could still be determined consistent and assigned a binary classifier of 1 to these areas (matching, consistent bin determinations). Further, some examiners noted that the method may be more beneficial with an inconclusive variable option or a weighing factor for scrim bins instead of just binary output ( 1 or 0 ). Those recommendations are currently being incorporated for future tests.

It was also determined that more training is needed to aid examiners with the interpretations in the use of the comparison edge qualifier. While expected ranges were set for ESS based on the assignment of comparison edge qualifiers according to previously determined score likelihood ratios (SLRs) ${ }^{4}$, many examiners did not provided qualifiers that were reasonable for certain ESS ranges.

Despite slight interpretation variation, the majority of ESS reported by participants were within approximate $\pm 20 \%$ ranges as compared to pre-distribution, consensus values with the exception of 15 out of 112 instances $(\mathrm{N}=16$ examiners overall, $\mathrm{n}=112$ total comparisons). No misclassifications were observed throughout the study by overall examiner conclusion per comparison pair. Observed error rates were as follows: 95\% true positives (61/64), $0 \%$ false negatives (0/48), $100 \%$ true negatives (48/48), and $0 \%$ false positives (0/64). The reduction in the true positive rate is the result of a $5 \%$ inconclusive rate (3 true positive samples were concluded as inconclusive across the sample set). When considering classification by the expected 50/50 non-match/match ESS threshold, overall error rates were as follows: 92\% true positives (59/64), $8 \%$ false negatives (5/48), 100\% true negatives (48/48), and $0 \%$ false positives (0/64).

Future work will include modification of the ESS method based upon examiner feedback received during the post-study survey to expand the binary outputs on the ESS scores and include further guidelines on macro assessments. Following optimization, expanded distribution of the interlaboratory study will be initiated in order to further validate the methodology for potential implementation into forensic laboratories. Utilization of the ESS method in duct tape physical fit examinations will uphold the high level of association offered by physical fits while reducing subjectivity and creating a more transparent review and interpretation process.

Future work will also include expanding upon a preliminary, linear mixed model fit by restricted maximum likelihood (REML) applied to the inter-laboratory ESS data in order to further assess the amount of variance existing between participant results. Within the model, sample groups by anticipated level of difficulty (expected comparison edge qualifier and ground truth) were utilized as the fixed effect. This resulted in three levels by sample group: easy true match $(\mathrm{M}+)$, difficult 
true match (M-), and true non-match (NM). The random effects on ESS results were described by two factors: the different sample groups by difficulty (3 levels) and the examiners participating in the study. In this manner, variance of study participants was able to be observed while correcting for the fact that different examiners were viewing different physical samples between the 3 kits. Application of the model to the current dataset revealed that variance between examiners was less than between different kits. However, this model is still in progress. As the current model does not apply significance testing and is descriptive of score variation alone, eventual expansion seeks to apply a Bayesian model to provide credible intervals for variation between examiners. In addition, fit of the model is expected to improve with a greater input of ESS data due to increased participants in future expanded distribution of the study kits.

The results from this ILS demonstrated that the proposed ESS method can provide support to examiner conclusions, offer systematic criteria that can lead to consensus-based methods, and allow for a quantitative assessment of factors influencing the quality of a fit as well as estimation of inter-examiner error rates. Examiners also recognized the method provides an avenue to conduct a systematic and transparent peer-review process, which is otherwise not possible with current examination protocols.

\section{References}

1. National Academy of Sciences (NAS). Strengthening Forensic Science in the United States: A Path Forward. 2009. doi:0.17226/12589

2. President's Council of Advisors on Science and Technology. Forensic Science in Criminal Courts: Ensuring Scientific Validity of Feature-Comparison Methods. 2016.

3. American Statistical Association. American Statistical Association Position on Statistical Statements for Forensic Evidence. [accessed 2019 Jan 30]. https://www.amstat.org/asa/files/pdfs/POL-ForensicScience.pdf

4. Prusinowski M, Brooks E, Trejos T. Development and validation of a systematic approach for the quantitative assessment of the quality of duct tape physical fits. Forensic Science International. $2020 ; 307$.

5. Bradley MJ, Keagy RL, Lowe PC, Rickenbach MP, Wright DM, LeBeau MA. A validation study for duct tape end matches. Journal of Forensic Sciences. 2006;51(3):504-508. doi:10.1111/j.1556-4029.2006.00106.x

6. McCabe KR, Tulleners FA, Braun J V, Currie G, Gorecho EN. A Quantitative Analysis of Torn and Cut Duct Tape Physical End Matching. Journal of Forensic Sciences. 2013;58(S1):S34-S42.

7. ISO/IEC 17043:2010 Conformity assessment - General requirements for proficiency testing. 2010 .

8. Ivanov AR, Colangelo CM, Dufresne CP, Friedman DB, Lilley KS, Mechtler K, Phinney BS, Rose KL, Rudnick PA, Searle BC, et al. Interlaboratory studies and initiatives developing standards for proteomics. Proteomics. 2013;13(6):904-909. doi:10.1002/pmic.201200532 
9. International Study Group. An inter-laboratory comparison of radiocarbon measurements in tree rings. Nature. 1982;298:619-623. doi:10.1038/298619a0

10. Chung JH, Cho K, Kim S, Jeon SH, Shin JH, Lee J, Ahn YG. Inter-Laboratory Validation of Method to Determine Residual Enrofloxacin in Chicken Meat. International Journal of Analytical Chemistry. 2018;2018. doi:10.1155/2018/6019549

11. Hoffman T, Corzo R, Weis P, Pollock E, van Es A, Wiarda W, Stryjnik A, Dorn H, Heydon A, Hoise E, et al. An inter-laboratory evaluation of LA-ICP-MS analysis of glass and the use of a database for the interpretation of glass evidence. Forensic Chemistry. 2018;11:65-76. doi:10.1016/j.forc.2018.10.001

12. Lucidarme D, Decoster A, Delamare C, Schmitt C, Kozlowski D, Harbonnier J, Jacob C, Cyran C, Forzy G, Defer C, et al. An inter-laboratory study of anti-HCV antibody detection in salavary samples. Gastroenterology. 2003;124(4):A705.

13. Hund E, Massart DL, Smeyers-Verbeke J. Inter-laboratory studies in analytical chemistry. Analytica Chimica Acta. 2000;423(2):145-165. doi:10.1016/S0003-2670(00)01115-6

14. ASTM International. ASTM E177 - 19 Standard Practice for Use of the Terms Precision and Bias in ASTM Test Methods. 2019:1-12. doi:10.1520/E0177-10.2

15. ASTM International. ASTM E691 - 19e1 Standard Practice for Conducting an Interlaboratory Study to Determine the Precision of a Test Method. 2019:1-26. doi:10.1080/00224065.1993.11979478

16. National Commission on Forensic Science. National Commission on Forensic Science: Views of the Commission - Proficiency Testing in Forensic Science. 2016.

17. ISO/IEC 17025:2017 General requirements for the competence of testing and calibration laboratories. 2017.

18. ISO/IEC 17011:2017 Conformity assessment - Requirements for accreditation bodies accrediting conformity assessment bodies. 2017. 


\section{CHAPTER 2: APPENDIX A}

\section{i. Study Protocol}

W.WestVirginiaUniversity.

\section{WVU Forensic and Investigative Science Duct Tape Research}

\section{Inter-Laboratory Study Protocol}

Thank you for taking the time to complete a sample set for our inter-laboratory study and assist our research efforts. The following protocol demonstrates the method our research group utilizes to determine edge similarity scores (ESS) for a quantitative, percentage-based representation of the quality of a duct tape physical fit. We ask that you follow this protocol while working through the inter-laboratory study kit. The kit contains 7 comparison pairs. It is estimated that the kit will take about 1 to 2 hours to complete. Participation is voluntary and anonymous.

Duct Tape Fracture Comparison and Edge Similarity Score (ESS) Determination Protocol

1. Remove the tape samples from their envelope. Each pair to be compared will be packaged together. Refer to the labelling scheme to assure samples are paired correctly before performing a comparison. A tape pair will have the same ID number followed by an A or B.

2. You are asked to perform just one edge comparison per sample pair. Each sample will have a hand-torn edge and a distinctive scissorcut edge. The comparison should be done only on the hand-torn edges, as shown in Figure 1.

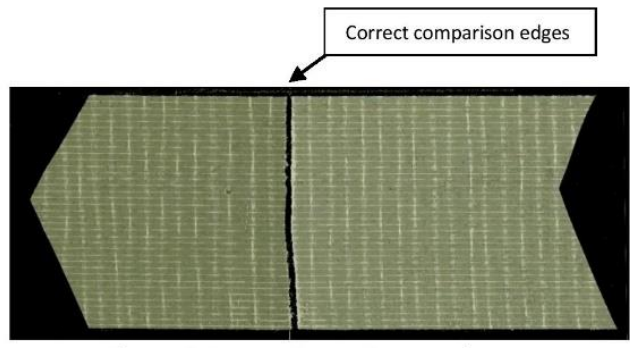

Figure 1. Comparison pair example

3. PLEASE conduct the observation of the duct tape edges without removing them from the transparency film, altering the edges in any way, or writing on the acetate film.
4. Examine the pairs under a stereomicroscope, both backing and scrim sides. The following lighting conditions are recommended:

a. Backing side: Reflected lighting. An oblique setting is best due to slight glare.

b. Scrim side: Alternate between reflected and transmitted lighting to view edge features of both the adhesive and scrim yarns as well as the backing through the adhesive surface.

5. First, observe the overall fractured edge on the backing side. Note any physical characteristics across the fracture that may help in alignment, or the lack of. These could include dimpling, calendering striations or general backing distortion (protrusions on sample A met by indentations on sample B, or vice versa). Align the top edge first (indicated by Sharpie-drawn arrows on the transparency film) to help with the physical fit assessment.

6. Flip the samples over and observe the overall fractured edge on the scrim side. Again, note any continuous/discontinuous physical features across the fractured edge. These could include warp scrim alignment, protruding warp yarns, general adhesive distortion (protrusions on sample A met by indentations on sample B, or vice versa), and continuation of scrim pattern. Observe any characteristics that could lead to an incompatible fracture fit such as warp scrim misalignment, double weft edge scrim (full weft yarns on each edge rather than partial on each), missing scrim, or inconsistent scrim pattern across the fracture (i.e. plain weave scrim pattern, warp yarn is over partial weft yarn on one edge but corresponding warp yarn is under a partial weft yarn on the edge of the other sample).

Please refer to example feature images in the PowerPoint presentation sent as a part of the study kit.

Note: Steps 7-10 that follow detail documentation of ESS during your examination. Both physical and digital templates are supplied as part of the study kit. Further detail is provided on the layout of each template in the "Template Information" section at the end of this document. 
WhestVirginiaUniversity.

7. Begin determining your ESS by counting the total number of scrim areas across the width of the tape pair. A scrim area is defined by the small areas formed by the warp yarns along the fractured edge. Include even those areas at the top and bottom edges of the tape that are only bordered by one warp yarn. If you count a total number of scrim areas less than or greater than the 37 allotted on the template, include those areas in your score consideration and mark your template accordingly.

Note: When counting scrim areas, your \#1 area when numbering 1-37 should be at the side of the tapes denoted by a Sharpie-drawn arrow, as shown in Figure 2 .

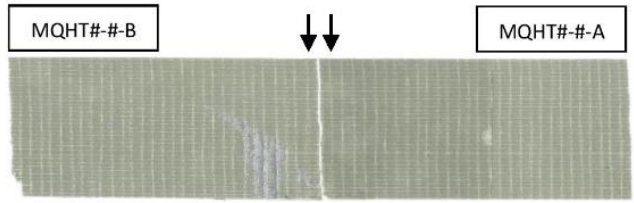

Figure 2. Scrim start point demonstration

8. Determine the scrim areas along the fracture edge that appear either consistent or inconsistent. Alternate between transmitted and reflected light to examine the backing and adhesive morphology at each scrim area. Flip the samples over to examine the backing as well as any scrim areas of discrepancy. Mark your conclusion per area on your template with a " 0 " for those considered to be inconsistent and a " 1 " for those considered to be consistent. If desired, sketch any specific morphology that led to your conclusion at a certain area in the provided space on the physical template. You may make notes on any specific features per area in the provided space on the template as well.

9. When you have finished making determinations at each area and annotating any comments or sketches, determine a qualitative description of the fit. These include the comparison edge qualifier and the comparison pair overall conclusion.

a. Comparison Edge Qualifier: A key denoting your confidence in drawing a conclusion of match, non-match or inconclusive. Options are as follows.

\author{
$\mathrm{M}+=$ Match with high confidence \\ $\mathrm{M}$ - = Match with low confidence \\ INC = Inconclusive \\ NM- = Non-match with low confidence \\ $\mathrm{NM}+=$ Non-match with high confidence
}

b. Comparison Pair Overall Conclusion: A code denoting your conclusion of the comparison pair.

1 = Match

INC = Inconclusive

$0=$ Non-match

Areas for these descriptors are provided at the end of each pair template on the physical template and under the "Total Edge Considerations" section on the digital template.

10. After documenting determinations at each scrim area, please refer to the digital template to determine your edge similarity score (ESS) per pair.

11. Please complete the brief, post-study examiner survey located on the final tab of the digital answer sheet template.

12. Re-package all tape samples. Please email the complete digital template to Evie Brooks at ekb0011@mix.wvu.edu

13. Using the pre-paid shipping label and FedEx envelope your study kit originally came in, please send the complete answer sheet templates and all tape samples back to:

Tatiana Trejos

Department of Forensic and Investigative

Science

208 Oglebay Hall

P.O. Box 6121

Morgantown, WV 26506-6121

Please reach out to Evie Brooks (email above) with any questions or concerns that may arise during examination. Thank you again for your time and participation! 
WhestVirginiaUniversity.

\section{Answer Sheet Template Information}

We have provided a digital template file for recording your results during your examination. For your convenience, we have also included a printed copy of the report template. If you prefer to use the hand-written template, we ask you to type the results into the electronic file. Please save the file as "ILS_Score_Template_" followed by your unique lab ID number (ex. If your lab ID is ILS-01, name the file as ILS_Score_Template_ILS-01). Your lab ID number is located on the label on the plastic sleeve your documents were received in.

Submit the complete digital template via email to: ekb0011@mix.wvu.edu

Printed Answer Sheet Template (Hard-Copy Version)

Refer to the printed template, 4 pages total. For each pair, a template is provided for documentation of the physical fit examination. For each scrim area across the width of the tape, a space is provided for documentation of the corresponding match code, a sketch, and comments.

Match Code: Binary numbers denoting if the scrim areas correspond over the fractured edge.

$$
\begin{aligned}
& 0=\text { Non-match } \\
& 1=\text { Match }
\end{aligned}
$$

Sketch: If desired, a sketch of the scrim area features leading to your conclusion

Comments: If desired, comments describing any features of the scrim area leading to your conclusion

At the bottom of each template, a space is provided to denote a comparison edge qualifier and overall conclusion.

Comparison Edge Qualifier: A key denoting your confidence in drawing a conclusion of match, non-match or inconclusive.

$$
\begin{aligned}
& M+=\text { Match with high certainty } \\
& M-=\text { Match with low certainty } \\
& \text { INC = Inconclusive } \\
& N M-=\text { Non-match with low certainty } \\
& N M+=\text { Non-match with high certainty }
\end{aligned}
$$

Comparison Pair Overall Conclusion: A code denoting your conclusion of the comparison pair. Options are as follows.

$$
\begin{aligned}
& 1=\text { Match } \\
& \text { INC = Inconclusive } \\
& 0=\text { Non-match }
\end{aligned}
$$

On Page 4 of the template, additional space is provided to write general comments for each pair regarding features along the fracture edge that helped you to draw a conclusion.

Digital Answer Sheet Template

Refer to the digital template (Excel file "ILS_Score_Template.xlsx"). The file contains 7 tabbed worksheets for each comparison pair, with the addition of one tab reserved for the post-study examiner survey. On each worksheet, the first 2 columns contain space to document the match code per scrim area as described above. To the right, the "Total Edge Considerations" section is provided to document the comparison edge qualifier and overall conclusion. The number of scrim areas considered matches, the scrim ratio and ESS should be populated automatically. On the far right, there is space to add any comments regarding features along the fracture edge that helped you to draw a conclusion. 


\section{ii. Physical scrim documentation template}

\begin{tabular}{|c|c|c|c|}
\hline \multicolumn{4}{|c|}{ Pair ID: } \\
\hline $\begin{array}{l}\text { Scrim } \\
\text { Area }\end{array}$ & 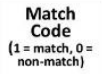 & Optional Sketch & Comments \\
\hline 1. & & & \\
\hline 2. & & & \\
\hline 3. & & & \\
\hline 4. & & & \\
\hline 5. & & & \\
\hline 6. & & & \\
\hline 7. & & & \\
\hline 8. & & & \\
\hline 9. & & & \\
\hline 10. & & & \\
\hline 11. & & & \\
\hline 12. & & & \\
\hline 13. & & & \\
\hline 14. & & & \\
\hline 15. & & & \\
\hline 16. & & & \\
\hline 17. & & & \\
\hline 18. & & & \\
\hline 19. & & & \\
\hline 20. & & & \\
\hline 21. & & & \\
\hline 22. & & & \\
\hline 23. & & & \\
\hline 24. & & & \\
\hline 25. & & & \\
\hline 26. & & & \\
\hline 27. & & & \\
\hline 28. & & & \\
\hline 29. & & & \\
\hline 30. & & & \\
\hline 31. & & & \\
\hline 32. & & & \\
\hline 33. & & & \\
\hline 34. & & & \\
\hline 35. & & & \\
\hline 36. & & & \\
\hline 37. & & & \\
\hline con & parison Edge & 2ualifier: & Comparison Pair Overall Conclusion: \\
\hline
\end{tabular}

WestVurginiaUniversity.

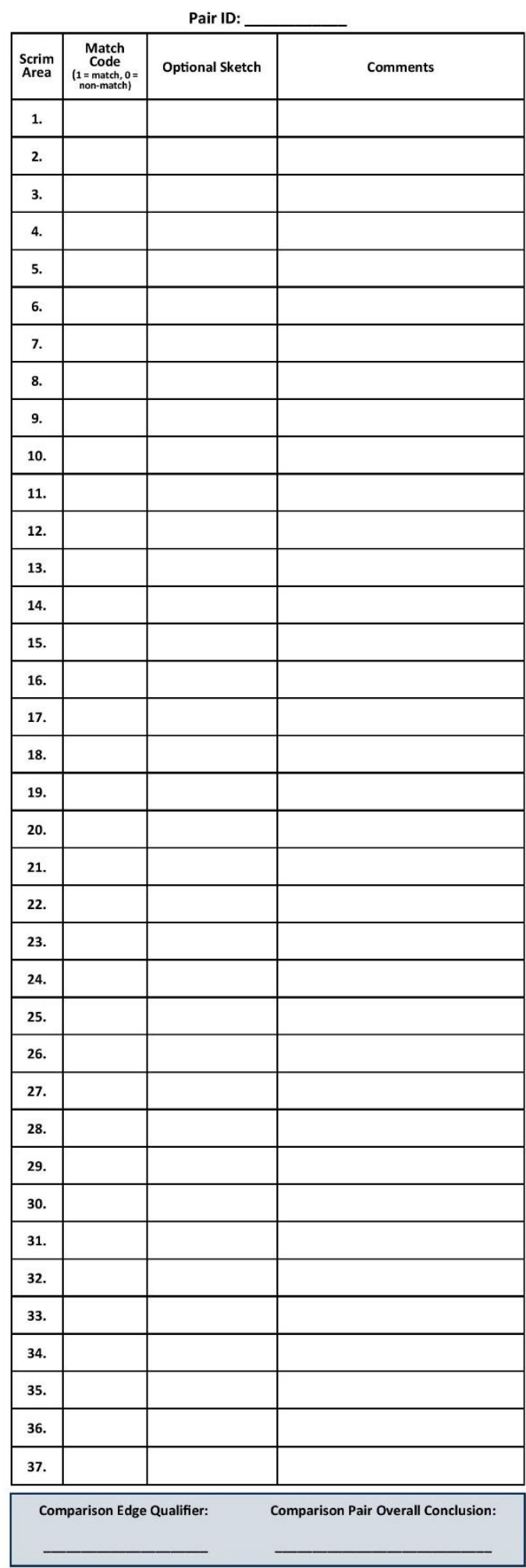

Lab ID:

Page 1 of 4 


\begin{tabular}{|c|c|c|c|}
\hline \multicolumn{4}{|c|}{ Pair ID:_ } \\
\hline $\begin{array}{l}\text { Scrim } \\
\text { Area }\end{array}$ & 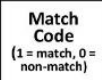 & Optional Sketch & Comments \\
\hline 1. & & & \\
\hline 2. & & & \\
\hline 3. & & & \\
\hline 4. & & & \\
\hline 5. & & & \\
\hline 6. & & & \\
\hline 7. & & & \\
\hline 8. & & & \\
\hline 9. & & & \\
\hline 10. & & & \\
\hline 11. & & & \\
\hline 12. & & & \\
\hline 13. & & & \\
\hline 14. & & & \\
\hline 15. & & & \\
\hline 16. & & & \\
\hline 17. & & & \\
\hline 18. & & & \\
\hline 19. & & & \\
\hline 20. & & & \\
\hline 21. & & & \\
\hline 22. & & & \\
\hline 23. & & & \\
\hline 24. & & & \\
\hline 25. & & & \\
\hline 26. & & & \\
\hline 27. & & & \\
\hline 28. & & & \\
\hline 29. & & & \\
\hline 30. & & & \\
\hline 31. & & & \\
\hline 32. & & & \\
\hline 33. & & & \\
\hline 34. & & & \\
\hline 35. & & & \\
\hline 36. & & & \\
\hline 37. & & & \\
\hline Con & nparison Edge & Qualifier: & Comparison Pair Overall Conclusion: \\
\hline
\end{tabular}

WestVirginiaUniversity.

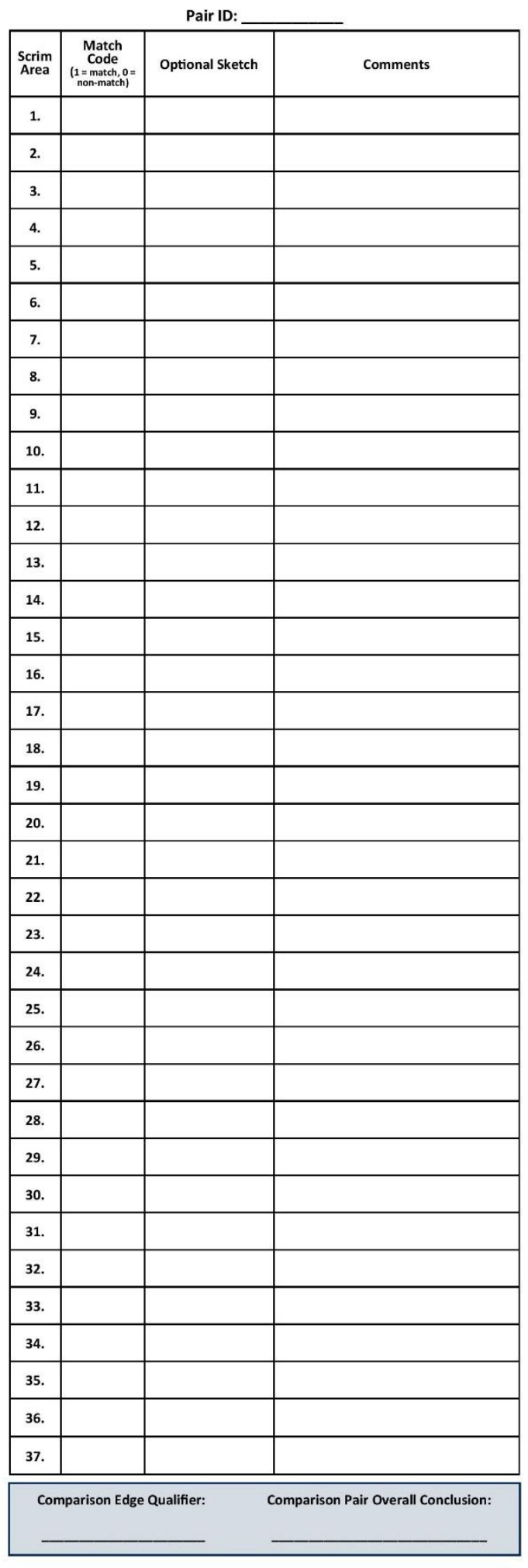

Lab ID:

Page 2 of 4 


\begin{tabular}{|c|c|c|c|}
\hline \multicolumn{4}{|c|}{ Pair ID:_ } \\
\hline $\begin{array}{l}\text { Scrim } \\
\text { Area }\end{array}$ & 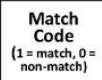 & Optional Sketch & Comments \\
\hline 1. & & & \\
\hline 2. & & & \\
\hline 3. & & & \\
\hline 4. & & & \\
\hline 5. & & & \\
\hline 6. & & & \\
\hline 7. & & & \\
\hline 8. & & & \\
\hline 9. & & & \\
\hline 10. & & & \\
\hline 11. & & & \\
\hline 12. & & & \\
\hline 13. & & & \\
\hline 14. & & & \\
\hline 15. & & & \\
\hline 16. & & & \\
\hline 17. & & & \\
\hline 18. & & & \\
\hline 19. & & & \\
\hline 20. & & & \\
\hline 21. & & & \\
\hline 22. & & & \\
\hline 23. & & & \\
\hline 24. & & & \\
\hline 25. & & & \\
\hline 26. & & & \\
\hline 27. & & & \\
\hline 28. & & & \\
\hline 29. & & & \\
\hline 30. & & & \\
\hline 31. & & & \\
\hline 32. & & & \\
\hline 33. & & & \\
\hline 34. & & & \\
\hline 35. & & & \\
\hline 36. & & & \\
\hline 37. & & & \\
\hline Con & nparison Edge & Qualifier: & Comparison Pair Overall Conclusion: \\
\hline
\end{tabular}

WestVirginiaUniversity.

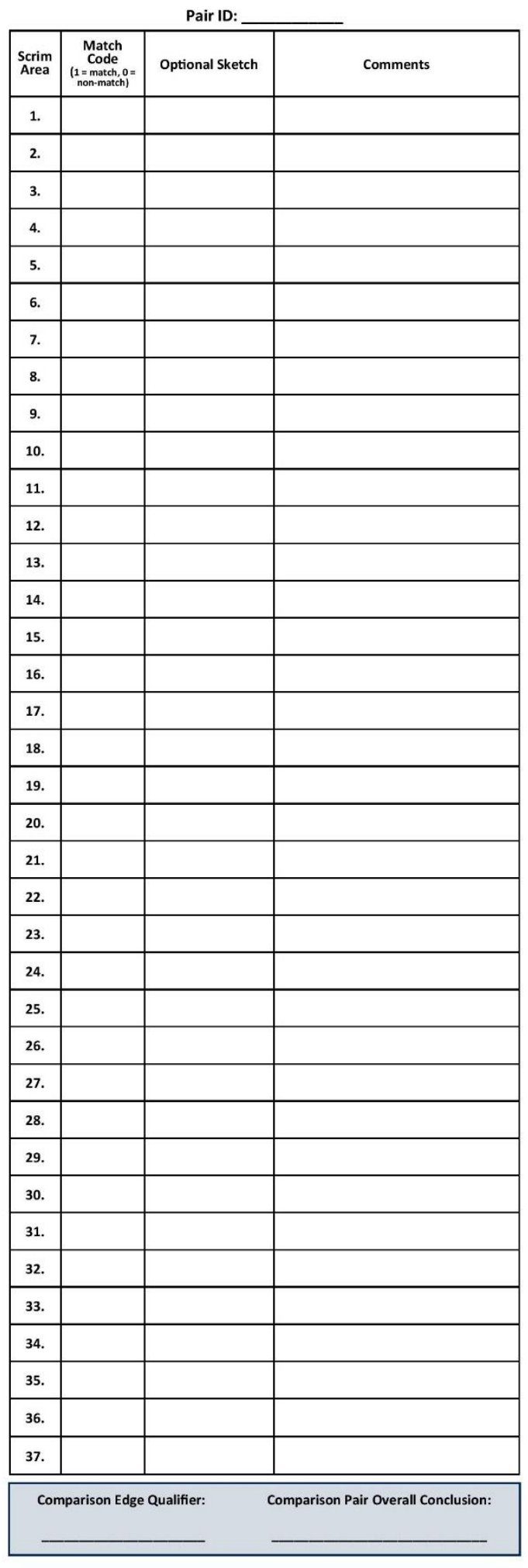

Lab ID:

Page 3 of 4 


\begin{tabular}{|c|c|c|c|}
\hline \multicolumn{4}{|c|}{ Pair ID: } \\
\hline $\begin{array}{l}\text { Scrim } \\
\text { Area }\end{array}$ & 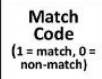 & Optional Sketch & Comments \\
\hline 1. & & & \\
\hline 2. & & & \\
\hline 3. & & & \\
\hline 4. & & & \\
\hline 5. & & & \\
\hline 6. & & & \\
\hline 7. & & & \\
\hline 8. & & & \\
\hline 9. & & & \\
\hline 10. & & & \\
\hline 11. & & & \\
\hline 12. & & & \\
\hline 13. & & & \\
\hline 14. & & & \\
\hline 15. & & & \\
\hline 16. & & & \\
\hline 17. & & & \\
\hline 18. & & & \\
\hline 19. & & & \\
\hline 20. & & & \\
\hline 21. & & & \\
\hline 22. & & & \\
\hline 23. & & & \\
\hline 24. & & & \\
\hline 25. & & & \\
\hline 26. & & & \\
\hline 27. & & & \\
\hline 28. & & & \\
\hline 29. & & & \\
\hline 30. & & & \\
\hline 31. & & & \\
\hline 32. & & & \\
\hline 33. & & & \\
\hline 34. & & & \\
\hline 35. & & & \\
\hline 36. & & & \\
\hline 37. & & & \\
\hline com & १parison Edge & Qualifier: & Comparison Pair Overall Conclusion: \\
\hline
\end{tabular}

WestVirginiaUniversity.

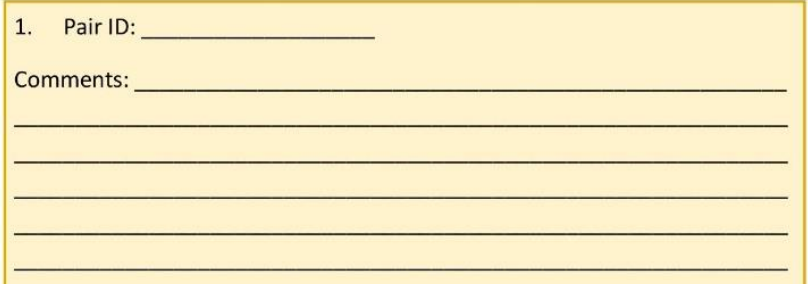

2. Pair ID:

Comments:

3. Pair ID

Comments:

4. Pair ID

Comments:

5. Pair ID:

Comments:

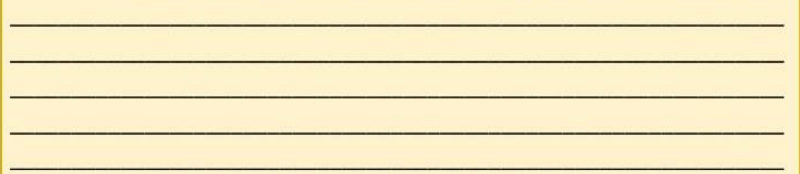

6. Pair ID:

Comments:

7. Pair ID:

Comments:

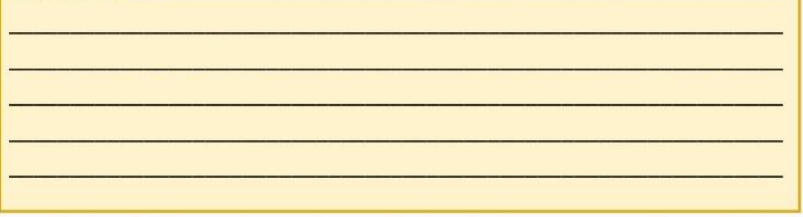

Lab ID:

Page 4 of 4 
iii. Digital scrim documentation template (1 of 8 worksheets, one per pair and a final survey tab)

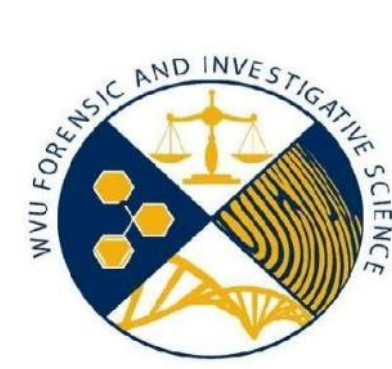

Total Edge Consideration Column Guidelines:

WEST

1. Cick the respective cell for a drop-down menu of companison edge qualier options, a qualtative representation of your certainty of the fit.

3-5. These cells should automatically populate once you have entered your match codes per scrim area in the pair template highlighted in blue. This will calculate your ESS for the comparison pair-

6. This cell provides space for any general comments on the comparison pair you may have written on your printed (hard-copy) answer sheet, or serves as a space to write comments now.
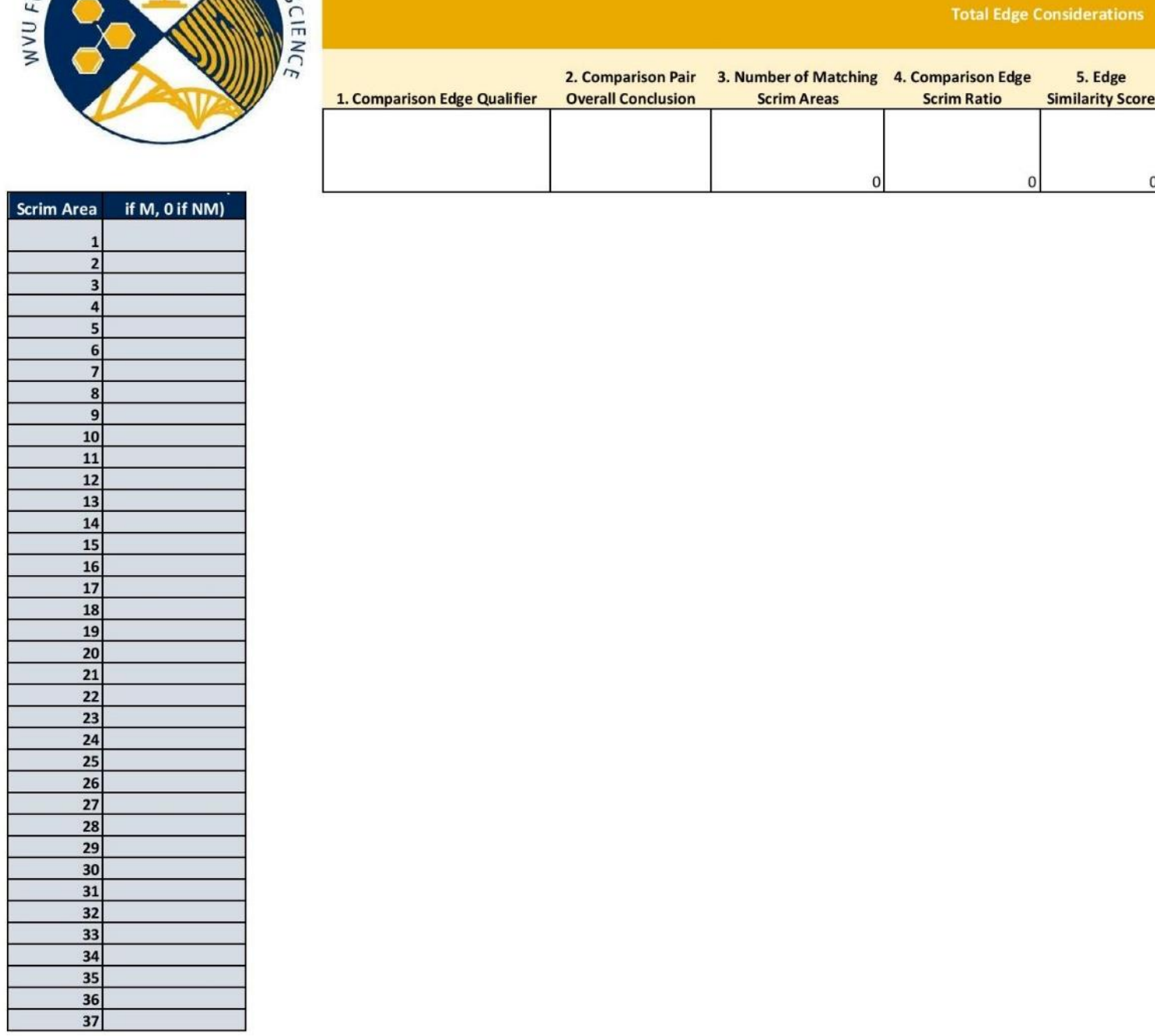

2. Comparison Pair 3. Number of Matching 4. Comparison Edge

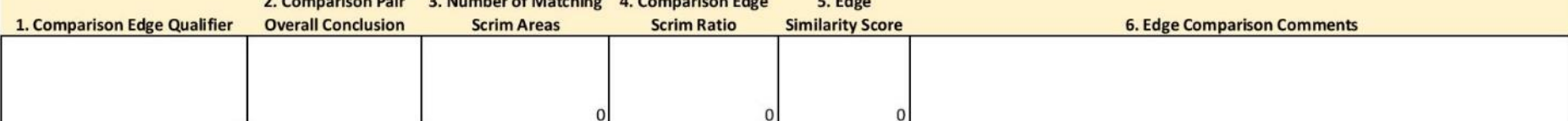




\section{iv. Instructional PowerPoint presentation}

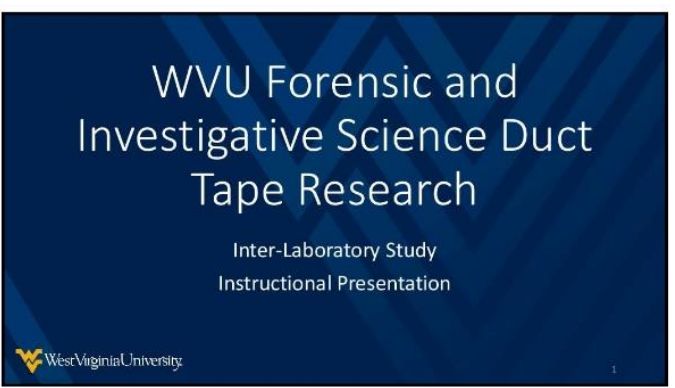

1

\section{Overview}

- Kit contains 7 comparison pairs

- Each tape has only one edge to be examined, meaning 1 comparison per pair

- Kit and documentation estimated to take 1.5 - 2

hours to complete

- Participation is voluntary and anonymous

\section{WWikschignniaUniversily}

\section{Step 1}

- Each comparison pair will be packaged in a folded paper sleeve, all within a labeled envelope. Assure samples are paired correctly before beginning your comparison.

- i.e. Envelope labeled with Sample ID: MQHT1-1 (A,B) should contain samples MOHT1-1-A and MOHT1-1-B

\section{WWeschiginiaUniversity}

\section{Step 2}

- You are asked to perform just one edge comparison per sample pair. Each sample will have a hand-torn edge and a distinctive scissor-cut edge. The comparison should be done only on the hand-torn edges, as shown below.

$$
\text { Tape edees to be compared }
$$

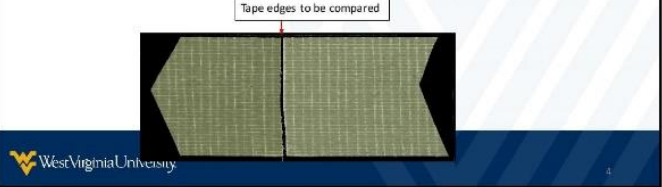

4

\section{Step 3}

- PLEASE conduct the observation of the duct tape edges without removing them from the transparency film or altering the edges in any way.

- Please note that since the tape ends are mounted on clear transparency films, they can be aligned and flipped back and forth without worrying about the edges and adhesive being stuck or altered. The clear films allows for observation of adhesive and scrim features through the microscope or magnification.

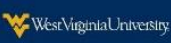

5

\section{Note:}

- You don't need to do physical examination other than the edge comparison (e.g., no need to measure thickness, width, scrim count). For purposes of this study, assume no physical differences among each pair. Study is to be focused only on the fracture edge characteristics.

\section{WWeschiginauninesily}

6 


\section{Step 4}

- Examine the pairs under a stereomicroscope both backing and scrim sides. The following lighting conditions are recommended:

- Backing side: Reflected light. An oblique setting is best due to slight glare.

- Scrim side: Alternate between reflected and transmitted

light to view edge features of both the adhesive and

scrim yarns as well as the backing through the adhesive surface.

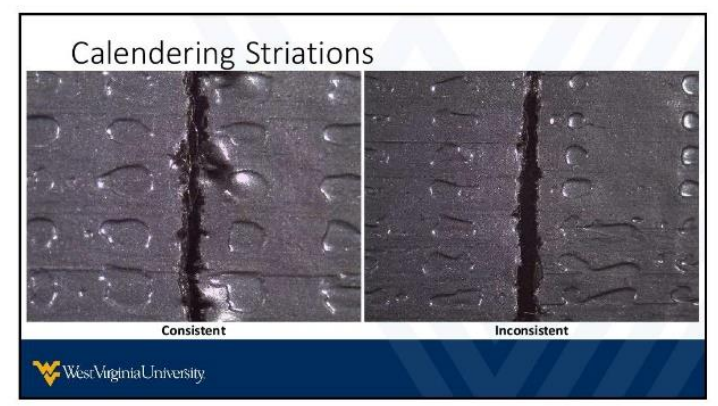

10

\section{Step 5}

- Observe the overall fractured

edge on the backing side.

- Align the top edge first

(indicated by Sharpie-drawn

arrows on the transparency film

to help with the physical fit

assessment.

- Note any physical characteristics

across the fracture that may help in alignment, or the lack of

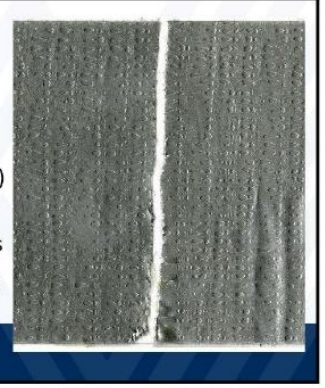

8

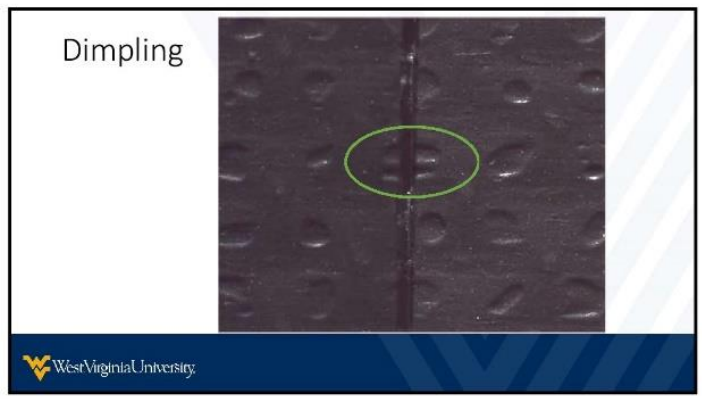

9

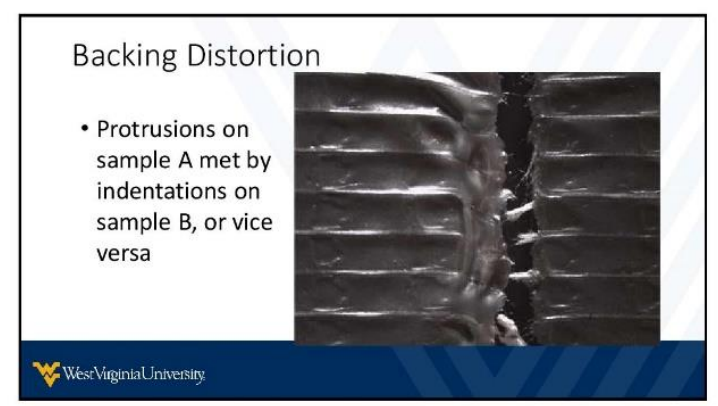

11

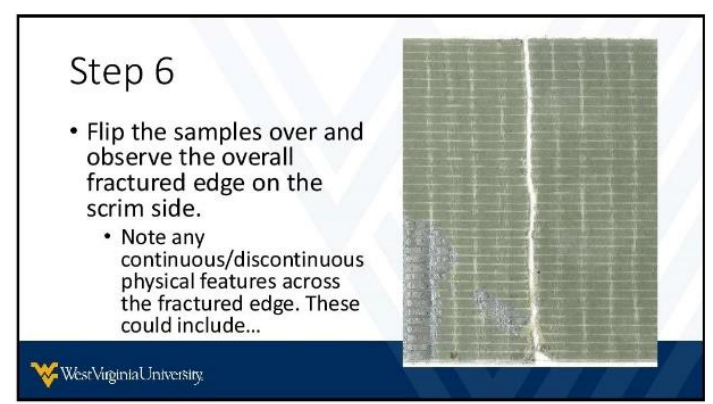

12 


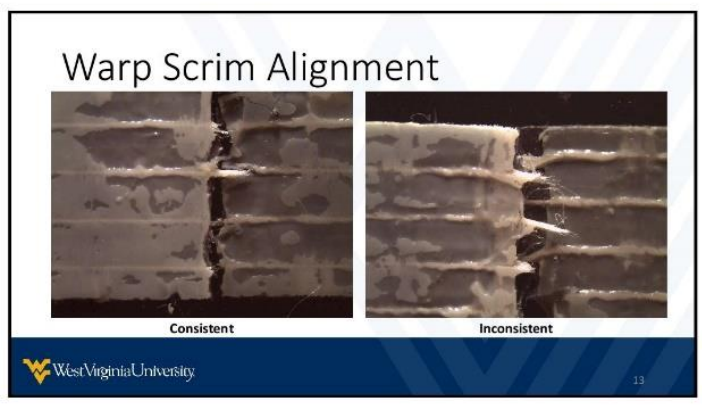

13

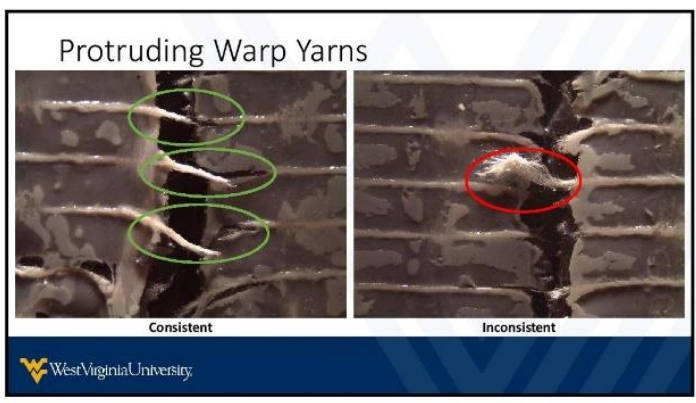

14

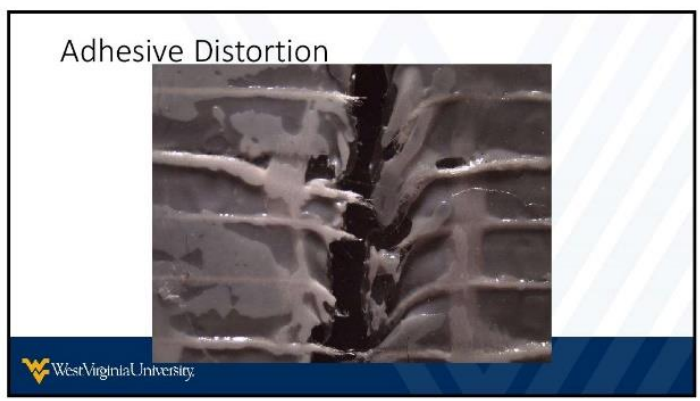

15
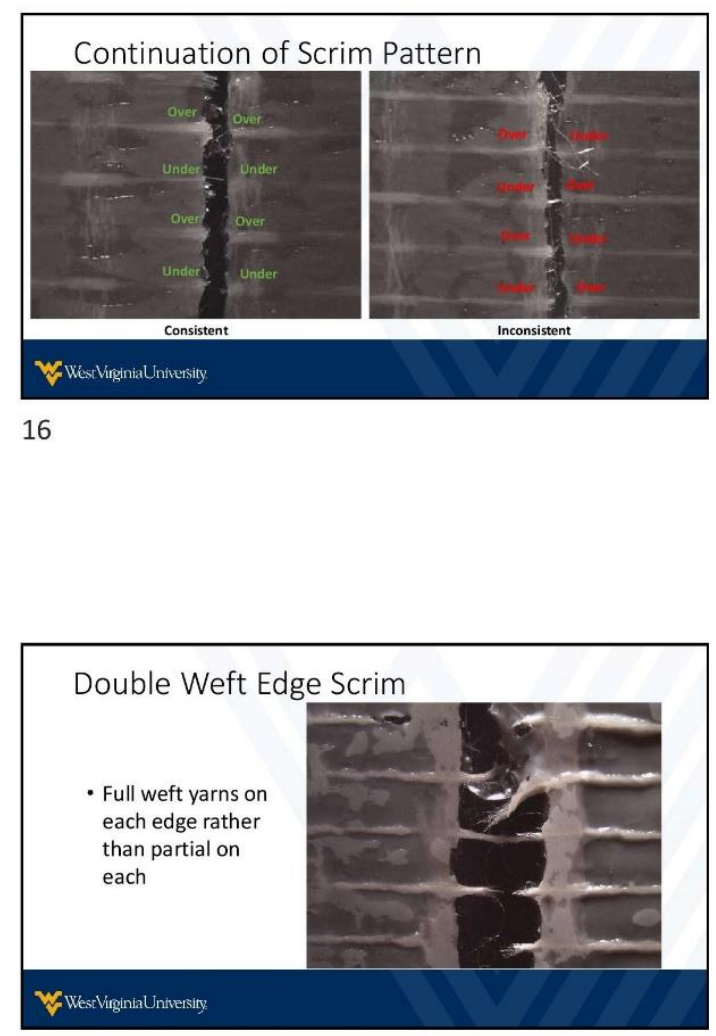

17

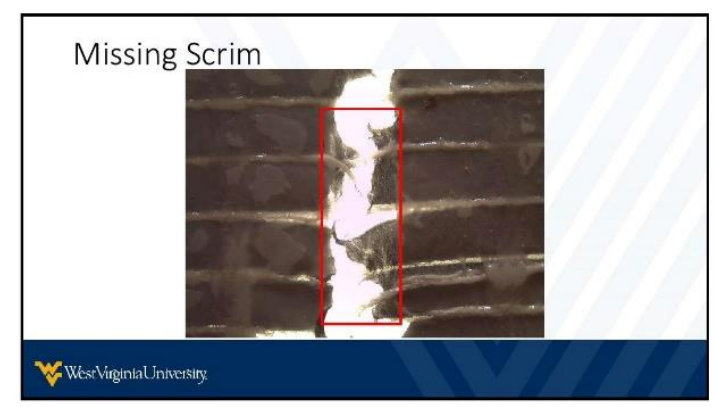

18 


\section{Step 7}

- Begin determining your ESS by counting the total number of scrim areas across the width of the

A scrim area is defined by the small areas formed by the warp yarns along the fractured edge. Include eve those areas at the top and bottom edges of the tape

that are only bordered by one warp yarn.
All the study pairs are expected to have 37 scrim areas. If you count a total number of scrim areas less than or greater than the 37 allotted on the template, include those areas in your score consideration and mark your template accordingly.

WW Westhiginia Univerisy

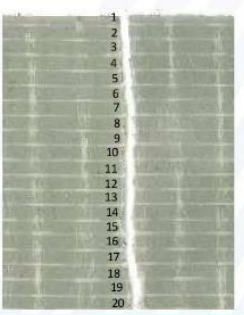

\section{Step 7 (continued)}

Note: When counting scrim areas, your \#1 area when numbering 1-37 should be at the side of the tapes denoted by a Sharpie-drawn arrow, as shown below.

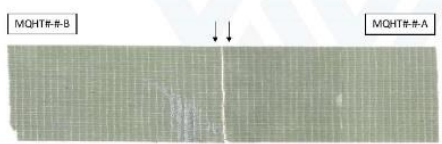

\section{WWeschiginnaUninerily:}

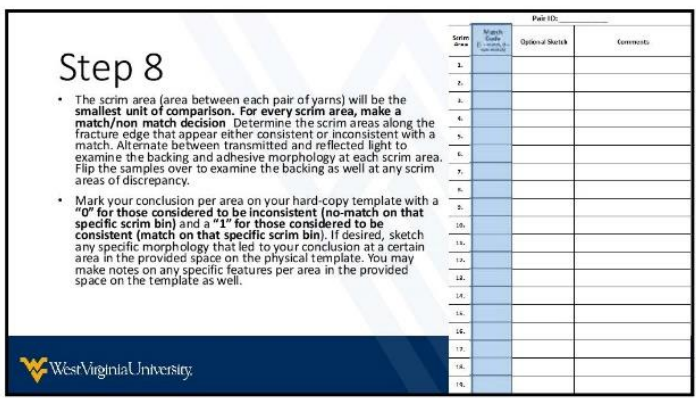

21

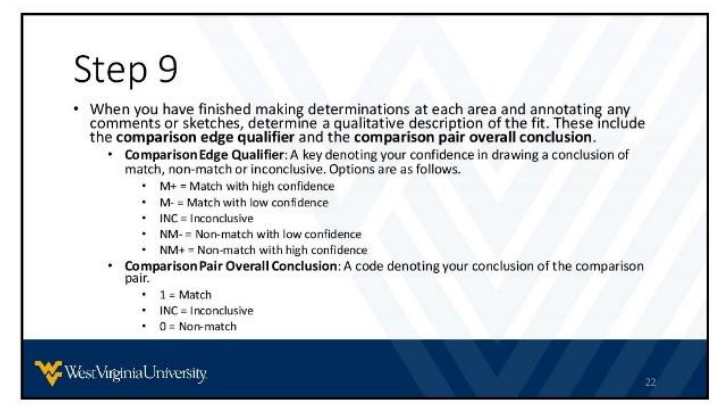

Step 9 (continued)

- On the hard-copy template, space to indicate qualifiers is provided
at the bottom of each pair's table.

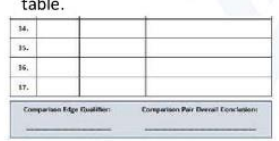

On the digital template, space to indicate
qualifiers is provided in the yellow table in columns 1 and 2 . Click the cell for

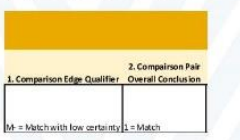

W-WeskigininiaUniverily,

23

\section{Step 10}

- Please transfer match code (1 or 0 assigned per scrim area) from your hard-copy template to your digital

template

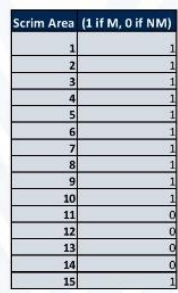

\section{WWesthiginal nnversily:}




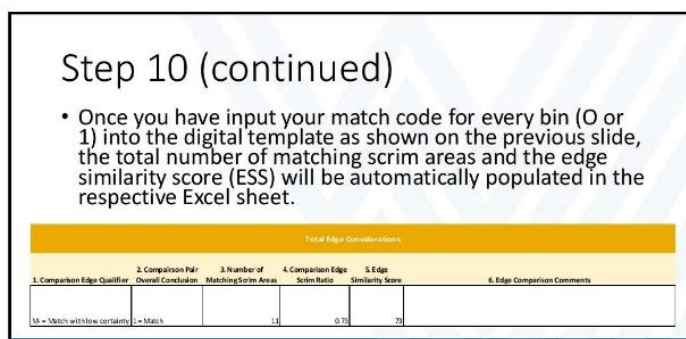

WWWesthiginia Univeriliy

25

\section{Step 10 (continued)}

- Both the digital and hard-copy templates allow space for comments on features observed that led to conclusions, discrepancies in the sample, or general feedback as shown below.

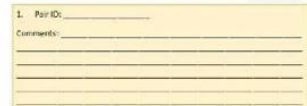

Hard-copyTemplate

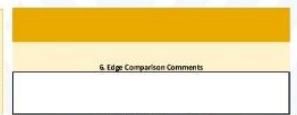

Digital Template

\section{WWeschiginniaUnineriliys}

26

\section{Step 11}

- Each tab in the Excel file will contain score templates for each comparison pair.

- Please complete the brief, post-study examiner survey located on the final tab of the digital answer sheet template.

\section{WWesthighna Unthesily:}

27

\section{Steps $12-13$}

- Re-package all tape samples. Please email the complete digital template to Evie Brooks at

ekb0011@mix.wvu.edu

- Using the pre-paid shipping label and FedEx envelope your study kit originally came in, please send the complete answer sheet templates and all tape samples back to:

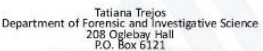

\section{WWesh hignial Universily}

28

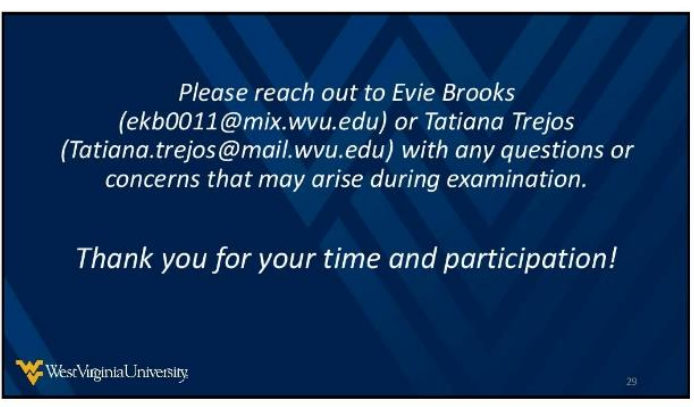

29 


\section{CHAPTER 2: APPENDIX B}

Is your lab accredited?

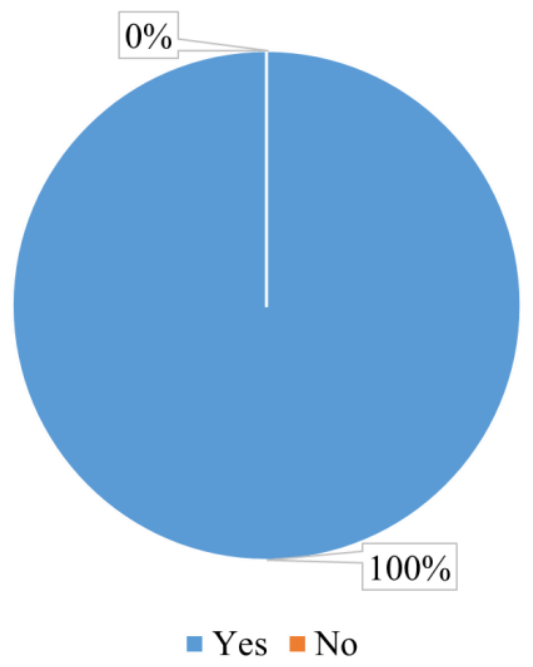

Figure i. Survey question 1 results

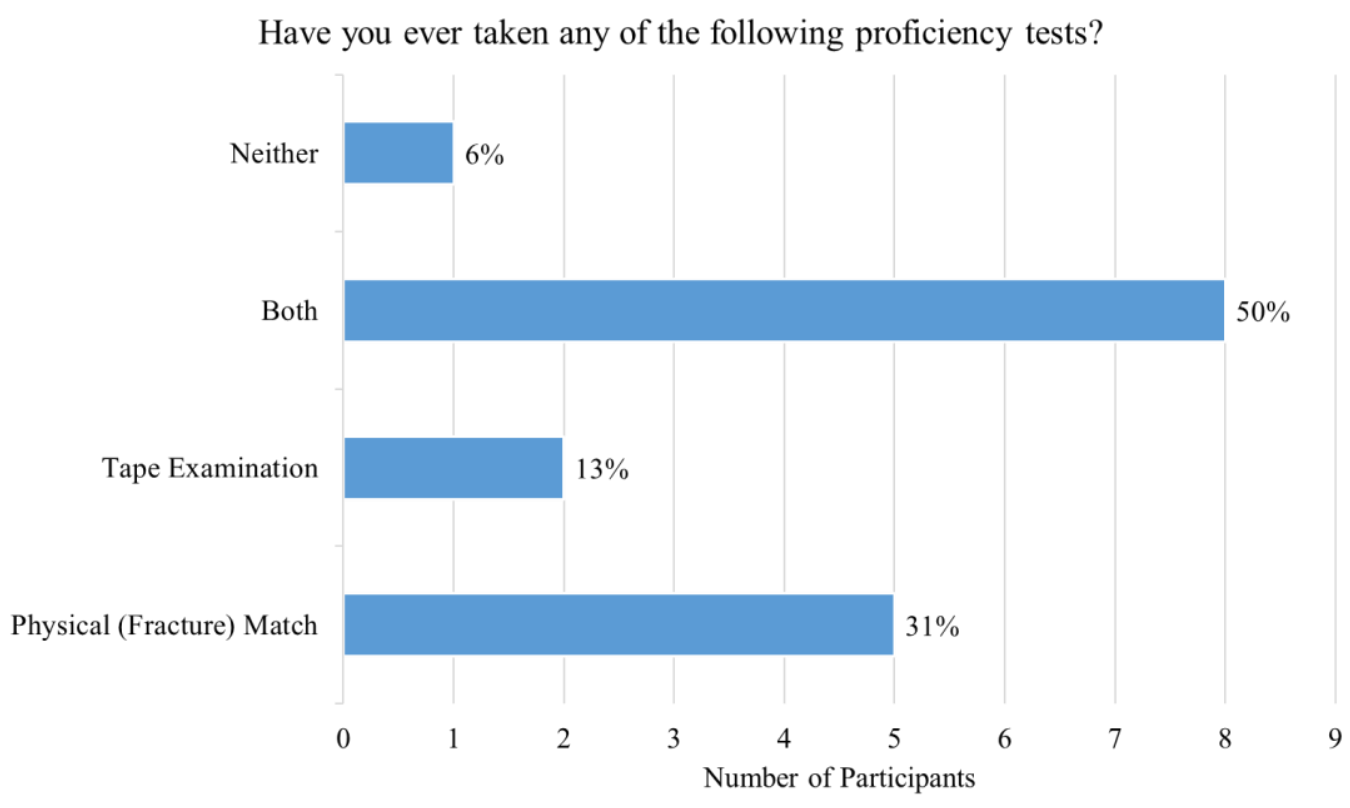

Figure ii. Survey question 2 results 
How much experience do you have with duct tape physical fits?

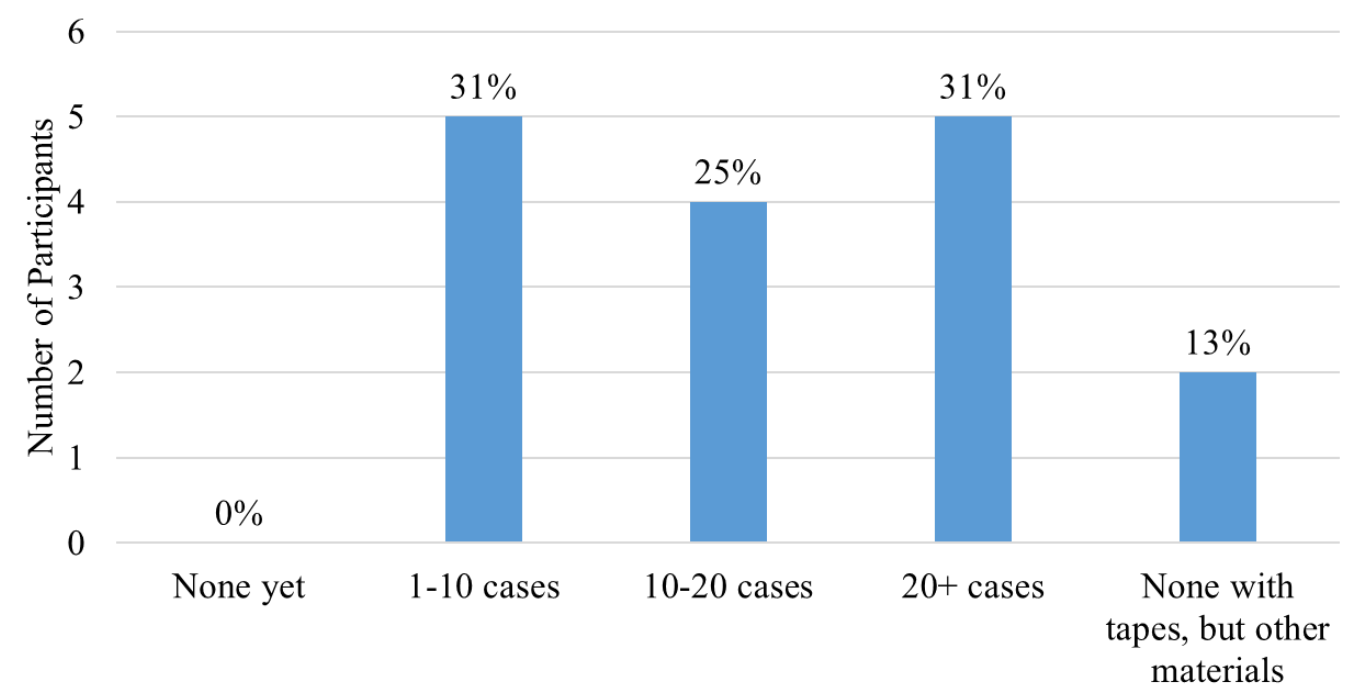

Figure iii. Survey question 3 results

How is a physical fit usually presented in court?

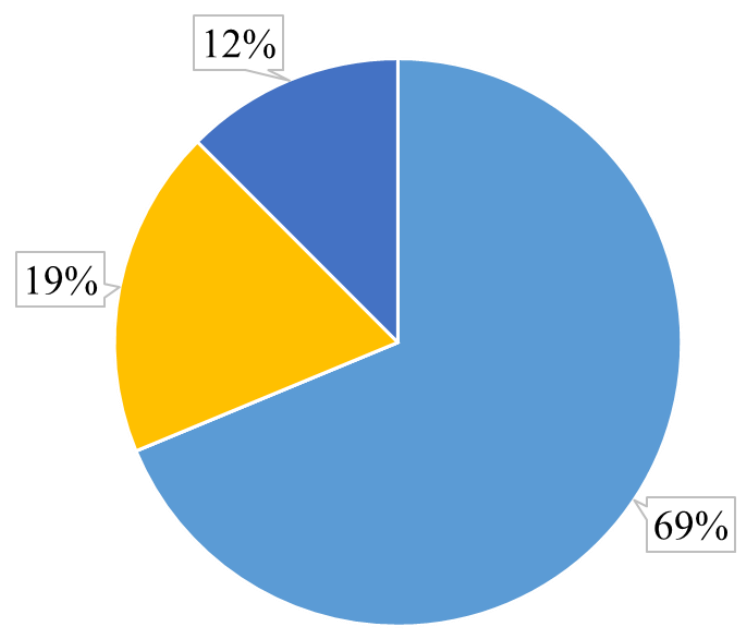
- Photo Documentation
- Sketches
- Models or Casts
$\square$ Physical samples brought in
- Other

Figure iv. Survey question 4 results 
How much time do you typically spend on a physical fit examination?

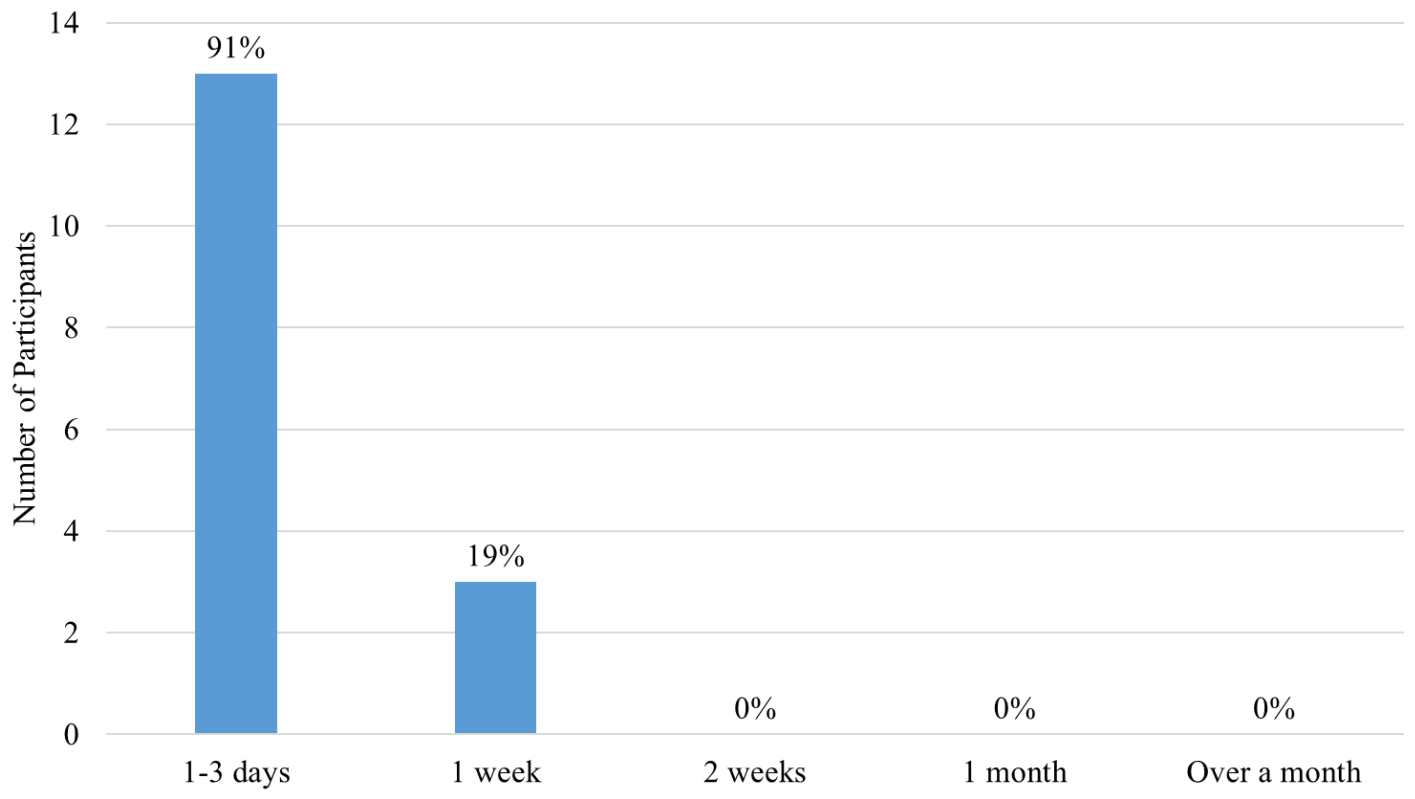

Figure v. Survey question 5 results

How long did it take you to work through the sample set?

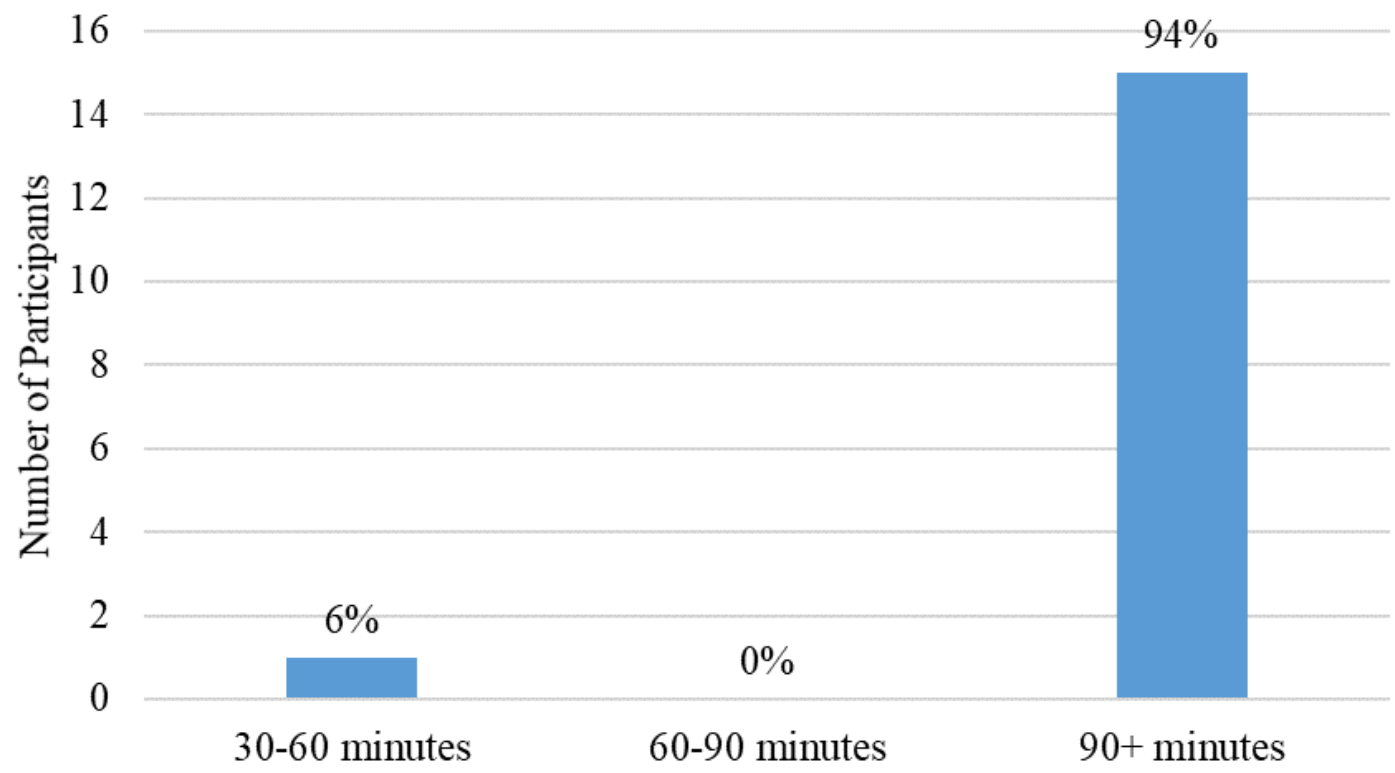

Figure vi. Survey question 6 results 
Did you find the ESS approach easy to follow for duct tape comparisons?

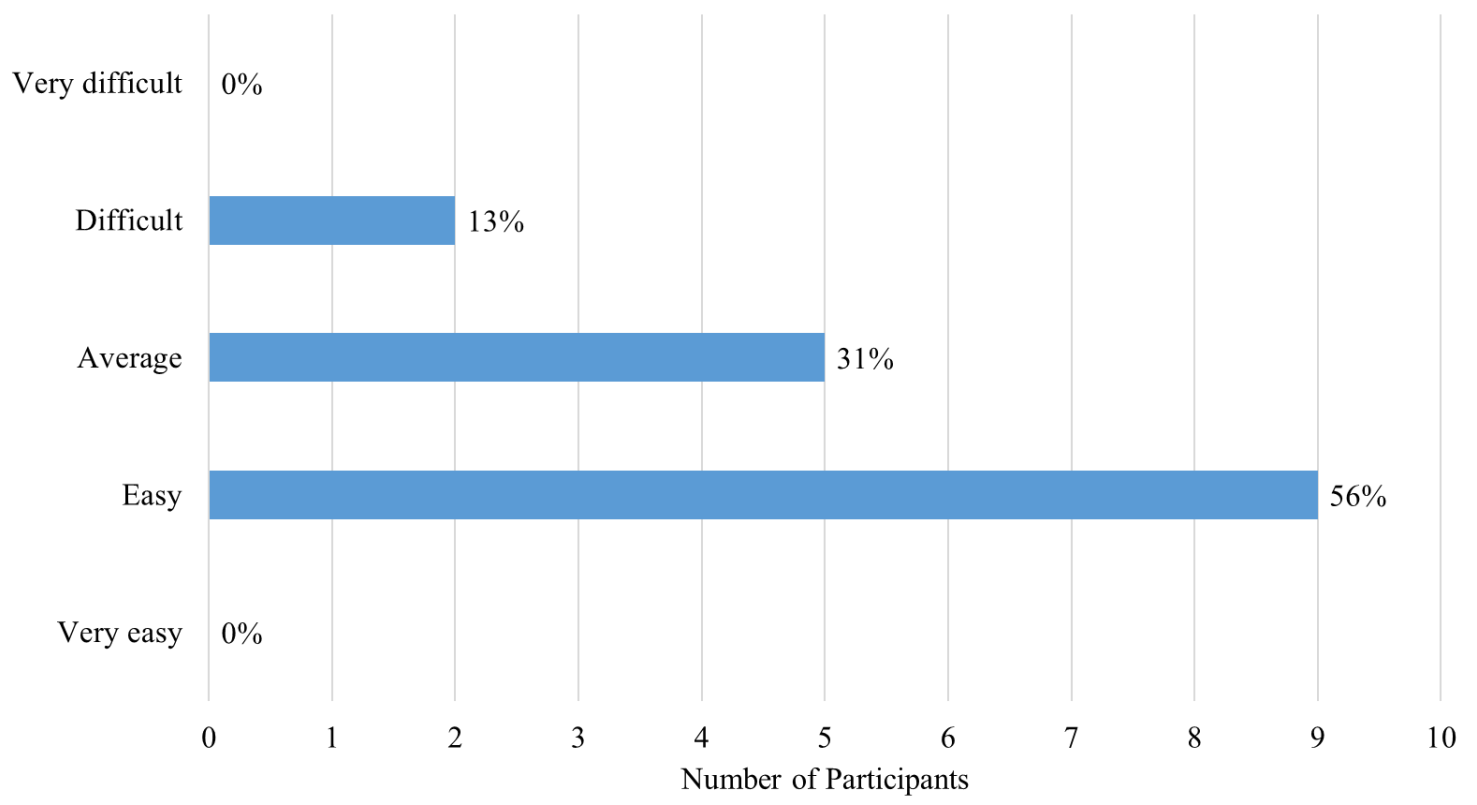

Figure vii. Survey question 7 results

Did you find the ESS metric useful to inform/support your opinion?

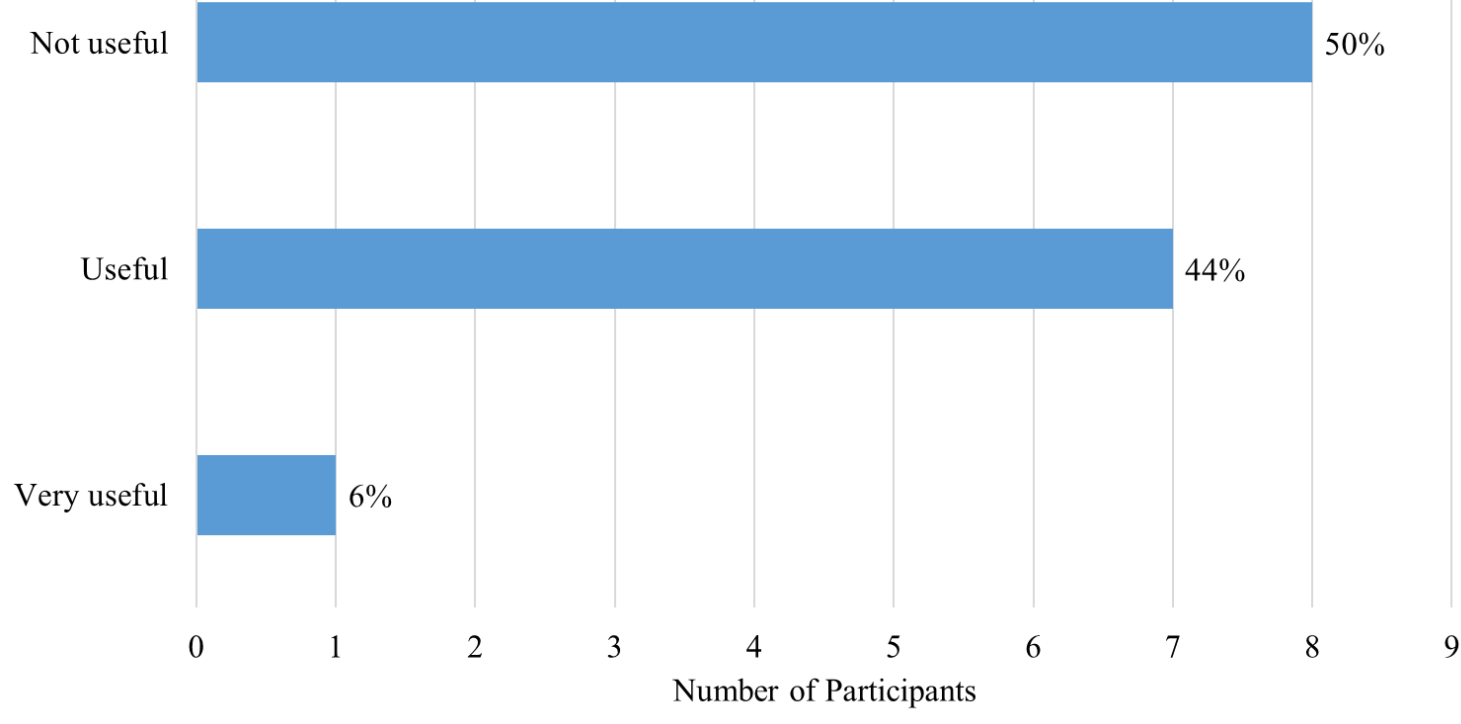

Figure viii. Survey question 8 results 
If you were to implement the ESS approach, would you find the report templates useful for a peer-review process?

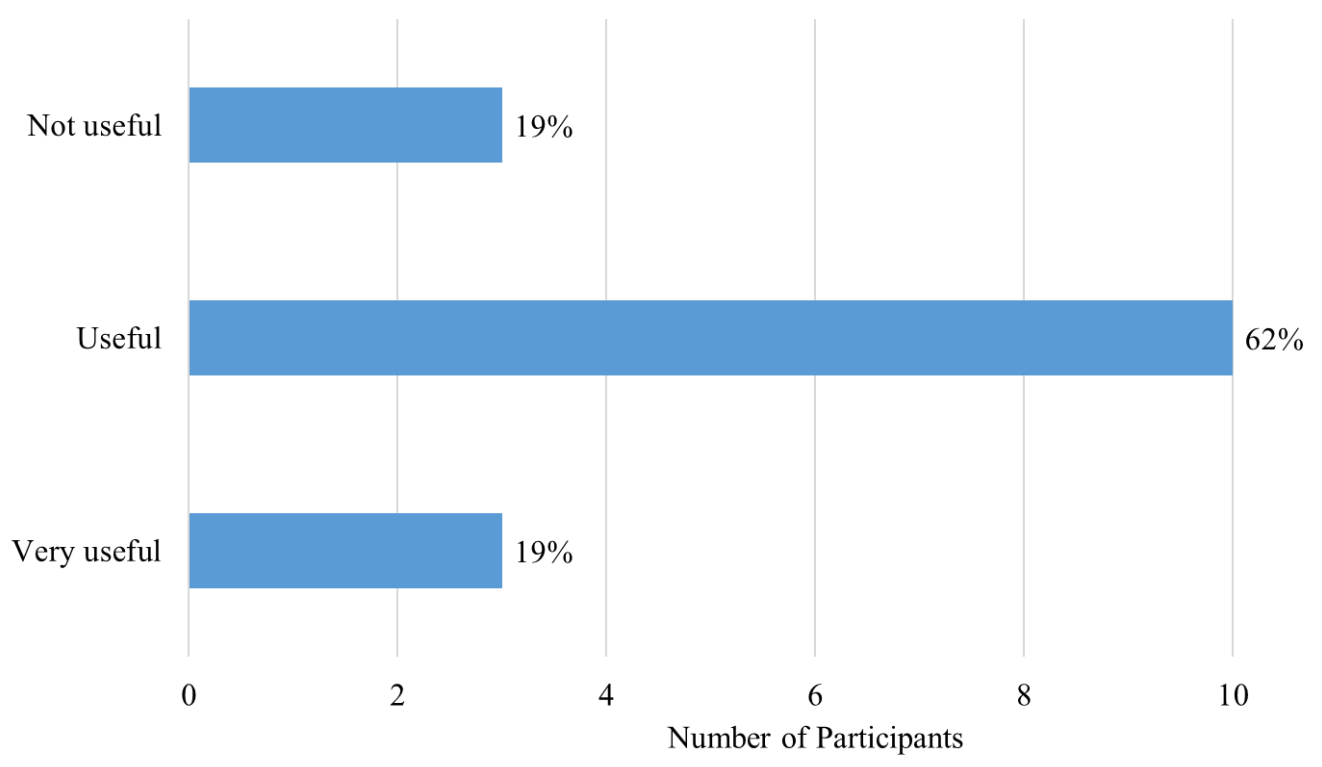

Figure ix. Survey question 9 results 


\section{CHAPTER THREE}

\section{Steps Toward Quantitative Assessment of Textile Physical Fits - Expansion of the Edge Similarity Score (ESS) Method}

\section{Overview of Textile Fracture Study}

Following the development of a systematic, quantitative, score-based edge similarity score (ESS) method of assessment for physical fits in duct tape samples by our research group, this project aims to extend assessment of the method's suitability into other trace material types. Textiles were selected as the initial material expansion due to their prevalence in clothing and household textile items, and their potential to be fractured during the commission of a crime. While the initial experimental design involved the assessment of 100 comparison pairs of hand-torn, $100 \%$ jerseyknit polyester, a high level of disagreement in overall physical fit conclusion was observed between two examiners in just the first 37 pairs of the sample set (74 comparisons, 37 per examiner). Likewise, unacceptable high false negatives (29 out of $46,63 \%$ false negative rate) were observed that required the evaluation of the causes of such error rates. Through this first dataset, it was evident that the assessment of suitability prior to examination of physical fits was imperative in textile samples. In the absence of consensus guides to assess suitability in current practice, the goal of our study was redirected to begin to answer more fundamental questions. Therefore, it was determined a baseline study assessing accuracy of the ESS method when applied to textile items of various compositions, constructions, and separation methods was needed in order to determine those textiles exhibiting sufficient distinctive edge characteristics for physical fit alignment.

A sample set of 100 comparison pairs was then created consisting of five textile items: 1) Item A, a pair of men's navy dress pants composed of $75 \%$ polyester and $25 \%$ cotton in a twill weave construction; 2) Item B, a pair of women's blue jeans composed of $60 \%$ cotton, $22 \%$ rayon, $17 \%$ polyester, and $1 \%$ spandex in a twill weave construction; 3) Item $\mathrm{C}$, a men's blue-striped, short sleeve button-up shirt composed of $100 \%$ cotton in a plain weave construction; 4) Item D, a beige women's tank top composed of $100 \%$ polyester in a satin weave construction; and 5) Item E, a blue and white patterned, short sleeve women's top composed of $93 \%$ rayon and $7 \%$ flax in a jersey knit construction. Twenty comparison pairs were prepared from each textile item, with ten each being separated through hand-tearing and stabbing, respectively. All sample pairs were relabelled and re-organized by external researchers who were not participating in pair assessment to reduce potential bias. Then, two examiners blind to the ground truth of the sample set participated in examination of the fracture edges and estimation of the ESS. The ESS method was adapted for textile examination as each edge was divided into 10 equal bins or units by overall fracture edge length. In addition to " 1 " (match) and " 0 " (non-match) decisions per unit, three weighting factors were potentially attributed to each bin due to the presence of distinctive characteristics described in further detail below. This led to the determination of an initial ESS, weighted ESS, and rarity ratio for each comparison pair. In addition, frequency of occurrence of all noted distinctive characteristics were documented as a preliminary effort to evaluate the rarity of observed features 
across the fracture edges.

Throughout the examination process, examiner notes indicated the following general characteristics that became useful in their edge assessments: color, fabric construction, general fiber size and shape, fiber twist, alignment of long and short threads, and general fluorescence. The following distinctive characteristics were noted as features attributing to the addition of weighting factors: pattern continuation across fracture, stains, fabric damage, protrusions or gaps, and partial pattern fluorescence.

Overall, 93\% accuracy was observed for the hand-torn set while 95\% accuracy was observed for the stabbed set. The hand-torn set resulted in an $8 \%$ false negative rate, $2 \%$ false positive rate, and $4 \%$ inconclusive (true match samples) rate. The stabbed set resulted in an $4 \%$ false negative rate, $0 \%$ false positive rate, $4 \%$ inconclusive (true match samples), and $2 \%$ inconclusive (true nonmatch samples) rate. A higher misclassification rate was observed in the hand-torn set due to the higher degree of distortion presented by the fraying and stretching contributed by the tearing process. In addition, most misclassifications occurred within samples associated to Items D and E, the women's tan tank top composed of $100 \%$ polyester and the navy patterned women's jerseyknit top. Both items attributed higher levels of stretch than the other garments. These results indicate that textile items with fabric types of higher elasticity, due to either fabric construction or fiber composition, may present limited fracture fit analysis capabilities and examiners should be aware of potential sources of uncertainty on their conclusions.

\section{Introduction}

Due to the prevalence of clothing items and household textiles in everyday use, textile items are materials commonly present at the scene of a crime. Depending upon the interaction of the textile item with individuals present during the commission of a crime, textile analysis can become a critical link between individuals, objects, and locations. In situations involving assault or homicide, both victim and suspect garments can become damaged and separated through tearing or shearing. Garments can also become damaged or fractured as the result of a hit-and-run, fire exposure, or long period of submersion in water. When violence occurs in the home, common household textiles such as bedsheets, curtains, or towels can become fractured as well. These situations lead to forensic textile examinations for the determination of textile damage source (i.e. stabbing, cutting, or tearing) as well as alignment of textile remains in the analysis of a potential fracture fit. Foreign fibers discovered at the scene or on collected textile materials can also be compared to known fibers collected from suspect garments to attribute a common source or to differentiate. ${ }^{1}$

Within the physical fit literature, case reports highlight the variety of situations in which a textile physical fit provided a useful link in an investigation. For example, Fisher et al. described multiple textile physical fit analyses: a case in which T-shirt fragments from the victim's hands were later compared to the suspect's recovered torn shirt; a situation in which a hit-and-run victim's torn coat was compared to a piece of fabric collected from the front fender of the suspect's car; and an 
additional scenario that involved a torn fabric fragment discovered at the point of entry of a burglary scene that was later compared to the suspect's torn clothing ${ }^{2}$. In addition to these, Shor $e t$ $a l$. shared a case in which a physical fit examination was responsible for the confirmation of stolen artwork. Examiners were able to physically fit questioned cut canvas edges to the known fragments remaining in the original frames due to the edge morphology features presented by the manipulated canvas $^{3}$.

When damaged textiles are received in a forensic laboratory, examination typically begins with visual examinations of the fracture at both the macro and microscopic levels to determine if a potential physical fit exists. Often, if the edges align and the textiles appear consistent in physical features such as color, construction, and weave/knit pattern, this will be considered the highest level of association and further analysis will not often occur ${ }^{4}$. Some laboratories will still carry out a full analytical scheme, documenting the physical properties of both the questioned and known textile samples as well as the optical properties and chemical composition properties through instrumental determination of polymer and dye type.

In addition to physical and chemical analysis, some laboratories will perform damage source determinations on the fractured textiles. This usually involves viewing fractured edge crosssectional morphology of textile fibers through either stereomicroscopy or scanning electron microscopy (SEM). Fiber cross-sectional shape after a fracture event has been shown to exhibit specific shapes, such as a "pinched" appearance following a shearing or a "mushroom cap" appearance following a tear. Source of damage analysis may also be accompanied by laboratorybased simulations or recreations of the suspected fracture event to compare fractured fiber morphology. ${ }^{5}$

Textile damage source determination is a well-researched niche within the trace evidence discipline. For example, Kemp et al. ${ }^{6}$ provided a damage determination study in apparel fabrics. The authors subjected two fabric types (cotton bull drill, more commonly known as denim, and cotton single jersey) at three levels of varying wear to stabbing events using three different weapons - a kitchen knife, hunting knife, and screwdriver. Stabbing events were delivered through two avenues: a human participant trial and an impact rig with each respective weapon. Fractured fabric ends were then examined through stereomicroscopy, digital photography, and Scanning Electron Microscopy (SEM) to determine if fabric morphology showed specific characteristics revealing weapon type. It was found that weapon type could be determined from differences in severance size and shape, degree of fabric distortion, position of severed yarn ends, loop snippets, curled yarns, and the morphology of the fractured fibers. Directionality of the stab could only be found if the upper and lower blade edges of the respective weapon had varying geometries, edge types, or degrees of sharpness and no tearing occurred during the fracture ${ }^{6}$. A similar SEM sourcedetermination study was presented for fibers by Pelton ${ }^{7}$. In this study, nylon fabric samples were cut in the weft direction with scissors, a carving knife, and an Elmendorf tear machine. Fibers were sampled from three different sites along the resulting fracture edge and analyzed through SEM for source determination. Of the 600 analyzed fiber ends, 322 were categorized based on their shearing $\operatorname{method}^{7}$. 
As highlighted in Chapter 1, forensic laboratories often have a single, general standard operating procedure for physical match as a whole rather than material-specific protocol ${ }^{4,8}$. These procedures usually recommend visual and stereomicroscopic viewing of the suspected physical fit pair. Consistent class and individual characteristics will be noted along with any specific similarities such as striations across the fracture edge or dissimilarities noted. Detailed documentation of similar characteristics and a digital photograph of the sample pair is typically recommended as well. However, Chapter 1 reviews two material-specific physical fit protocols in which direct recommendations for textile fracture analysis is provided. One described how to "side" and orient the fabric samples by their lengthwise (warp) and crosswise (weft) fibers. Both described macroscopic characteristics that could quickly eliminate a non-match. These included yarn thickness, printed design, or stains across the fractured edge. Microscopic characteristics are then mentioned for use of fracture edge alignment including color and construction of individual yarns and continuation of the weave/knit pattern.

The aim of this project was to expand the previously developed, systematic, quantitative technique of physical fit assessment, known as the edge similarity score (ESS) ${ }^{9}$, to other fractured material types - specifically textiles. The original experimental design of the project intended to minimize factors for assessment of the ESS method, followed by future expansion to additional fabric compositions and constructions. A preliminary set was created consisting of 100 hand-torn comparison pairs of $100 \%$ jersey-knit polyester. Two student examiners began the comparison set, blind to the ground truth of the comparison pairs. Due to fabric composition and construction, the samples experienced a high level of stretch and distortion.

The results highlighted the relevance of assessing suitability of the material for physical fits as the initial step of a physical fit examination. This is supported by the high disagreement levels exhibited in the preliminary set in only the first 37 samples, as well as the high false negative rate as further discussed with Section 4.1. However, to further demonstrate the varying accuracy in physical fit comparisons between fabric compositions and constructions, it was determined a proof of concept study was needed to assess which fabric types present sufficient features for accurate fracture fit examinations.

Therefore, the study was re-designed as an assessment of physical fit by both fabric type (composition and construction) and separation method. This was done to assess which fabric types present sufficient characteristics to be suitable for physical fit assessment in terms of relative error rates by examiners utilizing the ESS method. In this way, examiners were analyzing the comparison pairs in each of the same units or bins along the fractured edge, developing overall conclusions on the association or discrimination of a given sample pair as well as an ESS value and comparison edge qualifier supporting the examiner's confidence in the match. By observing the resulting ESS distributions per fabric type as well as separation method, the efficacy of the ESS method in revealing examiner consensus is shown. Further, error rates are established providing insight into the fabric types and separation methods exhibiting more difficult physical fit assessments to examiners and features are identified which may assist in comparison between textile samples of certain composition. 


\section{Materials and Methods}

\subsection{Preliminary dataset of jersey-knit fabric}

A set of 100 comparison pairs of hand-torn textile samples was created from tan, jersey-knit, 100\% polyester fabric. One hundred rectangles approximately $26 \mathrm{~cm}$ in length (in the fabric's wale direction) and $18 \mathrm{~cm}$ in width (in the fabric's course direction) were cut from bulk, bolt fabric. All samples were separated in the fabric's course direction by first performing a $3 \mathrm{~cm}$ scissor notch and then hand-tearing the remainder of the width of the fabric. All sample pairs were labeled according to their associated pairs by the research performing the separation. Pairs were later reorganized and re-labeled by a secondary researcher in order to keep the initial research blind to the ground truth of the established sample set. Due to sample edge curling, all samples were ironed prior to analysis. Each of two examiners completed analysis of $\mathrm{N}=37$ of the pairs in the sample set, resulting in a total of $\mathrm{N}=74$ total comparisons. Examiners utilized the ESS method, evaluating individual bins along the fractured edges by 10 equal divisions of the total fracture length.

\subsection{Suitability and performance assessment textile dataset}

A set of 100 comparisons of stabbed and hand-torn textile pairs was completed by each of two student examiners (Examiner A and B) for $\mathrm{N}=200$ total comparisons. The set was composed of five clothing items for purposes of assessment of multiple fabric compositions and constructions as summarized in Table 1 below.

Table 1. Textile item composition and construction summary

\begin{tabular}{|c|c|c|c|}
\hline Item & Description & Composition & Construction \\
\hline $\mathrm{A}$ & $\begin{array}{c}\text { Men's navy dress } \\
\text { pants }\end{array}$ & $\begin{array}{c}75 \% \text { polyester, 25\% } \\
\text { cotton }\end{array}$ & Twill weave \\
\hline $\mathrm{B}$ & $\begin{array}{c}\text { Women's blue } \\
\text { jeans }\end{array}$ & $\begin{array}{c}60 \% \text { cotton, 22\% } \\
\text { rayon, 17\% polyester, } \\
1 \% \text { spandex }\end{array}$ & Twill weave \\
\hline $\mathrm{C}$ & $\begin{array}{c}\text { Men's blue- } \\
\text { striped, short } \\
\text { sleeve button-up } \\
\text { shirt }\end{array}$ & $\begin{array}{c}100 \% \text { cotton } \\
\text { D }\end{array}$ & $\begin{array}{c}\text { Women's beige } \\
\text { tank top }\end{array}$ \\
\hline $\mathrm{E}$ & $\begin{array}{c}\text { Women's blue and } \\
\text { white patterned, } \\
\text { short sleeve top }\end{array}$ & $93 \%$ rayon, 7\% flax & Satin weave \\
\hline
\end{tabular}


In an attempt to simulate fracturing scenarios in the course of a criminal event, each garment was placed onto a foam human form cut from two layers of 3" solid charcoal firm foam (Foam Factory Inc.(C). An image of the foam form is provided in Figure 1, while Table 2 provides all measurements of the form pre-fracture.

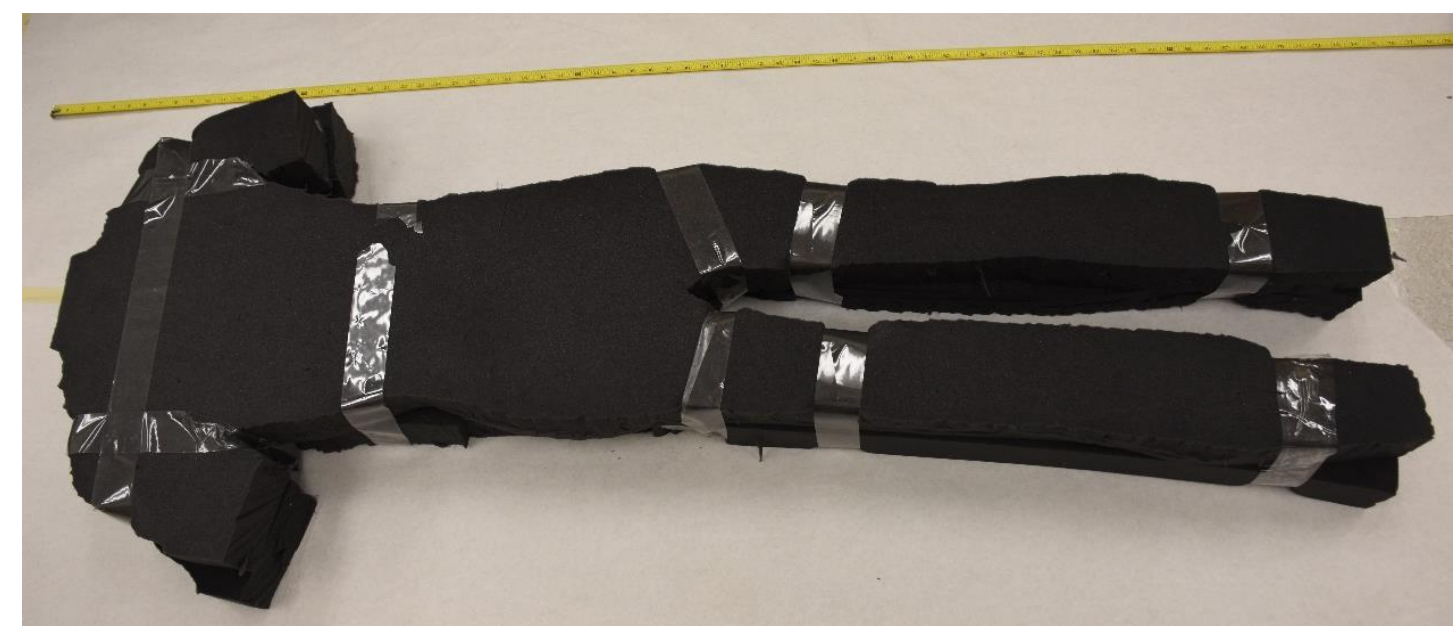

Figure 1. Foam human form fracturing substrate

Table 2. Measurements of the foam human form fracturing substrate

\begin{tabular}{|c|c|c|}
\hline \multicolumn{2}{|r|}{ Region } & Measurement (inches) \\
\hline \multirow{3}{*}{ Right arm } & Length (shoulder to wrist) & 26.0 \\
\hline & Width & 5.00 \\
\hline & Thickness & 5.75 \\
\hline \multirow{3}{*}{ Left arm } & Length (shoulder to wrist) & 25.8 \\
\hline & Width & 5.25 \\
\hline & Thickness & 6.00 \\
\hline \multirow{5}{*}{ Torso } & Length (neck to hips) & 25.5 \\
\hline & Width (between shoulders) & 22.5 \\
\hline & Width (waist) & 11.0 \\
\hline & Width (between armpits) & 12.5 \\
\hline & Thickness & 6.00 \\
\hline \multirow{3}{*}{ Right leg } & Length (hips to ankle) & 35.0 \\
\hline & Width & 4.50 \\
\hline & Thickness & 5.75 \\
\hline \multirow{3}{*}{ Left leg } & Length (hips to ankle) & 34.8 \\
\hline & Width & 4.50 \\
\hline & Thickness & 5.75 \\
\hline \multicolumn{2}{|c|}{ Overall height (neck to ankle) } & 61.5 \\
\hline \multicolumn{3}{|c|}{ Measurements following shortening of arms for Item D* } \\
\hline \multicolumn{2}{|r|}{ Region } & Measurement (inches) \\
\hline Right arm & Length (shoulder to wrist) & 9.50 \\
\hline Left arm & Length (shoulder to wrist) & 8.88 \\
\hline
\end{tabular}

*In order to facilitate the placement of Item D on the foam human form, the arms had to be cut to shorten the distance the sleeves of the tank top had to be stretched. Item D was the last garment fractured due to this implication. 
The front of each garment was stabbed ten times with a Cuisinart ${ }^{\circledR}$ Classic 8" chef's knife at five each of horizontal and vertical orientations. A plastic guard was adhered to the blade at 2.5 " from the tip to maintain consistent stab depth. Between stabbings, the plastic guard was repositioned to its original distance if any movement had occurred. Measurements were taken of the plastic guard position both pre- and post-stabbing to assess movement. Mean distance travelled by the guard during all stabbing events was $1.39 \pm 0.38$ inches.

A single researcher performed each stabbing with their right arm oriented at a $90^{\circ}$ angle, with distance from knife tip to "chest" surface measured with each replicate to maintain consistency. Distance of knife tip to garment surface was measured prior to each stabbing event. Mean distance through the stabbing process was $19.25 \pm 1.56$ ". Each item was then hand-torn ten times on different locations, at five each of horizontal and vertical orientations by a secondary researcher. A pair of scissors was used to create a 0.75 " notch in the tear location and the researcher proceeded by pulling each edge of the notch apart to create the hand tear.

All fractures were cut from the garments, reorganized, and labelled by student volunteers so examiners would remain blind to the ground truth of the fractured sample pairs. An inventory of the original identification numbers was then created to maintain the traceability of the samples, and a random number generator was used to relabel the items with a unique identifier and to mix the fracture edges to generate a relatively balanced number of true mated and true non-mated samples. Two examiners then completed the physical examination of the sample set of 100 comparison pairs, 20 pairs per garment with 10 each of stabbed and hand-torn fractures. A schematic of the experimental design can be observed in Figure 2 below. 


\section{Hand-torn/Stabbed Set $(\mathrm{N}=100,2$ examiners, $\mathrm{n}=200$ comparisons)}

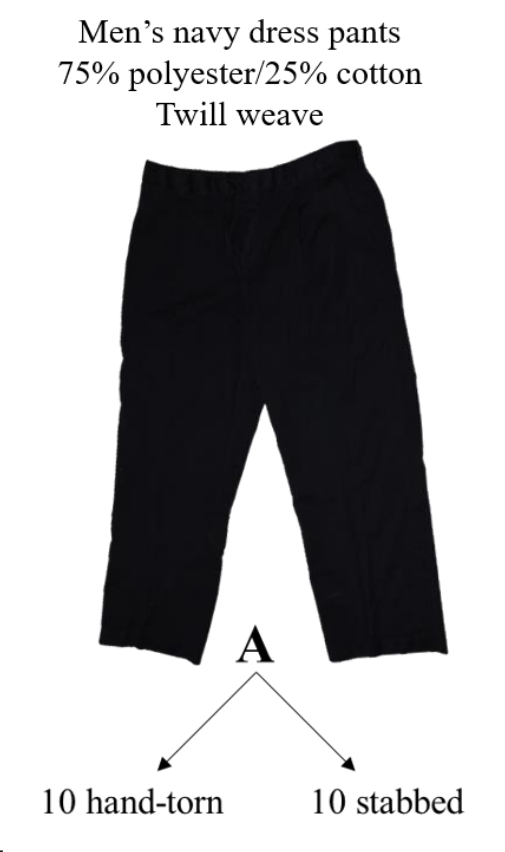

Women's blue jeans $60 \%$ cotton $/ 22 \%$ rayon $/ 17 \%$ polyester/ $1 \%$ spandex Twill weave

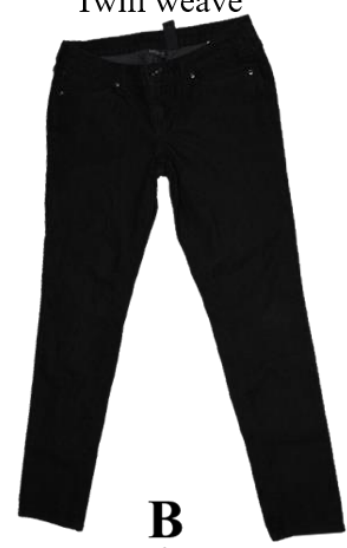

10 hand-torn
Men's blue-striped, short sleeve button-up shirt $100 \%$ cotton Plain weave

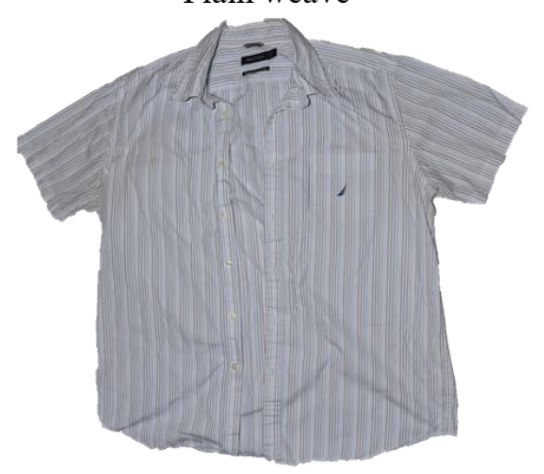
C

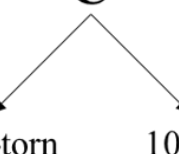

Women's beige tank top $100 \%$ polyester Satin weave

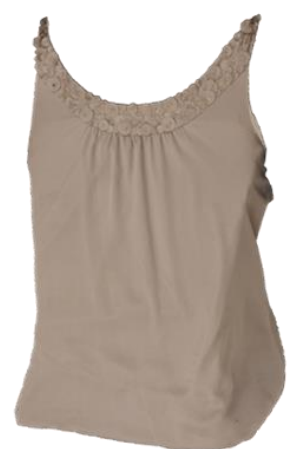

D

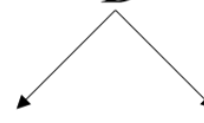

Women's blue and white patterned, short sleeve top $93 \%$ rayon $/ 7 \%$ flax Jersey knit

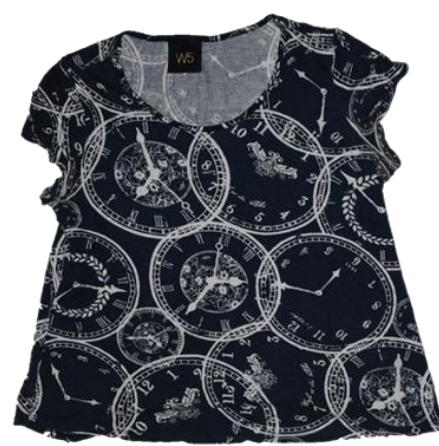

$\mathbf{E}$

Independently examined by $\mathrm{N}=2$ examiners using a blind process

Figure 2. Textile sample set experimental design schematic 
The sample set was analyzed by two student examiners. Samples were compared under a Leica@ EZ4 stereomicroscope using reflected lighting. Along with overall fracture edge morphology, examiners were also instructed to consider any observed alignment features of two types: general characteristics common to both samples as well as distinctive characteristics consistent across both fractured edges in the sample pair. Observed alignment features are provided in Table 3 below. Figures 3-12 below provide examples of each noted feature.

Table 3. Observed alignment feature summary

\begin{tabular}{|c|c|}
\hline General Characteristics & Distinctive Characteristics \\
\hline Color & Pattern continuation \\
\hline Fabric construction & Separation characteristics* \\
\hline General fiber size/shape & Partial pattern fluorescence \\
\hline Fiber twist & \\
\hline Alignment of long/short threads & \\
\hline General fluorescence & \\
\hline
\end{tabular}

*Separation characteristics include any protrusions/gaps consistent across fractured edge along with any consistent damage (i.e. "gather" across fabric)

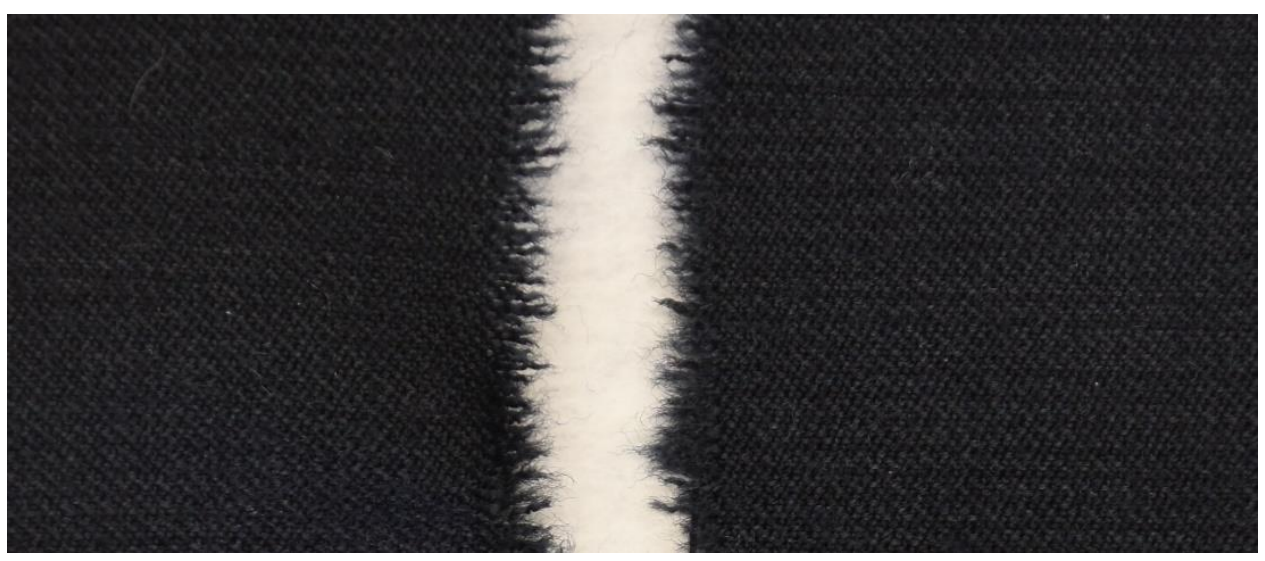

Figure 3. General characteristic example - color 


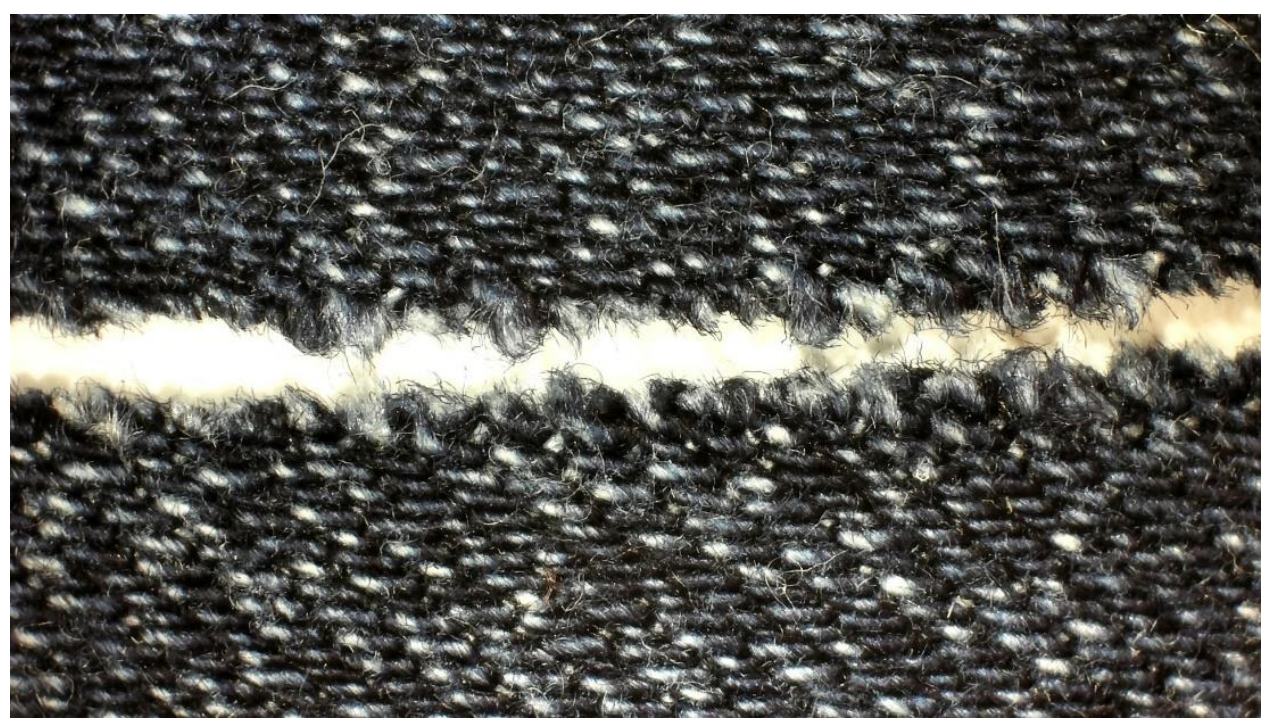

Figure 4. General characteristic example - fabric construction (twill weave)

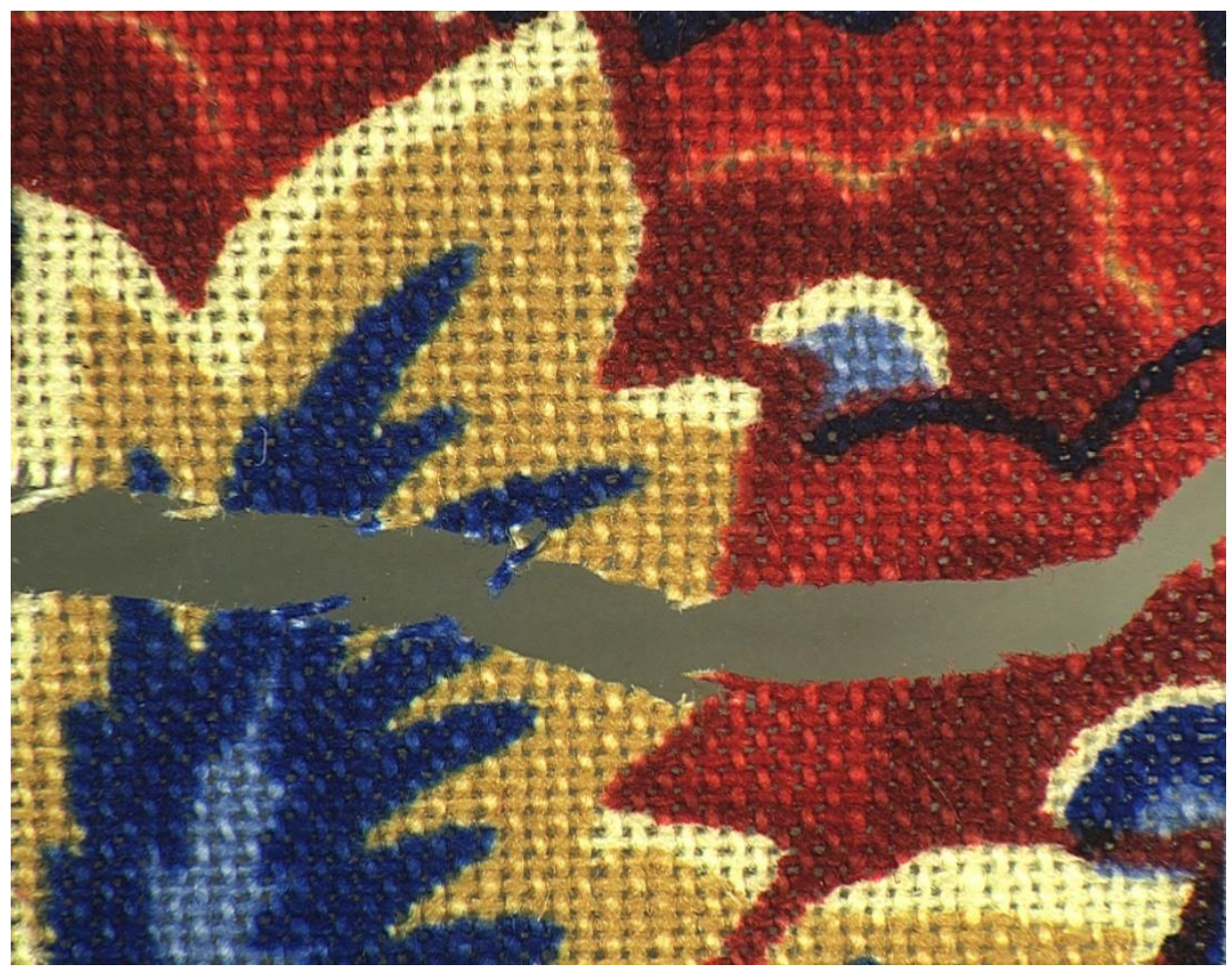

Figure 5. General characteristic example - general fiber size/shape 


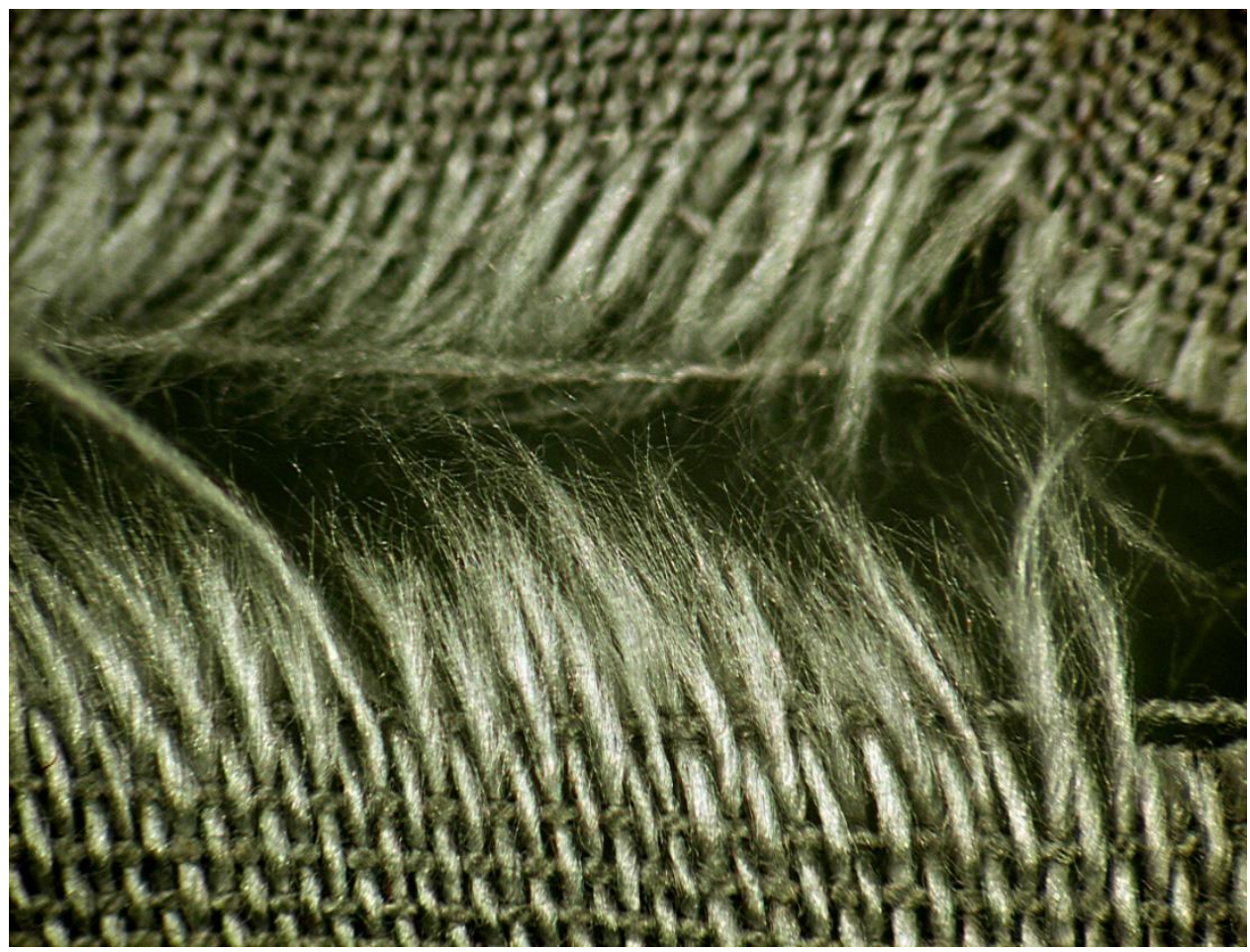

Figure 6. General characteristic example - fiber twist (" $Z$ ” twist)

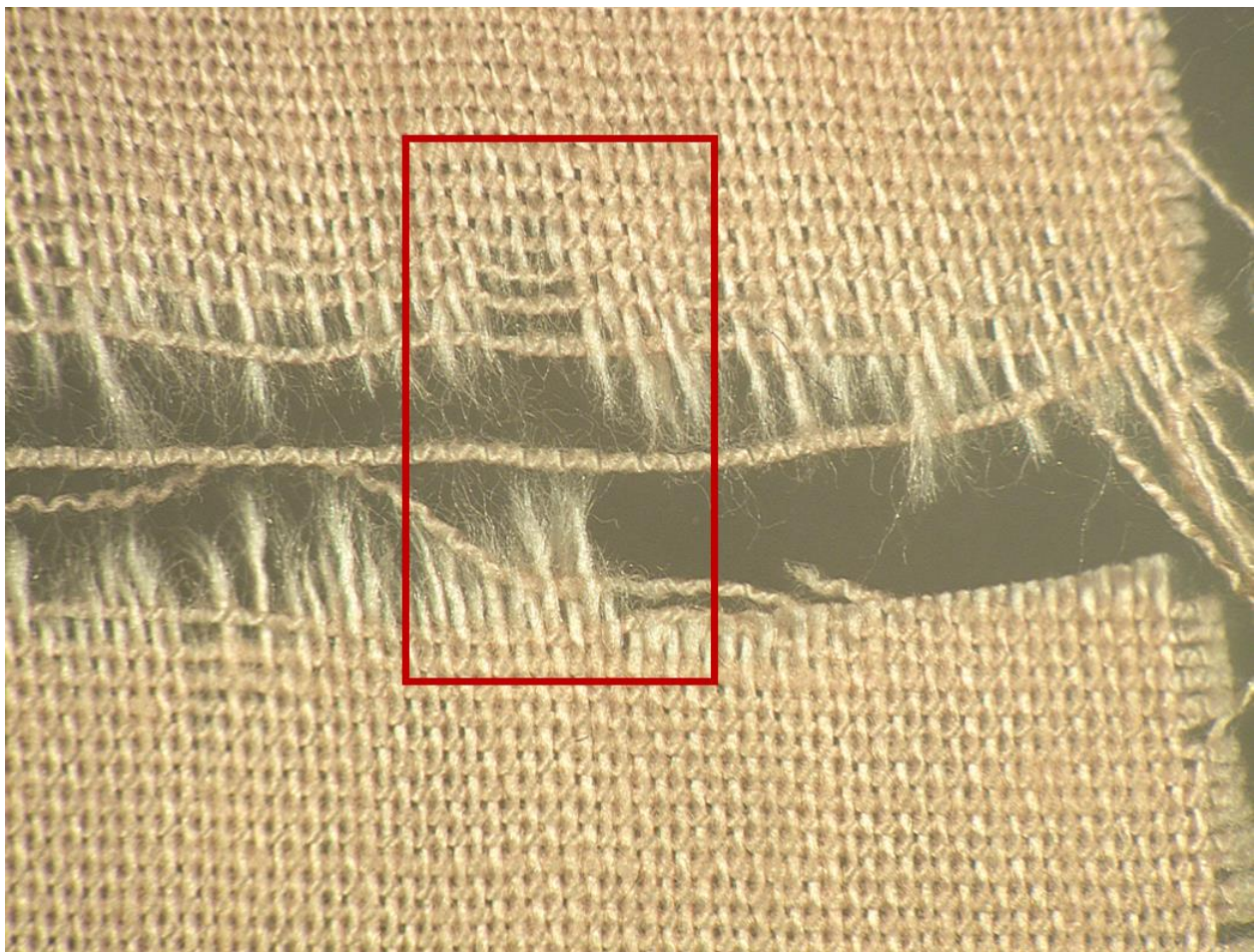

Figure 7. General characteristic example - alignment of long short threads. Note: Region highlighted indicates an area considered a distinctive characteristic (i.e. gap/protrusion) 


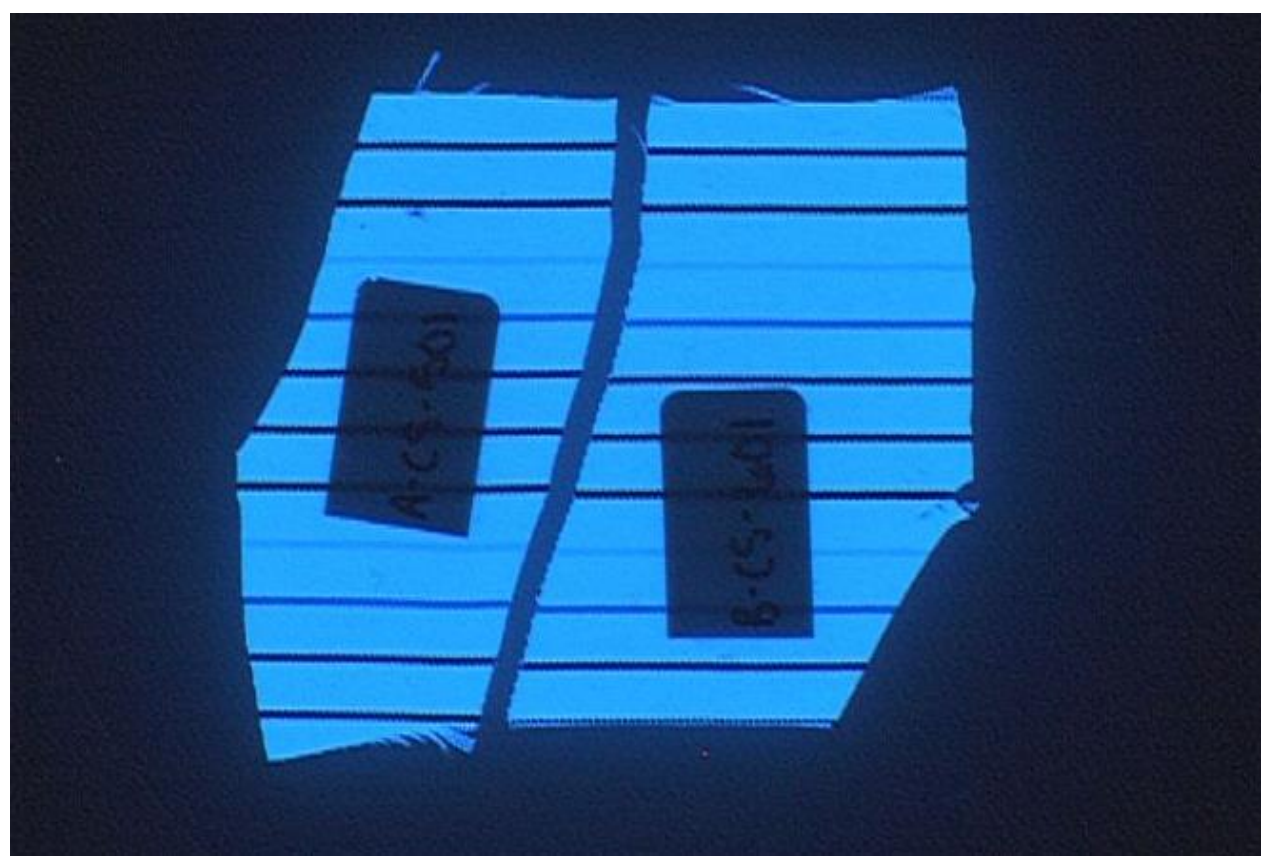

Figure 8. General characteristic example - general fluorescence (Note: The dark square regions on the right and left image are sample labels, not a region within the fabric's pattern.)

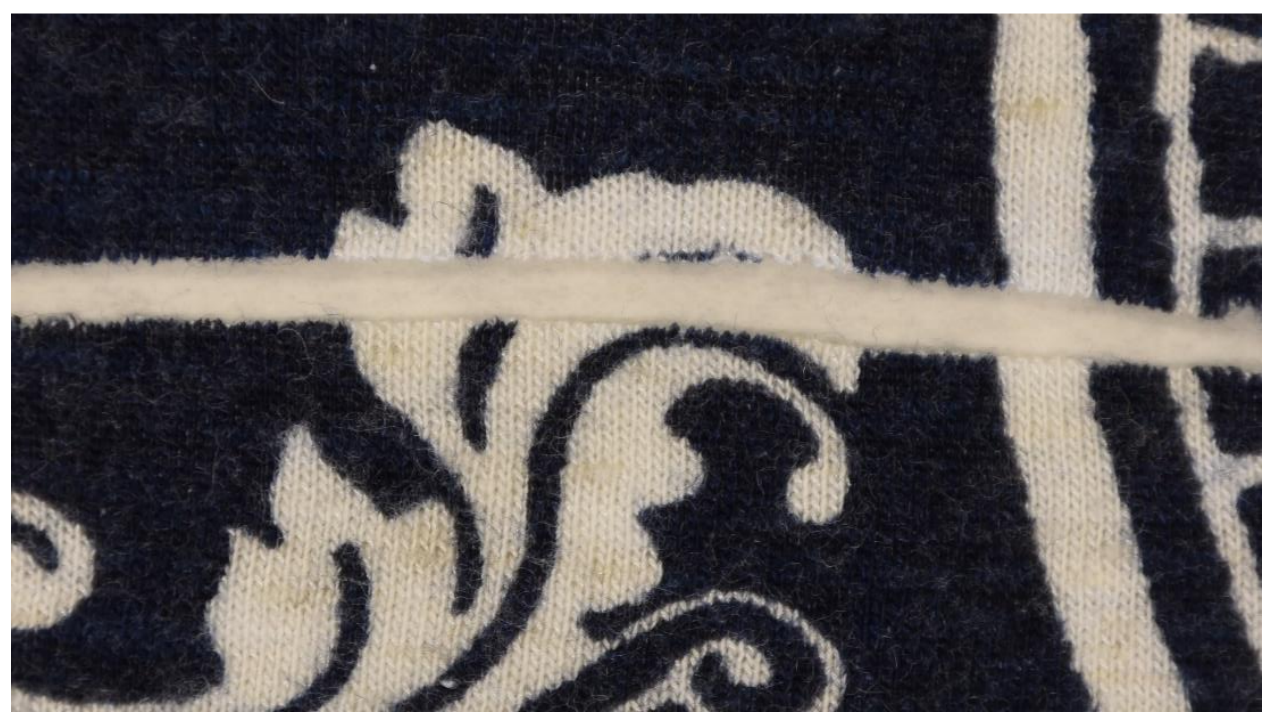

Figure 9. Distinctive characteristic example - pattern continuation across fracture 


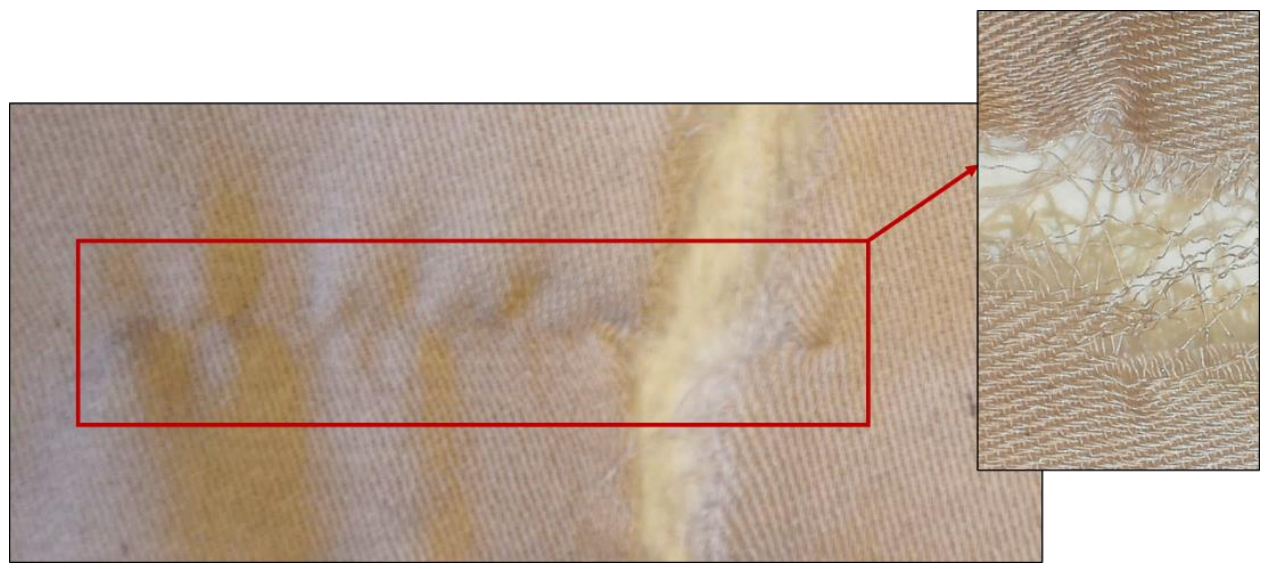

Figure 10. Distinctive characteristic example - separation characteristics (e.g. fabric damage continuation across fracture - a "gather" or pulled thread within the fabric weave)

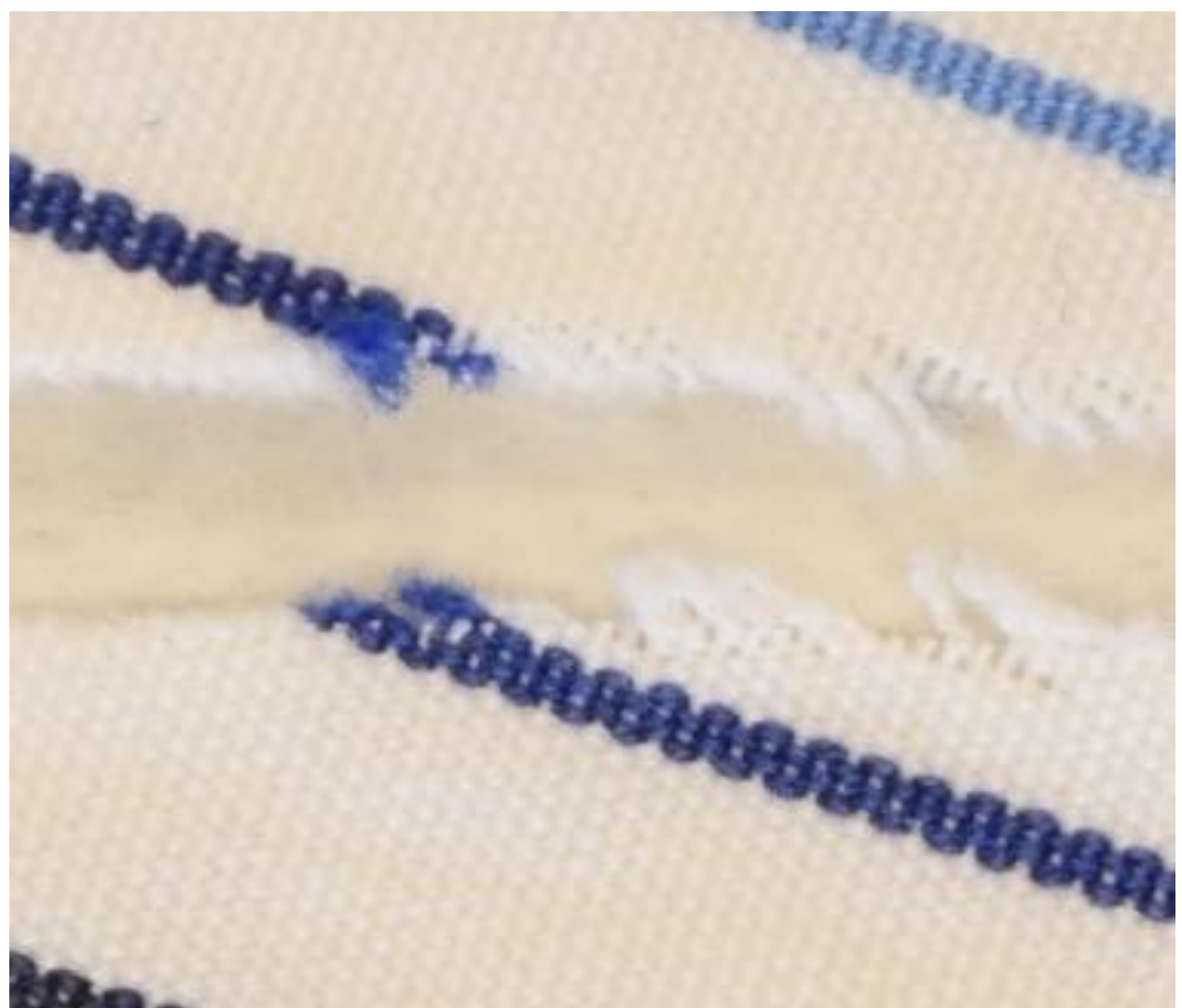

Figure 11. Distinctive characteristic example - separation characteristics (e.g. protrusions/gaps consistent across fracture) 


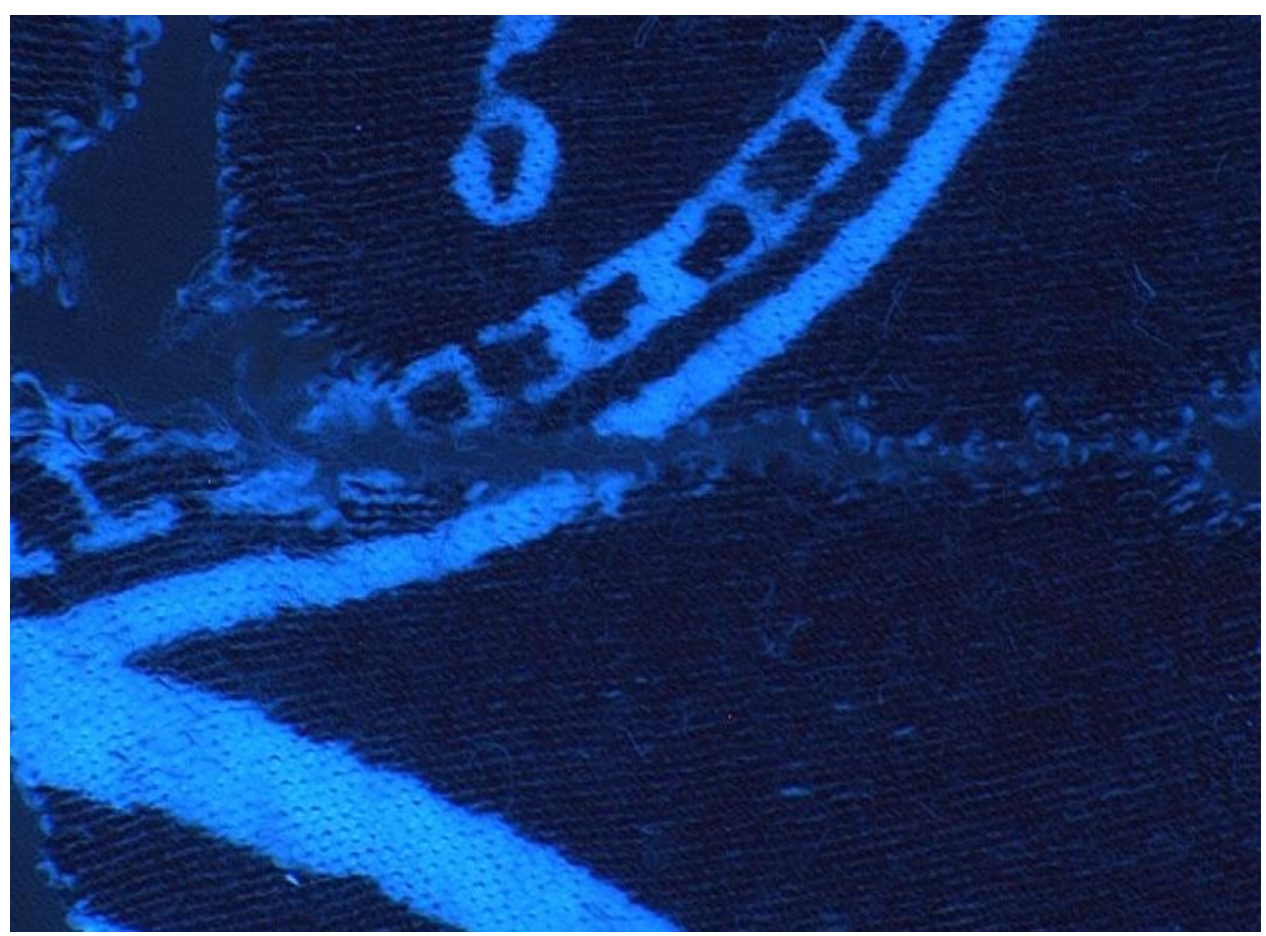

Figure 12. Distinctive characteristic example - partial pattern fluorescence

As observed in Figures 8 and 12, fluorescence became an important feature for consideration during the physical fit comparison procedure, specifically for Item E. In order to check for fluorescence, all textile samples were examined under a Foster \& Freeman video spectral comparator VSC 6000 (Foster and Freeman, VA, USA) using 365 nm UV lighting. All images were taken via the built-in instrument camera.

To keep comparison units constant for ESS determination, each sample was considered through 10 units taken as equal divisions of the total fracture edge length. Examiners first determined overall match " 1 " or non-match " 0 " decisions per comparison unit in order to determine an initial ESS according to Equation 1 below.

$$
\text { Edge similarity score }(E S S)=\frac{\text { Number of consistent bins }}{\text { Total number of bins }(\text { always } 10 \text { of equal length })} * 100
$$

Due to the increased level of features exhibited during textile fracture, weighting factor options were developed in the application of ESS to textile in order to allow for a better score representation of the added confidence any present edge features may add to the overall edge assessment. Following overall bin determination, examiners had the option of three weighting factors for distinctive characteristics observed within each unit. These consisted of pattern continuation across fracture, the presence of separation characteristics such as stains or any consistent damage across fracture, and the continuation of fluorescence across fracture, as outlined in Table 3. If any of the three features were determined present, they were assigned a " 2 " multiplication factor. If a feature was not present, a " 1 " was assigned. All weighting factors were multiplied together per bin with the overall bin determination factor of " 1 " vs " 0 ". For example, a 
single bin determined to be consistent (i.e. "1") with all three weighting factors assigned (i.e. three "2"s assigned) would result in an overall result of 8 (i.e. $1 * 2 * 2 * 2=8$ ). Therefore, the maximum score for all weighting factors assigned for all bins would be $80 \%$. The weighted ESS was then determined as an additive score to the initial ESS according to Equation 2 below, with a theoretical maximum of $180 \%$.

$$
\text { Weighted ESS }=\frac{\text { Sum of multiplied weighting factors per bin }}{80 \text { (highest possible weighting factor sum })} * 100+\text { Initial ESS }
$$

With the addition of a weighted ESS, a rarity ratio was determined as the ratio between the weighted ESS and non-weighted ESS. The rarity ratio was determined according to Equation 3 below, with a theoretical maximum of 1.8. However, no rarity ratios in the current study surpassed 1.55. In addition to the ESS, weighted ESS, and rarity ratios, examiners also determined an overall conclusion and comparison edge qualifier for each sample pair as is performed in the duct tape methodology. Options for each are as follows in Table 4.

$$
\text { Rarity Ratio }=\frac{\text { Weighted ESS }}{\text { Non-weighted ESS }}
$$

Table 4. Options for comparison pair overall conclusions and comparison edge qualifiers

\begin{tabular}{|c|c|}
\hline Comparison Pair Overall Conclusion & Comparison Edge Qualifier \\
\hline $1=$ Match & M+ = Match with high certainty \\
\hline INC = Inconclusive & M- = Match with low certainty \\
\hline $0=$ Non-match & INC = Inconclusive \\
\hline & NM- = Non-match with low certainty \\
\hline & NM+ = Non-match with high certainty \\
\hline
\end{tabular}

Following examiner determination of ESS, weighted ESS, and rarity ratios, data analysis consisted of performance rate assessment both by overall separation method as well as per textile item; distributions of ESS per separation method through boxplots; distribution of rarity ratios for determination of relevant interpretation thresholds; and observation of frequency of occurrence of distinctive features assigned weighting factors throughout the dataset. Data analysis mainly consists of assessments of initial ESS and rarity ratio, as the weighted ESS is considered an intermediate step in reaching the rarity ratio value. Performance rates assessed across the dataset include accuracy, sensitivity, specificity, false positive rate (FPR), false negative rate (FNR), true positive rate (TPR), true negative rate (TNR), as well as two inconclusive rate varieties - that of true positive samples concluded as INC as well as true negative samples concluded as INC. Equations used to determine these values are provided in Table 5 below. 
Table 5. Performance rate equation summary

\begin{tabular}{|c|c|}
\hline Performance rate & Equation \\
\hline Accuracy & Accuracy $=\frac{T P+T N}{T P+T N+F P+F N+I N C} * 100$ \\
\hline Sensitivity & Sensitivity $=\frac{T P}{T P+F N} * 100$ \\
\hline Specificity & Specificity $=\frac{T N}{T N+F P} * 100$ \\
\hline False Positive Rate (FPR) & FPR $=\frac{F P}{F P+T N+I N C} * 100$ \\
\hline False Negative Rate (FNR) & $F N R=\frac{F N}{T P+F N+I N C} * 100$ \\
\hline True Positive Rate (TPR) & $T P R=\frac{T P}{T P+F N+I N C} * 100$ \\
\hline True Negative Rate (TNR) & $T N R=\frac{T N}{T N+F P+I N C} * 100$ \\
\hline Inconclusive Rate (TP) & $I N C=\frac{I N C}{T P+F N+I N C} * 100$ \\
\hline Inconclusive Rate (TN) & $I N C=\frac{I N C}{T N+F P+I N C} * 100$ \\
\hline
\end{tabular}

\section{Results and Discussion}

\subsection{Preliminary $100 \%$, Jersey-Knit Polyester Set}

Prior to examination, all samples were ironed to aid in observation of any fracture edge features. Due to the elasticity of the fabric, the hand-torn edges tended to curl away from one another when examining a sample pair. An example of this curling is provided in Figure $\mathbf{1 3}$ below.

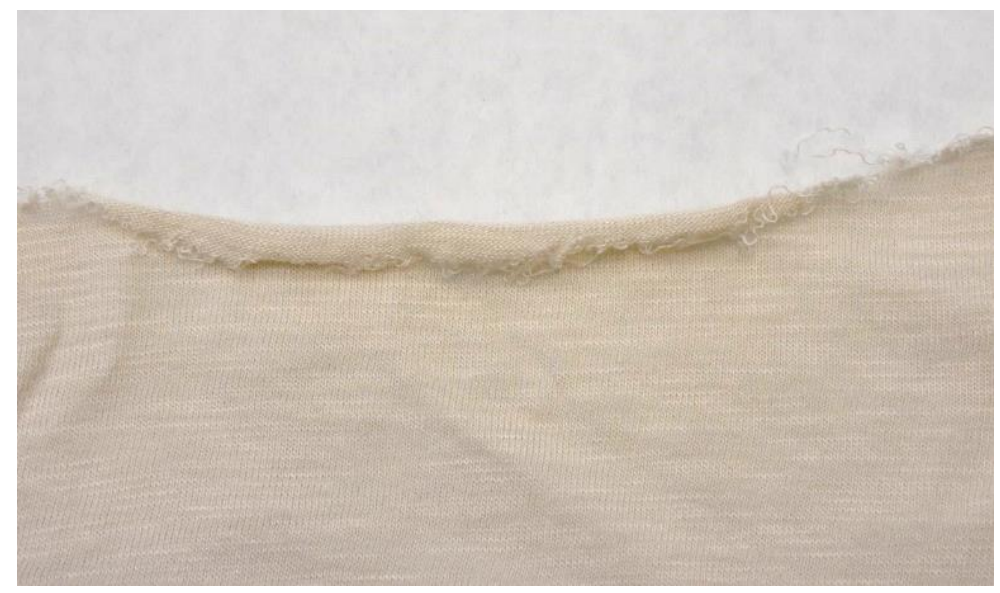

Figure 13. Edge curling in preliminary set fabric 
However, due to the distortion imparted prior to ironing, one sample often appeared longer in length than the corresponding mate. In addition, this stretching often distorted alignment features. Because of these observations, examiner conclusions were compared after both had examined 37 of the 100 sample pairs. In overall conclusion alone, a 30\% disagreement rate was observed (one called a non-match while the other called a match and vice versa). The remaining $70 \%$ of samples were assigned the same conclusion, however $31 \%$ of these samples were assigned varying comparison edge qualifiers. A visual comparison of examiner conclusions is provided in Figure 14 below.

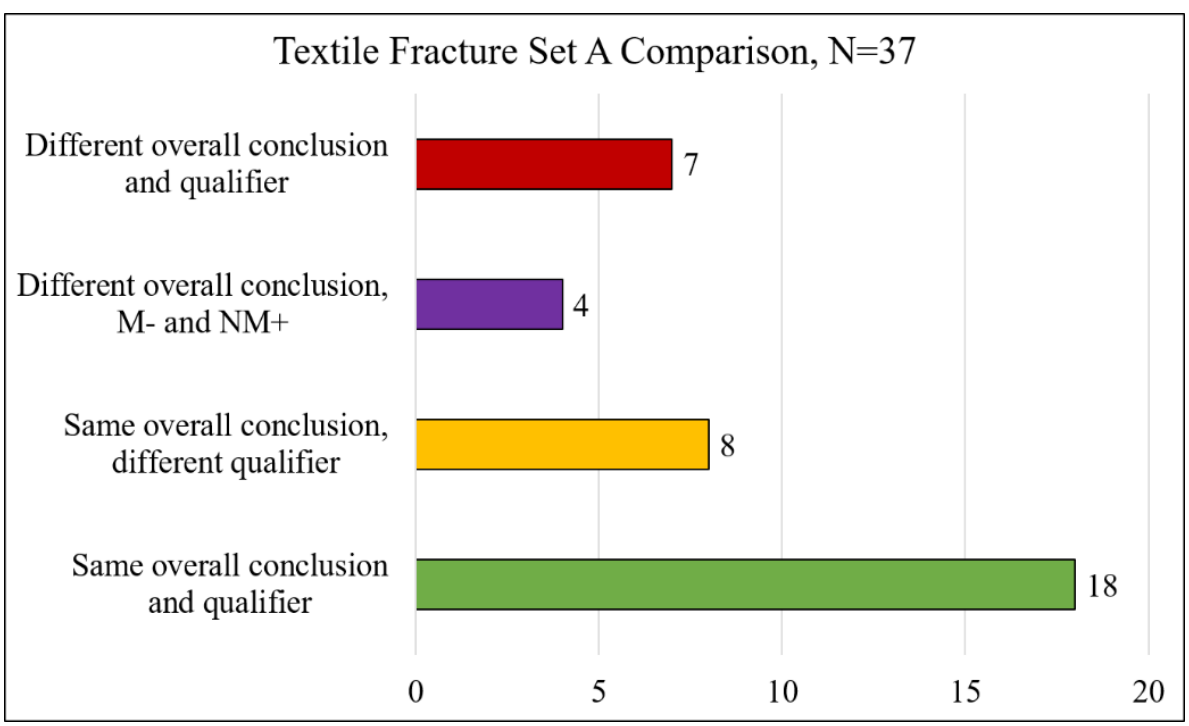

Figure 14. Overall conclusion and comparison edge qualifier comparison between two examiners, preliminary Set A (100\% hand-torn, jersey knit polyester)

In terms of ground truth, a high false negative rate (29 out of 46 true matching samples, 63\%) was observed between both examiners within the first 37 samples of the preliminary set. Table 6 below summarizes the resulting overall error rates. No false positives were noted in the examined results. Figure 15 below provides four examples of sample pairs concluded as false negatives by at least one examiner. Although all samples are true matches, the distortion imparted by hand-tearing can be observed in the images.

Table 6. Preliminary textile set error rates, N=74 total comparisons

\begin{tabular}{|c|c|c|c|c|}
\hline & $\begin{array}{c}\text { Reported } \\
\text { Non-match }\end{array}$ & Reported Match & $\begin{array}{c}\text { Reported } \\
\text { Inconclusive }\end{array}$ & $\begin{array}{c}\text { Total } \\
\text { comparisons } \\
\text { (N=2 examiners) }\end{array}$ \\
\hline $\begin{array}{c}\text { True } \\
\text { Non-match }\end{array}$ & $\begin{array}{c}28 \text { (out of } 28, \mathbf{1 0 0 \%} \\
\text { True negatives }\end{array}$ & $\begin{array}{c}\text { (out of } 28, \mathbf{0 \%}) \\
\text { False positives }\end{array}$ & 0 (out of $28, \mathbf{0 \%})$ & 28 \\
\hline True Match & $\begin{array}{c}29 \text { (out of } 46, \mathbf{6 3} \%) \\
\text { False negatives }\end{array}$ & $\begin{array}{c}17 \text { (out of } 46, \mathbf{3 7 \%}) \\
\text { True Positives }\end{array}$ & 0 (out of 46, 0\%) & 46 \\
\hline
\end{tabular}



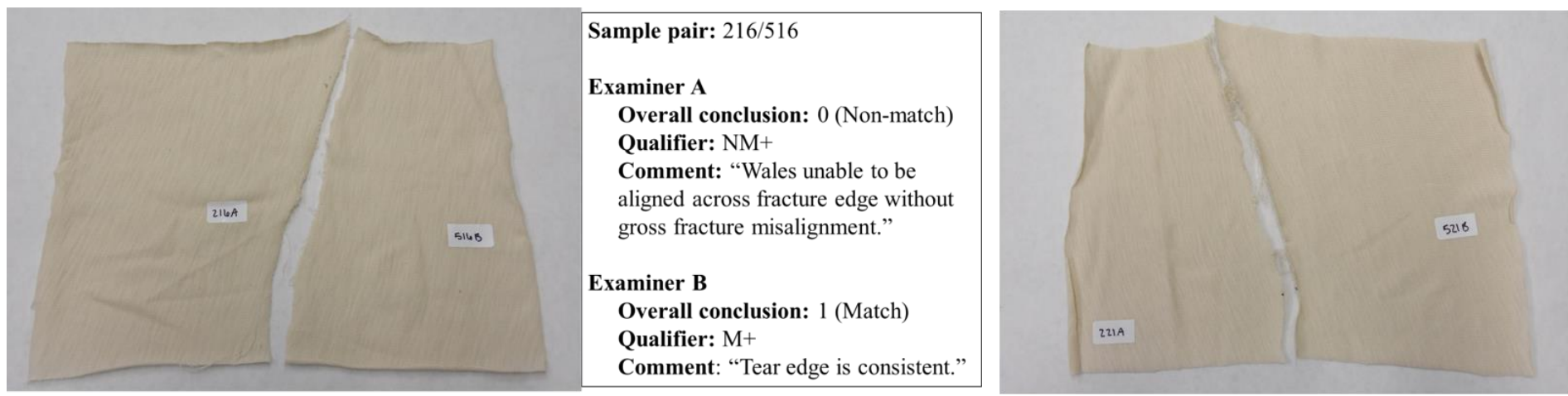

\begin{tabular}{|l|}
\hline Sample pair: $221 / 521$ \\
Examiner A \\
Overall conclusion: 1 (Match) \\
Qualifier: M+ \\
Comment: NA \\
Examiner B \\
Overall conclusion: 0 (Non-match) \\
Qualifier: NM+ \\
Comment: "Significant stretching \\
and unraveled fragments."
\end{tabular}
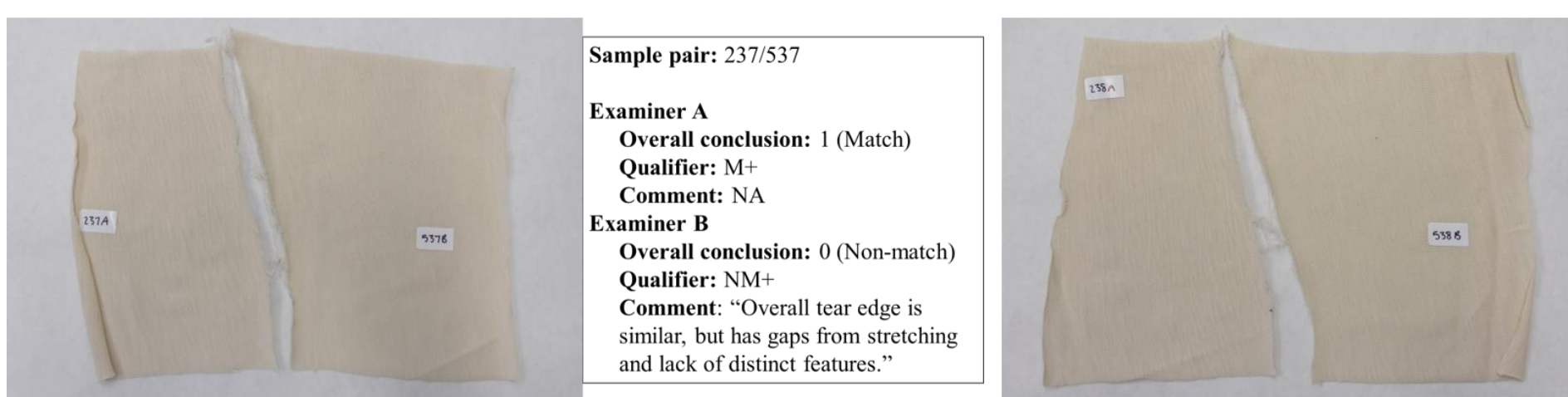

Sample pair: $238 / 538$

Examiner A

Overall conclusion: 1 (Match)

Qualifier: $\mathrm{M}+$

Comment: NA

\section{Examiner B}

Overall conclusion: 0 (Non-match) Qualifier: NM+

Comment: "Overall tear pattern has similar directionality but gaps from stretching exist."

Figure 15. Preliminary textile set false negative examples 


\subsection{Performance Rate Assessment}

\subsubsection{Performance rates by overall separation method}

Table 7 below provides a summary of performance rates calculated for overall comparison conclusion by both examiners, as compared between separation method. Each examiner conducted 50 comparisons per method of separation, the results presented in the Table 7 are the result of 100 comparisons per method by both examiners. Overall, both sets resulted in high accuracy rates with minimal misclassifications. As shown, the stabbed samples resulted in overall higher accuracy and lower misclassifications (false positives, false negatives) than the hand-torn samples. While the overall hand-torn set analysis resulted in one false positive, four false negatives, and two inconclusive responses, the stabbed set analysis resulted in no false positives, two false negatives, and 3 inconclusive responses. A further breakdown of overall performance rates is provided in Tables 8 and 9 below.

Table 7. Performance rate summary by separation method

\begin{tabular}{|c|c|c|}
\hline Performance rate & $\begin{array}{c}\text { Overall rates for } \\
\text { hand-torn samples }\end{array}$ & $\begin{array}{c}\text { Overall rates for } \\
\text { stabbed samples }\end{array}$ \\
\hline Accuracy & 93 & 95 \\
\hline Sensitivity & 88 & 92 \\
\hline Specificity & 98 & 98 \\
\hline FPR & 2 & 0 \\
\hline FNR & 8 & 4 \\
\hline TPR & 88 & 92 \\
\hline TNR & 98 & 4 \\
\hline Inconclusive Rate (TP) & 4 & 2 \\
\hline Inconclusive Rate (TN) & NA & \\
\hline
\end{tabular}

Table 8. Performance rate breakdown - hand-torn samples

\begin{tabular}{|c|c|c|c|c|}
\hline & $\begin{array}{c}\text { Reported } \\
\text { Non-match }\end{array}$ & Reported Match & $\begin{array}{c}\text { Total } \\
\text { Reported } \\
\text { Inconclusive }\end{array}$ & $\begin{array}{c}\text { comparisons } \\
\text { (N=2 } \\
\text { examiners) }\end{array}$ \\
\hline $\begin{array}{c}\text { True } \\
\text { Non-match }\end{array}$ & $\begin{array}{c}47 \text { (out of } 48, \mathbf{9 8 \%} \\
\text { True negatives }\end{array}$ & $\begin{array}{c}1 \text { (out of } 48, \mathbf{2 \%}) \\
\text { False positives }\end{array}$ & 0 (out of 48, 0\%) & 48 \\
\hline True Match & $\begin{array}{c}4 \text { (out of } 52, \mathbf{8 \%}) \\
\text { False negatives }\end{array}$ & $\begin{array}{c}46 \text { (out of } 52, \mathbf{8 8} \%) \\
\text { True Positives }\end{array}$ & 2 (out of 52, 4\%) & 52 \\
\hline
\end{tabular}


Table 9. Performance rate breakdown - stabbed samples

\begin{tabular}{|c|c|c|c|c|}
\hline & $\begin{array}{c}\text { Reported } \\
\text { Non-match }\end{array}$ & Reported Match & $\begin{array}{c}\text { Reported } \\
\text { Inconclusive }\end{array}$ & $\begin{array}{c}\text { Total comparisons } \\
\text { (N=2 examiners) }\end{array}$ \\
\hline $\begin{array}{c}\text { True } \\
\text { Non-match }\end{array}$ & $\begin{array}{c}47 \text { (out of 48, 98\%) } \\
\text { True negatives }\end{array}$ & $\begin{array}{c}0 \text { (out of 48, 0\%) } \\
\text { False positives }\end{array}$ & 1 (out of 48, 2\%) & 48 \\
\hline True Match & $\begin{array}{c}2 \text { (out of 52, 4\%) } \\
\text { False negatives }\end{array}$ & $\begin{array}{c}48 \text { (out of 52, 92\%) } \\
\text { True Positives }\end{array}$ & 2 (out of 52, 4\%) & 52 \\
\hline
\end{tabular}

The discrepancy in accuracy between the sets is likely due to the lower amount of distortion presented to samples during stabbing than in hand-tearing. During the stabbing process, the blade passed quickly through the textile items into the foam form with minimal resistance. However, during the hand-tearing process, samples were much more stretched and manipulated in order to initiate the separation. This was especially noticed in the twill woven items (Item A, the men's navy dress pants and Item B, the women's blue jeans), as the tight weave presented more difficulty to initiating a tear, leading to more stretch and pull throughout the fracture. The fracturing mechanisms translated to distortion of the edge features at the microscopic level.

On the other hand, it was observed the stabbed samples presented a higher number of inconclusive conclusions than the hand-torn samples. This is likely due to a lack of distinctive features in some of the comparison bins. As previously mentioned, it was observed that during the stabbing process, the blade quickly passed through all textile items. No drag or hanging of the blade on the fabric edges was experienced that may have introduced additional distinctive edge morphology features. Therefore, relatively less distinctive edge morphology was present in the stabbed samples, making examinations more difficult when edges were observed to be mostly featureless. The appearance of featureless edges typically leads to inconclusive conclusions. An example of the varying edge morphology between true match hand-torn and stabbed textile samples is provided in Figure 16. It is worth noting, however, that even on stabbed edges, small changes in directionality and observations of fabric construction alignment and some other distinctive features were still possible, depending on fabric type. 


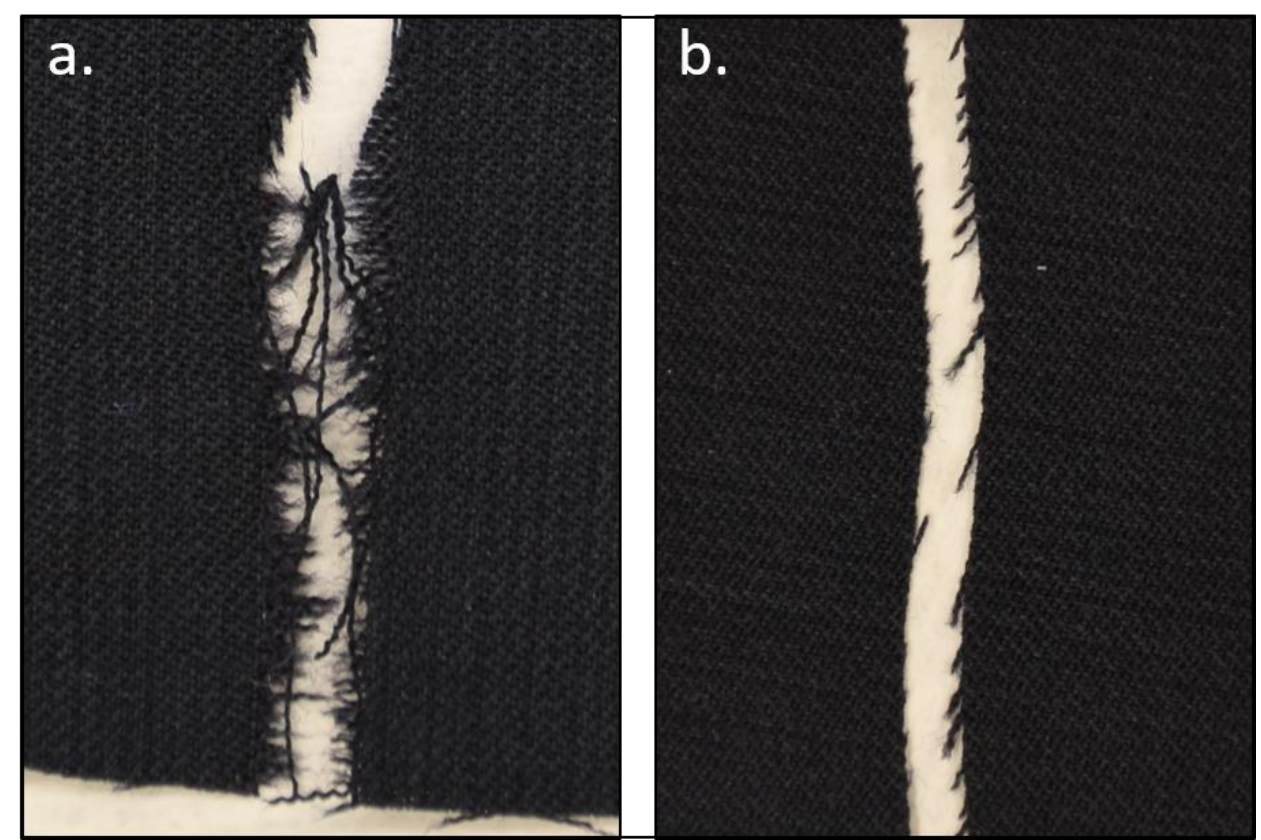

Figure 16. Item A edge morphology true match examples - a) hand-torn edges, b) stabbed edges

\subsubsection{Performance rates by textile item}

Table 10 below provides performance rates broken down by each textile item for the hand-torn set. It is observed that throughout items A, B, and C, perfect accuracy was achieved with no misclassifications noted. However, accuracy decreases to 85 and $80 \%$ respectively for Items D and E. The decrease in accuracy in Item $D$ is due to one instance each of a false positive, false negative, and inconclusive. The decrease in accuracy in Item $\mathrm{E}$ is due to three instances of false negatives and one instance of an inconclusive conclusion. This accuracy deterioration appears to follow the trend observed in the preliminary textile fracture experimentation involving jersey knit, $100 \%$ polyester fabric. Specifically, Item D is composed of $100 \%$ polyester, while Item E is of jersey knit construction. It should be noted that the polyester composition and jersey knit construction are only represented by Items D and $\mathrm{E}$ in the dataset and neither are present in Items A, B, or C. Therefore, the increase in error rates noted in the preliminary textile experimentation due to specific fabric composition and construction is supported by the results of hand-torn data set. Again, increased error rates are noted due to enhanced distortion presented by the jersey knit construction and polyester composition. 
Table 10. Performance rate summary by textile item - hand-torn samples

\begin{tabular}{|c|c|c|c|c|c|}
\hline Performance rate & Item A & Item B & Item C & Item D & Item $\mathrm{E}$ \\
\hline Accuracy & 100 & 100 & 100 & 85 & 80 \\
\hline Sensitivity & 100 & 100 & 100 & 83 & 50 \\
\hline Specificity & 100 & 100 & 100 & 88 & 100 \\
\hline FPR & 0 & 0 & 0 & 13 & 0 \\
\hline FNR & 0 & 0 & 0 & 8 & 38 \\
\hline TPR & 100 & 100 & 100 & 83 & 50 \\
\hline TNR & 100 & 100 & 100 & 88 & 100 \\
\hline $\begin{array}{c}\text { Inconclusive Rate } \\
\text { (TP) }\end{array}$ & 0 & 0 & 0 & 8 & 13 \\
\hline $\begin{array}{c}\text { Inconclusive Rate } \\
(\mathrm{TN})\end{array}$ & NA & NA & NA & NA & NA \\
\hline
\end{tabular}

Table 11 below provides performance rates per textile item for the stabbed set. Interestingly, Item E now presented superior accuracy with no misclassifications observed. Items A through D presented accuracy rates from 90-95\%. No false positives were observed in the stabbed set, although one false negative each was observed in Items $C$ and D. However, it was determined the false negative in Item $\mathrm{C}$ was due to the examiner comparing the incorrect edges of the sample pair and can be omitted for purposes of interpretation (gross error rather than a random error).

Table 11. Performance rate summary by textile item - stabbed samples

\begin{tabular}{|c|c|c|c|c|c|}
\hline Performance rate & Item A & Item B & Item C & Item D & Item E \\
\hline Accuracy & 95 & 90 & 95 & 95 & 100 \\
\hline $\begin{array}{c}\text { Sensitivity } \\
\text { Specificity }\end{array}$ & 100 & 83 & 90 & 90 & 100 \\
\hline FPR & 0 & 0 & 0 & 0 & 0 \\
\hline FNR & 0 & 0 & 10 & 10 & 0 \\
\hline TPR & 100 & 83 & 90 & 90 & 100 \\
\hline TNR & 88 & 100 & 100 & 100 & 100 \\
\hline $\begin{array}{c}\text { Inconclusive Rate } \\
\text { (TP) }\end{array}$ & 0 & 17 & 0 & 0 & 0 \\
\hline $\begin{array}{c}\text { Inconclusive Rate } \\
\text { (TN) }\end{array}$ & 13 & 0 & 0 & 0 & 0 \\
\hline
\end{tabular}


The inverse relationship between accuracy rate and separation method as observed in Item E can be explained due to the lower distortion and stretching exhibited by stabbing as compared to handtearing. Due to its construction (jersey knit), Item E exhibited distortion, affecting resulting accuracy of sample pairs within the hand-torn set. However, when no distortion was exhibited through stabbing, accuracy seems to increase due to the presence of a pattern on the fabric that was able to be aligned across the fracture in many sample pairs. This is greatly observed in examiner notes throughout the sample set. This higher accuracy due to pattern is also observed in the only other textile item with a pattern in the data set - Item C. As the FNR for Item C can be disregarded for interpretation purposes, Items $\mathrm{C}$ and $\mathrm{E}$ exhibited highest accuracy across the stabbed sample set due to the increase distinctiveness of pattern across the fractured edges. As the stabbing process typically left "featureless" edges with less distinctive edge morphology, the presence of a pattern assisted examiners in aligned true match sample pairs to one another, as well as quickly identifying true non-match samples through a lack of pattern continuation in these specific items.

\subsubsection{Misclassification examples}

Across the overall data set, 12 instances of misclassifications or inconclusive conclusions were observed. Three of these were instances of true negatives in which it was determined that one or both examiners had compared the incorrect edges of the textile sample pair. For that reason, they will be excluded from the following discussion. The example images below document the remaining 9 instances (5 hand-torn, 4 stabbed) of misclassifications across the data set, presented by separation method.

\subsubsection{Hand-torn sample set misclassifications}

Figure 17 below displays a sample pair from Item $D$ that resulted in the only false positive across the textile study. While both examiners noted bins of dissimilarity, Examiner A assigned an ESS of 0\% with a NM- qualifier while Examiner B assigned a 70\% and M-. As shown in the image, the macro edge morphology gave the illusion of a potential fit, while micro features noted by Examiner A revealed inconsistencies. Specifically, these inconsistencies appeared in the form of gathers in the fabric (i.e. damage) as well as the overall weave pattern alignment between samples. This example highlights the relevance of informing the examiner's opinion with micro-bin observations and quantitative assessment of the quality of a match. If only macroscopic general alignment features are considered during an examination (as most current examination protocols) the risk for false positives is more latent. 


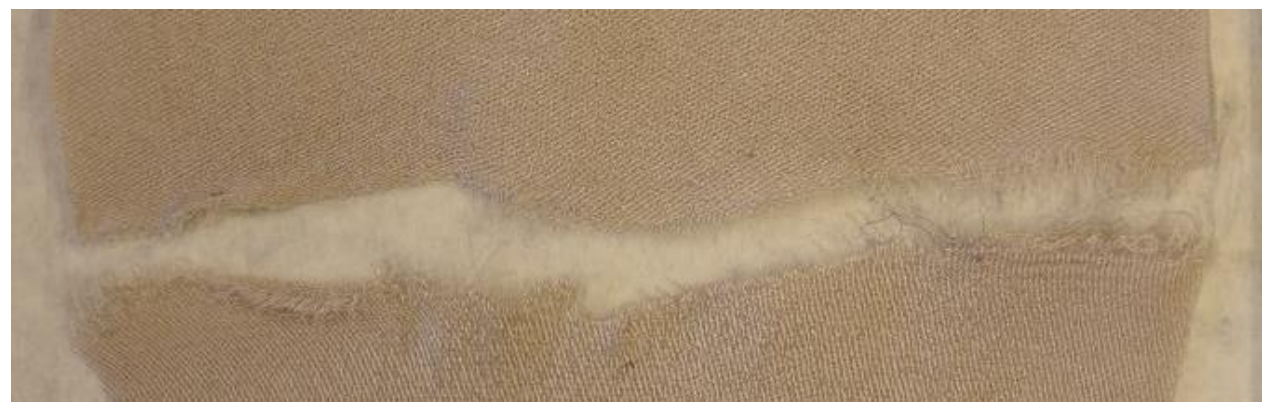

Figure 17. Examiner B false positive - Item D

Figure 18 below displays an example of a false negative conclusion by Examiner B. This sample pair presented a high level of distortion making for a difficult fracture fit assessment. While Examiner A assigned an 80\% ESS with a M- qualifier, Examiner B assigned a $40 \%$ and NM-. Upon technical review of misclassified samples, it was discovered that in instances of gaps as shown in the bottom sample in Figure 18, Examiner B considered these inconsistencies if there was no accompanying protrusion in the other sample. Examiner A tended to engage in more manipulation of the sample, meaning more movement of the edges for possible realignment during the comparison of edges, for an understanding of how the item may have separated from itself in these areas rather than from the other sample. While this discrepancy is attributed to variation in experience levels, the practice of manipulating sample edges to observe various orientations of potential alignment prevented misclassifications. Figures 19 and 20 below are additional instances in which this discrepancy between examiner methodology is also demonstrated due to large distortion and gaps in the samples. Figure 19 is another false negative example (Examiner A: 100\% ESS, M+; Examiner B: 10\%, NM-) while the sample pair in Figure 20 resulted in an inconclusive conclusion (Examiner A: 100\% ESS, M+; Examiner B $=30 \%$, INC). This is less detrimental as further chemical and physical analyses would likely be performed on a material in which a physical fit cannot be determined.

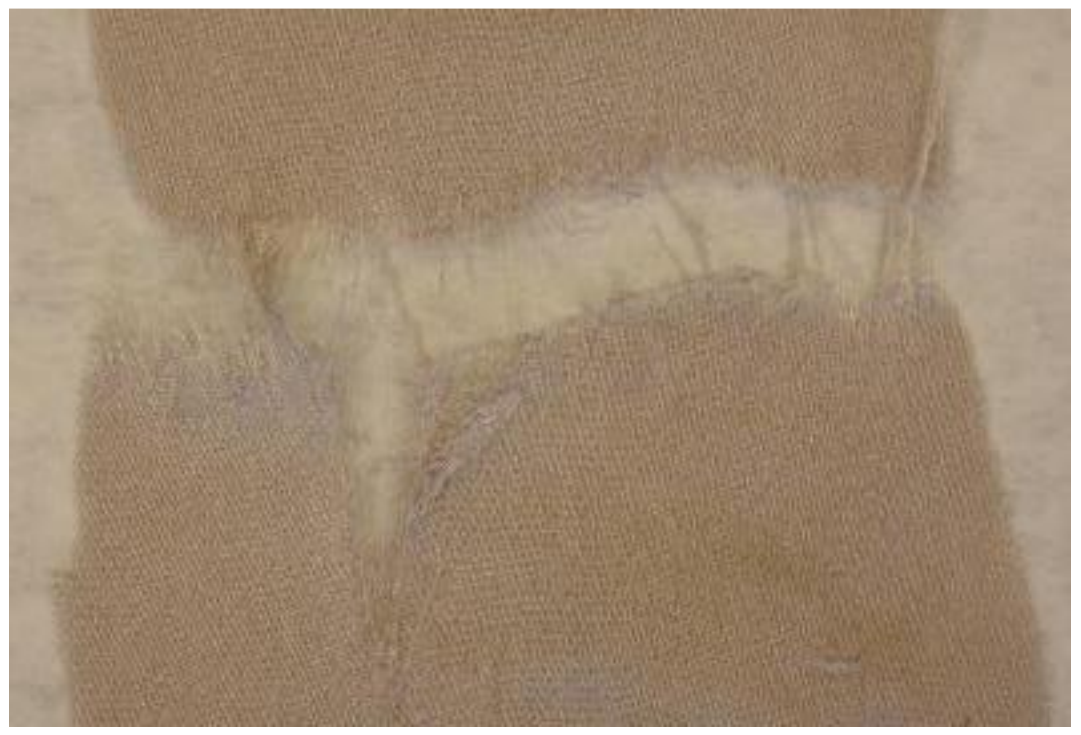

Figure 18. Examiner B false negative - Item D 


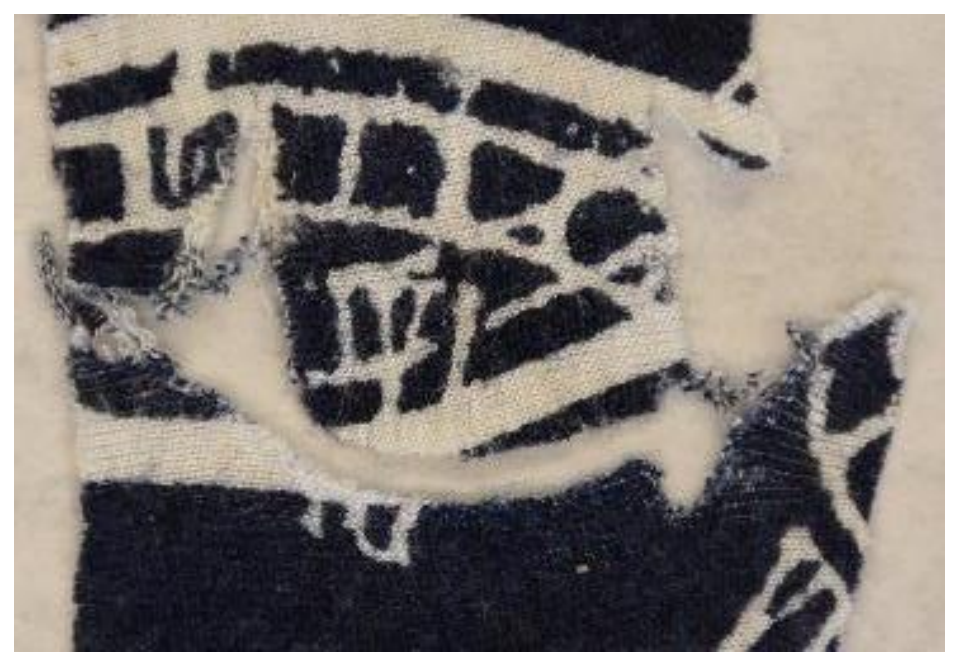

Figure 19. Examiner $B$ false negative - Item $E$

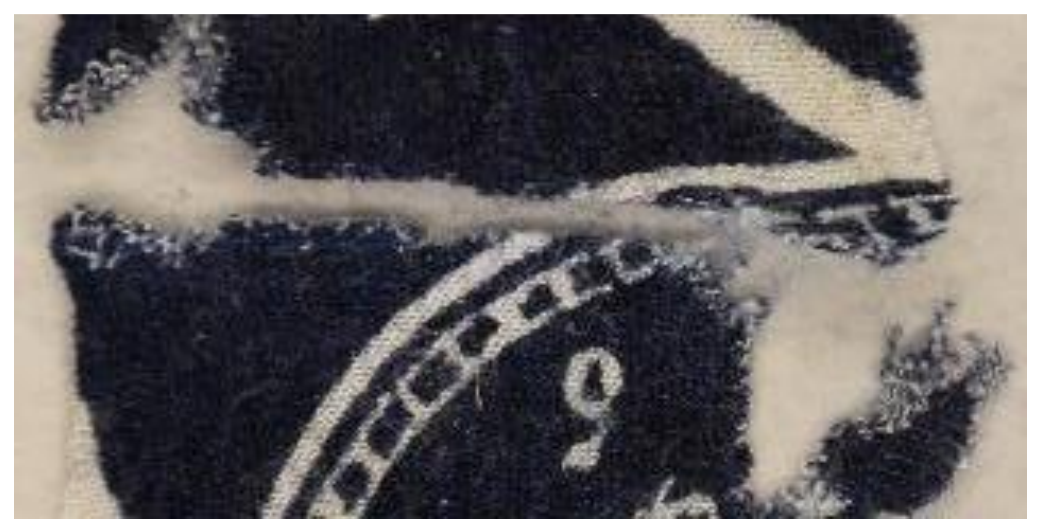

Figure 20. Examiner B inconclusive (true match sample) - Item E

Figure 21 below provides a true match sample pair in which an inconclusive conclusion was reported by Examiner B. While Examiner A assigned a 100\% ESS and a M+ qualifier, Examiner $\mathrm{B}$ assigned a $40 \%$ and INC. This was another discovered examiner discrepancy arising from unequal fracture edge length between two samples. While Examiner A would determine ESS by dividing 10 bins based upon the smaller of the two samples, Examiner B would take bin divisions across the longer of the two and consider the portion of the longer pair without corresponding material on the other item to be non-matching (" 0 ") bins. This discrepancy can easily be corrected with specification of this criteria prior to analysis in future studies. 


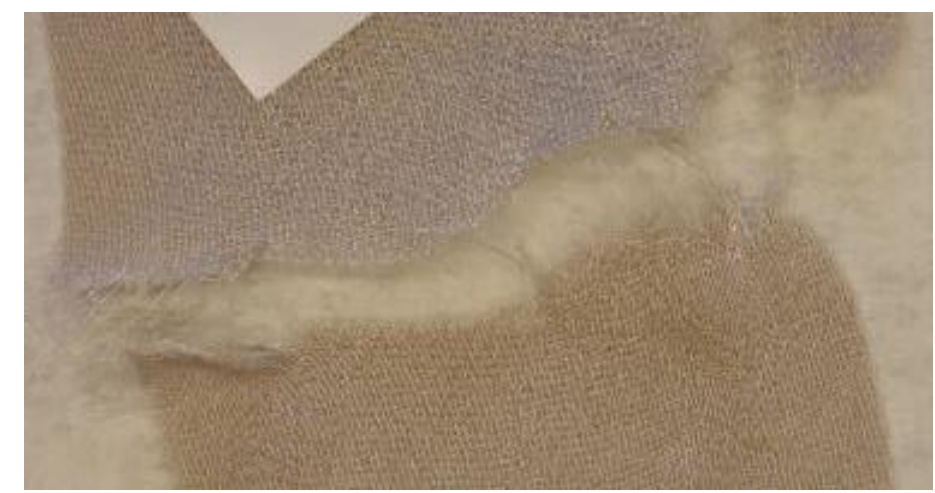

Figure 21. Examiner B inconclusive (true match sample) - Item D

\subsubsection{Hand-torn sample set misclassifications}

Figure 22 below provides an image of a sample pair resulting in a false negative conclusion by Examiner B. This instance is especially interesting as Examiner A reported the most confident possible match conclusion criteria (100\% ESS, M+ qualifier) while Examiner B reported the most confident possible non-match conclusion criteria (0\% ESS, NM+). While Examiner A noted consistent protruding fibers (i.e. separation characteristics) across the sample pair, Examiner B reported that alignment attempts in one portion of a sample resulted in one sample being overlaid across the other in another portion of the sample, meaning an overall fit could not be established. This issue led to their non-match conclusion. This appears to be a situation in which micro-level characteristics may have been overlooked.

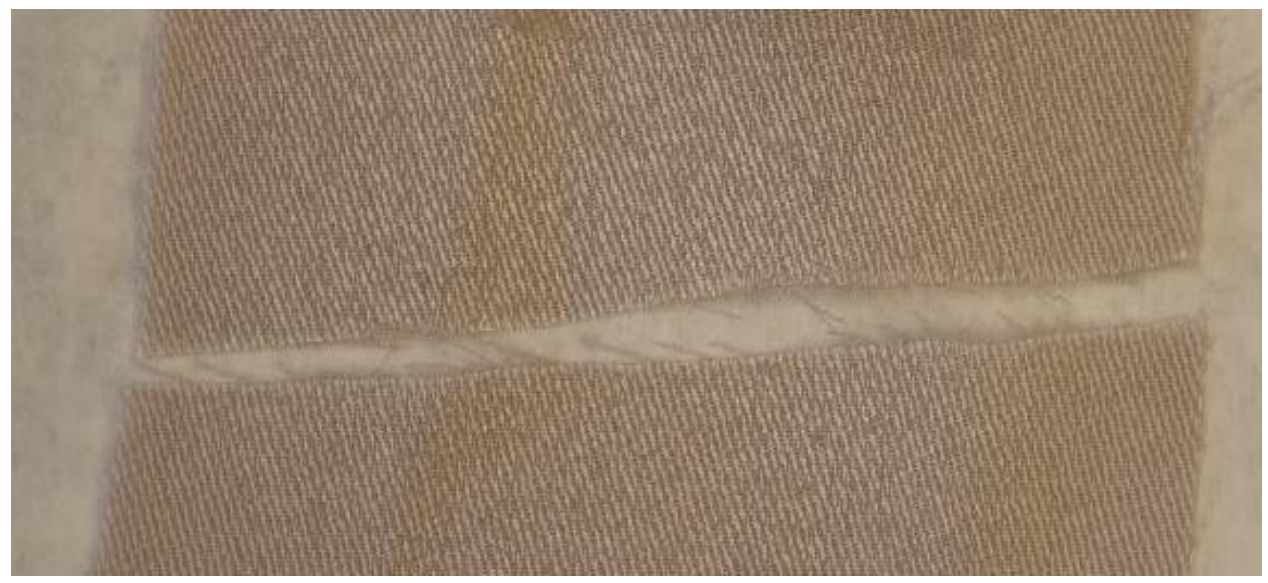

Figure 22. Examiner B false negative - Item D

Figure 23 below provides another interesting instance in which Examiner B labeled a true nonmatch sample as an inconclusive with a relatively high ESS value of 70\%, while Examiner A reported the most confident non-match criteria (0\% ESS, NM+). While both examiners note overall edge morphology does not align, Examiner A notes complete misalignment and Examiner B only noted partial misalignment. Specifically, Examiner B felt the ends of the overall fracture aligned while the middle portion did not. 


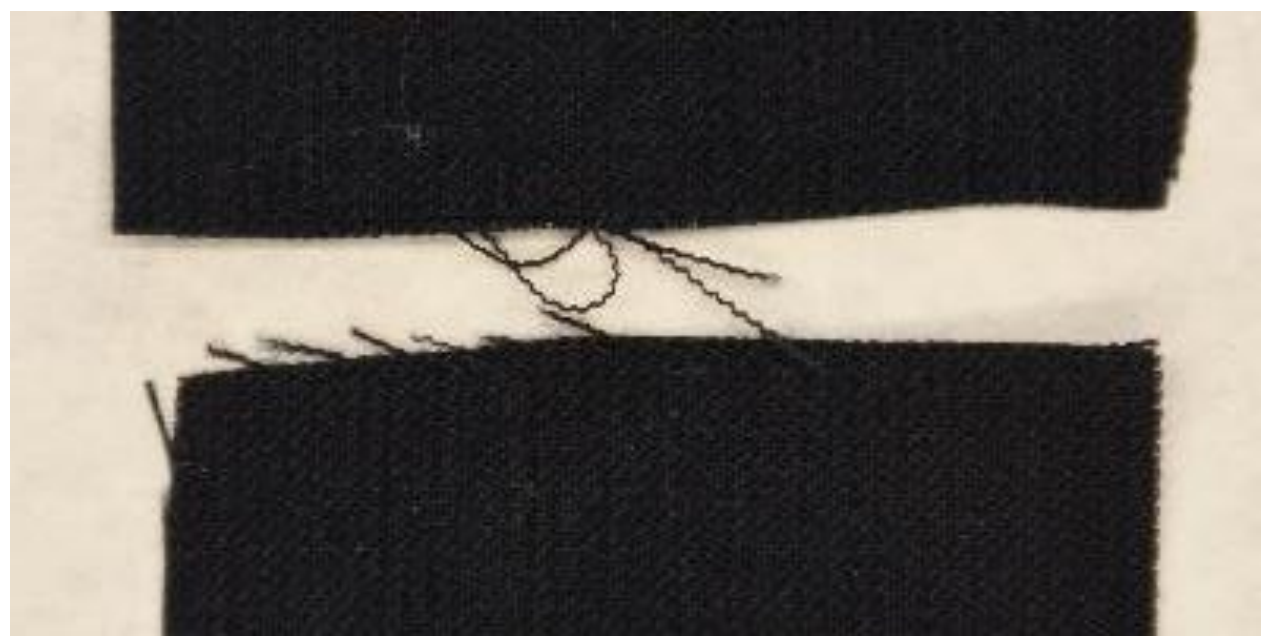

Figure 23. Examiner B inconclusive (true non-match sample) - Item A

Both sample pairs displayed in Figures 24 and 25 below are instances in which one examiner reported an inconclusive while the other examiner noted significant fiber protrusion (i.e. separation characteristics) to be in alignment, thus determining the true positive nature of the samples. Figure 24 displays a situation in which Examiner A determined an ESS of 70\% with a M- qualifier while Examiner B determined a 50\% ESS and INC qualifier. Figure 25 displays a sample pair in which Examiner A determined a 40\% ESS and INC qualifier while Examiner B determined a 70\% ESS and M- qualifier.

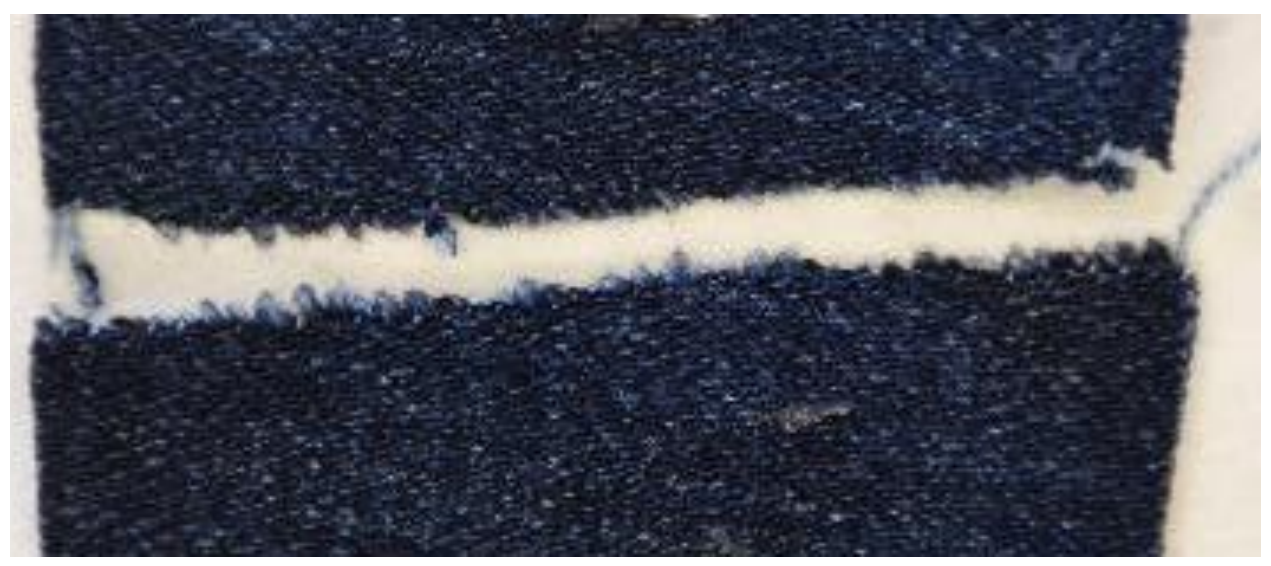

Figure 24. Examiner B inconclusive (true match sample) - Item B 


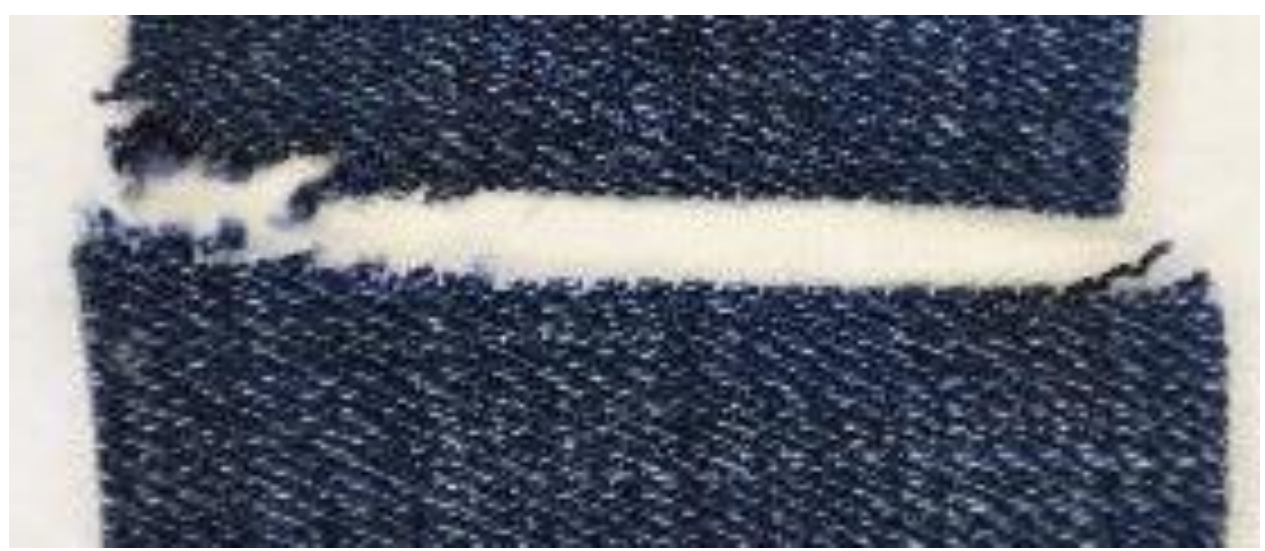

Figure 25. Examiner A inconclusive (true match sample) - Item B

Overall, the misclassification examples revealed how challenging the physical comparison of textile's fractured edges could become and how relevant the development of consensus criteria can be for the identification and documentation of features during the examination. The implementation of methods that allow for the assessment of the quality of a match seem particularly important to facilitate the peer review process and to support the basis for a conclusion.

\subsection{Boxplots of ESS Distributions by Separation Method}

Figures 26 and 27 below provide boxplot representations of the ESS distribution per separation method for the overall set as well as each individual textile item. Throughout all sets, good separation between true positive (blue) and true negative (green) samples is observed, with the exception of Item E in the hand-torn set. The comparison of Item E ESS distributions between the hand-torn and stabbed sample sets allows further visualization of the previously described inverse relationship between accuracy rate and separation method. Again, as Item E was of jersey knit construction, it experienced greater distortion throughout the hand-tearing separation process resulting in lower accuracy in the edge comparison examination. However, as Item E also exhibited a pattern, it had enhanced capacity for alignment as compared to other non-patterned textile items when faced with "featureless", stabbed edges. It is also noted in the ESS distribution boxplots that Item A exhibited a broader true negative sample distribution as compared to the other textile items, in which true negative samples were more often assigned ESS of 0\%. This is likely attributed to the lack of edge features noted by examiners within samples originating from Item $\mathrm{A}$ in comparison to other items. While other items exhibited characteristics such as pattern or edge protrusions/gaps allowing quicker identifications of true negative pairs, Item A provided more "featureless" edges. This observation can be observed in the low frequency of occurrence of weighting factors in Item A as discussed in Section 4.4. 


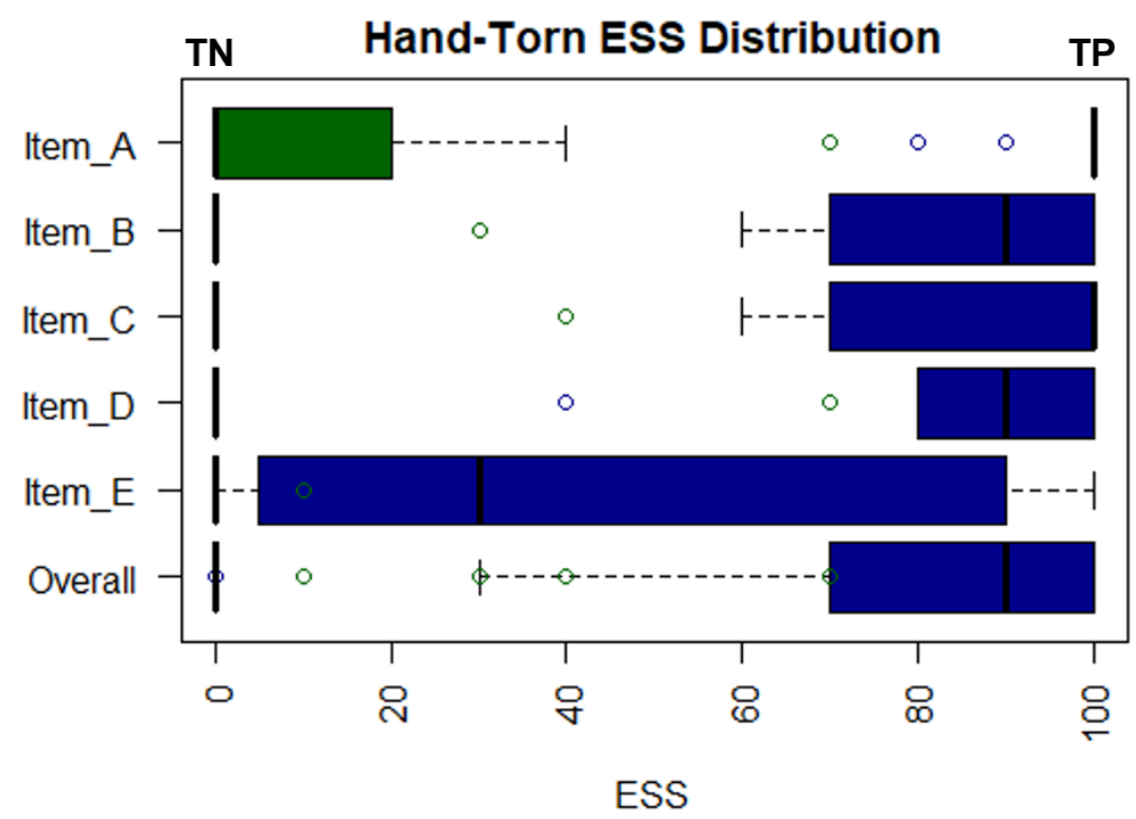

Figure 26. Hand-torn sample set ESS distribution boxplots

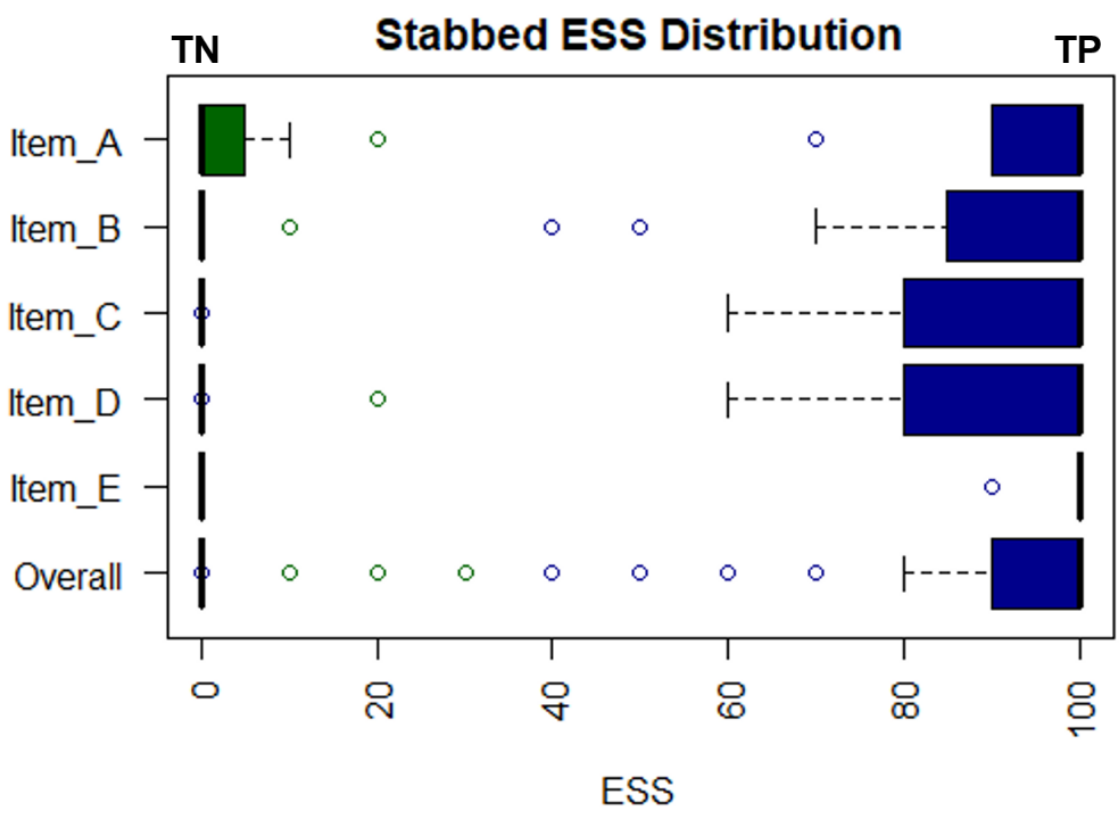

Figure 27. Stabbed sample set ESS distribution boxplots 


\subsection{Distribution of Rarity Ratios and Interpretation Thresholds}

Figures 28 and 29 below provide distributions of the rarity ratios calculated between weighted and non-weighted ESS for both the hand-torn and stabbed sample sets. The rarity ratio was introduced in this study as an interpretation method for the additional weighting factors added to the ESS in an attempt to better represent the varying confidence levels attributed to textile physical fits due to the presence or absence of distinctive edge features. Three potential weighting factors were possible due to the presence of pattern continuation across fracture, the presence of separation characteristics such as stains or any consistent damage across fracture, and the continuation of fluorescence across fracture. Theoretically, the greater the weighted ESS, the higher the rarity ratio. While the rarity ratio had a theoretical maximum of 1.80 , none of the ratios in the study surpassed values of 1.55. As shown by their distributions, both the hand-torn and stabbed sample sets experienced clear separation in rarity ratios between values either less than 0.05 or greater than 1.1. Greater distribution is shown in rarity ratios of the true positive samples per item, as the majority of true negative pairs across the sample set were assigned ESS values of $0 \%$.

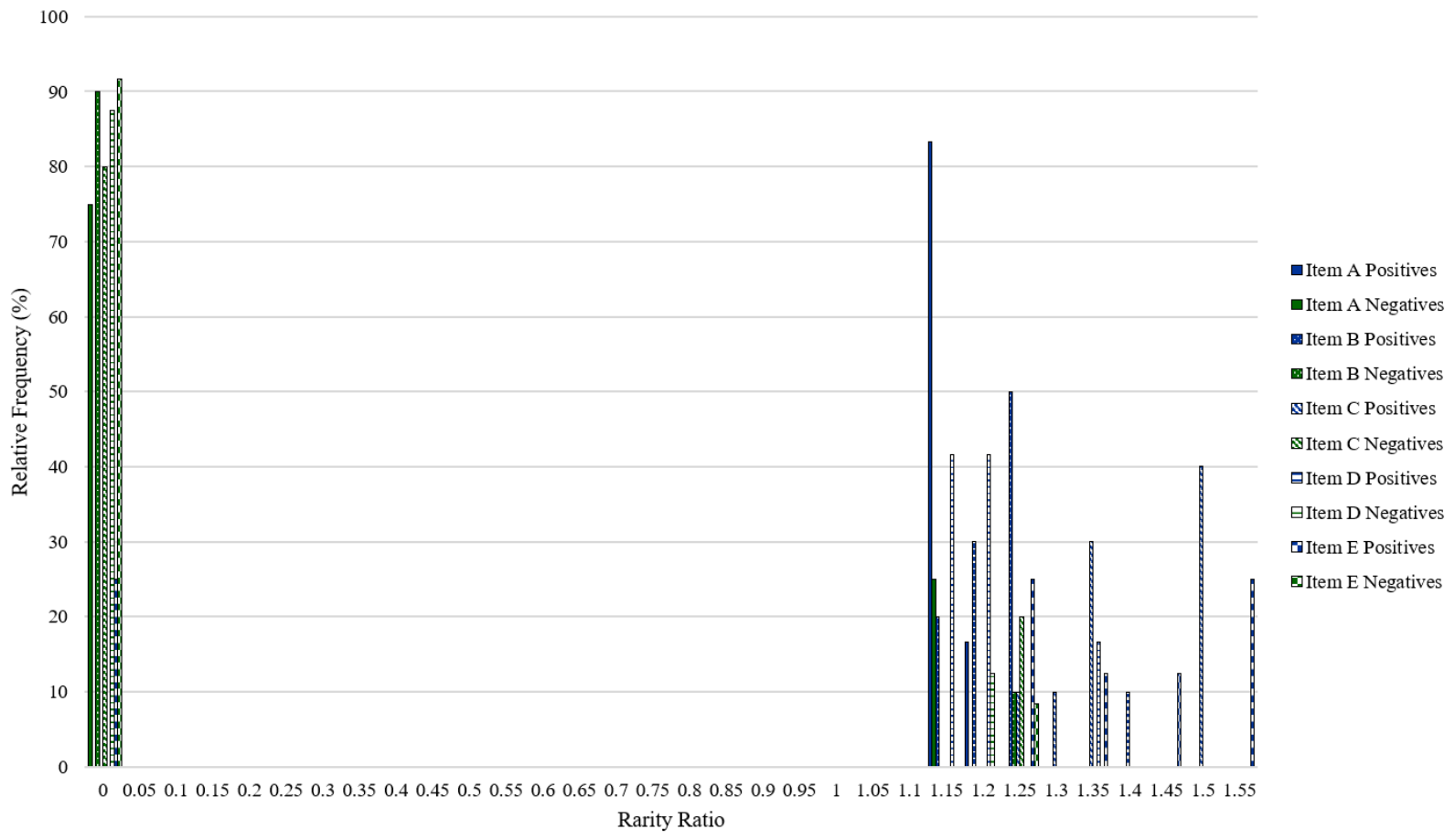

Figure 28. Rarity ratio distribution - hand-torn sample set 


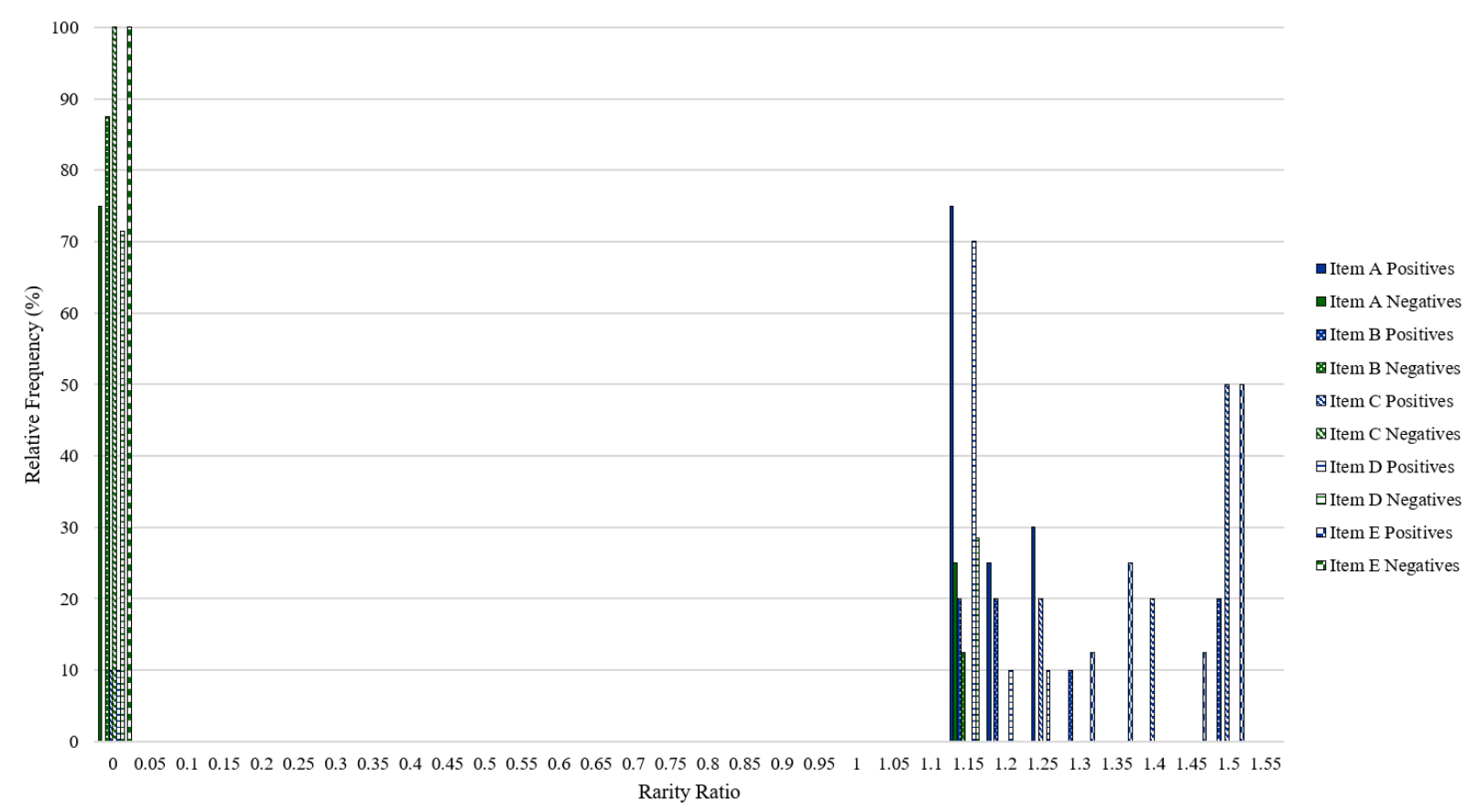

Figure 29. Rarity ratio distribution - stabbed sample set

As shown in the above figures, the majority of Item A rarity ratios remained within values of 11.2 regardless of separation method. Similarly, rarity ratios for Item $C$ true positives fell within the same ranges (1.25-1.5) regardless of separation method. In the hand-torn sample set, Item B true positive rarity ratios fell within the range of 1-1.25 as compared to an increased range of 1-1.5 in the stabbed sample set. This increased range indicates that more distinctive edge features were noted in Item B in the stabbed sample set as compared to the hand-torn set. This is likely due to the lower amount of distortion prohibiting the examiner from viewing any imparted edge features. The inverse of this was observed in Item D true positives, as the rarity ratio range decreased in the stabbed sample set (1.15-1.25) as compared to its range within the hand-torn sample set (1.151.35). Despite the distortion exhibited in the hand-torn set, Item D commonly experienced damage in the form of fabric "gathers" that were either consistent or inconsistent across the fracture edge, leading to the increased range of rarity ratios. An example of this damage is provided in Figure 10. Finally, the rarity ratios in Item E remained similar throughout both separation methods, with only a slight shift from a range of 1.25-1.55 in the hand-torn set to 1.3-1.5 in the stabbed set. Item E presented a greater capacity for assignment of weighting factors overall as regardless of separation method leading to separation characteristics (i.e. damage or protrusions/gaps), Item E exhibited both a pattern as well as fluorescence at the overall (class) and partial (distinctive) level.

Based on observations of rarity ratio distribution between the data sets, a verbal interpretation scale of rarity ratio thresholds is proposed as provided in Table 12. It should be noted that the verbal scale is utilized for a means of assessing the edge features present between textile types rather than an assessment of match vs. non-match. The range of 0-0.5, as shown by the majority of the true negative samples, indicates the absence of rare edge features that could be used to add weight to 
fracture fit conclusions. The range of 0.5-1 indicates that no additional information could be provided from weighting factors, as is evident in the sample set as no values fell within this range. The range of 1-1.55 indicates that rare features were observed between the sample pair and can then be further broken down into three levels of assessment based on the quantity of rare features observed, and therefore the representation of increased examiner confidence in their decision of match or non-match.

Table 12. Proposed rarity ratio thresholds for verbal interpretation scale

\begin{tabular}{|c|c|c|c|}
\hline Rarity ratio range & Interpretation of sample & Range sub-divisions & $\begin{array}{c}\text { Sub-division } \\
\text { interpretation }\end{array}$ \\
\hline $0-0.5$ & Absence of rare features & & \\
\hline \multirow{2}{*}{$0.5-1$} & $\begin{array}{c}\text { No additional } \\
\text { information from } \\
\text { weighting factors }\end{array}$ & $1-1.2$ & $\begin{array}{c}\text { Fracture edges with } \\
\text { added rare features }\end{array}$ \\
\cline { 3 - 4 } & & $1.2-1.4$ & $\begin{array}{c}\text { Fracture edges with } \\
\text { prevalent rare } \\
\text { features }\end{array}$ \\
\cline { 3 - 4 } & Rare features observed & $1.4-1.55$ & $\begin{array}{c}\text { Fracture edges with } \\
\text { highly prevalent } \\
\text { rare features }\end{array}$ \\
\cline { 3 - 4 } & & \multicolumn{2}{|c}{} \\
\hline
\end{tabular}

While most rarity ratios of true negative samples were in the $0-0.5$ "Absence of rare features" range, it is noted that a few non-match sample pairs fell in the 1-1.2 "Fracture edges with added rare features" range. While these were non-matching samples, they were still attributed weighting factors as distinctive characteristics were noted that assisted the examiner in determining the samples were not same source. Therefore, the pair did experience in increase in ESS between nonweighted and weighted, however both scores remained below 50\%. This demonstrates that the rarity ratio is intended to be used for interpretation of pair rarity within the sample set, regardless of ground truth. While the ESS and overall examiner conclusion signify the determination of match or non-match, the rarity ratio provides a verbal scale for the rarity of the observed edge features, indicating the strength of the respective match or non-match conclusion.

\subsection{Frequency of Occurrence of Distinctive Characteristics}

In order to further examine distinctive characteristics present per item across the data set, the relative frequency of occurrence of associated weighting factors was calculated. Relative frequencies are provided in Table $\mathbf{1 3}$ below and results are provided graphically in Figure 30. Relative frequencies were calculated from total number of examination bins present across the data set (20 pairs per item of 10 bins each, $n=200$ ). As shown, all items attributed some degree of separation characteristics through damage, gaps, or protrusions observed across fracture edges. Item B had the highest proportion of separation characteristics (25\%). Item C had the highest 
proportion of assigned weighting factor due to pattern continuation (47\%). This is expected even though both Items $\mathrm{C}$ and $\mathrm{E}$ exhibited patterns. As Item $\mathrm{C}$ consisted of vertical, multi-color stripes, the pattern was present in every bin compared across the total length of the fractured edges. Alternatively, Item E exhibited a randomly oriented clockface pattern, so pattern was not always present in every examination bin. Item $\mathrm{E}$ was the only textile that was initially observed to exhibit both overall and partial pattern fluorescence; therefore, it was the only item assigned weighting factors due to partial pattern fluorescence across an examination bin. However, it should be noted partial pattern fluorescence was also observed on Item B, and overall on Item C. Future work will include re-examination of Item B partial pattern fluorescence.

Items $\mathrm{D}$ and $\mathrm{E}$ had the lowest proportions of separation characteristics (6\% and 5\% respectively). Again, this was expected due to Item D being composed of $100 \%$ polyester and Item $E$ being of jersey knit construction. According to preliminary data, these two specifications led to greater distortion obstructing alignment features along fractured sample edges.

Table 13. Relative frequency of occurrence of weighting factor assignment

\begin{tabular}{|c|c|c|c|}
\hline & $\begin{array}{c}\text { Pattern } \\
\text { Continuation }\end{array}$ & $\begin{array}{c}\text { Separation } \\
\text { Characteristics }\end{array}$ & $\begin{array}{c}\text { Fluorescence } \\
\text { Continuation }\end{array}$ \\
\hline $\begin{array}{c}\text { Item A } \\
(\mathrm{n}=200)\end{array}$ & $0 \%$ & $10 \%$ & $0 \%$ \\
\hline $\begin{array}{c}\text { Item B } \\
(\mathrm{n}=200)\end{array}$ & $2 \%$ & $25 \%$ & $0 \%$ \\
\hline $\begin{array}{c}\text { Item C } \\
(\mathrm{n}=200)\end{array}$ & $47 \%$ & $13 \%$ & $0 \%$ \\
\hline $\begin{array}{c}\text { Item D } \\
(\mathrm{n}=200)\end{array}$ & $0 \%$ & $6 \%$ & $0 \%$ \\
\hline $\begin{array}{c}\text { Item E } \\
(\mathrm{n}=200)\end{array}$ & $21 \%$ & $5 \%$ & $18 \%$ \\
\hline $\begin{array}{c}\text { Overall } \\
(\mathrm{N}=1000)\end{array}$ & $14 \%$ & $12 \%$ & $4 \%$ \\
\hline
\end{tabular}




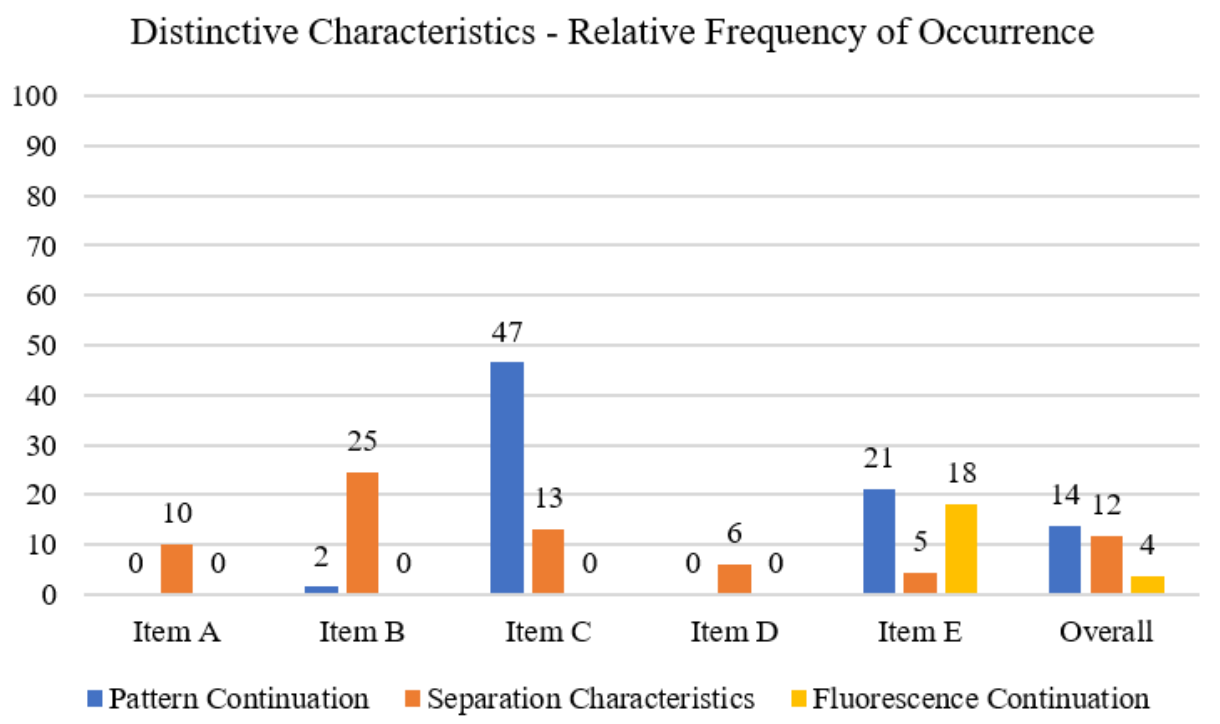

Figure 30. Graphical display of relative frequency of occurrence of weighting factor assignment (Note: fluorescence observations for Item B are being revisited in future work)

\section{Conclusions and Future Work}

Overall, this study represents the first time a quantitative, score-based method of physical fit assessment has been applied to textile materials. This study provides the foundation from which future textile physical fit research may expand and draws attention towards textile compositions and constructions that may be unsuitable for physical fit analysis due to high levels of disagreement between examiners caused by unpredictable distortions of the fractured edges that lead to both misclassification instances. This was shown through the preliminary jersey knit, $100 \%$ polyester set and supported by the lower accuracy resulting from textile items of similar composition and construction in the current study. In addition, this study proposes a novel verbal scale for the interpretation of distinctive alignment edge features present on fractured textile items for additional support of the strength of an examiner's match or non-match conclusion. Preliminary findings reveal a 3-step process is needed for textiles fracture edge comparison: 1) macroscopic observation of edge alignment and general characteristics, 2) microscopic examination and estimation of the ESS, and 3) computation of rare features per bin to estimate additional rarity ratio. This study presents a first attempt to define the description and examination of features that may be relevant in the assessment of textile fits and in future consensus-based methods.

Both the hand-torn and stabbed sample sets presented low error rates with accuracies ranging from 85-100\% depending on textile item. Lower accuracy rates were observed for items of either polyester composition (Item D) or jersey knit construction (Item E) for the hand-torn set, while woven, non-polyester items exhibited higher accuracy rates. This was attributed to higher distortion in the polyester or jersey knit items obstructing the examiners' view of edge alignment features. Frequency of occurrence results in distinctive characteristics across the sample set 
support this, as woven materials tended to exhibit a greater percentage of separation characteristics than other materials. For the stabbed sample set, it was observed that patterned materials (Items C and E) exhibited higher accuracy rates than solid-colored items. This was attributed to the added potential of pattern alignment (or misalignment) on items presenting otherwise "featureless" edges due to the stabbing separation mechanism.

Further analysis of examiner notes revealed two main methodology discrepancies dealing with treatment of gaps within a sample as well as inconsistent fracture edge length between two items. While Examiner A tended to manipulate samples to gain an understanding if gaps were due to an item separating from itself rather than another item, Examiner B treated these gaps as inconsistencies between the pair if the other item did not have a corresponding protrusion. In addition, Examiner A tended to take bin divisions from the smaller fracture edge length of two compared items while Examiner B tended to take bin divisions from the larger of the two. Both of these methodology discrepancies may be alleviated through further examiner training and specific distinction of bin division criteria prior to sample analysis, which may be implemented in a future study. Regardless of examiner discrepancies, only 12 misclassifications were observed across the entire data set. Only one of these was a false positive, with the remainder consisted of false negatives and inconclusive conclusions (not true misclassifications). These results are less detrimental to casework as negative or inconclusive samples would typically be taken through further physical and chemical analysis in a forensic laboratory.

This study represents a successful first expansion of the previously developed duct tape physical fit ESS methodology to an additional material. The results highlighted the relevance of development of material-specific approaches, as the factors that influence the quality of a match and error rates varied widely between duct tapes and textiles. Future work will include studies of expanded textile factors such as additional compositions, constructions, and external factors such as degree of wear. This work will identify any needed modifications to the ESS method to best account for additional encountered separation characteristics due to fabric type. Expanded work and increased sample sets will also assist in the fine-tuning of the proposed verbal interpretation based upon rarity ratio thresholds. Finally, an inter-laboratory study will be initiated to validate the now developed textile ESS methodology.

\section{References}

1. Grieve M, Houck MM. Introduction. In: Houck MM, editor. Trace Evidence Analysis: More Cases in Mute Witnesses. Burlington, MA: ElSevier Academic Press; 2004. p. 1-26.

2. Fisher BAJ, Svensson A, Wendel O. Techniques of Crime Scene Investigation. 4th ed. Fisher BAJ, editor. New York, NY: Elsevier Science Publishing Co., Inc.; 1987.

3. Shor Y, Novoselsky Y, Klein A, Lurie DJ, Levi JA, Vinokurov A, Levin N. The Identification of Stolen Paintings Using Comparison of Various Marks. Journal of Forensic Sciences. 2002:633637. 
4. Gross S. NIST-OSAC Materials (Trace) Subcommittee, physical fit task group, 2020 physical fit survey.

5. Dann T, Malbon C. Tearing or Ripping of Fabrics. In: Carr D, editor. Forensic Textile Science. Cambridge, MA: Woodhead Publishing; 2017. p. 169-180.

6. Kemp SE, Carr DJ, Kieser J, Niven BE, Taylor MC. Forensic evidence in apparel fabrics due to stab events. Forensic Science International. 2009;191:86-96. doi:10.1016/j.forsciint.2009.06.013

7. Pelton WR. Distinguishing the Cause of Textile Fiber Damage Using the Scanning Electron Microscope (SEM). Journal of Forensic Sciences. 1995;40(5):874-882.

8. Brooks E, Prusinowski M, Gross S, Trejos T. Forensic physical fits in the trace evidence discipline: A review. Forensic Science International. 2020. doi:10.1016/j.biteb.2019.100321

9. Prusinowski M, Brooks E, Trejos T. Development and validation of a systematic approach for the quantitative assessment of the quality of duct tape physical fits. Forensic Science International. $2020 ; 307$. 


\title{
V. CHAPTER FOUR
}

\section{Optimization and Evaluation of Spectral Comparisons of Electrical Tape Backings by X-ray Fluorescence}

\begin{abstract}
:
Electrical tape can be relevant forensic evidence in high-profile casework involving shootings or explosive devices. It is critical that practitioners have access to rapid, informative, and minimally invasive techniques of analysis to best support these investigations. The characterization of electrical tape backings through X-ray Fluorescence (XRF) Spectroscopy has been shown to be a highly discriminatory, non-destructive method of analysis requiring limited sample preparation. This study describes the process of parameter optimization of an XRF method for casework use. The work expands upon previous discrimination studies by broadening the total sample set of characterized tapes and evaluating the use of spectral overlay, spectral contrast angle, and Quadratic Discriminant Analysis (QDA) for the comparison of XRF spectra. The expanded sample set consisted of 114 samples, 94 from different sources of which 90 were previously analyzed, and 20 from the same roll. For each sample, replicate measurements on different locations of the tape were analyzed $(n=3)$ to assess the intra-roll variability. XRF provided superior discrimination to Scanning Electron Microscopy with Energy Dispersive Spectroscopy (SEM-EDS) on the expanded dataset and a more comprehensive elemental characterization (15 elements by XRF vs. 8 by SEM-EDS). While previous SEM-EDS analysis of the 90 electrical tapes resulted in 15 distinct groups and a discrimination power of $87.3 \%$, current XRF analysis considering the equivalent 90 electrical tapes resulted in 61 distinct groups with further subgroups providing a discrimination power of $96.7 \%$.

Duplicate controls and tape fragments from the same roll were also analyzed to assess inter-day, intra-day, and intra-roll variability $(n=20)$. Parameter optimization included comparison of atmospheric conditions, collection times, and instrumental filters. A study of the effects of adhesive and backing thickness on spectrum collection revealed key implications to the method that required modification to the sample support material. As an electrical tape standard reference material does not currently exist, NIST SRM 1831, a standard soda-lime glass, was found to be an adequate reference material for daily performance assessment of the instrument.

In addition, figures of merit assessed included accuracy and discrimination over time, precision, sensitivity, and selectivity. The performance of different methods for comparing and contrasting spectra was also evaluated. The optimization of this method was part of an assessment to incorporate XRF to a forensic laboratory protocol for rapid, highly informative elemental analysis of electrical tape backings and to expand examiners' casework capabilities.
\end{abstract}

\section{Introduction}

Pressure-sensitive tapes are often involved in the commission of a crime due to their low cost, ease of use, and their readily available nature. Specifically, electrical tape is commonly submitted to forensic laboratories in reference to crimes such as shootings (e.g., tape used for modifications to 
weapons) or bombing events (e.g., tape remaining from an improvised explosive device). It is critical that forensic scientists have access to rapid, highly discriminatory techniques to best utilize the potential of this type of physical evidence.

In a typical analytical scheme for electrical tape comparative analysis, examinations begin with physical characteristics and continue to chemical analysis if a discrimination is not made between items. Examination of physical characteristics includes documentation of color and thickness of respective backing and adhesive layers, as well as the overall width and surface texture. ${ }^{1}$ A full analytical scheme also consists of a combination of chemical and elemental techniques to provide a comprehensive characterization of all components of a tape sample. All-encompassing analytical schemes for electrical tapes are well-established in the literature. ${ }^{1-7}$

Electrical tape is composed of a backing and adhesive layer. Backing components can include the main polymer, plasticizers, fillers, pigments, flame retardants, stabilizers, and lubricants. The most common polymer used for electrical tape backings is polyvinyl chloride (PVC), but other polymers such as polyethylene, polypropylene, polyester, and polyimide are also used. ${ }^{3,4}$ Plasticizers are often added to soften the polymer to provide flexibility to the tape backing. These include aromatic plasticizers such as dialkyl phthalate esters or trialkyl trimellitate esters, or aliphatic plasticizers such as dialkyl adipate esters or tricresyl phosphates. ${ }^{6}$ Other components such as carbon black, calcium carbonate, titanium dioxide, barium sulfate, kaolin, talc, and dolomite are used as opacifiers, colorants and fillers. ${ }^{4,6}$ Flame retardants reduce the flammability of electrical tape due to the added plasticizers. Some common flame retardants include antimony oxide and aluminum hydroxide. ${ }^{6}$ Stabilizers, such as lead carbonate and lead sulfate, are added to prevent decomposition or ultraviolet irradiation degradation. ${ }^{6}$ Finally, adhesive components include a base elastomer (e.g., polyisoprene, polybutadiene), copolymers [e.g., poly(styrene-co-isoprene) or poly(styrene-co-butadiene), and poly(butylacrylate], and tackifying resins (e.g., wood rosin, terpene resins, and petroleum resins), along with aromatic and/or aliphatic plasticizers, antioxidants, flame retardants, and fillers., 4

Chemical analysis techniques vary depending upon the availability of the instruments and associated sample size. For example, Fourier-Transform Infrared Spectroscopy (FTIR) is a nondestructive method that reveals information on organic and some inorganic components of a tape sample, while Pyrolysis Gas Chromatography/Mass Spectrometry (py-GC/MS) can provide further characterization of the polymeric components. However, if there is a desire to preserve an evidence item of limited size, py-GC/MS may not be utilized as it is a destructive method. ${ }^{5}$ Elemental methods are used to characterize the inorganic components of the tape sample such as stabilizers, flame retardants, and fillers. ${ }^{6}$ Common methodology for electrical tapes includes Scanning Electron Microscopy with Energy Dispersive X-Ray Spectroscopy (SEM-EDS), ${ }^{3}$ which provides both an elemental profile of the sample and a topographic image of the scanned surface. ${ }^{5,6}$

This traditional analytical scheme was employed in a previous study by Mehltretter et $a l .{ }^{4}$ in which a set of 90 black electrical tapes was characterized by the physical and chemical characteristics of their backings. Physical examination resulted in a discrimination power of $64 \%$, while FTIR, pyGC/MS, and SEM-EDS analyses resulted in discrimination powers of $83 \%, 81 \%$, and $87 \%$, respectively. Considering the overall analytical scheme of the tape backings, the authors achieved 
94\% discrimination. ${ }^{4}$ Combining the adhesive with the backing results for the same sample set, the discrimination was raised to $96 \% .^{3}$

While high discrimination was achieved in the Mehltretter studies, ${ }^{3,4}$ a full analytical scheme for both the adhesive and backing of all tape samples was required. Additional research has reported on rapid techniques that are able to achieve high discrimination as a screening method to complement conventional analytical schemes such as X-ray Fluorescence Spectroscopy (XRF) ${ }^{8-}$ 10 and Laser Ablation - Inductively Coupled Plasma - Mass Spectrometry (LA-ICP-MS). ${ }^{11,12}$ Of these methods, XRF is easier to operate, non-destructive, and more widely available in forensic laboratories.

X-ray Fluorescence (XRF) Spectroscopy utilizes an X-ray beam to initiate photoelectric absorption in atoms present in the sample. This energy absorption occurs if the energy of the X-ray photons irradiating the sample is larger than the binding energy of the inner electron orbitals of a given atom, and results in inelastic ejection of an electron from its inner shells within the orbital. As an outer orbital electron transfers to fill this vacancy to restore the system stability, an X-ray photon is produced with an energy equivalent to the energy difference between the initial and final quantum states of the electron. Characteristic X-ray emission lines correspond to peaks within the resulting spectrum that can be used to identify the elemental composition of the sample in question. $^{13}$

XRF was previously utilized by Kee in the characterization of 131 black PVC electrical tape backing samples obtained through casework from 1980 to 1981. One-centimeter length tape segments were cut from respective rolls. Their backings were wiped with hexane prior to analysis, and samples were mounted on Mylar film held by a plastic sample cup. Only the top surface of the tape backing was analyzed. Four major classes were identified due to the presence or absence of lead and calcium, with further discrimination into 15 subclasses due to the presence of additional phosphorus, antimony, silicon, sulfur, and titanium. ${ }^{8}$ XRF analysis was also utilized in a study by Keto in which two rolls each of six tape brands were characterized according to the presence or absence of ten elements: aluminum, silicon, sulfur, chlorine, antimony, calcium, titanium, iron, zinc, and lead. Means and standard deviations of resulting counts were assessed to determine low within-brand variability and sufficient variability between brands to allow for discrimination. ${ }^{9}$

In a previous study by Prusinowski et al., the authors utilized three different XRF instrumental configurations to compare discrimination power when characterizing a set of 40 electrical tape backings. ${ }^{10}$ The results were compared to those of previous studies examining the same set of electrical tapes. ${ }^{4,11}$ XRF was found to be comparable to LA-ICP-MS when considering N=40 overall samples, with the most sensitive XRF configuration achieving a discrimination power of $90.1 \%$ as opposed to LA-ICP-MS at $84.6 \%$. The difference in discrimination power was noted to likely be a result of the presence of iron in the XRF spectra, whereas iron can be difficult to detect on standard quadrupole LA-ICP-MS instruments due to common polyatomic interferences. The enhanced discrimination by XRF was also attributed to an instrumental configuration with a larger spot size (e.g., $1 \mathrm{~cm}$ vs. 100-300 $\mu \mathrm{m}$ ). In addition, the Prusinowski study ${ }^{10}$ evaluated a semiquantitative method to compare samples. The relative area under the relevant elemental peaks in the XRF spectra was calculated and compared using Analysis of Variance (ANOVA) to determine which sample signal-to-noise ratios (SNRs) were significantly different. ${ }^{10}$ 
The aim of the current study was to evaluate the XRF method for use within a forensic laboratory by optimizing each selected parameter including atmospheric condition, collection time, sample support material, filters used, adhesive effects, and backing thickness effects. Further experimentation was then performed utilizing optimized parameters for assessments of accuracy and discrimination over time, precision, sensitivity, and selectivity. In addition, the previous sample set of 40 electrical tapes ${ }^{10}$ was increased to a full characterization of 94 samples originating from different-product rolls as well as an intra-roll variability study consisting of 20 same roll samples.

Following data collection, data analyses performed included spectral overlay comparison, estimation of spectral contrast angle ratios, and Quadratic Discriminant Analysis (QDA).

Spectral overlay and contrast angle comparison methods are useful for determining if respective XRF spectra demonstrate two tape samples originated from different sources. Likewise, a spectral comparison is informative in determining if two samples known to originate from the same source (e.g., same roll) produce indistinguishable spectra. When the ground truth of sample origin is known, these methods can be applied to evaluate false positives, false negatives, and accuracy. When the source of the sample is unknown, as in casework, the comparison methods serve to inform the examiner's opinion about whether or not the samples of interest could have originated from a common source.

During XRF spectral overlay comparisons, the spectra are superimposed to determine if the observed variability within the same source (i.e., replicate spectra of the known tape and replicate spectra of the questioned sample) is smaller than the variability between the compared items (e.g., spectra of known versus questioned tape). The variability of XRF spectra is assessed by differences of spectral shape or location (x-axis) and differences in the relative intensity of the peaks (y-axis). When those spectral differences between the compared samples are outside the variability of spectra originating from the same source, the samples are distinguished. Spectral overlay is a fast and intuitive method of comparison that provides simple distinction of large differences between samples. The method is widely used in forensic science and in spectrochemical comparisons in general.

However, when the compared spectra are similar and differences between samples are much smaller (i.e., a peak intensity (y-axis) difference only and no peak shape/location (x-axis) differences), it becomes more difficult for the examiner to determine if these differences are sufficient to distinguish or associate two samples. As a result, there are several alternative methods and software features that can aid in the quantitative and automated assessment of the similarities and differences between spectra. In this study, we proposed to evaluate the use a well-known and straightforward comparison method using spectral contrast angles to establish the level of similarity among spectra. In this method, each XRF spectrum can be represented as a vector whose length and orientation are determined by the peak energy (x-axis, keV) and intensities (y-axis, counts) of the spectrum. Then, the angle between the vectors of the compared spectra is calculated. The smaller the angle between the compared vectors, the more similar the spectra and vice versa. For instance, if two identical spectra were compared, the respective vectors would superimpose each other, resulting in a zero-degree angle. On the other hand, if two very different spectra were compared, the known and questioned vectors could show a difference as large as a 90-degree 
angle. ${ }^{14}$ Therefore, the contrast angle is utilized in this paper as a means to evaluate the similarity between spectra and complement the examiner's observations using visual spectral overlay comparisons. The utility of this method is assessed in this study as a proof of concept, but additional research would be needed before adopting it in casework.

Additionally, by evaluating spectral data by country of origin, valuable information pertaining to elemental differences by source may be achieved, assisting in the explanation of sample differences. Although not used in current practice, another research question of interest in this study is whether or not the XRF profile of electrical tapes can provide information about a potential source of origin. In this study, we use a fundamental classification method based on quadratic discriminant analysis (QDA) to identify if the samples can be reasonably grouped by country of origin based on their elemental composition. The objective of QDA is to use an algorithm that recognizes the maximum variation between classes or groups and use these features as variables to provide a plot of group clustering. Usually, the classes of the training set, such as country of origin, are known (i.e., supervised classification that learns a pattern based on predetermined categories). Discriminant analysis is a well-known supervised classification method for multivariate data that can be used to predict the grouping of a new sample or to gain insight into the relationships that may exist among the variables. In other words, discriminant analysis can become useful for variable selection to determine which set of features (e.g., specific elements) can best determine group membership or to identify what classification model best separates the groups of interest.

\section{Methods}

\subsection{Instrumentation}

The instrument used in this study was a Thermo Scientific ARL QUANT'X energy dispersive XRF spectrometer with specifications as shown in Table 1.

Table 1. XRF instrumental specifications

\begin{tabular}{|c|c|}
\hline X-ray Source & Rh \\
\hline Detector & SiLi (PCD) \\
\hline Spot Size Diameter & $\sim 1 \mathrm{~cm}$ \\
\hline Voltage $(\mathrm{kV})$ & Low $12 \mathrm{kV}$, Mid $28 \mathrm{kV}$, High 50 kV \\
\hline Current $(\mu \mathrm{A})$ & Low $200 \mu \mathrm{A}$, Mid $100 \mu \mathrm{A}$, High $300 \mu \mathrm{A}$ \\
\hline Working Distance & $54.1 \mathrm{~mm}$ \\
\hline Target Dead Time & $50 \%$ \\
\hline
\end{tabular}

\subsection{Sample Collection and Preparation}

A set of 90 electrical tapes, as previously characterized by Mehltretter et al. ${ }^{3,4}$ and Martinez et $a l .,{ }^{11}$ with the addition of four rolls purchased in 2019 to assess more contemporary formulations, 
was characterized with optimized XRF parameters. Product information for the expanded sample set $(\mathrm{N}=94)$ is provided in Table A.1 of the Appendix.

Full width tape samples $\sim 5-6 \mathrm{~cm}$ in length were cut from each roll. A sample size of at least $2 \mathrm{~cm}$ in length was ideal to account for interaction of the detector aperture diameter with the tape. However, smaller portions can be analyzed with the use of polypropylene or Mylar film, although not assessed in this study. Adhesive was removed from the backing in a region $\sim 2-3 \mathrm{~cm}$ in length and across the full tape width to provide a large enough area for the $\sim 1 \mathrm{~cm}$ beam diameter. This becomes critical when attempting replicates of the same sample in various areas of the adhesiveremoved region. Adhesive removal took place with acetone or hexane. Samples were placed on glass microscope slides within square Petri dishes for transportation and storage.

Samples were loaded into the instrument by positioning the tape over the detector aperture with the adhesive-free region centered. The remaining adhesive on each end of the tape sample was used to adhere the sample to the stage edges surrounding the detector aperture. A lucite planchet was placed on top of the tape sample to reduce X-ray interaction with the chamber material. A minimum of three replicates were collected when analyzing each tape sample. Replicates were collected by shifting and rotating the sample over the detector aperture between runs to expose different areas within the adhesive-free region of the tape sample.

\subsection{Daily Performance}

Each day an energy verification was performed as recommended by the instrument manufacturer. This consisted of analysis of an oxygen-free high thermal conductivity (OFHC) copper standard. A successful verification resulted in gain settings with a difference no greater than 100 between previous and current settings as well as a full width at half maximum not exceeding $195 \mathrm{eV}$.

Daily performance throughout the study consisted of both morning and afternoon runs of a previously selected, blind duplicate tape sample along with standard soda-lime glass NIST SRM 1831. The $\mathrm{Cl} / \mathrm{Ca}$ ratio was monitored in the daily tape sample to assess any extraneous variability, while Ti (low filter only) and Sr (mid and high filters only) peaks were monitored in NIST SRM 1831 according to guidelines set in ASTM E2926-17 ${ }^{15}$.

\subsection{Parameter Optimization Experiments}

Although the method had been previously developed by Prusinowski et al. ${ }^{10}$ all parameters were tested to assure optimal conditions were selected as appropriate for casework implementation.

\subsubsection{Atmospheric Conditions}

Six tape samples (tapes 45, 68, 85, 91, 93, and 94) were run both in air and under vacuum for 60 live seconds, with three replicates each of the aluminum (low Zc), thick palladium (mid Zc), and thick copper (high $\mathrm{Zb}$ ) filters. These tapes represented three previously characterized samples as well as three recently acquired samples, all with an expected range of both low and high $\mathrm{Z}$ elements as per previous publications. ${ }^{10}$ It should be noted that prior to filter comparison experimentation (Section 2.4.4.), filters selected in the previous study were used to keep parameters constant. 
Spectral overlays were performed after analysis to determine at which atmospheric condition peaks were best detected and resolved.

\subsubsection{Collection Time}

The six tape samples in Section 2.4.1. were run under vacuum for 20, 60, and 100 live seconds, collected in triplicate at each filter. Spectral overlays were then performed to determine at which collection time element peaks were best resolved with highest counts, while still adhering to an efficient overall analysis time.

\subsubsection{Sample Support Material Analysis}

To assure the sample support material was not contributing any extraneous peaks to sample spectra, the beryllium planchet used as the support material in the previous study was analyzed under vacuum in triplicate using each filter. For comparative purposes, a lucite planchet was also run under the same conditions.

\subsubsection{Filter Comparison}

The six tape samples described in Section 2.4.1. were each run in triplicate under vacuum for 60 live seconds with each of the filtering conditions given below:

a. As recommended by Prusinowski et al.: ${ }^{10} \mathrm{Al}$ (low Zc), thick $\mathrm{Pd}$ (mid Zc), and thick $\mathrm{Cu}$ (high Zb)

b. Additional filters as recommended for common electrical tape elements by instrument manufacturer excitation filter guide: No filter (low Za), cellulose (low Zb), thin Pd (mid $\mathrm{Za}$ ), medium $\mathrm{Pd}$ (mid $\mathrm{Zb}$ ), and thin $\mathrm{Cu}$ (high $\mathrm{Za}$ )

Spectral overlays were performed to examine any elemental signal lost or gained due to filter selection.

\subsubsection{Adhesive Effects}

Six tape samples of various adhesive composition (as determined by both color and SEM-EDS characterization by Mehltretter et al. ${ }^{3}$ ) were analyzed both before and after adhesive removal. The six tape samples selected were tapes representing various adhesive colors and compositions as follows:

a. Clear, colorless: 3,42

b. Clear with brown tint: 33,62

c. Opaque, black: 12,47

Samples were run in triplicate under vacuum for 60 live seconds at each filter. Spectral overlays were performed to determine if any interferences occurred due to the presence of the adhesive, which would require its removal before backing analysis.

\subsubsection{Backing Thickness Effects}

The six tape samples in Section 2.4.5. were analyzed (post adhesive-removal) both before and after hand-stretching to simulate common sample conditions in a casework scenario. Samples were run in triplicate under vacuum for 60 live seconds at each filter. Spectral overlays were performed to determine if any interferences were caused by thinner, stretched backings. 


\subsubsection{NIST SRM 1831 Analysis}

NIST SRM 1831 was run under the same conditions as previously run tape samples ${ }^{10}$ to assess suitability for a performance standard by observing if $\mathrm{Na}, \mathrm{Mg}, \mathrm{Al}, \mathrm{K}, \mathrm{Ca}, \mathrm{Ti}, \mathrm{Mn}, \mathrm{Fe}, \mathrm{Rd}, \mathrm{Sr}$, and $\mathrm{Zr}$ were detected. ${ }^{15}$ Runs took place under vacuum for 60 live seconds, collected in triplicate at each filter.

\subsection{Method Evaluation Using Optimized Parameters}

Following the optimization of the method, additional experiments were performed utilizing the optimized parameters, along with the tape set characterization and intra-roll variability studies.

\subsubsection{Accuracy and Discrimination Over Time}

\subsubsection{NIST SRM 1831}

The glass standard was run under optimized conditions in 24 replicates to confirm all elements detected by the method were consistent with ASTM Standard Method E2926-17 ${ }^{15}$ quality control recommendations. All peaks observed in the spectra were integrated according to the method described by Ernst et al. ${ }^{16}$ Elements with a signal-to-noise (SNR) ratio above 3 were considered present. Table 2 below provides the energy ranges used for NIST 1831 SNR calculations.

Table 2. Energy ranges (keV) for NIST SRM 1831 elements

\begin{tabular}{|c|c|c|c|}
\hline Element & Pre-peak & Peak & Post-peak \\
\hline $\mathrm{Na}$ & $0.58-0.76$ & $0.94-1.12$ & NA \\
\hline $\mathrm{Mg}$ & $1.04-1.18$ & $1.20-1.34$ & $\mathrm{NA}$ \\
\hline $\mathrm{Al}$ & $1.32-1.42$ & $1.46-1.56$ & $\mathrm{NA}$ \\
\hline $\mathrm{Si}$ & $1.32-1.40$ & $1.66-1.84$ & $1.86-1.94$ \\
\hline $\mathrm{K}$ & $2.94-3.16$ & $3.20-3.42$ & $\mathrm{NA}$ \\
\hline $\mathrm{Ca}$ & $3.32-3.54$ & $3.58-3.80$ & $\mathrm{NA}$ \\
\hline $\mathrm{Ti}$ & $4.24-4.34$ & $4.38-4.60$ & $4.64-4.74$ \\
\hline $\mathrm{Mn}$ & $5.48-5.70$ & $5.76-5.98$ & $\mathrm{NA}$ \\
\hline $\mathrm{Fe}$ & $6.18-6.28$ & $6.32-6.54$ & $6.58-6.68$ \\
\hline $\mathrm{Rb}$ & $\mathrm{NA}$ & $13.22-13.52$ & $13.56-13.86$ \\
\hline $\mathrm{Sr}$ & $13.76-13.92$ & $13.96-14.30$ & $14.34-14.50$ \\
\hline $\mathrm{Zr}$ & $15.34-15.52$ & $15.56-15.94$ & $15.98-16.16$ \\
\hline
\end{tabular}

\subsubsection{2. $\quad$ Tape Samples}

Three previously characterized tape samples were run under optimal conditions in triplicate. Results were compared to elemental composition as reported via SEM-EDS, XRF (iBeam, Quant'X, Bruker), and LA-ICP-MS. ${ }^{4,10,11}$

The selected tapes were samples 6, 8 , and 36 as they were previously reported to encompass all elements commonly found in electrical tapes including $\mathrm{Al}, \mathrm{Si}, \mathrm{Cl}, \mathrm{Ca}, \mathrm{Sb}, \mathrm{Ba}, \mathrm{Ti}, \mathrm{Fe}, \mathrm{Zn}, \mathrm{Pb}, \mathrm{Br}$, $\mathrm{Cd}, \mathrm{Cr}$, and Mo. 


\subsubsection{Sensitivity}

\subsubsection{NIST SRM 1831}

NIST SRM 1831 was analyzed under optimal conditions in 24 replicates. Limits of detection (LOD) were estimated for detected elements.

\subsubsection{Tape Samples}

The tape samples from Section 2.5.1.2. with the addition of tape sample 91 (a contemporary formulation) were analyzed under optimal conditions in triplicate. Results from SEM-EDS, other XRF instruments, and LA-ICP-MS were compared for each element to evaluate differences in sensitivity between techniques.

\subsubsection{Precision}

\subsubsection{Tape Samples}

Tape sample 10, the same tape selected as the blind duplicate in the previous study, ${ }^{10}$ was run under the same conditions both in the morning and afternoon for ten days over three weeks of the study. The $\mathrm{Cl} / \mathrm{Ca}$ ratio was selected for monitoring of repeatability and intermediate precision, as this ratio had the greatest variation between samples. The assessment was performed through spectral overlay and analysis of relative standard deviation values.

\subsubsection{Selectivity}

Tape samples determined to exhibit either $\mathrm{Ca} / \mathrm{Sb}$ or $\mathrm{Ba} / \mathrm{Ti}$ interferences during the previous study were re-analyzed under optimal conditions to determine if any of these elements were resolved. Selected samples are provided below:
a. $\mathrm{Ba} / \mathrm{Ti}$ only: Sample 6
b. $\mathrm{Ba} / \mathrm{Ti}$ and $\mathrm{Ca} / \mathrm{Sb}$ : Sample 8
c. $\mathrm{Ca} / \mathrm{Sb}$ only: Sample 36

\subsection{Tape Set Characterization and Discrimination ( $\mathrm{N}=94)$}

Each tape sample in the set of 94 was run in triplicate under optimal conditions. All peaks observed in the spectra were integrated according to the method described by Ernst et al. ${ }^{16}$ Elements with a signal-to-noise (SNR) ratio above 3 were used for comparisons. Table 3 below provides the energy ranges used for tape element calculations. Examples of peak appearance for various SNR values both below, near, and above the selected threshold of 3 are provided in Figures A.1-A.3 of the Appendix. 
Table 3. Energy ranges $(\mathrm{keV})$ for tape elements

\begin{tabular}{|c|c|c|c|}
\hline Element & Pre-peak & Peak & Post-peak \\
\hline $\mathrm{Al}$ & $1.32-1.42$ & $1.46-1.56$ & NA \\
\hline $\mathrm{Si}$ & $1.32-1.40$ & $1.66-1.84$ & $1.86-1.94$ \\
\hline $\mathrm{Cl}$ & $2.28-2.38$ & $2.52-2.74$ & $2.90-3.00$ \\
\hline $\mathrm{Ca} / \mathrm{Sb}$ & $3.32-3.54$ & $3.58-3.80$ & NA \\
\hline $\mathrm{Ba} / \mathrm{Ti}$ & $4.24-4.34$ & $4.38-4.60$ & $4.64-4.74$ \\
\hline $\mathrm{Cr}$ & $5.18-5.28$ & $5.30-5.52$ & $5.58-5.68$ \\
\hline $\mathrm{Fe}$ & $6.18-6.28$ & $6.32-6.54$ & $6.58-6.68$ \\
\hline $\mathrm{Zn}$ & $8.32-8.46$ & $8.50-8.80$ & $8.84-8.98$ \\
\hline $\mathrm{Pb}$ & $10.08-10.28$ & $10.32-10.74$ & $10.78-10.98$ \\
\hline $\mathrm{Br}$ & $11.72-11.80$ & $11.84-12.02$ & $12.06-12.14$ \\
\hline $\mathrm{Sr}$ & $13.76-13.92$ & $13.96-14.30$ & $14.34-14.50$ \\
\hline $\mathrm{Mo}$ & $16.98-17.16$ & $17.26-17.64$ & $17.68-18.86$ \\
\hline $\mathrm{Cd}$ & $22.60-22.78$ & $22.90-23.28$ & $23.44-23.62$ \\
\hline $\mathrm{Sb}^{*}$ & $25.40-25.76$ & $25.86-26.60$ & $26.64-27.00$ \\
\hline $\mathrm{Ba}^{*}$ & $31.36-31.60$ & $31.90-32.40$ & $32.80-33.04$ \\
\hline
\end{tabular}

*Elements denoted with an asterisk indicate those resolved with the thick copper (high $\mathrm{Zb}$ ) filter.

Samples were initially grouped by spectral overlay comparisons depending upon the presence/absence of elements. Groups were then further discriminated into subgroups based on spectral overlay differences in peak height between samples as performed in past studies. ${ }^{10,11}$

These groupings were confirmed by spectral contrast angle comparison, first by determining the contrast angle between every possible combination of replicates within the same sample (intra-roll contrast angle). The contrast angle was then calculated between every combination of replicates between two compared samples (between-samples contrast angle). Averages were taken of each. This calculation was performed according to Equation $\mathbf{1}^{14}$ below for every $x-y$ data-point of a spectrum, where $i$ indicates the maximum $\mathrm{x}$-axis energy $(\mathrm{keV})$ value for the spectra being considered ( $i=20.46$ for low Zc filtered spectra; $i=40.94$ for mid $\mathrm{Zc}$ or high $\mathrm{Zb}$ filtered spectra). Therefore, in Equation 1, ${ }^{14}$ Spectrum $1_{i}$ refers to the counts or intensity value at every energy increment of the x-axis of Spectrum 1. Likewise, Spectrum2 $i$ refers to the counts or intensity value at every energy increment of the x-axis in Spectrum 2. In this way, overall contrast angle equation is able to provide a comparison value considering every data point of each spectrum.

$$
\begin{gathered}
\cos \theta=\frac{\sum_{i} \text { Spectrum } 1_{i} \text { Spectrum }_{i}}{\sqrt{\sum_{i} \text { Spectrum } 1_{i}^{2} \sum_{i} \text { Spectrum } 2_{i}^{2}}} \\
\text { Spectral Contrast Angle Ratio }=\frac{\text { Mean } \theta \text { (between-samples) }}{\text { Mean } \theta \text { (within-samples) }}
\end{gathered}
$$

Following determination of average contrast angles both within and between samples, a ratio between the two values was taken as a representation of the relative similarity between compared spectra, as shown in Equation 2. For instance, to estimate the contrast ratio of three replicates of 
sample A (A1, A2, A3) and three replicates of sample B (B1, B2, B3), the numerator will be calculated from the mean contrast angle of all comparison pairs between the two spectra. That is, the between-sample contrast angle will be the mean of the contrast angle of the following spectral comparisons: A1-B1, A1-B2, A1-B3, A2-B1, A2-B2, A2-B3, A3-B1, A3-B2 and A3-B3. Then, the denominator is calculated as the mean of all comparisons within the same sample (A1-A2, A1A3, A2-A3, and B1-B2, B1-B3 and B2-B3). A larger value indicates greater between-sample difference relative to the intra-roll variation, while a smaller value indicates more similarities between the compared samples.

The intra-sample contrast angle ratio was determined for all possible comparison pairs of samples considered indistinguishable through spectral overlay from groups 4b, 5, 9a-d, 15, 17, 19a, 23, and 31a (see Table A.2 in the Appendix, $n=132$ comparison pairs) and from all possible comparison pairs from the 20 fragments originating from the same roll ( $\mathrm{n}=190$ comparison pairs). The mean and standard deviation of the ratio values were determined to establish an expected range of an "indistinguishable sample" contrast ratio (e.g., same source, same group, same roll). Inter-sample contrast angle ratios were then determined between samples considered distinguished by spectral overlay, one from each subgroup (e.g., different source samples $\mathrm{n}=21$ comparisons) and all possible comparison pairs between samples of different groups ( $\mathrm{n}=794$ comparisons). The intrasample ratio was then used as a threshold to estimate similarity between spectra. If the mean contrast angle for the samples compared fell outside the range of intra-samples, the samples were considered different by XRF. All calculations were conducted in Microsoft Excel (Version 19.08) and R Studio (Version 3.6.1) and a copy of the calculation templates is provided in the Supplementary Material.

Quadratic Discriminant Analysis (QDA) was also performed on the overall dataset to observe clustering due to elemental similarities or differences between varying tape countries of manufacture. QDA was performed in JMP® Pro Software Version 14.0.0. It should be noted that all spectral comparisons, both overlays and statistical analyses, were performed on spectra with normalized counts.

\subsection{Intra-roll Variability Study}

In a similar manner to the previous study, an additional tape roll (Super 33+, Scotch $3 \mathrm{M}^{\circledR}$, Saint Paul, MN) was selected to analyze intra-source variability with newly optimized parameters. Twenty samples were taken from the roll, with the first sample being 38 " from the starting edge of the roll and the remaining 19 taken every 38" into the roll. These increments were selected to account for evenly spaced samplings across the entire length of the roll. All samples were analyzed in triplicate under optimal conditions. Data analysis consisted of spectral overlay and spectral contrast angle ratio comparisons between intra-roll samples, per filtering condition, to determine any exclusionary differences. 


\section{Results}

\subsection{Parameter Optimization Experiments}

\subsubsection{Atmospheric Conditions}

Overall enhanced counts, mostly at lower energy peaks, were observed under vacuum as compared to in air. An example of this elemental enhancement is shown in Figure 1. For this reason, optimal atmospheric condition was determined to be vacuum. This parameter is consistent with the previous study. ${ }^{10}$

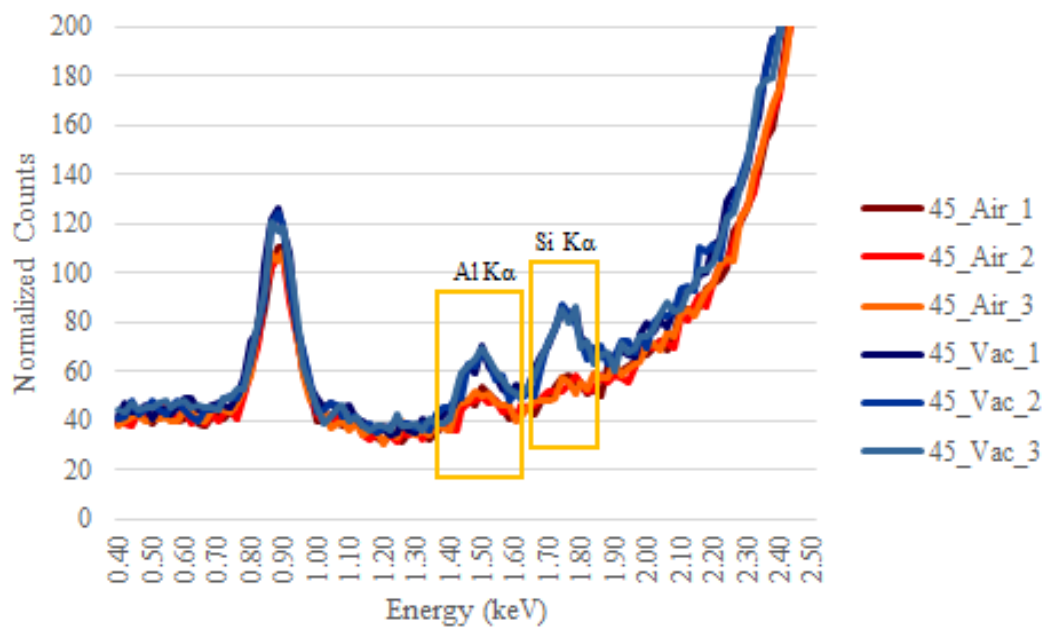

Figure 1. Spectra overlay comparison of tape 45 run both in air (3 reps) and under vacuum (3 reps), low Zc filter

\subsubsection{Collection Time}

Highest overall counts and respective SNRs were observed with 60 live seconds as compared to 20. While a 100 second collection time resulted in higher overall counts, no additional elements were observed beyond 60 seconds. Therefore, for the purposes of this study, 60 seconds was selected as the optimal collection time for a compromise of sensitivity and speed of analysis. However, during casework an examiner may choose to increase collection time for enhanced counts if desired. The selected collection time is meant to serve as a minimum value.

\subsubsection{Sample Support Material Analysis}

As the instrument's beam penetration depth has the capability to surpass the typical thickness of electrical tape backing material/polymer, a planchet must be used with the tape sample to prevent any interference from the sample chamber; the planchet is placed behind the sample relative to the beam. In the previous study, a beryllium planchet was used for this purpose. After analyzing the Be planchet alone as a blank with the newly optimized conditions, some peaks were detected corresponding to $\mathrm{Fe}, \mathrm{Ni}$, and $\mathrm{Cu}$. These elements did not come from the system itself. As these elements may be detected in tape samples, the trace amounts in the planchet could cause 
interference. It is important to note, however, that the new optimized conditions increased the acquisition time 3-fold, which can make the detection of $\mathrm{Fe}, \mathrm{Ni}$, and $\mathrm{Cu}$ from the planchet more prevalent above noise levels. Also, different tape segments were being analyzed as compared to the initial study, opening the possibility for a difference due to intra-roll variation. To confirm this, the planchet was analyzed on an additional XRF instrument of different source geometry and spot size. These elements were once again detected. In addition, tape 47 was run on the instrument using the Be planchet. According to LA-ICP-MS data, ${ }^{11}$ tape 47 does not contain $\mathrm{Fe}, \mathrm{Ni}$, or $\mathrm{Cu}$. However, when run with the Be planchet on the Quant'X XRF instrument, these three elements were observed. Therefore, it was determined the Be planchet was contributing interferences to the tape sample and is not a suitable sample support material.

A lucite planchet was then analyzed to determine its suitability as a support material under the current acquisition parameters. Negligible aluminum and calcium were observed with the aluminum (Low Zc) filter. However, observed counts were much lower than peaks observed in typical tape samples (i.e., $\sim 50$ counts vs. $\sim 500$ counts). Similarly, calcium counts were much lower than typical electrical tape calcium levels (i.e., $\sim 40$ counts vs. $~ 1600$ counts). In addition, these peaks were also present in the Be planchet and considered negligible as well. As seen in Figure 2, the lucite planchet presented no potential interferences beyond the negligible $\mathrm{Al}$ and $\mathrm{Ca}$ traces. Therefore, the lucite planchet was determined to be a more suitable support material within this study. It should be noted that these count differences were observed while viewing non-normalized spectra in instrumental software, but the differences were negligible in normalized data.

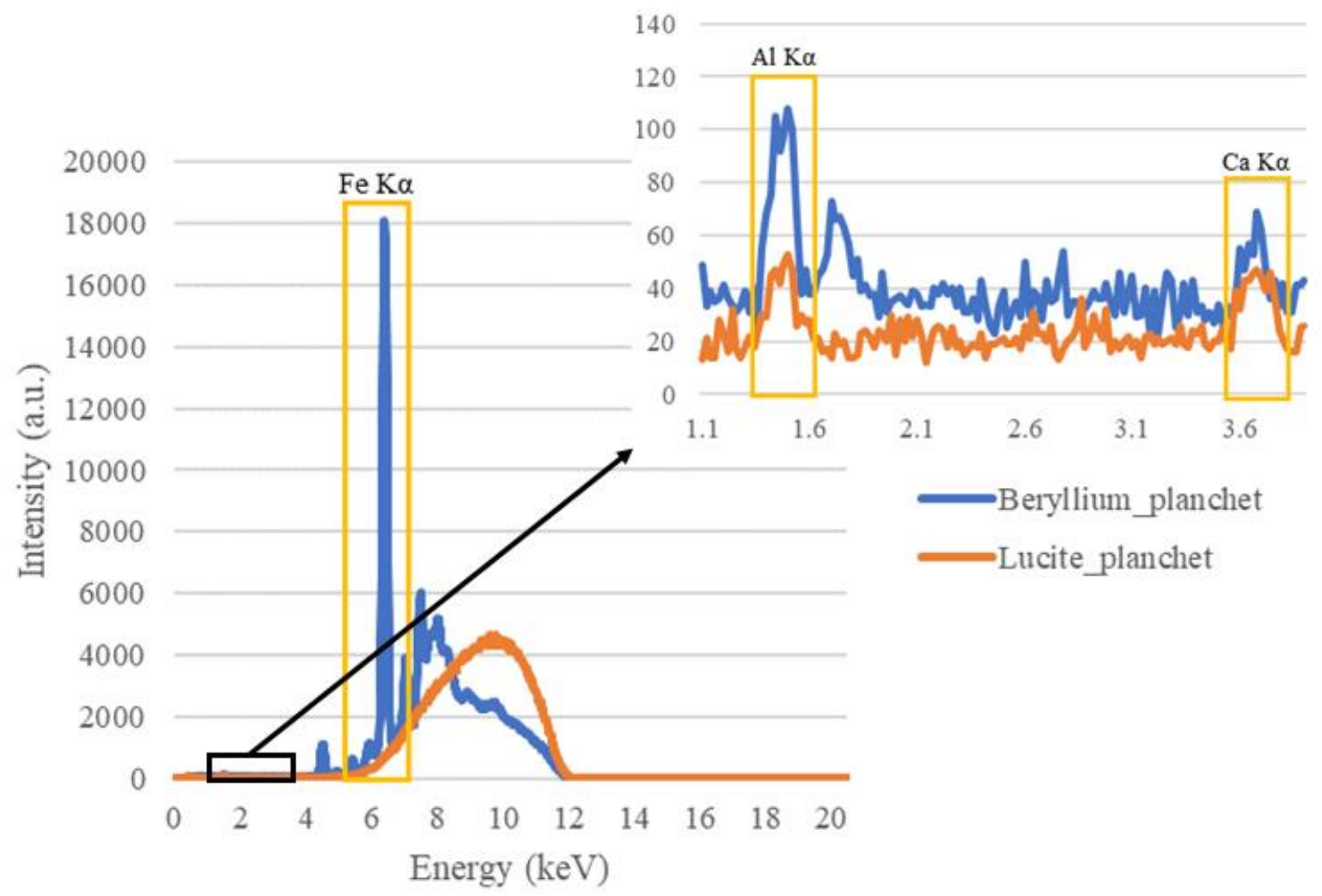

Figure 2. Spectra overlay of Be and lucite planchets, low Zc filter 


\subsubsection{Filter Comparison}

The filters provided in Table 4 were compared to filters used in the previous study ${ }^{10}$ due to their suitability according to manufacturer excitation filter guidance for common electrical tape elements..

Table 4. Filter comparison experiment results

\begin{tabular}{|c|c|c|c|}
\hline Elements & $\begin{array}{c}\text { Manufacturer } \\
\text { Recommended Filters }\end{array}$ & $\begin{array}{c}\text { Filters Used } \\
\text { Previously }\end{array}$ & Results \\
\hline $\begin{array}{l}\mathrm{Al}, \mathrm{Si} \\
\mathrm{Cl}, \mathrm{Ca}\end{array}$ & $\begin{array}{l}\text { No filter, cellulose, } \\
\text { aluminum }\end{array}$ & Aluminum & $\begin{array}{c}\mathrm{Ca}(\text { or } \mathrm{Ca} / \mathrm{Sb} \text { ) and } \mathrm{Ti} \text { (or } \mathrm{Ba} / \mathrm{Ti} \text { ) peaks detected } \\
\text { only with cellulose or } \mathrm{Al} \text { filters. Al filter offered } \\
\text { expanded elemental detection of } \mathrm{Fe}, \mathrm{Ni}, \mathrm{Cu} \text {, and } \\
\mathrm{Zn} \text {. }\end{array}$ \\
\hline $\mathrm{Sb}, \mathrm{Ba}$ & $\begin{array}{l}\text { No filter, aluminum, } \\
\text { thick } \mathrm{Cu}\end{array}$ & $\begin{array}{l}\text { Aluminum, } \\
\text { Thick } \mathrm{Cu}\end{array}$ & $\begin{array}{l}\mathrm{Sb}(\mathrm{Ca} / \mathrm{Sb}) \text { and } \mathrm{Ba}(\mathrm{Ba} / \mathrm{Ti}) \text { detected with the } \mathrm{Al} \\
\text { filter only, but in unresolved forms. However, } \\
\text { thick } \mathrm{Cu} \text { filter allowed for resolved detection of } \\
\mathrm{Sb} \text { and } \mathrm{Ba} \text {. }\end{array}$ \\
\hline $\mathrm{Ba} / \mathrm{Ti}, \mathrm{Fe}$ & $\begin{array}{l}\text { Aluminum, thin } \mathrm{Pd} \text {, } \\
\text { med. Pd }\end{array}$ & Aluminum & $\begin{array}{l}\text { Al filter resulted in higher background, but } \\
\mathrm{Ba} / \mathrm{Ti} \text { detection optimal. Thin or med. Pd } \\
\text { offered lower baselines and optimal SNR for Fe, } \\
\text { although Fe still detected in Al filter. Si lost with } \\
\text { thin Pd filter. }\end{array}$ \\
\hline $\begin{array}{l}\mathrm{Zn}, \mathrm{Pb}, \\
\mathrm{Br}, \mathrm{Sr} \\
\mathrm{Mo}\end{array}$ & $\begin{array}{l}\text { Med. Pd, thick Pd, } \\
\text { thin } \mathrm{Cu}\end{array}$ & Thick Pd & $\begin{array}{l}\mathrm{Pb}, \mathrm{Br}, \mathrm{Sr} \text {, and Mo only detected with thick Pd } \\
\text { filter. } \mathrm{Zn} \text { SNR optimal using thin Pd, but still } \\
\text { detected with thick Pd. }\end{array}$ \\
\hline $\mathrm{Cd}$ & No filter, thin $\mathrm{Cu}$ & Thick $\mathrm{Cu}$ & $\begin{array}{c}\text { Cd detected with thin or thick } \mathrm{Cu} \text { filters only. } \\
\text { Thick Cu offered better baseline shape than thin } \\
\mathrm{Cu} \text {. }\end{array}$ \\
\hline $\mathrm{Cr}$ & Aluminum, thin Pd & $\begin{array}{l}\text { Aluminum, } \\
\text { Thick Pd, Thick } \\
\text { Cu }\end{array}$ & $\begin{array}{l}\text { Cr detected in all filters except thick } \mathrm{Cu} \text {. In } \\
\text { addition, thin Pd offered increased element } \\
\text { detection and better SNRs in the } \sim 6-15 \mathrm{keV} \\
\text { region. However, to prevent addition of a } 4^{\text {th }} \\
\text { filter to the method, and therefore overall } \\
\text { increase in analysis time, } \mathrm{Al} \text { was chosen. }\end{array}$ \\
\hline
\end{tabular}

Due to the above findings, the following filters were determined to be optimal for the listed common electrical tape elements. It should be noted that to account for the full elemental range potential, all filters must be used. Analysis per sample involves three runs, one run per filter.

a. Low Zc: Aluminum

Optimized for: $\mathrm{Al}^{*}, \mathrm{Si}^{*}, \mathrm{Cl}, \mathrm{Ca} / \mathrm{Sb}, \mathrm{Ba} / \mathrm{Ti}, \mathrm{Cr}, \mathrm{Fe}, \mathrm{Zn}$

b. Mid Zc: Thick Pd

Optimized for: $\mathrm{Cl}, \mathrm{Ca} / \mathrm{Sb}, \mathrm{Cr}, \mathrm{Fe}, \mathrm{Zn}, \mathrm{Br} *, \mathrm{Sr}, \mathrm{Mo}, \mathrm{Pb}$

c. High $\mathrm{Zb}$ : Thick $\mathrm{Cu}$

Optimized for: $\mathrm{Cl}, \mathrm{Zn}, \mathrm{Sr}, \mathrm{Cd} *, \mathrm{Mo}, \mathrm{Pb}, \mathrm{Sb}$ (resolved)*, $\mathrm{Ba}$ (resolved)*

Elements only detected within the listed filter are denoted above with an asterisk. These filtering conditions are consistent with Prusinowski et al. ${ }^{10}$ 


\subsubsection{Adhesive Effects}

With adhesive still present on tape samples, higher $\mathrm{Cl}$ counts and lower counts of $\mathrm{Ca}, \mathrm{Fe}, \mathrm{Zn}, \mathrm{Ba}$, or $\mathrm{Pb}$ were typically observed as compared to adhesive-removed samples. Different elements also occurred in one tape sample. The presence of adhesive contributed $\mathrm{Ca}$ and $\mathrm{Zn}$ to sample 33, in which these elements were not detected with adhesive removed. The overlay of these spectra is provided in Figure 3.

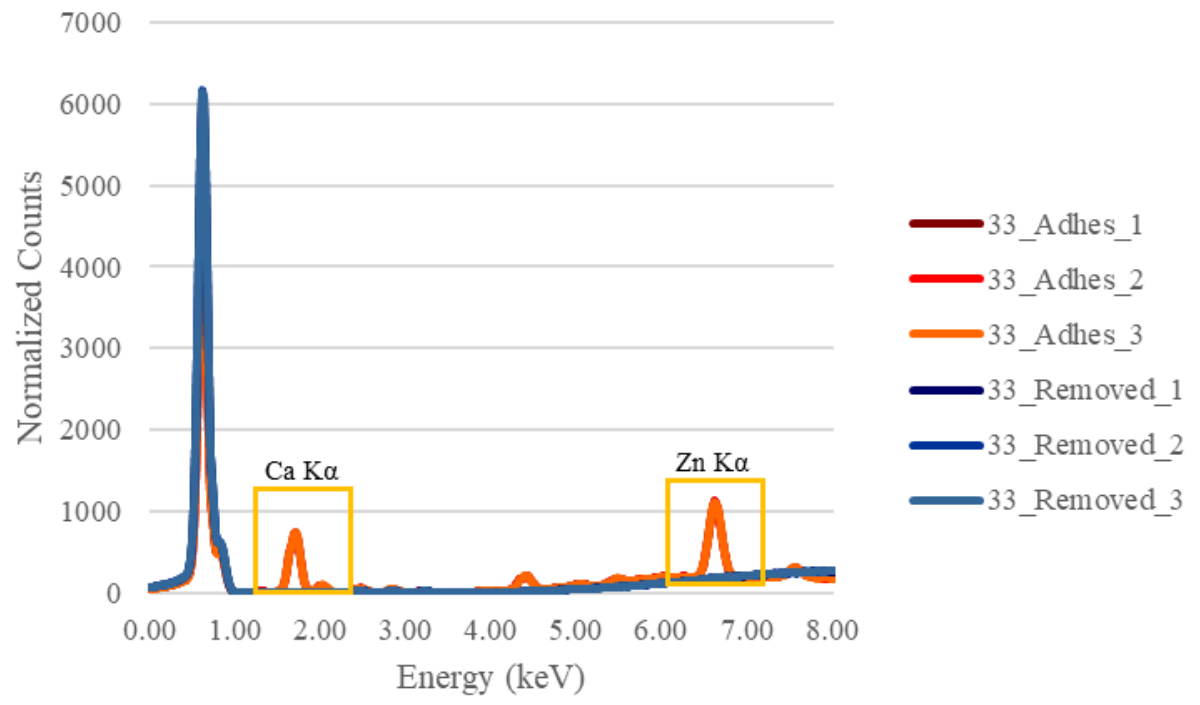

Figure 3. Spectra overlay comparison of tape 33 run both with adhesive ( 3 reps) and without adhesive (3 reps), low Zc filter

A scraping of the adhesive from sample 33 was run over Mylar film in an XRF sample cup (film and sample cup without adhesive scrapings were also run to account for any background scatter in the adhesive spectrum) under the same conditions previously used for the tapes. Both $\mathrm{Ca}$ and $\mathrm{Zn}$ were present in the adhesive, indicating they had contributed the peaks in the tape spectra without the adhesive removed, as they were not present in the adhesive-removed sample spectra. It should be noted that these elements were also present in the run of the sample cup alone, however with the addition of the adhesive scrapings the counts were much higher than that of the cup alone. Further, sample 33 exhibited brown-tinted adhesive in comparison to the other colorless and black adhesives. The attribution of the $\mathrm{Ca}$ and $\mathrm{Zn}$ may be due to the different adhesive formulation. It should be noted that sample 62 was also assessed in this experiment, and also exhibited a browntinted adhesive. However, $\mathrm{Ca}$ and $\mathrm{Zn}$ were detected in the backing of sample 62, so any additional attribution from the adhesive would not have been apparent. Overall, removal of the adhesive before the analysis of backings is recommended to avoid unwanted contributions to the elemental profiles due to the penetration of the X-Ray beam through the tape layers.

\subsubsection{Backing Thickness Effects}

Elemental differences were observed in stretched samples as compared to pristine samples when utilizing the Be planchet as the sample support material. For example, increased $\mathrm{Fe}, \mathrm{Ni}$, and $\mathrm{Cu}$ were detected in stretched sample 12 as compared to the pristine sample. This assisted in the 
confirmation of Be planchet interference as the thinner backing samples were allowing for greater beam penetration into the sample support material. Stretched sample 12 was then reanalyzed utilizing the lucite planchet as the sample support material. $\mathrm{Fe}, \mathrm{Ni}$, and $\mathrm{Cu}$ were not detected. Figure 4 provides a spectral overlay of stretched and pristine sample 12 with the Be planchet. These results indicate it is critical that any trace element interferences are minimized to negligible levels in the sample support material, as thinner tape backings (due to manipulation or natural thickness) are subject to full penetration by the X-ray beam.

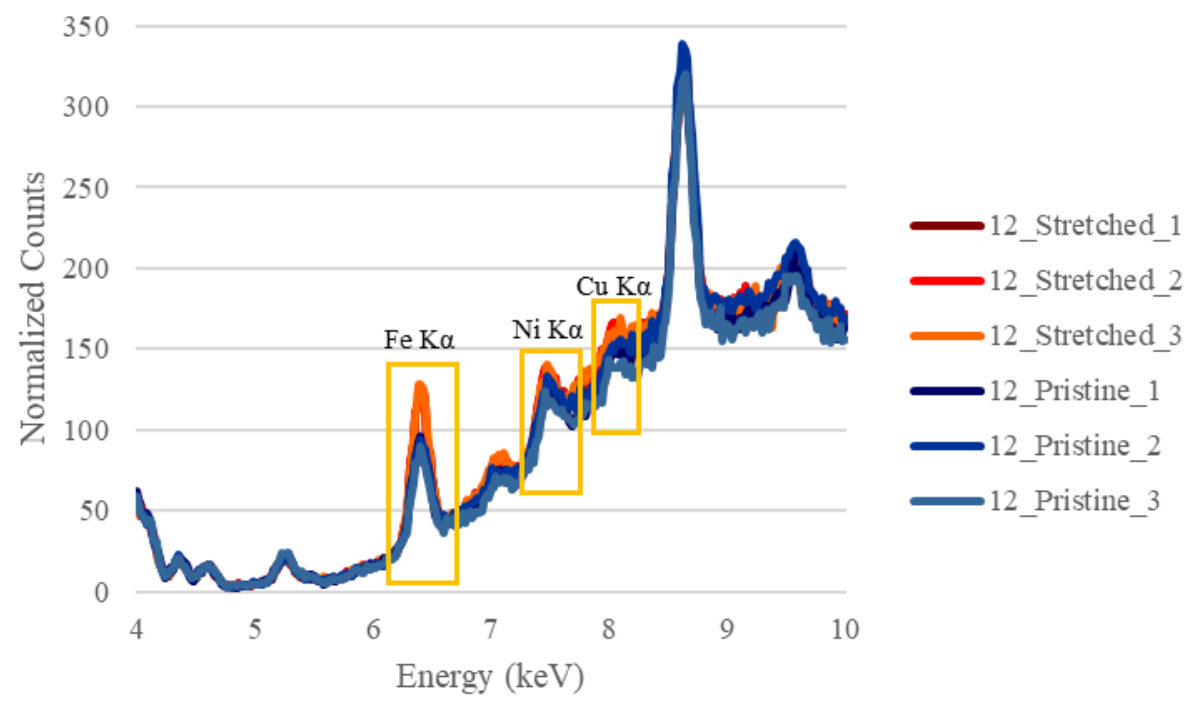

Figure 4. Spectra overlay of stretched and pristine sample 12 run with the Be planchet, low Zc filter

\subsubsection{NIST SRM 1831 Analysis}

All ASTM reported ${ }^{15}$ elements were detected when NIST SRM 1831 was run under the same optimal conditions for electrical tape backing analysis. Elements were detected at each filter as given below:

a. Aluminum (Low Zc): $\mathrm{Na}, \mathrm{Mg}, \mathrm{Al}, \mathrm{K}, \mathrm{Ca}, \mathrm{Ti}, \mathrm{Mn}, \mathrm{Fe}$

b. Thick Pd (Mid Zc): Rb

c. Thick $\mathrm{Cu}$ (High Zb): $\mathrm{Sr}, \mathrm{Zr}$

NIST SRM 1831 was determined to be a suitable reference material as the tape method parameters were able to detect the expected elemental composition for monitoring instrumental variability. 


\subsection{Method Evaluation Using Optimized Parameters}

\subsubsection{Accuracy and Discrimination Over Time}

\subsubsection{NIST SRM 1831}

Table 5 provides mean SNR and relative standard deviation (\%RSD) values per element for NIST SRM 1831 analysis over 24 replicates. It should be noted that elements are reported according to their optimal filter in Table 5.

Table 5. NIST SRM 1831 mean SNRs per element over all filters $(n=24)$

\begin{tabular}{|c|c|c|c|}
\hline Filter & Element & Mean SNR & RSD \\
\hline \multirow{4}{*}{ Aluminum (Low Zc) } & $\mathrm{Na}$ & 9.3 & 6.1 \\
\cline { 2 - 4 } & $\mathrm{Mg}$ & 9.4 & 5.6 \\
\cline { 2 - 4 } & $\mathrm{Al}$ & 20 & 4.6 \\
\cline { 2 - 4 } & $\mathrm{K}$ & 78 & 1.9 \\
\cline { 2 - 4 } & $\mathrm{Ca}$ & 1100 & 0.46 \\
\cline { 2 - 4 } & $\mathrm{Ti}$ & 26 & 4.4 \\
\cline { 2 - 4 } & $\mathrm{Mn}$ & 78 & 1.8 \\
\hline Thick Pd (Mid Zc) & $\mathrm{Fe}$ & 8.6 & 9.8 \\
\hline \multirow{2}{*}{ Thick Cu (High Zb) } & $\mathrm{Rb}$ & 14 & 7.5 \\
\cline { 2 - 4 } & $\mathrm{Sr}$ & 13 & 7.3 \\
\hline
\end{tabular}

\subsubsection{2. $\quad$ Tape Samples}

Table 6 outlines elements detected for each of samples 6, 8, and 36 through current XRF data as compared to previous SEM-EDS, XRF (iBeam, Quant'X, and Bruker), and LA-ICP-MS data., ${ }^{4,10,11}$ This data confirms the reproducibility of the present method through comparison to previous characterizations of the same samples, as any differences between instrumental methods were explainable depending upon parameter modifications in the current study. 
Table 6. Comparison of elements detected in different methods and instrumental configurations

\begin{tabular}{|c|c|c|c|}
\hline \multicolumn{4}{|c|}{ Sample 6} \\
\hline Method & \multicolumn{3}{|c|}{ Detected Elements } \\
\hline \multirow{2}{*}{$\begin{array}{c}\text { Current Quant'X } \\
\text { XRF }\end{array}$} & $\mathrm{Al}($ Low Zc) & Thick Pd (Mid Zc) & Thick $\mathrm{Cu}$ (High Zb) \\
\hline & $\mathrm{Al}, \mathrm{Cl}, \mathrm{Ca}, \mathrm{Ba} / \mathrm{Ti}, \mathrm{Fe}$ & $\begin{array}{l}\mathrm{Cl}, \mathrm{Ca}, \mathrm{Ti}, \mathrm{Fe}, \mathrm{Zn}, \\
\text { Pb, } \mathrm{Sr}^{*}\end{array}$ & $\mathrm{Cl}, \mathrm{Ca}, \mathrm{Pb}, \mathrm{Cd}, \mathrm{Ba}$ \\
\hline SEM-EDS $^{4}$ & \multicolumn{3}{|c|}{$\mathrm{Cl}, \mathrm{Ca}$} \\
\hline iBeam $X^{2} F^{10}$ & \multicolumn{3}{|c|}{$\mathrm{Cl}, \mathrm{Ca}, \mathrm{Ba} / \mathrm{Ti}, \mathrm{Pb}$} \\
\hline \multirow[b]{2}{*}{ Quant'X XRF ${ }^{10}$} & $\mathrm{Al}($ Low Zc) & Thick Pd (Mid Zc) & Thick $\mathrm{Cu}$ (High Zb) \\
\hline & $\begin{array}{l}\mathrm{Al}, \mathrm{Cl}, \mathrm{Ca}, \mathrm{Ba} / \mathrm{Ti}, \mathrm{Fe} \\
\mathrm{Ni}^{*}\end{array}$ & $\begin{array}{l}\mathrm{Cl}, \mathrm{Ca}, \mathrm{Ba} / \mathrm{Ti}^{*}, \mathrm{Fe} \\
\mathrm{Ni}^{*}, \mathrm{Cu}^{*}, \mathrm{Zn}, \mathrm{Pb}\end{array}$ & $\begin{array}{c}\mathrm{Cl}, \mathrm{Ca}, \mathrm{Fe}^{*}, \mathrm{~Pb}, \mathrm{Cd} \\
\mathrm{Ba}\end{array}$ \\
\hline Bruker $\mathrm{XRF}^{10}$ & \multicolumn{3}{|c|}{$\mathrm{Cl}, \mathrm{Ca} / \mathrm{Sb}, \mathrm{Ba} / \mathrm{Ti}, \mathrm{Fe}, \mathrm{Zn}, \mathrm{Pb}$} \\
\hline LA-ICP-MS $^{11}$ & \multicolumn{3}{|c|}{$\mathrm{Li}, \mathrm{B}, \mathrm{Na}, \mathrm{Mg}, \mathrm{Al}, \mathrm{S}, \mathrm{P}, \mathrm{Cl}, \mathrm{K}, \mathrm{Ca}, \mathrm{Ti}, \mathrm{Zn}, \mathrm{Sr}, \mathrm{Sn}, \mathrm{Sb}, \mathrm{Cd}, \mathrm{Ba}, \mathrm{Pb}$} \\
\hline \multicolumn{4}{|c|}{ Sample 8} \\
\hline Method & \multicolumn{3}{|c|}{ Detected Elements } \\
\hline \multirow{2}{*}{$\begin{array}{l}\text { Current Quant'X } \\
\text { XRF }\end{array}$} & $\mathrm{Al}($ Low Zc) & Thick Pd (Mid Zc) & Thick $\mathrm{Cu}$ (High $\mathrm{Zb})$ \\
\hline & $\begin{array}{c}\mathrm{Al}, \mathrm{Si}, \mathrm{Cl}, \mathrm{Ca}, \mathrm{Ba} / \mathrm{Ti} \\
\mathrm{Fe}\end{array}$ & $\mathrm{Cl}, \mathrm{Ca}, \mathrm{Pb}, \mathrm{Br}$ & $\mathrm{Cl}, \mathrm{Pb}, \mathrm{Br}^{*}, \mathrm{Sb}$ \\
\hline SEM-EDS $^{4}$ & \multicolumn{3}{|c|}{$\mathrm{Al}, \mathrm{Si}, \mathrm{Cl}, \mathrm{Ca}$} \\
\hline iBeam XRF $\mathrm{XR}^{10}$ & \multicolumn{3}{|c|}{$\mathrm{Al}, \mathrm{Si}, \mathrm{Cl}, \mathrm{Ca}, \mathrm{Ti}, \mathrm{Fe}$} \\
\hline \multirow[b]{2}{*}{ Quant'X XRF ${ }^{10}$} & $\mathrm{Al}($ Low Zc) & Thick Pd (Mid Zc) & Thick Cu (High Zb) \\
\hline & $\begin{array}{l}\mathrm{Al}, \mathrm{Si}, \mathrm{Cl}, \mathrm{Ca}, \mathrm{Ba} / \mathrm{Ti} \\
\quad \mathrm{Fe}, \mathrm{Ni}^{*}, \mathrm{Cu}^{*}\end{array}$ & $\begin{array}{c}\mathrm{Al}^{*}, \mathrm{Si}^{*}, \mathrm{Cl}, \mathrm{Ca}, \\
\mathrm{Ba} / \mathrm{Ti}^{*}, \mathrm{Fe}^{*}, \mathrm{Ni}^{*}, \\
\mathrm{Cu}^{*}, \mathrm{Br}\end{array}$ & $\begin{array}{l}\mathrm{Cl}, \mathrm{Ca}^{*}, \mathrm{Fe}^{*}, \mathrm{Ni}^{*} \\
\mathrm{~Pb}, \mathrm{Sb}\end{array}$ \\
\hline Bruker $\mathrm{XRF}^{10}$ & \multicolumn{3}{|c|}{$\mathrm{Al}, \mathrm{Si}, \mathrm{Cl}, \mathrm{Ca} / \mathrm{Sb}, \mathrm{Ba} / \mathrm{Ti}, \mathrm{Fe}, \mathrm{Pb}, \mathrm{Br}$} \\
\hline LA-ICP-MS ${ }^{11}$ & \multicolumn{3}{|c|}{$\begin{array}{c}\mathrm{Li}, \mathrm{Na}, \mathrm{Mg}, \mathrm{Al}, \mathrm{Si}, \mathrm{S}, \mathrm{Cl}, \mathrm{K}, \mathrm{Ca}, \mathrm{Ti}, \mathrm{Fe}, \mathrm{Cu}, \mathrm{Zn}, \mathrm{Ga}, \mathrm{Sr}, \mathrm{Sn}, \mathrm{Sb}, \mathrm{Ba} \\
\text { Pb, Th, U, Nb, Zr }\end{array}$} \\
\hline \multicolumn{4}{|c|}{ Sample 36} \\
\hline Method & \multicolumn{3}{|c|}{ Detected Elements } \\
\hline \multirow{2}{*}{$\begin{array}{l}\text { Current Quant'X } \\
\text { XRF }\end{array}$} & $\mathrm{Al}($ Low Zc) & Thick Pd (Mid Zc) & Thick $\mathrm{Cu}($ High $\mathrm{Zb})$ \\
\hline & $\begin{array}{c}\mathrm{Al}, \mathrm{Cl}, \mathrm{Ca} / \mathrm{Sb}, \mathrm{Cr}, \mathrm{Fe}, \\
\mathrm{Zn}\end{array}$ & $\mathrm{Cl}, \mathrm{Ca}^{*}, \mathrm{Zn}, \mathrm{Pb}, \mathrm{Mo}$ & $\mathrm{Cl}, \mathrm{Zn}, \mathrm{Pb}, \mathrm{Mo}, \mathrm{Sb}$ \\
\hline SEM-EDS $^{4}$ & \multicolumn{3}{|c|}{$\mathrm{Cl}, \mathrm{Ca} / \mathrm{Sb}, \mathrm{Pb}$} \\
\hline iBeam XRF ${ }^{10}$ & \multicolumn{3}{|c|}{$\mathrm{Cl}, \mathrm{Ca} / \mathrm{Sb}, \mathrm{Zn}, \mathrm{Pb}$} \\
\hline \multirow[b]{2}{*}{ Quant'X XRF ${ }^{10}$} & $\mathrm{Al}$ (Low Zc) & Thick Pd (Mid Zc) & Thick Cu (High Zb) \\
\hline & $\mathrm{Cl}, \mathrm{Ca} / \mathrm{Sb}, \mathrm{Zn}, \mathrm{Pb}, \mathrm{Cr}$ & $\begin{array}{c}\mathrm{Cl}, \mathrm{Fe}^{*}, \mathrm{Ni}^{*}, \mathrm{Cu}^{*}, \\
\mathrm{Zn}, \mathrm{Pb}, \mathrm{Mo}\end{array}$ & $\mathrm{Cl}, \mathrm{Zn}, \mathrm{Pb}, \mathrm{Mo}, \mathrm{Sb}$ \\
\hline Bruker $\mathrm{XRF}^{10}$ & \multicolumn{3}{|c|}{$\mathrm{Cl}, \mathrm{Ca} / \mathrm{Sb}, \mathrm{Cr}, \mathrm{Zn}, \mathrm{Pb}, \mathrm{Mo}$} \\
\hline LA-ICP-MS $^{11}$ & \multicolumn{3}{|c|}{$\mathrm{Na}, \mathrm{Mg}, \mathrm{Al}, \mathrm{P}, \mathrm{Cl}, \mathrm{K}, \mathrm{Ca}, \mathrm{Cr}, \mathrm{Zn}, \mathrm{Mo}, \mathrm{Sb}, \mathrm{Ba}, \mathrm{La}, \mathrm{Pb}$} \\
\hline
\end{tabular}

*Differences are attributed to changes in acquisition parameters or sample support planchets between studies. 


\subsubsection{Sensitivity}

\subsubsection{NIST SRM 1831}

Table 7 provides mean LOD and \%RSD values over 24 replicates for detected elements in the NIST SRM 1831 reference material. It should be noted that concentrations for elements $\mathrm{Na}, \mathrm{Mg}$, $\mathrm{Al}, \mathrm{K}, \mathrm{Ca}$, Ti, and Fe were obtained from the NIST SRM certificate, ${ }^{17}$ while concentrations for $\mathrm{Mn}, \mathrm{Rb}, \mathrm{Sr}$, and Zr were obtained from ASTM method E2330-19. ${ }^{18}$ In addition, elements are only reported at their optimized filters in Table 7. It should be noted that NIST SRM 1831 analysis was only used for quality control purposes and instrumental conditions were optimized for tape, not glass. For example, samples were run with the low $\mathrm{Zc}$ filter at an accelerating voltage of $12 \mathrm{kV}$, while the recommended voltage for glass is at least $35 \mathrm{kV} .{ }^{15}$ Therefore, LODs, especially in the low $\mathrm{Z}$ elements, are inferior to what is reported for glass examinations. ${ }^{15}$ Further, LODs are shown simply to establish NIST 1831 as a suitable quality control standard for the tape method due to the lack of electrical tape standard reference material, not to suggest the method is currently a quantitative technique for electrical tapes.

Table 7. Estimated LODs for NIST SRM 1831 as a quality control standard for daily instrument performance $(\mathrm{n}=24)$

\begin{tabular}{|c|c|c|c|}
\hline Filter & Element & Mean LOD $(\mathrm{ppm})$ & \%RSD \\
\hline \multirow{4}{*}{ Aluminum (Low Zc) } & $\mathrm{Na}$ & 32000 & 5.8 \\
\cline { 2 - 4 } & $\mathrm{Mg}$ & 6700 & 5.6 \\
\cline { 2 - 4 } & $\mathrm{Al}$ & 970 & 4.6 \\
\cline { 2 - 4 } & $\mathrm{K}$ & 110 & 1.9 \\
\cline { 2 - 4 } & $\mathrm{Ca}$ & 160 & 0.46 \\
\cline { 2 - 4 } & $\mathrm{Ti}$ & 22 & 5.2 \\
\cline { 2 - 4 } & $\mathrm{Mn}$ & 1.7 & 4.7 \\
\hline Thick Pd (Mid Zc) & $\mathrm{Fe}$ & 23 & 1.8 \\
\hline \multirow{2}{*}{ Thick Cu (High Zb) } & $\mathrm{Rb}$ & 19 & 10 \\
\cline { 2 - 4 } & $\mathrm{Sr}$ & 10 & 7.4 \\
\hline
\end{tabular}

\subsubsection{Tape Samples}

As an electrical tape standard reference material is not currently available, quantitative elemental assessment through LOD calculations were not determined for the tape samples. For the purposes of this study, sensitivity will be discussed in terms of detection capability differences between SEM-EDS and LA-ICP-MS data from previous studies. ${ }^{4,11}$ Due to the addition of four electrical tape samples to the overall set, and the fact that each of these four was discriminated in the current study, four of the 61 groups were not applicable for comparison to previous methods.

As compared to SEM-EDS groups, ${ }^{4}$ the XRF groups were either equivalently or further discriminated, yielding 57 groups. As compared to LA-ICP-MS groups, ${ }^{11} 55$ out of the 57 XRF groups were either equivalently or further discriminated. The remaining two groups were further discriminated by LA-ICP-MS. When considering comparable discrimination power excluding the four additional samples ( $\mathrm{N}=90$ overall), SEM-EDS had a discrimination power of $87.3 \%,{ }^{4} \mathrm{XRF}$ of 
96.7\%, and LA-ICP-MS of $93.9 \% .{ }^{11}$ This data indicates that the current XRF method has high sensitivity resulting in comparable discrimination with LA-ICP-MS for the specific tape set. However, LA-ICP-MS allows for the detection of a larger number of elements.

\subsubsection{Precision}

\subsubsection{1. $\quad$ Tape Samples}

A spectral overlay of both morning and afternoon runs per day for 10 days over three weeks revealed small variation between blind duplicate tape spectra. Mean SNR and \%RSD values for $\mathrm{Cl} / \mathrm{Ca}$ ratios per day of the study are provided in Table $\mathbf{8}$. When considering both morning and afternoon replicates, high \%RSD was observed in day 4. This sample experienced higher background overall, potentially due to incorrect positioning of the tape sample over the detector aperture. This illustrated the relevance of running daily performance tests to identify any immediate, gross errors. Due to this, $\mathrm{Cl} / \mathrm{Ca}$ peak ratio replicates were analyzed for outliers using the Grubbs' test. It was determined that the afternoon run of day 4 was an outlier caused by a gross error. Therefore, this replicate was eliminated from the overall mean. This ratio is denoted with an asterisk in Table 8.

Table 8. $\mathrm{Cl} / \mathrm{Ca}$ repeatability and intermediate precision: sample 10

\begin{tabular}{|c|c|c|}
\hline Day & Mean Cl/Ca & \%RSD \\
\hline 1 & 9.0 & 2.3 \\
\hline 2 & 9.2 & 1.4 \\
\hline 3 & 8.9 & 4.4 \\
\hline $4^{*}$ & 9.1 & NA \\
\hline 5 & 9.1 & 3.3 \\
\hline 6 & 9.1 & 0.87 \\
\hline 7 & 9.0 & 0.58 \\
\hline 8 & 9.1 & 2.0 \\
\hline 9 & 9.0 & 2.3 \\
\hline 10 & 9.0 & 0.61 \\
\hline Inter-day & 9.0 & 0.81 \\
\hline
\end{tabular}

* One replicate removed from day 4 mean due to outlier (ratio value of 0.005 )

\subsubsection{Selectivity}

Due to the close proximity of X-ray emission lines, two interferences were observed in electrical tape spectra: an overlap of $\mathrm{Ba}$ and $\mathrm{Ti}$ as well as $\mathrm{Ca}$ and $\mathrm{Sb}$ in the low $\mathrm{Zc}$ filter. Samples 6, 8, and 36 (samples previously shown to exhibit these interferences ${ }^{10}$ ) as well as sample 91 were analyzed to determine if optimized conditions could provide better resolution of these peaks. While interferences were still shown in the low Zc filter, the high $\mathrm{Zb}$ filter could be used to confirm the presence of $\mathrm{Ba}$ and $\mathrm{Sb}$ in the sample.

Sample 6 demonstrated the $\mathrm{Ba} \mathrm{K} \alpha$ peak in the high $\mathrm{Zb}$ filter, resolving the $\mathrm{Ba} / \mathrm{Ti}$ interference from the low Zc filter. Similarly, sample 36 demonstrated the $\mathrm{Sb} \mathrm{K} \alpha$ peak in the high $\mathrm{Zb}$ filter, resolving the $\mathrm{Ca} / \mathrm{Sb}$ interference at low energies. 
Likewise, sample 8 was previously reported to exhibit both the $\mathrm{Ca} / \mathrm{Sb}$ and $\mathrm{Ba} / \mathrm{Ti}$ interferences. ${ }^{10}$ The $\mathrm{Ca} / \mathrm{Sb}$ interference was shown in the low Zc filter and a peak that corresponds to either $\mathrm{Ba}$ or Ti. Ba was not detected at high energies, indicating that the $\mathrm{Ba} / \mathrm{Ti}$ designation in the low energy filter represented only Ti. $\mathrm{Sb} \mathrm{K} \alpha$ was resolved in the high $\mathrm{Zb}$ filter. For demonstrative purposes, Figure 5 shows both the $\mathrm{Ca} / \mathrm{Sb}$ interference in the low $\mathrm{Zc}$ filter as well as $\mathrm{Sb}$ in its resolved form in the high $\mathrm{Zb}$ filter as shown by sample 91.
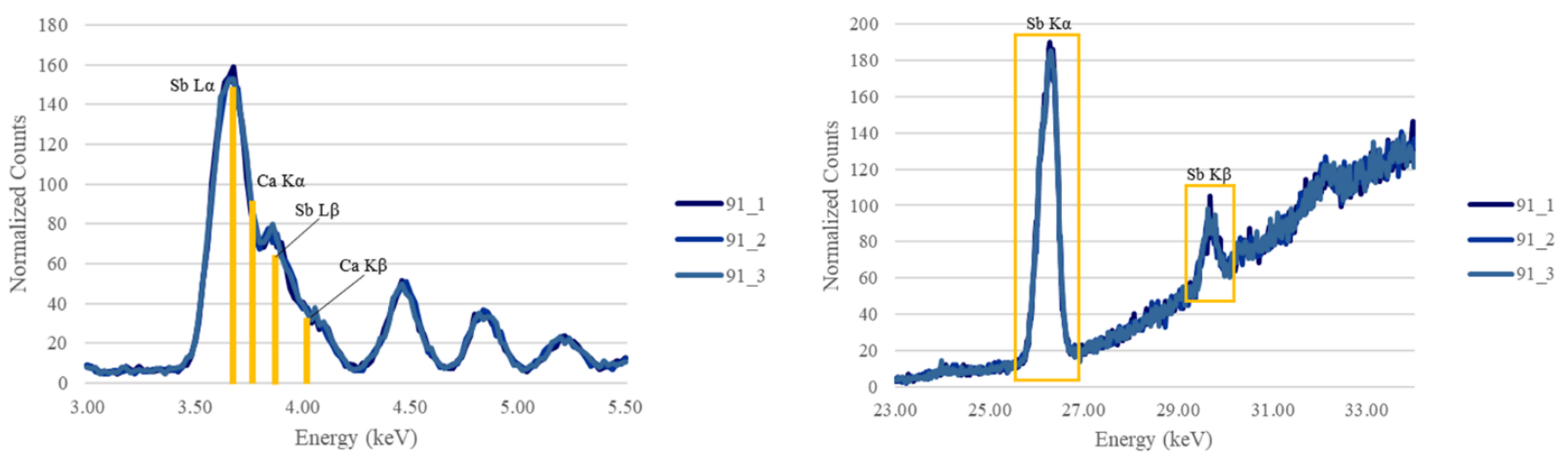

Figure 5. $\mathrm{Ca} / \mathrm{Sb}$ low $\mathrm{Zc}$ interference and high $\mathrm{Zb}$ resolved $\mathrm{Sb}$, sample 91

\subsection{Tape Set Characterization and Discrimination $(\mathrm{N}=94)$}

Samples were characterized according to the presence/absence of elements as well as peak shape or height differences and placed into 61 distinctive sub-groups according to their respective similarities and differences. From these, 41 groups showed obvious differences in the elements present due to SNR >3 criteria (e.g., SNR >3 indicated presence of elements). The additional differences between groups were a result of relative differences in peak size and shape as determined by consistent differences from multiple replicates from each comparison sample. The overall discriminatory power was $97.0 \%$ for $\mathrm{N}=94$ and $96.7 \%$ for $\mathrm{N}=90$. Table 9 displays final sample groupings.

Table 9. Tape set (N=94) XRF characterization groups

\begin{tabular}{|c|c|c|c|}
\hline Group & Elements & Samples & Subgroups and Main Observed Differences \\
\hline 1 & $\mathrm{Al}, \mathrm{Cl}, \mathrm{Ca} / \mathrm{Sb}, \mathrm{Zn}, \mathrm{Sb}$ & 1,49 & \\
\hline 2 & $\mathrm{Al}, \mathrm{Cl}, \mathrm{Ca} / \mathrm{Sb}, \mathrm{Fe}, \mathrm{Zn}, \mathrm{Pb}, \mathrm{Sb}$ & 2 & \\
\hline 3 & $\begin{array}{c}\mathrm{Al}, \mathrm{Cl}, \mathrm{Ca} / \mathrm{Sb}, \mathrm{Ba} / \mathrm{Ti}, \mathrm{Fe}, \mathrm{Zn}, \mathrm{Pb}, \\
\mathrm{Ba}\end{array}$ & 3 & \\
\hline \multirow{5}{*}{4} & \multirow{5}{*}{$\mathrm{Al}, \mathrm{Si}, \mathrm{Cl}, \mathrm{Ca} / \mathrm{Sb}, \mathrm{Ba} / \mathrm{Ti}, \mathrm{Fe}, \mathrm{Pb}$} & 4 & 4A. Lower $\mathrm{Pb}$ than 4B-D \\
\hline & & 42,51 & 4B. $\mathrm{Mid} \mathrm{Pb}$ \\
\hline & & 53 & 4C. Higher $\mathrm{Ca} / \mathrm{Sb}$ than $4 \mathrm{~A}, \mathrm{~B}, \mathrm{D}, \mathrm{E}$ \\
\hline & & 56 & 4D. Higher $\mathrm{Pb}$ than $4 \mathrm{~B}-\mathrm{E}$ \\
\hline & & 70 & 4E. Higher $\mathrm{Ba} / \mathrm{Ti}$ than $4 \mathrm{~A}-\mathrm{D}$, lower $\mathrm{Pb}$ than 4B-D \\
\hline 5 & $\mathrm{Al}, \mathrm{Cl}, \mathrm{Ca} / \mathrm{Sb}, \mathrm{Fe}, \mathrm{Zn}, \mathrm{Sb}$ & 5,7 & \\
\hline 6 & $\begin{array}{c}\mathrm{Al}, \mathrm{Cl}, \mathrm{Ca} / \mathrm{Sb}, \mathrm{Ba} / \mathrm{Ti}, \mathrm{Fe}, \mathrm{Zn}, \mathrm{Pb}, \\
\mathrm{Cd}, \mathrm{Ba}\end{array}$ & 6 & \\
\hline
\end{tabular}




\begin{tabular}{|c|c|c|c|}
\hline \multirow{5}{*}{7} & \multirow{5}{*}{$\begin{array}{c}\mathrm{Al}, \mathrm{Si}, \mathrm{Cl}, \mathrm{Ca} / \mathrm{Sb}, \mathrm{Ba} / \mathrm{Ti}, \mathrm{Fe}, \mathrm{Pb} \\
\mathrm{Br}, \mathrm{Sb}\end{array}$} & 8 & 7A. Higher $\mathrm{Ca} / \mathrm{Sb}$ than 7B-D, lower Fe than 7B-E \\
\hline & & 21 & $\begin{array}{l}\text { 7B. Lower } \mathrm{Ca} / \mathrm{Sb} \text { than } 7 \mathrm{~A}, \mathrm{E} \text {, higher Fe than 7A,D,E, and } \\
\text { higher } \mathrm{Sb} \text { than 7A,C,D }\end{array}$ \\
\hline & & 38 & 7C. Lower $\mathrm{Ca} / \mathrm{Sb}$ than 7A,E, higher Fe than 7A,D,E \\
\hline & & 67 & 7D. Lower $\mathrm{Ca} / \mathrm{Sb}$ than $7 \mathrm{~A}, \mathrm{E}$ \\
\hline & & 81 & 7E. Higher $\mathrm{Ca} / \mathrm{Sb}$ than $7 \mathrm{~B}-\mathrm{D}$, higher $\mathrm{Sb}$ than $7 \mathrm{~A}, \mathrm{C}, \mathrm{D}$ \\
\hline 8 & $\mathrm{Al}, \mathrm{Cl}, \mathrm{Ca} / \mathrm{Sb}, \mathrm{Ba} / \mathrm{Ti}, \mathrm{Pb}$ & 9 & \\
\hline \multirow{6}{*}{9} & \multirow{6}{*}{$\mathrm{Al}, \mathrm{Cl}, \mathrm{Ca} / \mathrm{Sb}, \mathrm{Zn}, \mathrm{Pb}, \mathrm{Sb}, \mathrm{Mo}$} & $10,17,23,24,63$ & $\begin{array}{l}\text { 9A. Higher } \mathrm{Pb} \text { than 9B-F, higher Mo than 9C,E, and } \\
\text { higher Sb than 9F }\end{array}$ \\
\hline & & $\begin{array}{c}11-13,15,18-20,25,26,41,54 \\
61,64,68\end{array}$ & 9B. Higher Mo than $9 \mathrm{C}, \mathrm{E}$ and higher $\mathrm{Sb}$ than $9 \mathrm{~F}$ \\
\hline & & $16,29,30,34,43,44,47$ & $\begin{array}{l}\text { 9C. Lower Pb than 9A,E, lower Mo than 9A,B,D,F, and } \\
\text { lower Sb than 9A,B,E }\end{array}$ \\
\hline & & 27,28 & $\begin{array}{l}\text { 9D. Lower } \mathrm{Pb} \text { than 9A,E, higher Mo than 9C,E and higher } \\
\mathrm{Sb} \text { than 9F }\end{array}$ \\
\hline & & 39 & 9E. Lower Mo than 9A,B,D,F, higher $\mathrm{Sb}$ than $9 \mathrm{~F}$ \\
\hline & & 40 & $\begin{array}{l}\text { 9F. Lower Pb than 9A,E, lower } \mathrm{Sb} \text { than 9A,B,E, higher } \\
\text { Mo than 9C,E }\end{array}$ \\
\hline 10 & $\begin{array}{c}\mathrm{Al}, \mathrm{Si}, \mathrm{Cl}, \mathrm{Ca} / \mathrm{Sb}, \mathrm{Ba} / \mathrm{Ti}, \mathrm{Fe}, \mathrm{Pb}, \\
\mathrm{Cd}, \mathrm{Sb}\end{array}$ & 14 & \\
\hline 11 & $\mathrm{Al}, \mathrm{Cl}, \mathrm{Ca} / \mathrm{Sb}, \mathrm{Ba} / \mathrm{Ti}, \mathrm{Pb}, \mathrm{Br}, \mathrm{Sb}$ & 22 & \\
\hline 12 & $\mathrm{Al}, \mathrm{Cl}, \mathrm{Ca} / \mathrm{Sb}, \mathrm{Pb}$ & 31 & \\
\hline 13 & $\begin{array}{c}\mathrm{Al}, \mathrm{Si}, \mathrm{Cl}, \mathrm{Ca} / \mathrm{Sb}, \mathrm{Ba} / \mathrm{Ti}, \mathrm{Fe}, \mathrm{Pb}, \\
\mathrm{Sb}\end{array}$ & 32 & \\
\hline 14 & $\mathrm{Al}, \mathrm{Cl}, \mathrm{Ca} / \mathrm{Sb}, \mathrm{Ba} / \mathrm{Ti}, \mathrm{Pb}, \mathrm{Ba}$ & 33 & \\
\hline 15 & $\begin{array}{c}\mathrm{Al}, \mathrm{Si}, \mathrm{Cl}, \mathrm{Ca} / \mathrm{Sb}, \mathrm{Ba} / \mathrm{Ti}, \mathrm{Fe}, \mathrm{Pb}, \\
\mathrm{Cr}, \mathrm{Cd}, \mathrm{Sb}\end{array}$ & 35,37 & \\
\hline 16 & $\begin{array}{c}\mathrm{Al}, \mathrm{Cl}, \mathrm{Ca} / \mathrm{Sb}, \mathrm{Zn}, \mathrm{Pb}, \mathrm{Cr}, \mathrm{Sb} \\
\mathrm{Mo}\end{array}$ & 36 & \\
\hline 17 & $\begin{array}{c}\mathrm{Al}, \mathrm{Si}, \mathrm{Cl}, \mathrm{Ca} / \mathrm{Sb}, \mathrm{Ba} / \mathrm{Ti}, \mathrm{Fe}, \mathrm{Pb} \\
\mathrm{Br}, \mathrm{Cd}\end{array}$ & 45,55 & \\
\hline 18 & $\begin{array}{c}\mathrm{Al}, \mathrm{Si}, \mathrm{Cl}, \mathrm{Ca} / \mathrm{Sb}, \mathrm{Ba} / \mathrm{Ti}, \mathrm{Fe}, \mathrm{Pb} \\
\mathrm{Cr}, \mathrm{Br}, \mathrm{Sb}\end{array}$ & 46 & \\
\hline \multirow{3}{*}{19} & \multirow{3}{*}{$\mathrm{Al}, \mathrm{Cl}, \mathrm{Ca} / \mathrm{Sb}, \mathrm{Ba} / \mathrm{Ti}, \mathrm{Zn}, \mathrm{Sb}$} & 48,57 & 19A. Higher $\mathrm{Ca} / \mathrm{Sb}$ than 19B-C \\
\hline & & 72 & 19B. Lower $\mathrm{Ca} / \mathrm{Sb}$ than $19 \mathrm{~A}$ \\
\hline & & 79 & 19C. Lower $\mathrm{Ca} / \mathrm{Sb}$ than $19 \mathrm{~A}$, lowest $\mathrm{Zn}$, highest $\mathrm{Ba} / \mathrm{Ti}$ \\
\hline 20 & $\begin{array}{l}\mathrm{Al}, \mathrm{Si}, \mathrm{Cl}, \mathrm{Ca} / \mathrm{Sb}, \mathrm{Ba} / \mathrm{Ti}, \mathrm{Fe}, \mathrm{Zn}, \\
\mathrm{Pb}, \mathrm{Cr}, \mathrm{Cd}, \mathrm{Sb}\end{array}$ & 50 & \\
\hline 21 & $\begin{array}{c}\mathrm{Al}, \mathrm{Si}, \mathrm{Cl}, \mathrm{Ca} / \mathrm{Sb}, \mathrm{Ba} / \mathrm{Ti}, \mathrm{Fe}, \mathrm{Zn}, \\
\mathrm{Pb}, \mathrm{Sb}, \mathrm{Mo}\end{array}$ & 52 & \\
\hline \multirow{2}{*}{22} & \multirow{2}{*}{$\begin{array}{c}\mathrm{Al}, \mathrm{Si}, \mathrm{Cl}, \mathrm{Ca} / \mathrm{Sb}, \mathrm{Ba} / \mathrm{Ti}, \mathrm{Fe}, \mathrm{Pb}, \\
\mathrm{Br}\end{array}$} & 58 & 22A. Lower Fe than 22B \\
\hline & & 86 & 22B. Lower $\mathrm{Pb}$ than $22 \mathrm{~A}$ \\
\hline 23 & $\mathrm{Al}, \mathrm{Ca} / \mathrm{Sb}, \mathrm{Ba} / \mathrm{Ti}$ & 59,60 & \\
\hline 24 & $\begin{array}{c}\mathrm{Al}, \mathrm{Cl}, \mathrm{Ca} / \mathrm{Sb}, \mathrm{Ba} / \mathrm{Ti}, \mathrm{Zn}, \mathrm{Pb}, \mathrm{Cr}, \\
\mathrm{Cd}, \mathrm{Sb}\end{array}$ & 62 & \\
\hline \multirow{2}{*}{25} & \multirow{2}{*}{$\mathrm{Al}, \mathrm{Cl}, \mathrm{Ca} / \mathrm{Sb}, \mathrm{Pb}, \mathrm{Sb}$} & 65 & 25A. Higher $\mathrm{Pb}$ and lower $\mathrm{Sb}$ than $25 \mathrm{~B}$ \\
\hline & & 69 & 25B. Lower $\mathrm{Pb}$ and lower $\mathrm{Sb}$ than $25 \mathrm{~A}$ \\
\hline 26 & $\mathrm{Al}, \mathrm{Si}, \mathrm{Cl}, \mathrm{Ba} / \mathrm{Ti}, \mathrm{Fe}, \mathrm{Zn}, \mathrm{Cd}$ & 66 & \\
\hline 27 & $\mathrm{Al}, \mathrm{Cl}, \mathrm{Ca} / \mathrm{Sb}, \mathrm{Ba} / \mathrm{Ti}, \mathrm{Fe}, \mathrm{Pb}, \mathrm{Cd}$ & 71 & \\
\hline 28 & $\begin{array}{l}\mathrm{Al}, \mathrm{Cl}, \mathrm{Ca} / \mathrm{Sb}, \mathrm{Ba} / \mathrm{Ti}, \mathrm{Fe}, \mathrm{Zn}, \mathrm{Pb}, \\
\mathrm{Sr}, \mathrm{Cd}, \mathrm{Ba}, \mathrm{Sb}\end{array}$ & 73 & \\
\hline 29 & $\mathrm{Al}, \mathrm{Cl}, \mathrm{Ca} / \mathrm{Sb}, \mathrm{Ba} / \mathrm{Ti}, \mathrm{Zn}, \mathrm{Ba}, \mathrm{Sb}$ & 74 & \\
\hline 30 & $\mathrm{Al}, \mathrm{Ca} / \mathrm{Sb}, \mathrm{Fe}, \mathrm{Zn}$ & 75 & \\
\hline
\end{tabular}




\begin{tabular}{|c|c|c|c|}
\hline \multirow{4}{*}{31} & \multirow{4}{*}{$\begin{array}{c}\mathrm{Al}, \mathrm{Cl}, \mathrm{Ca} / \mathrm{Sb}, \mathrm{Ba} / \mathrm{Ti}, \mathrm{Zn}, \mathrm{Ba}, \mathrm{Sb}, \\
\mathrm{Mo}\end{array}$} & $76,77,83$ & 31A. Lower $\mathrm{Sb}$ than 31B \\
\hline & & 80 & 31B. Higher $\mathrm{Sb}$ than $31 \mathrm{~A}$ \\
\hline & & 78 & 31C. Lowest $\mathrm{Ca} / \mathrm{Sb}, \mathrm{Mo}$, and $\mathrm{Sb}$, highest $\mathrm{Cl}$ \\
\hline & & 91 & 31D. Lower $\mathrm{Sb}$ than $31 \mathrm{~A}-\mathrm{B}$ \\
\hline 32 & $\begin{array}{c}\mathrm{Al}, \mathrm{Si}, \mathrm{Cl}, \mathrm{Ca} / \mathrm{Sb}, \mathrm{Ba} / \mathrm{Ti}, \mathrm{Fe}, \mathrm{Zn}, \\
\mathrm{Pb}, \mathrm{Br}\end{array}$ & 82 & \\
\hline 33 & $\mathrm{Al}, \mathrm{Cl}, \mathrm{Ca} / \mathrm{Sb}, \mathrm{Ba} / \mathrm{Ti}, \mathrm{Zn}, \mathrm{Br}, \mathrm{Sb}$ & 84 & \\
\hline 34 & $\begin{array}{c}\mathrm{Al}, \mathrm{Cl}, \mathrm{Ca} / \mathrm{Sb}, \mathrm{Ba} / \mathrm{Ti}, \mathrm{Fe}, \mathrm{Zn}, \mathrm{Pb}, \\
\mathrm{Cr}, \mathrm{Cd}, \mathrm{Sb}\end{array}$ & 85 & \\
\hline 35 & $\mathrm{Al}, \mathrm{Cl}, \mathrm{Ca} / \mathrm{Sb}, \mathrm{Ba} / \mathrm{Ti}, \mathrm{Zn}$ & 87 & \\
\hline 36 & $\mathrm{Al}, \mathrm{Cl}, \mathrm{Ca} / \mathrm{Sb}, \mathrm{Ba} / \mathrm{Ti}, \mathrm{Fe}, \mathrm{Zn}$ & 88 & \\
\hline 37 & $\begin{array}{c}\mathrm{Al}, \mathrm{Cl}, \mathrm{Ca} / \mathrm{Sb}, \mathrm{Ba} / \mathrm{Ti}, \mathrm{Zn}, \mathrm{Pb}, \mathrm{Cd}, \\
\mathrm{Sb}\end{array}$ & 89 & \\
\hline 38 & $\begin{array}{c}\mathrm{Al}, \mathrm{Cl}, \mathrm{Ca} / \mathrm{Sb}, \mathrm{Ba} / \mathrm{Ti}, \mathrm{Fe}, \mathrm{Pb}, \mathrm{Cd}, \\
\mathrm{Sb}\end{array}$ & 90 & \\
\hline 39 & $\begin{array}{c}\mathrm{Al}, \mathrm{Cl}, \mathrm{Ca} / \mathrm{Sb}, \mathrm{Ba} / \mathrm{Ti}, \mathrm{Zn}, \mathrm{Pb}, \mathrm{Ba}, \\
\mathrm{Sb}, \mathrm{Mo}\end{array}$ & 92 & \\
\hline 40 & $\begin{array}{c}\mathrm{Al}, \mathrm{Cl}, \mathrm{Ca} / \mathrm{Sb}, \mathrm{Ba} / \mathrm{Ti}, \mathrm{Fe}, \mathrm{Zn}, \mathrm{Sr} \\
\mathrm{Br}, \mathrm{Ba}, \mathrm{Sb}\end{array}$ & 93 & \\
\hline 41 & $\mathrm{Al}, \mathrm{Cl}, \mathrm{Ca} / \mathrm{Sb}, \mathrm{Ba} / \mathrm{Ti}, \mathrm{Fe}, \mathrm{Zn}, \mathrm{Sb}$ & 94 & \\
\hline
\end{tabular}

\subsubsection{Spectral Contrast Angle Comparison}

Spectral overlay is a recognized method for the comparison of EDS spectra (e.g., SEM-EDS and $\mathrm{XRF})^{3,4,10}$ and is widely implemented in forensic laboratories as the first step for identifying spectral differences or similarities. Replicates of the known and questioned spectra are overlaid to assess variability of each sample. When variability of spectral shape and intensity of the questioned sample is greater than the intra-roll variability of the known sample, then the samples are distinguished by EDS or XRF. Large differences between samples are easy to detect by this method. However, comparing spectra by visual methods, such as spectral overlay, becomes more challenging with increased similarity between spectra. As a result, the judgment of similarity of spectra becomes more complex and adds subjectivity. This is a common problem not only in forensic science but in spectrochemical comparisons in general.

To deal with these situations, analytical scientists have reported alternative methods for the comparison of spectra. ${ }^{14,19,20}$ In this study, we exhibit a complementary method for the confirmation of spectra overlay by applying well known, vector-based spectral comparison using contrast angles. This method is widely applied in spectral library searching (e.g., FTIR, mass spectra). ${ }^{14,19}$ However, unlike spectral overlay, the contrast angle ratio is not yet applied for routine tape comparisons. This study aims to evaluate the utility of spectral contrast angle as a potential complementary tool that could be used in the future to support examiner opinion.

In order to confirm sub-groups made by observed spectral differences (spectra overlay), the spectral contrast angle was found in every combination both within sample replicates and between sample replicates. These values were used to create a ratio of between-sample mean contrast angle to intra-roll mean contrast angle. Ratios were determined between all combinations of sample pairs considered indistinguishable through spectral overlay and through samples from the same roll. Ratios were also determined between those samples determined to be distinguishable, and thus 
separated into subgroups as indicated in Table 9. Each spectral contrast ratio for each pair considered distinguishable through spectral overlay (e.g., between-pairs) fell outside the range of the mean ratio for all pairs considered indistinguishable (e.g., within-pairs, within-roll), indicating the observed differences were large enough for group and subgroup distinction. In general, the greater the dissimilarity, the higher the contrast angle ratio estimated. There was one comparison pair (samples 1 and 49) that had a ratio with the contrast angle ratio overlapping the indistinguishable, same-source range. Therefore, a decision was made to maintain samples 1 and 49 within the same group. The range of indistinguishable within-group ratios (e.g., intra-subgroup samples, replicates, blind duplicate samples, and intra-roll samples) ranged from 0.92 to1.36 while between-group ratios ranged from 1.08 to 82.45 and between-subgroup ratios ranged from 1.43 to 8.09. It should be noted that although there is wide variation in between-group ratios, there is only an overlap of five out of the 794 inter-group samples with the indistinguishable range, indicating a false inclusion rate of only $0.6 \%$. Contrast angle ratio values are summarized in Table A.2 of the Appendix and displayed in Figure 6.

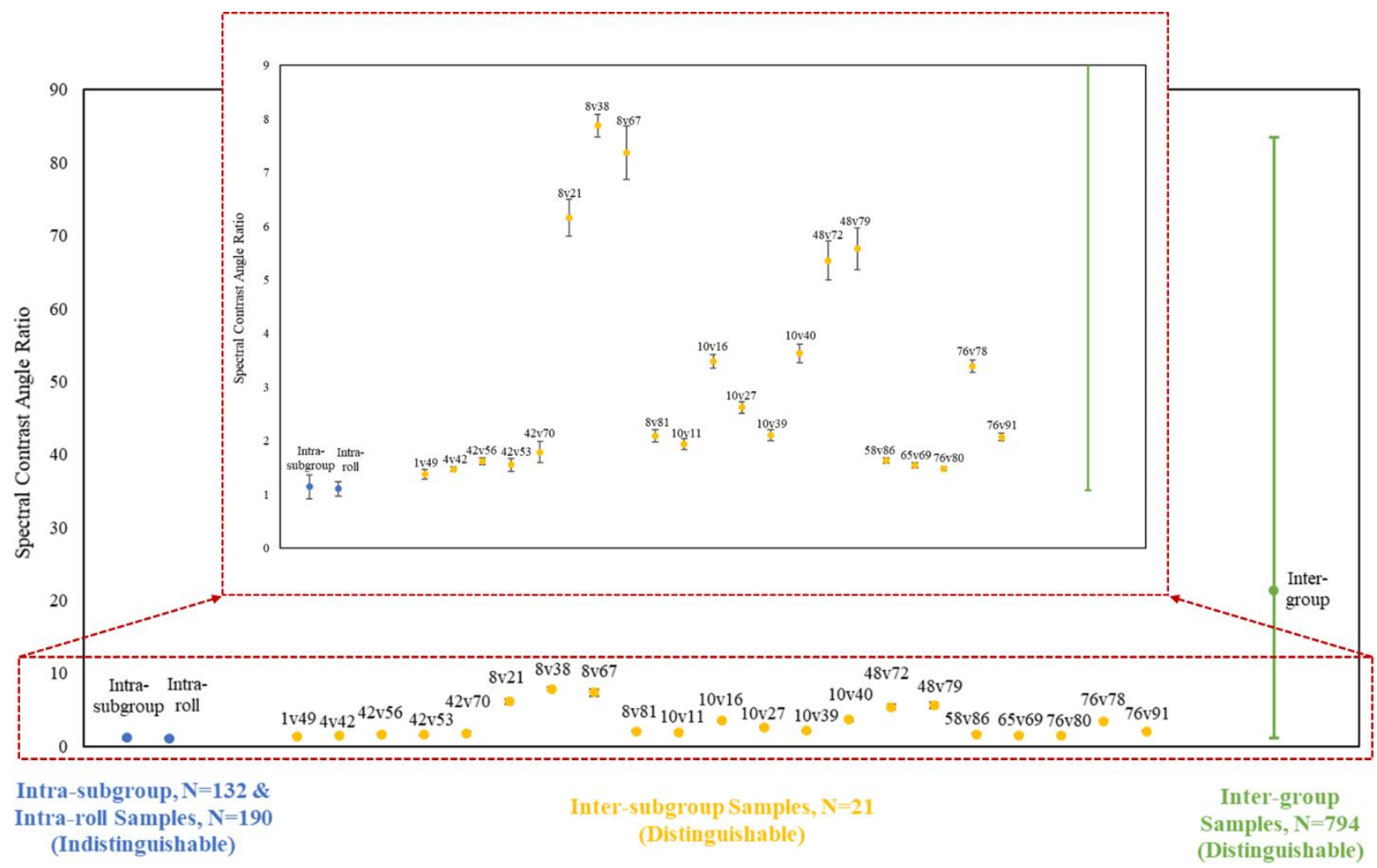

Figure 6. Comparison of ranges of contrast angle ratios variation for intra-samples (indistinguishable subgroup samples, same roll samples), and inter-samples (between groups and between subgroup samples). The inset shows a zoomed area of the plot.

\subsubsection{Quadratic Discriminant Analysis (QDA)}

QDA is a statistical method used to discriminate between groups based upon the individual covariance for each class in a dataset. This method is included as a technique of exploratory data analysis of the fully characterized dataset. It is not intended, however, to be used in casework, as larger data sets would be needed to provide further evidence of the classification capabilities. 
In order to reduce dimensionality of the data, SNRs of selected elements were used as numerical input rather than all spectral x-y data points. SNRs per element for each tape sample in the dataset $(\mathrm{N}=94)$ were subjected to QDA for classification according to country of manufacture. Analysis results are displayed in the form of a canonical plot in which samples are represented by points corresponding to their multivariate means and are plotted in terms of the first two canonical variables. These variables represent the canonical correlation between the levels of the dataset or the indicator variables (e.g., countries of manufacture) and the covariates or characteristics of the dataset (e.g., SNRs per element). The first two canonical variables represent the dimensions of optimal separation for the dataset. In order to examine the loadings of these canonical variables, or the weight each covariate holds in relation to a canonical variable, biplot rays are observed. For this study, the rays represent which elemental SNR is responsible for the variance in a given direction of the QDA canonical plot. QDA is a useful method for the visualization of which elements are most responsible for variation between the countries of manufacture for the dataset. $^{21,22}$

In order to examine classification potential of XRF elemental composition by country of manufacture, quadratic discriminant analysis (QDA) was performed on a data set containing sample data with SNRs only from the optimal filter per element. By observing the number of misclassified samples by the predicted algorithm based upon individual country covariance matrices for elemental composition at each filter, it was observed that only one sample was misclassified by QDA. In this instance, one of the 36 samples manufactured in Taiwan was classified as originating in the US. However, the sample misclassified by this method was Sample 77 , which was manufactured by $3 \mathrm{M} 囚$. It was observed that the majority of the samples outside the US and Taiwan confidence intervals in the canonical plot shown in Figure 7 were of $3 \mathrm{M} ®$ branding. It should be noted that sample 2, the only sample originating from England, was removed from this dataset for ease of view of country clustering. QDA biplots displaying the loadings (vectors showing by which elements samples are most variable) for the data set are provided in Figure A.4 of the Appendix. 


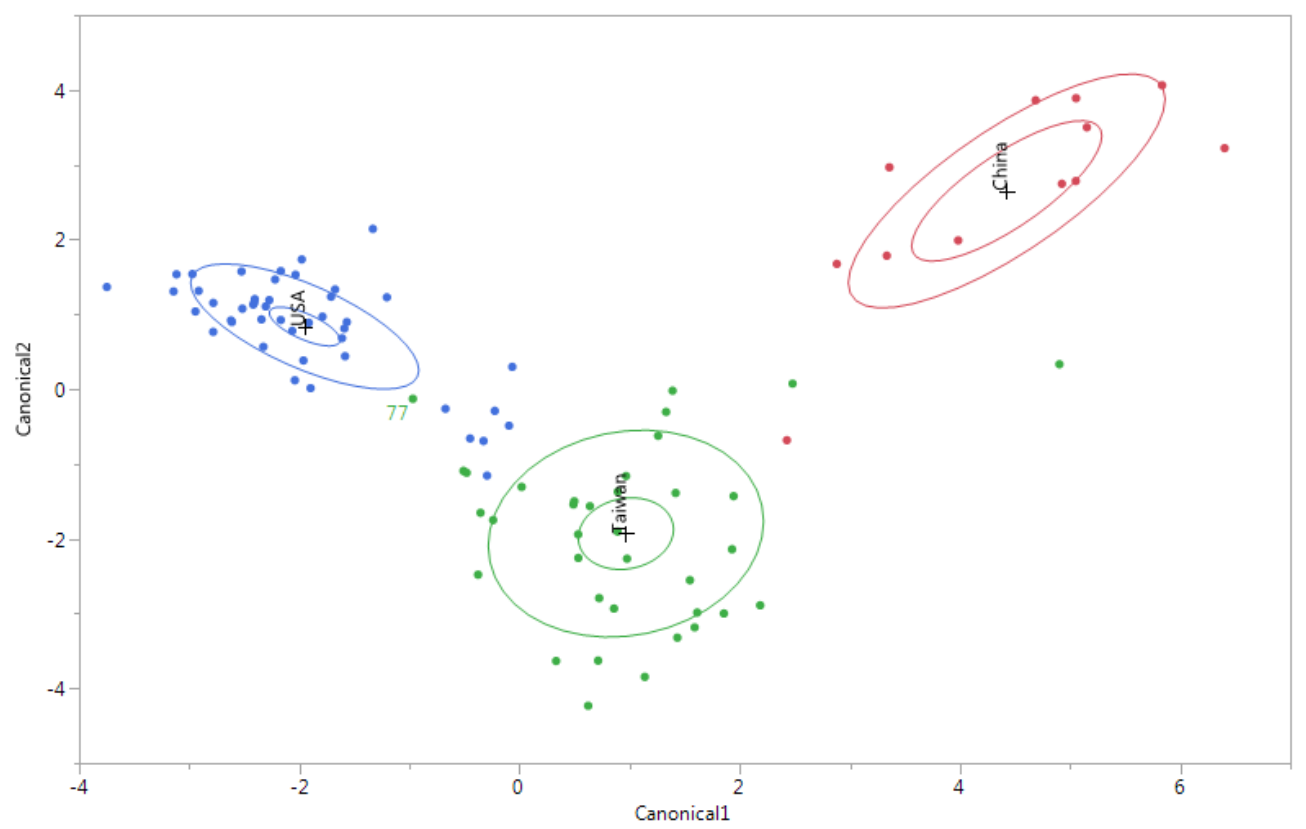

Figure 7. QDA canonical plot by manufacturing origin for optimized filter overall tape data set $(\mathrm{N}=94)$

According to group means by country, general trends showed that Chinese samples were attributed lower SNRs for $\mathrm{Cl}$ and higher $\mathrm{SNRs}$ for $\mathrm{Ca} / \mathrm{Sb}$ as compared to samples manufactured in other countries. Group means also showed that samples manufactured in England or the US displayed low $\mathrm{Ba} / \mathrm{Ti}$ and high $\mathrm{Pb}$ and $\mathrm{Sb}$ as compared to samples from other countries. Samples manufactured in the US typically showed higher $\mathrm{Zn}$ and Mo than other samples, while samples from China showed higher $\mathrm{Cd}$. These exploratory results indicate XRF could be a feasible technique for providing potential sourcing information for investigative leads, as first suggested with LA-ICPMS electrical tape characterization. ${ }^{11}$ However, the classification findings cannot be generalized as larger population sets would be needed.

\subsection{Intra-roll Variability Study}

\subsubsection{Spectral Contrast Angle Comparison}

Spectral contrast angle ratios were determined between every possible combination of the 20 intraroll variability sample runs ( $\mathrm{N}=190$ pairs). Ratios were determined at each of the low $\mathrm{Zc}$, mid Zc, and high $\mathrm{Zb}$ intra-roll data sets. The highest mean spectral contrast ratio and associated relative standard deviation were observed for the low Zc dataset, indicating highest variability between replicates at this filter. On the other hand, the lowest mean spectral contrast ratio and associated relative standard deviation were observed for the high $\mathrm{Zb}$ dataset, indicating lowest variability between replicates at this filter. Figure $\mathbf{8 b}$ provides the distributions of spectral contrast ratios for the low $\mathrm{Zc}$ and high $\mathrm{Zb}$ filtered data sets while Figure 8a provides a comparison of these values to the inter-group ratio range as determined in section 3.3.1. As observed in Figure 8, most-intra roll comparisons produced ratios lower than 1.24 , with only 5 intra-roll compared samples at the low 
Zc filter overlapping with the inter-group ratio range. According to outlier analysis via the Grubbs' test, one of these samples was determined to be an outlier (a ratio value of 1.62 as compared to a mean of $1.10 \pm 0.14$ ). Figure 8 also displays that at best-case variability (e.g., high Zc filter data), no overlaps with the inter-group ratio range were observed. Therefore, this data indicates that 376 out of 380 comparison pairs were determined indistinguishable for samples originating from the same roll (98.9\% correct association, $1.1 \%$ false exclusion).

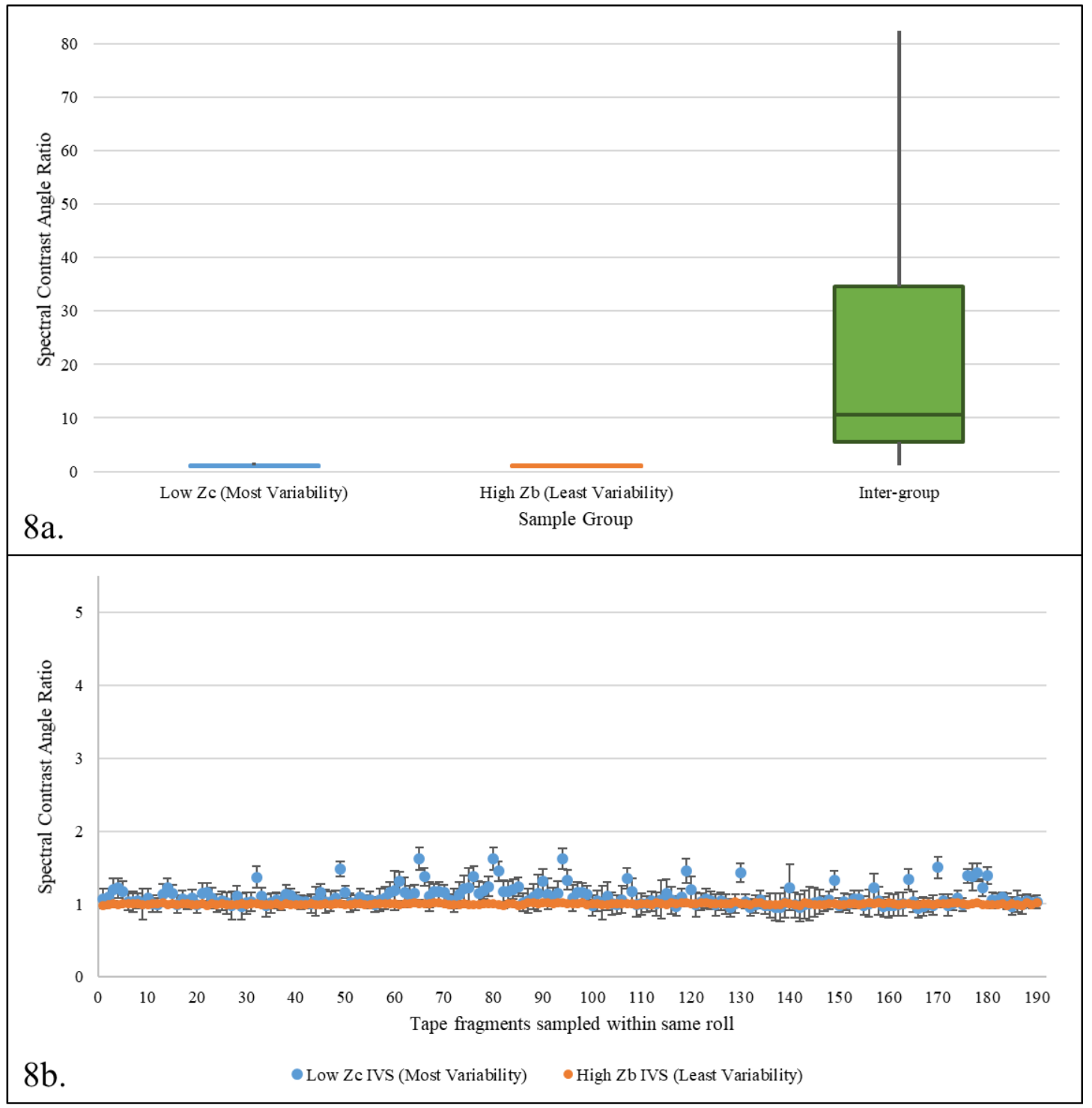

Figure 8. Spectral contrast angle intra-roll sample variation as compared to inter-group variation. 8a: Box plots of intra-roll (low Zc and high Zb and inter-group. 8b: Display of spectral contrast angle ratio for 190 comparison pairs of tape samples from the same roll. 


\section{Conclusions}

XRF is a rapid, sensitive addition for highly discriminatory electrical tape backing analysis. The discrimination achieved through XRF analysis alone, as demonstrated in this study, is comparable to discrimination achieved both through a full analytical scheme (physical observations and measurements, FTIR, py-GC/MS, and SEM-EDS) for electrical tape backings and LA-ICP-MS characterization (i.e., for $\mathrm{N}=90,96.7 \%$ as compared to $94.3 \%$ and $93.9 \%$, respectively). ${ }^{4,11}$ This technique is well suited for quick screening with accuracy and discrimination over time, precision, sensitivity, and selectivity.

This study also highlighted the high inter-sample variability and low intra-sample variability of electrical tape backings as characterized through the optimized XRF method. While these metrics were only measured on a set of 94 tapes, this set represents a variety of tapes from various brands and four different countries of manufacture including the US, China, Taiwan, and England. Therefore, this data provides insight into the expected variation both between electrical tape types as well as within a single roll.

It is critical for forensic examiners to have access to rapid, highly discriminatory techniques for optimal utilization of the probative value of submitted evidence items. This method provides an additional tool to traditional electrical tape chemical analysis. The optimization process described through this study suggests proper parameters for XRF electrical tape analysis, and the additional experiments using those optimized parameters provides a model of the key factors and potential interferences to assess when attempting to adapt this method for use in other forensic laboratories. Further, the application of spectral contrast angle interpretation to spectral comparison has been demonstrated to be a useful tool for supporting examiner opinion and complementing spectral overlay comparisons. Future work using additional tape datasets is recommended to test these findings further and evaluate the potential adoption of contrast ratios comparisons to casework.

\section{Acknowledgements}

The acknowledgements below are included as they would appear once the submission of this chapter is accepted for publication by Forensic Chemistry:

The authors would like to thank Susan M. Marvin of the Laboratory Division of the Federal Bureau of Investigation for her assistance in instrumental training and expertise in data interpretation. The authors would also like to acknowledge Ilan Geerlof-Vidavsky for sharing the Microsoft Excel macro for calculation of contrast angles used in his publication. ${ }^{14}$ Also, the authors acknowledge the valuable feedback provided by Diana Wright, Maureen Bottrell and Jason Brewer during the revision of the manuscript.

This is publication number 20-54 of the FBI Laboratory Division. Names of commercial manufacturers are provided for identification purposes only, and inclusion does not imply endorsement of the manufacturer, or its products or services by the FBI. The views expressed are those of the authors and do not necessarily reflect the official policy or position of the FBI or the U.S. Government. 


\section{References}

1. Scientific Working Group for Materials Analysis (SWGMAT). Guideline for Assessing Physical Characteristics in Forensic Tape Examinations. Journal of the American Society of Trace Evidence Examiners. 2014;5(1):34-41.

2. Blackledge RD. Tapes with Adhesive Backings. In: Mitchell, John J, editor. Appl. Polym. Anal. Charact. Munich: Hanser; 1987. p. 413-421.

3. Mehltretter AH, Bradley MJ, Wright DM. Analysis and Discrimination of Electrical Tapes: Part I. Adhesives. Journal of Forensic Sciences. 2011;56(1):82-94. doi:10.1111/j.15564029.2010.01560.x

4. Mehltretter AH, Bradley MJ, Wright DM. Analysis and discrimination of electrical tapes: Part II. Backings. Journal of Forensic Sciences. 2011;56(6):1493-1504. doi:10.1111/j.15564029.2011.01873.x

5. Scientific Working Group on Materials Analysis (SWGMAT). Guideline for Forensic Examination of Pressure Sensitive Tapes. Journal of the American Society of Trace Evidence Examiners. 2011;2(1):88-97.

6. Goodpaster J V., Sturdevant AB, Andrews KL, Brun-Conti L. Identification and comparison of electrical tapes using instrumental and statistical techniques: I. Microscopic surface texture and elemental composition. Journal of Forensic Sciences. 2007;52(3):610-629. doi:10.1111/j.15564029.2007.00406.x

7. Goodpaster J V., Sturdevant AB, Andrews KL, Briley EM, Brun-Conti L. Identification and comparison of electrical tapes using instrumental and statistical techniques: II. Organic composition of the tape backing and adhesive. Journal of Forensic Sciences. 2009;54(2):328-338. doi:10.1111/j.1556-4029.2008.00969.x

8. Kee TG. The Characterization of PVC Adhesive Tape. In: Proceedings of International Symposium on the Analysis and Identification of Polymers. FBI Academy, Quantico, VA; 1984. p. $77-85$.

9. Keto RO. Forensic characterization of black polyvinyl chloride electrical tape. Crime Laboratory Digest. 1984;11(4).

10. Prusinowski M, Mehltretter A, Martinez-Lopez C, Almirall J, Trejos T. Assessment of the utility of X-ray Fluorescence for the chemical characterization and comparison of black electrical tape backings. Forensic Chemistry. 2019;13(January):100146. doi:10.1016/j.forc.2019.100146

11. Martinez-Lopez C, Trejos T, Mehltretter AH, Almirall JR. Elemental analysis and characterization of electrical tape backings by LA-ICP-MS. Forensic Chemistry. 2017;4:96-107. doi:10.1016/j.forc.2017.03.003

12. Kuczelinis F, Weis P, Bings NH. Forensic comparison of PVC tape backings using time resolved LA-ICP-MS analysis. Forensic Chemistry. 2019;12(July 2018):33-41. doi:10.1016/j.forc.2018.11.004 
13. Margui E, Grieken R Van. Ch. 1 Introduction. In: X-Ray Fluorescence Spectrometry and Related Techniques: An Introduction. Momentum Press; 2013.

14. Wan KX, Vidavsky I, Gross ML. Comparing Similar Spectra: From Similarity Index to Spectral Contrast Angle. Journal of the American Society for Mass Spectrometry. 2002;13(1):8588.

15. ASTM International. ASTM E2926-17: Standard Test Method for Forensic Comparison of Glass Using Micro X-ray Fluorescence ( $\mu$-XRF) Spectrometry. 2017.

16. Ernst T, Berman T, Buscaglia J, Eckert-Lumsdon T, Hanlon C, Olsson K, Palenik C, Ryland $\mathrm{S}$, Trejos T, Valadez M, et al. Signal-to-noise ratios in forensic glass analysis by micro X-ray fluorescence spectrometry. X-Ray Spectrometry. 2014;43(1):13-21. doi:10.1002/xrs.2437

17. National Institute of Standards \& Technology (NIST). Certificate of Analysis: Standard Reference Material 1831. 2017.

18. ASTM International. ASTM E2330-19: Standard Test Method for Determination of Concentrations of Elements in Glass Samples Using Inductively Coupled Plasma Mass Spectrometry (ICP-MS) for Forensic Comparisons. 2019:1-7. doi:10.1520/E2330-12.Copyright

19. Stein SE, Scott DR. Optimization and Testing of Mass Spectral Library Search Algorithms for Compound Identification. Journal of the American Society for Mass Spectrometry. 1994;5(9):859-866.

20. Swartz ME, Brown PR. Use of Mathematically Enhanced Spectral Analysis and Spectral Contrast Techniques for the Liquid Chromatographic and Capillary Electrophoretic Detection and Identification of Pharmaceutical Compounds. Chirality. 1996;8(1):67-76.

21. Härdle WK, Simar L. Discriminant Analysis. In: Applied Multivariate Statistical Analysis. 3rd ed. Berlin, Germany: Springer-Verlag; 2012. p. 331-350.

22. Brereton RG. Two Class Classifiers. In: Chemometrics for Pattern Recognition. 1st ed. West Sussex, UK: John Wiley \& Sons, Ltd; 2009. p. 177-232. 
CHAPTER 4: SUPPLEMENTARY MATERIAL

i. Spectral contrast angle ratio calculation template

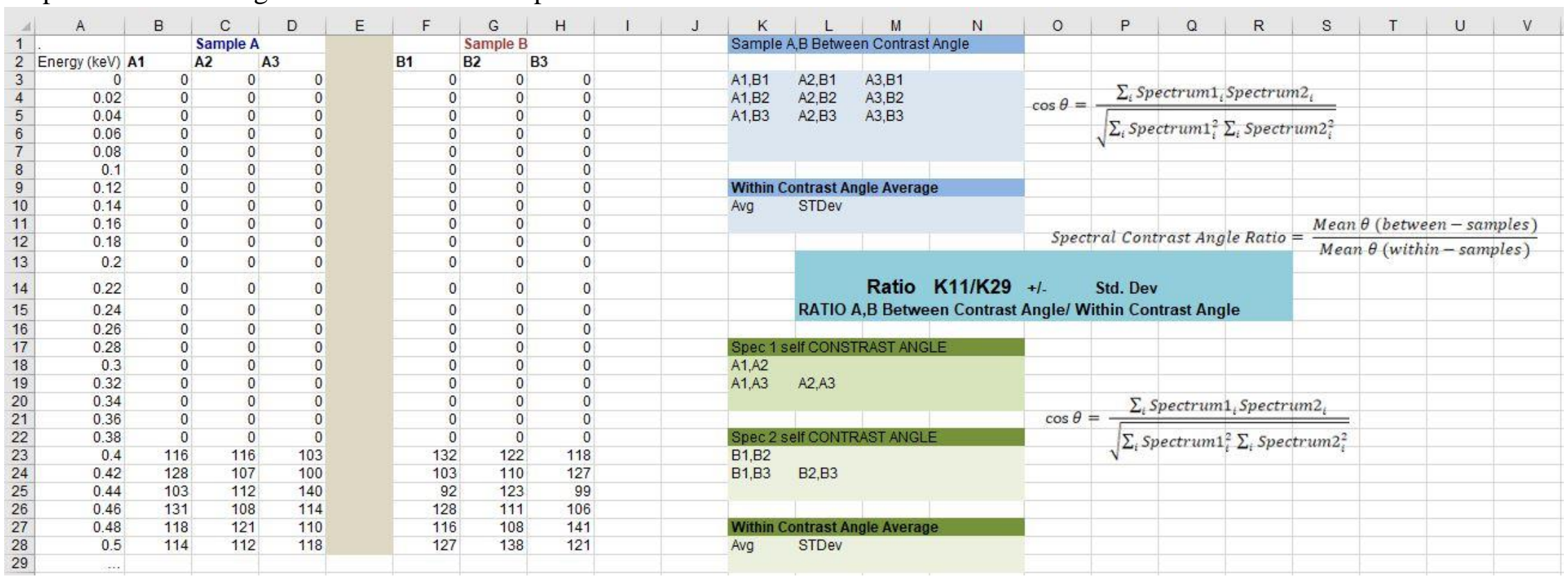




\section{CHAPTER 4: APPENDIX}

Table A.1. Tape set product information for samples originating from different sources

\begin{tabular}{|c|c|c|c|}
\hline Sample & Brand & Product & Country \\
\hline 1 & Marcy Enterprises, Inc. & MA 750 & Taiwan \\
\hline 2 & Advance $\AA$ & AT7, BS3924, 31/90Tp & England \\
\hline 3 & Work SaverTM (Royal Tools) & Stock no. 55, 5 color PVC Tape Assortment & China \\
\hline 4 & tesa tape, Inc. & 40201, No. 111 E52811A & Taiwan \\
\hline 5 & Tape It, Inc. & E-60 & Taiwan \\
\hline 6 & Qualpack® & 1346, 6-Color & China \\
\hline 7 & Marcy Enterprises, Inc. & MA 750 & Taiwan \\
\hline 8 & Manco $\circledR$ & $200 \mathrm{MPH}, \mathrm{AE}-66$ & Taiwan \\
\hline 9 & Archer ${ }^{\circledR}$ (Radio Shack) & $64-2349$ & Taiwan \\
\hline 10 & $3 \mathrm{M} \mathrm{S} \operatorname{cotch}^{\mathrm{TM}}$ & Super 88, 054007-06143 & USA \\
\hline 11 & $3 \mathrm{M} \mathrm{S} \operatorname{cotch}^{\mathrm{TM}}$ & Super $33+, 10414$ NA & USA \\
\hline 12 & $3 \mathrm{M} \mathrm{S} \operatorname{cotch}^{\mathrm{TM}}$ & Super $33+, 10455 \mathrm{NA}$ & USA \\
\hline 13 & $3 \mathrm{M} \mathrm{S} \operatorname{cotch}^{\mathrm{TM}}$ & Super 33+ & USA \\
\hline 14 & Frost King® & ET60 & Taiwan \\
\hline 15 & $3 \mathrm{M} \mathrm{S} \operatorname{cotch}^{\mathrm{TM}}$ & Super $33+, 10455$ NA & USA \\
\hline 16 & $3 \mathrm{M}$ & $\operatorname{Tartan}^{\mathrm{TM}} 1710$, part no. 05400749656 & USA \\
\hline 17 & $3 \mathrm{M} \mathrm{S} \operatorname{cotch}^{\mathrm{TM}}$ & Super $88,054007-06143$ & USA \\
\hline 18 & $3 \mathrm{M} \mathrm{S} \operatorname{cotch}^{\mathrm{TM}}$ & Super 33+, Cat. 195NA & USA \\
\hline 19 & $3 \mathrm{M} \mathrm{S} \operatorname{cotch}^{\mathrm{TM}}$ & Super 33+, Cat. 194NA & USA \\
\hline 20 & $3 \mathrm{M} \mathrm{S} \operatorname{cotch}^{\mathrm{TM}}$ & Super $33+, 10414$ NA & USA \\
\hline 21 & Manco® & P-66 & Taiwan \\
\hline 22 & Manco® & 667 Pro Series ${ }^{\mathrm{TM}}$ & Taiwan \\
\hline 23 & $3 \mathrm{M} \mathrm{S} \operatorname{cotch}^{\mathrm{TM}}$ & Super $88,054007-06143$ & USA \\
\hline 24 & $3 \mathrm{M} \mathrm{S} \operatorname{cotch}^{\mathrm{TM}}$ & Super $88,054007-06143$ & USA \\
\hline 25 & $3 \mathrm{M} \mathrm{S} \operatorname{cotch}^{\mathrm{TM}}$ & Super 33+ 054007-06132 & USA \\
\hline 26 & $3 \mathrm{M} \mathrm{S} \operatorname{cotch}^{\mathrm{TM}}$ & Super 33+ 054007-06132 & USA \\
\hline 27 & $3 \mathrm{M}$ & $\operatorname{Tartan}^{\mathrm{TM}} 1710$, part no. 05400749656 & USA \\
\hline 28 & $3 \mathrm{M}$ & $\operatorname{Tartan}^{\mathrm{TM}} 1710$, part no. 05400749656 & USA \\
\hline 29 & $3 \mathrm{M}$ & Temflex ${ }^{\mathrm{TM}}, 1700,54007-69764$ & USA \\
\hline 30 & $3 \mathrm{M}$ & Temflex ${ }^{\mathrm{TM}}, 1700,54007-69764$ & USA \\
\hline 31 & Regal® & Model ET-6 & Taiwan \\
\hline 32 & GE & GE2472-3DD & Taiwan \\
\hline 33 & $3 \mathrm{M}$ Scotch ${ }^{\mathrm{TM}}$ & Cat. 190 & USA \\
\hline 34 & $3 \mathrm{M}$ & $\operatorname{Tartan}^{\mathrm{TM}} 1710$, part no. 05400749656 & USA \\
\hline 35 & Frost King® & ET60 & Taiwan \\
\hline 36 & $3 \mathrm{M}$ & $\operatorname{Tartan}^{\mathrm{TM}} 1710$, part no. 49656 & USA \\
\hline 37 & National & All-Purpose Grade & Taiwan \\
\hline 38 & Manco® & P-660 & Taiwan \\
\hline 39 & $3 \mathrm{M} \mathrm{S} \operatorname{cotch}^{\mathrm{TM}}$ & Super $33+, 3744 N A$ & USA \\
\hline 40 & $3 \mathrm{M}$ & $\operatorname{Tartan}^{\mathrm{TM}} 1710$, part no. 05400749656 & USA \\
\hline 41 & $3 \mathrm{M} \mathrm{S} \operatorname{cotch}^{\mathrm{TM}}$ & Super $33+, 200 N A$ & USA \\
\hline 42 & National & All-Purpose & Taiwan \\
\hline 43 & $3 \mathrm{M}$ & $\operatorname{Tartan}^{\mathrm{TM}} 1710$, part no. 05400749656 & USA \\
\hline 44 & $3 \mathrm{M}$ & $\operatorname{Tartan}^{\mathrm{TM}} 1710$, part no. 05400749656 & USA \\
\hline 45 & Calterm® & 49605 & Taiwan \\
\hline 46 & Manco $®$ & $\mathrm{P}-20$ & Taiwan \\
\hline 47 & $3 \mathrm{M}$ & $\operatorname{Tartan}^{\mathrm{TM}} 1710$, part no. 05400749656 & USA \\
\hline 48 & Tape It, Inc. & $36-\mathrm{T}$ & USA \\
\hline
\end{tabular}




\begin{tabular}{|c|c|c|c|}
\hline 49 & Tape It, Inc. & 36- $\mathrm{T}$ & USA \\
\hline 50 & GE & GE2472-31D & Taiwan \\
\hline 51 & National & No. 101, E52811A & Taiwan \\
\hline 52 & Frost King ${ }^{\circledR}$ & ET60FR & USA \\
\hline 53 & National & No. 101, E52811A & Taiwan \\
\hline 54 & $3 \mathrm{M} \mathrm{S} \operatorname{cotch}^{\mathrm{TM}}$ & Super $33+, 03404 N A$ & USA \\
\hline 55 & Manco® & $1219-60$ & Taiwan \\
\hline 56 & $\begin{array}{l}\text { Victor Automotive Products } \\
\text { (Thermoflex) }\end{array}$ & 33-UL60, No. 101 E52811A & Taiwan \\
\hline 57 & United Tape Company & UT-602 & Taiwan \\
\hline 58 & Frost King ${ }^{\circledR}$ & ET60 & Taiwan \\
\hline 59 & Tuff ${ }^{\mathrm{TM}}$ Hand Tools & & China \\
\hline 60 & Tuff'TM Hand Tools & & China \\
\hline 61 & $3 \mathrm{M} \mathrm{S} \operatorname{cotch}^{\mathrm{TM}}$ & $88 \mathrm{~T}$ & USA \\
\hline 62 & Nitto Denko & No. 228 & Taiwan \\
\hline 63 & $3 \mathrm{M} \mathrm{S} \operatorname{cotch}^{\mathrm{TM}}$ & Super $88,054007-06143$ & USA \\
\hline 64 & $3 \mathrm{M} \mathrm{S} \operatorname{cotch}^{\mathrm{TM}}$ & Super $33+, 10455 N A$ & USA \\
\hline 65 & $3 \mathrm{M} \mathrm{S} \operatorname{cotch}^{\mathrm{TM}}$ & 700 Commercial Grade, 054007-04218 & USA \\
\hline 66 & L.G. Sourcing, Inc. & 19453 & Taiwan \\
\hline 67 & Manco & $\mathrm{P}-66$ & Taiwan \\
\hline 68 & $3 \mathrm{M} \mathrm{S} \operatorname{cotch}^{\mathrm{TM}}$ & Super 33+ & USA \\
\hline 69 & $3 \mathrm{M}$ & $\operatorname{Tartan}^{\mathrm{TM}} 1710$, part no. 05400749656 & Taiwan \\
\hline 70 & Tyco Adhesives (National) & No. 101, E52811A & Taiwan \\
\hline 71 & Qualpack® & 1346, 6-Color & China \\
\hline 72 & Nitto Denko & Nitto $₫$ No. 228 & Taiwan \\
\hline 73 & Frost King $₫$ & ET60FR & China \\
\hline 74 & $3 \mathrm{M} \mathrm{Scotch} \AA$ & 700 Commercial Grade, 054007-04218 & USA \\
\hline 75 & $3 \mathrm{M} \mathrm{S} \operatorname{cotch}^{\mathrm{TM}}$ & Linerless Electrical Rubber Splicing Tape, 2242, 06165 & USA \\
\hline 76 & $3 \mathrm{M} \mathrm{Scotch} \AA$ & Super 33+, Cold Weather Electrical Tape, 16736NA & USA \\
\hline 77 & $3 \mathrm{M} \mathrm{S} \operatorname{cotch} \circledast$ & Super 33+, 054007-06132 & USA \\
\hline 78 & $3 \mathrm{M}$ & Tartan $^{\mathrm{TM}} 1710$ General Use, $054007-49656$ & Taiwan \\
\hline 79 & $3 \mathrm{M} \mathrm{S} \operatorname{cotch} \circledast$ & 700 Commercial Grade, 054007-04218 & USA \\
\hline 80 & $3 \mathrm{M} \mathrm{S} \operatorname{cotch} \circledast$ & Super $88,054007-06143$ & USA \\
\hline 81 & Ace (Henkel) & All Weather & Taiwan \\
\hline 82 & Ace (Henkel) & Weather Resistant & Taiwan \\
\hline 83 & $3 \mathrm{M} \mathrm{S} \operatorname{cotch} \circledast$ & Super $33+, 10414 \mathrm{NA}$ & USA \\
\hline 84 & $3 \mathrm{M}$ & $\operatorname{Tartan}^{\mathrm{TM}} 1710$ General Use, $054007-49656$ & Taiwan \\
\hline 85 & Frost King ${ }^{\circledR}$ & ET60FR & China \\
\hline 86 & Duck (Henkel) & Vinyl Electrical Tape & Taiwan \\
\hline 87 & Nitto Denko & No. 21E & China \\
\hline 88 & Frost King $₫$ & ET60FR & China \\
\hline 89 & Power Pro Craft & ETF & China \\
\hline 90 & Duck (Henkel) & Extra wide electrical tape & China \\
\hline 91 & $3 \mathrm{M} \mathrm{S} \operatorname{cotch} \circledast$ & Super 33+ & USA \\
\hline 92 & $3 \mathrm{M} \mathrm{S} \operatorname{cotch} \circledast$ & Super 88 & USA \\
\hline 93 & $\begin{array}{l}\text { Commercial Electric } \\
\text { (Home Depot) }\end{array}$ & EE-100 & China \\
\hline 94 & $3 \mathrm{M}$ & 3M Economy 1400 & Taiwan \\
\hline
\end{tabular}


Table A.2. Examples of spectral contrast angle ratio comparison. Refer to table 10 for subgroup additional information

\begin{tabular}{|c|c|c|c|}
\hline & Sample Pair & $\begin{array}{c}\text { Spectral } \\
\text { Contrast Ratio }\end{array}$ & $\begin{array}{r}\text { Standard } \\
\text { Deviation }\end{array}$ \\
\hline 1. Indistinguishable Pairs $(\mathrm{N}=132)$ & Mean & 1.14 & 0.22 \\
\hline $\begin{array}{l}\text { 2. Intra-roll Pairs }(\mathrm{N}=380) \\
\text { a. Low Zc pairs }(\mathrm{N}=190) \\
\text { b. High Zb pairs }(\mathrm{N}=190)\end{array}$ & Mean & $\begin{array}{l}1.10 \\
1.00\end{array}$ & $\begin{array}{l}0.14 \\
0.02\end{array}$ \\
\hline 3. Inter-subgroups $(\mathrm{N}=20)$ & & & \\
\hline \multirow{4}{*}{$\begin{array}{l}\text { a. Sub-groups 4A-4E } \\
\text { Distinguishable Pairs }\end{array}$} & $4 \mathrm{v} 42$ & 1.47 & 0.04 \\
\hline & $42 \mathrm{v} 53$ & 1.55 & 0.12 \\
\hline & $42 \mathrm{v} 56$ & 1.62 & 0.06 \\
\hline & $42 \mathrm{v} 70$ & 1.79 & 0.20 \\
\hline \multirow{4}{*}{$\begin{array}{l}\text { b. Sub-groups 7A-7E } \\
\text { Distinguishable Pairs }\end{array}$} & $8 \mathrm{v} 21$ & 6.16 & 0.34 \\
\hline & $8 \mathrm{v} 38$ & 7.88 & 0.21 \\
\hline & $8 \mathrm{v} 67$ & 7.37 & 0.50 \\
\hline & $8 \mathrm{v} 81$ & 2.09 & 0.11 \\
\hline \multirow{5}{*}{ 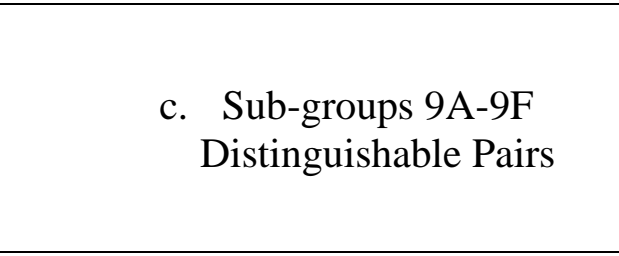 } & $10 \mathrm{v} 11$ & 1.94 & 0.10 \\
\hline & $10 \mathrm{v} 16$ & 3.48 & 0.13 \\
\hline & $10 \mathrm{v} 27$ & 2.62 & 0.11 \\
\hline & 10v39 & 2.10 & 0.10 \\
\hline & $10 \mathrm{v} 40$ & 3.63 & 0.17 \\
\hline \multirow{2}{*}{$\begin{array}{l}\text { d. Sub-groups 19A-19C } \\
\text { Distinguishable Pairs }\end{array}$} & $48 \mathrm{v} 72$ & 5.36 & 0.36 \\
\hline & $48 \mathrm{v} 79$ & 5.58 & 0.39 \\
\hline $\begin{array}{ll}\text { e. } & \text { Sub-groups 22A-22B } \\
\text { Distinguishable Pairs }\end{array}$ & $58 v 86$ & 1.63 & 0.05 \\
\hline 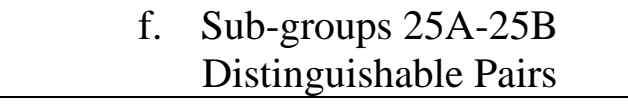 & $65 \mathrm{v} 69$ & 1.54 & 0.05 \\
\hline \multirow{3}{*}{$\begin{array}{l}\text { g. Sub-groups 31A-31D } \\
\text { Distinguishable Pairs }\end{array}$} & $76 \mathrm{v} 78$ & 3.39 & 0.11 \\
\hline & $76 \mathrm{v} 80$ & 1.48 & 0.04 \\
\hline & 76v91 & 2.07 & 0.07 \\
\hline 4. Inter-group Pairs $(N=794)$ & Mean & 21.4 & 22.0 \\
\hline
\end{tabular}

Note: Indistinguishable pair ratios originated from mid Zc filter runs of intra-subgroup samples, intra-roll pair ratios originated from low Zc filter runs of intra-roll variability study samples, inter-subgroup pair ratios originated from the filtered data at which differences were observed during spectral overlay, and inter-group pair ratios originated from low Zc filter runs. Ratios were established according to the filter at which worst-case variability was observed. 


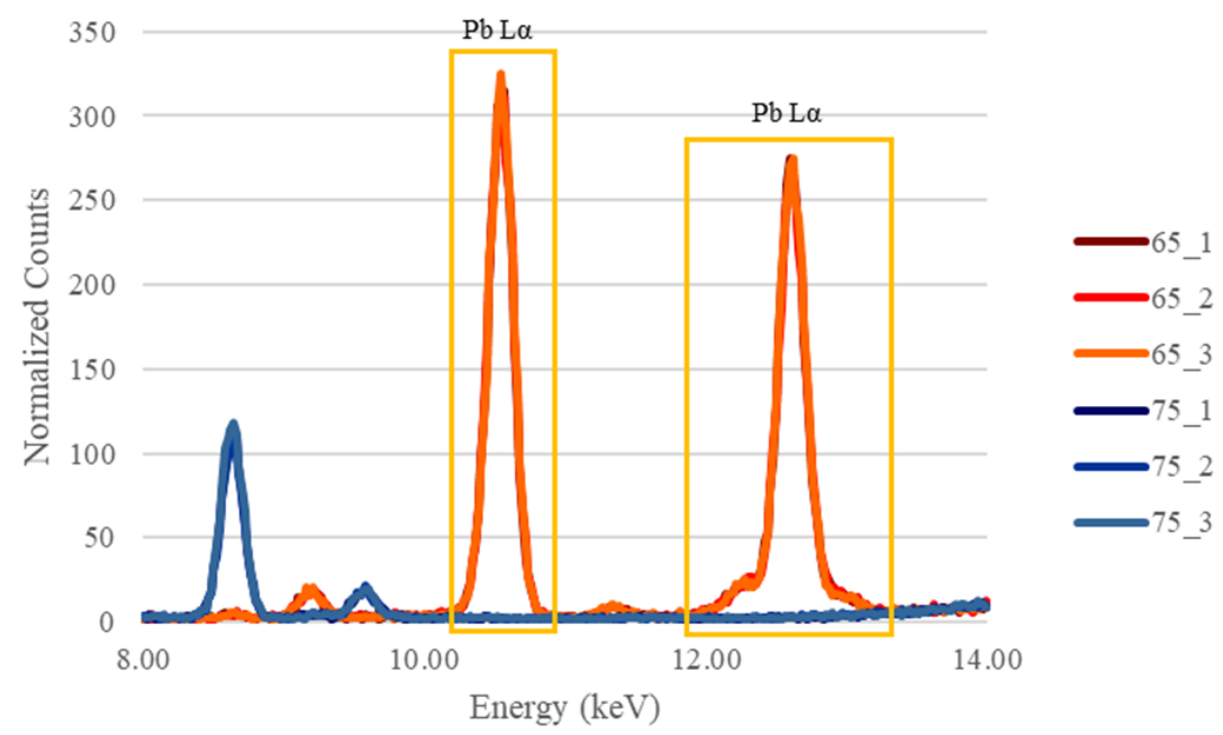

Figure A.1. Inter-group SNR differences in present vs. absent elements: sample 65 ( $\mathrm{Pb}$ present with $\mathrm{SNR}=301.28)$ and sample $75(\mathrm{~Pb}$ absent with $\mathrm{SNR}=0.74)$, mid $\mathrm{Zc}$ filter

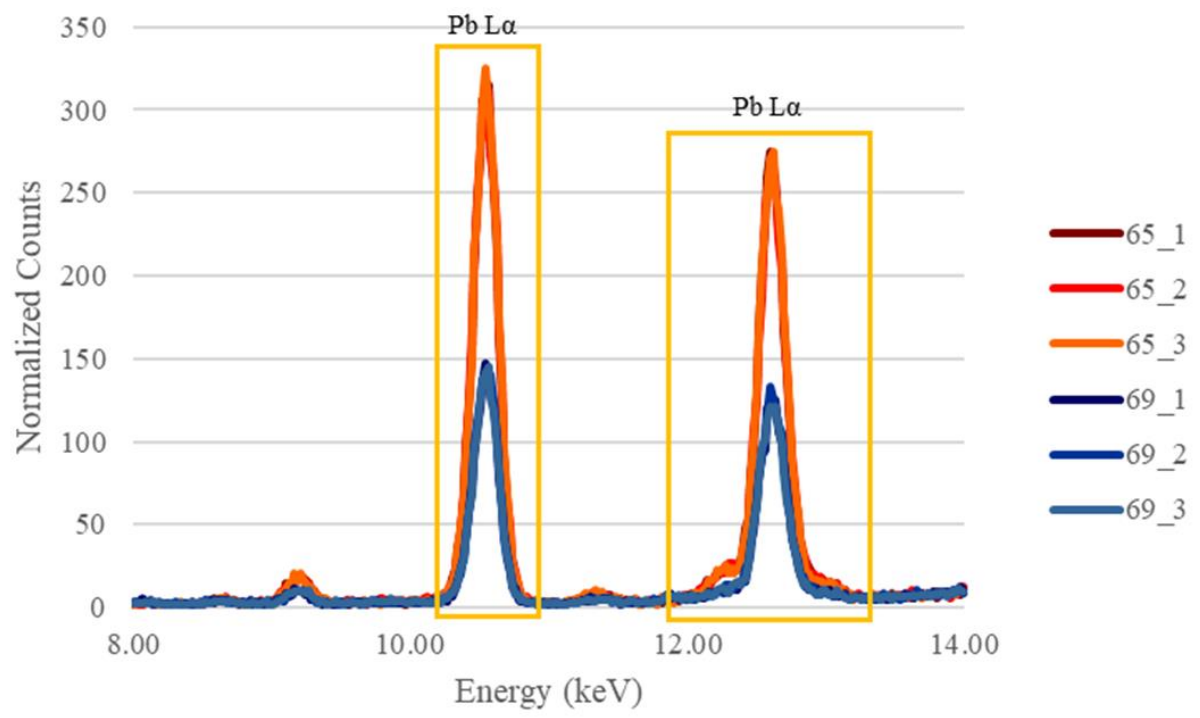

Figure A.2. Inter-subgroup SNR difference in peak height/shape: sample 65 (higher Pb with $\mathrm{SNR}=301.28$ ) and sample 69 (lower $\mathrm{Pb}$ with $\mathrm{SNR}=167.67$ ), mid Zc filter 


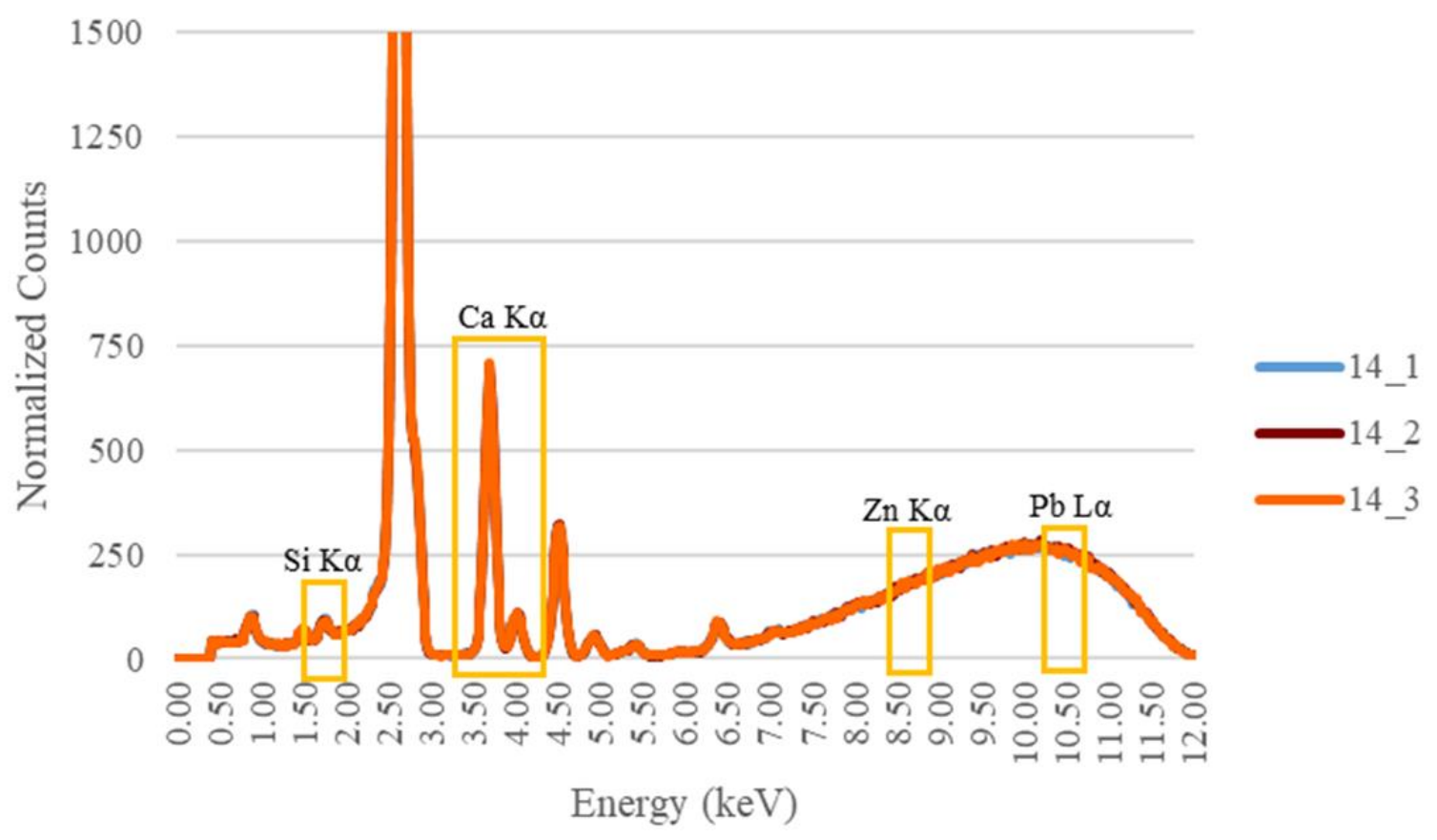

Figure A.3. Sample 14 - various SNR value examples: SNR < 3 (Zn SNR=1.36), SNR 3 (Pb $\mathrm{SNR}=2.98), \mathrm{SNR}>3(\mathrm{Si} \mathrm{SNR}=12.9), \mathrm{SNR}>3(\mathrm{Ca} \mathrm{SNR}=522)$

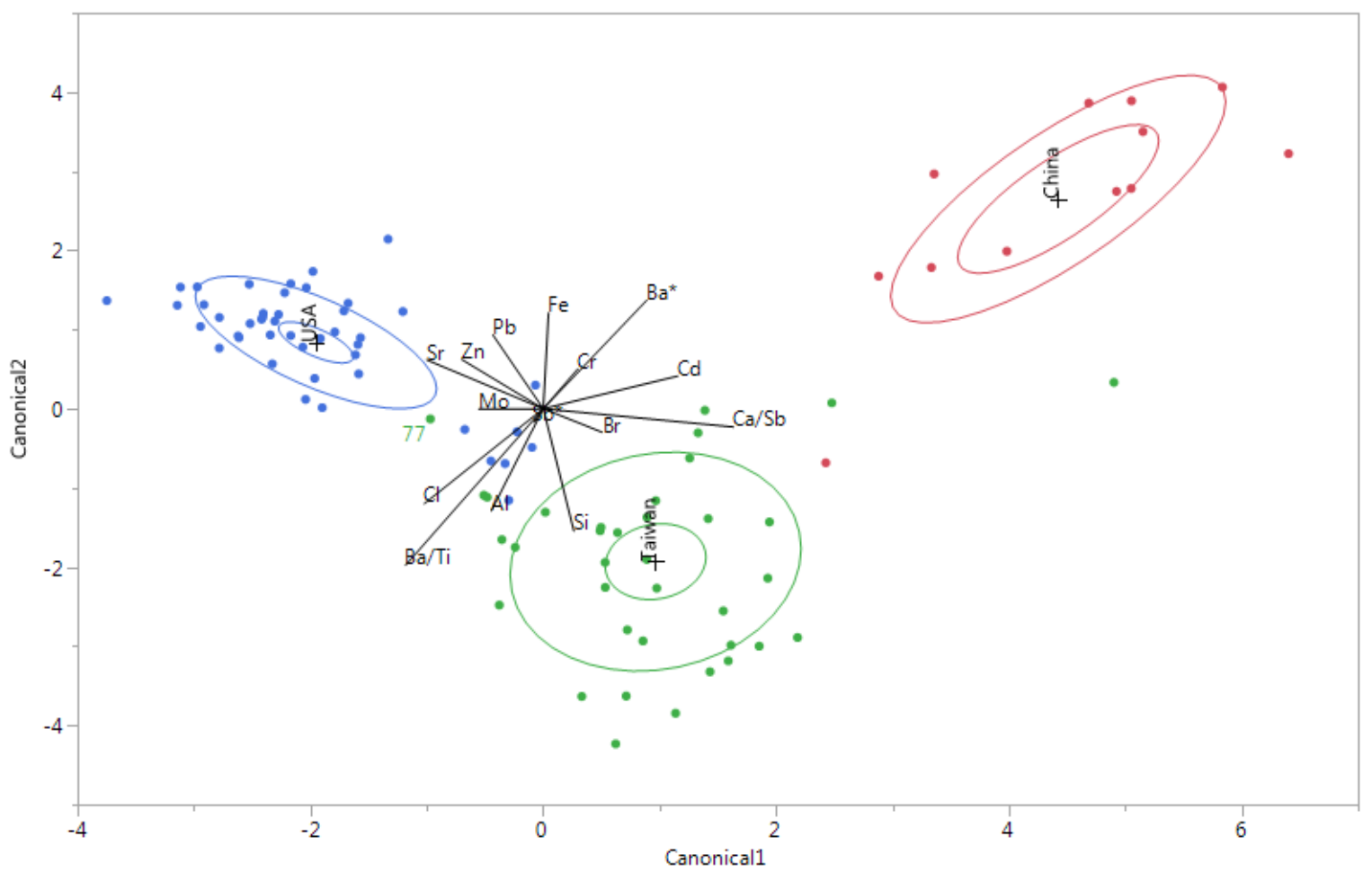

Figure A.4. QDA biplots displaying sample variation by element for optimized filter overall tape data set $(\mathrm{N}=94)$ 


\section{OVERALL CONCLUSIONS AND FUTURE WORK}

The forensic fracture fit discipline has a vast and well-established case report foundation, providing documentation of the value these evidential linkages have supplied to forensic casework dating back as far as the 1700s. ${ }^{13}$ The physical fit research base continues to evolve to meet the modern demands faced by the forensic field. Many different approaches have been taken to study physical fits including, generally, case reports, fractography or qualitative-based studies, and quantitative-based studies. Case reports are typically published by forensic practitioners and allow the authors to document and share their casework experiences with others in the field, providing innovative methodology for unusual material types ${ }^{5,6}$ and assisting researchers in understanding the prevalence of certain items in casework. Fractography studies attempt to shed light into the nature of fractures of specific materials to provide qualitative features that examiners may incorporate in their physical fit assessments to demonstrate either alignment or inconsistency between two items. Quantitative-based studies have expanded recently, with studies emerging for performance assessment through examiner error rates during physical fit assessments, ${ }^{21,22}$ scorebased reporting and quantitative assessment through the score likelihood ratio, ${ }^{14}$ statistical interpretations through attempts at populational frequency studies, ${ }^{23,24}$ and most recently the expansion of automated algorithms for more objective fracture fit application and support. ${ }^{25,26}$ Growth in these quantitative aspects aims to substantiate the scientific validity of one of the oldest and seemingly straightforward forensic analyses, advocating for the discipline in response to NAS, PCAST, ASA, and NIST-OSAC recommendations ${ }^{8-11}$.

To attribute to the need for quantitative approaches to physical fit examinations, the pilot interlaboratory study conducted in this thesis was designed to take steps towards validation of systematic, score-based ESS methodology previously developed by Prusinowski et al. ${ }^{14}$ The ESS values, comparison edge qualifiers, and overall examiner conclusions from 16 participants were assessed for inter-examiner agreement, examiner error rates, variance from consensus means, and survey feedback to facilitate future adoption of the method to their laboratories. Overall, interexaminer agreement with reporting ESS scores within $20 \%$ of the mean consensus values was observed, with participants accuracy ranging from 88 to 100\%. Moreover, the inter-laboratory study highlighted the utility of the ESS score method to enhance future physical fit practice in several aspects including increased objectivity, consensus between examiners, peer-review process, proficiency testing, and strengthened scientific reliability.

A thorough review of participant scrim templates, examination notes, and feedback left within the post-study survey revealed three main observations. First, those participants that did not participate in formal method training through either the in-person method presentation or teleconference tended to exhibit statistically significant score differences from the consensus, pre-distribution mean ESS. This was shown through results of the Dunnett's test as well as distribution of scores. Second, variance was observed in how participants interpreted a featureless or distorted scrim bin for ESS assignment. While some assigned a " 0 " binary classifier to those areas to signify they had interpreted it as a non-matching, inconsistent bin, others assigned a " 1 " binary classifier to indicate the bin was interpreted as a matching, consistent area. When facing this discrepancy, some 
examiners recommended the option of an "inconclusive" qualifier for scrim bins. The third observation was an apparent misunderstanding in application of the comparison edge qualifier. Expected ranges were set for ESS based on the assignment of comparison edge qualifiers according to previously determined score likelihood ratios (SLRs) ${ }^{14}$, and many examiners did not provide qualifiers that were reasonable for certain ESS ranges. As a result, future work on expanded inter-laboratory studies will include more in-depth, mandatory training as a pre-requisite to participation, in addition to incorporation of the inconclusive scrim bin criteria. In addition, future work will include the application of a linear mixed model fit by restricted maximum likelihood (REML) to inter-laboratory study results as an input for Bayesian models to provide credible intervals for variation between examiners.

Along with the expansion of the duct tape ESS project, the application of the ESS to clothing items represents the first time a quantitative, score-based method of physical fit assessment has been applied to textile materials. The methodology allowed for quantitative assessment of examiner performance, and both the hand-torn and stabbed sample sets presented low error rates with accuracies ranging from $85-100 \%$ depending on textile item. One of the most significant discoveries in this study was the impact a fabric composition and construction type may have in the suitability of a physical fit. Lower accuracy rates were observed for items of either polyester composition (Item D) or jersey knit construction (Item E) for the hand-torn set, while woven, nonpolyester items exhibited higher accuracy rates. This was attributed to higher distortion in the polyester or jersey knit items, as was also observed in a preliminary set of 100 jersey knit, 100\% polyester comparison pairs, where unacceptable high error rates demonstrated the challenges of evaluation of fracture fits on these types of textiles. For the stabbed sample set, it was observed that patterned materials (Items $\mathrm{C}$ and $\mathrm{E}$ ) exhibited higher accuracy rates than solid-colored items. This was attributed to the added potential of pattern alignment (or misalignment) on items presenting otherwise "featureless" edges due to the stabbing separation mechanism.

Also, another relevant aspect of this study was the identification, documentation, and description of physical features that can lead to future standardization of examination protocols. Further analysis of examiner notes revealed two main methodology discrepancies dealing with treatment of gaps within a sample as well as treatment of inconsistent fracture edge length between two items. Regardless of examiner discrepancies, only 12 misclassifications were observed across the entire data set. While one false positive was observed, and later realized as an observation error by the examiner during peer review, the remaining 11 misclassifications consisted of false negative and inconclusive results. These results are less detrimental to casework as negative or inconclusive samples would typically be subject to further testing according to a forensic laboratory's associated analytical scheme.

The textile fracture study provided an important foundation from which future textile physical fit research may expand, as it established preliminary ESS data on various textile compositions, constructions, and separation methods. In addition, study data revealed that due to high disagreement rates between examiners, certain textiles may be unsuitable for physical fit analysis 
if lacking distinctive characteristics beyond general characteristics. The jersey knit construction and $100 \%$ polyester composition demonstrated to be unsuitable for fracture fit analysis as deformations lead to high rates of misclassification. These results raise awareness as to the need to further evaluate the effect of other textile types on error rates. Future work will include studies of expanded textile factors such as additional compositions, constructions, and external factors such as degree of wear, in order to determine if modifications to the textile ESS criteria are needed. In addition, future work and expanded datasets will assist in the fine-tuning of the proposed verbal interpretation scale based upon rarity ratio thresholds. Eventually, an inter-laboratory study is recommended to validate the now developed textile ESS methodology.

In the absence of physical fits, it is critical for forensic examiners to have access to highly discriminatory techniques for optimal utilization of the probative value of submitted evidence items. This becomes especially critical on items such as electrical tape that are more prone to deformation, with a lack of distinctive features on the fractured edges. As electrical tapes are amorphous materials exhibiting enough physical fit variability to cause the FBI to modify their physical match protocols, ${ }^{15}$ it is important that efficient methods are available to the examiner upon continued chemical analysis. The XRF method presented in this work provides an additional tool to traditional electrical tape chemical analysis.

The XRF study aimed to expand previous work into electrical tape XRF method development. ${ }^{18}$ The optimization process described through this study suggests proper parameters for XRF electrical tape analysis, and the additional experiments using those optimized parameters provides a model of the key factors and potential interferences to assess when attempting to adapt this method for use in other forensic laboratories. This experimentation established that this technique is well suited for quick screening with accuracy and discrimination over time, precision, sensitivity, and selectivity. This study also highlighted the high inter-sample variability and low intra-sample variability of electrical tape backings as characterized through the optimized XRF method. Further, results of the study support the application of spectral contrast angle interpretation to spectral comparison, as it has been demonstrated to be a useful tool for supporting examiner opinion and complementing spectral overlay comparisons. Future work using additional tape datasets is recommended to test these findings further and evaluate the potential adoption of contrast ratios comparisons to casework.

Physical fits are a complex research topic. Many factors influence the resulting fracture pattern and vary by material type. To name a few, the force of the fracture, directionality, object used to impart the break, manipulation following the breaking event, and even temperature may influence the resulting fracture edge features. However, this inherent randomization of physical fit events is precisely what adds significance to their occurrence. Therefore, it is critical experimental, quantitative, and systematic research bases be established for a wide variety of material types so that the strength of these potential evidential linkages is best represented and upheld in the court setting. In doing so, it must be stressed that physical fit examinations can never be truly objective, as the examiner's expert opinion is an essential input in the overall assessment. Although, with added quantitative interpretation, statistical capabilities, and automated algorithm support, the high 
associative power of physical fit examinations can be more transparently and credibly validated instances of forensic evidence.

This thesis research represents important steps towards meeting these means. By organizing and summarizing the vast physical fit research basis (Chapter 1), an understanding of the strength and history of the discipline is shared with the forensic community and beyond. The pilot interlaboratory study of the duct tape ESS method (Chapter 2) provides the first step into the implementation process, as examiner feedback and modification are crucial aspects to optimizing the methodology. As the long-term goals of our research group include expanding the ESS technique into multiple material types of trace evidence interest, the textile fracture study (Chapter 3) represents the novel application of the methodology to textile materials. Finally, in order to account for amorphous materials in which physical fits may not be feasible due to a lack of distinctive features, an XRF technique has been optimized for implementation into forensic laboratories for the rapid, highly discriminatory analysis of electrical tape backing samples. A systematic method for spectral comparison was also proposed and evaluated to help examiners in the decision-making process (Chapter 4). Future work will expand upon the groundwork laid for the growth of the physical fit discipline through this research. 


\section{OVERALL REFERENCES}

These references correspond to citations on the Overall Introduction (Section I) and Overall Conclusions/Future Work Sections (Section VI).

1. American Society of Trace Evidence Examiners (ASTEE). ASTEE Trace 101. 2018 [accessed 2018 Dec 12]. http://www.asteetrace.org/

2. Gummer T, Walsh K. Matching vehicle parts back to the vehicle: a study of the process. Forensic Science International. 1996;82:89-97. doi:10.1016/0379-0738(96)01970-6

3. Jayaprakash PT. Practical relevance of pattern uniqueness in forensic science. Forensic Science International. 2013;231:403.e1-403.e16. doi:10.1016/j.forsciint.2013.05.028

4. Ryland S, Houck MM. Only Circumstantial Evidence. In: Houck MM, editor. Mute Witnesses: Trace Evidence Analysis. San Diego, CA: Academic Press; 2001. p. 117-137.

5. Perper JA, Prichard W, McCommons P. Matching the Lost Skin of a Homicide Suspect. Forensic Science International. 1985;29:77-82.

6. Bisbing RE, Willmer JH, LaVoy TA, Berglund JS. A Fingernail Identification. AFTE Journal. 1980;12(1):27-28.

7. Scientific Working Group on Materials Analysis (SWGMAT). A 2012 Survey Regarding the Status of Forensic Tape Analysis. 2012.

8. Gross S. NIST-OSAC Materials (Trace) Subcommittee, physical fit task group, 2020 physical fit survey.

9. National Academy of Sciences (NAS). Strengthening Forensic Science in the United States: A Path Forward. 2009. doi:0.17226/12589

10. President's Council of Advisors on Science and Technology. Forensic Science in Criminal Courts: Ensuring Scientific Validity of Feature-Comparison Methods. 2016.

11. American Statistical Association. American Statistical Association Position on Statistical Statements for Forensic Evidence. [accessed 2019 Jan 30].

https://www.amstat.org/asa/files/pdfs/POL-ForensicScience.pdf

12. \{US Supreme Court\}. Daubert vs Merrell Dow Pharmaceuticals, Inc. 509 U.S. 579 (1993). JUSTIA US Supreme Couts. 1993.

13. Gehl R, Plecas D. Chapter 1: Introduction. In: Introduction to Criminal Investigation: Processes, Practices and Thinking. New Westminster, BC: Justice Institute of British Columbia; 2016. p. 1-10.

14. Prusinowski M, Brooks E, Trejos T. Development and validation of a systematic approach for the quantitative assessment of the quality of duct tape physical fits. Forensic Science International. 2020;307. 
15. Bradley MJ, Gauntt JM, Mehltretter AH, Lowe PC, Wright DM. A Validation Study for Vinyl Electrical Tape End Matches. Journal of Forensic Sciences. 2011;56(3):606-611. doi:10.1111/j.1556-4029.2011.01736.x

16. Kee TG. The Characterization of PVC Adhesive Tape. In: Proceedings of International Symposium on the Analysis and Identification of Polymers. FBI Academy, Quantico, VA; 1984. p. $77-85$.

17. Keto RO. Forensic characterization of black polyvinyl chloride electrical tape. Crime Laboratory Digest. 1984;11(4).

18. Prusinowski M, Mehltretter A, Martinez-Lopez C, Almirall J, Trejos T. Assessment of the utility of X-ray Fluorescence for the chemical characterization and comparison of black electrical tape backings. Forensic Chemistry. 2019;13(January):100146. doi:10.1016/j.forc.2019.100146

19. Martinez-Lopez C, Trejos T, Mehltretter AH, Almirall JR. Elemental analysis and characterization of electrical tape backings by LA-ICP-MS. Forensic Chemistry. 2017;4:96-107. doi:10.1016/j.forc.2017.03.003

20. Mehltretter AH, Bradley MJ, Wright DM. Analysis and discrimination of electrical tapes: Part II. Backings. Journal of Forensic Sciences. 2011;56(6):1493-1504. doi:10.1111/j.15564029.2011.01873.x

21. Bradley MJ, Keagy RL, Lowe PC, Rickenbach MP, Wright DM, LeBeau MA. A validation study for duct tape end matches. Journal of Forensic Sciences. 2006;51(3):504-508. doi:10.1111/j.1556-4029.2006.00106.x

22. Christensen AM, Sylvester AD. Physical Matches of Bone, Shell and Tooth Fragments: A Validation Study. Journal of Forensic Sciences. 2008;53(3):694-698. doi:10.1111/j.15564029.2008.00705.x

23. Lograsso BK. Physical Matching of Metals: Grain Orientation Association at Fracture Edge. Journal of Forensic Sciences. 2015;60(S1):S66-S75. doi:10.1111/1556-4029.12607

24. Stone RS. A Probabilistic Model of Fractures in Brittle Metals. AFTE Journal. 2004;36(4):297-301.

25. Yekutieli Y, Shor Y, Wiesner S, Tsach T. Physical Matching Verification. Final Report to United States Department of Justice on Grant 2005-IJ-R-051; National Criminal Justice Reference Service: Rockville, MD. 2012.

26. Ristenpart W, Tulleners FA, Alfter A. Quantitative Algorithm for the Digital Comparison of Torn Duct Tape. Final Report to the National Institute of Justice Grant 2013-R2-CX-K009; University of California at Davis: Davis, CA. 2017. 